\title{
Nickel-Catalyzed Four-Component Carbocarbonylation of Alkenes under 1 atm of $\mathrm{CO}$
}

\author{
Minqi Zhou, ${ }^{1, \uparrow}$ Hai-Yang Zhao, ${ }^{1, \dagger}$ Shu Zhang ${ }^{2}$, Yanxia Zhang ${ }^{1,}$ and Xingang Zhang ${ }^{1, *}$ \\ ${ }^{1}$ Key Laboratory of Organofluorine Chemistry, Center for Excellence in Molecular Synthesis, Shanghai Institute of \\ Organic Chemistry, University of Chinese Academy of Sciences, Chinese Academy of Sciences, 345 Lingling Road, \\ Shanghai 200032, China. \\ ${ }^{2}$ School of Materials and Energy, University of Electronic Science and Technology of China, 2006 Xiyuan Avenue, \\ West High-Tech Zone, Chengdu, Sichuan 611731, China.
}

${ }^{\dagger}$ Minqi Zhou and Hai-Yang Zhao contributed equally.

Correspondence to: $x$ gzhang@mail.sioc.ac.cn 


\section{Table of Contents}

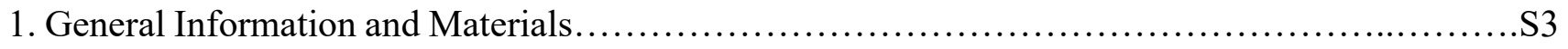

2. Optimization of the Ni-catalyzed four-component carbocarbonylation of $N$-vinylacetamide 1 a with

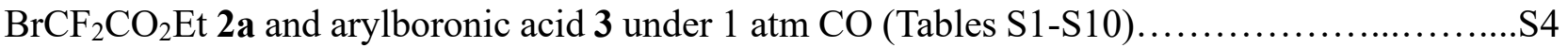

3. Screening of the loading amount of $\mathbf{2 e}$ and $\mathbf{3 e}$ in the Ni-catalyzed reaction of $\mathbf{1 a}$ with $\mathbf{2 e}$ and $3 \mathbf{e}$

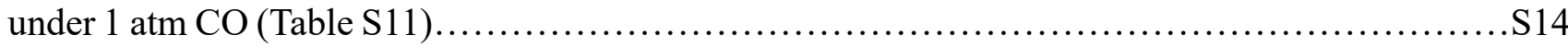

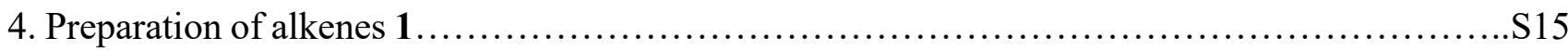

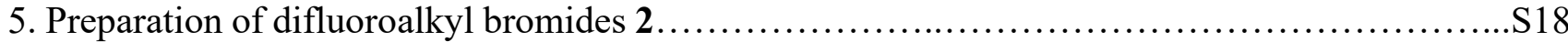

6. Ni-catalyzed four-component carbocarbonylation of alkenes $\mathbf{1}$ with difluoroalkyl bromides $\mathbf{2}$ and

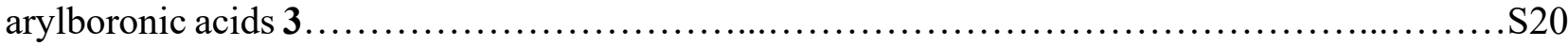

6.1. Ni-catalyzed four-component carbocarbonylation of alkenes $\mathbf{1}$ with difluoroalkyl bromides $\mathbf{2}$

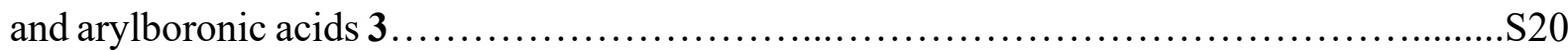

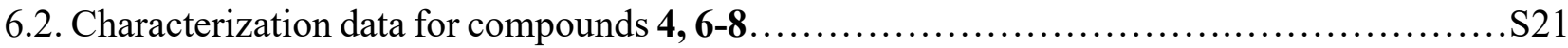

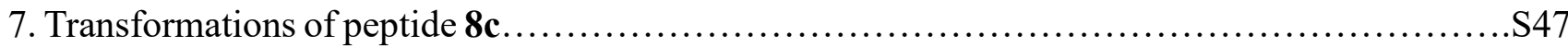

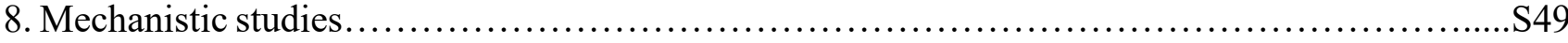

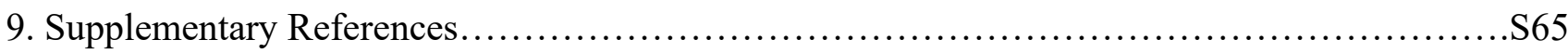

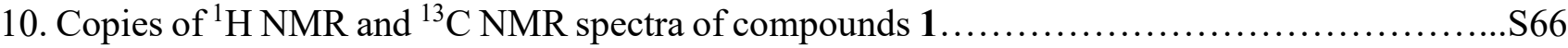

11. Copies of ${ }^{1} \mathrm{H}$ NMR, ${ }^{19} \mathrm{~F}$ NMR and ${ }^{13} \mathrm{C}$ NMR spectra of compounds $2 \ldots \ldots \ldots \ldots \ldots \ldots \ldots \ldots . . . . . . \ldots \ldots$

12. Copies of ${ }^{1} \mathrm{H}$ NMR, ${ }^{19} \mathrm{~F}$ NMR and ${ }^{13} \mathrm{C}$ NMR spectra of compounds 4-17 and B1 ...............S74 


\section{General information and materials}

General Information: ${ }^{1} \mathrm{H}$ NMR, ${ }^{19} \mathrm{~F}$ NMR and ${ }^{13} \mathrm{C}$ NMR spectra were recorded on an Agilent MR 400 and Agilent MR 500 spectrometer, and are calibrated using residual undeuterated solvent $\left(\mathrm{CHCl}_{3}\right.$ at $7.26 \mathrm{ppm}{ }^{1} \mathrm{H}$ NMR; $77.6 \mathrm{ppm}{ }^{13} \mathrm{C} \mathrm{NMR} ; \mathrm{CFCl}_{3}$ as an external standard and low field is positive). Chemical shifts $(\delta)$ are reported in ppm and coupling constants $(J)$ are in Hertz $(\mathrm{Hz})$. The following abbreviations were used to explain the multiplicities: $\mathrm{s}=$ singlet, $\mathrm{d}=$ doublet, $\mathrm{t}=$ triplet, $\mathrm{q}=$ quartet, $\mathrm{m}$ $=$ multiplet, $\mathrm{br}=$ broad. NMR yield was determined by ${ }^{19} \mathrm{~F}$ NMR using fluorobenzene as an internal standard before working up the reaction.

Materials: All reagents were used as received from commercial sources without further purification. Super dry 1,4-dioxane was purchased from Energy Chemical. $\mathrm{NiCl}_{2} \cdot \mathrm{DME}$ was purchased from Strem and Ligands were purchased from TCI chemistry and used as received. $\mathrm{K}_{2} \mathrm{CO}_{3}$ was purchased from TCI. Arylboronic acids were purchased from J\&K and Energy Chemical. 
2. Optimization of the Ni-catalyzed four-component carbocarbonylation of $N$-vinylacetamide 1 a with $\mathrm{BrCF}_{2} \mathrm{CO}_{2} \mathrm{Et} 2 \mathrm{a}$ and arylboronic acid 3 under $1 \mathrm{~atm} \mathrm{CO}$ (Tables S1-S10)

Table S1. Screening of the solvents ${ }^{a}$

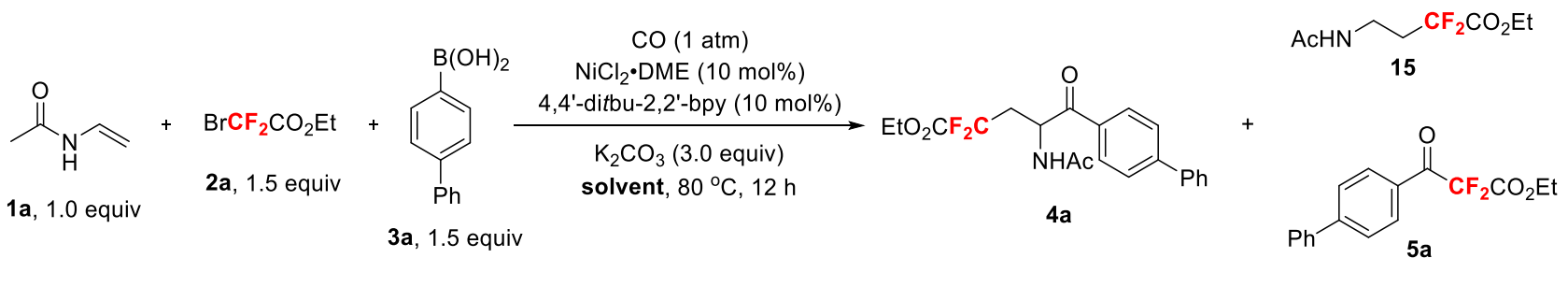

\begin{tabular}{ccccc}
\hline & & \multicolumn{3}{c}{ yield $(\%)^{b}$} \\
\cline { 3 - 5 } entry & solvent & $\mathbf{4 a}$ & $\mathbf{1 5}$ & $\mathbf{5 a}^{\mathbf{c}}$ \\
\hline $\mathbf{1}$ & dioxane & $\mathbf{2 9}$ & $\mathbf{1 3}$ & $\mathbf{5}$ \\
2 & $\mathrm{THF}$ & 16 & 19 & 2 \\
3 & $\mathrm{DME}$ & 17 & 9 & 1 \\
4 & $i \mathrm{Pr}_{2} \mathrm{O}$ & nd & nd & nd \\
5 & $\mathrm{MeCN}$ & 18 & trace & trace \\
6 & $\mathrm{DCE}$ & trace & nd & 5 \\
7 & DMF & nd & nd & nd \\
8 & toluene & nd & 15 & nd \\
\hline
\end{tabular}

${ }^{a}$ Reaction conditions (unless otherwise specified): 1a ( $0.3 \mathrm{mmol}, 1.0$ equiv), $\mathbf{2 a}$ (1.5 equiv), 3a (1.5 equiv), solvent $(3.0 \mathrm{~mL}), 80^{\circ} \mathrm{C}, 12 \mathrm{~h} .{ }^{b}$ Determined by ${ }^{19} \mathrm{~F}$ NMR using fluorobenzene as an internal standard. nd, not detected. ${ }^{c}$ The yield of $\mathbf{5 a}$ is based on $\mathbf{3 a}$. 


\section{Table S2. Screening of the ligands ${ }^{a}$}
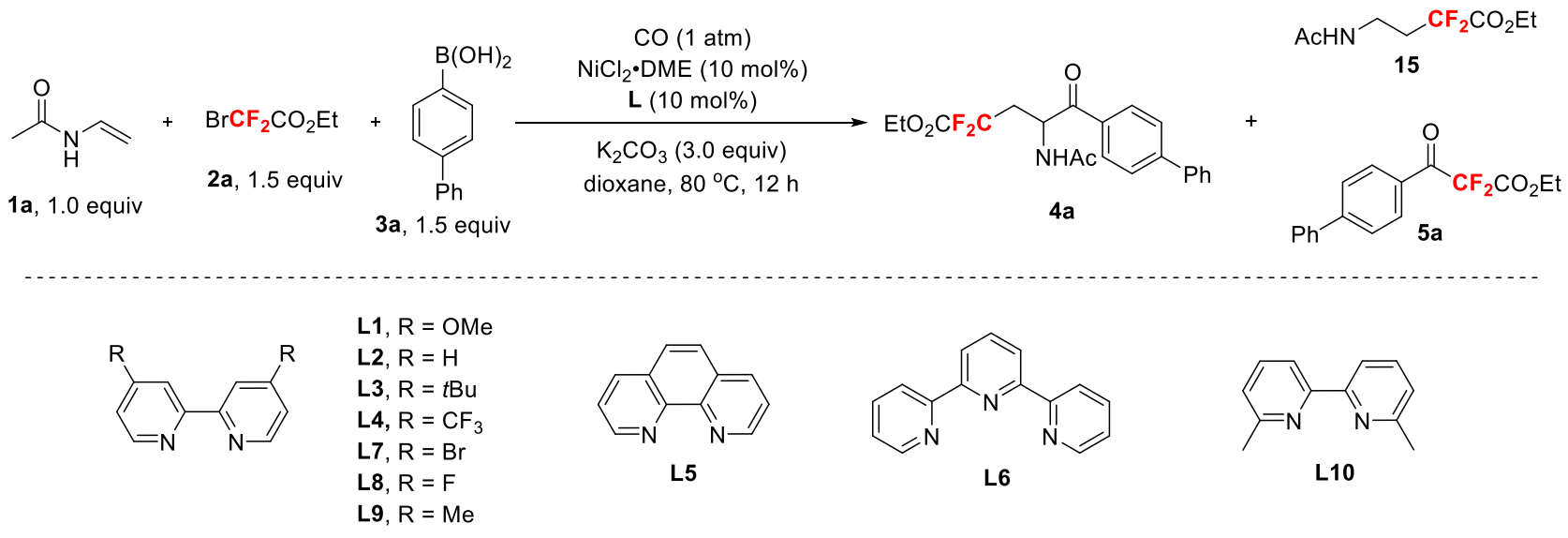

L9, $\mathrm{R}=\mathrm{Me}$

\begin{tabular}{ccccc}
\hline & & & yield $(\%)^{b}$ & \\
\cline { 3 - 5 } entry & ligand & $\mathbf{4 a}$ & $\mathbf{1 5}$ & $\mathbf{5 a}^{\mathbf{c}}$ \\
\hline $\mathbf{1}$ & $\mathbf{L 1}$ & $\mathbf{3 8}$ & $\mathbf{1 6}$ & $\mathbf{1 8}$ \\
2 & $\mathbf{L 2}$ & 30 & 16 & 15 \\
3 & $\mathbf{L 3}$ & 29 & 13 & 5 \\
4 & $\mathbf{L 4}$ & nd & nd & nd \\
5 & $\mathbf{L 5}$ & nd & 1 & nd \\
6 & $\mathbf{L 6}$ & nd & nd & nd \\
7 & $\mathbf{L 7}$ & trace & 30 & 3 \\
8 & $\mathbf{L 8}$ & trace & 10 & trace \\
9 & $\mathbf{L 9}$ & 35 & 14 & 23 \\
10 & $\mathbf{L 1 0}$ & nd & nd & nd \\
\hline
\end{tabular}

${ }^{a}$ Reaction conditions (unless otherwise specified): $1 \mathbf{a}(0.3 \mathrm{mmol}, 1.0$ equiv), $\mathbf{2 a}$ ( 1.5 equiv), $\mathbf{3 a}$ ( 1.5 equiv), dioxane $(3.0 \mathrm{~mL}), 80{ }^{\circ} \mathrm{C}, 12 \mathrm{~h} .{ }^{b}$ Determined by ${ }^{19} \mathrm{~F}$ NMR using fluorobenzene as an internal standard. nd, not detected. ${ }^{c}$ The yield of $\mathbf{5 a}$ is based on $\mathbf{3} \mathbf{a}$. 
Table S3. Screening of the reaction temperature ${ }^{a}$

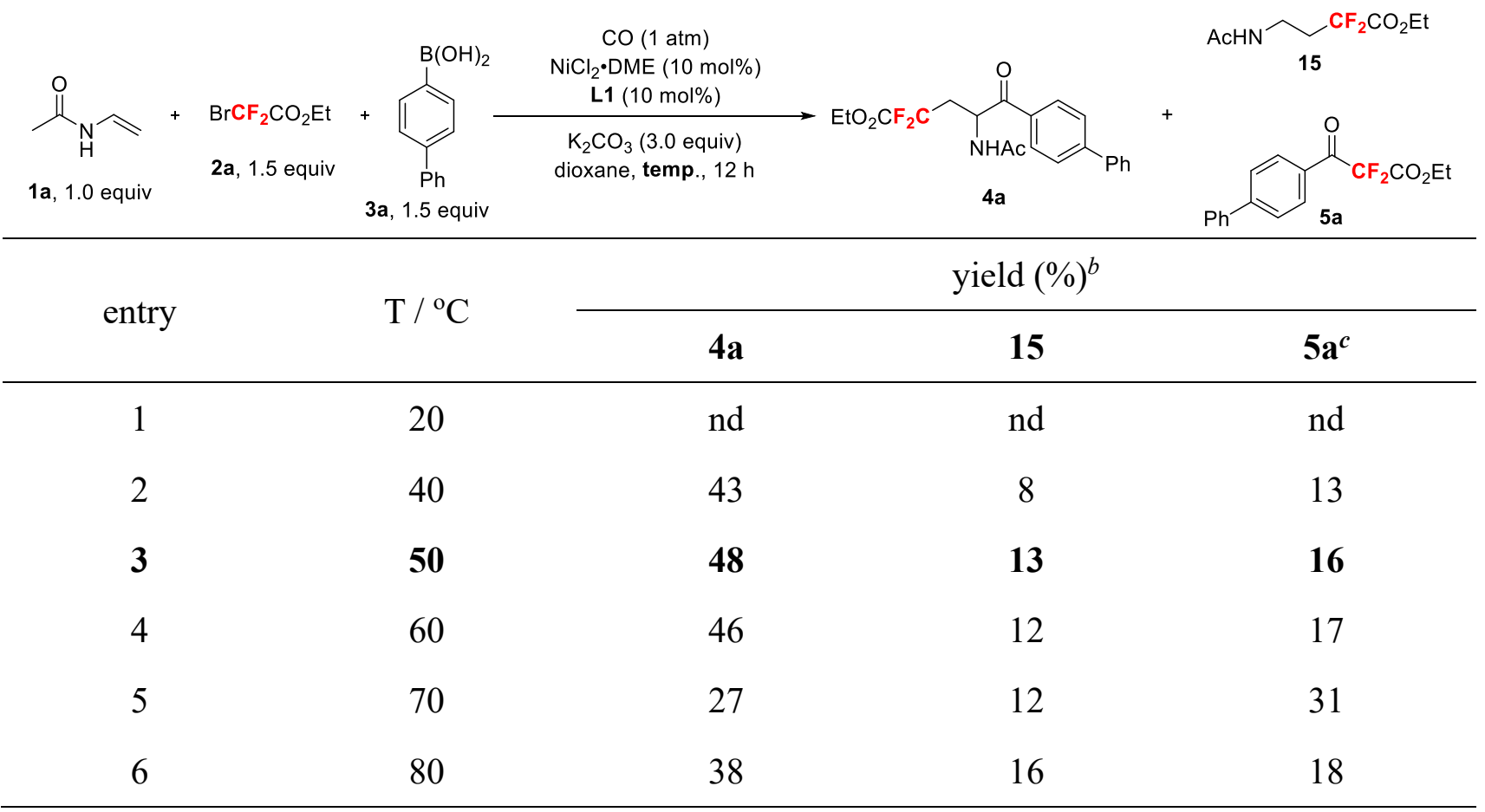

${ }^{a}$ Reaction conditions (unless otherwise specified): $\mathbf{1 a}(0.3 \mathrm{mmol}, 1.0$ equiv), $\mathbf{2 a}$ (1.5 equiv), $\mathbf{3 a}$ (1.5 equiv), dioxane $(3.0 \mathrm{~mL}), 12 \mathrm{~h} .{ }^{b}$ Determined by ${ }^{19} \mathrm{~F}$ NMR using fluorobenzene as an internal standard. nd, not detected. ${ }^{c}$ The yield of 5a is based on 3a. 
Table S4. Screening of the nickel sources ${ }^{a}$

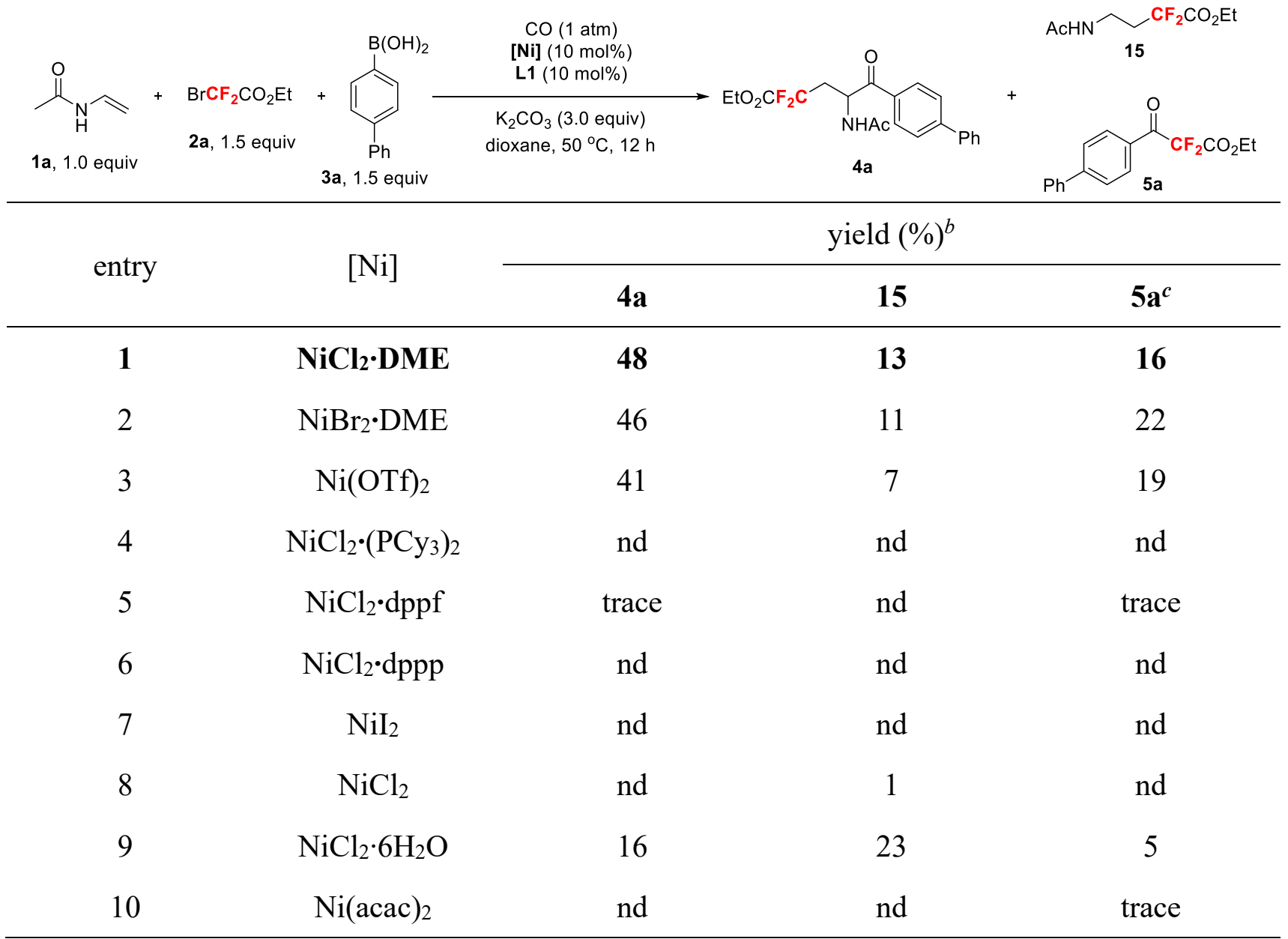

${ }^{a}$ Reaction conditions (unless otherwise specified): 1a ( $0.3 \mathrm{mmol}, 1.0$ equiv), $2 \mathbf{2 a}$ (1.5 equiv), $\mathbf{3 a}$ ( 1.5 equiv), dioxane $(3.0 \mathrm{~mL}), 50{ }^{\circ} \mathrm{C}, 12 \mathrm{~h} .{ }^{b}$ Determined by ${ }^{19} \mathrm{~F}$ NMR using fluorobenzene as an internal standard. nd, not detected. ${ }^{c}$ The yield of $\mathbf{5 a}$ is based on $\mathbf{3 a}$. 
Table S5. Screening of the bases ${ }^{a}$

\begin{tabular}{|c|c|c|c|c|c|}
\hline$\overbrace{\text { 1a, } 1.0 \text { equiv }}^{\mathrm{O}}$ & 2a, 1.5 equiv & $\begin{array}{c}\mathrm{CO}(1 \mathrm{~atm}) \\
\mathrm{NiCl}_{2} \cdot \mathrm{DME}(10 \mathrm{~mol} \%) \\
\mathrm{L1}(10 \mathrm{~mol} \%)\end{array}$ & ${ }_{2} 6$ & + & $\mathrm{CF}_{2} \mathrm{CO}_{2} \mathrm{Et}$ \\
\hline \multirow{2}{*}{ entry } & \multirow{2}{*}{ base } & \multirow{2}{*}{$\mathrm{x}$ equiv } & \multicolumn{3}{|c|}{ yield $(\%)^{b}$} \\
\hline & & & $4 a$ & 15 & $5 \mathbf{a}^{c}$ \\
\hline 1 & $\mathrm{Na}_{2} \mathrm{CO}_{3}$ & 3.0 & 14 & 3 & 5 \\
\hline 2 & $\mathrm{~K}_{2} \mathrm{CO}_{3}$ & 3.0 & 48 & 13 & 16 \\
\hline 3 & $\mathrm{Cs}_{2} \mathrm{CO}_{3}$ & 3.0 & nd & nd & nd \\
\hline 4 & $\mathrm{~K}_{3} \mathrm{PO}_{4}$ & 3.0 & trace & nd & nd \\
\hline 5 & $\mathrm{NaOAc}$ & 3.0 & trace & nd & nd \\
\hline 6 & $\mathrm{NaO} t \mathrm{Bu}$ & 3.0 & nd & nd & nd \\
\hline 7 & $\mathrm{KO} t \mathrm{Bu}$ & 3.0 & nd & nd & nd \\
\hline 8 & $\mathrm{~K}_{2} \mathrm{CO}_{3}$ & 2.0 & 38 & 1 & 33 \\
\hline 9 & $\mathrm{~K}_{2} \mathrm{CO}_{3}$ & 2.5 & 36 & 2 & 31 \\
\hline 10 & $\mathrm{~K}_{2} \mathrm{CO}_{3}$ & 3.5 & 51 & 3 & 39 \\
\hline 11 & $\mathrm{~K}_{2} \mathrm{CO}_{3}$ & 4.0 & 59 & 6 & 31 \\
\hline
\end{tabular}

${ }^{a}$ Reaction conditions (unless otherwise specified): 1a ( $0.3 \mathrm{mmol}, 1.0$ equiv), 2 a (1.5 equiv), 3a (1.5 equiv), dioxane $(3.0 \mathrm{~mL}), 50{ }^{\circ} \mathrm{C}, 12 \mathrm{~h} .{ }^{b}$ Determined by ${ }^{19} \mathrm{~F}$ NMR using fluorobenzene as an internal standard. nd, not detected. ${ }^{c}$ The yield of $\mathbf{5 a}$ is based on $\mathbf{3 a}$. 
Table S6. Screening of the loading amount of $2 \mathrm{a}$ and $3 \mathrm{a}^{a}$

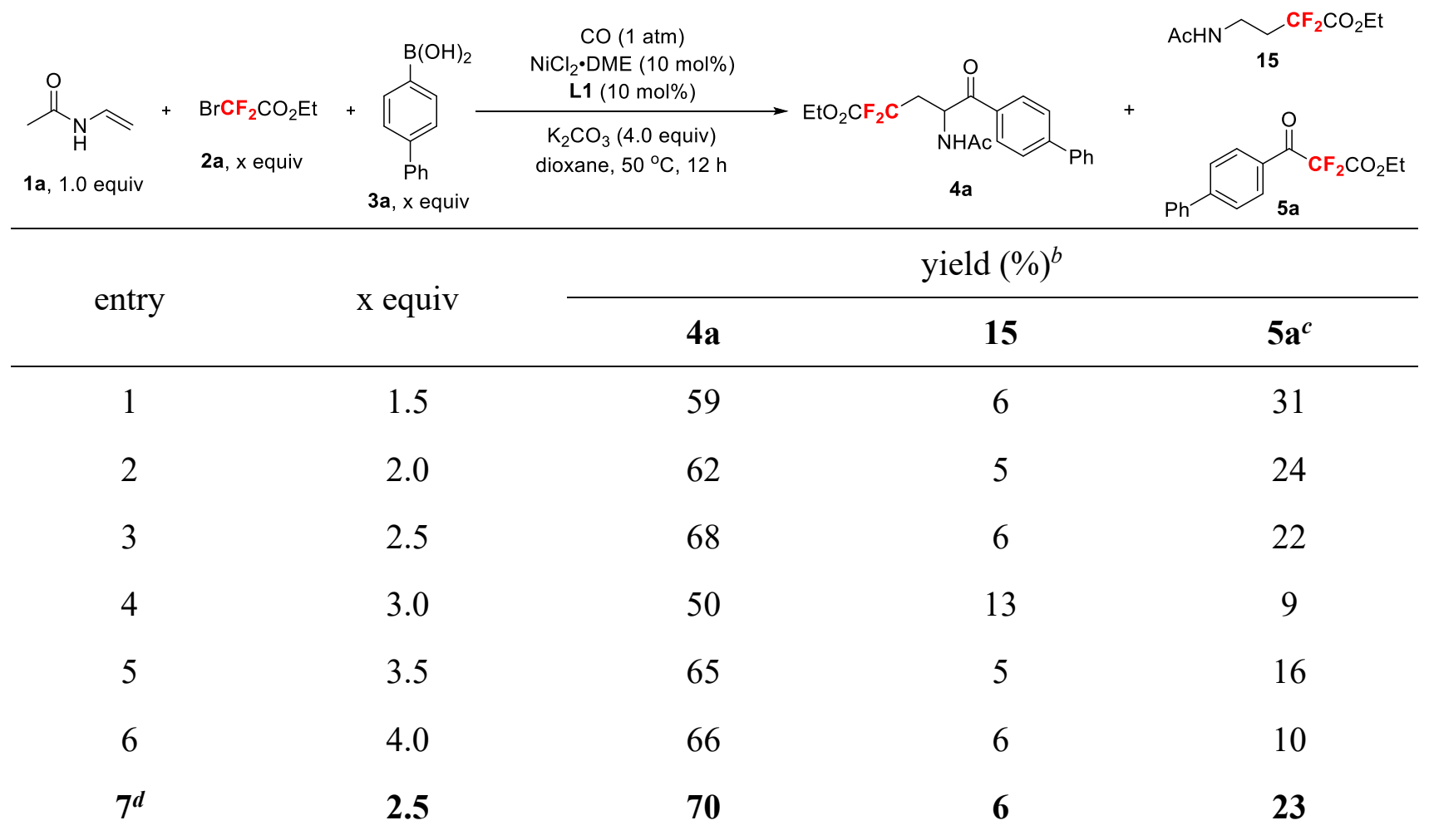

${ }^{a}$ Reaction conditions (unless otherwise specified): $\mathbf{1 a}(0.3 \mathrm{mmol}, 1.0$ equiv), $\mathbf{2 a}$ (x equiv), 3a (x equiv), dioxane (3.0 $\mathrm{mL}), 50{ }^{\circ} \mathrm{C}, 12 \mathrm{~h} .{ }^{b}$ Determined by ${ }^{19} \mathrm{~F}$ NMR using fluorobenzene as an internal standard. ${ }^{c}$ The yield of $\mathbf{5 a}$ is based on 3a. ${ }^{d}$ Reaction run for $18 \mathrm{~h}$. 
Table S7. Screening of the loading amount of additive $\mathrm{H}_{2} \mathrm{O}^{a}$

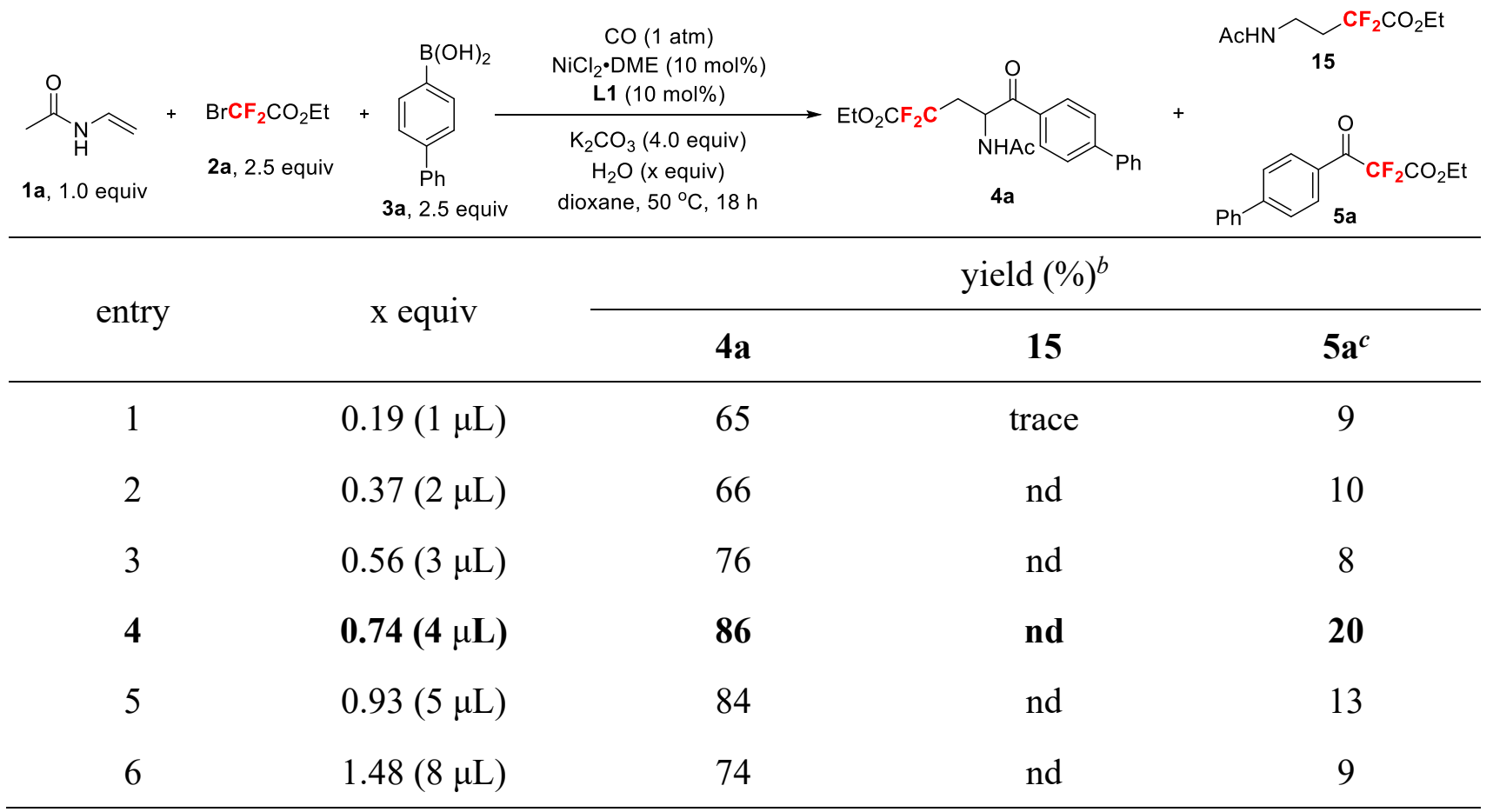

${ }^{a}$ Reaction conditions (unless otherwise specified): $\mathbf{1 a}\left(0.3 \mathrm{mmol}, 1.0\right.$ equiv), $\mathbf{2 a}$ (2.5 equiv), $\mathbf{3 a}$ ( 2.5 equiv), $\mathrm{H}_{2} \mathrm{O}$ (x equiv), dioxane $(3.0 \mathrm{~mL}), 50{ }^{\circ} \mathrm{C}, 18 \mathrm{~h} .{ }^{b}$ Determined by ${ }^{19} \mathrm{~F}$ NMR using fluorobenzene as an internal standard. nd, not detected. ${ }^{c}$ The yield of $\mathbf{5 a}$ is based on $\mathbf{3 a}$. 
Table S8. Control experiments ${ }^{a}$

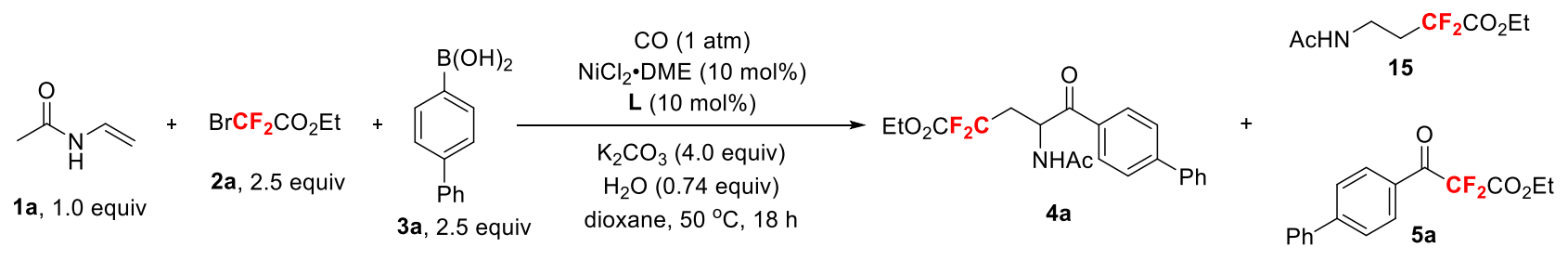

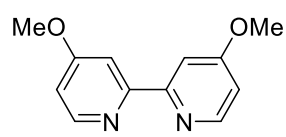

L1

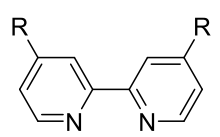

L2, $\mathrm{R}=\mathrm{H}$

$\mathrm{L} 3, \mathrm{R}=t \mathrm{Bu}$

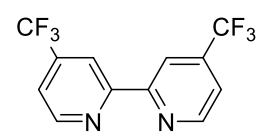

L4

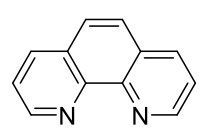

L5

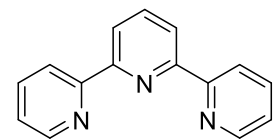

L6

\begin{tabular}{|c|c|c|c|c|}
\hline \multirow{2}{*}{ entry } & \multirow{2}{*}{ reaction conditions } & \multicolumn{3}{|c|}{ yield $(\%)^{b}$} \\
\hline & & $4 a$ & 15 & $5 \mathbf{a}^{c}$ \\
\hline 1 & $\mathbf{L 1}$ & $(86)$ & nd & 20 \\
\hline 2 & $\mathbf{L 2}$ & 61 & 2 & 15 \\
\hline 3 & $\mathbf{L 3}$ & 67 & 3 & 23 \\
\hline 4 & L4 & nd & nd & nd \\
\hline 5 & $\mathbf{L 5}$ & nd & nd & nd \\
\hline 6 & L6 & nd & nd & nd \\
\hline 7 & $\mathbf{L} \mathbf{1}, \mathrm{NiBr}_{2} \cdot \mathrm{DME}$ instead of $\mathrm{NiCl}_{2} \cdot \mathrm{DME}$ & 70 & trace & 12 \\
\hline 8 & $\mathbf{L} 1, \mathrm{NiCl}_{2}$ or $\mathrm{NiBr}_{2}$ instead of $\mathrm{NiCl}_{2} \cdot \mathrm{DME}$ & nd & nd & nd \\
\hline 9 & $\mathbf{L 1}, \mathrm{Ni}(\mathrm{COD})_{2}$ instead of $\mathrm{NiCl}_{2} \cdot \mathrm{DME}$ & 65 & 3 & 10 \\
\hline 10 & $\mathbf{L 1}, \mathrm{Ni}\left(\mathrm{PPh}_{3}\right)_{4}$ instead of $\mathrm{NiCl}_{2} \cdot \mathrm{DME}$ & nd & nd & nd \\
\hline 11 & without $\mathrm{H}_{2} \mathrm{O}$ & 68 & 5 & 22 \\
\hline 12 & without nickel catalyst & nd & nd & nd \\
\hline 13 & without ligand & nd & nd & nd \\
\hline 14 & without base & nd & nd & nd \\
\hline
\end{tabular}

${ }^{a}$ Reaction conditions (unless otherwise specified): $\mathbf{1 a}\left(0.3\right.$ mmol, 1.0 equiv), $\mathbf{2 a}$ ( 2.5 equiv), 3a (2.5 equiv), $\mathrm{H}_{2} \mathrm{O}(0.74$ equiv), dioxane $(3.0 \mathrm{~mL}), 50{ }^{\circ} \mathrm{C}, 18 \mathrm{~h} .{ }^{b}$ Determined by ${ }^{19} \mathrm{~F}$ NMR using fluorobenzene as an internal standard. nd, not detected. ${ }^{c}$ The yield of $\mathbf{5 a}$ is based on $\mathbf{3 a}$. 
Table S9. Optimization of Ni-catalyzed carbocarbonylation of alkene 1a with $2 \mathrm{a}$ and $3 \mathrm{j}$ under 1 $\operatorname{atm} \mathrm{CO}^{a}$

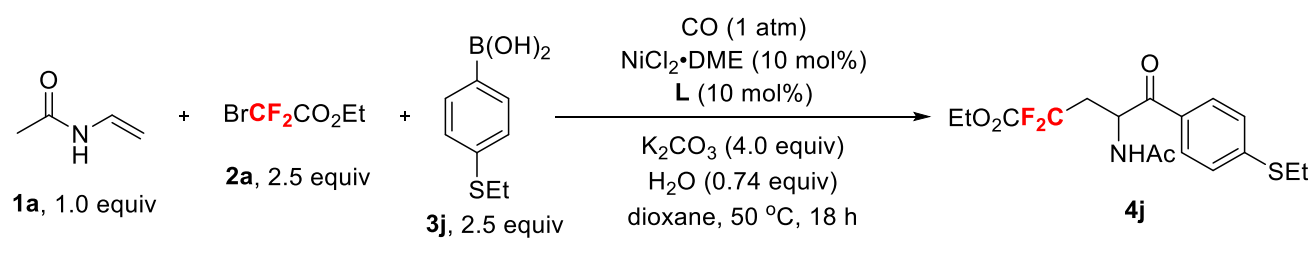

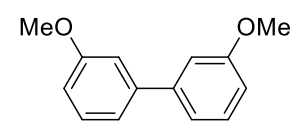

L1

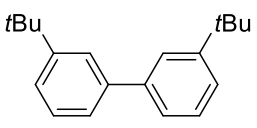

L3

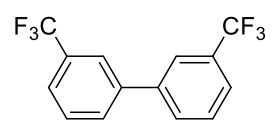

L4

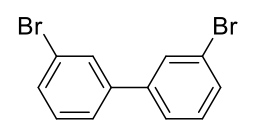

L7

\begin{tabular}{ccc}
\hline entry & ligand & yield $(\%)^{b}$ \\
\hline 1 & L1 & 31 \\
2 & $\mathbf{L 3}$ & 40 \\
3 & $\mathbf{L 4}$ & nd \\
$\mathbf{4}$ & $\mathbf{L 7}$ & $\mathbf{7 2}(\mathbf{7 4})$ \\
\hline
\end{tabular}

${ }^{a}$ Reaction conditions (unless otherwise specified): 1a ( $0.3 \mathrm{mmol}, 1.0$ equiv), $\mathbf{2 a}$ ( 2.5 equiv), $\mathbf{3 j}$ ( 2.5 equiv), dioxane $(3.0 \mathrm{~mL}), 50{ }^{\circ} \mathrm{C}, 18 \mathrm{~h} .{ }^{b}$ Determined by ${ }^{19} \mathrm{~F}$ NMR using fluorobenzene as an internal standard and the number in parenthesis is the isolated yield. nd, not detected. 
Table S10. Optimization of Ni-catalyzed carbocarbonylation of alkene 1a with 2 a and $3 \mathrm{~m}$ under $1 \mathrm{~atm} \mathrm{CO}^{a}$

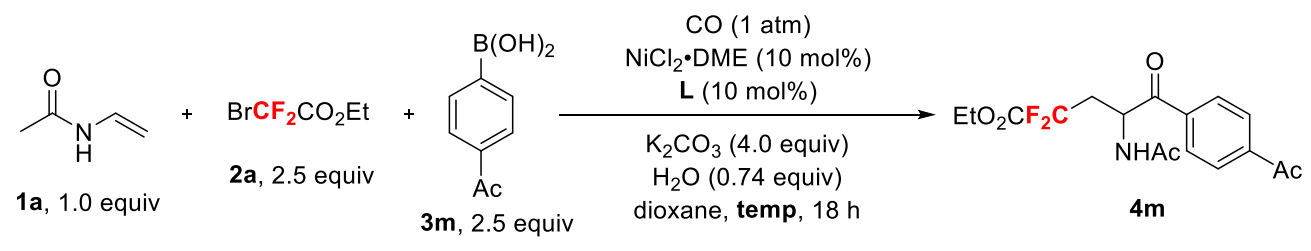

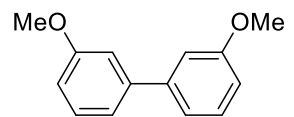

L1

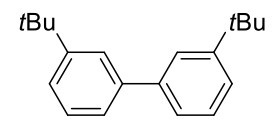

L3

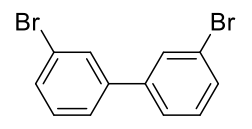

L7

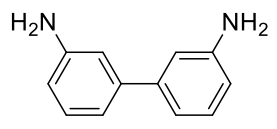

L11

\begin{tabular}{cccc}
\hline entry & $\mathrm{T}\left({ }^{\circ} \mathrm{C}\right)$ & ligand & yield $(\%)^{b}$ \\
\hline 1 & 50 & $\mathbf{L 1}$ & 10 \\
2 & 50 & $\mathbf{L 3}$ & trace \\
3 & 50 & $\mathbf{L 7}$ & nd \\
4 & 50 & $\mathbf{L 1 1}$ & nd \\
5 & 60 & $\mathbf{L 1}$ & 14 \\
6 & 70 & $\mathbf{L 1}$ & 48 \\
7 & $\mathbf{8 0}$ & $\mathbf{L 1}$ & $\mathbf{6 6}(\mathbf{6 4})$ \\
8 & 90 & $\mathbf{L 1}$ & 10
\end{tabular}

${ }^{a}$ Reaction conditions (unless otherwise specified): $\mathbf{1 a}(0.3 \mathrm{mmol}, 1.0$ equiv), $\mathbf{2 a}$ ( 2.5 equiv), $\mathbf{3 m}$ ( 2.5 equiv), dioxane $(3.0 \mathrm{~mL}), 50{ }^{\circ} \mathrm{C}, 18 \mathrm{~h} .{ }^{b}$ Determined by ${ }^{19} \mathrm{~F}$ NMR using fluorobenzene as an internal standard and the number in parenthesis is the isolated yield. nd, not detected. 
3. Table S11. Screening of the loading amount of $2 \mathrm{e}$ and $3 \mathrm{e}^{a}$

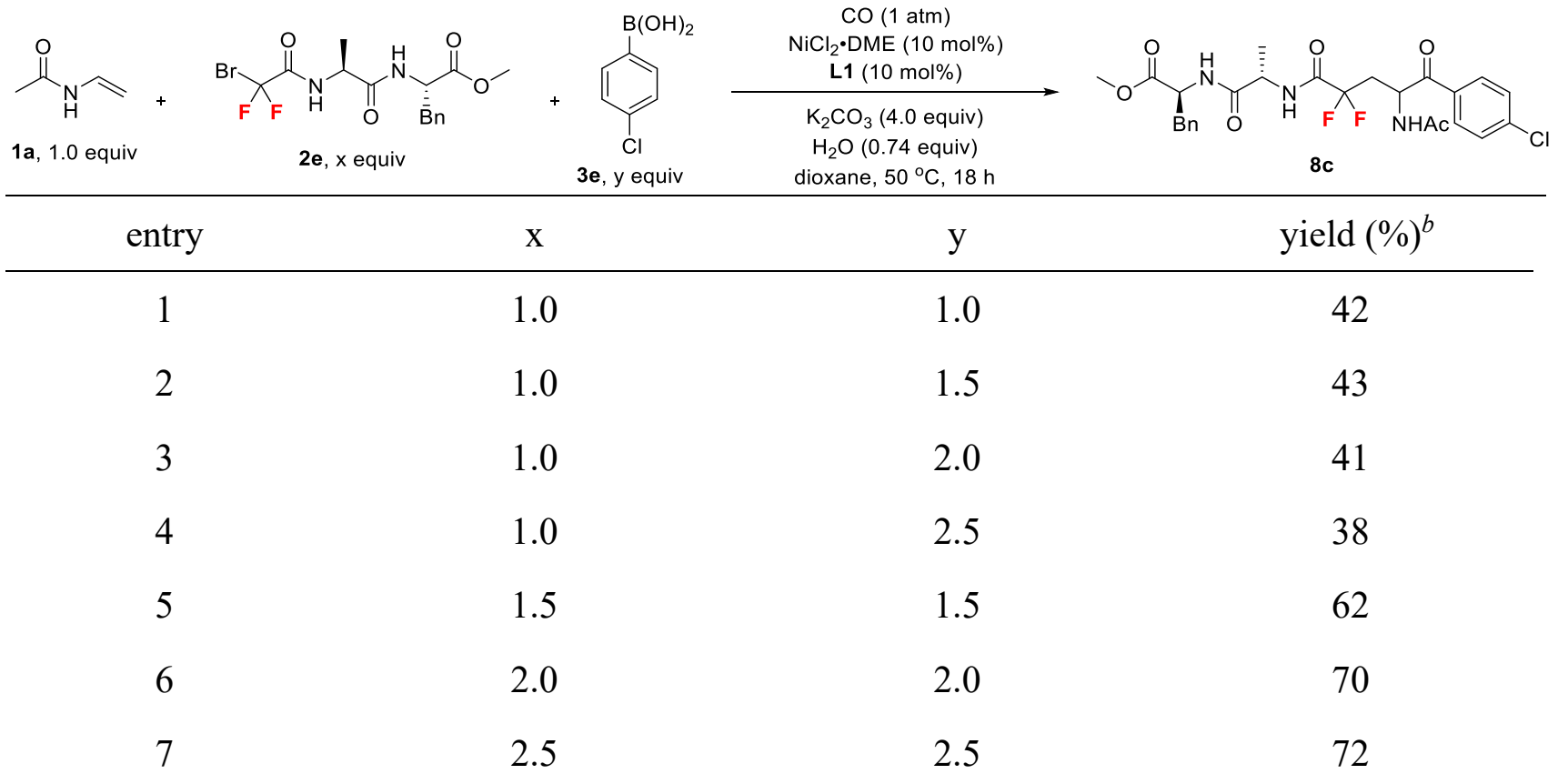

${ }^{a}$ Reaction conditions (unless otherwise specified): $1 \mathrm{a}\left(0.3 \mathrm{mmol}, 1.0\right.$ equiv), dioxane $(3.0 \mathrm{~mL}), 50{ }^{\circ} \mathrm{C}, 18 \mathrm{~h}$. ${ }^{b}$ Determined by ${ }^{19} \mathrm{~F}$ NMR using fluorobenzene as an internal standard and the number in parenthesis is the isolated yield. 
4. Preparation of alkenes 1 .<smiles>C=CNC(C)=O</smiles><smiles>C=COC(C)=O</smiles>

$1 e$<smiles>C=CCCNC(=O)c1ccccc1</smiles><smiles>C=CCNC(=O)[C@H](CCC(=O)OCCC)NC(=O)OCc1ccccc1</smiles><smiles>C=CNC(=O)OC(C)(C)C</smiles>

$1 \mathrm{~b}$<smiles>C=CCNC(=O)c1ccccc1</smiles><smiles>C=CCOC(=O)c1ccccc1</smiles><smiles>C=CNC(=O)c1ccccc1</smiles><smiles>C=CCNC(=O)OC(C)(C)C</smiles><smiles>C=CCOC(=O)COc1ccccc1</smiles><smiles>C=CN1CCCC1=O</smiles><smiles>C=CCNC(=O)C(F)(F)F</smiles>

1h<smiles>C=CCOC(C)=O</smiles>

11<smiles>C=CCNCCNC(=O)C(Br)NC(=O)OC(C)(C)C</smiles>

\section{Structures of alkenes}

Note: Alkenes 1a-e, 1g-h, 1j-l, 1p are commercially available.

\subsection{Preparation of $N$-allylbenzamide 1f.}
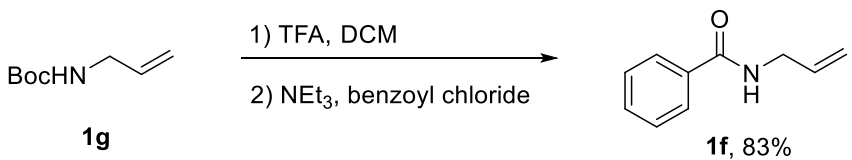

To a $250 \mathrm{~mL}$ round bottle equipped with a Teflon-coated magnetic stir bar were added $1 \mathrm{~g}$ (1.57 g, 10 mmol, 1.0 equiv) and $\mathrm{CH}_{2} \mathrm{Cl}_{2}$ (DCM, $\left.50 \mathrm{~mL}\right)$. Trifluoroacetic acid (9.12 g, $80 \mathrm{mmol}, 8.0$ equiv) was then added dropwise. The resulting mixture was stirred at room temperature for $8 \mathrm{~h}$ until the starting material was totally consumed. To the resulting mixture were added $\mathrm{NEt}_{3}(10.1 \mathrm{~g}, 100 \mathrm{mmol}, 10.0$ equiv), DMAP (122.2 mg, $1.0 \mathrm{mmol}, 0.1$ equiv) and benzoyl chloride (1.69 g, $12.0 \mathrm{mmol}, 1.2$ equiv). The resulting mixture was stirred at room temperature overnight. After the reaction was completed, the reaction mixture was washed with brine, dried over $\mathrm{Na}_{2} \mathrm{SO}_{4}$, filtered and concentrated. The alkene 1f (1.34 g, 83\% yield) as a yellow oil was purified with silica gel chromatography (Petroleum ether: Ethyl acetate =4: 1). Compound $\mathbf{1 f}$ is known. ${ }^{1}{ }^{1} \mathrm{H} \mathrm{NMR}\left(400 \mathrm{MHz}, \mathrm{CDCl}_{3}\right) \delta 7.78(\mathrm{~d}, J=7.2 \mathrm{~Hz}, 2 \mathrm{H})$, $7.47(\mathrm{t}, J=7.2 \mathrm{~Hz}, 1 \mathrm{H}), 7.38(\mathrm{t}, J=7.2 \mathrm{~Hz}, 2 \mathrm{H}), 6.63(\mathrm{~s}, 1 \mathrm{H}), 5.96-5.84(\mathrm{~m}, 1 \mathrm{H}), 5.22(\mathrm{~d}, J=17.2$ 
$\mathrm{Hz}, 1 \mathrm{H}), 5.14(\mathrm{~d}, J=10.4 \mathrm{~Hz}, 1 \mathrm{H}), 4.08-4.01(\mathrm{~m}, 2 \mathrm{H}) .{ }^{13} \mathrm{C} \mathrm{NMR}\left(101 \mathrm{MHz}, \mathrm{CDCl}_{3}\right) \delta 168.0,134.9$, $134.6,132.0,129.0,127.5,117.0,42.9$.

\subsection{Preparation of $N$-(but-3-en-1-yl)benzamide $1 i$}

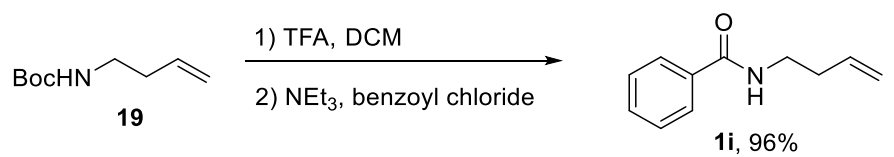

To a $250 \mathrm{~mL}$ round bottle equipped with a Teflon-coated magnetic stir bar were added tert-butyl but3-en-1-ylcarbamate 19 (3.42 g, 20 mmol, 1.0 equiv) and DCM (100 mL). TFA (18.24 g, $160 \mathrm{mmol}$, 8.0 equiv) was then added dropwise. The resulting mixture was stirred at room temperature for $8 \mathrm{~h}$ until the starting material was totally consumed. To the resulting mixture were added $\mathrm{NEt}_{3}(20.2 \mathrm{~g}, 200$ mmol, 10.0 equiv), DMAP (244.2 mg, $2.0 \mathrm{mmol}, 0.1$ equiv) and benzoyl chloride (3.37 g, $24.0 \mathrm{mmol}$, 1.2 equiv). The resulting mixture was stirred at room temperature overnight. After reaction was completed, the reaction mixture was washed with brine, dried over $\mathrm{Na}_{2} \mathrm{SO}_{4}$, filtered and concentrated. The alkene 1i (3.69 g, 96\% yield) as a colorless oil was purified with silica gel chromatography (Petroleum ether: Ethyl acetate $=3: 1)$. Compound $1 \mathbf{i}$ is known. ${ }^{2}{ }^{1} \mathrm{H} \mathrm{NMR}\left(400 \mathrm{MHz}, \mathrm{CDCl}_{3}\right) \delta 7.75$ $(\mathrm{d}, J=7.2 \mathrm{~Hz}, 2 \mathrm{H}), 7.42(\mathrm{t}, J=7.2 \mathrm{~Hz}, 1 \mathrm{H}), 7.33(\mathrm{t}, J=7.2 \mathrm{~Hz}, 2 \mathrm{H}), 6.91(\mathrm{~s}, 1 \mathrm{H}), 5.82-5.70(\mathrm{~m}, 1 \mathrm{H})$, $5.10-5.01(\mathrm{~m}, 2 \mathrm{H}), 3.48-3.41(\mathrm{~m}, 2 \mathrm{H}), 2.35-2.28(\mathrm{~m}, 2 \mathrm{H}) .{ }^{13} \mathrm{C} \mathrm{NMR}\left(101 \mathrm{MHz}, \mathrm{CDCl}_{3}\right) \delta 168.2$, $135.7,135.1,131.7,128.8,127.4,117.5,39.4,34.2$.

\subsection{Preparation of tert-butyl (S)-5-(allylamino)-4-(((benzyloxy)carbonyl)amino)-5-} oxopentanoate $1 \mathrm{~m}$.
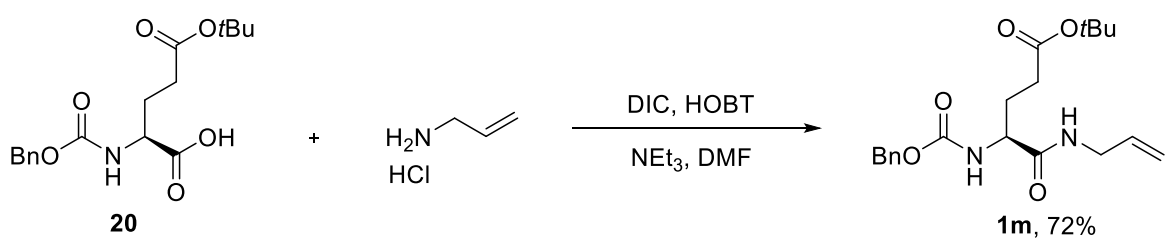

To a $250 \mathrm{~mL}$ round bottle equipped with a Teflon-coated magnetic stir bar were added protected amino acid 20 (3.37 g, $10 \mathrm{mmol}, 1.0$ equiv), HOBT (1.35 g, $10 \mathrm{mmol}, 1.0$ equiv) and DMF (50 mL). Subsequently, DIC (1.26 g, $10 \mathrm{mmol}, 1.0$ equiv), NEt 3 (2.02 g, $20 \mathrm{mmol}, 2.0$ equiv) and allylamine hydrochloride (1.41 g, $15 \mathrm{mmol}, 1.5$ equiv) were added. After the reaction was stirred at room temperature overnight, $\mathrm{H}_{2} \mathrm{O}(100 \mathrm{~mL})$ was added. The resulting mixture was extracted by ethyl acetate 
(EA) 5 times. The combined organic phase was dried over $\mathrm{Na}_{2} \mathrm{SO}_{4}$, filtered and concentrated. The alkene $1 \mathrm{~m}$ (2.71 g, 72\% yield) as a white solid (m.p. 66-69 ${ }^{\circ} \mathrm{C}$ ) was purified with silica gel chromatography (Petroleum ether: Ethyl acetate =3: 1). ${ }^{1} \mathrm{H}$ NMR $\left(400 \mathrm{MHz}, \mathrm{CDCl}_{3}\right) \delta 7.36-7.29(\mathrm{~m}$, 5H), $6.62(\mathrm{~m}, 1 \mathrm{H}), 5.90-5.72(\mathrm{~m}, 2 \mathrm{H}), 5.20-5.06(\mathrm{~m}, 4 \mathrm{H}), 4.28-4.17(\mathrm{~m}, 1 \mathrm{H}), 3.89-3.82(\mathrm{~m}, 2 \mathrm{H})$, $2.45-2.24(\mathrm{~m}, 2 \mathrm{H}), 2.13-2.02(\mathrm{~m}, 1 \mathrm{H}), 1.98-1.86(\mathrm{~m}, 1 \mathrm{H}), 1.42(\mathrm{~s}, 9 \mathrm{H}) .{ }^{13} \mathrm{C} \mathrm{NMR}(101 \mathrm{MHz}$, $\left.\mathrm{CDCl}_{3}\right) \delta 173.4,171.8,156.9,136.7,134.2,129.1,128.7,128.6,117.1,104.0,81.6,67.6,54.9,42.4$, 32.2, 28.6. MS (ESI): m/z (\%) $377(\mathrm{M}+\mathrm{H})^{+}$. HRMS (ESI): Calcd. for $\mathrm{C}_{20} \mathrm{H}_{29} \mathrm{O}_{5} \mathrm{~N}_{2}: 377.2071(\mathrm{M}+\mathrm{H})^{+}$; Found: $377.2068(\mathrm{M}+\mathrm{H})^{+}$.

\subsection{Preparation of tert-Butyl (S)-(1-(Allylamino)-1-oxo-3-phenylpropan-2-yl)carbamate $1 \mathrm{n}$.}

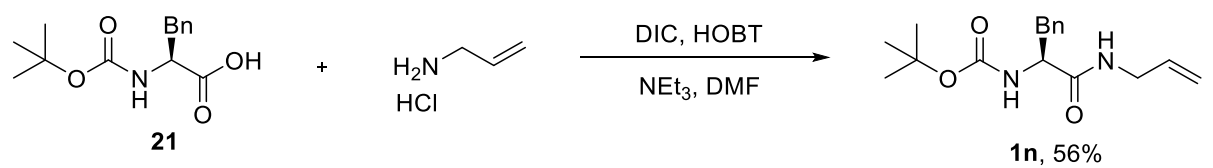

To a $250 \mathrm{~mL}$ round bottle equipped with a Teflon-coated magnetic stir bar were added protected amino acid 21 (2.65 g, $10 \mathrm{mmol}, 1.0$ equiv), HOBT (1.35 g, $10 \mathrm{mmol}, 1.0$ equiv) and DMF (50 mL). Subsequently, DIC (1.26 g, $10 \mathrm{mmol}, 1.0$ equiv), NEt 3 (2.02 g, $20 \mathrm{mmol}, 2.0$ equiv) and allylamine hydrochloride (1.41 g, $15 \mathrm{mmol}, 1.5$ equiv) were added. After the reaction was stirred at room temperature overnight, $\mathrm{H}_{2} \mathrm{O}(100 \mathrm{~mL})$ was added. The resulting mixture was then extracted by EA 5 times. The combined organic phase was dried over $\mathrm{Na}_{2} \mathrm{SO}_{4}$, filtered and concentrated. The alkene $\mathbf{1 n}$ (1.70 g, 56\% yield) as a white solid (m.p. $89-93{ }^{\circ} \mathrm{C}$ ) was purified with silica gel chromatography (Petroleum ether: Ethyl acetate = 2: 1). ${ }^{1} \mathrm{H}$ NMR (400 MHz, $\left.\mathrm{CDCl}_{3}\right) \delta 7.31-7.17(\mathrm{~m}, 5 \mathrm{H}), 6.42(\mathrm{~s}$, 1H), $5.75-5.61(\mathrm{~m}, 1 \mathrm{H}), 5.40(\mathrm{~d}, J=7.2 \mathrm{~Hz}, 1 \mathrm{H}), 5.12-4.98(\mathrm{~m}, 2 \mathrm{H}), 4.47-4.35(\mathrm{~m}, 1 \mathrm{H}), 3.88-$ $3.70(\mathrm{~m}, 2 \mathrm{H}), 3.09-2.98(\mathrm{~m}, 2 \mathrm{H}), 1.38(\mathrm{~s}, 9 \mathrm{H}) .{ }^{13} \mathrm{C} \mathrm{NMR}\left(101 \mathrm{MHz}, \mathrm{CDCl}_{3}\right) \delta 172.0,156.1,137.3$, 134.1, 129.9, 129.1, 127.3, 116.8, 80.6, 56.4, 42.3, 39.3, 28.8. MS (ESI): m/z (\%) $305(\mathrm{M}+\mathrm{H})^{+}$. HRMS (ESI): Calcd. for $\mathrm{C}_{17} \mathrm{H}_{25} \mathrm{O}_{3} \mathrm{~N}_{2}: 305.1860(\mathrm{M}+\mathrm{H})^{+}$; Found: $305.1866(\mathrm{M}+\mathrm{H})^{+}$. 


\subsection{Preparation of Benzyl ((S)-1-(((S)-1-(Allylamino)-4-methyl-1-oxopentan-2-yl)amino)-1-oxo-}

3-phenylpropan-2-yl)carbamate 10.

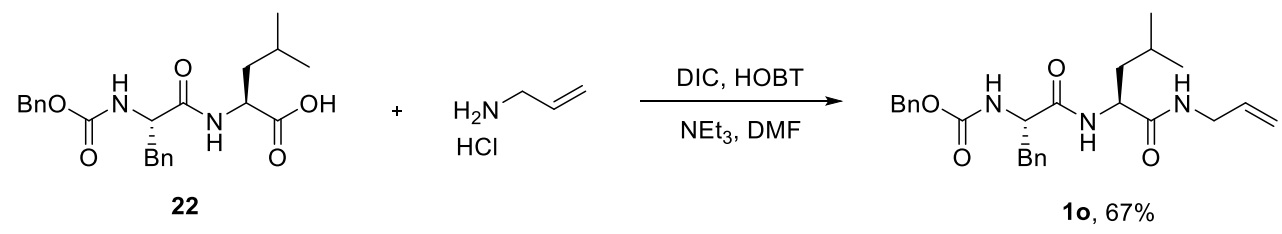

To a $250 \mathrm{~mL}$ round bottle equipped with a Teflon-coated magnetic stir bar were added protected amino acid 22 (4.12 g, $10 \mathrm{mmol}, 1.0$ equiv), HOBT (1.35 g, $10 \mathrm{mmol}, 1.0$ equiv) and DMF (50 mL). Subsequently, DIC (1.26 g, $10 \mathrm{mmol}, 1.0$ equiv), NEt 3 (2.02 g, $20 \mathrm{mmol}, 2.0$ equiv) and allylamine hydrochloride (1.41 g, $15 \mathrm{mmol}, 1.5$ equiv) were added. After the reaction was stirred at room temperature overnight, $\mathrm{H}_{2} \mathrm{O}(100 \mathrm{~mL})$ was added. The resulting mixture was then extracted by EA 5 times. The combined organic phase was dried over $\mathrm{Na}_{2} \mathrm{SO}_{4}$, filtered and concentrated. The alkene 10 (3.02 g, 67\% yield) as a white solid (m.p. 159-161 ${ }^{\circ} \mathrm{C}$ ) was purified with silica gel chromatography $\left(\right.$ Petroleum ether: Ethyl acetate = 2: 1). ${ }^{1} \mathrm{H}$ NMR $\left(400 \mathrm{MHz}, \mathrm{CDCl}_{3}\right) \delta 7.37-7.19(\mathrm{~m}, 8 \mathrm{H}), 7.15(\mathrm{~d}, J$ $=6.8 \mathrm{~Hz}, 2 \mathrm{H}), 6.64(\mathrm{~d}, J=8.0 \mathrm{~Hz}, 1 \mathrm{H}), 6.51-6.45(\mathrm{~m}, 1 \mathrm{H}), 5.83-5.71(\mathrm{~m}, 1 \mathrm{H}), 5.49(\mathrm{~d}, J=7.6 \mathrm{~Hz}$, $1 \mathrm{H}), 5.19-5.08(\mathrm{~m}, 2 \mathrm{H}), 5.05(\mathrm{~s}, 2 \mathrm{H}), 4.54-4.40(\mathrm{~m}, 2 \mathrm{H}), 3.87-3.71(\mathrm{~m}, 2 \mathrm{H}), 3.06(\mathrm{~d}, J=6.8 \mathrm{~Hz}$, 2H), $1.73-1.64(\mathrm{~m}, 1 \mathrm{H}), 1.56-1.40(\mathrm{~m}, 2 \mathrm{H}), 0.87(\mathrm{~d}, J=5.6 \mathrm{~Hz}, 6 \mathrm{H}) .{ }^{13} \mathrm{C} \mathrm{NMR}\left(126 \mathrm{MHz}, \mathrm{CDCl}_{3}\right)$ $\delta 171.3,171.1,156.2,136.04,135.97,133.9,129.3,128.8,128.6,128.3,128.1,127.2,116.3,67.3$, 56.3, 51.9, 41.9, 40.8, 38.2, 23.5, 22.9. MS (ESI): m/z (\%) $452(\mathrm{M}+\mathrm{H})^{+}$. HRMS (ESI): Calcd. for $\mathrm{C}_{26} \mathrm{H}_{34} \mathrm{O}_{4} \mathrm{~N}_{3}: 452.2544(\mathrm{M}+\mathrm{H})^{+}$; Found: $452.2539(\mathrm{M}+\mathrm{H})^{+}$.

\section{Preparation of difluoroalkyl bromides 2.}

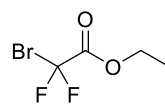

2a

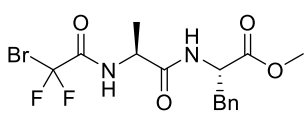

2e

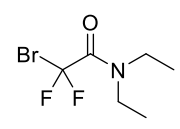

2b

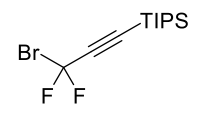

$2 c$

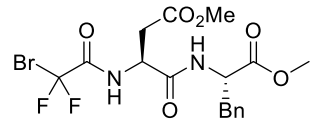

$2 f$

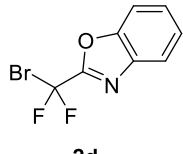

2d

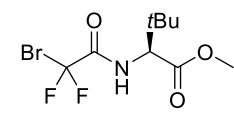

2g

Structures of difluoroalkyl bromides 2

Note: Compounds $\mathbf{2 b - d}$ and $\mathbf{2 g}$ were prepared according to the literature. ${ }^{3-6}$ 


\subsection{Preparation of Methyl (2-Bromo-2,2-difluoroacetyl)- $L$-alanyl- $L$-phenylalaninate 2 e.}
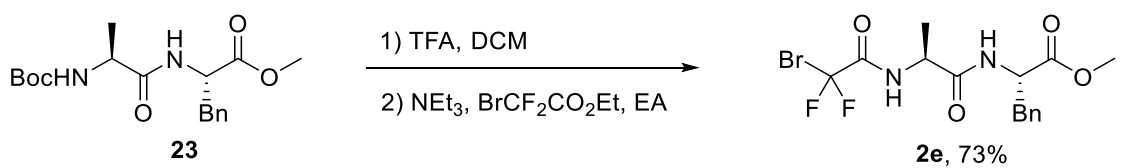

To a $250 \mathrm{~mL}$ round bottle equipped with a Teflon-coated magnetic stir bar were added Boc-Ala-PheOMe 23 (10.5 g, 30 mmol, 1.0 equiv) and DCM (100 mL). Subsequently, TFA (27.4 g, 240 mmol, 8.0 equiv) was added dropwise. After the reaction was stirred at room temperature overnight, the solvent was removed. The crude product was dissolved in EA (100 mL). NEt 3 (30.4 g, 300 mmol, 10 equiv) and $\mathrm{BrCF}_{2} \mathrm{CO}_{2} \mathrm{Et}$ (12.2 g, $60 \mathrm{mmol}, 2.0$ equiv) were added. The resulting mixture was stirred at room temperature overnight. The reaction mixture was diluted with EtOAc, washed with brine three times. The organic layer was dried over $\mathrm{Na}_{2} \mathrm{SO}_{4}$, filtered and concentrated. Compound $2 \mathrm{e}(8.9 \mathrm{~g}, 73 \%$ yield $)$ as a white solid (m.p. $118-122^{\circ} \mathrm{C}$ ) was purified with silica gel chromatography (Petroleum ether: Ethyl acetate = 3: 1). ${ }^{1} \mathrm{H}$ NMR $\left(400 \mathrm{MHz}, \mathrm{CDCl}_{3}\right) \delta 7.32-7.23(\mathrm{~m}, 3 \mathrm{H}), 7.16-7.05(\mathrm{~m}, 3 \mathrm{H}), 6.37(\mathrm{~d}, J=$ $7.6 \mathrm{~Hz}, 1 \mathrm{H}), 4.90-4.83(\mathrm{~m}, 1 \mathrm{H}), 4.50-4.41(\mathrm{~m}, 1 \mathrm{H}), 3.75(\mathrm{~s}, 3 \mathrm{H}), 3.13(\mathrm{qd}, J=13.6 \mathrm{~Hz}, 5.6 \mathrm{~Hz}, 2 \mathrm{H})$, $1.41(\mathrm{~d}, J=6.8 \mathrm{~Hz}, 3 \mathrm{H}) .{ }^{19} \mathrm{~F} \mathrm{NMR}\left(376 \mathrm{MHz}, \mathrm{CDCl}_{3}\right) \delta-58.3(\mathrm{~s}, 2 \mathrm{~F}) .{ }^{13} \mathrm{C} \mathrm{NMR}\left(101 \mathrm{MHz}, \mathrm{CDCl}_{3}\right) \delta$ $172.1,170.8,160.0(\mathrm{t}, J=28.3 \mathrm{~Hz}), 135.8,129.8,129.3,128.0,112.0(\mathrm{t}, J=317.1 \mathrm{~Hz}), 54.0,53.2$, 49.9, 38.3, 19.0. MS (ESI): m/z (\%) $407(\mathrm{M}+\mathrm{H})^{+}$. HRMS (ESI): Calcd. for $\mathrm{C}_{21} \mathrm{H}_{22} \mathrm{O}_{4} \mathrm{NF}_{2}: 407.0413$ $(\mathrm{M}+\mathrm{H})^{+}$; Found: $407.0410(\mathrm{M}+\mathrm{H})^{+}$.

\subsection{Preparation of Methyl (S)-3-(2-Bromo-2,2-difluoroacetamido)-4-(((S)-1-methoxy-1-oxo-3-} phenylpropan-2-yl)amino)-4-oxobutanoate $2 \mathrm{f}$.

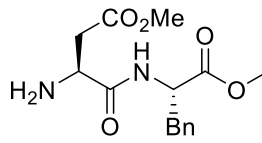

24

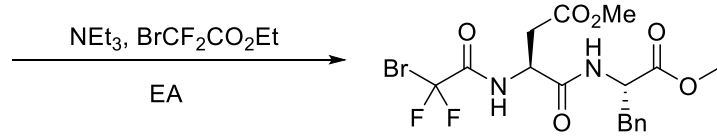

2f, $63 \%$

To a $250 \mathrm{~mL}$ round bottle equipped with a Teflon-coated magnetic stir bar were added protected Aspartame 24 (3.08 g, 10 mmol, 1.0 equiv), $\mathrm{NEt}_{3}$ (2.02 g, $20 \mathrm{mmol}, 20$ equiv), $\mathrm{BrCF}_{2} \mathrm{CO}_{2} \mathrm{Et}$ (4.06 g, $20 \mathrm{mmol}, 2.0$ equiv $)$ and $\mathrm{EA}(50 \mathrm{~mL})$. The reaction was stirred at room temperature overnight. The reaction mixture was diluted with EtOAc, washed with brine three times. The organic layer was dried over $\mathrm{Na}_{2} \mathrm{SO}_{4}$, filtered and concentrated. Compound $2 \mathbf{f}$ (2.93 g, 63\% yield) as a yellow solid (m.p. 109$112^{\circ} \mathrm{C}$ ) was purified with silica gel chromatography (Petroleum ether: Ethyl acetate =4: 1). ${ }^{1} \mathrm{H}$ NMR 
$\left(400 \mathrm{MHz}, \mathrm{CDCl}_{3}\right) \delta 7.67(\mathrm{~d}, J=7.2 \mathrm{~Hz}, 1 \mathrm{H}), 7.33-7.24(\mathrm{~m}, 3 \mathrm{H}), 7.15-7.09(\mathrm{~m}, 2 \mathrm{H}), 6.98(\mathrm{~d}, J=$ $8.0 \mathrm{~Hz}, 1 \mathrm{H}), 4.81-4.72(\mathrm{~m}, 2 \mathrm{H}), 3.72(\mathrm{~s}, 3 \mathrm{H}), 3.70(\mathrm{~s}, 3 \mathrm{H}), 3.17-3.03(\mathrm{~m}, 2 \mathrm{H}), 2.99-2.93(\mathrm{~m}, 1 \mathrm{H})$, $2.72-2.65(\mathrm{~m}, 1 \mathrm{H}) .{ }^{19} \mathrm{~F}$ NMR $\left(376 \mathrm{MHz}, \mathrm{CDCl}_{3}\right) \delta-60.8(\mathrm{~d}, J=161.7 \mathrm{~Hz}, 1 \mathrm{~F}),-61.4(\mathrm{~d}, J=161.7$ $\mathrm{Hz}, 1 \mathrm{~F}) .{ }^{13} \mathrm{C} \mathrm{NMR}\left(126 \mathrm{MHz}, \mathrm{CDCl}_{3}\right) \delta 172.6,171.8,169.1,160.2(\mathrm{t}, J=29.0 \mathrm{~Hz}), 135.9,129.7,129.3$, 127.9, $111.7(\mathrm{t}, J=316.3 \mathrm{~Hz}), 54.3,53.04,53.02,49.9,38.1,35.9 . \mathrm{MS}(\mathrm{ESI}): \mathrm{m} / \mathrm{z}(\%) 465(\mathrm{M}+\mathrm{H})^{+}$. HRMS (ESI): Calcd. for $\mathrm{C}_{17} \mathrm{H}_{20} \mathrm{O}_{6} \mathrm{~N}_{2} \mathrm{BrF}_{2}$ : $465.0467(\mathrm{M}+\mathrm{H})^{+}$; Found: $465.0464(\mathrm{M}+\mathrm{H})^{+}$.

6. Ni-catalyzed four-component carbocarbonylation of alkenes 1 with difluoroalkyl bromides 2 and arylboronic acids 3 .

6.1 General procedure for the Ni-catalyzed four-component carbocarbonylation of alkenes 1 with difluoroalkyl bromides 2 and arylboronic acids 3.
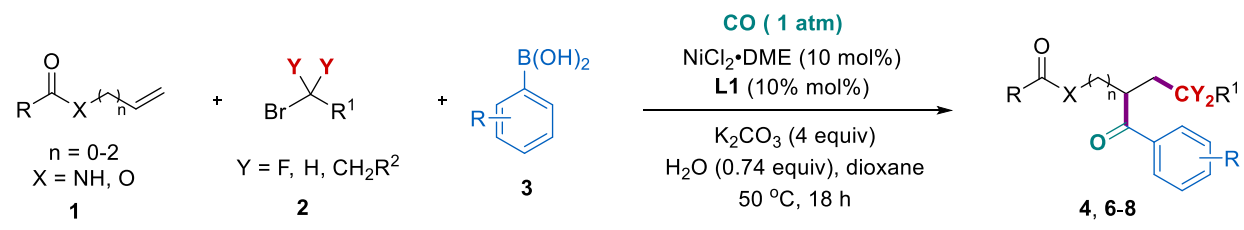

To a $25 \mathrm{~mL}$ of Schlenk tube were added alkene 1 ( $0.3 \mathrm{mmol}, 1.0$ equiv), arylboronic acid 3 ( 2.5 equiv), $\mathrm{NiCl}_{2}$-DME (10 mol\%), 4,4'-diOMe-2,2'-bpy $\mathbf{L 1}$ (10 mol \%) and $\mathrm{K}_{2} \mathrm{CO}_{3}$ (4.0 equiv) under Ar. The reaction mixture was then evacuated and backfilled with Ar (3 times). The reaction mixture was evacuated again and backfilled with carbon monoxide CO (1 atm, balloon), followed by addition of alkyl bromide 2 (2.5 equiv), $\mathrm{H}_{2} \mathrm{O}(0.74$ equiv, $4 \mu \mathrm{L})$ and dioxane $(3.0 \mathrm{~mL})$. The tube was screw-capped and heated to $50{ }^{\circ} \mathrm{C}$ (oil bath). After stirring for $18 \mathrm{~h}$, the reaction mixture was cooled to room temperature, and was diluted with ethyl acetate and $\mathrm{H}_{2} \mathrm{O}$. The resulting mixture was extracted with ethyl acetate, the combined organic layers were washed with brine, dried over $\mathrm{Na}_{2} \mathrm{SO}_{4}$, filtered and concentrated. The residue was purified with silica gel chromatography to give the products $\mathbf{4 , 6 - 8}$. 
6.2 Characterization data for compounds $4,6-8$

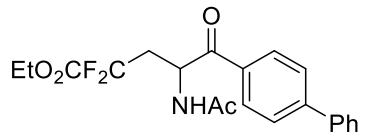

Ethyl 5-([1,1'-biphenyl]-4-yl)-4-acetamido-2,2-difluoro-5-oxopentanoate

was purified with silica gel chromatography (Petroleum ether: Ethyl acetate $=4: 1$ to 2:1). ${ }^{1} \mathrm{H}$ NMR $\left(400 \mathrm{MHz}, \mathrm{CDCl}_{3}\right) \delta 8.09(\mathrm{~d}, J=8.4 \mathrm{~Hz}, 2 \mathrm{H}), 7.69(\mathrm{~d}, J=8.4 \mathrm{~Hz}, 2 \mathrm{H}), 7.60(\mathrm{~d}, J=7.2 \mathrm{~Hz}, 2 \mathrm{H}), 7.45$ $(\mathrm{t}, J=7.2 \mathrm{~Hz}, 2 \mathrm{H}), 7.39$ (t, $J=7.2 \mathrm{~Hz}, 1 \mathrm{H}), 6.83(\mathrm{~d}, J=8.0 \mathrm{~Hz}, 1 \mathrm{H}), 5.95-5.88(\mathrm{~m}, 1 \mathrm{H}), 4.35-4.25$ (m, 2H), $2.92-2.75(\mathrm{~m}, 1 \mathrm{H}), 2.60-2.41(\mathrm{~m}, 1 \mathrm{H}), 2.02(\mathrm{~s}, 3 \mathrm{H}), 1.33(\mathrm{t}, J=7.2 \mathrm{~Hz}, 3 \mathrm{H}) .{ }^{19} \mathrm{~F}$ NMR $\left(376 \mathrm{MHz}, \mathrm{CDCl}_{3}\right) \delta-101.8(\mathrm{ddd}, J=269.6 \mathrm{~Hz}, 16.5 \mathrm{~Hz}, 14.3 \mathrm{~Hz}, 1 \mathrm{~F}),-103.4(\mathrm{dt}, J=269.6 \mathrm{~Hz}, 16.5$ $\mathrm{Hz}, 1 \mathrm{~F}) .{ }^{13} \mathrm{C} \mathrm{NMR}\left(101 \mathrm{MHz}, \mathrm{CDCl}_{3}\right) \delta 196.8,170.3,163.9$ (t, $\left.J=32.2 \mathrm{~Hz}\right), 147.2,139.9,133.0,129.9$, 129.5, 129.0, 128.0, 127.7, $115.5(\mathrm{t}, J=253.4 \mathrm{~Hz}), 63.7,48.7(\mathrm{t}, J=3.5 \mathrm{~Hz}), 37.1(\mathrm{t}, J=23.3 \mathrm{~Hz})$, 23.4, 14.3. MS (DART): $\mathrm{m} / \mathrm{z}(\%) 390(\mathrm{M}+\mathrm{H})^{+}$. HRMS (DART): Calcd. for $\mathrm{C}_{21} \mathrm{H}_{22} \mathrm{O}_{4} \mathrm{NF}_{2}: 390.1511$ $(\mathrm{M}+\mathrm{H})^{+}$; Found: $390.1513(\mathrm{M}+\mathrm{H})^{+}$.

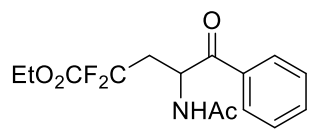

Ethyl 4-acetamido-2,2-difluoro-5-oxo-5-phenylpentanoate (4b). Compound

4b (64 mg, 68\% yield) as a yellow solid (m.p. 81-84 ${ }^{\circ} \mathrm{C}$ ) was purified with silica gel chromatography (Petroleum ether: Ethyl acetate $=4: 1$ to $2: 1) .{ }^{1} \mathrm{H}$ NMR $\left(400 \mathrm{MHz}, \mathrm{CDCl}_{3}\right) \delta 7.99$ $(\mathrm{d}, J=7.6 \mathrm{~Hz}, 2 \mathrm{H}), 7.59$ (t, $J=7.6 \mathrm{~Hz}, 1 \mathrm{H}), 7.47$ (t, $J=7.6 \mathrm{~Hz}, 2 \mathrm{H}), 6.70(\mathrm{~d}, J=8.4 \mathrm{~Hz}, 1 \mathrm{H}), 5.88-$ $5.81(\mathrm{~m}, 1 \mathrm{H}), 4.34-4.21(\mathrm{~m}, 2 \mathrm{H}), 2.85-2.70(\mathrm{~m}, 1 \mathrm{H}), 2.53-2.37(\mathrm{~m}, 1 \mathrm{H}), 1.98(\mathrm{~s}, 3 \mathrm{H}), 1.30(\mathrm{t}, J=$ $7.2 \mathrm{~Hz}, 3 \mathrm{H}) .{ }^{19} \mathrm{~F}$ NMR $\left(376 \mathrm{MHz}, \mathrm{CDCl}_{3}\right) \delta-101.9(\mathrm{ddd}, J=270.0 \mathrm{~Hz}, 16.9 \mathrm{~Hz}, 13.9 \mathrm{~Hz}, 1 \mathrm{~F}),-103.5$ (dt, $J=270.0 \mathrm{~Hz}, 16.5 \mathrm{~Hz}, 1 \mathrm{~F}) .{ }^{13} \mathrm{C} \mathrm{NMR}\left(101 \mathrm{MHz}, \mathrm{CDCl}_{3}\right) \delta 197.3,170.3,163.9(\mathrm{t}, J=32.5 \mathrm{~Hz})$, 134.7, 134.4, 129.5, 129.3, $115.4(\mathrm{t}, J=253.6 \mathrm{~Hz}), 63.8,48.8(\mathrm{t}, J=3.6 \mathrm{~Hz}), 37.1(\mathrm{t}, J=23.3 \mathrm{~Hz})$, 23.5, 14.3. MS (ESI): m/z (\%) $314(\mathrm{M}+\mathrm{H})^{+}$. HRMS (ESI): Calcd. for $\mathrm{C}_{15} \mathrm{H}_{18} \mathrm{O}_{4} \mathrm{NF}_{2}: 314.1198(\mathrm{M}+\mathrm{H})^{+}$; Found: $314.1198(\mathrm{M}+\mathrm{H})^{+}$.

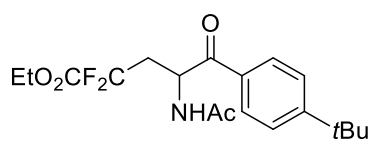

Ethyl 4-acetamido-5-(4-(tert-butyl)phenyl)-2,2-difluoro-5-oxopentanoate

(4c). Compound 4c (94 mg, 85\% yield) as a colorless oil was purified with silica gel chromatography (Petroleum ether: Ethyl acetate $=4: 1$ to 2: 1). ${ }^{1} \mathrm{H} \mathrm{NMR}\left(400 \mathrm{MHz}, \mathrm{CDCl}_{3}\right)$ $\delta 7.96(\mathrm{~d}, J=8.0 \mathrm{~Hz}, 2 \mathrm{H}), 7.51(\mathrm{~d}, J=8.0 \mathrm{~Hz}, 2 \mathrm{H}), 6.46(\mathrm{~d}, J=8.0 \mathrm{~Hz}, 1 \mathrm{H}), 5.90-5.82(\mathrm{~m}, 1 \mathrm{H}), 4.31$ $(\mathrm{q}, J=6.4 \mathrm{~Hz}, 2 \mathrm{H}), 2.86-2.70(\mathrm{~m}, 1 \mathrm{H}), 2.53-2.36(\mathrm{~m}, 1 \mathrm{H}), 2.01(\mathrm{~s}, 3 \mathrm{H}), 1.37-1.30(\mathrm{~m}, 12 \mathrm{H}) .{ }^{19} \mathrm{~F}$ 
NMR $\left(376 \mathrm{MHz}, \mathrm{CDCl}_{3}\right) \delta-101.8(\mathrm{ddd}, J=270.7 \mathrm{~Hz}, 16.2 \mathrm{~Hz}, 14.3 \mathrm{~Hz}, 1 \mathrm{~F}),-103.4$ (dt, $J=270.7 \mathrm{~Hz}$, $16.2 \mathrm{~Hz}, 1 \mathrm{~F}) .{ }^{13} \mathrm{C} \mathrm{NMR}\left(101 \mathrm{MHz}, \mathrm{CDCl}_{3}\right) \delta 196.8,170.2,164.0(\mathrm{t}, J=32.0 \mathrm{~Hz}), 158.8,131.6,129.4$, 126.6, 115.5 (t, $J=254.6 \mathrm{~Hz}), 63.8,48.8$ (t, $J=3.9 \mathrm{~Hz}), 37.5$ (t, $J=23.4 \mathrm{~Hz}), 35.8,31.6,23.6,14.4$. MS (ESI): m/z (\%) $370(\mathrm{M}+\mathrm{H})^{+}$. HRMS (ESI): Calcd. for $\mathrm{C}_{19} \mathrm{H}_{26} \mathrm{O}_{4} \mathrm{NF}_{2}$ : $370.1824(\mathrm{M}+\mathrm{H})^{+}$; Found: $370.1824(\mathrm{M}+\mathrm{H})^{+}$.

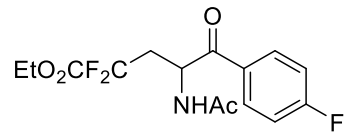

Ethyl 4-acetamido-2,2-difluoro-5-(4-fluorophenyl)-5-oxopentanoate (4d).

Compound 4d (83 mg, 83\% yield) as a white solid (m.p. 116-118 ${ }^{\circ} \mathrm{C}$ ) was purified with silica gel chromatography (Petroleum ether: Ethyl acetate $=5: 1$ to $3: 1) .{ }^{1} \mathrm{H}$ NMR $(400$ $\left.\mathrm{MHz}, \mathrm{CDCl}_{3}\right) \delta 8.05(\mathrm{dd}, J=8.8 \mathrm{~Hz}, 5.2 \mathrm{~Hz}, 2 \mathrm{H}), 7.15(\mathrm{t}, J=8.8 \mathrm{~Hz}, 2 \mathrm{H}), 6.64(\mathrm{~d}, J=8.4 \mathrm{~Hz}, 1 \mathrm{H})$, $5.86-5.78(\mathrm{~m}, 1 \mathrm{H}), 4.33-4.26(\mathrm{~m}, 2 \mathrm{H}), 2.82-2.67(\mathrm{~m}, 1 \mathrm{H}), 2.53-2.37(\mathrm{~m}, 1 \mathrm{H}), 1.99(\mathrm{~s}, 3 \mathrm{H}), 1.32$ $(\mathrm{t}, J=7.2 \mathrm{~Hz}, 3 \mathrm{H}) .{ }^{19} \mathrm{~F} \mathrm{NMR}\left(376 \mathrm{MHz}, \mathrm{CDCl}_{3}\right) \delta-101.9(\mathrm{ddd}, J=270.7 \mathrm{~Hz}, 16.9 \mathrm{~Hz}, 13.5 \mathrm{~Hz}, 1 \mathrm{~F})$, $102.8(\mathrm{~m}, 1 \mathrm{~F}),-103.6(\mathrm{dt}, J=270.7 \mathrm{~Hz}, 16.9 \mathrm{~Hz}, 1 \mathrm{~F}) .{ }^{13} \mathrm{C} \mathrm{NMR}\left(101 \mathrm{MHz}, \mathrm{CDCl}_{3}\right) \delta 195.8,170.5$, $166.9(\mathrm{~d}, J=258.3 \mathrm{~Hz}), 163.9(\mathrm{t}, J=32.0 \mathrm{~Hz}), 132.2(\mathrm{~d}, J=9.6 \mathrm{~Hz}), 130.8(\mathrm{~d}, J=2.9 \mathrm{~Hz}), 116.8(\mathrm{~d}$, $J=22.1 \mathrm{~Hz}), 115.4(\mathrm{t}, J=254.3 \mathrm{~Hz}), 63.9,48.7(\mathrm{t}, J=3.8 \mathrm{~Hz}), 37.1(\mathrm{t}, J=23.3 \mathrm{~Hz}), 23.5,14.4 . \mathrm{MS}$ (DART): $\mathrm{m} / \mathrm{z}(\%) 332(\mathrm{M}+\mathrm{H})^{+}$. HRMS (DART): Calcd. for $\mathrm{C}_{15} \mathrm{H}_{17} \mathrm{O}_{4} \mathrm{NF}_{3}: 332.1104(\mathrm{M}+\mathrm{H})^{+}$; Found: $332.1105(\mathrm{M}+\mathrm{H})^{+}$.

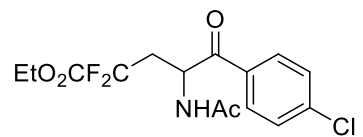

Ethyl 4-acetamido-5-(4-chlorophenyl)-2,2-difluoro-5-oxopentanoate (4e). Compound 4 e (84 mg, 80\% yield) as a white solid (m.p. $132-135{ }^{\circ} \mathrm{C}$ ) was purified with silica gel chromatography (Petroleum ether: Ethyl acetate $=5: 1$ to 3:1). ${ }^{1} \mathrm{H}$ NMR (400 $\left.\mathrm{MHz}, \mathrm{CDCl}_{3}\right) \delta 7.96(\mathrm{~d}, J=8.4 \mathrm{~Hz}, 2 \mathrm{H}), 7.48(\mathrm{~d}, J=8.4 \mathrm{~Hz}, 2 \mathrm{H}), 6.36(\mathrm{~d}, J=8.0 \mathrm{~Hz}, 1 \mathrm{H}), 5.86-5.76$ $(\mathrm{m}, 1 \mathrm{H}), 4.31(\mathrm{q}, J=7.2 \mathrm{~Hz}, 2 \mathrm{H}), 2.83-2.68(\mathrm{~m}, 1 \mathrm{H}), 2.53-2.37(\mathrm{~m}, 1 \mathrm{H}), 2.00(\mathrm{~s}, 3 \mathrm{H}), 1.35(\mathrm{t}, J=$ $7.2 \mathrm{~Hz}, 3 \mathrm{H}) .{ }^{19} \mathrm{~F}$ NMR $\left(376 \mathrm{MHz}, \mathrm{CDCl}_{3}\right) \delta-101.8(\mathrm{dt}, J=270.7 \mathrm{~Hz}, 15.4 \mathrm{~Hz}, 1 \mathrm{~F}),-103.4(\mathrm{dt}, J=$ 270.7 Hz, 16.2 Hz, 1F). ${ }^{13} \mathrm{C}$ NMR (101 MHz, $\left.\mathrm{CDCl}_{3}\right) \delta 196.2,170.2,163.9(\mathrm{t}, J=32.1 \mathrm{~Hz}), 141.3$, 132.8, 130.8, 129.9, $115.4(\mathrm{t}, J=253.5 \mathrm{~Hz}), 63.9,48.8(\mathrm{t}, J=3.2 \mathrm{~Hz}), 37.1(\mathrm{t}, J=23.3 \mathrm{~Hz}), 23.5,14.4$. MS (DART): m/z (\%) $348(\mathrm{M}+\mathrm{H})^{+}$. HRMS (DART): Calcd. for $\mathrm{C}_{15} \mathrm{H}_{17} \mathrm{O}_{4} \mathrm{NF}_{2}: 348.0809(\mathrm{M}+\mathrm{H})^{+}$; Found: $348.0810(\mathrm{M}+\mathrm{H})^{+}$. 


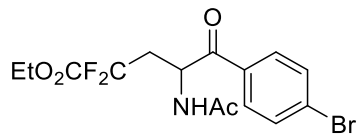

Ethyl 4-acetamido-5-(4-bromophenyl)-2,2-difluoro-5-oxopentanoate (4f).

Compound $4 f\left(62 \mathrm{mg}, 53 \%\right.$ yield) as a white solid (m.p. $141-143{ }^{\circ} \mathrm{C}$ ) was purified with silica gel chromatography (Petroleum ether: Ethyl acetate $=5: 1$ to $3: 1) .{ }^{1} \mathrm{H}$ NMR $(400$ MHz, DMSO-d 6$) \delta 8.70(\mathrm{~d}, J=8.8 \mathrm{~Hz}, 1 \mathrm{H}), 7.89(\mathrm{~d}, J=8.8 \mathrm{~Hz}, 2 \mathrm{H}), 7.78(\mathrm{~d}, J=8.8 \mathrm{~Hz}, 2 \mathrm{H}), 5.52-$ $5.43(\mathrm{~m}, 1 \mathrm{H}), 4.37-4.22(\mathrm{~m}, 2 \mathrm{H}), 2.90-2.73(\mathrm{~m}, 1 \mathrm{H}), 2.64-2.45(\mathrm{~m}, 1 \mathrm{H}), 1.78(\mathrm{~s}, 3 \mathrm{H}), 1.28(\mathrm{t}, J=$ $7.2 \mathrm{~Hz}, 3 \mathrm{H}) .{ }^{19} \mathrm{~F}$ NMR $\left(376 \mathrm{MHz}, \mathrm{CD}_{2} \mathrm{Cl}_{2}\right) \delta-102.6(\mathrm{ddd}, J=268.8 \mathrm{~Hz}, 17.7 \mathrm{~Hz}, 14.3 \mathrm{~Hz}, 1 \mathrm{~F}),-103.9$ (dt, $J=268.8 \mathrm{~Hz}, 16.5 \mathrm{~Hz}, 1 \mathrm{~F}) .{ }^{13} \mathrm{C}$ NMR $\left(101 \mathrm{MHz}, \mathrm{DMSO}_{6}\right) \delta 196.5,169.8,163.8$ (t, $\left.J=32.3 \mathrm{~Hz}\right)$, $134.5,132.7,131.2,128.5,116.3(\mathrm{t}, J=251.8 \mathrm{~Hz}), 64.0,48.5(\mathrm{t}, J=3.0 \mathrm{~Hz}), 35.3(\mathrm{t}, J=23.1 \mathrm{~Hz})$, 23.1, 14.5. MS (DART): $\mathrm{m} / \mathrm{z}(\%) 392(\mathrm{M}+\mathrm{H})^{+}$. HRMS (DART): Calcd. for $\mathrm{C}_{15} \mathrm{H}_{17} \mathrm{O}_{4} \mathrm{NBrF}_{2}: 392.0304$ $(\mathrm{M}+\mathrm{H})^{+}$; Found: $392.0304(\mathrm{M}+\mathrm{H})^{+}$.

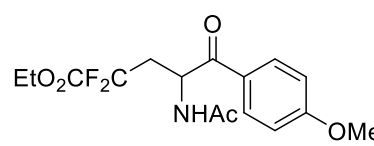

Ethyl 4-acetamido-2,2-difluoro-5-(4-methoxyphenyl)-5-oxopentanoate

$\mathrm{mg}, 85 \%$ yield) as a yellow solid (m.p. $74-79{ }^{\circ} \mathrm{C}$ ) was purified with silica gel chromatography (Petroleum ether: Ethyl acetate $=4: 1$ to $2: 1) .{ }^{1} \mathrm{H} \mathrm{NMR}\left(400 \mathrm{MHz}, \mathrm{CDCl}_{3}\right) \delta 8.00(\mathrm{~d}, J=8.8 \mathrm{~Hz}, 2 \mathrm{H})$, $6.95(\mathrm{~d}, J=8.8 \mathrm{~Hz}, 2 \mathrm{H}), 6.55(\mathrm{~d}, J=8.4 \mathrm{~Hz}, 1 \mathrm{H}), 5.88-5.78(\mathrm{~m}, 1 \mathrm{H}), 4.36-4.22(\mathrm{~m}, 2 \mathrm{H}), 3.86(\mathrm{~s}$, $3 \mathrm{H}), 2.81-2.66(\mathrm{~m}, 1 \mathrm{H}), 2.51-2.35(\mathrm{~m}, 1 \mathrm{H}), 1.98(\mathrm{~s}, 3 \mathrm{H}), 1.32(\mathrm{t}, J=7.2 \mathrm{~Hz}, 3 \mathrm{H}) .{ }^{19} \mathrm{~F}$ NMR $(376$ $\left.\mathrm{MHz}, \mathrm{CDCl}_{3}\right) \delta-101.7$ (ddd, $\left.J=270.7 \mathrm{~Hz}, 16.2 \mathrm{~Hz}, 14.3 \mathrm{~Hz}, 1 \mathrm{~F}\right),-103.4$ (dt, $J=270.7 \mathrm{~Hz}, 16.2 \mathrm{~Hz}$, 1F). ${ }^{13} \mathrm{C} \mathrm{NMR}\left(101 \mathrm{MHz}, \mathrm{CDCl}_{3}\right) \delta 195.6,170.2,165.0,164.0(\mathrm{t}, J=32.4 \mathrm{~Hz}), 131.8,127.1,115.5(\mathrm{t}$, $J=253.5 \mathrm{~Hz}), 114.8,63.8,56.1,48.3(\mathrm{t}, J=3.4 \mathrm{~Hz}), 37.6(\mathrm{t}, J=23.3 \mathrm{~Hz}), 23.6,14.4 . \mathrm{MS}(\mathrm{ESI}): \mathrm{m} / \mathrm{z}$ (\%) $344(\mathrm{M}+\mathrm{H})^{+}$. HRMS (ESI): Calcd. for $\mathrm{C}_{16} \mathrm{H}_{20} \mathrm{O}_{5} \mathrm{NF}_{2}: 344.1304(\mathrm{M}+\mathrm{H})^{+}$; Found: $344.1303(\mathrm{M}+\mathrm{H})^{+}$.

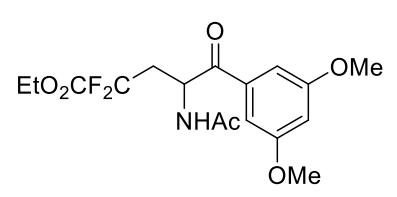

Ethyl 4-acetamido-5-(3,5-dimethoxyphenyl)-2,2-difluoro-5oxopentanoate (4h). The reaction was conducted with L7 as the ligand. Compound $4 \mathrm{~h}\left(85 \mathrm{mg}, 76 \%\right.$ yield) as a white solid (m.p. 123-129 $\left.{ }^{\circ} \mathrm{C}\right)$ was purified with silica gel chromatography (Petroleum ether: Ethyl acetate $=4: 1$ to $1: 1) .{ }^{1} \mathrm{H}$ NMR $(400$ $\left.\mathrm{MHz}, \mathrm{CDCl}_{3}\right) \delta 7.14(\mathrm{~s}, 2 \mathrm{H}), 6.68(\mathrm{~s}, 1 \mathrm{H}), 6.50(\mathrm{~d}, J=8.4 \mathrm{~Hz}, 1 \mathrm{H}), 5.83-5.74(\mathrm{~m}, 1 \mathrm{H}), 4.31(\mathrm{q}, J=$ $7.2 \mathrm{~Hz}, 2 \mathrm{H}), 3.82(\mathrm{~s}, 6 \mathrm{H}), 2.86-2.71(\mathrm{~m}, 1 \mathrm{H}), 2.51-2.34(\mathrm{~m}, 1 \mathrm{H}), 2.01(\mathrm{~s}, 3 \mathrm{H}), 1.34(\mathrm{t}, J=7.2 \mathrm{~Hz}$, 3H). ${ }^{19} \mathrm{~F}$ NMR $\left(376 \mathrm{MHz}, \mathrm{CDCl}_{3}\right) \delta-101.2(\mathrm{dt}, J=271.5 \mathrm{~Hz}, 14.7 \mathrm{~Hz}, 1 \mathrm{~F}),-103.6(\mathrm{dt}, J=271.5 \mathrm{~Hz}$, $16.5 \mathrm{~Hz}, 1 \mathrm{~F}) .{ }^{13} \mathrm{C} \mathrm{NMR}\left(101 \mathrm{MHz}, \mathrm{CDCl}_{3}\right) \delta 196.9,170.3,163.9(\mathrm{t}, J=32.2 \mathrm{~Hz}), 161.6,136.1,115.5$ 
$(\mathrm{dd}, J=254.7 \mathrm{~Hz}, 252.7 \mathrm{~Hz}), 107.4,106.9,63.8,56.2,49.2(\mathrm{t}, J=3.5 \mathrm{~Hz}), 37.3(\mathrm{t}, J=23.3 \mathrm{~Hz}), 23.5$, 14.4. MS (DART): $\mathrm{m} / \mathrm{z}(\%) 374(\mathrm{M}+\mathrm{H})^{+}$. HRMS (DART): Calcd. for $\mathrm{C}_{17} \mathrm{H}_{22} \mathrm{O}_{6} \mathrm{NF}_{2}: 374.1410(\mathrm{M}+\mathrm{H})^{+}$; Found: $374.1412(\mathrm{M}+\mathrm{H})^{+}$.

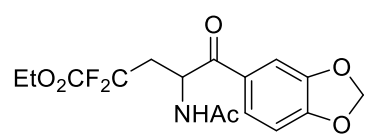

Ethyl

4-acetamido-5-(benzo $[d][1,3]$ dioxol-5-yl)-2,2-difluoro-5-

oxopentanoate (4i). The reaction was conducted with $\mathbf{L 7}$ as the ligand. Compound 4i (84 mg, 78\% yield) as a yellow oil was purified with silica gel chromatography (Petroleum ether: Ethyl acetate $=4: 1$ to $1: 1) .{ }^{1} \mathrm{H} \mathrm{NMR}\left(400 \mathrm{MHz}, \mathrm{CDCl}_{3}\right) \delta 7.66(\mathrm{~d}, J=8.0 \mathrm{~Hz}, 1 \mathrm{H})$, $7.45(\mathrm{~s}, 1 \mathrm{H}), 6.87(\mathrm{~d}, J=8.0 \mathrm{~Hz}, 1 \mathrm{H}), 6.52(\mathrm{~d}, J=8.4 \mathrm{~Hz}, 1 \mathrm{H}), 6.05(\mathrm{~s}, 2 \mathrm{H}), 5.82-5.75(\mathrm{~m}, 1 \mathrm{H}), 4.30$ (q, $J=7.2 \mathrm{~Hz}, 2 \mathrm{H}), 2.80-2.65(\mathrm{~m}, 1 \mathrm{H}), 2.50-2.35(\mathrm{~m}, 1 \mathrm{H}), 1.99(\mathrm{~s}, 3 \mathrm{H}), 1.33(\mathrm{t}, J=7.2 \mathrm{~Hz}, 3 \mathrm{H})$. ${ }^{19} \mathrm{~F}$ NMR $\left(376 \mathrm{MHz}, \mathrm{CDCl}_{3}\right) \delta-101.6(\mathrm{ddd}, J=271.5 \mathrm{~Hz}, 16.5 \mathrm{~Hz}, 13.9 \mathrm{~Hz}, 1 \mathrm{~F}),-103.5$ (dt, $J=271.5$ $\mathrm{Hz}, 16.5 \mathrm{~Hz}, 1 \mathrm{~F}) .{ }^{13} \mathrm{C} \mathrm{NMR}\left(101 \mathrm{MHz}, \mathrm{CDCl}_{3}\right) \delta 195.3,170.2,164.0(\mathrm{t}, J=32.2 \mathrm{~Hz}), 153.4,149.1$, 128.9, 126.1, $115.5(\mathrm{dd}, J=254.3 \mathrm{~Hz}, 252.9 \mathrm{~Hz}), 108.92,108.86,102.7,63.8,48.4(\mathrm{t}, J=3.8 \mathrm{~Hz})$, $37.6(\mathrm{t}, J=23.1 \mathrm{~Hz}), 23.6,14.4$. MS (ESI): $\mathrm{m} / \mathrm{z}(\%) 358(\mathrm{M}+\mathrm{H})^{+}$. HRMS (ESI): Calcd. for $\mathrm{C}_{16} \mathrm{H}_{18} \mathrm{O}_{6} \mathrm{NF}_{2}: 358.1097(\mathrm{M}+\mathrm{H})^{+}$; Found: $358.1097(\mathrm{M}+\mathrm{H})^{+}$.

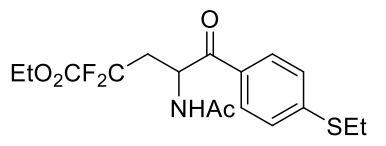

Ethyl 4-acetamido-5-(4-(ethylthio)phenyl)-2,2-difluoro-5-oxopentanoate

(4j). The reaction was conducted with $\mathbf{L} 7$ as the ligand. Compound $\mathbf{4 j}$ ( $83 \mathrm{mg}$, $74 \%$ yield) as a white solid (m.p. $132-135^{\circ} \mathrm{C}$ ) was purified with silica gel chromatography (Petroleum ether: Ethyl acetate $=4: 1$ to 3: 1). ${ }^{1} \mathrm{H}$ NMR $\left(400 \mathrm{MHz}, \mathrm{CDCl}_{3}\right) \delta 7.86(\mathrm{~d}, J=8.4 \mathrm{~Hz}, 2 \mathrm{H}), 7.25(\mathrm{~d}, J$ $=8.4 \mathrm{~Hz}, 2 \mathrm{H}), 6.71(\mathrm{~d}, J=8.4 \mathrm{~Hz}, 1 \mathrm{H}), 5.82-5.73(\mathrm{~m}, 1 \mathrm{H}), 4.32-4.18(\mathrm{~m}, 2 \mathrm{H}), 2.97$ (q, $J=7.2 \mathrm{~Hz}$, 2H), $2.79-2.64(\mathrm{~m}, 1 \mathrm{H}), 2.49-2.32(\mathrm{~m}, 1 \mathrm{H}), 1.94(\mathrm{~s}, 3 \mathrm{H}), 1.32(\mathrm{t}, J=7.6 \mathrm{~Hz}, 3 \mathrm{H}), 1.28(\mathrm{t}, J=7.2$ $\mathrm{Hz}, 3 \mathrm{H}) .{ }^{19} \mathrm{~F}$ NMR $\left(376 \mathrm{MHz}, \mathrm{CDCl}_{3}\right) \delta-101.6(\mathrm{dt}, J=270.7 \mathrm{~Hz}, 15.0 \mathrm{~Hz}, 1 \mathrm{~F}),-103.5(\mathrm{dt}, J=270.7$ $\mathrm{Hz}, 16.5 \mathrm{~Hz}, 1 \mathrm{~F}) .{ }^{13} \mathrm{C} \mathrm{NMR}\left(101 \mathrm{MHz}, \mathrm{CDCl}_{3}\right) \delta 196.1,170.2,163.9$ (t, $\left.J=32.3 \mathrm{~Hz}\right), 147.1,130.6$, 129.7, 126.6, $115.4(\mathrm{t}, J=253.3 \mathrm{~Hz}), 63.7,48.4,37.2(\mathrm{t}, J=23.2 \mathrm{~Hz}), 26.2,23.4,14.30,14.27 . \mathrm{MS}$ (DART): $\mathrm{m} / \mathrm{z}(\%) 374(\mathrm{M}+\mathrm{H})^{+}$. HRMS (DART): Calcd. for $\mathrm{C}_{17} \mathrm{H}_{22} \mathrm{O}_{4} \mathrm{NF}_{2} \mathrm{~S}: 374.1232(\mathrm{M}+\mathrm{H})^{+}$; Found: $374.1231(\mathrm{M}+\mathrm{H})^{+}$. 


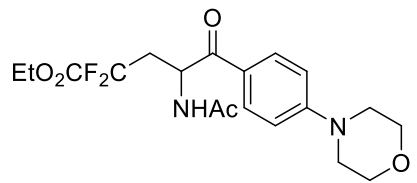

Ethyl

4-acetamido-2,2-difluoro-5-(4-morpholinophenyl)-5-

oxopentanoate (4k). The reaction was conducted with $\mathbf{L} 7$ as the ligand.

Compound $4 \mathbf{k}\left(60 \mathrm{mg}, 50 \%\right.$ yield) as a brown solid (m.p. 106-110 $\left.{ }^{\circ} \mathrm{C}\right)$ was

purified with silica gel chromatography (Petroleum ether: Ethyl acetate $=2: 1$ to 1:2). ${ }^{1} \mathrm{H}$ NMR (400 $\left.\mathrm{MHz}, \mathrm{CDCl}_{3}\right) \delta 7.93(\mathrm{~d}, J=8.8 \mathrm{~Hz}, 2 \mathrm{H}), 6.85(\mathrm{~d}, J=8.8 \mathrm{~Hz}, 2 \mathrm{H}), 6.64(\mathrm{~d}, J=8.4 \mathrm{~Hz}, 1 \mathrm{H}), 5.85-5.77$ (m, 1H), $4.32-4.23(\mathrm{~m}, 2 \mathrm{H}), 3.82(\mathrm{t}, J=4.4 \mathrm{~Hz}, 4 \mathrm{H}), 3.31(\mathrm{t}, J=4.4 \mathrm{~Hz}, 4 \mathrm{H}), 2.79-2.63(\mathrm{~m}, 1 \mathrm{H})$, $2.49-2.33(\mathrm{~m}, 1 \mathrm{H}), 1.97(\mathrm{~s}, 3 \mathrm{H}), 1.31(\mathrm{t}, J=7.2 \mathrm{~Hz}, 3 \mathrm{H}) .{ }^{19} \mathrm{~F}$ NMR $\left(376 \mathrm{MHz}, \mathrm{CDCl}_{3}\right) \delta-101.8(\mathrm{dt}$, $J=270.3 \mathrm{~Hz}, 15.0 \mathrm{~Hz}, 1 \mathrm{~F}),-103.4(\mathrm{dt}, J=270.3 \mathrm{~Hz}, 16.2 \mathrm{~Hz}, 1 \mathrm{~F}) .{ }^{13} \mathrm{C} \mathrm{NMR}\left(101 \mathrm{MHz}, \mathrm{CDCl}_{3}\right) \delta$ 194.9, 170.1, $164.0(\mathrm{t}, J=32.2 \mathrm{~Hz}), 155.3,131.6,124.3,115.5(\mathrm{t}, J=253.4 \mathrm{~Hz}), 113.7,66.9,63.7$, 47.9, 47.6, $37.7(\mathrm{t}, J=23.4 \mathrm{~Hz}), 23.6,14.3 . \mathrm{MS}(\mathrm{DART}): \mathrm{m} / \mathrm{z}(\%) 399(\mathrm{M}+\mathrm{H})^{+}$. HRMS (DART): Calcd. for $\mathrm{C}_{19} \mathrm{H}_{25} \mathrm{O}_{5} \mathrm{~N}_{2} \mathrm{~F}_{2}: 399.1726(\mathrm{M}+\mathrm{H})^{+}$; Found: $399.1724(\mathrm{M}+\mathrm{H})^{+}$.

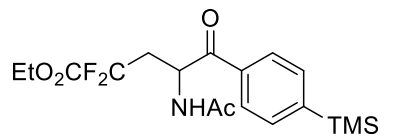

Ethyl

4-acetamido-2,2-difluoro-5-oxo-5-(4-

(trimethylsilyl)phenyl)pentanoate (4I). Compound $4 \mathrm{l}$ (59 $\mathrm{mg}, 51 \%$ yield) as a yellow oil was purified with silica gel chromatography (Petroleum ether: Ethyl acetate $=5: 1$ to 3: 1). ${ }^{1} \mathrm{H}$ NMR $\left(400 \mathrm{MHz}, \mathrm{CDCl}_{3}\right) \delta 7.95(\mathrm{~d}, J=8.0 \mathrm{~Hz}, 2 \mathrm{H}), 7.64(\mathrm{~d}, J=8.0 \mathrm{~Hz}, 2 \mathrm{H}), 6.61(\mathrm{~d}, J=8.0$ $\mathrm{Hz}, 1 \mathrm{H}), 5.90-5.82(\mathrm{~m}, 1 \mathrm{H}), 4.35-4.23(\mathrm{~m}, 2 \mathrm{H}), 2.86-2.71(\mathrm{~m}, 1 \mathrm{H}), 2.53-2.37(\mathrm{~m}, 1 \mathrm{H}), 2.00(\mathrm{~s}$, $3 \mathrm{H}), 1.32(\mathrm{t}, J=7.2 \mathrm{~Hz}, 3 \mathrm{H}), 0.28(\mathrm{~s}, 9 \mathrm{H}) .{ }^{19} \mathrm{~F} \mathrm{NMR}\left(376 \mathrm{MHz}, \mathrm{CDCl}_{3}\right) \delta-101.4(\mathrm{ddd}, J=271.5 \mathrm{~Hz}$, $16.5 \mathrm{~Hz}, 14.3 \mathrm{~Hz}, 1 \mathrm{~F}),-103.4(\mathrm{dt}, J=271.5 \mathrm{~Hz}, 16.5 \mathrm{~Hz}, 1 \mathrm{~F}) .{ }^{13} \mathrm{C}$ NMR $\left(101 \mathrm{MHz}, \mathrm{CDCl}_{3}\right) \delta 197.5$, 170.2, $164.0(\mathrm{t}, J=32.1 \mathrm{~Hz}), 149.4,134.41,134.36,128.2,115.5(\mathrm{t}, J=254.3 \mathrm{~Hz}), 63.8,48.9(\mathrm{t}, J=$ $3.4 \mathrm{~Hz}), 37.2(\mathrm{t}, J=23.4 \mathrm{~Hz}), 23.5,14.4,0.9 . \mathrm{MS}(\mathrm{DART}): \mathrm{m} / \mathrm{z}(\%) 386(\mathrm{M}+\mathrm{H})^{+}$. HRMS (DART): Calcd. for $\mathrm{C}_{18} \mathrm{H}_{26} \mathrm{O}_{4} \mathrm{NF}_{2} \mathrm{Si}: 386.1594(\mathrm{M}+\mathrm{H})^{+}$; Found: $386.1596(\mathrm{M}+\mathrm{H})^{+}$.

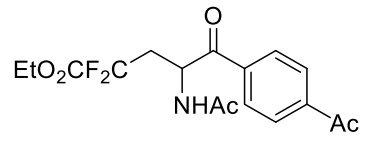

Ethyl 4-acetamido-5-(4-acetylphenyl)-2,2-difluoro-5-oxopentanoate (4m). white solid (m.p. 121-123 ${ }^{\circ} \mathrm{C}$ ) was purified with silica gel chromatography (Petroleum ether: Ethyl acetate $=4: 1$ to 2: 1$) .{ }^{1} \mathrm{H} \mathrm{NMR}\left(400 \mathrm{MHz}, \mathrm{CDCl}_{3}\right) \delta 8.06(\mathrm{~d}, J=8.4 \mathrm{~Hz}, 2 \mathrm{H}), 8.02(\mathrm{~d}, J=8.4 \mathrm{~Hz}, 2 \mathrm{H})$, $6.65(\mathrm{~d}, J=8.0 \mathrm{~Hz}, 1 \mathrm{H}), 5.85-5.76(\mathrm{~m}, 1 \mathrm{H}), 4.29(\mathrm{q}, J=7.2 \mathrm{~Hz}, 2 \mathrm{H}), 2.86-2.70(\mathrm{~m}, 1 \mathrm{H}), 2.62(\mathrm{~s}$, $3 \mathrm{H}), 2.55-2.39(\mathrm{~m}, 1 \mathrm{H}), 1.98(\mathrm{~s}, 3 \mathrm{H}), 1.32(\mathrm{t}, J=7.2 \mathrm{~Hz}, 3 \mathrm{H}) .{ }^{19} \mathrm{~F}$ NMR $\left(376 \mathrm{MHz}, \mathrm{CDCl}_{3}\right) \delta-101.9$ 
(ddd, $J=270.0 \mathrm{~Hz}, 17.7 \mathrm{~Hz}, 13.5 \mathrm{~Hz}, 1 \mathrm{~F}),-103.7$ (dt, $J=270.0 \mathrm{~Hz}, 16.5 \mathrm{~Hz}, 1 \mathrm{~F}) .{ }^{13} \mathrm{C} \mathrm{NMR}(101 \mathrm{MHz}$, $\left.\mathrm{CDCl}_{3}\right) \delta 197.9,196.9,170.3,163.9(\mathrm{t}, J=32.1 \mathrm{~Hz}), 141.3,137.9,129.5,129.2,115.4(\mathrm{t}, J=254.2$ $\mathrm{Hz}), 63.9,49.3(\mathrm{t}, J=3.5 \mathrm{~Hz}), 36.7(\mathrm{t}, J=23.3 \mathrm{~Hz}), 27.4,23.4,14.4$. MS (DART): m/z (\%) $356(\mathrm{M}+\mathrm{H})^{+}$. HRMS (DART): Calcd. for $\mathrm{C}_{17} \mathrm{H}_{20} \mathrm{O}_{5} \mathrm{NF}_{2}: 356.1304(\mathrm{M}+\mathrm{H})^{+}$; Found: $356.1302(\mathrm{M}+\mathrm{H})^{+}$.

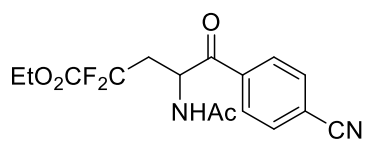

Ethyl 4-acetamido-5-(4-cyanophenyl)-2,2-difluoro-5-oxopentanoate (4n). yellow solid (m.p. $112-116^{\circ} \mathrm{C}$ ) was purified with silica gel chromatography (Petroleum ether: Ethyl acetate $=4: 1$ to $2: 1) .{ }^{1} \mathrm{H} \mathrm{NMR}\left(400 \mathrm{MHz} \mathrm{CDCl}_{3}\right) \delta 8.10(\mathrm{~d}, J=8.4 \mathrm{~Hz}, 2 \mathrm{H}), 7.79(\mathrm{~d}, J=8.4 \mathrm{~Hz}, 2 \mathrm{H})$, $6.57(\mathrm{~d}, J=8.4 \mathrm{~Hz}, 1 \mathrm{H}), 5.79-5.71(\mathrm{~m}, 1 \mathrm{H}), 4.30(\mathrm{q}, J=7.2 \mathrm{~Hz}, 2 \mathrm{H}), 2.85-2.70(\mathrm{~m}, 1 \mathrm{H}), 2.56-$ $2.41(\mathrm{~m}, 1 \mathrm{H}), 1.98(\mathrm{~s}, 3 \mathrm{H}), 1.33(\mathrm{t}, J=7.2 \mathrm{~Hz}, 3 \mathrm{H}) .{ }^{19} \mathrm{~F}$ NMR $\left(376 \mathrm{MHz}, \mathrm{CDCl}_{3}\right) \delta-102.1(\mathrm{ddd}, J=$ $270.0 \mathrm{~Hz}, 18.0 \mathrm{~Hz}, 13.2 \mathrm{~Hz}, 1 \mathrm{~F}),-103.7$ (dt, $J=270.0 \mathrm{~Hz}, 16.5 \mathrm{~Hz}, 1 \mathrm{~F}) .{ }^{13} \mathrm{C} \mathrm{NMR}\left(101 \mathrm{MHz}, \mathrm{CDCl}_{3}\right)$ $\delta 196.3,170.3,163.9(\mathrm{t}, J=32.0 \mathrm{~Hz}), 137.9,133.3,129.7,118.2,117.7,115.3(\mathrm{t}, J=254.1 \mathrm{~Hz}), 64.0$, $49.1(\mathrm{t}, J=3.5 \mathrm{~Hz}), 36.5(\mathrm{t}, J=23.3 \mathrm{~Hz}), 23.4,14.4$. MS (DART): m/z (\%) $339(\mathrm{M}+\mathrm{H})^{+}$. HRMS (DART): Calcd. for $\mathrm{C}_{16} \mathrm{H}_{17} \mathrm{O}_{4} \mathrm{~N}_{2} \mathrm{~F}_{2}: 339.1151(\mathrm{M}+\mathrm{H})^{+}$; Found: $339.1149(\mathrm{M}+\mathrm{H})^{+}$.

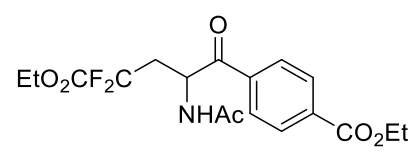

\section{Ethyl 4-(2-acetamido-5-ethoxy-4,4-difluoro-5-oxopentanoyl)benzoate}

(4o). The reaction was conducted at $80^{\circ} \mathrm{C}$. Compound $40(76 \mathrm{mg}, 66 \%$ yield) as a yellow oil was purified with silica gel chromatography (Petroleum ether: Ethyl acetate $=4: 1$ to $2: 1) .{ }^{1} \mathrm{H}$ NMR $\left(400 \mathrm{MHz}, \mathrm{CDCl}_{3}\right) \delta 8.12(\mathrm{~d}, J=8.4 \mathrm{~Hz}, 2 \mathrm{H}), 8.03(\mathrm{~d}, J=8.4$ $\mathrm{Hz}, 2 \mathrm{H}), 6.75(\mathrm{~d}, J=8.0 \mathrm{~Hz}, 1 \mathrm{H}), 5.85-5.77(\mathrm{~m}, 1 \mathrm{H}), 4.38(\mathrm{q}, J=7.2 \mathrm{~Hz}, 2 \mathrm{H}), 4.32-4.24(\mathrm{~m}, 2 \mathrm{H})$, $2.86-2.69(\mathrm{~m}, 1 \mathrm{H}), 2.55-2.39(\mathrm{~m}, 1 \mathrm{H}), 1.98(\mathrm{~s}, 3 \mathrm{H}), 1.38(\mathrm{t}, J=7.2 \mathrm{~Hz}, 3 \mathrm{H}), 1.31(\mathrm{t}, J=7.2 \mathrm{~Hz}$, 3H). ${ }^{19} \mathrm{~F}$ NMR $\left(376 \mathrm{MHz}, \mathrm{CDCl}_{3}\right) \delta-101.9(\mathrm{ddd}, J=270.7 \mathrm{~Hz}, 17.3 \mathrm{~Hz}, 13.2 \mathrm{~Hz}, 1 \mathrm{~F}),-103.7(\mathrm{dt}, J=$ $270.7 \mathrm{~Hz}, 16.5 \mathrm{~Hz}, 1 \mathrm{~F}) .{ }^{13} \mathrm{C} \mathrm{NMR}\left(101 \mathrm{MHz}, \mathrm{CDCl}_{3}\right) \delta 197.0,170.5,166.0,163.9$ (t, $\left.J=32.2 \mathrm{~Hz}\right)$, $137.8,135.6,130.5,129.2,115.4(\mathrm{t}, J=253.4 \mathrm{~Hz}), 63.9,62.1,49.3(\mathrm{t}, J=3.7 \mathrm{~Hz}), 36.8(\mathrm{t}, J=23.4$ $\mathrm{Hz}), 23.4,14.7,14.3$. MS (DART): $\mathrm{m} / \mathrm{z}(\%) 386(\mathrm{M}+\mathrm{H})^{+}$. HRMS (DART): Calcd. for $\mathrm{C}_{18} \mathrm{H}_{22} \mathrm{O}_{6} \mathrm{NF}_{2}$ : $386.1410(\mathrm{M}+\mathrm{H})^{+}$; Found: $386.1408(\mathrm{M}+\mathrm{H})^{+}$. 
$\mathrm{EtO}_{2} \mathrm{CF}_{2} \mathrm{C}$

Ethyl 3-(2-acetamido-5-ethoxy-4,4-difluoro-5-oxopentanoyl)benzoate

(4p). The reaction was conducted at $80^{\circ} \mathrm{C}$. Compound $4 \mathbf{p}(60 \mathrm{mg}, 52 \%$ yield) as a yellow solid (m.p. $90-94{ }^{\circ} \mathrm{C}$ ) was purified with silica gel chromatography (Petroleum ether: Ethyl acetate $=4: 1$ to $2: 1) .{ }^{1} \mathrm{H}$ NMR $\left(400 \mathrm{MHz}, \mathrm{CDCl}_{3}\right) \delta 8.61(\mathrm{~s}, 1 \mathrm{H}), 8.25(\mathrm{~d}, J=7.6 \mathrm{~Hz}, 1 \mathrm{H}), 8.18(\mathrm{~d}, J$ $=7.6 \mathrm{~Hz}, 1 \mathrm{H}), 7.57(\mathrm{t}, J=7.6 \mathrm{~Hz}, 1 \mathrm{H}), 6.66(\mathrm{~d}, J=8.0 \mathrm{~Hz}, 1 \mathrm{H}), 5.85-5.78(\mathrm{~m}, 1 \mathrm{H}), 4.39(\mathrm{q}, J=7.2$ $\mathrm{Hz}, 2 \mathrm{H}), 4.39$ (q, $J=7.2 \mathrm{~Hz}, 2 \mathrm{H}), 2.88-2.72(\mathrm{~m}, 1 \mathrm{H}), 2.56-2.40(\mathrm{~m}, 1 \mathrm{H}), 1.99(\mathrm{~s}, 3 \mathrm{H}), 1.39$ (t, $J=$ $7.2 \mathrm{~Hz}, 3 \mathrm{H}), 1.32(\mathrm{t}, J=7.2 \mathrm{~Hz}, 3 \mathrm{H}) .{ }^{19} \mathrm{~F}$ NMR $\left(376 \mathrm{MHz}, \mathrm{CDCl}_{3}\right) \delta-101.7$ (ddd, $J=270.0 \mathrm{~Hz}, 17.3$ $\mathrm{Hz}, 13.2 \mathrm{~Hz}, 1 \mathrm{~F}),-103.6(\mathrm{dt}, J=270.0 \mathrm{~Hz}, 16.5 \mathrm{~Hz}, 1 \mathrm{~F}) .{ }^{13} \mathrm{C} \mathrm{NMR}\left(101 \mathrm{MHz}, \mathrm{CDCl}_{3}\right) \delta 196.7,170.4$, 166.0, 163.9 (t, $J=32.4 \mathrm{~Hz}), 135.3,134.8,133.3,132.0,130.2,129.8,115.4$ (t, $J=254.1 \mathrm{~Hz}), 63.9$, 62.1, $49.3(\mathrm{t}, J=3.4 \mathrm{~Hz}), 36.9(\mathrm{t}, J=23.4 \mathrm{~Hz}), 23.5,14.8,14.4$. MS (DART): $\mathrm{m} / \mathrm{z}(\%) 386(\mathrm{M}+\mathrm{H})^{+}$. HRMS (DART): Calcd. for $\mathrm{C}_{18} \mathrm{H}_{22} \mathrm{O}_{6} \mathrm{NF}_{2}: 386.1410(\mathrm{M}+\mathrm{H})^{+}$; Found: $386.1408(\mathrm{M}+\mathrm{H})^{+}$.

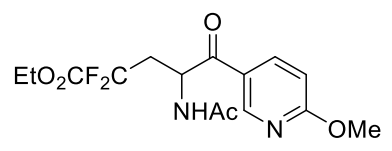

Ethyl

4-acetamido-2,2-difluoro-5-(6-methoxypyridin-3-yl)-5-

oxopentanoate $(\mathbf{4 q})$. The reaction was conducted with $\mathbf{L 7}$ as the ligand. Compound 4q (73 mg, 71\% yield) as a pink solid (m.p. 99-103 ${ }^{\circ} \mathrm{C}$ ) was purified with silica gel chromatography (Petroleum ether: Ethyl acetate $=4: 1$ to $2: 1) .{ }^{1} \mathrm{H}$ NMR $\left(400 \mathrm{MHz}, \mathrm{CDCl}_{3}\right) \delta 8.85$ (s, 1H), $8.14(\mathrm{~d}, J=8.8 \mathrm{~Hz}, 1 \mathrm{H}), 6.77(\mathrm{~d}, J=8.8 \mathrm{~Hz}, 2 \mathrm{H}), 5.77-5.67(\mathrm{~m}, 1 \mathrm{H}), 4.34-4.21(\mathrm{~m}, 2 \mathrm{H}), 3.97$ $(\mathrm{s}, 3 \mathrm{H}), 2.82-2.66(\mathrm{~m}, 1 \mathrm{H}), 2.53-2.37(\mathrm{~m}, 1 \mathrm{H}), 1.96(\mathrm{~s}, 3 \mathrm{H}), 1.30(\mathrm{t}, J=7.2 \mathrm{~Hz}, 3 \mathrm{H}) .{ }^{19} \mathrm{~F} \mathrm{NMR}(376$ $\left.\mathrm{MHz} \mathrm{CDCl}_{3}\right) \delta-102.1$ (ddd, $\left.J=269.6 \mathrm{~Hz}, 16.5 \mathrm{~Hz}, 14.3 \mathrm{~Hz}, 1 \mathrm{~F}\right),-103.6$ (dt, $J=269.6 \mathrm{~Hz}, 16.2 \mathrm{~Hz}$, 1F). ${ }^{13} \mathrm{C} \mathrm{NMR}\left(101 \mathrm{MHz}, \mathrm{CDCl}_{3}\right) \delta 195.1,170.3,167.8,163.9(\mathrm{t}, J=31.9 \mathrm{~Hz}), 150.5,139.2,124.3$, $115.4(\mathrm{t}, J=253.3 \mathrm{~Hz}), 112.0,63.8,54.7,48.6(\mathrm{t}, J=3.0 \mathrm{~Hz}), 37.0(\mathrm{t}, J=23.4 \mathrm{~Hz}), 23.4,14.3$. MS (DART): $\mathrm{m} / \mathrm{z}(\%) 345(\mathrm{M}+\mathrm{H})^{+}$. HRMS (DART): Calcd. for $\mathrm{C}_{15} \mathrm{H}_{19} \mathrm{O}_{5} \mathrm{~N}_{2} \mathrm{~F}_{2}: 345.1257(\mathrm{M}+\mathrm{H})^{+}$; Found: $345.1255(\mathrm{M}+\mathrm{H})^{+}$.

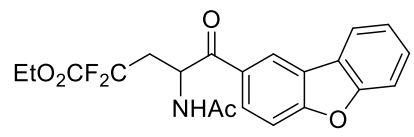

\section{Ethyl}

4-acetamido-5-(dibenzo[b,d] furan-2-yl)-2,2-difluoro-5-

oxopentanoate (4r). The reaction was conducted at $80{ }^{\circ} \mathrm{C}$. Compound $4 \mathbf{r}$

(86 mg, 75\% yield) as a yellow solid (m.p. 136-138 ${ }^{\circ} \mathrm{C}$ ) was purified with silica gel chromatography $($ Petroleum ether: Ethyl acetate $=4: 1$ to $2: 1) .{ }^{1} \mathrm{H} \mathrm{NMR}\left(400 \mathrm{MHz}, \mathrm{CDCl}_{3}\right) \delta 8.67(\mathrm{~d}, J=1.2 \mathrm{~Hz}, 1 \mathrm{H})$, $8.15(\mathrm{dd}, J=8.4 \mathrm{~Hz}, 1.2 \mathrm{~Hz}, 1 \mathrm{H}), 7.99$ (d, $J=8.0 \mathrm{~Hz}, 1 \mathrm{H}), 7.61(\mathrm{~d}, J=8.4 \mathrm{~Hz}, 1 \mathrm{H}), 7.57(\mathrm{~d}, J=8.0$ 
$\mathrm{Hz}, 1 \mathrm{H}), 7.49$ (t, $J=8.0 \mathrm{~Hz}, 1 \mathrm{H}), 7.38(\mathrm{t}, J=8.0 \mathrm{~Hz}, 1 \mathrm{H}), 6.70(\mathrm{~d}, J=8.4 \mathrm{~Hz}, 1 \mathrm{H}), 6.06-5.99$ (m, $1 \mathrm{H}), 4.39-4.25(\mathrm{~m}, 2 \mathrm{H}), 2.94-2.79(\mathrm{~m}, 1 \mathrm{H}), 2.62-2.46(\mathrm{~m}, 1 \mathrm{H}), 2.05(\mathrm{~s}, 3 \mathrm{H}), 1.34(\mathrm{t}, J=7.2 \mathrm{~Hz}$, 3H). ${ }^{19} \mathrm{~F}$ NMR $\left(376 \mathrm{MHz} \mathrm{CDCl}_{3}\right) \delta-101.6(\mathrm{ddd}, J=271.1 \mathrm{~Hz}, 16.9 \mathrm{~Hz}, 13.5 \mathrm{~Hz}, 1 \mathrm{~F}),-103.5(\mathrm{dt}, J=$ 271.1 Hz, 16.5 Hz, 1F). ${ }^{13} \mathrm{C}$ NMR (101 MHz, $\left.\mathrm{CDCl}_{3}\right) \delta 196.5,170.3,164.0(\mathrm{t}, J=32.3 \mathrm{~Hz}), 160.0$, $157.4,129.5,128.9,128.8,125.6,124.1,123.9,122.9,121.7,115.5(\mathrm{t}, J=254.4 \mathrm{~Hz}), 112.7,112.5$, $63.8,48.9(\mathrm{t}, J=3.6 \mathrm{~Hz}), 37.6(\mathrm{t}, J=23.5 \mathrm{~Hz}), 23.6,14.4$. MS (DART): m/z (\%) $404(\mathrm{M}+\mathrm{H})^{+}$. HRMS (DART): Calcd. for $\mathrm{C}_{21} \mathrm{H}_{20} \mathrm{O}_{5} \mathrm{NF}_{2}$ : $404.1304(\mathrm{M}+\mathrm{H})^{+}$; Found: $404.1302(\mathrm{M}+\mathrm{H})^{+}$.

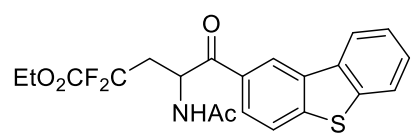

\section{Ethyl}

4-acetamido-5-(dibenzo[b,d] thiophen-2-yl)-2,2-difluoro-5-

oxopentanoate (4s). The reaction was conducted at $80{ }^{\circ} \mathrm{C}$. Compound $4 \mathrm{~s}$ (104 mg, 83\% yield) as a white solid (m.p. 161-163 ${ }^{\circ} \mathrm{C}$ ) was purified with silica gel chromatography (Petroleum ether: Ethyl acetate $=3: 1$ to 2: 1$).{ }^{1} \mathrm{H}$ NMR $\left(400 \mathrm{MHz}, \mathrm{DMSO}-\mathrm{d}_{6}\right) \delta 9.00(\mathrm{~s}, 1 \mathrm{H}), 8.82(\mathrm{~d}$, $J=8.8 \mathrm{~Hz}, 1 \mathrm{H}), 8.48-8.40(\mathrm{~m}, 1 \mathrm{H}), 8.21(\mathrm{~d}, J=8.4 \mathrm{~Hz}, 1 \mathrm{H}), 8.13-8.05(\mathrm{~m}, 2 \mathrm{H}), 7.66-7.56(\mathrm{~m}$, $2 \mathrm{H}), 5.76-5.66(\mathrm{~m}, 1 \mathrm{H}), 4.39-4.24(\mathrm{~m}, 2 \mathrm{H}), 3.01-2.85(\mathrm{~m}, 1 \mathrm{H}), 2.75-2.58(\mathrm{~m}, 1 \mathrm{H}), 1.81(\mathrm{~s}, 3 \mathrm{H})$, $1.29(\mathrm{t}, J=7.2 \mathrm{~Hz}, 3 \mathrm{H}) .{ }^{19} \mathrm{~F}$ NMR (376 MHz, DMSO-d 6 ) $\delta-102.0(\mathrm{dt}, J=259.8 \mathrm{~Hz}, 16.5 \mathrm{~Hz}, 1 \mathrm{~F}),-$ $103.5(\mathrm{dt}, J=269.6 \mathrm{~Hz}, 17.3 \mathrm{~Hz}, 1 \mathrm{~F}) .{ }^{13} \mathrm{C}$ NMR $\left(101 \mathrm{MHz}, \mathrm{DMSO}-\mathrm{d}_{6}\right) \delta 196.6,169.8,164.0(\mathrm{t}, J=$ $32.1 \mathrm{~Hz}), 144.9,139.9,135.9,135.5,132.0,128.7,127.2,126.2,124.23,124.19,123.1,123.0,116.5$ (t, $J=251.3 \mathrm{~Hz}), 64.0,48.8,35.6(\mathrm{t}, J=22.8 \mathrm{~Hz}), 23.2,14.5 . \mathrm{MS}(\mathrm{ESI}): \mathrm{m} / \mathrm{z}(\%) 420(\mathrm{M}+\mathrm{H})^{+} . \mathrm{HRMS}$ (ESI): Calcd. for $\mathrm{C}_{21} \mathrm{H}_{20} \mathrm{O}_{4} \mathrm{NF}_{2} \mathrm{~S}$ : $420.1076(\mathrm{M}+\mathrm{H})^{+}$; Found: $420.1077(\mathrm{M}+\mathrm{H})^{+}$.

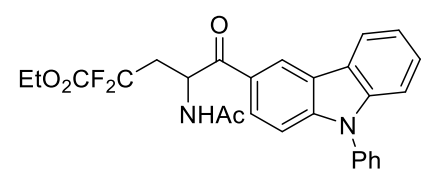

\section{Ethyl 4-acetamido-2,2-difluoro-5-oxo-5-(9-phenyl-9H-carbazol-3-} yl)pentanoate (4t). The reaction was conducted at $80^{\circ} \mathrm{C}$. Compound $4 \mathbf{t}(85$ $\mathrm{mg}, 59 \%$ yield) as a white solid was purified with silica gel chromatography (Petroleum ether: Ethyl acetate $=3: 1$ to $1: 1) .{ }^{1} \mathrm{H}$ NMR $\left(400 \mathrm{MHz}, \mathrm{DMSO}-\mathrm{d}_{6}\right) \delta 9.00(\mathrm{~d}, J=1.2 \mathrm{~Hz}$, $1 \mathrm{H}), 8.74(\mathrm{~d}, J=8.8 \mathrm{~Hz}, 1 \mathrm{H}), 8.35(\mathrm{~d}, J=7.6 \mathrm{~Hz}, 1 \mathrm{H}), 8.11(\mathrm{dd}, J=8.4 \mathrm{~Hz}, 1.2 \mathrm{~Hz}, 1 \mathrm{H}), 7.74(\mathrm{t}, J=$ $7.6 \mathrm{~Hz}, 2 \mathrm{H}), 7.66(\mathrm{~d}, J=7.2 \mathrm{~Hz}, 2 \mathrm{H}), 7.62(\mathrm{t}, J=7.2 \mathrm{~Hz}, 1 \mathrm{H}), 7.53(\mathrm{t}, J=7.2 \mathrm{~Hz}, 1 \mathrm{H}), 7.46(\mathrm{~d}, J=8.8$ $\mathrm{Hz}, 1 \mathrm{H}), 7.44-7.38(\mathrm{~m}, 2 \mathrm{H}), 5.85-5.76(\mathrm{~m}, 1 \mathrm{H}), 4.39-4.24(\mathrm{~m}, 2 \mathrm{H}), 2.99-2.83(\mathrm{~m}, 1 \mathrm{H}), 2.71-$ $2.55(\mathrm{~m}, 1 \mathrm{H}), 1.85(\mathrm{~s}, 3 \mathrm{H}), 1.29(\mathrm{t}, J=7.2 \mathrm{~Hz}, 3 \mathrm{H}) .{ }^{19} \mathrm{~F}$ NMR $\left(376 \mathrm{MHz}, \mathrm{DMSO}-\mathrm{d}_{6}\right) \delta-102.4(\mathrm{dt}, J=$ $259.4 \mathrm{~Hz}, 16.5 \mathrm{~Hz}, 1 \mathrm{~F}),-103.2(\mathrm{dt}, J=259.4 \mathrm{~Hz}, 16.9 \mathrm{~Hz}, 1 \mathrm{~F}) .{ }^{13} \mathrm{C}$ NMR $\left(101 \mathrm{MHz}, \mathrm{DMSO}-\mathrm{d}_{6}\right) \delta$ 
196.1, 169.7, 163.9 (t, $J=32.4 \mathrm{~Hz}), 143.9,142.0,137.0,131.2,129.2,128.1,127.8,127.7,127.5$, $123.7,123.5,123.0,122.1,121.6,116.4$ (t, $J=251.1 \mathrm{~Hz}), 111.1,110.7,64.0,48.2,36.1(\mathrm{t}, J=23.0$ $\mathrm{Hz}), 23.3,14.5$. MS (ESI): m/z (\%) $479(\mathrm{M}+\mathrm{H})^{+}$. HRMS (ESI): Calcd. for $\mathrm{C}_{27} \mathrm{H}_{24} \mathrm{O}_{4} \mathrm{~N}_{2} \mathrm{~F}_{2}: 479.1777$ $(\mathrm{M}+\mathrm{H})^{+}$; Found: $479.1774(\mathrm{M}+\mathrm{H})^{+}$.

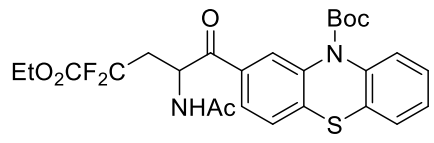

tert-Butyl 2-(2-acetamido-5-ethoxy-4,4-difluoro-5-oxopentanoyl)10H-phenothiazine-10-carboxylate (4u). The reaction was conducted at $80{ }^{\circ} \mathrm{C}$. Compound $4 \mathbf{u}(80 \mathrm{mg}, 50 \%$ yield) as a yellow oil was purified with silica gel chromatography (Petroleum ether: Ethyl acetate = 3: 1 to 1: 1). ${ }^{1} \mathrm{H}$ NMR $\left(400 \mathrm{MHz}, \mathrm{CDCl}_{3}\right) \delta 8.13(\mathrm{~d}, J=1.6 \mathrm{~Hz}, 1 \mathrm{H})$, $7.78(\mathrm{dd}, J=8.0 \mathrm{~Hz}, J=1.2 \mathrm{~Hz}, 1 \mathrm{H}), 7.52(\mathrm{~d}, J=8.0 \mathrm{~Hz}, 1 \mathrm{H}), 7.40(\mathrm{~d}, J=8.0 \mathrm{~Hz}, 1 \mathrm{H}), 7.32-7.27$ (m, 2H), $7.15(\mathrm{t}, J=7.2 \mathrm{~Hz}, 1 \mathrm{H}), 6.71(\mathrm{t}, J=8.4 \mathrm{~Hz}, 1 \mathrm{H}), 5.84-5.76(\mathrm{~m}, 1 \mathrm{H}), 4.35-4.21(\mathrm{~m}, 2 \mathrm{H})$, $2.89-2.74(\mathrm{~m}, 1 \mathrm{H}), 2.55-2.40(\mathrm{~m}, 1 \mathrm{H}), 1.98(\mathrm{~s}, 3 \mathrm{H}), 1.47(\mathrm{~s}, 9 \mathrm{H}), 1.31(\mathrm{t}, J=7.2 \mathrm{~Hz}, 3 \mathrm{H}) .{ }^{19} \mathrm{~F}$ NMR $\left(376 \mathrm{MHz}, \mathrm{CDCl}_{3}\right) \delta-101.7(\mathrm{ddd}, J=270.0 \mathrm{~Hz}, 16.5 \mathrm{~Hz}, 14.3 \mathrm{~Hz}, 1 \mathrm{~F}),-103.6(\mathrm{dt}, J=270.0 \mathrm{~Hz}, 16.5$ $\mathrm{Hz}, 1 \mathrm{~F}) .{ }^{13} \mathrm{C} \mathrm{NMR}\left(101 \mathrm{MHz}, \mathrm{CDCl}_{3}\right) \delta 196.0,170.3,163.9$ (t, $\left.J=31.9 \mathrm{~Hz}\right), 152.5,140.4,139.4,138.4$, $132.8,130.9,128.1,128.0,127.8,127.6,126.9,126.5,115.4(\mathrm{t}, J=252.7 \mathrm{~Hz}), 83.3,63.7,48.9(\mathrm{t}, J=$ $2.9 \mathrm{~Hz}), 37.0(\mathrm{t}, J=23.2 \mathrm{~Hz}), 28.5,23.4,14.3 . \mathrm{MS}(\mathrm{ESI}): \mathrm{m} / \mathrm{z}(\%) 557(\mathrm{M}+\mathrm{Na})^{+}$. HRMS (ESI): Calcd. for $\mathrm{C}_{26} \mathrm{H}_{29} \mathrm{O}_{6} \mathrm{~N}_{2} \mathrm{~F}_{2} \mathrm{~S}: 535.1709(\mathrm{M}+\mathrm{H})^{+}$; Found: $535.1712(\mathrm{M}+\mathrm{H})^{+}$.

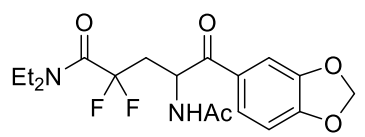

\section{4-Acetamido-5-(benzo $[d][1,3]$ dioxol-5-yl)- $N, N$-diethyl-2,2-difluoro-5-}

oxopentanamide (6a). The reaction was conducted with $\mathbf{L 7}$ as the ligand.

Compound 6a (81 mg, 70\% yield) as a yellow oil was purified with silica gel chromatography (Petroleum ether: Ethyl acetate $=4: 1$ to $1: 1) .{ }^{1} \mathrm{H}$ NMR $\left(400 \mathrm{MHz}, \mathrm{CDCl}_{3}\right) \delta 7.73(\mathrm{dd}, J=8.4 \mathrm{~Hz}, 1.6$ $\mathrm{Hz}, 1 \mathrm{H}), 7.48(\mathrm{~d}, J=1.6 \mathrm{~Hz}, 1 \mathrm{H}), 6.83(\mathrm{~d}, J=8.4 \mathrm{~Hz}, 1 \mathrm{H}), 6.76(\mathrm{~d}, J=8.0 \mathrm{~Hz}, 1 \mathrm{H}), 6.00(\mathrm{~s}, 2 \mathrm{H}), 5.82$ $(\mathrm{td}, J=8.4 \mathrm{~Hz}, 3.6 \mathrm{~Hz}, 1 \mathrm{H}), 3.54-3.25(\mathrm{~m}, 4 \mathrm{H}), 2.78-2.62(\mathrm{~m}, 1 \mathrm{H}), 2.52-2.36(\mathrm{~m}, 1 \mathrm{H}), 1.95$ (s, $3 \mathrm{H}), 1.14(\mathrm{t}, J=7.2 \mathrm{~Hz}, 3 \mathrm{H}), 1.11(\mathrm{t}, J=7.2 \mathrm{~Hz}, 3 \mathrm{H}) .{ }^{19} \mathrm{~F}$ NMR $\left(376 \mathrm{MHz}, \mathrm{CDCl}_{3}\right) \delta-96.5(\mathrm{dt}, J=$ $286.1 \mathrm{~Hz}, 16.2 \mathrm{~Hz}, 1 \mathrm{~F}),-97.6(\mathrm{dt}, J=285.1 \mathrm{~Hz}, 16.9 \mathrm{~Hz}, 1 \mathrm{~F}) .{ }^{13} \mathrm{C} \mathrm{NMR}\left(101 \mathrm{MHz}, \mathrm{CDCl}_{3}\right) \delta 196.0$, $169.9,162.6(\mathrm{t}, J=28.6 \mathrm{~Hz}), 153.0,148.9,129.3,126.1,119.2(\mathrm{t}, J=258.8 \mathrm{~Hz}), 109.0,108.7,102.5$, $48.7(\mathrm{t}, J=3.7 \mathrm{~Hz}), 42.4(\mathrm{t}, J=6.5 \mathrm{~Hz}), 42.2,37.9(\mathrm{t}, J=23.3 \mathrm{~Hz}), 23.5,14.6,12.7 . \mathrm{MS}(\mathrm{ESI}): \mathrm{m} / \mathrm{z}$ 
(\%) $385(\mathrm{M}+\mathrm{H})^{+}$. HRMS (ESI): Calcd. for $\mathrm{C}_{18} \mathrm{H}_{23} \mathrm{O}_{5} \mathrm{~N}_{2} \mathrm{~F}_{2}$ : $385.1570(\mathrm{M}+\mathrm{H})^{+}$; Found: 385.1570 $(\mathrm{M}+\mathrm{H})^{+}$.

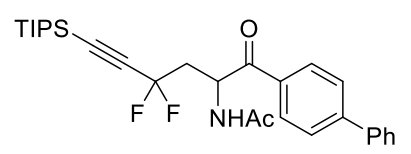

\section{N-(1-([1,1'-biphenyl]-4-yl)-4,4-difluoro-1-oxo-6-(triisopropylsilyl)hex-5-}

yn-2-yl)acetamide (6b). Compound $6 \mathbf{b}(81 \mathrm{mg}, 54 \%$ yield) as a white solid (m.p. 95-100 ${ }^{\circ} \mathrm{C}$ ) was purified with silica gel chromatography (Petroleum ether: Ethyl acetate $=6: 1$ to 3: 1). ${ }^{1} \mathrm{H}$ NMR $\left(400 \mathrm{MHz}, \mathrm{CDCl}_{3}\right) \delta 8.12(\mathrm{~d}, J=8.4 \mathrm{~Hz}, 2 \mathrm{H}), 7.70(\mathrm{~d}, J=8.4 \mathrm{~Hz}, 2 \mathrm{H}), 7.61(\mathrm{~d}, J=7.2$ $\mathrm{Hz}, 2 \mathrm{H}), 7.47$ (t, $J=7.2 \mathrm{~Hz}, 2 \mathrm{H}), 7.40$ (t, $J=7.2 \mathrm{~Hz}, 1 \mathrm{H}), 6.63(\mathrm{~d}, J=8.0 \mathrm{~Hz}, 1 \mathrm{H}), 6.03-5.93(\mathrm{~m}$, 1H), $2.87-2.72(\mathrm{~m}, 1 \mathrm{H}), 2.59-2.44(\mathrm{~m}, 1 \mathrm{H}), 2.04(\mathrm{~s}, 3 \mathrm{H}), 1.09(\mathrm{~m}, 21 \mathrm{H}) .{ }^{19} \mathrm{~F} \mathrm{NMR}\left(376 \mathrm{MHz}, \mathrm{CDCl}_{3}\right)$ $\delta-79.1(\mathrm{ddd}, J=275.2 \mathrm{~Hz}, 15.8 \mathrm{~Hz}, 10.5 \mathrm{~Hz}, 1 \mathrm{~F}),-80.7$ (dt, $J=275.2 \mathrm{~Hz}, 15.8 \mathrm{~Hz}, 1 \mathrm{~F}) .{ }^{13} \mathrm{C} \mathrm{NMR}(101$ $\left.\mathrm{MHz}, \mathrm{CDCl}_{3}\right) \delta 197.3,170.0,147.2,140.1,133.5,129.9,129.5,129.0,128.0,127.8,112.8(\mathrm{t}, J=234.6$ Hz), 98.5 (t, $J=37.5 \mathrm{~Hz}), 92.2$ (t, $J=5.5 \mathrm{~Hz}), 49.4,41.9$ (t, $J=26.9 \mathrm{~Hz}), 23.6,18.9$, 11.4. MS (ESI): $\mathrm{m} / \mathrm{z}(\%) 498(\mathrm{M}+\mathrm{H})^{+}$. HRMS (ESI): Calcd. for $\mathrm{C}_{29} \mathrm{H}_{38} \mathrm{O}_{2} \mathrm{NF}_{2} \mathrm{Si}: 498.2634(\mathrm{M}+\mathrm{H})^{+}$; Found: 498.2637 $(\mathrm{M}+\mathrm{H})^{+}$.

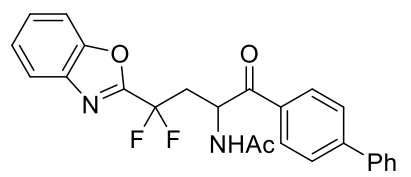

\section{$N-(1-([1,1 '-B i p h e n y l]-4-y l)-4-(b e n z o[d]$ oxazol-2-yl)-4,4-difluoro-1-} oxobutan-2-yl)acetamide (6c). Compound 6 c (75.6 $\mathrm{mg}, 58 \%$ yield) as a yellow solid (m.p. 161-163 ${ }^{\circ} \mathrm{C}$ ) was purified with silica gel chromatography (Petroleum ether: Ethyl acetate $=5: 1$ to $3: 1) .{ }^{1} \mathrm{H}$ NMR $\left(400 \mathrm{MHz}, \mathrm{DMSO}-\mathrm{d}_{6}\right) \delta 8.73(\mathrm{~d}, J=8.4 \mathrm{~Hz}$, 1H), $8.08(\mathrm{~d}, \mathrm{~J}=8.0 \mathrm{~Hz}, 2 \mathrm{H}), 7.95(\mathrm{~d}, \mathrm{~J}=8.0 \mathrm{~Hz}, 1 \mathrm{H}), 7.90(\mathrm{~d}, \mathrm{~J}=8.0 \mathrm{~Hz}, 1 \mathrm{H}), 7.86(\mathrm{~d}, \mathrm{~J}=8.0 \mathrm{~Hz}$, 2H), $7.78(\mathrm{~d}, \mathrm{~J}=8.0 \mathrm{~Hz}, 2 \mathrm{H}), 7.60(\mathrm{t}, \mathrm{J}=8.0 \mathrm{~Hz}, 1 \mathrm{H}), 7.54(\mathrm{t}, \mathrm{J}=8.0 \mathrm{~Hz}, 3 \mathrm{H}), 7.46(\mathrm{t}, \mathrm{J}=8.0 \mathrm{~Hz}, 1 \mathrm{H})$, $5.81-5.74(\mathrm{~m}, 1 \mathrm{H}), 3.34-3.17(\mathrm{~m}, 1 \mathrm{H}), 3.04-2.87(\mathrm{~m}, 1 \mathrm{H}), 1.65(\mathrm{~s}, 3 \mathrm{H}) .{ }^{19} \mathrm{~F}$ NMR $(376 \mathrm{MHz}$, DMSO-d 6$) \delta-94.6(\mathrm{dt}, J=274.5 \mathrm{~Hz}, 16.5 \mathrm{~Hz}, 1 \mathrm{~F}),-96.0(\mathrm{dt}, J=274.5 \mathrm{~Hz}, 16.5 \mathrm{~Hz}, 1 \mathrm{~F}) .{ }^{13} \mathrm{C}$ NMR $\left(101 \mathrm{MHz}, \mathrm{DMSO}-\mathrm{d}_{6}\right) \delta 196.6,169.6,157.8(\mathrm{t}, J=32.9 \mathrm{~Hz}), 151.0,145.8,140.3,139.7,134.1,130.0$ (2C), 129.4, 128.3, 127.9, 127.8, 126.6, 122.0, 117.3 (t, $J=242.6 \mathrm{~Hz}), 112.7,48.6,37.0$ (t, $J=22.5$ $\mathrm{Hz}$ ), 23.0. MS (DART): m/z (\%) $435(\mathrm{M}+\mathrm{H})^{+}$. HRMS (DART): Calcd. for $\mathrm{C}_{25} \mathrm{H}_{21} \mathrm{O}_{3} \mathrm{~N}_{2} \mathrm{~F}_{2}: 435.1515$ $(\mathrm{M}+\mathrm{H})^{+}$; Found: $435.1512(\mathrm{M}+\mathrm{H})^{+}$. 
$\mathrm{EtO}_{2} \mathrm{CF}_{2} \mathrm{C}$

Ethyl

5-([1,1'-biphenyl]-4-yl)-4-((tert-butoxycarbonyl)amino)-2,2-

difluoro-5-oxopentanoate (6d). Compound $6 \mathbf{d}$ (86 $\mathrm{mg}$, 64\% yield) as a

yellow oil was purified with silica gel chromatography (Petroleum ether: Ethyl acetate $=7: 1$ ). ${ }^{1} \mathrm{H}$ NMR (400 MHz, $\left.\mathrm{CDCl}_{3}\right) \delta 8.10(\mathrm{~d}, J=8.0 \mathrm{~Hz}, 2 \mathrm{H}), 7.71(\mathrm{~d}, J=8.0 \mathrm{~Hz}, 2 \mathrm{H}), 7.62(\mathrm{~d}, J=7.2 \mathrm{~Hz}, 2 \mathrm{H})$, $7.53-7.35(\mathrm{~m}, 3 \mathrm{H}), 5.68-5.54(\mathrm{~m}, 1 \mathrm{H}), 5.51-5.32(\mathrm{~m}, 1 \mathrm{H}), 4.42-4.26(\mathrm{~m}, 2 \mathrm{H}), 2.68-2.68(\mathrm{~m}$, 1H), $2.57-2.38(\mathrm{~m}, 1 \mathrm{H}), 1.44(\mathrm{~s}, 9 \mathrm{H}), 1.37(\mathrm{t}, J=6.8 \mathrm{~Hz}, 3 \mathrm{H}) .{ }^{19} \mathrm{~F} \mathrm{NMR}\left(376 \mathrm{MHz}, \mathrm{CDCl}_{3}\right) \delta-102.0$ $--103.8(\mathrm{~m}, 2 \mathrm{~F}) .{ }^{13} \mathrm{C}$ NMR $\left(101 \mathrm{MHz}, \mathrm{CDCl}_{3}\right) \delta 197.1,164.0(\mathrm{t}, J=32.5 \mathrm{~Hz}), 155.5,147.2,140.0$, 133.0, 129.9, 129.5, 129.0, 128.0, 127.8, 115.5 (t, $J=253.2 \mathrm{~Hz}), 80.9,63.7,50.3,37.7(\mathrm{t}, J=23.8 \mathrm{~Hz})$, 28.8, 14.4. $\mathrm{MS}(\mathrm{ESI}): \mathrm{m} / \mathrm{z}(\%) 448(\mathrm{M}+\mathrm{H})^{+}$. $\mathrm{HRMS}(\mathrm{ESI}):$ Calcd. for $\mathrm{C}_{24} \mathrm{H}_{28} \mathrm{O}_{5} \mathrm{NF}_{2}: 448.1930(\mathrm{M}+\mathrm{H})^{+}$; Found: $448.1927(\mathrm{M}+\mathrm{H})^{+}$.

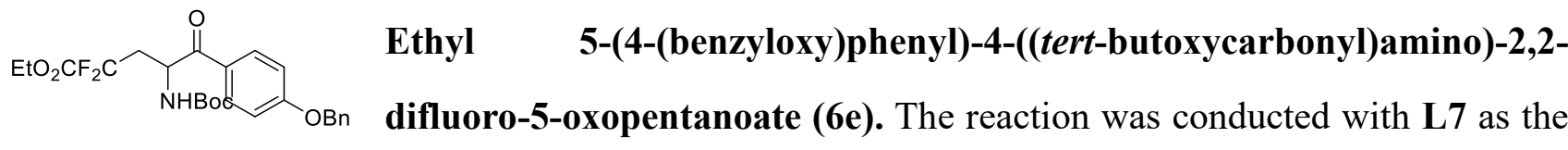
ligand. Compound $6 \mathrm{e}$ (79 $\mathrm{mg}, 55 \%$ yield) as a yellow oil was purified with silica gel chromatography $\left(\right.$ Petroleum ether: Ethyl acetate = 5: 1). ${ }^{1} \mathrm{H}$ NMR $\left(400 \mathrm{MHz}, \mathrm{CDCl}_{3}\right) \delta 8.01(\mathrm{~d}, J=8.0 \mathrm{~Hz}, 2 \mathrm{H}), 7.46-$ $7.30(\mathrm{~m}, 5 \mathrm{H}), 7.03(\mathrm{~d}, J=8.0 \mathrm{~Hz}, 2 \mathrm{H}), 5.60-5.49(\mathrm{~m}, 1 \mathrm{H}), 5.47-5.37(\mathrm{~m}, 1 \mathrm{H}), 5.16-5.85(\mathrm{~m}, 2 \mathrm{H})$, $4.40-4.24(\mathrm{~m}, 2 \mathrm{H}), 2.78-2.61(\mathrm{~m}, 1 \mathrm{H}), 2.52-2.32(\mathrm{~m}, 1 \mathrm{H}), 1.43(\mathrm{~s}, 9 \mathrm{H}), 1.35(\mathrm{t}, J=6.8 \mathrm{~Hz}, 3 \mathrm{H})$. ${ }^{19} \mathrm{~F}$ NMR $\left(376 \mathrm{MHz}, \mathrm{CDCl}_{3}\right) \delta-102.8--103.0(\mathrm{~m}, 2 \mathrm{~F}) .{ }^{13} \mathrm{C} \mathrm{NMR}\left(101 \mathrm{MHz}, \mathrm{CDCl}_{3}\right) \delta 195.8,164.0$ (t, $J=32.2 \mathrm{~Hz}), 163.9,155.4,136.4,131.7,129.2,128.8,128.0,127.3,115.5(\mathrm{t}, J=254.6 \mathrm{~Hz}), 115.5$, 80.7, 70.7, 63.6, 49.7, 37.9 (t, $J=22.7 \mathrm{~Hz}), 28.7,14.3 . \mathrm{MS}(\mathrm{ESI}): \mathrm{m} / \mathrm{z}(\%) 478(\mathrm{M}+\mathrm{H})^{+}$. HRMS (ESI): Calcd. for $\mathrm{C}_{25} \mathrm{H}_{30} \mathrm{O}_{6} \mathrm{NF}_{2}$ : $478.2036(\mathrm{M}+\mathrm{H})^{+}$; Found: $478.2031(\mathrm{M}+\mathrm{H})^{+}$.

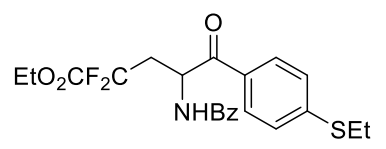

Ethyl 4-benzamido-5-(4-(ethylthio)phenyl)-2,2-difluoro-5-oxopentanoate

(6f). The reaction was conducted with $\mathbf{L} 7$ as the ligand. Compound $\mathbf{6 f}(82 \mathrm{mg}$, $63 \%$ yield) as a yellow solid (m.p. $108-109^{\circ} \mathrm{C}$ ) was purified with silica gel chromatography (Petroleum ether: Ethyl acetate $=4: 1$ to 2: 1$).{ }^{1} \mathrm{H}$ NMR $\left(400 \mathrm{MHz}, \mathrm{CDCl}_{3}\right) \delta 7.94(\mathrm{~d}, J=8.4 \mathrm{~Hz}, 2 \mathrm{H}), 7.78(\mathrm{~d}, J$ $=8.4 \mathrm{~Hz}, 2 \mathrm{H}), 7.48(\mathrm{t}, J=7.2 \mathrm{~Hz}, 1 \mathrm{H}), 7.38(\mathrm{t}, J=7.2 \mathrm{~Hz}, 2 \mathrm{H}), 7.33-7.27(\mathrm{~m}, 3 \mathrm{H}), 6.04-5.96(\mathrm{~m}$, 1H), $4.25-4.11(\mathrm{~m}, 2 \mathrm{H}), 3.00(\mathrm{q}, J=7.6 \mathrm{~Hz}, 2 \mathrm{H}), 2.95-2.80(\mathrm{~m}, 1 \mathrm{H}), 2.70-2.55(\mathrm{~m}, 1 \mathrm{H}), 1.36(\mathrm{t}$, $J=7.6 \mathrm{~Hz}, 3 \mathrm{H}), 1.23(\mathrm{t}, J=7.2 \mathrm{~Hz}, 3 \mathrm{H}) .{ }^{19} \mathrm{~F} \mathrm{NMR}\left(376 \mathrm{MHz}, \mathrm{CDCl}_{3}\right) \delta-101.9(\mathrm{dt}, J=270.0 \mathrm{~Hz}, 15.4$ 
$\mathrm{Hz}, 1 \mathrm{~F}),-103.0(\mathrm{dt}, J=270.0 \mathrm{~Hz}, 15.8 \mathrm{~Hz}, 1 \mathrm{~F}) .{ }^{13} \mathrm{C} \mathrm{NMR}\left(101 \mathrm{MHz}, \mathrm{CDCl}_{3}\right) \delta 196.1,167.3,164.0(\mathrm{t}$, $J=32.3 \mathrm{~Hz}$ ), 147.2, 133.9, 132.5, 130.6, 129.7, 129.1, 127.6, 126.7, 115.5 (t, $J=253.4 \mathrm{~Hz}), 63.8,49.1$ (t, $J=3.8 \mathrm{~Hz}), 37.3(\mathrm{t}, J=23.4 \mathrm{~Hz}), 26.3,14.31,14.26 . \mathrm{MS}(\mathrm{DART}): \mathrm{m} / \mathrm{z}(\%) 436(\mathrm{M}+\mathrm{H})^{+} . \mathrm{HRMS}$ (DART): Calcd. for $\mathrm{C}_{22} \mathrm{H}_{24} \mathrm{O}_{4} \mathrm{NF}_{2} \mathrm{~S}: 436.1389(\mathrm{M}+\mathrm{H})^{+}$; Found: $436.1388(\mathrm{M}+\mathrm{H})^{+}$.

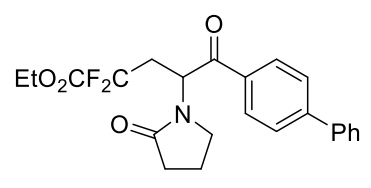

\section{Ethyl 5-([1,1'-biphenyl]-4-yl)-2,2-difluoro-5-oxo-4-(2-oxopyrrolidin-1-} yl)pentanoate (6g). Compound $\mathbf{6 g}(97 \mathrm{mg}, 78 \%$ yield) as a white solid (m.p. $122-128{ }^{\circ} \mathrm{C}$ ) was purified with silica gel chromatography (Petroleum ether: Ethyl acetate $=10: 1$ to 5: 1). ${ }^{1} \mathrm{H}$ NMR $\left(400 \mathrm{MHz}, \mathrm{CD}_{2} \mathrm{Cl}_{2}\right) \delta 8.11(\mathrm{~d}, J=8.4 \mathrm{~Hz}, 2 \mathrm{H}), 7.73(\mathrm{~d}, J=8.4$ $\mathrm{Hz}, 2 \mathrm{H}), 7.65(\mathrm{~d}, J=7.6 \mathrm{~Hz}, 2 \mathrm{H}), 7.48(\mathrm{t}, J=7.6 \mathrm{~Hz}, 2 \mathrm{H}), 7.41(\mathrm{t}, J=7.6 \mathrm{~Hz}, 1 \mathrm{H}), 6.01(\mathrm{dd}, J=8.0$ $\mathrm{Hz}, 5.2 \mathrm{~Hz}, 1 \mathrm{H}), 4.38-4.24(\mathrm{~m}, 2 \mathrm{H}), 3.40-3.32(\mathrm{~m}, 1 \mathrm{H}), 3.07-3.00(\mathrm{~m}, 1 \mathrm{H}), 2.96-2.80(\mathrm{~m}, 1 \mathrm{H})$, $2.68-2.52(\mathrm{~m}, 1 \mathrm{H}), 2.37-2.17(\mathrm{~m}, 2 \mathrm{H}), 2.01-1.76(\mathrm{~m}, 2 \mathrm{H}), 1.33(\mathrm{t}, J=7.2 \mathrm{~Hz}, 3 \mathrm{H}) .{ }^{19} \mathrm{~F}$ NMR $(376$ MHz, DMSO-d 6 ) $\delta-103.1(\mathrm{dt}, J=260.6 \mathrm{~Hz}, 17.3 \mathrm{~Hz}, 1 \mathrm{~F}),-103.9$ (ddd, $J=260.6 \mathrm{~Hz}, 18.4 \mathrm{~Hz}, 15.8$ $\mathrm{Hz}, 1 \mathrm{~F}) .{ }^{13} \mathrm{C}$ NMR $\left(101 \mathrm{MHz}, \mathrm{DMSO}-\mathrm{d}_{6}\right) \delta 195.2,174.7,163.8(\mathrm{t}, J=32.4 \mathrm{~Hz}), 146.2,139.5,133.9$, 130.0, 129.9, 129.5, 128.0, $116.4(\mathrm{t}, J=251.8 \mathrm{~Hz}), 64.1,49.8,44.0,32.4(\mathrm{t}, J=23.3 \mathrm{~Hz}), 30.9,18.5$, 14.5. MS (ESI): $\mathrm{m} / \mathrm{z}(\%) 416(\mathrm{M}+\mathrm{H})^{+}$. HRMS (ESI): Calcd. for $\mathrm{C}_{23} \mathrm{H}_{24} \mathrm{O}_{4} \mathrm{NF}_{2}$ : $416.1668(\mathrm{M}+\mathrm{H})^{+}$; Found: $416.1669(\mathrm{M}+\mathrm{H})^{+}$.

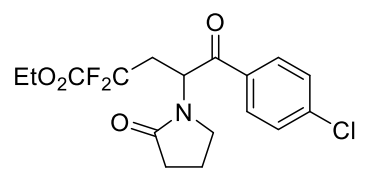

Ethyl 5-(4-chlorophenyl)-2,2-difluoro-5-oxo-4-(2-oxopyrrolidin-1-

yl)pentanoate (6h). Compound $\mathbf{6 h}(81 \mathrm{mg}, 72 \%$ yield) as a yellow solid (m.p. 202-205 ${ }^{\circ} \mathrm{C}$ ) was purified with silica gel chromatography (Petroleum ether: Ethyl acetate $=10: 1$ to $6: 1) .{ }^{1} \mathrm{H}$ NMR $\left(400 \mathrm{MHz}, \mathrm{CDCl}_{3}\right) \delta 7.96(\mathrm{~d}, J=8.4 \mathrm{~Hz}, 2 \mathrm{H}), 7.41(\mathrm{~d}, J=8.4$ Hz, 2H), $5.94(\mathrm{dd}, J=8.4,5.2 \mathrm{~Hz}, 1 \mathrm{H}), 4.35-4.22(\mathrm{~m}, 2 \mathrm{H}), 3.37-3.29(\mathrm{~m}, 1 \mathrm{H}), 3.02-2.94(\mathrm{~m}, 1 \mathrm{H})$, $2.86-2.70(\mathrm{~m}, 1 \mathrm{H}), 2.62-2.46(\mathrm{~m}, 1 \mathrm{H}), 2.39-2.17(\mathrm{~m}, 2 \mathrm{H}), 2.00-1.75(\mathrm{~m}, 2 \mathrm{H}), 1.31(\mathrm{t}, J=7.2 \mathrm{~Hz}$, 3H). ${ }^{19} \mathrm{~F} \mathrm{NMR}\left(376 \mathrm{MHz}, \mathrm{CDCl}_{3}\right) \delta-103.6(\mathrm{ddd}, J=268.5 \mathrm{~Hz}, 18.4 \mathrm{~Hz}, 12.8 \mathrm{~Hz}, 1 \mathrm{~F}),-105.0(\mathrm{dt}, J=$ $268.5 \mathrm{~Hz}, 16.5 \mathrm{~Hz}, 1 \mathrm{~F}) .{ }^{13} \mathrm{C} \mathrm{NMR}\left(101 \mathrm{MHz}, \mathrm{CDCl}_{3}\right) \delta 194.5,174.9,163.9(\mathrm{t}, J=32.8 \mathrm{~Hz}), 141.2$, $132.9,130.7,129.8,115.5(\mathrm{t}, J=253.3 \mathrm{~Hz}), 63.8,49.5,43.6,32.4(\mathrm{t}, J=23.4 \mathrm{~Hz}), 30.9,18.5,14.3$. MS (ESI): m/z (\%) $374(\mathrm{M}+\mathrm{H})^{+}$. HRMS (ESI): Calcd. for $\mathrm{C}_{17} \mathrm{H}_{19} \mathrm{O}_{4} \mathrm{NClF}_{2}: 374.0965(\mathrm{M}+\mathrm{H})^{+}$; Found: $374.0967(\mathrm{M}+\mathrm{H})^{+}$. 
$\mathrm{EtO}_{2} \mathrm{CF}_{2} \mathrm{C}$

\section{Ethyl 5-([1,1'-biphenyl]-4-yl)-4-acetoxy-2,2-difluoro-5-oxopentanoate (6i).}

Compound $6 \mathbf{i}\left(82 \mathrm{mg}, 70 \%\right.$ yield) as a yellow solid (m.p. $56-60{ }^{\circ} \mathrm{C}$ ) was purified with silica gel chromatography (Petroleum ether: Ethyl acetate $=10: 1) .{ }^{1} \mathrm{H}$ NMR $(400 \mathrm{MHz}$, $\left.\mathrm{CDCl}_{3}\right) \delta 8.04(\mathrm{~d}, J=8.0 \mathrm{~Hz}, 2 \mathrm{H}), 7.72(\mathrm{~d}, J=8.0 \mathrm{~Hz}, 2 \mathrm{H}), 7.62(\mathrm{~d}, J=7.6 \mathrm{~Hz}, 2 \mathrm{H}), 7.48(\mathrm{t}, J=7.6$ $\mathrm{Hz}, 2 \mathrm{H}), 7.41(\mathrm{t}, J=7.6 \mathrm{~Hz}, 1 \mathrm{H}), 6.24(\mathrm{t}, J=6.8 \mathrm{~Hz}, 1 \mathrm{H}), 4.36(\mathrm{q}, J=7.2 \mathrm{~Hz}, 2 \mathrm{H}), 2.78-2.66(\mathrm{~m}$, 2H), $2.13(\mathrm{~s}, 3 \mathrm{H}), 1.38(\mathrm{t}, J=7.2 \mathrm{~Hz}, 3 \mathrm{H}) .{ }^{19} \mathrm{~F} \mathrm{NMR}\left(376 \mathrm{MHz}, \mathrm{CDCl}_{3}\right) \delta-103.0(\mathrm{dt}, J=265.1 \mathrm{~Hz}$, $13.9 \mathrm{~Hz}, 1 \mathrm{~F}),-106.1(\mathrm{dt}, J=265.1 \mathrm{~Hz}, 16.5 \mathrm{~Hz}, 1 \mathrm{~F}) .{ }^{13} \mathrm{C} \mathrm{NMR}\left(101 \mathrm{MHz}, \mathrm{CDCl}_{3}\right) \delta 194.0,170.2$, $163.8(\mathrm{t}, J=31.3 \mathrm{~Hz}), 147.3,140.0,132.8,129.7,129.5,129.0,128.1,127.8,114.9(\mathrm{t}, J=253.4 \mathrm{~Hz})$, $69.5(\mathrm{dd}, J=3.1 \mathrm{~Hz}, 5.5 \mathrm{~Hz}), 63.8,36.4(\mathrm{t}, J=24.4 \mathrm{~Hz}), 20.9,14.5 . \mathrm{MS}(\mathrm{DART}): \mathrm{m} / \mathrm{z}(\%) 391(\mathrm{M}+\mathrm{H})^{+}$. HRMS (DART): Calcd. for $\mathrm{C}_{21} \mathrm{H}_{21} \mathrm{O}_{5} \mathrm{~F}_{2}: 391.1352(\mathrm{M}+\mathrm{H})^{+}$; Found: $391.1350(\mathrm{M}+\mathrm{H})^{+}$.

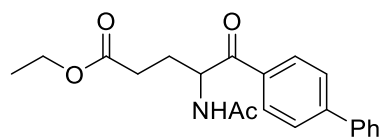

\section{Ethyl 5-([1,1'-biphenyl]-4-yl)-4-acetamido-5-oxopentanoate}

$(6 \mathbf{j})$.

Compound $\mathbf{6 j}$ (45.6 mg, 43\% yield) as a white solid (m.p. 97-101 ${ }^{\circ} \mathrm{C}$ ) was purified with silica gel chromatography (Petroleum ether: Ethyl acetate $=5: 1) .{ }^{1} \mathrm{H} \mathrm{NMR}(400 \mathrm{MHz}$, $\left.\mathrm{CDCl}_{3}\right) \delta 8.14(\mathrm{~d}, J=8.0 \mathrm{~Hz}, 2 \mathrm{H}), 7.71(\mathrm{~d}, J=8.0 \mathrm{~Hz}, 2 \mathrm{H}), 7.61(\mathrm{~d}, J=7.6 \mathrm{~Hz}, 2 \mathrm{H}), 7.45(\mathrm{t}, J=7.6$ $\mathrm{Hz}, 2 \mathrm{H}), 7.39$ (t, $J=7.6 \mathrm{~Hz}, 1 \mathrm{H}), 6.70(\mathrm{~d}, J=7.6 \mathrm{~Hz}, 1 \mathrm{H}), 5.77-5.69(\mathrm{~m}, 1 \mathrm{H}), 4.13(\mathrm{q}, J=7.2 \mathrm{~Hz}$ 2H), $2.55-2.41(\mathrm{~m}, 1 \mathrm{H}), 2.41-2.29(\mathrm{~m}, 2 \mathrm{H}), 2.06(\mathrm{~s}, 3 \mathrm{H}), 1.86-1.75(\mathrm{~m}, 1 \mathrm{H}), 1.24(\mathrm{t}, J=7.2 \mathrm{~Hz}$, 3H). ${ }^{13} \mathrm{C}$ NMR $\left(101 \mathrm{MHz}, \mathrm{CDCl}_{3}\right) \delta 198.5,173.3,170.7,147.2,140.1,133.2,130.0,129.5,128.9$, 128.0, 127.8, 61.2, 53.4, 30.4, 29.3, 23.8, 14.7. MS (ESI): m/z (\%) $354(\mathrm{M}+\mathrm{H})^{+}$. HRMS (DART): Calcd. for $\mathrm{C}_{21} \mathrm{H}_{24} \mathrm{O}_{4} \mathrm{~N}$ : $354.1700(\mathrm{M}+\mathrm{H})^{+}$; Found: $354.1697(\mathrm{M}+\mathrm{H})^{+}$.

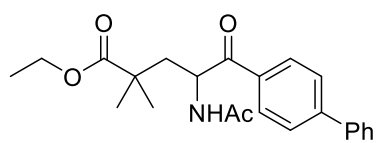

\section{Ethyl}

5-([1,1'-biphenyl]-4-yl)-4-acetamido-2,2-dimethyl-5-

oxopentanoate (6k). Compound 6k (60 mg, 52\% yield) as a white solid (m.p. $62-66{ }^{\circ} \mathrm{C}$ ) was purified with silica gel chromatography (Petroleum ether: Ethyl acetate =2: 1). ${ }^{1} \mathrm{H}$ NMR $\left(400 \mathrm{MHz}, \mathrm{CDCl}_{3}\right) \delta 8.09(\mathrm{~d}, J=8.0 \mathrm{~Hz}, 2 \mathrm{H}), 7.70(\mathrm{~d}, J=8.0 \mathrm{~Hz}, 2 \mathrm{H}), 7.61(\mathrm{~d}, J=7.6 \mathrm{~Hz}, 2 \mathrm{H}), 7.45$ (t, $J=7.6 \mathrm{~Hz}, 2 \mathrm{H}), 7.39(\mathrm{t}, J=7.6 \mathrm{~Hz}, 1 \mathrm{H}), 6.39(\mathrm{~d}, J=8.8 \mathrm{~Hz}, 1 \mathrm{H}), 5.80-5.71(\mathrm{~m}, 1 \mathrm{H}), 4.12(\mathrm{q}, J=$ $7.2 \mathrm{~Hz}, 2 \mathrm{H}), 2.10-1.89(\mathrm{~m}, 5 \mathrm{H}), 1.32(\mathrm{~s}, 3 \mathrm{H}), 1.27-1.21(\mathrm{~m}, 6 \mathrm{H}) .{ }^{13} \mathrm{C} \mathrm{NMR}\left(101 \mathrm{MHz}, \mathrm{CDCl}_{3}\right) \delta$ $199.3,178.1,170.3,147.0,140.1,133.6,129.8,129.5,128.9,128.0,127.8,61.3,51.4,42.2,41.8,28.0$, 
24.5, 23.7, 14.6. MS (ESI): m/z (\%) $382(\mathrm{M}+\mathrm{H})^{+}$. HRMS (ESI): Calcd. for $\mathrm{C}_{23} \mathrm{H}_{28} \mathrm{O}_{4} \mathrm{~N}: 382.2013$ $(\mathrm{M}+\mathrm{H})^{+}$; Found: $382.2007(\mathrm{M}+\mathrm{H})^{+}$.

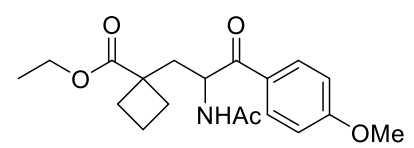

\section{Ethyl 1-(2-acetamido-3-(4-methoxyphenyl)-3-oxopropyl)cyclobutane-1-} carboxylate (61). The reaction was conducted with $\mathbf{L 3}$ as the ligand. Compound 61 (39 mg, 37\% yield) as a colorless oil was purified with silica gel chromatography (Petroleum ether: Ethyl acetate $=2: 1) .{ }^{1} \mathrm{H}$ NMR $\left(500 \mathrm{MHz}, \mathrm{CDCl}_{3}\right) \delta 8.05(\mathrm{~d}, J$ $=9.0 \mathrm{~Hz}, 2 \mathrm{H}), 6.96(\mathrm{~d}, J=9.0 \mathrm{~Hz}, 2 \mathrm{H}), 6.34(\mathrm{~d}, J=8.5 \mathrm{~Hz}, 1 \mathrm{H}), 5.72-5.66(\mathrm{~m}, 1 \mathrm{H}), 4.26-4.12(\mathrm{~m}$, 2H), $3.87(\mathrm{~s}, 3 \mathrm{H}), 2.46-2.32(\mathrm{~m}, 3 \mathrm{H}), 2.07-1.95(\mathrm{~m}, 4 \mathrm{H}), 1.95(\mathrm{~s}, 3 \mathrm{H}), 1.91-1.82(\mathrm{~m}, 1 \mathrm{H}), 1.28(\mathrm{t}$, $J=7.5 \mathrm{~Hz}, 3 \mathrm{H}) .{ }^{13} \mathrm{C}$ NMR $\left(126 \mathrm{MHz}, \mathrm{CDCl}_{3}\right) \delta 197.8,176.9,170.1,164.7,131.8,127.7,114.6,61.3$, 56.1, 51.7, 46.8, 41.4, 31.7, 31.6, 23.7, 17.0, 14.8. MS (ESI): m/z (\%) $348(\mathrm{M}+\mathrm{H})^{+}$. HRMS (ESI): Calcd. for $\mathrm{C}_{19} \mathrm{H}_{26} \mathrm{O}_{5} \mathrm{~N}$ : $348.1805(\mathrm{M}+\mathrm{H})^{+}$; Found: $348.1800(\mathrm{M}+\mathrm{H})^{+}$.

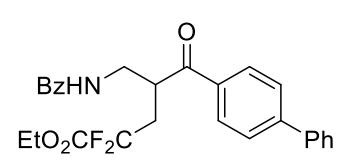

Ethyl

5-([1,1'-biphenyl]-4-yl)-4-(benzamidomethyl)-2,2-difluoro-5oxopentanoate (7a). Compound $7 \mathbf{a}(99 \mathrm{mg}, 71 \%$ yield) as a colorless oil was purified with silica gel chromatography (Petroleum ether: Ethyl acetate $=5: 1$ to 3: 1). ${ }^{1} \mathrm{H}$ NMR $\left(400 \mathrm{MHz}, \mathrm{CDCl}_{3}\right) \delta 8.13(\mathrm{~d}, J=8.4 \mathrm{~Hz}, 2 \mathrm{H}), 7.68(\mathrm{~d}, J=8.0 \mathrm{~Hz}, 2 \mathrm{H}), 7.65(\mathrm{~d}, J=8.4$ $\mathrm{Hz}, 2 \mathrm{H}), 7.57$ (d, J=7.2 Hz, 2H), $7.48-7.36(\mathrm{~m}, 4 \mathrm{H}), 7.33$ (t, $J=7.2 \mathrm{~Hz}, 2 \mathrm{H}), 7.09$ (s, 1H), $4.45-$ $4.35(\mathrm{~m}, 1 \mathrm{H}), 4.29-4.12(\mathrm{~m}, 2 \mathrm{H}), 3.90-3.79(\mathrm{~m}, 1 \mathrm{H}), 3.63-3.53(\mathrm{~m}, 1 \mathrm{H}), 3.01-2.82(\mathrm{~m}, 1 \mathrm{H}), 2.48$ $-2.31(\mathrm{~m}, 1 \mathrm{H}), 1.23(\mathrm{t}, J=7.2 \mathrm{~Hz}, 3 \mathrm{H}) .{ }^{19} \mathrm{~F} \mathrm{NMR}\left(376 \mathrm{MHz}, \mathrm{CDCl}_{3}\right) \delta-102.9--104.6(\mathrm{~m}, 2 \mathrm{~F}) .{ }^{13} \mathrm{C}$ NMR (101 MHz, $\left.\mathrm{CDCl}_{3}\right) \delta 201.0,168.7,164.2$ (t, $\left.J=32.6 \mathrm{~Hz}\right), 146.9,140.0,135.1,134.4,132.1$, $129.7,129.4,129.0,128.8,127.9,127.7,127.4,115.8(\mathrm{t}, J=252.3 \mathrm{~Hz}), 63.6,42.9,39.8,34.8(\mathrm{t}, J=$ 23.8 Hz), 14.2. MS (ESI): m/z (\%) $466(\mathrm{M}+\mathrm{H})^{+}$. HRMS (ESI): Calcd. for $\mathrm{C}_{27} \mathrm{H}_{26} \mathrm{O}_{4} \mathrm{NF}_{2}: 466.1824$ $(\mathrm{M}+\mathrm{H})^{+}$; Found: $466.1824(\mathrm{M}+\mathrm{H})^{+}$.

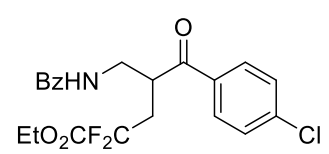

Ethyl

4-(benzamidomethyl)-5-(4-chlorophenyl)-2,2-difluoro-5oxopentanoate (7b). Compound $7 \mathbf{b}(89 \mathrm{mg}, 72 \%$ yield) as a yellow oil was purified with silica gel chromatography (Petroleum ether: Ethyl acetate $=4: 1$ ). ${ }^{1} \mathrm{H}$ NMR $\left(400 \mathrm{MHz}, \mathrm{CDCl}_{3}\right) \delta 7.99(\mathrm{~d}, J=8.8 \mathrm{~Hz}, 2 \mathrm{H}), 7.65(\mathrm{~d}, J=7.2 \mathrm{~Hz}, 2 \mathrm{H}), 7.46(\mathrm{t}, J=7.2 \mathrm{~Hz}$, 
1H), $7.40(\mathrm{~d}, J=8.8 \mathrm{~Hz}, 2 \mathrm{H}), 7.35(\mathrm{~d}, J=7.2 \mathrm{~Hz}, 2 \mathrm{H}), 6.91(\mathrm{t}, J=5.6 \mathrm{~Hz}, 1 \mathrm{H}), 4.33-4.14(\mathrm{~m}, 3 \mathrm{H})$, $3.81-3.73(\mathrm{~m}, 1 \mathrm{H}), 3.54-3.46(\mathrm{~m}, 1 \mathrm{H}), 2.91-2.74(\mathrm{~m}, 1 \mathrm{H}), 2.41-2.26(\mathrm{~m}, 1 \mathrm{H}), 1.23(\mathrm{t}, J=7.2 \mathrm{~Hz}$, 3H). ${ }^{19} \mathrm{~F}$ NMR $\left(376 \mathrm{MHz}, \mathrm{CDCl}_{3}\right) \delta-103.9(\mathrm{t}, J=17.3 \mathrm{~Hz}, 2 \mathrm{~F}) .{ }^{13} \mathrm{C} \mathrm{NMR}\left(101 \mathrm{MHz}, \mathrm{CDCl}_{3}\right) \delta 200.4$, $168.8,164.1(\mathrm{t}, J=32.7 \mathrm{~Hz}), 140.9,134.8,134.3,132.3,130.6,129.7,129.1,127.4,115.7(\mathrm{t}, J=252.5$ $\mathrm{Hz}), 63.7,42.9,39.8,34.9(\mathrm{t}, J=23.5 \mathrm{~Hz}), 14.3$. MS (ESI): m/z (\%) $446(\mathrm{M}+\mathrm{Na})^{+}$. HRMS (ESI): Calcd. for $\mathrm{C}_{21} \mathrm{H}_{21} \mathrm{O}_{4} \mathrm{NClF}_{2}$ : $424.1122(\mathrm{M}+\mathrm{H})^{+}$; Found: $424.1123(\mathrm{M}+\mathrm{H})^{+}$.

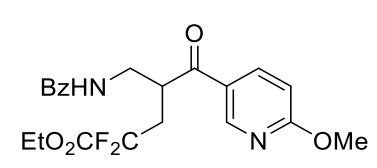

\section{Ethyl 4-(benzamidomethyl)-2,2-difluoro-5-(6-methoxypyridin-3-yl)-5-} oxopentanoate (7c). The reaction was conducted with $\mathbf{L 7}$ as the ligand. Compound $7 \mathrm{c}$ (77 mg, 61\% yield) as a yellow oil was purified with silica gel chromatography (Petroleum ether: Ethyl acetate = 3: 1). ${ }^{1} \mathrm{H}$ NMR $\left(400 \mathrm{MHz}, \mathrm{CDCl}_{3}\right) \delta 8.91(\mathrm{~s}, 1 \mathrm{H})$, $8.20(\mathrm{~d}, J=8.4 \mathrm{~Hz}, 1 \mathrm{H}), 7.67(\mathrm{~d}, J=7.6 \mathrm{~Hz}, 2 \mathrm{H}), 7.46(\mathrm{t}, J=7.6 \mathrm{~Hz}, 1 \mathrm{H}), 7.36(\mathrm{t}, J=7.6 \mathrm{~Hz}, 2 \mathrm{H})$, $6.85(\mathrm{~s}, 1 \mathrm{H}), 6.76(\mathrm{~d}, J=8.4 \mathrm{~Hz}, 1 \mathrm{H}), 4.31-4.13(\mathrm{~m}, 3 \mathrm{H}), 3.97(\mathrm{~s}, 3 \mathrm{H}), 3.86-3.75(\mathrm{~m}, 1 \mathrm{H}), 3.58-$ $3.48(\mathrm{~m}, 1 \mathrm{H}), 2.92-2.73(\mathrm{~m}, 1 \mathrm{H}), 2.43-2.25(\mathrm{~m}, 1 \mathrm{H}), 1.25(\mathrm{t}, J=7.2 \mathrm{~Hz}, 3 \mathrm{H}) .{ }^{19} \mathrm{~F}$ NMR $(376 \mathrm{MHz}$, $\left.\mathrm{CDCl}_{3}\right) \delta-103.2--104.8(\mathrm{~m}, 2 \mathrm{~F}) .{ }^{13} \mathrm{C} \mathrm{NMR}\left(101 \mathrm{MHz}, \mathrm{CDCl}_{3}\right) \delta 199.3,168.6,167.8,164.2(\mathrm{t}, J=$ $32.6 \mathrm{~Hz}), 150.4$, 139.1, 134.3, 132.3, 129.1, 127.4, 126.2, 115.8 (t, $J=252.9 \mathrm{~Hz}), 111.9,63.8,54.7$, 42.7, 39.7, $34.8(\mathrm{t}, J=23.4 \mathrm{~Hz}), 14.3$. MS (ESI): $\mathrm{m} / \mathrm{z}(\%) 421(\mathrm{M}+\mathrm{H})^{+}$. HRMS (ESI): Calcd. for $\mathrm{C}_{21} \mathrm{H}_{23} \mathrm{O}_{5} \mathrm{~N}_{2} \mathrm{~F}_{2}$ : $421.1570(\mathrm{M}+\mathrm{H})^{+}$; Found: $421.1566(\mathrm{M}+\mathrm{H})^{+}$.

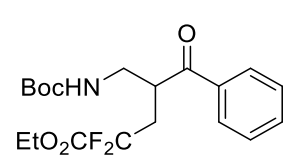

Ethyl 4-(((tert-butoxycarbonyl)amino)methyl)-2,2-difluoro-5-oxo-5-

phenylpentanoate (7d). The reaction was conducted with $\mathbf{L 8}$ as the ligand. Compound 7d (71 mg, 61\% yield) as a yellow oil was purified with silica gel chromatography (Petroleum ether: Ethyl acetate $=8: 1$ to 5: 1). ${ }^{1} \mathrm{H}$ NMR $\left(400 \mathrm{MHz}, \mathrm{CDCl}_{3}\right) \delta 8.00(\mathrm{~d}$, $J=7.2 \mathrm{~Hz}, 2 \mathrm{H}), 7.57(\mathrm{t}, J=7.2 \mathrm{~Hz}, 1 \mathrm{H}), 7.46(\mathrm{t}, J=7.2 \mathrm{~Hz}, 2 \mathrm{H}), 4.96-4.74(\mathrm{~m}, 1 \mathrm{H}), 4.35-4.12(\mathrm{~m}$, $3 \mathrm{H}), 3.48-3.39(\mathrm{~m}, 1 \mathrm{H}), 3.30-3.21(\mathrm{~m}, 1 \mathrm{H}), 2.86-2.67(\mathrm{~m}, 1 \mathrm{H}), 2.34-2.17(\mathrm{~m}, 1 \mathrm{H}), 1.44-1.33$ $(\mathrm{m}, 9 \mathrm{H}), 1.24(\mathrm{t}, J=7.2 \mathrm{~Hz}, 3 \mathrm{H}) .{ }^{19} \mathrm{~F}$ NMR $\left(376 \mathrm{MHz}, \mathrm{CDCl}_{3}\right) \delta-103.2--104.9(\mathrm{~m}, 2 \mathrm{~F}) .{ }^{13} \mathrm{C}$ NMR $\left(101 \mathrm{MHz}, \mathrm{CDCl}_{3}\right) \delta 201.5,164.2(\mathrm{t}, J=32.6 \mathrm{~Hz}), 156.4,136.6,134.2,129.3,129.1,115.8(\mathrm{t}, J=$ $252.4 \mathrm{~Hz}), 80.2,63.6,43.6,40.0,34.6(\mathrm{t}, J=23.8 \mathrm{~Hz}), 28.8,14.3 . \mathrm{MS}(\mathrm{ESI}): \mathrm{m} / \mathrm{z}(\%) 408(\mathrm{M}+\mathrm{Na})^{+}$. HRMS (ESI): Calcd. for $\mathrm{C}_{19} \mathrm{H}_{26} \mathrm{O}_{5} \mathrm{NF}_{2}: 386.1774(\mathrm{M}+\mathrm{H})^{+}$; Found: $386.1771(\mathrm{M}+\mathrm{H})^{+}$. 
Gram-Scale Synthesis of Compound 7d: To a 1 L of Schlenk tube were added $t$-butyl $N$ allylcarbamate (1.0 g, 1.0 equiv), phenylboronic acid 3a (1.94 g, 2.5 equiv), $\mathrm{NiCl}_{2} \cdot \mathrm{DME}$ (139.9 mg, $10 \mathrm{~mol} \%$ ), 4,4'-diF-2,2'-bpy (122.4 mg, $10 \mathrm{~mol} \%$ ) and $\mathrm{K}_{2} \mathrm{CO}_{3}$ (4.39 g, 4.0 equiv) under Ar. The reaction mixture was then evacuated and backfilled with $\operatorname{Ar}(3$ times). The mixture was evacuated again and backfilled with carbon monoxide $\mathrm{CO}$ (1 atm), followed by addition of ethyl bromodifluoroacetate $2 \mathbf{a}\left(3.2 \mathrm{~g}, 2.5\right.$ equiv), $\mathrm{H}_{2} \mathrm{O}(85 \mu \mathrm{L})$ and dioxane $(75 \mathrm{~mL})$. The tube was screwcapped and heated to $50{ }^{\circ} \mathrm{C}$ (oil bath). After stirring for $48 \mathrm{~h}$, the reaction mixture was cooled to room temperature, the reaction mixture was diluted with ethyl acetate and $\mathrm{H}_{2} \mathrm{O}$. The resulting mixture was extracted with ethyl acetate, the combined organic layers were washed with brine, dried over $\mathrm{Na}_{2} \mathrm{SO}_{4}$, filtered and concentrated. The residue was purified with silica gel chromatography to give the product $7 \mathbf{d}(1.37 \mathrm{~g}, 56 \%)$.

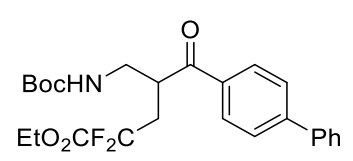

Ethyl 5-([1,1'-biphenyl]-4-yl)-4-(((tert-butoxycarbonyl)amino)methyl)-2,2difluoro-5-oxopentanoate (7e). Compound 7e (100 mg, 72\% yield) as a yellow oil was purified with silica gel chromatography (Petroleum ether: Ethyl acetate $=8: 1$ to 5: 1). ${ }^{1} \mathrm{H}$ NMR (400 MHz, $\left.\mathrm{CDCl}_{3}\right) \delta 8.10(\mathrm{~d}, J=8.4 \mathrm{~Hz}, 2 \mathrm{H}), 7.70(\mathrm{~d}, J=8.4 \mathrm{~Hz}, 2 \mathrm{H}), 7.62$ $(\mathrm{d}, J=7.2 \mathrm{~Hz}, 2 \mathrm{H}), 7.47(\mathrm{t}, J=7.2 \mathrm{~Hz}, 2 \mathrm{H}), 7.40$ (t, $J=7.2 \mathrm{~Hz}, 1 \mathrm{H}), 5.00-4.77(\mathrm{~m}, 1 \mathrm{H}), 4.36-4.15$ (m, 3H), $3.54-3.42(\mathrm{~m}, 1 \mathrm{H}), 3.35-3.24(\mathrm{~m}, 1 \mathrm{H}), 2.92-2.71(\mathrm{~m}, 1 \mathrm{H}), 2.38-2.20(\mathrm{~m}, 1 \mathrm{H}), 1.50-$ $1.34(\mathrm{~m}, 9 \mathrm{H}), 1.27$ (t, $J=7.2 \mathrm{~Hz}, 3 \mathrm{H}) .{ }^{19} \mathrm{~F}$ NMR $\left(376 \mathrm{MHz}, \mathrm{CDCl}_{3}\right) \delta-103.6(\mathrm{dt}, J=259.4 \mathrm{~Hz}, 18.8$ $\mathrm{Hz}, 1 \mathrm{~F}),-104.4(\mathrm{dt}, J=259.4 \mathrm{~Hz}, 18.8 \mathrm{~Hz}, 1 \mathrm{~F}) .{ }^{13} \mathrm{C} \mathrm{NMR}\left(101 \mathrm{MHz}, \mathrm{CDCl}_{3}\right) \delta 201.1,164.3(\mathrm{t}, J=$ $32.5 \mathrm{~Hz}), 156.4,146.8,140.2,135.3,129.7,129.5,128.9,128.0,127.8,115.8(\mathrm{t}, J=252.4 \mathrm{~Hz}), 80.2$, 63.6, 43.7, 40.0, 34.7 (t, $J=23.4 \mathrm{~Hz}), 28.8,14.3$. MS (ESI): m/z (\%) $484(\mathrm{M}+\mathrm{Na})^{+}$. HRMS (ESI): Calcd. for $\mathrm{C}_{25} \mathrm{H}_{30} \mathrm{O}_{5} \mathrm{NF}_{2}: 462.2087(\mathrm{M}+\mathrm{H})^{+}$; Found: $462.2083(\mathrm{M}+\mathrm{H})^{+}$.

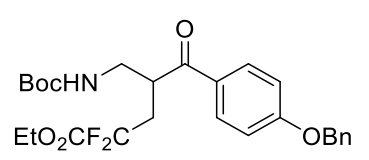

Ethyl 5-(4-(benzyloxy)phenyl)-4-(((tert-butoxycarbonyl)amino)methyl)2,2-difluoro-5-oxopentanoate (7f). The reaction was conducted with $\mathbf{L} 7$ as the ligand. Compound $\mathbf{7 f}(91 \mathrm{mg}, 62 \%$ yield) as a yellow oil was purified with silica gel chromatography (Petroleum ether: Ethyl acetate $=8: 1$ to 4: 1). ${ }^{1} \mathrm{H}$ NMR $\left(400 \mathrm{MHz}, \mathrm{CDCl}_{3}\right) \delta 8.01$ $(\mathrm{d}, J=8.4 \mathrm{~Hz}, 2 \mathrm{H}), 7.50-7.30(\mathrm{~m}, 5 \mathrm{H}), 7.02(\mathrm{~d}, J=8.4 \mathrm{~Hz}, 2 \mathrm{H}), 5.12(\mathrm{~s}, 2 \mathrm{H}), 4.92(\mathrm{~s}, 1 \mathrm{H}), 4.40-$ 
$3.98(\mathrm{~m}, 3 \mathrm{H}), 3.48-3.38(\mathrm{~m}, 1 \mathrm{H}), 3.29-3.19(\mathrm{~m}, 1 \mathrm{H}), 2.87-2.68(\mathrm{~m}, 1 \mathrm{H}), 2.33-2.16(\mathrm{~m}, 1 \mathrm{H}), 1.50$ - $1.35(\mathrm{~m}, 9 \mathrm{H}), 1.25(\mathrm{t}, J=7.2 \mathrm{~Hz}, 3 \mathrm{H}) .{ }^{19} \mathrm{~F} \mathrm{NMR}\left(376 \mathrm{MHz}, \mathrm{CDCl}_{3}\right) \delta-103.6$ (ddd, $J=259.4 \mathrm{~Hz}$, 19.2 Hz, 15.0 Hz, 1F), -104.6 (dt, $J=259.4 \mathrm{~Hz}, 18.8 \mathrm{~Hz}, 1 \mathrm{~F}) .{ }^{13} \mathrm{C}$ NMR $\left(101 \mathrm{MHz}, \mathrm{CDCl}_{3}\right) \delta 199.7$, $164.3(\mathrm{t}, J=33.0 \mathrm{~Hz}), 163.6,156.4,136.6,131.5,129.9,129.2,128.8,128.0,115.9$ (t, $J=252.3 \mathrm{~Hz})$, 115.4, 80.1, 70.7, 63.5, 43.8, 39.6, 34.7 (t, $J=23.7 \mathrm{~Hz}), 28.8,14.3 . \mathrm{MS}(\mathrm{ESI}): \mathrm{m} / \mathrm{z}(\%) 514(\mathrm{M}+\mathrm{Na})^{+}$. HRMS (ESI): Calcd. for $\mathrm{C}_{26} \mathrm{H}_{31} \mathrm{O}_{6} \mathrm{NF}_{2} \mathrm{Na}$ : $514.2012(\mathrm{M}+\mathrm{Na})^{+}$; Found: $514.2007(\mathrm{M}+\mathrm{Na})^{+}$.

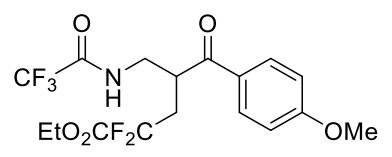

\section{Ethyl}

2,2-difluoro-5-(4-methoxyphenyl)-5-oxo-4-((2,2,2-

trifluoroacetamido)methyl)pentanoate $(\mathbf{7 g})$. The reaction was conducted with $\mathbf{L 3}$ as the ligand. Compound $7 \mathbf{g}$ (102 mg, 83\% yield) as an orange oil was purified with silica gel chromatography (Petroleum ether: Ethyl acetate $=4: 1) .{ }^{1} \mathrm{H}$ NMR $\left(400 \mathrm{MHz}, \mathrm{CDCl}_{3}\right) \delta 7.95(\mathrm{~d}, J=$ $8.8 \mathrm{~Hz}, 2 \mathrm{H}), 7.32(\mathrm{~s}, 1 \mathrm{H}), 6.94(\mathrm{~d}, J=8.8 \mathrm{~Hz}, 2 \mathrm{H}), 4.30-4.11(\mathrm{~m}, 3 \mathrm{H}), 3.85(\mathrm{~m}, 3 \mathrm{H}), 3.75-3.65(\mathrm{~m}$, $1 \mathrm{H}), 3.57-3.48(\mathrm{~m}, 1 \mathrm{H}), 2.80-2.63(\mathrm{~m}, 1 \mathrm{H}), 2.33-2.17(\mathrm{~m}, 1 \mathrm{H}), 1.26(\mathrm{t}, J=7.2 \mathrm{~Hz}, 3 \mathrm{H}) .{ }^{19} \mathrm{~F} \mathrm{NMR}$ $\left(376 \mathrm{MHz} \mathrm{CDCl}_{3}\right) \delta-76.1(\mathrm{~s}, 3 \mathrm{~F}),-103.8(\mathrm{ddd}, J=261.7 \mathrm{~Hz}, 19.9 \mathrm{~Hz}, 14.3 \mathrm{~Hz}, 1 \mathrm{~F}),-104.6(\mathrm{ddd}, J=$ 261.7 Hz, 19.9 Hz, 15.4 Hz, 1F). $\left.{ }^{13} \mathrm{C} \mathrm{NMR} \mathrm{(101} \mathrm{MHz,} \mathrm{CDCl}_{3}\right) \delta 199.2,165.0,164.0$ (t, $\left.J=32.5 \mathrm{~Hz}\right)$, $158.4(\mathrm{q}, J=37.6 \mathrm{~Hz}), 131.6,128.8,116.2(\mathrm{q}, J=288.8 \mathrm{~Hz}), 115.6(\mathrm{t}, J=253.0 \mathrm{~Hz}), 114.8,63.9,56.1$, 42.2, $38.4(\mathrm{t}, J=2.0 \mathrm{~Hz}), 34.9(\mathrm{t}, J=23.7 \mathrm{~Hz}), 14.2$. MS (ESI): m/z (\%) $412(\mathrm{M}+\mathrm{H})^{+}$. HRMS (ESI): Calcd. for $\mathrm{C}_{17} \mathrm{H}_{19} \mathrm{O}_{5} \mathrm{NF}_{5}: 412.1178(\mathrm{M}+\mathrm{H})^{+}$; Found: $412.1178(\mathrm{M}+\mathrm{H})^{+}$.

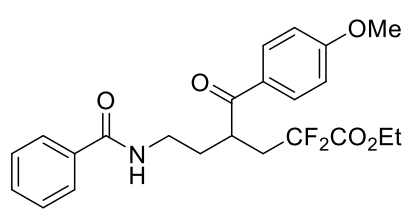

Ethyl 6-benzamido-2,2-difluoro-4-(4-methoxybenzoyl)hexanoate (7h). The reaction was conducted with $\mathbf{L 3}$ as the ligand. Compound $\mathbf{7 h}$ ( $74 \mathrm{mg}$, $57 \%$ yield) as a yellow oil was purified with silica gel chromatography (Petroleum ether: Ethyl acetate $=4: 1$ to $1.5: 1) .{ }^{1} \mathrm{H} \mathrm{NMR}\left(400 \mathrm{MHz}, \mathrm{CDCl}_{3}\right) \delta 7.90(\mathrm{~d}, J=8.8 \mathrm{~Hz}$, 2H), $7.65(\mathrm{~d}, J=7.6 \mathrm{~Hz}, 2 \mathrm{H}), 7.44(\mathrm{t}, J=7.2 \mathrm{~Hz}, 1 \mathrm{H}), 7.35$ (t, $J=7.6 \mathrm{~Hz}, 2 \mathrm{H}), 6.86(\mathrm{~d}, J=8.8 \mathrm{~Hz}$, 2H), $6.59(\mathrm{~m}, 1 \mathrm{H}), 4.23-4.13(\mathrm{~m}, 2 \mathrm{H}), 3.89-3.82(\mathrm{~m}, 1 \mathrm{H}), 3.81(\mathrm{~s}, 3 \mathrm{H}), 3.49-3.31(\mathrm{~m}, 2 \mathrm{H}), 2.93-$ $2.75(\mathrm{~m}, 1 \mathrm{H}), 2.31-2.15(\mathrm{~m}, 1 \mathrm{H}), 2.14-2.03(\mathrm{~m}, 1 \mathrm{H}), 1.92-1.81(\mathrm{~m}, 1 \mathrm{H}), 1.22(\mathrm{t}, J=7.2 \mathrm{~Hz}, 3 \mathrm{H})$. ${ }^{19} \mathrm{~F}$ NMR $\left(376 \mathrm{MHz}, \mathrm{CDCl}_{3}\right) \delta-103.8(\mathrm{ddd}, J=259.4 \mathrm{~Hz}, 22.6 \mathrm{~Hz}, 15.0 \mathrm{~Hz}, 1 \mathrm{~F}),-104.8(\mathrm{ddd}, J=$ $259.4 \mathrm{~Hz}, 22.6 \mathrm{~Hz}, 15.0 \mathrm{~Hz}, 1 \mathrm{~F}) .{ }^{13} \mathrm{C}$ NMR $\left(126 \mathrm{MHz} \mathrm{CDCl}_{3}\right) \delta 200.5,168.0,164.4,164.3(\mathrm{t}, J=31.5$ Hz), 134.6, 132.0, 131.3, 129.2, 129.0, $127.3,115.9$ (t, $J=252.0 \mathrm{~Hz}), 114.5,63.6,56.0,38.2,37.4(\mathrm{t}$, 
$J=2.5 \mathrm{~Hz}), 36.5(\mathrm{t}, J=23.9 \mathrm{~Hz}), 33.4,14.3 . \mathrm{MS}(\mathrm{ESI}): \mathrm{m} / \mathrm{z}(\%) 434(\mathrm{M}+\mathrm{H})^{+}$. HRMS (ESI): Calcd. for $\mathrm{C}_{26} \mathrm{H}_{25} \mathrm{O}_{4} \mathrm{NF}_{2}$ : $434.1762(\mathrm{M}+\mathrm{H})^{+}$; Found: $434.1764(\mathrm{M}+\mathrm{H})^{+}$.

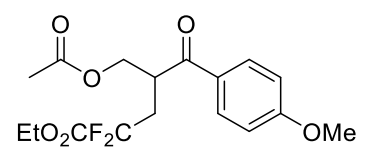

Ethyl

4-(acetoxymethyl)-2,2-difluoro-5-(4-methoxyphenyl)-5-

Compound $7 \mathbf{i}$ (77 $\mathrm{mg}, 72 \%$ yield) as a yellow oil was purified with silica gel chromatography $\left(\right.$ Petroleum ether: Ethyl acetate = 10: 1). ${ }^{1} \mathrm{H}$ NMR $\left(400 \mathrm{MHz}, \mathrm{CDCl}_{3}\right) \delta 7.98(\mathrm{~d}, J=8.8 \mathrm{~Hz}, 2 \mathrm{H}), 6.95$ $(\mathrm{d}, J=8.8 \mathrm{~Hz}, 2 \mathrm{H}), 4.38-4.31(\mathrm{~m}, 1 \mathrm{H}), 4.28-4.17(\mathrm{~m}, 2 \mathrm{H}), 4.16-4.07(\mathrm{~m}, 2 \mathrm{H}), 3.86(\mathrm{~s}, 3 \mathrm{H}), 2.93$ $-2.76(\mathrm{~m}, 1 \mathrm{H}), 2.38-2.23(\mathrm{~m}, 1 \mathrm{H}), 1.95(\mathrm{~s}, 3 \mathrm{H}), 1.26(\mathrm{t}, J=7.2 \mathrm{~Hz}, 3 \mathrm{H}) .{ }^{19} \mathrm{~F} \mathrm{NMR}\left(376 \mathrm{MHz}, \mathrm{CDCl}_{3}\right)$ $\delta$-103.9 (ddd, $J=260.6 \mathrm{~Hz}, 20.3 \mathrm{~Hz}, 13.5 \mathrm{~Hz}, 1 \mathrm{~F}),-105.0$ (ddd, $J=260.6 \mathrm{~Hz}, 20.3 \mathrm{~Hz}, 15.0 \mathrm{~Hz}, 1 \mathrm{~F}$ ). ${ }^{13} \mathrm{C} \mathrm{NMR}\left(101 \mathrm{MHz}, \mathrm{CDCl}_{3}\right) \delta 197.4,171.1,164.5,164.1(\mathrm{t}, J=32.6 \mathrm{~Hz}), 131.4,129.4,115.7(\mathrm{t}, J=$ 252.4 Hz), 114.5, 65.4, 63.6, 56.0, $39.4(\mathrm{t}, J=2.9 \mathrm{~Hz}), 33.8$ (t, $J=23.6 \mathrm{~Hz}), 21.1,14.3 . \mathrm{MS}(\mathrm{ESI}): \mathrm{m} / \mathrm{z}$ (\%) $359(\mathrm{M}+\mathrm{H})^{+}$. HRMS (ESI): Calcd. for $\mathrm{C}_{17} \mathrm{H}_{21} \mathrm{O}_{6} \mathrm{~F}_{2}$ : $359.1301(\mathrm{M}+\mathrm{H})^{+}$; Found: $359.1301(\mathrm{M}+\mathrm{H})^{+}$.

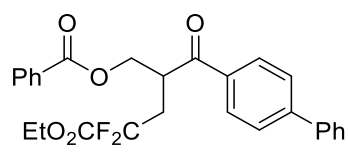

\section{2-([1,1'-Biphenyl]-4-carbonyl)-5-ethoxy-4,4-difluoro-5-oxopentyl benzoate}

(7j). Compound $7 \mathbf{j}$ (83 mg, 59\% yield) as a colorless oil was purified with silica gel chromatography (Petroleum ether: Ethyl acetate $=20: 1) .{ }^{1} \mathrm{H}$ NMR $(400$ $\left.\mathrm{MHz} \mathrm{CDCl}_{3}\right) \delta 8.14(\mathrm{~d}, J=8.4 \mathrm{~Hz}, 2 \mathrm{H}), 7.85(\mathrm{~d}, J=7.2 \mathrm{~Hz}, 2 \mathrm{H}), 7.72(\mathrm{~d}, J=8.4 \mathrm{~Hz}, 2 \mathrm{H}), 7.62(\mathrm{~d}, J$ $=7.2 \mathrm{~Hz}, 2 \mathrm{H}), 7.54-7.45(\mathrm{~m}, 3 \mathrm{H}), 7.44-7.39(\mathrm{~m}, 1 \mathrm{H}), 7.35(\mathrm{t}, J=7.6 \mathrm{~Hz}, 2 \mathrm{H}), 4.61(\mathrm{dd}, J=10.8$ $\mathrm{Hz}, 6.8 \mathrm{~Hz}, 1 \mathrm{H}), 4.48(\mathrm{dd}, J=10.8 \mathrm{~Hz}, 6.4 \mathrm{~Hz}, 1 \mathrm{H}), 4.44-4.36(\mathrm{~m}, 1 \mathrm{H}), 4.33-4.18$ (m, 2H), $3.13-$ $2.97(\mathrm{~m}, 1 \mathrm{H}), 2.54-2.38(\mathrm{~m}, 1 \mathrm{H}), 1.28(\mathrm{t}, J=7.2 \mathrm{~Hz}, 3 \mathrm{H}) .{ }^{19} \mathrm{~F} \mathrm{NMR}\left(376 \mathrm{MHz}, \mathrm{CDCl}_{3}\right) \delta-103.6(\mathrm{ddd}$, $J=260.6 \mathrm{~Hz}, 20.3 \mathrm{~Hz}, 13.5 \mathrm{~Hz}, 1 \mathrm{~F}),-104.8(\mathrm{ddd}, J=260.6 \mathrm{~Hz}, 20.3 \mathrm{~Hz}, 15.4 \mathrm{~Hz}, 1 \mathrm{~F}) .{ }^{13} \mathrm{C}$ NMR $(101$ $\left.\mathrm{MHz}, \mathrm{CDCl}_{3}\right) \delta 198.9,166.5,164.2(\mathrm{t}, J=32.5 \mathrm{~Hz}), 146.9,140.2,135.4,133.8,130.1,129.8,129.7$, 129.5, 128.9, 128.0, 127.8, $115.7(\mathrm{t}, J=252.4 \mathrm{~Hz}), 66.0,63.7,39.9(\mathrm{t}, J=2.7 \mathrm{~Hz}), 33.9(\mathrm{t}, J=23.9$ $\mathrm{Hz})$, 14.3. MS (ESI): m/z (\%) $489(\mathrm{M}+\mathrm{Na})^{+}$. HRMS (ESI): Calcd. for $\mathrm{C}_{27} \mathrm{H}_{25} \mathrm{O}_{5} \mathrm{~F}_{2}: 467.1665(\mathrm{M}+\mathrm{H})^{+}$; Found: $467.1667(\mathrm{M}+\mathrm{H})^{+}$. 


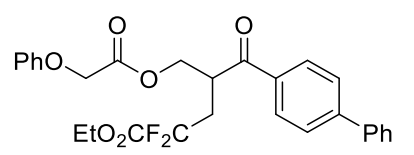

Ethyl

5-([1,1'-biphenyl]-4-yl)-2,2-difluoro-5-oxo-4-((2phenoxyacetoxy)methyl)pentanoate (7k). Compound $7 \mathbf{k}(86 \mathrm{mg}, 58 \%$ yield) as a yellow oil was purified with silica gel chromatography (Petroleum ether: Ethyl acetate $=15: 1$ to 10: 1). ${ }^{1} \mathrm{H}$ NMR $\left(400 \mathrm{MHz}, \mathrm{CDCl}_{3}\right) \delta 8.05(\mathrm{~d}, J=8.4 \mathrm{~Hz}, 2 \mathrm{H}), 7.69(\mathrm{~d}$, $J=8.4 \mathrm{~Hz}, 2 \mathrm{H}), 7.61(\mathrm{~d}, J=7.2 \mathrm{~Hz}, 2 \mathrm{H}), 7.46(\mathrm{t}, J=7.2 \mathrm{~Hz}, 2 \mathrm{H}), 7.40(\mathrm{t}, J=7.2 \mathrm{~Hz}, 1 \mathrm{H}), 7.24(\mathrm{t}, J=$ $8.4 \mathrm{~Hz}, 2 \mathrm{H}), 6.96(\mathrm{t}, J=7.2 \mathrm{~Hz}, 1 \mathrm{H}), 6.82(\mathrm{~d}, J=8.0 \mathrm{~Hz}, 2 \mathrm{H}), 4.59-4.48(\mathrm{~m}, 3 \mathrm{H}), 4.34-4.17(\mathrm{~m}$, 4H), $2.96-2.80(\mathrm{~m}, 1 \mathrm{H}), 2.40-2.24(\mathrm{~m}, 1 \mathrm{H}), 1.27(\mathrm{t}, J=7.2 \mathrm{~Hz}, 3 \mathrm{H}) .{ }^{19} \mathrm{~F}$ NMR $\left(376 \mathrm{MHz}, \mathrm{CDCl}_{3}\right)$ $\delta$-103.7 (ddd, $J=261.7 \mathrm{~Hz}, 20.3 \mathrm{~Hz}, 13.5 \mathrm{~Hz}, 1 \mathrm{~F}$ ), -104.7 (ddd, $J=261.7 \mathrm{~Hz}, 20.3 \mathrm{~Hz}, 15.0 \mathrm{~Hz}, 1 \mathrm{~F}$ ). ${ }^{13} \mathrm{C} \mathrm{NMR}\left(101 \mathrm{MHz}, \mathrm{CDCl}_{3}\right) \delta 198.3,169.2,164.0(\mathrm{t}, J=32.4 \mathrm{~Hz}), 158.1,147.0,140.1,135.0,130.1$, $129.7,129.5,129.0,128.0,127.8,122.3,115.6(\mathrm{t}, J=252.6 \mathrm{~Hz}), 115.0,65.9,65.5,63.7,39.7(\mathrm{t}, J=$ $2.8 \mathrm{~Hz}), 33.8(\mathrm{t}, J=23.6 \mathrm{~Hz}), 14.3$. MS (ESI): m/z (\%) $497(\mathrm{M}+\mathrm{H})^{+}$. HRMS (ESI): Calcd. for $\mathrm{C}_{28} \mathrm{H}_{27} \mathrm{O}_{6} \mathrm{~F}_{2}: 497.1770(\mathrm{M}+\mathrm{H})^{+}$; Found: $497.1771(\mathrm{M}+\mathrm{H})^{+}$.

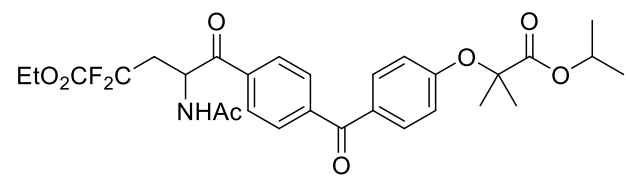

\section{Ethyl 4-acetamido-2,2-difluoro-5-(4-(4-((1-isopropoxy-2- methyl-1-oxopropan-2-yl)oxy)benzoyl)phenyl)-5-}

oxopentanoate (8a). The reaction was conducted at $80{ }^{\circ} \mathrm{C}$.

Compound 8a (131 mg, 77\% yield) as a yellow oil was purified with silica gel chromatography (Petroleum ether: Ethyl acetate $=4: 1$ to $2: 1) .{ }^{1} \mathrm{H} \mathrm{NMR}\left(400 \mathrm{MHz}, \mathrm{CDCl}_{3}\right) \delta 8.07(\mathrm{~d}, J=8.0 \mathrm{~Hz}, 2 \mathrm{H})$, $7.78(\mathrm{~d}, J=8.0 \mathrm{~Hz}, 2 \mathrm{H}), 7.72(\mathrm{~d}, J=8.8 \mathrm{~Hz}, 2 \mathrm{H}), 6.83(\mathrm{~d}, J=8.8 \mathrm{~Hz}, 2 \mathrm{H}), 6.79(\mathrm{~d}, J=8.4 \mathrm{~Hz}, 1 \mathrm{H})$, $5.86-5.78(\mathrm{~m}, 1 \mathrm{H}), 5.10-4.98(\mathrm{~m}, 1 \mathrm{H}), 4.32-4.23(\mathrm{~m}, 2 \mathrm{H}), 2.87-2.71(\mathrm{~m}, 1 \mathrm{H}), 2.55-2.39(\mathrm{~m}$, 1H), $1.96(\mathrm{~s}, 3 \mathrm{H}), 1.63(\mathrm{~s}, 6 \mathrm{H}), 1.30(\mathrm{t}, J=7.2 \mathrm{~Hz}, 3 \mathrm{H}), 1.16(\mathrm{~d}, J=6.4 \mathrm{~Hz}, 6 \mathrm{H}) .{ }^{19} \mathrm{~F}$ NMR $(376 \mathrm{MHz}$, $\left.\mathrm{CDCl}_{3}\right) \delta-101.9(\mathrm{ddd}, J=269.6 \mathrm{~Hz}, 17.3 \mathrm{~Hz}, 13.5 \mathrm{~Hz}, 1 \mathrm{~F}),-103.7(\mathrm{dt}, J=269.6 \mathrm{~Hz}, 16.5 \mathrm{~Hz}, 1 \mathrm{~F}) .{ }^{13} \mathrm{C}$ NMR $\left(101 \mathrm{MHz}, \mathrm{CDCl}_{3}\right) \delta 196.8,194.9,173.5,170.3,163.9(\mathrm{t}, J=32.4 \mathrm{~Hz}), 160.6,143.3,136.8$, 132.6, 130.3, 130.1, 129.1, 117.8, 115.4 (t, $J=253.8 \mathrm{~Hz}), 80.0,69.9,63.8,49.1(\mathrm{t}, J=3.0 \mathrm{~Hz}), 36.7(\mathrm{t}$, $J=23.4 \mathrm{~Hz}), 25.8,23.4,22.0,14.3$. MS (ESI): $\mathrm{m} / \mathrm{z}(\%) 562(\mathrm{M}+\mathrm{H})^{+}$. HRMS (ESI): Calcd. for $\mathrm{C}_{29} \mathrm{H}_{34} \mathrm{O}_{8} \mathrm{NF}_{2}: 562.2247(\mathrm{M}+\mathrm{H})^{+}$; Found: $562.2249(\mathrm{M}+\mathrm{H})^{+}$. 


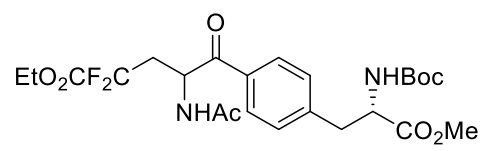

Ethyl 4-acetamido-5-(4-((S)-2-((tert-butoxycarbonyl)amino)-3methoxy-3-oxopropyl)phenyl)-2,2-difluoro-5-oxopentanoate (8b).

The reaction was conducted at $80{ }^{\circ} \mathrm{C}$. Compound $\mathbf{8 b}$ (92 mg, 60\% yield) as a yellow solid (m.p. 120$140{ }^{\circ} \mathrm{C}$ ) was purified with silica gel chromatography (Petroleum ether: Ethyl acetate $=4: 1$ to $2: 1$ ). ${ }^{1} \mathrm{H}$ NMR $\left(400 \mathrm{MHz}, \mathrm{CDCl}_{3}\right) \delta 7.91(\mathrm{~d}, J=8.0 \mathrm{~Hz}, 2 \mathrm{H}), 7.23(\mathrm{~d}, J=8.0 \mathrm{~Hz}, 2 \mathrm{H}), 6.62(\mathrm{~d}, J=7.2 \mathrm{~Hz}, 1 \mathrm{H})$, $5.84-5.75(\mathrm{~m}, 1 \mathrm{H}), 5.09(\mathrm{~d}, J=7.6 \mathrm{~Hz}, 1 \mathrm{H}), 4.57(\mathrm{q}, J=6.8 \mathrm{~Hz}, 1 \mathrm{H}), 4.27-4.24(\mathrm{~m}, 2 \mathrm{H}), 3.67$ (s, 3H), $3.16(\mathrm{dd}, J=13.6 \mathrm{~Hz}, 5.6 \mathrm{~Hz}, 1 \mathrm{H}), 3.05(\mathrm{dd}, J=13.6 \mathrm{~Hz}, 6.4 \mathrm{~Hz}, 1 \mathrm{H}), 2.80-2.63(\mathrm{~m}, 1 \mathrm{H}), 2.48$ $-2.32(\mathrm{~m}, 1 \mathrm{H}), 1.95(\mathrm{~s}, 3 \mathrm{H}), 1.35(\mathrm{~s}, 9 \mathrm{H}), 1.29(\mathrm{t}, J=7.2 \mathrm{~Hz}, 3 \mathrm{H}) .{ }^{19} \mathrm{~F}$ NMR $\left(376 \mathrm{MHz}, \mathrm{CDCl}_{3}\right) \delta-$ $101.8(\mathrm{dt}, J=270.3 \mathrm{~Hz}, 16.2 \mathrm{~Hz}, 1 \mathrm{~F}),-103.0--104.0(\mathrm{~m}, 1 \mathrm{~F}) .{ }^{13} \mathrm{C} \mathrm{NMR}\left(101 \mathrm{MHz}, \mathrm{CDCl}_{3}\right) \delta 196.8$, $172.4,170.2,163.9$ (t, $J=31.9 \mathrm{~Hz}), 155.5,143.8,133.1,130.5,129.6,115.4$ (t, $J=254.3 \mathrm{~Hz}), 80.7$, 63.8, 54.6, 52.9, 48.7, 38.9, $37.2(\mathrm{t}, J=23.8 \mathrm{~Hz}), 28.7,23.5,14.3 . \mathrm{MS}(\mathrm{DART}): \mathrm{m} / \mathrm{z}(\%) 537(\mathrm{M}+\mathrm{Na})^{+}$. HRMS (DART): Calcd. for $\mathrm{C}_{24} \mathrm{H}_{33} \mathrm{O}_{8} \mathrm{~N}_{2} \mathrm{~F}_{2}: 515.2199(\mathrm{M}+\mathrm{H})^{+}$; Found: $515.2195(\mathrm{M}+\mathrm{H})^{+}$.

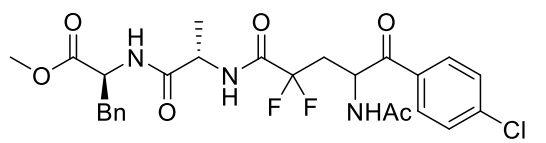

\section{Methyl}

(4-acetamido-5-(4-chlorophenyl)-2,2-difluoro-5oxopentanoyl)- $L$-alanyl- $L$-phenylalaninate $(8 \mathrm{c})$. Compounds $8 \mathrm{c}$ $(\mathrm{dr}=1.8: 1)$ is a mixture of diastereomers $\mathbf{8 c - 1}$ and $\mathbf{8 c - 2}$, which could be separated by silica gel chromatography. Compound 8c-1 (76.3 mg, 46\% yield) as a white solid (m.p. 169-172 ${ }^{\circ} \mathrm{C}$ ) was purified with silica gel chromatography (Petroleum ether: Ethyl acetate = 1: 1). ${ }^{1} \mathrm{H}$ NMR (400 MHz, DMSOd $) \delta 8.76(\mathrm{~d}, J=7.2 \mathrm{~Hz}, 1 \mathrm{H}), 8.62(\mathrm{~d}, J=8.0 \mathrm{~Hz}, 1 \mathrm{H}), 8.49(\mathrm{t}, J=7.6 \mathrm{~Hz}, 1 \mathrm{H}), 7.97(\mathrm{~d}, J=8.4 \mathrm{~Hz}$, 2H), $7.63(\mathrm{~d}, J=8.4 \mathrm{~Hz}, 2 \mathrm{H}), 7.32-7.20(\mathrm{~m}, 5 \mathrm{H}), 5.54-5.45(\mathrm{~m}, 1 \mathrm{H}), 4.55-4.46(\mathrm{~m}, 1 \mathrm{H}), 4.39-$ $4.29(\mathrm{~m}, 1 \mathrm{H}), 3.60(\mathrm{~s}, 3 \mathrm{H}), 3.11-2.95(\mathrm{~m}, 2 \mathrm{H}), 2.81-2.64(\mathrm{~m}, 1 \mathrm{H}), 2.50-2.35(\mathrm{~m}, 1 \mathrm{H}), 1.80(\mathrm{~s}, 3 \mathrm{H})$, $1.31(\mathrm{~d}, J=7.2 \mathrm{~Hz}, 3 \mathrm{H}) .{ }^{19} \mathrm{~F}$ NMR $\left(376 \mathrm{MHz}, \mathrm{DMSO}-\mathrm{d}_{6}\right) \delta-102.5(\mathrm{ddd}, J=252.7 \mathrm{~Hz}, 18.0 \mathrm{~Hz}, 14.3$ $\mathrm{Hz}, 1 \mathrm{~F}),-104.0(\mathrm{dt}, J=252.7 \mathrm{~Hz}, 18.0 \mathrm{~Hz}, 1 \mathrm{~F}) .{ }^{13} \mathrm{C} \mathrm{NMR}\left(101 \mathrm{MHz}, \mathrm{DMSO}-\mathrm{d}_{6}\right) \delta 196.5,172.6,172.5$, $169.9,163.7(\mathrm{t}, J=29.3 \mathrm{~Hz}), 139.3,137.9$, 134.3, 131.1, 130.0, 129.7, 129.2, $127.5,117.8$ (t, $J=253.2$ $\mathrm{Hz}), 54.7,52.8,49.4,48.7,37.4,35.5$ (t, $J=23.2 \mathrm{~Hz}), 23.2,18.4$. MS (ESI): m/z (\%) $552(\mathrm{M}+\mathrm{H})^{+}$. HRMS (ESI): Calcd. for $\mathrm{C}_{26} \mathrm{H}_{29} \mathrm{O}_{6} \mathrm{~N}_{3} \mathrm{~F}_{2} \mathrm{Cl}: 552.1707(\mathrm{M}+\mathrm{H})^{+}$; Found: $552.1703(\mathrm{M}+\mathrm{H})^{+}$.

Compound 8c-2 (42 mg, 26\% yield) as a white solid (m.p. $122-125^{\circ} \mathrm{C}$ ) was purified with silica gel chromatography (Petroleum ether: Ethyl acetate =1: 1). ${ }^{1} \mathrm{H}$ NMR $\left(400 \mathrm{MHz}, \mathrm{CDCl}_{3}\right) \delta 7.96(\mathrm{~d}, J=$ 
$8.0 \mathrm{~Hz}, 2 \mathrm{H}), 7.47$ (d, $J=8.0 \mathrm{~Hz}, 2 \mathrm{H}), 7.31-7.18(\mathrm{~m}, 4 \mathrm{H}), 7.16-7.09$ (m, 2H), 6.93 (d, J= 7.2 Hz, $1 \mathrm{H}), 6.78(\mathrm{~d}, J=8.0 \mathrm{~Hz}, 1 \mathrm{H}), 5.80-5.69(\mathrm{~m}, 1 \mathrm{H}), 4.88-4.78(\mathrm{~m}, 1 \mathrm{H}), 4.56-4.44(\mathrm{~m}, 1 \mathrm{H}), 3.72(\mathrm{~s}$, $3 \mathrm{H}), 3.21-3.03(\mathrm{~m}, 2 \mathrm{H}), 2.80-2.61(\mathrm{~m}, 1 \mathrm{H}), 2.56-2.38(\mathrm{~m}, 1 \mathrm{H}), 1.97(\mathrm{~s}, 3 \mathrm{H}), 1.40(\mathrm{~d}, J=6.4 \mathrm{~Hz}$, 3H). ${ }^{19} \mathrm{~F}$ NMR $\left(376 \mathrm{MHz}, \mathrm{CDCl}_{3}\right) \delta-102.3(\mathrm{t}, J=15.4 \mathrm{~Hz}, 2 \mathrm{~F}) .{ }^{13} \mathrm{C} \mathrm{NMR}\left(101 \mathrm{MHz}, \mathrm{CDCl}_{3}\right) \delta 196.1$, 172.3, 171.3, 170.6, 163.7 (t, $J=29.4 \mathrm{~Hz}), 141.3,136.3,132.7,130.8,130.0,129.8,129.1,127.7$, $117.3(\mathrm{t}, J=255.6 \mathrm{~Hz}), 54.2,53.0,49.6,48.9,38.2,36.6(\mathrm{t}, J=23.3 \mathrm{~Hz}), 23.5,18.5 . \mathrm{MS}(\mathrm{ESI}): \mathrm{m} / \mathrm{z}$ (\%) $552(\mathrm{M}+\mathrm{H})^{+}$. HRMS (ESI): Calcd. for $\mathrm{C}_{26} \mathrm{H}_{29} \mathrm{O}_{6} \mathrm{~N}_{3} \mathrm{~F}_{2} \mathrm{Cl}: 552.1707(\mathrm{M}+\mathrm{H})^{+}$; Found: 552.1703 $(\mathrm{M}+\mathrm{H})^{+}$.

Gram-Scale Synthesis of 8c with 2.5 equiv of $2 \mathrm{e}$ and 2.5 equiv of arylboronic acid: To a $500 \mathrm{~mL}$ of Schlenk tube were added $N$-vinylacetamide (425.6 mg, $5 \mathrm{mmol}, 1.0$ equiv), bromodifluoroalkane 2e (5.09 g, 2.5 equiv), 4-chlorophenylboronic acid (1.95 g, 2.5 equiv), $\mathrm{NiCl}_{2} \cdot \mathrm{DME}$ (109.9 mg, 10 mol\%), 4,4'-diOMe-2,2'-bpy (108.1 mg, $10 \mathrm{~mol} \%$ ) and $\mathrm{K}_{2} \mathrm{CO}_{3}$ (2.76 g, 4.0 equiv) under Ar. The reaction mixture was then evacuated and backfilled with Ar (3 times). The mixture was evacuated again and backfilled with carbon monoxide $\mathrm{CO}(1 \mathrm{~atm})$, followed by addition of $\mathrm{H}_{2} \mathrm{O}(65 \mu \mathrm{L})$ and dioxane $(50 \mathrm{~mL})$. The tube was screw-capped and heated to $50{ }^{\circ} \mathrm{C}$ (oil bath). After stirring for $24 \mathrm{~h}$, the reaction mixture was cooled to room temperature, the reaction mixture was diluted with ethyl acetate and $\mathrm{H}_{2} \mathrm{O}$. The resulting mixture was extracted with ethyl acetate, the combined organic layers were washed with brine, dried over $\mathrm{Na}_{2} \mathrm{SO}_{4}$, filtered and concentrated. The residue was purified with silica gel chromatography to give the product $8 \mathrm{c}(1.65 \mathrm{~g}, 60 \%)$ and recover starting material $2 \mathrm{e}(2.13 \mathrm{~g}, 42 \%)$.

Gram-Scale Synthesis of 8c with 1.5 equiv of $2 \mathrm{e}$ and 1.5 equiv of arylboronic acid: To a $500 \mathrm{~mL}$ of Schlenk tube were added $N$-vinylacetamide (425.6 mg, $5 \mathrm{mmol}, 1.0$ equiv), bromodifluoroalkane 2e (3.05 g, 1.5 equiv), 4-chlorophenylboronic acid (1.17 g, 1.5 equiv), $\mathrm{NiCl}_{2} \cdot \mathrm{DME}$ (109.9 mg, 10 mol\%), 4,4'-diOMe-2,2'-bpy (108.1 mg, $10 \mathrm{~mol} \%$ ) and $\mathrm{K}_{2} \mathrm{CO}_{3}$ (2.76 g, 4.0 equiv) under Ar. The reaction mixture was then evacuated and backfilled with Ar (3 times). The mixture was evacuated again and backfilled with carbon monoxide $\mathrm{CO}(1 \mathrm{~atm})$, followed by addition of $\mathrm{H}_{2} \mathrm{O}(65 \mu \mathrm{L})$ and dioxane $(50 \mathrm{~mL})$. The tube was screw-capped and heated to $50^{\circ} \mathrm{C}$ (oil bath). After stirring for $24 \mathrm{~h}$, the reaction mixture was cooled to room temperature, the reaction mixture was diluted with ethyl acetate 
and $\mathrm{H}_{2} \mathrm{O}$. The resulting mixture was extracted with ethyl acetate, the combined organic layers were washed with brine, dried over $\mathrm{Na}_{2} \mathrm{SO}_{4}$, filtered and concentrated. The residue was purified with silica gel chromatography to give the product $8 \mathrm{c}(1.44 \mathrm{~g}, 53 \%)$.

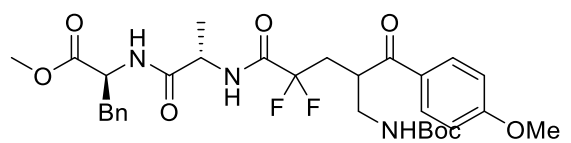

\section{Methyl (4-(((tert-butoxycarbonyl)amino)methyl)-2,2-difluoro- 5-(4-methoxyphenyl)-5-oxopentanoyl)- $L$-alanyl- $L$ -}

phenylalaninate (8d). The reaction was conducted with $\mathbf{L 3}$ as the ligand. Compounds $8 \mathbf{d}$ (90 mg, 53\% yield, $\mathrm{dr}=1: 1.1)$ as a mixture of diastereomers $\mathbf{8 d - 1}$ and $\mathbf{8 d - 2}$ were purified with silica gel chromatography (Petroleum ether: Ethyl acetate $=1$ : 1). 8d-1 and 8d-2 could be separated by preparative chiral HPLC on PC-3 column (Phenomenex Lux 5u Cellulose-3, 250*4.60 mm, acetonitrile: water $=45: 55,0.7 \mathrm{~mL} / \mathrm{min}, \mathrm{UV}$ detection at $254 \mathrm{~nm}) . \mathbf{8 d - 1}$ : yellow oil. ${ }^{1} \mathrm{H}$ NMR $(400$ $\left.\mathrm{MHz}, \mathrm{CDCl}_{3}\right) \delta 8.00(\mathrm{~d}, J=8.4 \mathrm{~Hz}, 2 \mathrm{H}), 7.26-7.18(\mathrm{~m}, 3 \mathrm{H}), 7.13-7.05(\mathrm{~m}, 3 \mathrm{H}), 6.92(\mathrm{~d}, J=8.8 \mathrm{~Hz}$, 2H), $6.63(\mathrm{~d}, J=7.6 \mathrm{~Hz}, 1 \mathrm{H}), 5.08-5.00(\mathrm{~m}, 1 \mathrm{H}), 4.86-4.78(\mathrm{~m}, 1 \mathrm{H}), 4.47-4.38(\mathrm{~m}, 1 \mathrm{H}), 4.18-$ $4.09(\mathrm{~m}, 1 \mathrm{H}), 3.83(\mathrm{~s}, 3 \mathrm{H}), 3.69(\mathrm{~s}, 3 \mathrm{H}), 3.48-3.38(\mathrm{~m}, 1 \mathrm{H}), 3.31-3.21(\mathrm{~m}, 1 \mathrm{H}), 3.15-3.00(\mathrm{~m}, 2 \mathrm{H})$, $2.77-2.58(\mathrm{~m}, 1 \mathrm{H}), 2.38-2.20(\mathrm{~m}, 1 \mathrm{H}), 1.38(\mathrm{~s}, 9 \mathrm{H}), 1.33(\mathrm{~d}, J=6.8 \mathrm{~Hz}, 3 \mathrm{H}) .{ }^{19} \mathrm{~F}$ NMR $(376 \mathrm{MHz}$, $\left.\mathrm{CDCl}_{3}\right) \delta-104.1(\mathrm{~m}, 2 \mathrm{~F}) .{ }^{13} \mathrm{C} \mathrm{NMR}\left(126 \mathrm{MHz}, \mathrm{CDCl}_{3}\right) \delta 200.0,172.2,171.3,164.5,164.1(\mathrm{t}, J=29.0$ Hz), 156.5, 136.1, 131.6, 129.74, 129.66, 129.2, 127.8, $117.6(\mathrm{t}, J=253.2 \mathrm{~Hz}), 114.5,80.1,56.0,53.9$, 53.0, 49.5, 43.3, 39.8, 38.3, $34.3(\mathrm{t}, J=23.9 \mathrm{~Hz}), 28.8,18.7 . \mathrm{MS}(\mathrm{ESI}): \mathrm{m} / \mathrm{z}(\%) 620(\mathrm{M}+\mathrm{H})^{+} . \mathrm{HRMS}$ (ESI): Calcd. for $\mathrm{C}_{31} \mathrm{H}_{40} \mathrm{O}_{8} \mathrm{~N}_{3} \mathrm{~F}_{2}: 620.2778(\mathrm{M}+\mathrm{H})^{+}$; Found: $620.2778(\mathrm{M}+\mathrm{H})^{+}$.

8d-2: yellow oil. ${ }^{1} \mathrm{H}$ NMR $\left(400 \mathrm{MHz}, \mathrm{CDCl}_{3}\right) \delta 8.02(\mathrm{~d}, J=8.8 \mathrm{~Hz}, 2 \mathrm{H}), 7.26-7.17(\mathrm{~m}, 3 \mathrm{H}), 7.11-$ $7.06(\mathrm{~m}, 2 \mathrm{H}), 7.05(\mathrm{~d}, J=7.6 \mathrm{~Hz}, 1 \mathrm{H}), 6.94(\mathrm{~d}, J=8.8 \mathrm{~Hz}, 2 \mathrm{H}), 6.66(\mathrm{~d}, J=8.0 \mathrm{~Hz}, 1 \mathrm{H}), 5.02-4.95$ $(\mathrm{m}, 1 \mathrm{H}), 4.86-4.79(\mathrm{~m}, 1 \mathrm{H}), 4.47-4.37(\mathrm{~m}, 1 \mathrm{H}), 4.17-4.08(\mathrm{~m}, 1 \mathrm{H}), 3.85(\mathrm{~s}, 3 \mathrm{H}), 3.70(\mathrm{~s}, 3 \mathrm{H}), 3.47$ $-3.37(\mathrm{~m}, 1 \mathrm{H}), 3.30-3.21(\mathrm{~m}, 1 \mathrm{H}), 3.16-3.02(\mathrm{~m}, 2 \mathrm{H}), 2.81-2.63(\mathrm{~m}, 1 \mathrm{H}), 2.36-2.19(\mathrm{~m}, 1 \mathrm{H})$, $1.37(\mathrm{~s}, 9 \mathrm{H}), 1.30(\mathrm{~d}, J=6.8 \mathrm{~Hz}, 3 \mathrm{H}) .{ }^{19} \mathrm{~F}$ NMR $\left(376 \mathrm{MHz}, \mathrm{CDCl}_{3}\right) \delta-104.0(\mathrm{ddd}, J=255.7 \mathrm{~Hz}, 22.6$ $\mathrm{Hz}, 15.0 \mathrm{~Hz}, 1 \mathrm{~F}),-105.0$ (ddd, $J=255.7 \mathrm{~Hz}, 22.6 \mathrm{~Hz}, 15.0 \mathrm{~Hz}, 1 \mathrm{~F}) .{ }^{13} \mathrm{C} \mathrm{NMR}\left(126 \mathrm{MHz}, \mathrm{CDCl}_{3}\right) \delta$ 200.0, 172.2, 171.2, 164.5, $164.1(\mathrm{t}, J=29.0 \mathrm{~Hz}), 156.5,136.1,131.6,129.8,129.7,129.2,127.8$, $117.6(\mathrm{t}, J=253.3 \mathrm{~Hz}), 114.6,80.1,56.1,54.0,53.0,49.4,43.5,39.7,38.3,34.2(\mathrm{t}, J=23.9 \mathrm{~Hz}), 28.8$, 
18.6. MS (ESI): $\mathrm{m} / \mathrm{z}(\%) 620(\mathrm{M}+\mathrm{H})^{+}$. HRMS (ESI): Calcd. for $\mathrm{C}_{31} \mathrm{H}_{40} \mathrm{O}_{8} \mathrm{~N}_{3} \mathrm{~F}_{2}: 620.2778(\mathrm{M}+\mathrm{H})^{+}$; Found: $620.2775(\mathrm{M}+\mathrm{H})^{+}$.

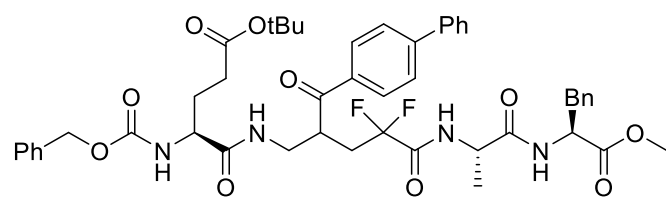

\section{Methyl (5S,14S,17S)-9-([1,1'-biphenyl]-4-carbonyl)-17- benzyl-5-(3-(tert-butoxy)-3-oxopropyl)-11,11-difluoro- \\ 14-methyl-3,6,12,15-tetraoxo-1-phenyl-2-oxa-4,7,13,16-}

tetraazaoctadecan-18-oate (8e). Compounds 8 e (130 $\mathrm{mg}, 49 \%$ yield, $\mathrm{dr}=1: 1.0)$ as a mixture of diastereomers 8e-1 and 8e-2 were purified with silica gel chromatography (Petroleum ether: Ethyl acetate $=1: 1)$. 8e-1 and $\mathbf{8 e - 2}$ could be separated by preparative chiral HPLC on PC-4 column (Phenomenex Lux 5u Cellulose-4, 250*4.60 mm, acetonitrile:water $=95: 5,0.7 \mathrm{~mL} / \mathrm{min}$, UV detection at $254 \mathrm{~nm}) .8 \mathrm{e}-1$ : yellow oil. ${ }^{1} \mathrm{H}$ NMR $\left(400 \mathrm{MHz}, \mathrm{CDCl}_{3}\right) \delta 8.17(\mathrm{~d}, J=8.0 \mathrm{~Hz}, 2 \mathrm{H}), 7.69(\mathrm{~d}, J=8.4$ $\mathrm{Hz}, 2 \mathrm{H}), 7.60(\mathrm{~d}, J=7.6 \mathrm{~Hz}, 2 \mathrm{H}), 7.52-7.42(\mathrm{~m}, 3 \mathrm{H}), 7.42-7.35(\mathrm{~m}, 2 \mathrm{H}), 7.33-7.27(\mathrm{~m}, 5 \mathrm{H}), 7.25$ - $7.19(\mathrm{~m}, 3 \mathrm{H}), 7.12-7.08(\mathrm{~m}, 2 \mathrm{H}), 6.88(\mathrm{~d}, J=8.0 \mathrm{~Hz}, 1 \mathrm{H}), 5.88(\mathrm{~d}, J=8.4 \mathrm{~Hz}, 1 \mathrm{H}), 5.10-4.96(\mathrm{~m}$, 2H), $4.89-4.81(\mathrm{~m}, 1 \mathrm{H}), 4.51-4.42(\mathrm{~m}, 1 \mathrm{H}), 4.28-4.20(\mathrm{~m}, 1 \mathrm{H}), 4.20-4.12(\mathrm{~m}, 1 \mathrm{H}), 3.69(\mathrm{~s}, 3 \mathrm{H})$, $3.27-3.18(\mathrm{~m}, 1 \mathrm{H}), 3.17-3.03(\mathrm{~m}, 2 \mathrm{H}), 2.89-2.71(\mathrm{~m}, 1 \mathrm{H}), 2.46-2.16(\mathrm{~m}, 4 \mathrm{H}), 2.01-1.91(\mathrm{~m}$, 1H), $1.88-1.77(\mathrm{~m}, 1 \mathrm{H}), 1.39(\mathrm{~s}, 9 \mathrm{H}), 1.34(\mathrm{~d}, J=6.8 \mathrm{~Hz}, 3 \mathrm{H}) .{ }^{19} \mathrm{~F}$ NMR $\left(376 \mathrm{MHz}, \mathrm{CDCl}_{3}\right) \delta-103.1$ (ddd, $J=251.9 \mathrm{~Hz}, 22.6 \mathrm{~Hz}, 11.3 \mathrm{~Hz}, 1 \mathrm{~F}),-106.7$ (ddd, $J=251.9 \mathrm{~Hz}, 22.6 \mathrm{~Hz}, 11.3 \mathrm{~Hz}, 1 \mathrm{~F}) .{ }^{13} \mathrm{C}$ NMR $\left(126 \mathrm{MHz}, \mathrm{CDCl}_{3}\right) \delta 200.2,173.1,172.9,172.5,171.7164 .3(\mathrm{t}, J=30.2 \mathrm{~Hz}), 156.9,146.9,140.2$, $136.6,136.2,134.9,129.9,129.8,129.5,129.1,129.0,128.8,128.7,128.5,128.0,127.8,127.7,117.6$ (t, $J=254.5 \mathrm{~Hz}), 81.5,67.6,54.8,54.0,53.0,49.7,42.2,39.9,38.3,34.0(\mathrm{t}, J=22.7 \mathrm{~Hz}), 32.1,28.5$, 28.2, 18.4. MS (ESI): $\mathrm{m} / \mathrm{z}(\%) 885(\mathrm{M}+\mathrm{H})^{+}$. HRMS (ESI): Calcd. for $\mathrm{C}_{48} \mathrm{H}_{55} \mathrm{O}_{10} \mathrm{~N}_{4} \mathrm{~F}_{2}: 885.3881$ $(\mathrm{M}+\mathrm{H})^{+}$; Found: $885.3886(\mathrm{M}+\mathrm{H})^{+}$.

8e-2: colorless oil. ${ }^{1} \mathrm{H}$ NMR $\left(400 \mathrm{MHz}, \mathrm{CDCl}_{3}\right) \delta 8.09(\mathrm{~d}, J=8.0 \mathrm{~Hz}, 2 \mathrm{H}), 7.65-7.59(\mathrm{~m}, 3 \mathrm{H}), 7.52$ $(\mathrm{d}, J=7.2 \mathrm{~Hz}, 2 \mathrm{H}), 7.45-7.34(\mathrm{~m}, 4 \mathrm{H}), 7.32-7.26(\mathrm{~m}, 5 \mathrm{H}), 7.26-7.18(\mathrm{~m}, 3 \mathrm{H}), 7.13(\mathrm{~d}, J=7.2 \mathrm{~Hz}$, 2H), $6.88(\mathrm{~d}, J=7.2 \mathrm{~Hz}, 1 \mathrm{H}), 5.84(\mathrm{~d}, J=7.2 \mathrm{~Hz}, 1 \mathrm{H}), 5.06(\mathrm{~d}, J=12.0 \mathrm{~Hz}, 1 \mathrm{H}), 4.95(\mathrm{~d}, J=12.4 \mathrm{~Hz}$, $1 \mathrm{H}), 4.90-4.83(\mathrm{~m}, 1 \mathrm{H}), 4.53-4.43(\mathrm{~m}, 1 \mathrm{H}), 4.17-4.06(\mathrm{~m}, 1 \mathrm{H}), 3.66(\mathrm{~s}, 3 \mathrm{H}), 3.56-3.46(\mathrm{~m}, 2 \mathrm{H})$, $3.17-3.02(\mathrm{~m}, 2 \mathrm{H}), 2.90-2.71(\mathrm{~m}, 1 \mathrm{H}), 2.42-2.19(\mathrm{~m}, 4 \mathrm{H}), 2.08-1.97(\mathrm{~m}, 1 \mathrm{H}), 1.92-1.80(\mathrm{~m}$, 1H), $1.39(\mathrm{~s}, 9 \mathrm{H}), 1.34(\mathrm{~d}, J=6.8 \mathrm{~Hz}, 3 \mathrm{H}) .{ }^{19} \mathrm{~F}$ NMR $\left(376 \mathrm{MHz}, \mathrm{CDCl}_{3}\right) \delta-103.2(\mathrm{~m}, 2 \mathrm{~F}) .{ }^{13} \mathrm{C}$ NMR 
$\left(126 \mathrm{MHz}, \mathrm{CDCl}_{3}\right) \delta 200.1,173.0,172.8,172.2,172.0164 .5(\mathrm{t}, J=29.0 \mathrm{~Hz}), 156.9,146.8,140.2$, $136.6,136.1,134.9,129.83,129.80,129.4,129.2,129.0,128.8,128.7,128.5,128.0,127.8,117.8$ (t, $J$ $=253.3 \mathrm{~Hz}), 81.4,67.6,55.1,54.0,52.9,50.0,41.5,40.6,38.3,34.4(\mathrm{t}, J=23.9 \mathrm{~Hz}), 32.1,28.5,28.0$, 18.3. MS (ESI): $\mathrm{m} / \mathrm{z}(\%) 885(\mathrm{M}+\mathrm{H})^{+}$. HRMS (ESI): Calcd. for $\mathrm{C}_{48} \mathrm{H}_{55} \mathrm{O}_{10} \mathrm{~N}_{4} \mathrm{~F}_{2}: 885.3881(\mathrm{M}+\mathrm{H})^{+}$; Found: $885.3881(\mathrm{M}+\mathrm{H})^{+}$.

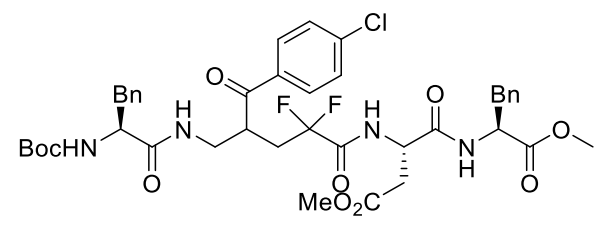

\section{Methyl $\quad(6 S, 15 S, 18 S)-6,18-d i b e n z y l-10-(4-c h l o r o b e n z o y l)-$} 12,12-difluoro-15-(2-methoxy-2-oxoethyl)-2,2-dimethyl4,7,13,16-tetraoxo-3-oxa-5,8,14,17-tetraazanonadecan-19oate (8f). Compounds $8 f(152 \mathrm{mg}, 61 \%$ yield, $\mathrm{dr}=1.2: 1)$ as a mixture of diastereomers $8 \mathbf{f}-\mathbf{1}$ and $\mathbf{8 f - 2}$ were purified with silica gel chromatography (Petroleum ether: Ethyl acetate =1: 1). $\mathbf{8 f - 1}$ and $\mathbf{8 f - 2}$ could be separated by preparative HPLC on IC column (Chiralpak IC: $5 \mu \mathrm{m}, 250 * 4.60 \mathrm{~mm}$, hexane: isopropyl alcohol $=50: 50,6.0 \mathrm{~mL} / \mathrm{min}$, $U V$ detection at $254 \mathrm{~nm}) .8 f-1:$ yellow solid, m.p. 165-168 ${ }^{\circ} \mathrm{C}$. ${ }^{1} \mathrm{H}$ NMR $\left(500 \mathrm{MHz}, \mathrm{CDCl}_{3}\right) \delta 8.02(\mathrm{~d}, J=8.0 \mathrm{~Hz}, 2 \mathrm{H}), 7.84-7.75(\mathrm{~m}, 1 \mathrm{H}), 7.48(\mathrm{~d}, J=8.5 \mathrm{~Hz}, 2 \mathrm{H})$, $7.33-7.28(\mathrm{~m}, 2 \mathrm{H}), 7.27-7.22(\mathrm{~m}, 3 \mathrm{H}), 7.20-7.12(\mathrm{~m}, 5 \mathrm{H}), 6.79(\mathrm{~s}, 1 \mathrm{H}), 5.12(\mathrm{~d}, J=6.5 \mathrm{~Hz}, 1 \mathrm{H})$, $4.82(\mathrm{q}, J=6.5 \mathrm{~Hz}, 1 \mathrm{H}), 4.77-4.71(\mathrm{~m}, 1 \mathrm{H}), 4.37-4.25(\mathrm{~m}, 1 \mathrm{H}), 4.11-4.03(\mathrm{~m}, 1 \mathrm{H}), 3.73(\mathrm{~s}, 3 \mathrm{H})$, $3.68(\mathrm{~s}, 3 \mathrm{H}), 3.57-3.48(\mathrm{~m}, 1 \mathrm{H}), 3.23-3.09(\mathrm{~m}, 3 \mathrm{H}), 3.05-2.99(\mathrm{~m}, 1 \mathrm{H}), 2.98-2.91(\mathrm{~m}, 2 \mathrm{H}), 2.71$ $-2.56(\mathrm{~m}, 2 \mathrm{H}), 2.38-2.25(\mathrm{~m}, 1 \mathrm{H}), 1.96(\mathrm{~s}, 1 \mathrm{H}), 1.37(\mathrm{~s}, 9 \mathrm{H}) .{ }^{19} \mathrm{~F}$ NMR $\left(471 \mathrm{MHz}, \mathrm{CDCl}_{3}\right) \delta-100.4$ $(\mathrm{dm}, J=259.1 \mathrm{~Hz}, 1 \mathrm{~F}),-104.9(\mathrm{dm}, J=259.1 \mathrm{~Hz}, 1 \mathrm{~F}) .{ }^{13} \mathrm{C} \mathrm{NMR}\left(126 \mathrm{MHz}, \mathrm{CDCl}_{3}\right) \delta$ 199.6, 172.9, $172.6,172.1,169.8,164.3(\mathrm{t}, J=29.0 \mathrm{~Hz}), 156.1,140.9,137.1,136.2,134.4,130.8,129.8,129.7$, 129.23, 129.15, 127.8, 127.5, $117.4(\mathrm{t}, J=253.3 \mathrm{~Hz}), 80.9$, 56.2, 54.3, 53.0, 52.8, 49.8, 41.7, 40.3, 38.8, 38.0, 35.7, $34.1(\mathrm{t}, J=23.9 \mathrm{~Hz}), 28.7 . \mathrm{MS}(\mathrm{ESI}): \mathrm{m} / \mathrm{z}(\%) 829$ (M+H) ${ }^{+}$. HRMS (ESI): Calcd. for $\mathrm{C}_{41} \mathrm{H}_{48} \mathrm{O}_{10} \mathrm{~N}_{4} \mathrm{ClF}_{2}: 829.3022(\mathrm{M}+\mathrm{H})^{+}$; Found: $829.3019(\mathrm{M}+\mathrm{H})^{+}$.

8f-2: yellow solid, m.p. 166-169 ${ }^{\circ} \mathrm{C} .{ }^{1} \mathrm{H}$ NMR $\left(500 \mathrm{MHz}, \mathrm{CDCl}_{3}\right) \delta 7.92(\mathrm{~d}, J=8.5 \mathrm{~Hz}, 2 \mathrm{H}), 7.41(\mathrm{~d}$, $J=8.0 \mathrm{~Hz}, 2 \mathrm{H}), 7.31-7.26(\mathrm{~m}, 4 \mathrm{H}), 7.24-7.19(\mathrm{~m}, 2 \mathrm{H}), 7.17-7.13(\mathrm{~m}, 5 \mathrm{H}), 6.79(\mathrm{~s}, 1 \mathrm{H}), 5.08(\mathrm{~d}$, $J=5.5 \mathrm{~Hz}, 1 \mathrm{H}), 4.83-4.74(\mathrm{~m}, 2 \mathrm{H}), 4.29-4.21(\mathrm{~m}, 1 \mathrm{H}), 3.90(\mathrm{~s}, 1 \mathrm{H}), 3.70(\mathrm{~s}, 3 \mathrm{H}), 3.67(\mathrm{~s}, 3 \mathrm{H}), 3.48$ - $3.41(\mathrm{~m}, 1 \mathrm{H}), 3.38-3.30(\mathrm{~m}, 1 \mathrm{H}), 3.18-3.06(\mathrm{~m}, 2 \mathrm{H}), 3.04-2.97(\mathrm{~m}, 1 \mathrm{H}), 2.95-2.88(\mathrm{~m}, 2 \mathrm{H})$, $2.76-2.69(\mathrm{~m}, 1 \mathrm{H}), 2.66-2.56(\mathrm{~m}, 1 \mathrm{H}), 2.25-2.11(\mathrm{~m}, 1 \mathrm{H}), 1.92(\mathrm{~s}, 1 \mathrm{H}), 1.36(\mathrm{~s}, 9 \mathrm{H}) .{ }^{19} \mathrm{~F}$ NMR 
$\left(471 \mathrm{MHz}, \mathrm{CDCl}_{3}\right) \delta-101.8(\mathrm{dm}, J=259.1 \mathrm{~Hz}, 1 \mathrm{~F}),-102.8(\mathrm{dt}, J=259.1 \mathrm{~Hz}, 18.8 \mathrm{~Hz}, 1 \mathrm{~F}) .{ }^{13} \mathrm{C} \mathrm{NMR}$ $\left(126 \mathrm{MHz}, \mathrm{CDCl}_{3}\right) \delta 199.6,172.8,172.4,172.0,170.1,164.4(\mathrm{t}, J=29.0 \mathrm{~Hz}), 156.2,140.8,137.1$, $136.1,134.5,130.7,129.74,129.72,129.70,129.3,129.2,127.8,127.5,117.5(\mathrm{t}, J=253.3 \mathrm{~Hz}), 81.0$, 56.5, 54.3, 53.0, 52.9, 49.9, 41.3, 40.5, 38.8, 38.0, 36.0, 34.5 (t, $J=23.9$ Hz), 28.7. MS (ESI): m/z (\%) $829(\mathrm{M}+\mathrm{H})^{+}$. HRMS (ESI): Calcd. for $\mathrm{C}_{41} \mathrm{H}_{48} \mathrm{O}_{10} \mathrm{~N}_{4} \mathrm{ClF}_{2}: 829.3022(\mathrm{M}+\mathrm{H})^{+}$; Found: $829.3023(\mathrm{M}+\mathrm{H})^{+}$.

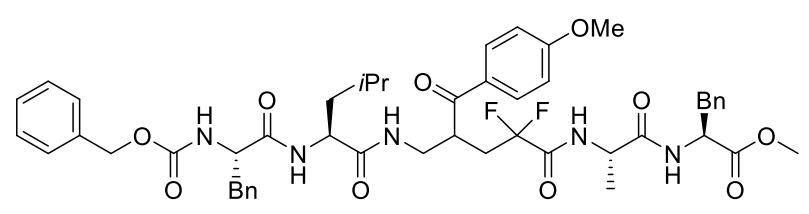

Methyl

((S)-4-(((S)-2-((S)-2-

(((benzyloxy)carbonyl)amino)-3-

phenylpropanamido)-4-

methylpentanamido)methyl)-2,2-difluoro-5-(4-methoxyphenyl)-5-oxopentanoyl)- $L$-alanyl- $L$ -

phenylalaninate (8g). The reaction was conducted with $\mathbf{L 3}$ as the ligand. Compounds $8 \mathbf{g}$ (145 mg, $53 \%$ yield, $\mathrm{dr}=1.1: 1$ ) as a mixture of diastereomers $\mathbf{8 g - 1}$ and $\mathbf{8 g - 2}$ were purified with silica gel chromatography (Petroleum ether: Ethyl acetate $=2: 1$ to Ethyl acetate). 8g-1 and $\mathbf{8 g - 2}$ could be separated by preparative chiral HPLC on PC-2 column (Phenomenex Lux 5u Cellulose-2, 250*4.60 $\mathrm{mm}$, acetonitrile: water $=55: 45,0.7 \mathrm{~mL} / \mathrm{min}, \mathrm{UV}$ detection at $214 \mathrm{~nm}) .8 \mathrm{8g}-\mathbf{1}$ : yellow solid, m.p. 179$184{ }^{\circ} \mathrm{C} .{ }^{1} \mathrm{H}$ NMR $\left(600 \mathrm{MHz}, \mathrm{CDCl}_{3}\right) \delta 8.10(\mathrm{~d}, J=9.0 \mathrm{~Hz}, 2 \mathrm{H}), 7.39-7.26(\mathrm{~m}, 10 \mathrm{H}), 7.26-7.20(\mathrm{~m}$, $3 \mathrm{H}), 7.17(\mathrm{~d}, J=7.2 \mathrm{~Hz}, 2 \mathrm{H}), 7.14-7.09(\mathrm{~m}, 3 \mathrm{H}), 6.93(\mathrm{~d}, J=8.4 \mathrm{~Hz}, 2 \mathrm{H}), 6.65(\mathrm{~d}, J=7.8 \mathrm{~Hz}, 1 \mathrm{H})$, $6.55(\mathrm{~d}, J=7.8 \mathrm{~Hz}, 1 \mathrm{H}), 5.47(\mathrm{~d}, J=6.0 \mathrm{~Hz}, 1 \mathrm{H}), 5.09-5.01(\mathrm{~m}, 2 \mathrm{H}), 4.88-4.83(\mathrm{~m}, 1 \mathrm{H}), 4.46-$ $4.38(\mathrm{~m}, 2 \mathrm{H}), 4.36-4.31(\mathrm{~m}, 1 \mathrm{H}), 4.19-4.12(\mathrm{~m}, 1 \mathrm{H}), 3.84(\mathrm{~s}, 3 \mathrm{H}), 3.71(\mathrm{~s}, 3 \mathrm{H}), 3.65-3.57(\mathrm{~m}, 1 \mathrm{H})$, $3.17-3.04(\mathrm{~m}, 5 \mathrm{H}), 2.40-2.27(\mathrm{~m}, 1 \mathrm{H}), 1.41-1.33(\mathrm{~m}, 5 \mathrm{H}), 0.91-0.83(\mathrm{~m}, 1 \mathrm{H}), 0.78(\mathrm{~d}, J=5.4$ $\mathrm{Hz}, 6 \mathrm{H}) .{ }^{19} \mathrm{~F}$ NMR $\left(471 \mathrm{MHz}, \mathrm{CDCl}_{3}\right) \delta-102.2(\mathrm{dm}, J=254.3 \mathrm{~Hz}, 1 \mathrm{~F}),-106.8(\mathrm{dt}, J=254.3 \mathrm{~Hz}, 18.8$ $\mathrm{Hz}, 1 \mathrm{~F}) .{ }^{13} \mathrm{C} \mathrm{NMR}\left(151 \mathrm{MHz}, \mathrm{CDCl}_{3}\right) \delta 199.1,172.9,172.5,171.9,171.6,164.6,164.4(\mathrm{t}, J=30.2$ Hz), 156.8, 136.7, 136.5, 136.2, 131.8, 129.9, 129.4, 129.3, 129.2, 129.1, 128.9, 128.7, 127.82, 127.76, $117.7(\mathrm{t}, J=253.7 \mathrm{~Hz}), 114.6,67.8,57.0,56.1,54.0,53.1,52.3,49.9,42.4,41.1,39.3,38.4,34.0$ (t, $J$ $=24.2 \mathrm{~Hz}), 25.2,23.3,22.5,18.4$. MS (ESI): m/z (\%) $914(\mathrm{M}+\mathrm{H})^{+}$. HRMS (ESI): Calcd. for $\mathrm{C}_{49} \mathrm{H}_{58} \mathrm{O}_{10} \mathrm{~N}_{5} \mathrm{~F}_{2}: 914.4146(\mathrm{M}+\mathrm{H})^{+}$; Found: $914.4143(\mathrm{M}+\mathrm{H})^{+}$.

8g-2: yellow solid, m.p. 153-158 ${ }^{\circ} \mathrm{C} .{ }^{1} \mathrm{H}$ NMR $\left(500 \mathrm{MHz}, \mathrm{CDCl}_{3}\right) \delta 8.05(\mathrm{~d}, J=9.0 \mathrm{~Hz}, 2 \mathrm{H}), 7.59-$ $7.53(\mathrm{~m}, 1 \mathrm{H}), 7.42-7.36(\mathrm{~m}, 1 \mathrm{H}), 7.34-7.26(\mathrm{~m}, 7 \mathrm{H}), 7.26-7.20(\mathrm{~m}, 5 \mathrm{H}), 7.15-7.12(\mathrm{~m}, 4 \mathrm{H}), 6.88$ 
$(\mathrm{d}, J=8.5 \mathrm{~Hz}, 2 \mathrm{H}), 6.81(\mathrm{~d}, J=7.5 \mathrm{~Hz}, 1 \mathrm{H}), 6.49(\mathrm{~d}, J=8.0 \mathrm{~Hz}, 1 \mathrm{H}), 5.45(\mathrm{~d}, J=6.5 \mathrm{~Hz}, 1 \mathrm{H}), 5.07-$ $5.00(\mathrm{~m}, 2 \mathrm{H}), 4.90-4.85(\mathrm{~m}, 1 \mathrm{H}), 4.54-4.47(\mathrm{~m}, 1 \mathrm{H}), 4.42-4.31(\mathrm{~m}, 2 \mathrm{H}), 3.78(\mathrm{~s}, 3 \mathrm{H}), 3.68(\mathrm{~s}, 3 \mathrm{H})$, $3.54-3.46(\mathrm{~m}, 1 \mathrm{H}), 3.37-3.29(\mathrm{~m}, 1 \mathrm{H}), 3.17-3.06(\mathrm{~m}, 2 \mathrm{H}), 3.05-2.93(\mathrm{~m}, 2 \mathrm{H}), 2.86-2.72(\mathrm{~m}$, $1 \mathrm{H}), 2.38-2.25(\mathrm{~m}, 1 \mathrm{H}), 1.64-1.56(\mathrm{~m}, 1 \mathrm{H}), 1.54-1.45(\mathrm{~m}, 1 \mathrm{H}), 1.41-1.36(\mathrm{~m}, 1 \mathrm{H}), 1.35(\mathrm{~d}, J=$ $7.0 \mathrm{~Hz}, 3 \mathrm{H}), 0.85(\mathrm{dd}, J=9.5 \mathrm{~Hz}, 6.5 \mathrm{~Hz}, 6 \mathrm{H}) .{ }^{19} \mathrm{~F}$ NMR $\left(471 \mathrm{MHz}, \mathrm{CDCl}_{3}\right) \delta-102.7(\mathrm{dt}, J=254.3$ $\mathrm{Hz}, 18.8 \mathrm{~Hz}, 1 \mathrm{~F}),-104.3(\mathrm{dm}, J=254.3 \mathrm{~Hz}, 1 \mathrm{~F}) .{ }^{13} \mathrm{C} \mathrm{NMR}\left(126 \mathrm{MHz}, \mathrm{CDCl}_{3}\right) \delta 198.8,172.8,172.3$, 171.93, 171.90, 164.6 (t, $J=29.0 \mathrm{~Hz}), 164.5,156.8,136.60,136.56,136.0,131.7,129.9,129.8,129.30$, 129.27, 129.1, 128.8, 128.7, 127.9, 127.7, 117.8 (t, $J=253.3 \mathrm{~Hz}), 114.6,67.8,56.8,56.0,54.0,53.0$, 52.7, 49.9, 41.8, 41.4, 40.0, 38.5, $34.4(\mathrm{t}, J=22.7 \mathrm{~Hz}), 25.2,23.2,22.6,18.5 . \mathrm{MS}(\mathrm{ESI}): \mathrm{m} / \mathrm{z}(\%) 914$ $(\mathrm{M}+\mathrm{H})^{+}$. HRMS (ESI): Calcd. for $\mathrm{C}_{49} \mathrm{H}_{58} \mathrm{O}_{10} \mathrm{~N}_{5} \mathrm{~F}_{2}$ : $914.4146(\mathrm{M}+\mathrm{H})^{+}$; Found: $914.4139(\mathrm{M}+\mathrm{H})^{+}$.

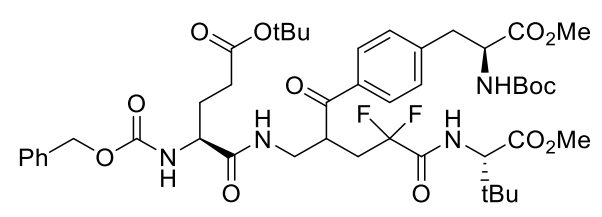

\section{Methyl (5S,14S)-5-(3-(tert-butoxy)-3-oxopropyl)-9-(4-((S)-2-} ((tert-butoxycarbonyl)amino)-3-methoxy-3oxopropyl)benzoyl)-14-(tert-butyl)-11,11-difluoro-3,6,12-

trioxo-1-phenyl-2-oxa-4,7,13-triazapentadecan-15-oate $(\mathbf{8 h})$. The reaction was conducted at $80{ }^{\circ} \mathrm{C}$. Compounds $\mathbf{8 h}$ (100.0 mg, 37\% yield, dr=1.2: 1) as a mixture of diastereomers $\mathbf{8 h - 1}$ and $\mathbf{8 h - 2}$ were purified with silica gel chromatography (Petroleum ether: Ethyl acetate =1: 1). $\mathbf{8 h - 1}$ and $\mathbf{8 h - 2}$ could be separated by preparative chiral HPLC on PC-4 column (Phenomenex Lux 5u Cellulose-4, 250*4.60 $\mathrm{mm}$, acetonitrile:water $=60: 40,0.7 \mathrm{~mL} / \mathrm{min}, \mathrm{UV}$ detection at $254 \mathrm{~nm}) . \mathbf{8 h}-\mathbf{1}$ : brown oil. ${ }^{1} \mathrm{H}$ NMR $(600$ $\left.\mathrm{MHz}, \mathrm{CDCl}_{3}\right) \delta 7.95(\mathrm{~d}, J=7.8 \mathrm{~Hz}, 2 \mathrm{H}), 7.35-7.33(\mathrm{~m}, 5 \mathrm{H}), 7.32-7.29(\mathrm{~m}, 2 \mathrm{H}), 7.24$ (d, J=8.4 Hz, 2H), $6.87(\mathrm{~d}, J=9.6 \mathrm{~Hz}, 1 \mathrm{H}), 6.82(\mathrm{~m}, 1 \mathrm{H}), 5.68(\mathrm{~d}, J=6.6 \mathrm{~Hz}, 1 \mathrm{H}), 5.11-5.05(\mathrm{~m}, 2 \mathrm{H}), 4.65-4.59$ (m, 1H), $4.13-4.07(\mathrm{~m}, 2 \mathrm{H}), 3.74(\mathrm{~s}, 3 \mathrm{H}), 3.72(\mathrm{~s}, 3 \mathrm{H}), 3.67-3.61(\mathrm{~m}, 1 \mathrm{H}), 3.34-3.27(\mathrm{~m}, 1 \mathrm{H}), 3.21$ - $3.16(\mathrm{~m}, 1 \mathrm{H}), 3.12-3.07(\mathrm{~m}, 1 \mathrm{H}), 2.81-2.69(\mathrm{~m}, 1 \mathrm{H}), 2.38-2.29(\mathrm{~m}, 2 \mathrm{H}), 2.26-2.19(\mathrm{~m}, 1 \mathrm{H})$, $1.98-1.92(\mathrm{~m}, 1 \mathrm{H}), 1.83-1.77(\mathrm{~m}, 1 \mathrm{H}), 1.42(\mathrm{~s}, 18 \mathrm{H}), 0.97(\mathrm{~s}, 9 \mathrm{H}) .{ }^{19} \mathrm{~F} \mathrm{NMR}\left(565 \mathrm{MHz}, \mathrm{CDCl}_{3}\right) \delta$ $102.8(\mathrm{ddd}, J=254.3 \mathrm{~Hz}, 22.6 \mathrm{~Hz}, 11.3 \mathrm{~Hz}, 1 \mathrm{~F}),-105.8(\mathrm{dm}, J=254.3 \mathrm{~Hz}, 1 \mathrm{~F}) .{ }^{13} \mathrm{C} \mathrm{NMR}(151 \mathrm{MHz}$, $\left.\mathrm{CDCl}_{3}\right) \delta 200.4,173.3,172.53,172.50,171.5,164.1(\mathrm{t}, J=28.7 \mathrm{~Hz}), 156.9,155.6,143.1,136.8,135.2$, $130.5,129.5,129.1,128.8,128.7,117.8(\mathrm{t}, J=253.7 \mathrm{~Hz}), 81.6,80.8,67.7,60.8,54.9,54.7,53.0,52.7$, 42.1, 39.8, 38.9, 35.6, $33.9(\mathrm{t}, J=22.7 \mathrm{~Hz}), 32.3,28.9,28.6,28.0,27.0 . \mathrm{MS}(\mathrm{ESI}): \mathrm{m} / \mathrm{z}(\%) 905(\mathrm{M}+\mathrm{H})^{+}$. HRMS (ESI): Calcd. for $\mathrm{C}_{45} \mathrm{H}_{63} \mathrm{O}_{13} \mathrm{~N}_{4} \mathrm{~F}_{2}$ : $905.4354(\mathrm{M}+\mathrm{H})^{+}$; Found: $905.4348(\mathrm{M}+\mathrm{H})^{+}$. 
8h-2: yellow oil. ${ }^{1} \mathrm{H}$ NMR $\left(600 \mathrm{MHz}, \mathrm{CDCl}_{3}\right) \delta 7.89(\mathrm{~d}, J=7.8 \mathrm{~Hz}, 2 \mathrm{H}), 7.38-7.29(\mathrm{~m}, 7 \mathrm{H}), 7.20(\mathrm{~d}$, $J=7.2 \mathrm{~Hz}, 2 \mathrm{H}), 6.89(\mathrm{~d}, J=9.0 \mathrm{~Hz}, 1 \mathrm{H}), 6.80(\mathrm{~m}, 1 \mathrm{H}), 5.57-5.52(\mathrm{~m}, 1 \mathrm{H}), 5.12-5.07(\mathrm{~m}, 1 \mathrm{H}), 5.07$ - $5.03(\mathrm{~m}, 2 \mathrm{H}), 4.67-4.61(\mathrm{~m}, 1 \mathrm{H}), 4.22-4.15(\mathrm{~m}, 1 \mathrm{H}), 4.07-4.01(\mathrm{~m}, 1 \mathrm{H}), 3.72(\mathrm{~s}, 6 \mathrm{H}), 3.56-$ $3.48(\mathrm{~m}, 1 \mathrm{H}), 3.48-3.41(\mathrm{~m}, 1 \mathrm{H}), 3.19-3.13(\mathrm{~m}, 1 \mathrm{H}), 3.12-3.06(\mathrm{~m}, 1 \mathrm{H}), 2.80-2.67(\mathrm{~m}, 1 \mathrm{H}), 2.40$ $-2.23(\mathrm{~m}, 3 \mathrm{H}), 1.82-1.67(\mathrm{~m}, 1 \mathrm{H}), 1.41(\mathrm{~s}, 18 \mathrm{H}), 0.96(\mathrm{~s}, 9 \mathrm{H}) .{ }^{19} \mathrm{~F} \mathrm{NMR}\left(565 \mathrm{MHz}, \mathrm{CDCl}_{3}\right) \delta-103.0$ $(\mathrm{dm}, J=259.9 \mathrm{~Hz}, 1 \mathrm{~F}),-104.1(\mathrm{dm}, J=259.9 \mathrm{~Hz}, 1 \mathrm{~F}) .{ }^{13} \mathrm{C} \mathrm{NMR}\left(151 \mathrm{MHz}, \mathrm{CDCl}_{3}\right) \delta 200.8,173.3$, $172.7,172.5,171.5,164.1(\mathrm{t}, J=28.7 \mathrm{~Hz}), 156.9$, 155.6, 143.0, 136.8, 135.4, 130.5, 129.4, 129.1, 128.8, 128.7, 117.7 (t, $J=253.7 \mathrm{~Hz}), 81.6,80.8,67.7,60.7,54.9,54.7,53.0,52.7,42.2,39.5,38.9$, 35.6, $34.2(\mathrm{t}, J=22.7 \mathrm{~Hz}), 32.3,28.9,28.6,27.9,27.0 . \mathrm{MS}(\mathrm{ESI}): \mathrm{m} / \mathrm{z}(\%) 905(\mathrm{M}+\mathrm{H})^{+}$. HRMS (ESI): Calcd. for $\mathrm{C}_{45} \mathrm{H}_{63} \mathrm{O}_{13} \mathrm{~N}_{4} \mathrm{~F}_{2}$ : $905.4354(\mathrm{M}+\mathrm{H})^{+}$; Found: $905.4353(\mathrm{M}+\mathrm{H})^{+}$.

\section{Transformations of peptide $8 \mathrm{c}$}

\subsection{Reduction of 8c-1 with $\mathrm{NaBH}_{4}$}

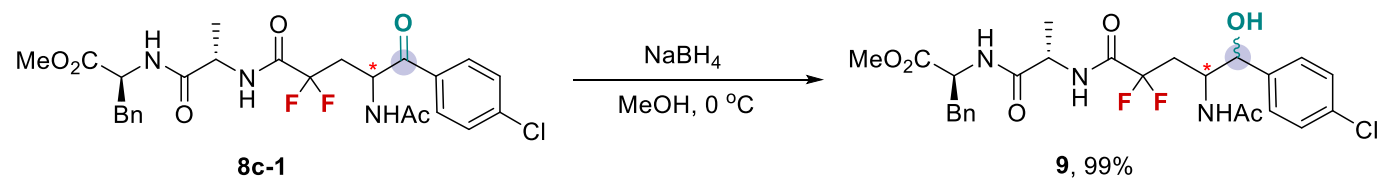

\section{Methyl (4-acetamido-5-(4-chlorophenyl)-2,2-difluoro-5-hydroxypentanoyl)- $L$-alanyl- $L$ -}

phenylalaninate (9). To a $25 \mathrm{~mL}$ of round bottomed flask were added compound 8c-1 (20 mg, 0.036 mmol, 1.0 equiv) and methanol $(5 \mathrm{~mL}) . \mathrm{NaBH}_{4}(1.4 \mathrm{mg}, 0.036 \mathrm{mmol}, 1.0$ equiv) was then added at 0 ${ }^{\circ} \mathrm{C}$. After stirring for $1 \mathrm{~h}$ at $0{ }^{\circ} \mathrm{C}$, the reaction mixture was quenched by diluted hydrochloric acid, and diluted with DCM and $\mathrm{H}_{2} \mathrm{O}$. The resulting mixture was extracted with DCM for three times, the combined organic layers were dried over $\mathrm{Na}_{2} \mathrm{SO}_{4}$, filtered and concentrated to give a white solid 9 (20 mg, 99\% yield). ${ }^{1} \mathrm{H}$ NMR (400 MHz, DMSO-d 6 ) $\delta 8.61-8.47(\mathrm{~m}, 1 \mathrm{H}), 8.42(\mathrm{t}, J=7.6 \mathrm{~Hz}, 1 \mathrm{H}), 7.85$ - $7.60(\mathrm{~m}, 1 \mathrm{H}), 7.38$ - $7.18(\mathrm{~m}, 9 \mathrm{H}), 5.81-5.74(\mathrm{~m}, 1 \mathrm{H}), 4.68-4.41(\mathrm{~m}, 2 \mathrm{H}), 4.32-4.04(\mathrm{~m}, 2 \mathrm{H})$, 3.57 (s, 3H), $3.05-2.90(\mathrm{~m}, 2 \mathrm{H}), 2.43-2.02(\mathrm{~m}, 2 \mathrm{H}), 1.66(\mathrm{~s}, 3 \mathrm{H}), 1.26-1.17(\mathrm{~m}, 3 \mathrm{H}) .{ }^{19} \mathrm{~F}$ NMR (376 MHz, DMSO-d 6 ) $\delta-104.4$ - -105.3 (m, 2F). ${ }^{13} \mathrm{C}$ NMR (126 MHz, DMSO-d 6 ) $\delta ~ 171.7, ~ 171.6$, 168.83, 168.75, 168.5, 163.08 (t, $J=29.0 \mathrm{~Hz}), 162.97(\mathrm{t}, J=29.0 \mathrm{~Hz}), 141.62,141.58,141.24,141.20$, $137.04,137.00,131.42,131.40,129.09,129.07,128.4,128.3,127.8,127.5,126.6,117.52(\mathrm{t}, J=252.0$ $\mathrm{Hz}), 117.48(\mathrm{t}, J=252.0 \mathrm{~Hz}), 73.6,73.5,72.4,72.3,53.8,53.7,51.8,48.43,48.36,48.34,48.26,36.51$, 
36.49, 35.00 (t, $J=23.9 \mathrm{~Hz}), 34.99$ (t, $J=23.9 \mathrm{~Hz}), 22.6,22.5,22.34,22.30,17.51,17.45$. MS (ESI): m/z (\%) $554(\mathrm{M}+\mathrm{H})^{+}$. HRMS (ESI): Calcd. for $\mathrm{C}_{26} \mathrm{H}_{31} \mathrm{O}_{6} \mathrm{~N}_{3} \mathrm{ClF}_{2}: 554.1864(\mathrm{M}+\mathrm{H})^{+}$; Found: 554.1866 $(\mathrm{M}+\mathrm{H})^{+}$.

\subsection{Synthesis of biotin-ligated peptide 10 with $8 \mathrm{c}-2$}
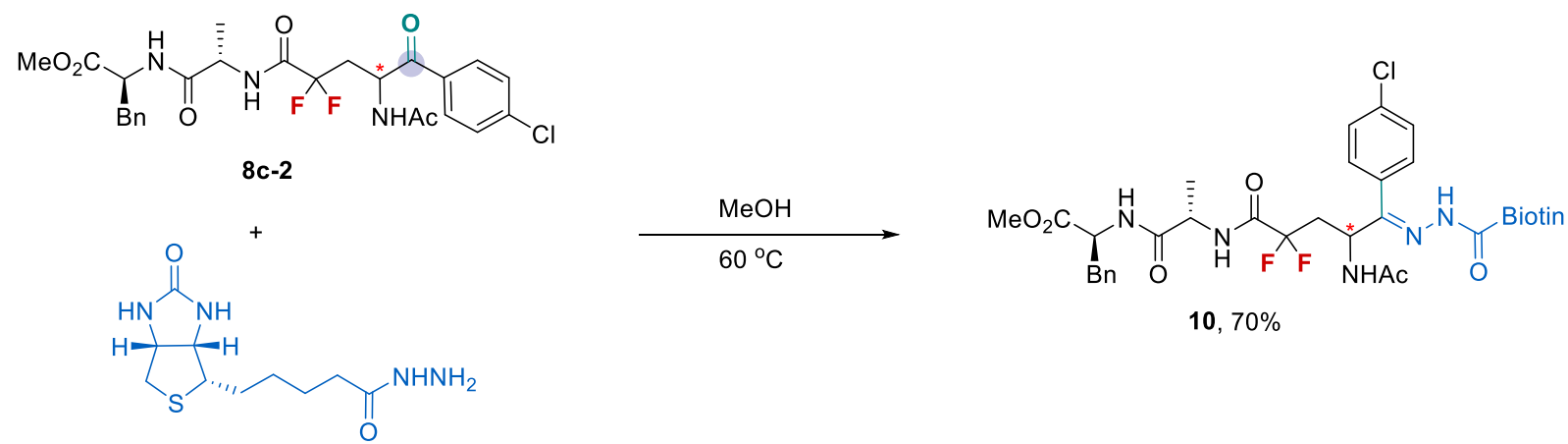

$10,70 \%$

Methyl

((E)-4-acetamido-5-(4-chlorophenyl)-2,2-difluoro-5-(2-(5-((3aS,4S,6aR)-2oxohexahydro- $1 H$-thieno[3,4- $d]$ imidazol-4-yl)pentanoyl)hydrazineylidene)pentanoyl)- $L$-alanyl$\boldsymbol{L}$-phenylalaninate (10). To a $25 \mathrm{~mL}$ of round bottomed flask were added compound $\mathbf{8 c - 2}$ (20 mg, $0.036 \mathrm{mmol}, 1.0$ equiv), biotin hydrazide (10.2 $\mathrm{mg}, 0.04 \mathrm{mmol}, 1.1$ equiv) and methanol ( $3 \mathrm{~mL})$. TFA was then added. The resulting mixture was stirred at $60{ }^{\circ} \mathrm{C}$ for 3 days. The solution was concentrated to afford a mixture. The formation of compound $\mathbf{1 0}$ was analyzed by LC-MS and ${ }^{19} \mathrm{~F}$ NMR with fluorobenzene (1.0 equiv) as an internal standard. The yield of $\mathbf{1 0}$ is $70 \%$ yield $(E / Z=4.5: 1)$ determined by ${ }^{19} \mathrm{~F}$ NMR using fluorobenzene as an internal standard in DMSO-d6. ${ }^{19} \mathrm{~F}$ NMR $(376 \mathrm{MHz}$, $\left.\mathrm{CDCl}_{3}\right) \delta-97.6(\mathrm{dm}, J=259.4 \mathrm{~Hz}, 1 \mathrm{~F}$, minor), $-102.2(\mathrm{dm}, J=259.4 \mathrm{~Hz}, 1 \mathrm{~F}$, major), $-102.2(\mathrm{dt}, J=$ 259.4 Hz, 15.0 Hz, 1F, major), -106.1 (dm, $J=259.4 \mathrm{~Hz}, 1 \mathrm{~F}$, minor). MS (ESI): m/z (\%) $792(\mathrm{M}+\mathrm{H})^{+}$. HRMS (ESI): Calcd. for $\mathrm{C}_{36} \mathrm{H}_{45} \mathrm{O}_{7} \mathrm{~N}_{7} \mathrm{ClF}_{2} \mathrm{~S}$ : $792.2752(\mathrm{M}+\mathrm{H})^{+}$; Found: $792.2748(\mathrm{M}+\mathrm{H})^{+}$. 


\section{Mechanistic studies}

\subsection{Radical inhibition experiments:}

1,4 -dinitrobenzene $(0.2$ equiv)
Entry
1

${ }^{a}$ Reaction conditions (unless otherwise specified): $N$-vinylacetamide 1a ( $0.3 \mathrm{mmol}, 1.0$ equiv), 3a ( $0.75 \mathrm{mmol}, 2.5$ equiv), $\mathrm{BrCF}_{2} \mathrm{CO}_{2} \mathrm{Et} \mathbf{2 a}$ ( $0.75 \mathrm{mmol}, 2.5$ equiv), dioxane $(3 \mathrm{~mL}) .{ }^{b}$ Determined by ${ }^{19} \mathrm{~F}$ NMR using fluorobenzene as an internal standard.

Procedure: To a $25 \mathrm{~mL}$ of Schlenck tube were added arylboronic acid 3a (0.75 mmol, 2.5 equiv), $\mathrm{NiCl}_{2} \cdot \operatorname{DME}(10 \mathrm{~mol} \%), 4,4^{\prime}$-diOMebpy $\mathbf{L 1}(10 \mathrm{~mol} \%)$ and $N$-vinylacetamide $\mathbf{1 a}(0.3 \mathrm{mmol}, 1.0$ equiv $)$ under Ar, followed by $\mathrm{K}_{2} \mathrm{CO}_{3}$ (1.2 mmol, 4.0 equiv) and 1,4-dinitrobenzene (0.2-1.0 equiv) under air. The mixture was then evacuated and backfilled with Ar (3 times). The mixture was evacuated again and backfilled with carbon monoxide $\mathrm{CO}$ (1 atm, balloon), $\mathrm{BrCF}_{2} \mathrm{CO}_{2} \mathrm{Et}$ 2a $(0.75 \mathrm{mmol}, 2.5$ equiv), $\mathrm{H}_{2} \mathrm{O}(4 \mu \mathrm{L})$ and dioxane $(3 \mathrm{~mL})$ were added subsequently. The tube was screw capped and put into a preheated oil bath $\left(50^{\circ} \mathrm{C}\right)$. After stirring for $24 \mathrm{~h}$, the reaction mixture was cooled to room temperature. The yield was determined by ${ }^{19} \mathrm{~F}$ NMR using fluorobenzene as an internal standard.

\subsection{Radical clock experiment}<smiles>C=CNC(C)=O</smiles><smiles>O[Ga]c1ccc(-c2ccccc2)cc1</smiles>

3a

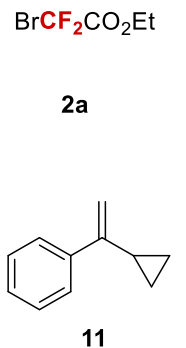

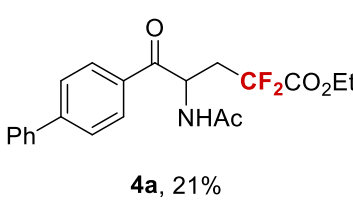

$4 a, 21 \%$

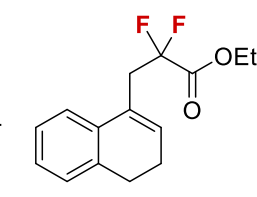

$12,25 \%$

Procedure: To a $25 \mathrm{~mL}$ of Schlenck tube were added arylboronic acid 3a (0.75 mmol, 2.5 equiv), $\mathrm{NiCl}_{2} \cdot \operatorname{DME}(10 \mathrm{~mol} \%), 4,4^{\prime}$-diOMebpy L1 (10 mol \%) and $N$-vinylacetamide 1a (0.3 mmol, 1.0 
equiv) under $\mathrm{Ar}$, followed by $\mathrm{K}_{2} \mathrm{CO}_{3}(1.2 \mathrm{mmol}, 4.0$ equiv) under air. The mixture was then evacuated and backfilled with Ar (3 times). The mixture was evacuated again and backfilled with carbon monoxide $\mathrm{CO}$ (1 atm, balloon), $\mathrm{BrCF}_{2} \mathrm{CO}_{2} \mathrm{Et} 2 \mathrm{a}$ (0.75 mmol, 2.5 equiv), 11 (0.75 mmol, 2.5 equiv), $\mathrm{H}_{2} \mathrm{O}(4 \mu \mathrm{L})$ and dioxane $(3 \mathrm{~mL})$ were added subsequently. The tube was screw capped and put into a preheated oil bath $\left(50^{\circ} \mathrm{C}\right)$. After stirring for $24 \mathrm{~h}$, the reaction mixture was cooled to room temperature. The yield was determined by ${ }^{19} \mathrm{~F}$ NMR using fluorobenzene as an internal standard.

Ethyl 3-(3,4-dihydronaphthalen-1-yl)-2,2-difluoro propanoate (12). Compound 12 (50 mg, 25\% yield) as a yellow oil was purified with silica gel chromatography (Petroleum ether: Ethyl acetate = 10: 1). Compound 12 is known. ${ }^{7}{ }^{1} \mathrm{H}$ NMR (400 MHz, $\left.\mathrm{CDCl}_{3}\right) \delta 7.40-6.95(\mathrm{~m}, 4 \mathrm{H}), 6.07$ (t, $J=4.4$ $\mathrm{Hz}, 1 \mathrm{H}), 4.13(\mathrm{q}, J=7.2 \mathrm{~Hz}, 2 \mathrm{H}), 3.23(\mathrm{t}, J=15.6 \mathrm{~Hz}, 2 \mathrm{H}), 2.73$ (t, $J=8.4 \mathrm{~Hz}, 2 \mathrm{H}), 2.27$ (m, $2 \mathrm{H})$, $1.20(\mathrm{t}, J=7.2 \mathrm{~Hz}, 3 \mathrm{H}) .{ }^{19} \mathrm{~F}$ NMR $\left(376 \mathrm{MHz}, \mathrm{CDCl}_{3}\right) \delta-99.17(\mathrm{t}, J=15.0 \mathrm{~Hz}) .{ }^{13} \mathrm{C} \mathrm{NMR}(101 \mathrm{MHz}$, $\left.\mathrm{CDCl}_{3}\right) \delta 164.1(\mathrm{t}, J=32.6 \mathrm{~Hz}), 136.3,133.9,131.6,127.6,127.3(\mathrm{t}, J=4.3 \mathrm{~Hz}), 127.2,126.3,122.8$, $115.5(\mathrm{t}, J=252.3 \mathrm{~Hz}), 62.7,37.3(\mathrm{t}, J=24.2 \mathrm{~Hz}), 28.0,23.2,13.8$.

\subsection{EPR studies}

\section{A. Reaction of 1a with 2a and $3 a$ in the presence of PBN under 1 atm of CO.}

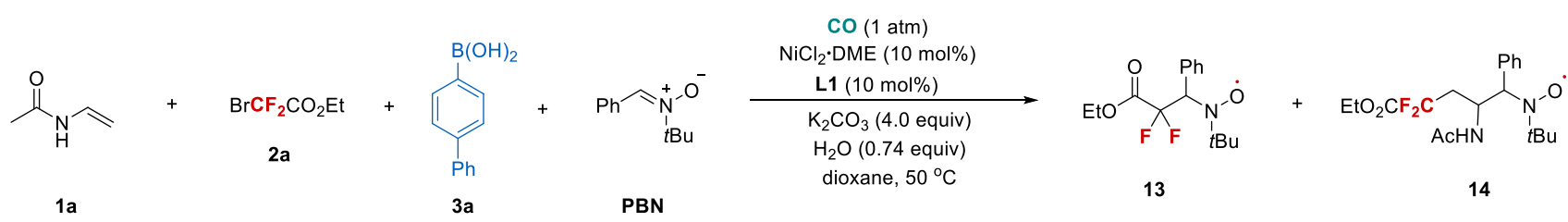

Procedure: To a $25 \mathrm{~mL}$ of Schlenk tube were added alkene $\mathbf{1 a}(0.3 \mathrm{mmol}, 1.0$ equiv), arylboronic acid 3a (0.75 mmol, 2.5 equiv), $\mathrm{NiCl}_{2} \cdot \mathrm{DME}$ (10 mol\%), L1 (10 mol\%), PBN (0.6 mmol, 2.0 equiv) and $\mathrm{K}_{2} \mathrm{CO}_{3}$ (1.2 mmol, 4.0 equiv) under Ar. The reaction mixture was then evacuated and backfilled with $\operatorname{Ar}$ (3 times). The reaction mixture was evacuated again and backfilled with carbon monoxide CO (1 atm, balloon), followed by addition of $\mathbf{2 a}\left(0.75 \mathrm{mmol}, 2.5\right.$ equiv), $\mathrm{H}_{2} \mathrm{O}(0.74$ equiv, $4 \mu \mathrm{L})$ and dioxane $\left(3 \mathrm{~mL}\right.$ ). The tube was screw-capped and heated to $50{ }^{\circ} \mathrm{C}$ (oil bath). After stirring for $8 \mathrm{~h}$, the resulting mixture was analyzed by EPR. The EPR showed an e.p.r. spectrum of nitroxides 13 and 14 (13: $g=$ 2.0056; $\mathrm{a}_{\mathrm{N}}=14.48 \mathrm{G}, \mathrm{a}_{H}^{\beta}=4.93 \mathrm{G} .14: \mathrm{g}=2.00567 ; \mathrm{a}_{\mathrm{N}}=14.61 \mathrm{G}, \mathrm{a}_{H}^{\beta}=2.52 \mathrm{G}$.) (Figure $\mathrm{S} 1$ ). 


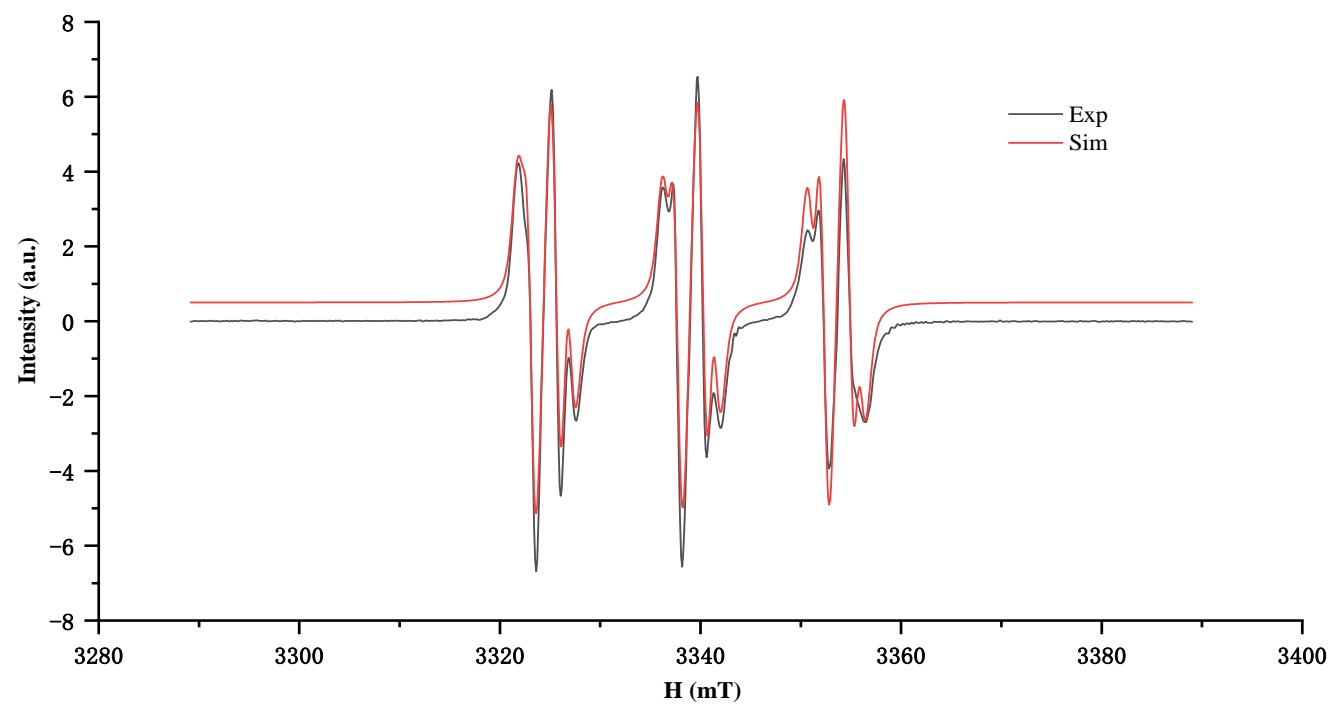

Figure S1. The EPR spectrum of a mixture of PBN, 1a, 2a and 3a under standard reaction conditions. 


\section{B. Reaction of $2 \mathrm{a}$ with $3 \mathrm{a}$ in the presence of PBN under $1 \mathrm{~atm}$ of $\mathrm{CO}$.}

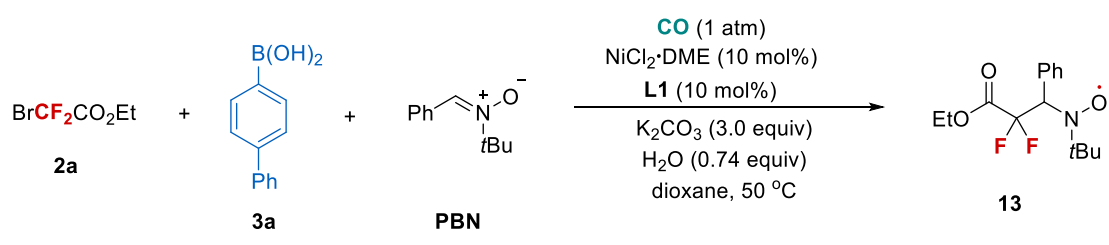

Procedure: To a $25 \mathrm{~mL}$ of Schlenk tube were added arylboronic acid 3a (0.45 mmol, 1.5 equiv), $\mathrm{NiCl}_{2} \cdot \mathrm{DME}(10 \mathrm{~mol} \%), \mathrm{L1}$ (10 mol\%), $\mathrm{PBN}$ (0.6 mmol, 2.0 equiv), and $\mathrm{K}_{2} \mathrm{CO}_{3}(0.9 \mathrm{mmol}, 3.0$ equiv) under Ar. The reaction mixture was then evacuated and backfilled with Ar ( 3 times). The reaction mixture was evacuated again and backfilled with carbon monoxide CO ( $1 \mathrm{~atm}$, balloon), followed by addition of $2 \mathrm{a}\left(0.3 \mathrm{mmol}, 1.0\right.$ equiv), $\mathrm{H}_{2} \mathrm{O}(0.74$ equiv, $4 \mu \mathrm{L})$ and dioxane $(3 \mathrm{~mL})$. The tube was screwcapped and heated to $50^{\circ} \mathrm{C}$ (oil bath). After stirring for $8 \mathrm{~h}$, the resulting mixture was analyzed by EPR. The EPR showed an e.p.r. spectrum of nitroxide $13\left(\mathrm{~g}=2.00575 ; \mathrm{a}_{\mathrm{N}}=14.52 \mathrm{G}, \mathrm{a}_{H}^{\beta}=6.48 \mathrm{G}\right)$ (Figure S2).

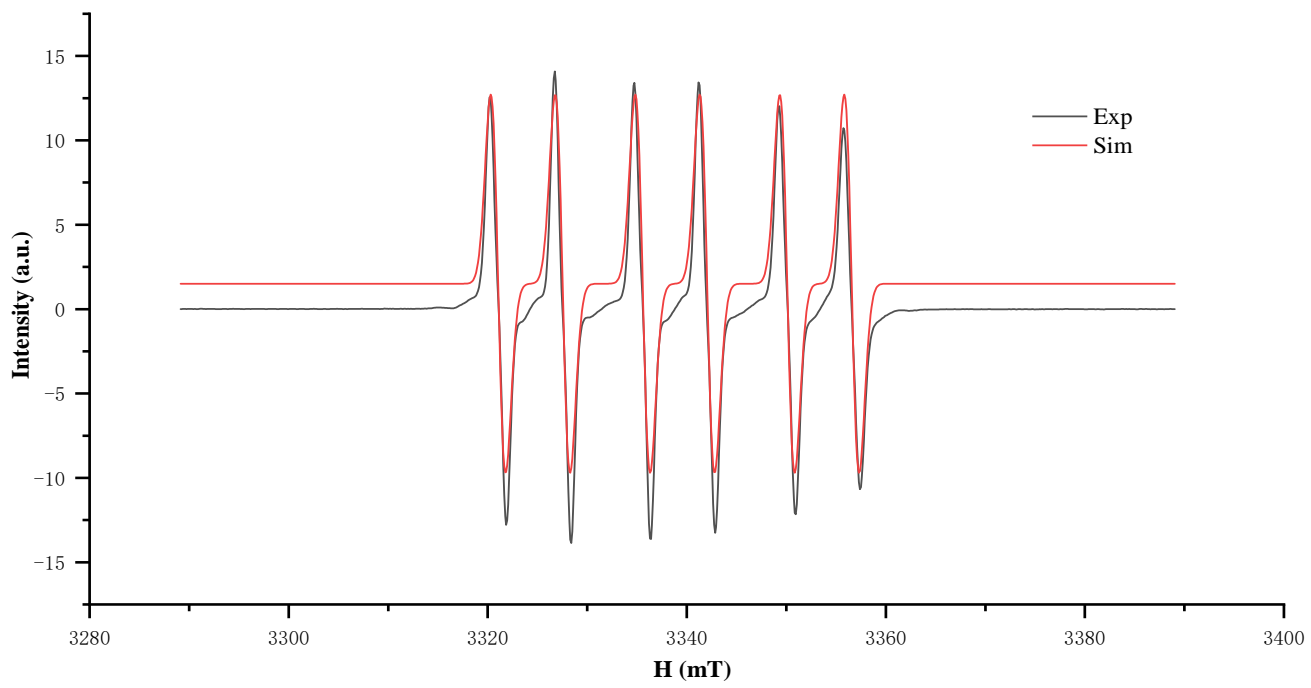

Figure S2. The EPR spectrum of a mixture of PBN, 2a and 3a under standard reaction conditions. 


\section{Reaction of 2a with PBN under 1 atm of CO.}

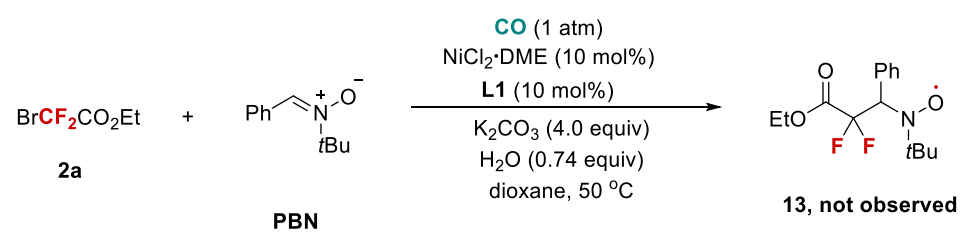

Procedure: To a $25 \mathrm{~mL}$ of Schlenk tube were added $\mathrm{NiCl}_{2} \cdot \mathrm{DME}(10 \mathrm{~mol} \%)$, L1 (10 mol\%), PBN (0.6 mmol, 2.0 equiv), and $\mathrm{K}_{2} \mathrm{CO}_{3}$ ( $0.9 \mathrm{mmol}, 3.0$ equiv) under $\mathrm{Ar}$. The reaction mixture was then evacuated and backfilled with $\operatorname{Ar}$ (3 times). The reaction mixture was evacuated again and backfilled with carbon monoxide $\mathrm{CO}$ ( $1 \mathrm{~atm}$, balloon), followed by addition of $\mathbf{2 a}\left(0.3 \mathrm{mmol}, 1.0\right.$ equiv), $\mathrm{H}_{2} \mathrm{O}(0.74$ equiv, 4 $\mu \mathrm{L})$ and dioxane $(3 \mathrm{~mL})$. The tube was screw-capped and heated to $50{ }^{\circ} \mathrm{C}$ (oil bath). After stirring for $8 \mathrm{~h}$, the resulting mixture was analyzed by EPR. The EPR showed no e.p.r. signal of nitroxide $\mathbf{1 3}$ (Figure S3). This result demonstrates that arylboronic acid is likely involved in the initial step to generate the radical.

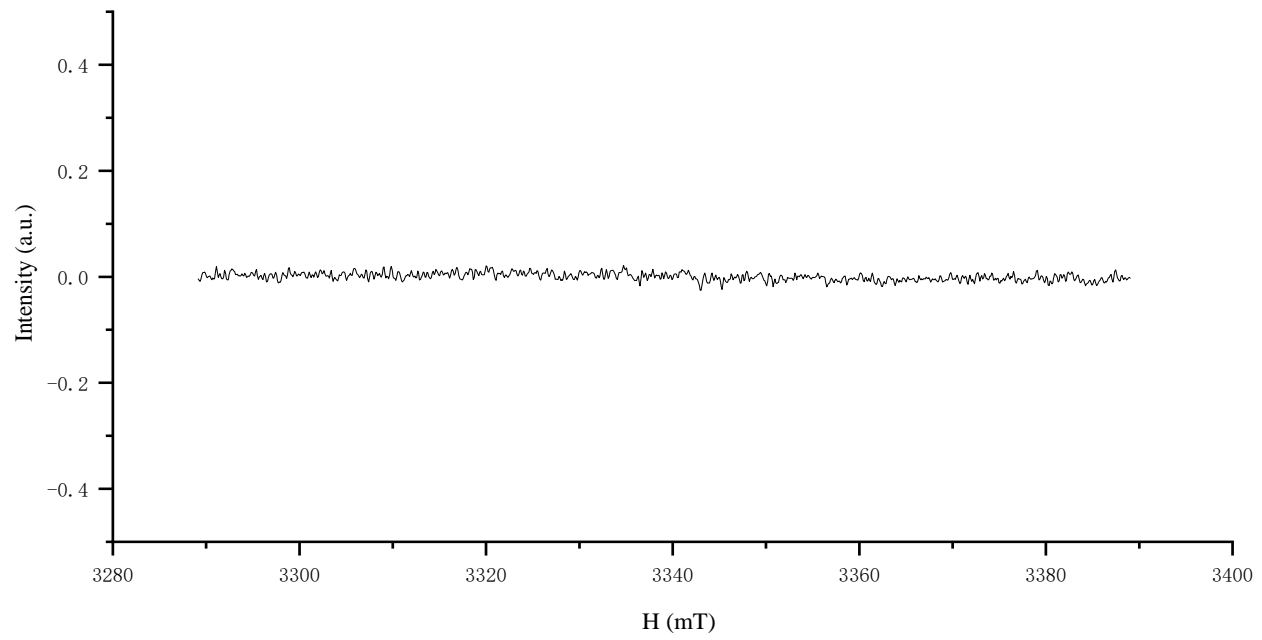

Figure S3. The EPR spectrum of a mixture of PBN and 2a under standard reaction conditions. 


\section{Reaction of 1a with PBN under Ar.}

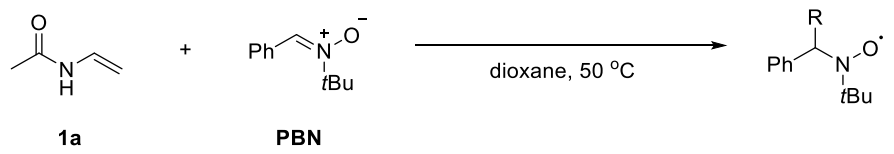

To a $25 \mathrm{~mL}$ of Schlenk tube were added alkene 1a (0.3 mmol, 1.0 equiv) and PBN (0.6 mmol, 2.0 equiv) under Ar. The reaction mixture was evacuated and backfilled with Ar (3 times) and dioxane (3 $\mathrm{mL}$ ) was added. The tube was screw-capped and heated to $50{ }^{\circ} \mathrm{C}$ (oil bath). After stirring for $8 \mathrm{~h}$, the resulting mixture was analyzed by EPR. The EPR showed a weak e.p.r. signal (Figure S4), but it does not influence the analysis of the standard reaction.

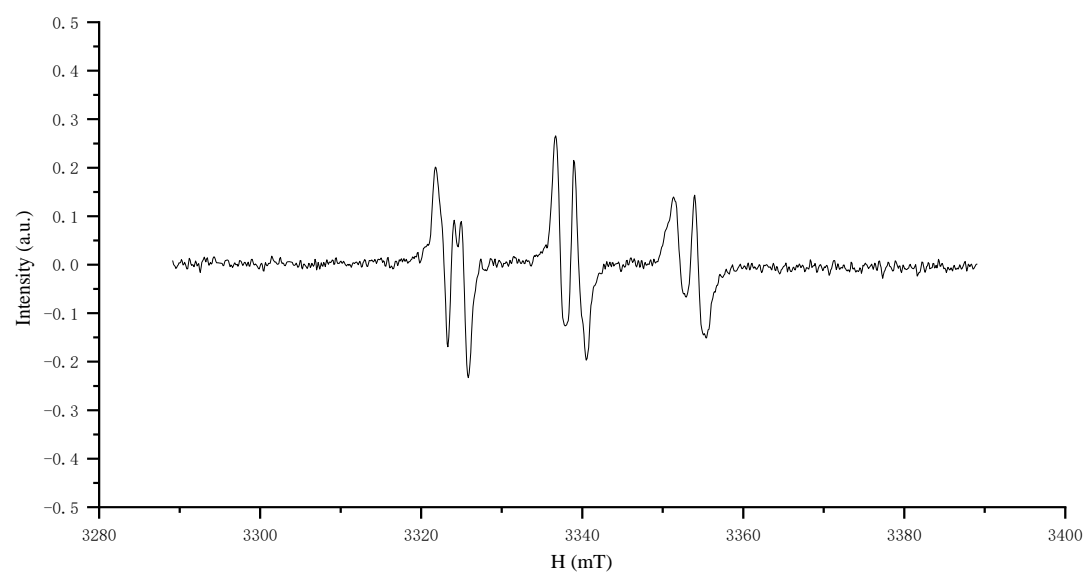

Figure S4. The EPR spectrum of a mixture of PBN and 1a in dioxane. 


\section{E. Reaction of 1a with 2a and 3a in the presence of PBN without $\mathrm{CO}$ under standard reaction}

conditions.

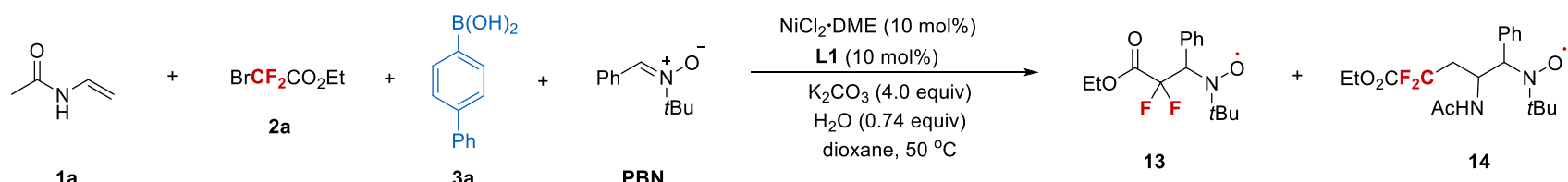

Procedure: To a $25 \mathrm{~mL}$ of Schlenk tube were added alkene 1a $(0.3 \mathrm{mmol}, 1.0$ equiv), arylboronic acid 3a (0.75 mmol, 2.5 equiv), $\mathrm{NiCl}_{2} \cdot \mathrm{DME}(10 \mathrm{~mol} \%), \mathbf{L 1}(10 \mathrm{~mol} \%), \mathrm{PBN}(0.6 \mathrm{mmol}, 2.0$ equiv), and $\mathrm{K}_{2} \mathrm{CO}_{3}$ (1.2 mmol, 4.0 equiv) under Ar. The reaction mixture was then evacuated and backfilled with $\operatorname{Ar}\left(3\right.$ times). The reaction mixture was added by $\mathbf{2 a}\left(0.75\right.$ mmol, 2.5 equiv), $\mathrm{H}_{2} \mathrm{O}(0.74$ equiv, $4 \mu \mathrm{L})$ and dioxane $(3 \mathrm{~mL})$ under Ar. The tube was screw-capped and heated to $50{ }^{\circ} \mathrm{C}$ (oil bath). After stirring for $8 \mathrm{~h}$, the resulting mixture was analyzed by EPR. The EPR showed an e.p.r. spectrum of nitroxides 13 and 14 (Figure S5), which is similar with Figure S1.

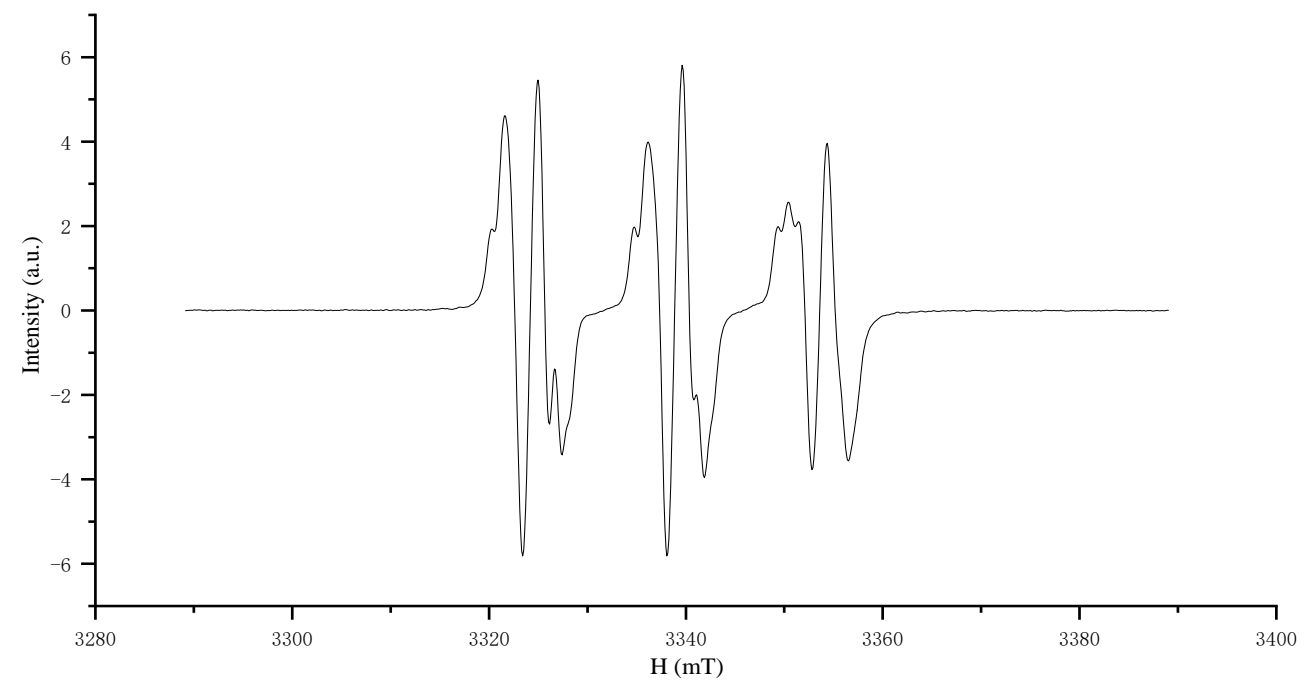

Figure S5. The EPR spectrum of a mixture of 1a, 2a, 3a, and PBN without $C O$ under standard reaction conditions. 


\section{F. Reaction of 1a with 2a in the presence of PBN under 1 atm of $\mathrm{CO}$}

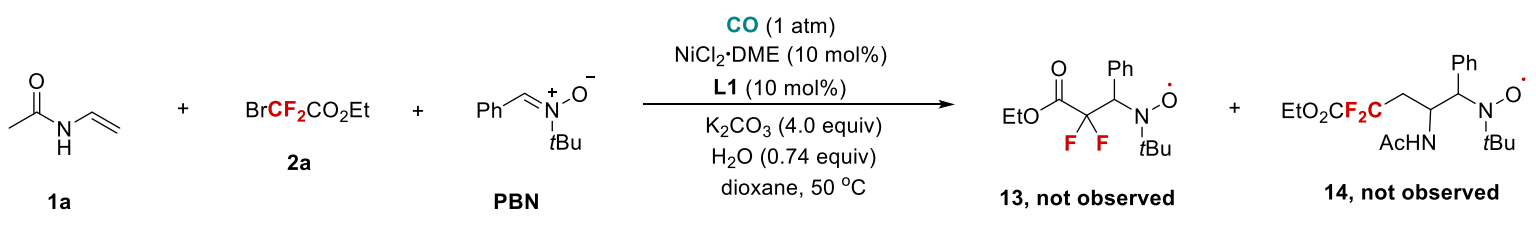

Procedure: To a $25 \mathrm{~mL}$ of Schlenk tube were added alkene 1a $\left(0.3 \mathrm{mmol}, 1.0\right.$ equiv), $\mathrm{NiCl}_{2} \cdot \mathrm{DME}(10$ mol\%), L1 (10 mol\%), PBN (0.6 mmol, 2.0 equiv), and $\mathrm{K}_{2} \mathrm{CO}_{3}$ (1.2 mmol, 4.0 equiv) under Ar. The reaction mixture was then evacuated and backfilled with Ar (3 times). The reaction mixture was evacuated again and backfilled with carbon monoxide CO (1 atm, balloon), followed by addition of 2a ( $0.75 \mathrm{mmol}, 2.5$ equiv), $\mathrm{H}_{2} \mathrm{O}(0.74$ equiv, $4 \mu \mathrm{L})$ and dioxane $(3 \mathrm{~mL})$. The tube was screw-capped and heated to $50{ }^{\circ} \mathrm{C}$ (oil bath). After stirring for $8 \mathrm{~h}$, the resulting mixture was analyzed by EPR. The EPR showed a weak e.p.r. signal (Figure S6), which is not corresponding to $\mathbf{1 3}$ and $\mathbf{1 4}$. This result demonstrates that arylboronic acid is essential in promoting the reaction.

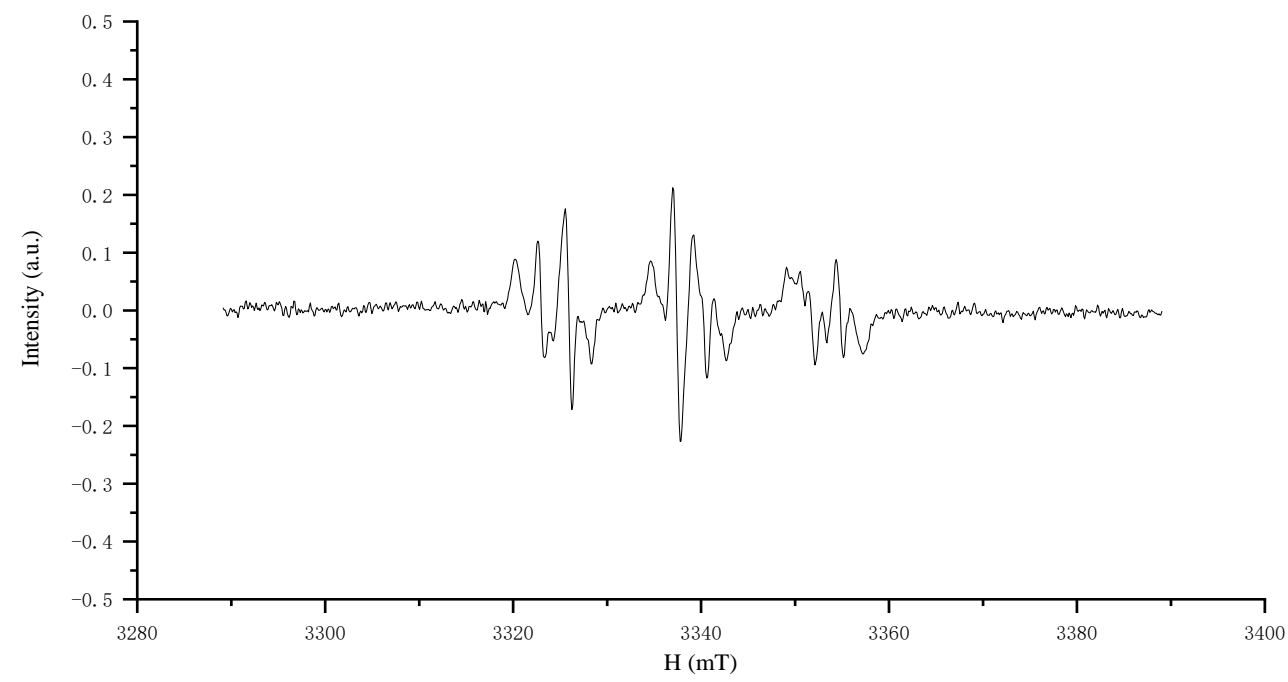

Figure S6. The EPR spectrum of a mixture of PBN, 1a and 2a under standard reaction conditions. 


\section{G. Reaction of 1a with 2a and 3a in the presence of PBN under $1 \mathrm{~atm}$ of $\mathrm{CO}$ without $[\mathrm{Ni} / \mathrm{L}]$}

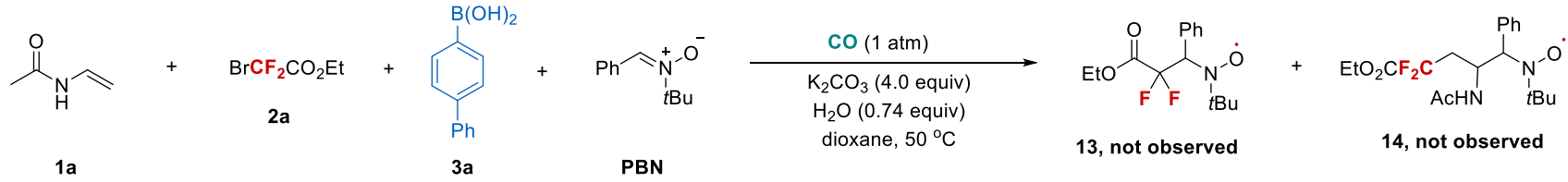

Procedure: To a $25 \mathrm{~mL}$ of Schlenk tube were added alkene $1 \mathbf{a}(0.3 \mathrm{mmol}, 1.0$ equiv), arylboronic acid 3a (0.75 mmol, 2.5 equiv), $\mathrm{PBN}$ (0.6 mmol, 2.0 equiv), and $\mathrm{K}_{2} \mathrm{CO}_{3}$ (1.2 mmol, 4.0 equiv) under Ar. The reaction mixture was then evacuated and backfilled with Ar ( 3 times). The reaction mixture was evacuated again and backfilled with carbon monoxide CO (1 atm, balloon), followed by addition of 2a $\left(0.75 \mathrm{mmol}, 2.5\right.$ equiv), $\mathrm{H}_{2} \mathrm{O}(0.74$ equiv, $4 \mu \mathrm{L})$ and dioxane $(3 \mathrm{~mL})$. The tube was screw-capped and heated to $50{ }^{\circ} \mathrm{C}$ (oil bath). After stirring for $8 \mathrm{~h}$, the resulting mixture was analyzed by EPR. Almost no e.p.r. signal was observed (Figure S7), demonstrating that $[\mathrm{Ni} / \mathrm{L}]$ is essential in promoting the reaction.

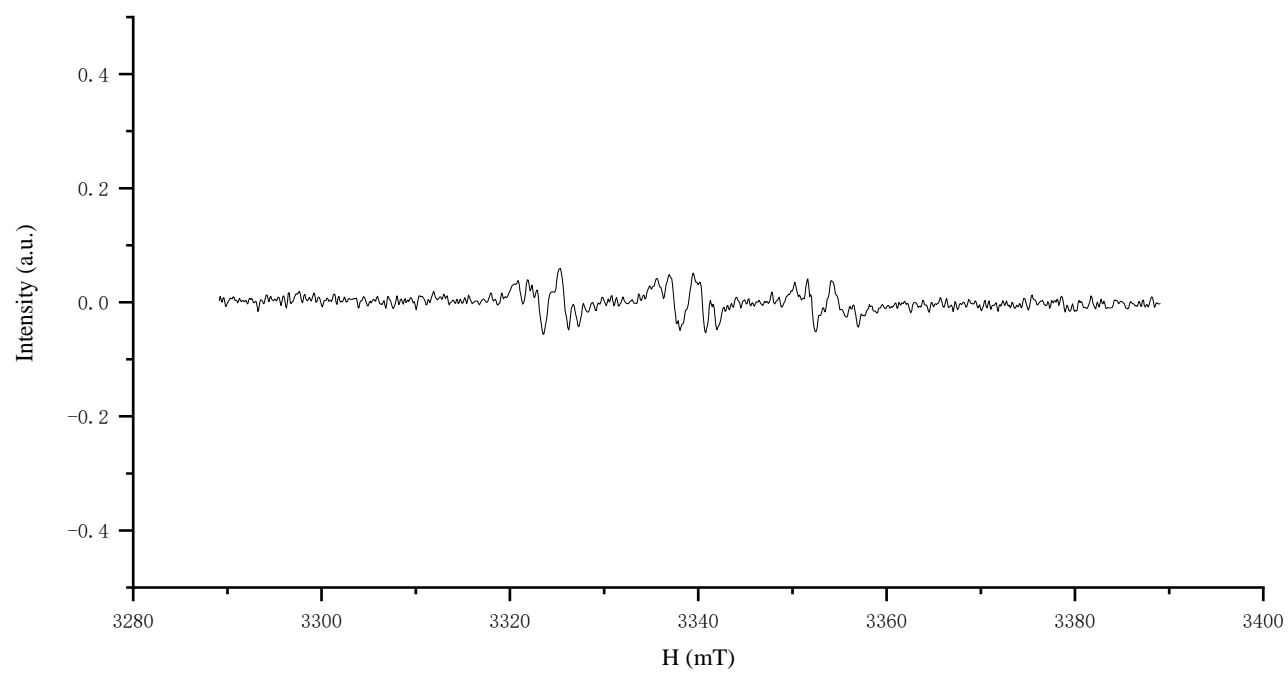

Figure S7. The EPR spectrum of a mixture of PBN, 1a, 2a and 3a without $[\mathrm{Ni} / \mathbf{L}]$. 


\subsection{Stoichiometric reaction of $B 1$ with $1 \mathrm{a}$ and $2 \mathrm{a}$}

\subsubsection{Preparation of nickel complex B1}

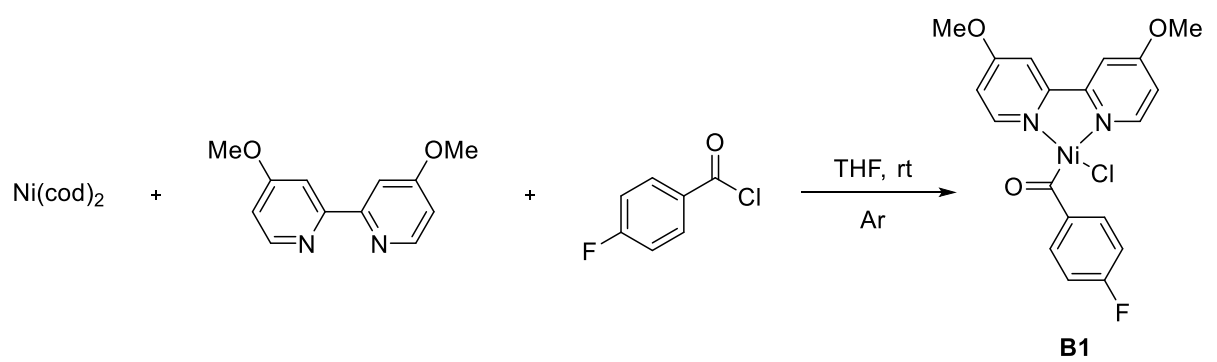

Procedure: To a Schlenk tube was charged with $\mathrm{Ni}(\mathrm{COD})_{2}(550 \mathrm{mg}, 2.0 \mathrm{mmol}), 4,4^{\prime}$-dimethoxyl-2,2'pyridine $(432 \mathrm{mg}, 2.0 \mathrm{mmol})$ and degassed dry THF $(5 \mathrm{~mL})$ under Ar. The resulting deep purple solution was left to stir for 1 hour at ambient temperature. 4-Fluorobenzoyl chloride $(2.1 \mathrm{mmol}, 1.05$ equiv) was then added and the reaction mixture was stirred for $10 \mathrm{~min}$. The resulting dark red solution was triturated with degassed dry pentane and the precipitate was collected on a frit immediately. And then the complex rinsed with pentane and dried under vacuum to give nickel complex (B1) as a dark orange powder (662 mg, 76\% yield). ${ }^{1} \mathrm{H}$ NMR (400 MHz, $\left.\mathrm{CD}_{2} \mathrm{Cl}_{2}\right) \delta 8.72-8.60(\mathrm{~m}, 3 \mathrm{H}), 7.55-7.40$ (m, 3H), $7.15-7.07$ (m, 2H), 6.96 (br, 1H), 6.66 (br, 1H), 3.99 (s, 3H), $3.93(\mathrm{~s}, 3 \mathrm{H}) .{ }^{19} \mathrm{~F}$ NMR $(376$ $\left.\mathrm{MHz}, \mathrm{CD}_{2} \mathrm{Cl}_{2}\right) \delta-109.5(\mathrm{~s}, 1 \mathrm{~F})$.

\subsubsection{Stoichiometric reaction of $B 1$ with $1 \mathrm{a}$ and $2 \mathrm{a}$}
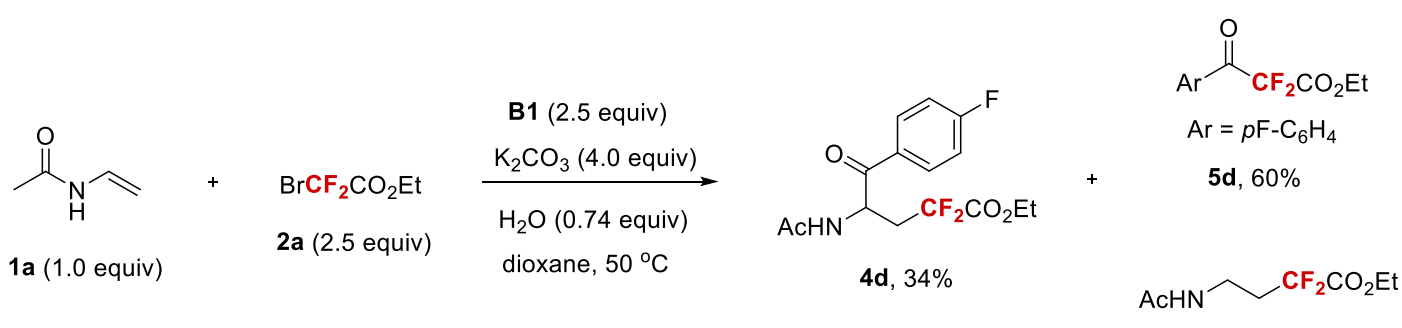

$15,23 \%$

Procedure: To a $25 \mathrm{~mL}$ of Schlenck tube was added alkene 1a $(0.3 \mathrm{mmol}, 1.0$ equiv), B1 (0.75 mmol, 2.5 equiv) and $\mathrm{K}_{2} \mathrm{CO}_{3}$ (4.0 equiv) under Ar. The reaction mixture was then evacuated and backfilled with $\mathrm{Ar}$ (3 times). The reaction mixture was evacuated again and backfilled with carbon monoxide $\mathrm{CO}$ ( 1 atm, balloon), followed by addition of $\mathbf{2 a}\left(0.75\right.$ mmol, 2.5 equiv), $\mathrm{H}_{2} \mathrm{O}(0.74$ equiv, $4 \mu \mathrm{L})$ and dioxane $(3 \mathrm{~mL})$. The tube was screw-capped and heated to $50{ }^{\circ} \mathrm{C}$ (oil bath). After stirring for $18 \mathrm{~h}$, the reaction mixture was cooled to room temperature. The yield was determined by ${ }^{19} \mathrm{~F}$ NMR using fluorobenzene as an internal standard. The yield of $\mathbf{5 d}$ is based on $\mathbf{2 a}$. 


\subsection{Complex B1-catalyzed Reaction of $1 \mathrm{a}$ with $2 \mathrm{a}$ and $3 d$}

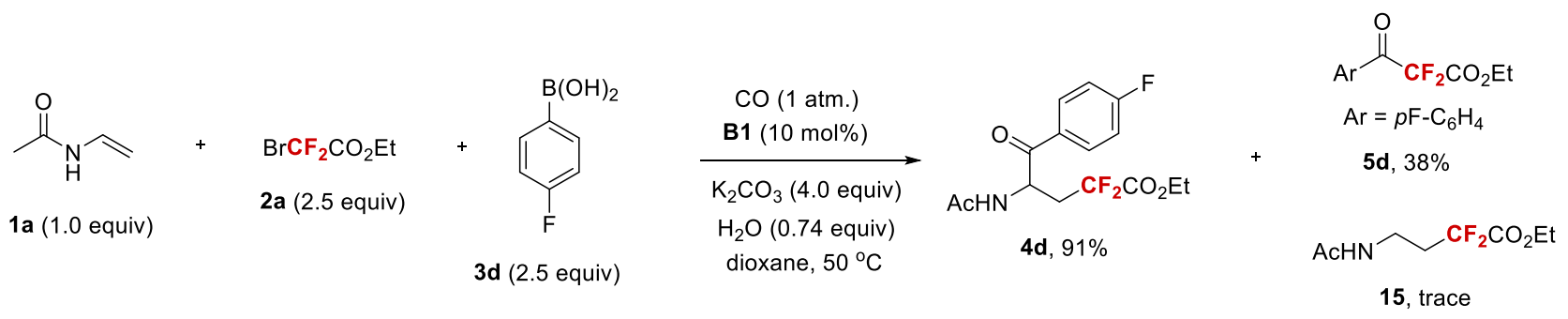

Procedure: To a $25 \mathrm{~mL}$ of Schlenck tube was added alkene 1a ( $0.3 \mathrm{mmol}, 1.0$ equiv), $3 \mathbf{d}(0.75 \mathrm{mmol}$, 2.5 equiv), $\mathbf{B} 1$ ( $0.03 \mathrm{mmol}, 10 \mathrm{~mol} \%$ ) and $\mathrm{K}_{2} \mathrm{CO}_{3}$ (4.0 equiv) under Ar. The reaction mixture was then evacuated and backfilled with Ar (3 times). The reaction mixture was evacuated again and backfilled with carbon monoxide $\mathrm{CO}$ ( $1 \mathrm{~atm}$, balloon), followed by addition of $\mathbf{2 a}\left(0.75 \mathrm{mmol}, 2.5\right.$ equiv), $\mathrm{H}_{2} \mathrm{O}$ (0.74 equiv, $4 \mu \mathrm{L})$ and dioxane $(3 \mathrm{~mL})$. The tube was screw-capped and heated to $50{ }^{\circ} \mathrm{C}$ (oil bath). After stirring for $18 \mathrm{~h}$, the reaction mixture was cooled to room temperature. The yield was determined by ${ }^{19} \mathrm{~F}$ NMR using fluorobenzene as an internal standard. The yield of $\mathbf{5 d}$ was calculated based on $\mathbf{2 a}$. The reaction mixture was diluted with ethyl acetate and $\mathrm{H}_{2} \mathrm{O}$. The resulting mixture was then extracted with ethyl acetate, the combined organic layers were washed with brine, dried over $\mathrm{Na}_{2} \mathrm{SO}_{4}$, filtered, and concentrated. The residue was purified with silica gel chromatography (Petroleum ether: Ethyl acetate $=4: 1)$ to give product $\mathbf{4 d}(91 \mathrm{mg}, 91 \%$ yield $)$ as a white solid.

\subsection{Reaction of alkene 1a with bromodifluoroacetate 2a under standard conditions}

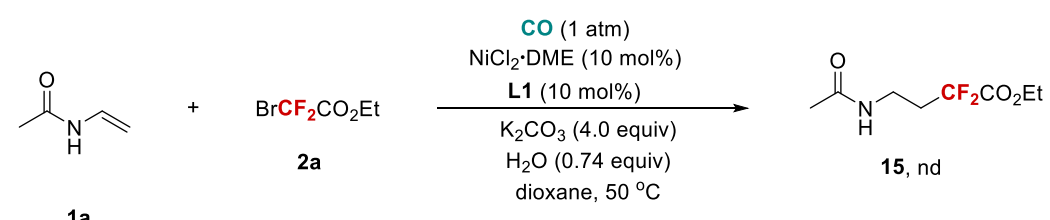

Procedure: To a $25 \mathrm{~mL}$ of Schlenck tube were added alkene 1a $\left(0.3 \mathrm{mmol}, 1.0\right.$ equiv), $\mathrm{NiCl}_{2} \cdot \mathrm{DME}$ (0.03 mmol, 0.1 equiv), $\mathbf{L 1}$ ( $0.03 \mathrm{mmol}, 0.1$ equiv) and $\mathrm{K}_{2} \mathrm{CO}_{3}$ (4.0 equiv). The reaction mixture was then evacuated and backfilled with Ar (3 times). The resulting mixture was evacuated again and backfilled with CO (1 atm, balloon). To the Schlenck tube were added difluoroalkyl bromide $2 \mathbf{a}(0.3$ mmol, 1.0 equiv), $\mathrm{H}_{2} \mathrm{O}(0.74$ equiv, $4 \mu \mathrm{L})$ and dioxane $(3 \mathrm{~mL})$. The tube was screw capped and put into a preheated oil bath $\left(50^{\circ} \mathrm{C}\right)$. After stirring for $18 \mathrm{~h}$, the reaction mixture was cooled to room temperature. The resulting mixture was analyzed by ${ }^{19} \mathrm{~F}$ NMR and GC-MS, and no desired product 15 
was observed.

\subsection{Reaction of 2a with B1}

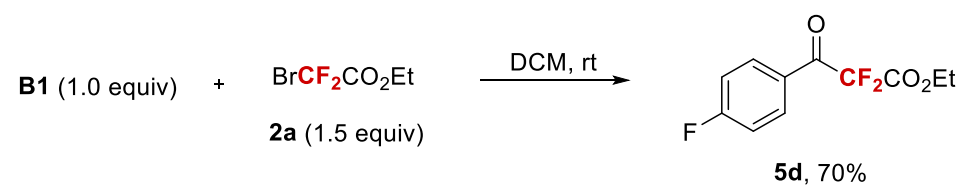

Procedure: To a $25 \mathrm{~mL}$ of Schlenck tube was added B1 (0.2 mmol, 1.0 equiv), 2a (0.3 mmol, 1.5 equiv) and DCM (2 mL) under Ar. The tube was screw-capped and reacted at room temperature. After stirring for $12 \mathrm{~h}$, the reaction mixture was diluted with ethyl acetate and $\mathrm{H}_{2} \mathrm{O}$. The resulting mixture was then extracted with ethyl acetate, the combined organic layers were washed with brine, dried over $\mathrm{Na}_{2} \mathrm{SO}_{4}$, filtered, and concentrated. The residue was purified with silica gel chromatography (DCM) to give product $\mathbf{5 d}$ (34 mg, 70\% yield) as a yellow oil. Compound $\mathbf{5 d}$ is known. ${ }^{9}{ }^{1} \mathrm{H}$ NMR (400 MHz, $\left.\mathrm{CDCl}_{3}\right) \delta 8.13(\mathrm{~m}, 2 \mathrm{H}), 7.19(\mathrm{t}, J=8.8 \mathrm{~Hz}, 2 \mathrm{H}), 4.39(\mathrm{q}, J=7.2 \mathrm{~Hz}, 2 \mathrm{H}), 1.32(\mathrm{t}, J=7.2 \mathrm{~Hz}, 3 \mathrm{H}) .{ }^{19} \mathrm{~F}$ NMR (376 MHz, $\left.\mathrm{CDCl}_{3}\right) \delta-100.6(\mathrm{~m}, 1 \mathrm{~F}),-107.6(\mathrm{~s}, 2 \mathrm{~F}) .{ }^{13} \mathrm{C} \mathrm{NMR}\left(101 \mathrm{MHz}, \mathrm{CDCl}_{3}\right) \delta 184.6(\mathrm{t}, J$ $=27.3 \mathrm{~Hz}), 167.4(\mathrm{~d}, J=259.6 \mathrm{~Hz}), 162.2(\mathrm{t}, J=30.3 \mathrm{~Hz}), 133.5(\mathrm{dt}, J=10.1 \mathrm{~Hz}, 2.0 \mathrm{~Hz}), 128.1(\mathrm{t}, J$ $=2.0 \mathrm{~Hz}), 117.0(\mathrm{~d}, J=22.2 \mathrm{~Hz}), 110.4(\mathrm{t}, J=266.6 \mathrm{~Hz}), 64.4,14.4$.

\subsection{Reaction of arylboronic acid 3a with $\mathrm{MeOH}$ under 1atm of $\mathrm{CO}$ in the presence of $\mathrm{NiCl}$. DME/L1}

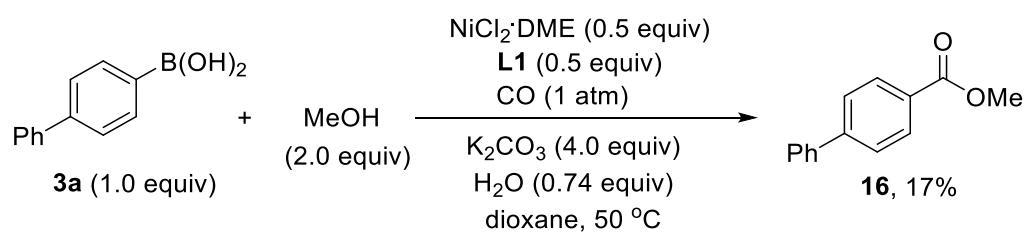

Procedure: To a $25 \mathrm{~mL}$ of Schlenck tube were added aryl boronic acid 3a ( $0.3 \mathrm{mmol}, 1.0$ equiv), $\mathrm{NiCl}_{2} \cdot \mathrm{DME}(50 \mathrm{~mol} \%)$, $\mathbf{L 1}(50 \mathrm{~mol} \%)$, and $\mathrm{K}_{2} \mathrm{CO}_{3}$ (4.0 equiv). The reaction mixture was then evacuated and backfilled with $\operatorname{Ar}$ (3 times). The resulting mixture was evacuated again and backfilled with $\mathrm{CO}$ ( 1 atm, balloon). To the Schlenck tube were added $\mathrm{MeOH}(0.6$ mmol, 2.0 equiv $), \mathrm{H}_{2} \mathrm{O}(0.74$ equiv, $4 \mu \mathrm{L})$ and dioxane $(2 \mathrm{~mL})$. The tube was screw capped and put into a preheated oil bath $\left(50{ }^{\circ} \mathrm{C}\right)$. After stirring for $18 \mathrm{~h}$, the reaction mixture was cooled to room temperature. The reaction mixture was diluted with ethyl acetate and $\mathrm{H}_{2} \mathrm{O}$. The resulting mixture was then extracted with ethyl acetate, the 
combined organic layers were washed with brine, dried over $\mathrm{Na}_{2} \mathrm{SO}_{4}$, filtered, and concentrated. The residue was purified with silica gel chromatography to give product $\mathbf{1 7}(11 \mathrm{mg}, 17 \%$ yield) as a white solid. Compound 16 is known. ${ }^{8}{ }^{1} \mathrm{H}$ NMR $\left(400 \mathrm{MHz}, \mathrm{CDCl}_{3}\right) \delta 8.12(\mathrm{~d}, J=8.0 \mathrm{~Hz}, 2 \mathrm{H}), 7.67(\mathrm{~d}, J=$ $8.0 \mathrm{~Hz}, 2 \mathrm{H}), 7.63$ (d, $J=7.6 \mathrm{~Hz}, 2 \mathrm{H}), 7.47$ (t, $J=7.6 \mathrm{~Hz}, 2 \mathrm{H}), 7.40$ (t, $J=7.6 \mathrm{~Hz}, 1 \mathrm{H}), 3.95$ (s, 3H). ${ }^{13} \mathrm{C}$ NMR $\left(101 \mathrm{MHz} \mathrm{CDCl}_{3}\right) \delta 167.5,146.1,140.5,130.6,129.5,129.4,128.7,127.8,127.6,52.7$.

\subsection{Reaction of bromodifluoroacetate $2 \mathrm{a}$ with $\mathrm{MeOH}$ under $1 \mathrm{~atm}$ of $\mathrm{CO}$ in the presence of}

\section{$\mathrm{NiCl} \cdot \mathbf{D M E} / \mathrm{L} 1$}

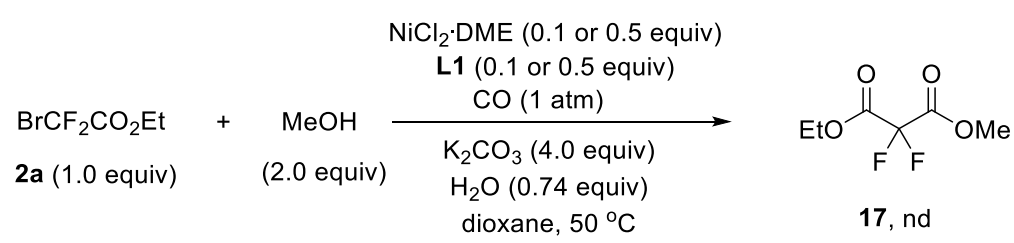

Procedure: To a $25 \mathrm{~mL}$ of Schlenck tube were added $\mathrm{NiCl}_{2} \cdot \mathrm{DME}(0.1$ or 0.5 equiv), $\mathbf{L 1}(0.1$ or 0.5 equiv), and $\mathrm{K}_{2} \mathrm{CO}_{3}$ (4.0 equiv). The reaction mixture was then evacuated and backfilled with Ar (3 times). The resulting mixture was evacuated again and backfilled with $\mathrm{CO}$ ( $1 \mathrm{~atm}$, balloon). To the Schlenck tube were added 2a (0.3 mmol, 1.0 equiv), $\mathrm{H}_{2} \mathrm{O}$ (0.74 equiv, $\left.4 \mu \mathrm{L}\right), \mathrm{MeOH}(2.0$ equiv) and dioxane $(2 \mathrm{~mL})$. The tube was screw capped and put into a preheated oil bath $\left(50{ }^{\circ} \mathrm{C}\right)$. After stirring for $18 \mathrm{~h}$, the reaction mixture was cooled to room temperature. The resulting mixture was analyzed by ${ }^{19} \mathrm{~F}$ NMR and GC-MS, and no desired product 17 was observed.

\subsection{Reaction of bromodifluoroacetate $2 \mathrm{a}$ with $\mathrm{MeOH}$ under $1 \mathrm{~atm}$ of $\mathrm{CO}$ in the presence of} $\mathrm{Ni}(\mathrm{COD})_{2} / \mathrm{L} 1$

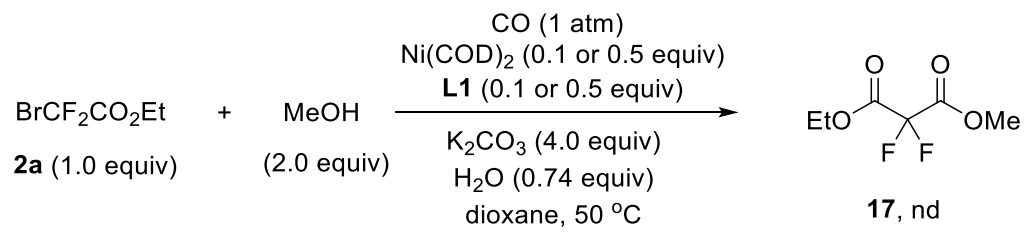

Procedure: To a $25 \mathrm{~mL}$ of Schlenck tube were added $\mathrm{Ni}(\mathrm{COD})_{2}(0.1$ or 0.5 equiv), $\mathbf{L 1}(0.1$ or 0.5 equiv), and $\mathrm{K}_{2} \mathrm{CO}_{3}$ (4.0 equiv). The reaction mixture was then evacuated and backfilled with $\operatorname{Ar}(3$ times). The resulting mixture was evacuated again and backfilled with $\mathrm{CO}$ ( $1 \mathrm{~atm}$, balloon). To the Schlenck tube were added difluoroalkyl bromide 2a (0.3 mmol, 1.0 equiv), $\mathrm{MeOH}$ (2.0 equiv) and 
dioxane $(2 \mathrm{~mL})$. The tube was screw capped and put into a preheated oil bath $\left(50^{\circ} \mathrm{C}\right)$. After stirring for $18 \mathrm{~h}$, the reaction mixture was cooled to room temperature. The resulting mixture was analyzed by ${ }^{19} \mathrm{~F}$ NMR and GC-MS, and no desired product 17 was observed.

\subsection{Reaction of alkene $1 p$, bromodifluoroacetate 2 a with arylboronic acid $3 a$ under standard} reaction conditions

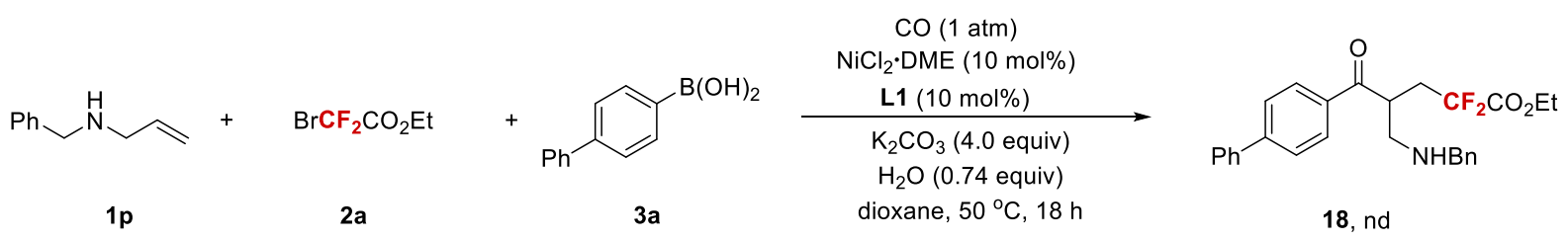

Figure S8. The use of benzyl protected allyl amine 1p as a coupling partner

Procedure: To a $25 \mathrm{~mL}$ of Schlenck tube was added arylboronic acid 3a (0.75 mmol, 2.5 equiv), $\mathrm{NiCl}_{2} \cdot \mathrm{DME}(10 \mathrm{~mol} \%), \mathbf{L 1}(10 \mathrm{~mol} \%)$ and $\mathrm{K}_{2} \mathrm{CO}_{3}$ (4.0 equiv) under Ar. The reaction mixture was then evacuated and backfilled with Ar (3 times). The reaction mixture was evacuated again and backfilled with carbon monoxide CO (1 atm, balloon), followed by addition of alkene 1p ( $0.3 \mathrm{mmol}, 1.0$ equiv), 2a (0.75 mmol, 2.5 equiv), $\mathrm{H}_{2} \mathrm{O}(0.74$ equiv, $4 \mu \mathrm{L})$ and dioxane $(3 \mathrm{~mL})$. The tube was screw-capped and heated to $50{ }^{\circ} \mathrm{C}$ (oil bath). After stirring for $18 \mathrm{~h}$, the reaction mixture was cooled to room temperature. The resulting mixture was analyzed by ${ }^{19} \mathrm{~F}$ NMR and LC-MS, and no desired product 18 was observed. 


\subsection{Kinetic Studies of Nickel-Catalyzed Reaction of 1a with 2 a and $3 d$.}

8.12.1. Kinetic Study of Nickel-Catalzyed Reaction of 1a with 2a and 3d under Standard Reaction Conditions ${ }^{a}$

\begin{tabular}{|c|c|c|c|c|}
\hline 1a (1.0 equiv) & $\begin{array}{l}\mathrm{BrCF}_{2} \mathrm{CO}_{2} \mathrm{Et} \\
\text { 2a (2.5 equiv) }\end{array}$ & 3d (2.5 equiv) & $\begin{array}{c}\mathrm{CO}(1 \mathrm{~atm}) \\
\mathrm{NiCl}_{2} \cdot \mathrm{DME}(10 \mathrm{~mol} \%) \\
\mathrm{L1}(10 \mathrm{~mol} \%) \\
\mathrm{K}_{2} \mathrm{CO}_{3}(4.0 \text { equiv }) \\
\mathrm{H}_{2} \mathrm{O}(0.74 \text { equiv }) \\
\text { dioxane, } 50^{\circ} \mathrm{C}\end{array}$ & ${ }_{4 \mathbf{d}}$ \\
\hline entry & & reaction tim & & yield $(\%)^{b}$ \\
\hline 1 & & $15 \mathrm{~min}$ & & nd \\
\hline 2 & & $30 \mathrm{~min}$ & & nd \\
\hline 3 & & $60 \mathrm{~min}$ & & nd \\
\hline 4 & & $90 \mathrm{~min}$ & & nd \\
\hline 5 & & $120 \mathrm{~min}$ & & 5 \\
\hline 6 & & $150 \mathrm{~min}$ & & 18 \\
\hline 7 & & $180 \mathrm{~min}$ & & 29 \\
\hline 8 & & $210 \mathrm{~min}$ & & 37 \\
\hline 9 & & $240 \mathrm{~min}$ & & 41 \\
\hline
\end{tabular}

${ }^{a}$ Reaction conditions (unless otherwise specified): $1 \mathbf{a}$ ( 0.3 mmol, 1.0 equiv), $2 \mathbf{2 a}$ ( 2.5 equiv), $\mathbf{3 d}$ ( 2.5 equiv), dioxane $(3.0 \mathrm{~mL}), 50{ }^{\circ} \mathrm{C}, 18 \mathrm{~h} .{ }^{b}$ Determined by ${ }^{19} \mathrm{~F}$ NMR using fluorobenzene as an internal standard and the number in parenthesis is the isolated yield. nd, not detected.

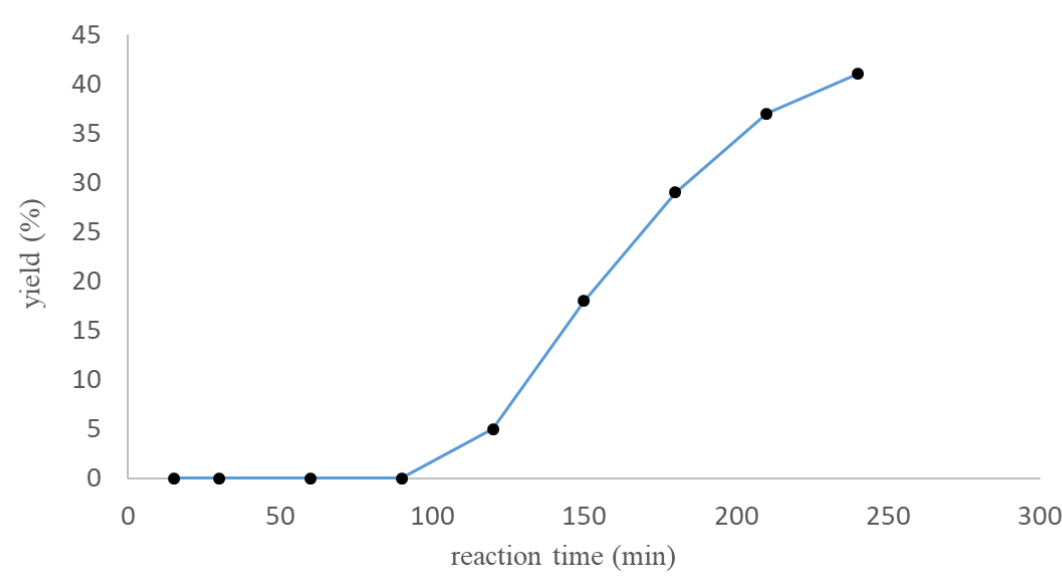

Figure S9. Kinetic Study of Nickel-Catalzyed Reaction of 1a with 2a and 3d under Standard Reaction Conditions. 


\subsubsection{Kinetic Studies of B1-Catalzyed Reaction of 1a with 2a and 3d}

\begin{tabular}{|c|c|c|c|c|}
\hline$\overbrace{\mathrm{H}}^{\mathrm{O}} \mathrm{N}_{\mathrm{H}(1.0 \text { equiv })}$ & $\begin{array}{l}\mathrm{BrCF}_{2} \mathrm{CO}_{2} \mathrm{Et} \\
\text { 2a (2.5 equiv) }\end{array}$ & 3d (2.5 equiv) & $\begin{array}{c}\mathrm{CO}(1 \mathrm{~atm} .) \\
\text { B1 }(10 \mathrm{~mol} \%) \\
\mathrm{K}_{2} \mathrm{CO}_{3}(4.0 \text { equiv }) \\
\mathrm{H}_{2} \mathrm{O}(0.74 \text { equiv }) \\
\text { dioxane, } 50^{\circ} \mathrm{C}\end{array}$ & ${ }_{4 d}$ \\
\hline entry & & reaction time & & yield $(\%)^{b}$ \\
\hline 1 & & $3 \mathrm{~min}$ & & 2 \\
\hline 2 & & $5 \mathrm{~min}$ & & 2 \\
\hline 3 & & $10 \mathrm{~min}$ & & 6 \\
\hline 4 & & $15 \mathrm{~min}$ & & 13 \\
\hline 5 & & $20 \mathrm{~min}$ & & 17 \\
\hline 6 & & $30 \mathrm{~min}$ & & 25 \\
\hline 7 & & $60 \mathrm{~min}$ & & 28 \\
\hline 8 & & $120 \mathrm{~min}$ & & 39 \\
\hline
\end{tabular}

${ }^{a}$ Reaction conditions (unless otherwise specified): $1 \mathbf{a}$ ( $0.3 \mathrm{mmol}, 1.0$ equiv), $\mathbf{2 a}$ ( 2.5 equiv), $\mathbf{3 d}$ ( 2.5 equiv), dioxane $(3.0 \mathrm{~mL}), 50{ }^{\circ} \mathrm{C}, 18 \mathrm{~h} .{ }^{b}$ Determined by ${ }^{19} \mathrm{~F}$ NMR using fluorobenzene as an internal standard and the number in parenthesis is the isolated yield.

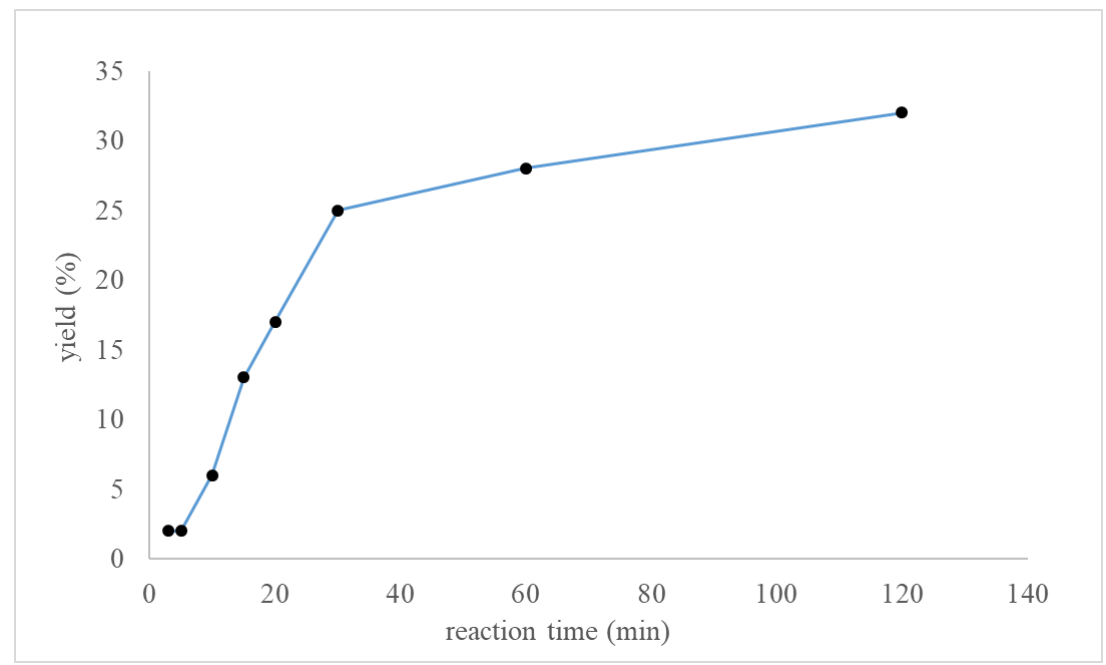

Figure S10. Kinetic studies of B1-catalzyed reaction of 1a with 2a and 3d. 


\section{References}

1. Hemming, D. S.; Talbot, E. P.; Steel, P. G. A mild copper catalyzed method for the selective deprotection of aryl allyl ethers. Tetrahedron Lett. 2017, 58, 17-20.

2. Mizuta, S.; Verhoog, S.; Engle, K. M.; Khotavivattana, T.; O’Duill, M.; Wheelhouse, K.; Rassias, G.; Medebielle, M.; Gouverneur, V. Catalytic hydrotrifluoromethylation of unactivated alkenes. $J$. Am. Chem. Soc. 2013, 135, 2505-2508.

3. Andersen, T. L.; Frederiksen, M. W.; Domino, K.; Skrydstrup, T. Direct access to $\alpha, \alpha-$ difluoroacylated arenes by palladium-catalyzed carbonylation of (hetero)aryl boronic acid derivatives. Angew. Chem. Int. Ed. 2016, 55, $10396-10400$.

4. Chang, C.-F.; Mfuh, A.; Gao, J.; Wu, H.-Y.; Woo, C. M. Synthesis of an electronically-tuned minimally interfering alkynyl photo-affinity label to measure small molecule-protein interactions. Tetrahedron 2018, 74, 3273-3277.

5. Jiang, H.; Yuan, S.; Cai, Y.; Wan, W.; Zhu, S.; Hao, J. A Facile preparation of 2bromodifluoromethyl benzo-1,3-diazoles and its application in the synthesis of gemdifluoromethylene linked aryl ether compounds. J. Fluorine Chem. 2012, 133, 167-170.

6. Xu, C.; Cheng, R.; Luo, Y.-C.; Wang, M.-K.; Zhang, X. trans-Selective aryldifluoroalkylation of endocyclic enecarbamates and enamides via nickel catalysis. Angew. Chem. Int. Ed. 2020, 59, DOI:10.1002/anie.202008498.

7. Feng, Z.; Min, Q.-Q.; Zhang, X. Palladium-catalyzed Heck-type difluoroalkylation of alkenes with functionalized difluoromethyl bromides. Synthesis 2015, 47, 2912-2923.

8. Zhao, H.-Y.; Gao, X.; Zhang, S.; Zhang, X. Nickel-catalyzed carbonylation of difluoroalkyl bromides with arylboronic acids. Org. Lett. 2019, 21, 1031-1036.

9. Yang, M.-H.; Orsi, D. L.; Altman, R. A. Ligand-controlled regiodivergent palladium-catalyzed decarboxylative allylation reaction to access $\alpha, \alpha$-difluoroketones. Angew. Chem. Int. Ed. 2015, 54, $2361-2365$. 
10. Copies of ${ }^{1} \mathrm{H}$ NMR and ${ }^{13} \mathrm{C}$ NMR spectra of compounds 1

$\mathrm{N}$-allylbenzamide (1f).

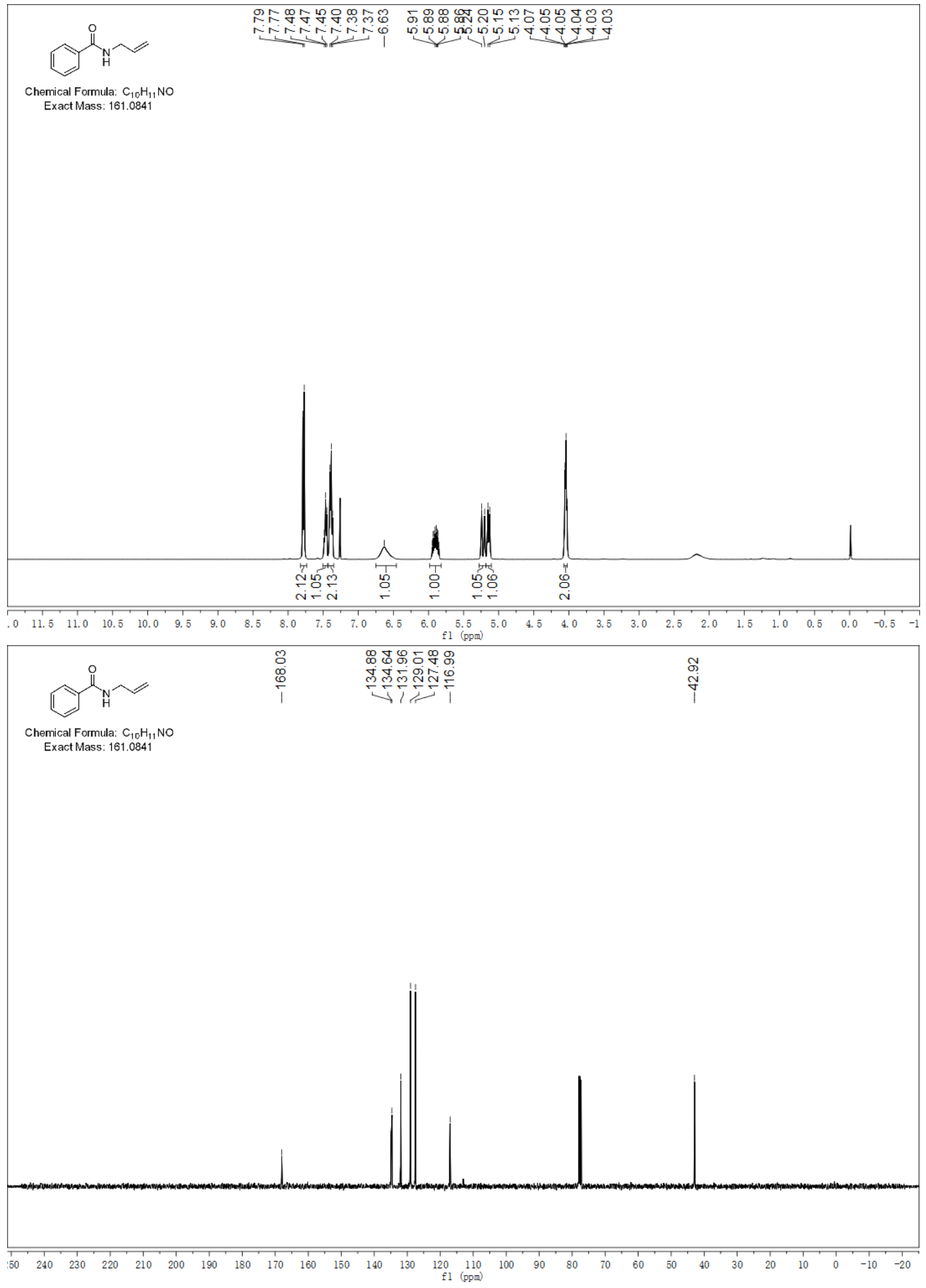


$N$-(but-3-en-1-yl)benzamide (1i).

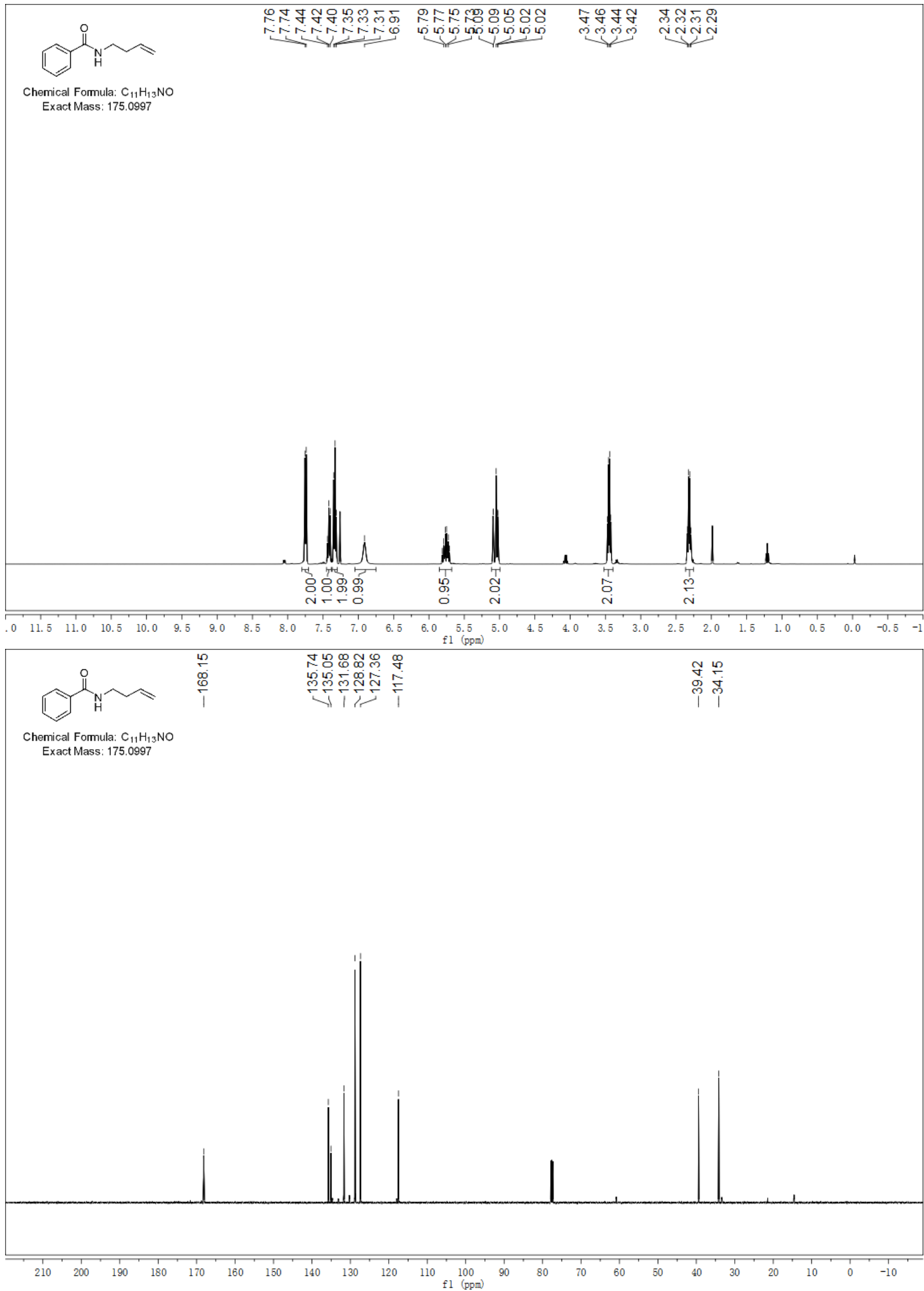


tert-Butyl (S)-5-(allylamino)-4-(((benzyloxy)carbonyl)amino)-5-oxopentanoate $(1 \mathrm{~m})$.

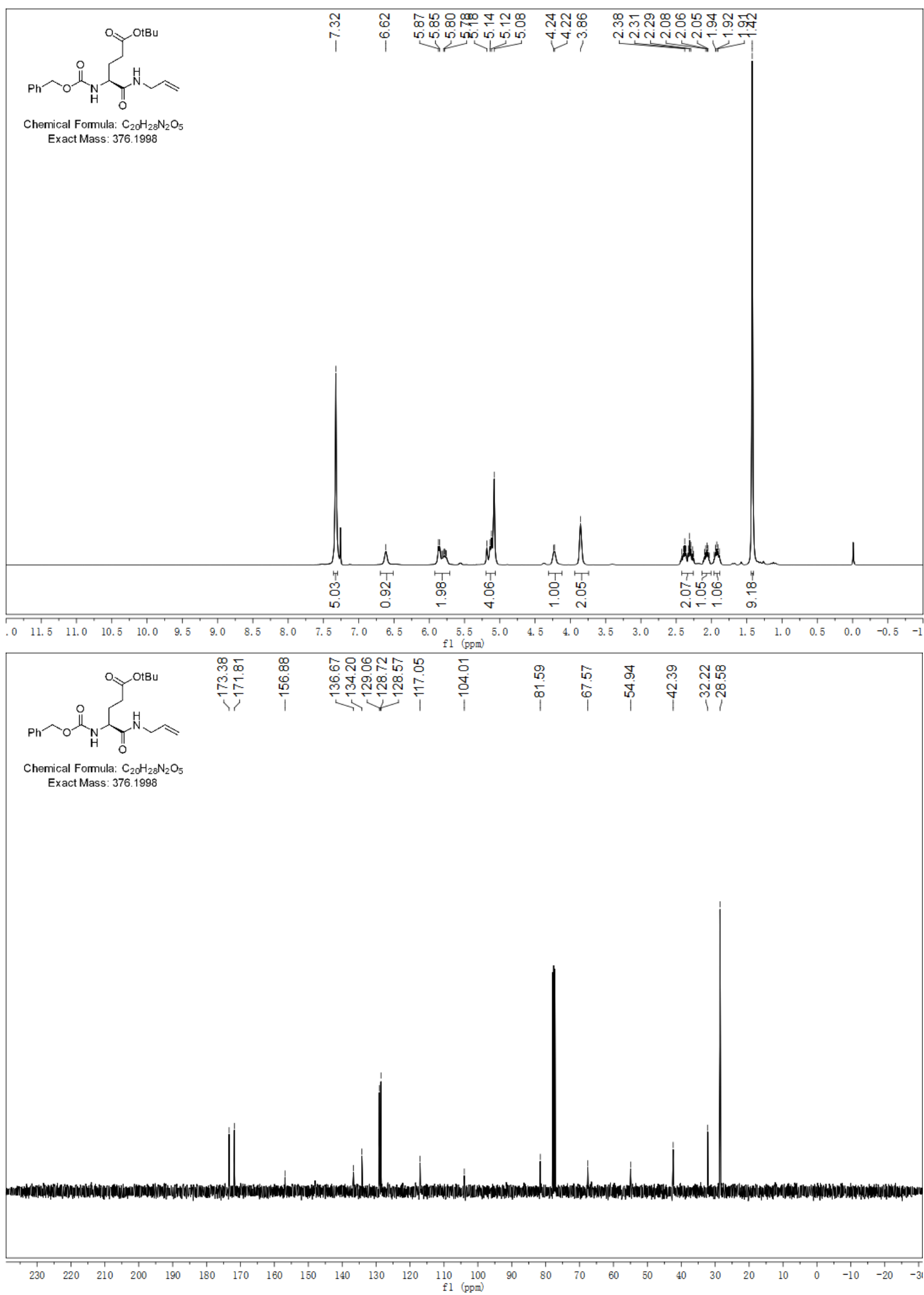


tert-Butyl (S)-(1-(Allylamino)-1-oxo-3-phenylpropan-2-yl)carbamate (1n).

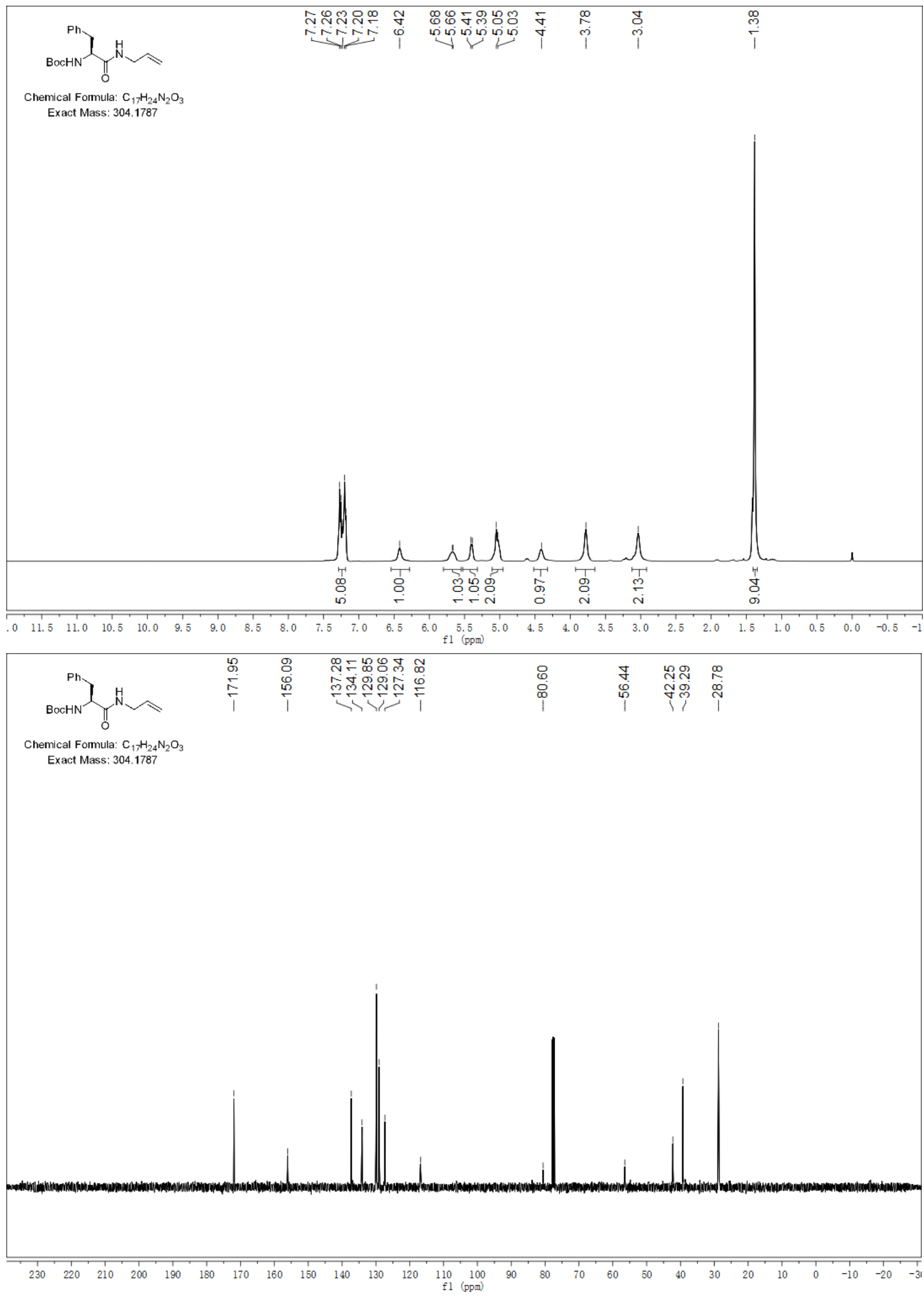


Benzyl ((S)-1-(((S)-1-(Allylamino)-4-methyl-1-oxopentan-2-yl)amino)-1-oxo-3-phenylpropan-2-

yl)carbamate (10).

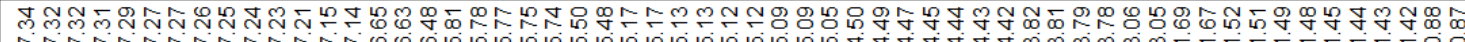
等<smiles>C=CCNC(=O)C(CC(C)C)NC(=O)[C@H](Br)NC(=O)OCc1ccccc1</smiles>

Exact Mass: 451.2471

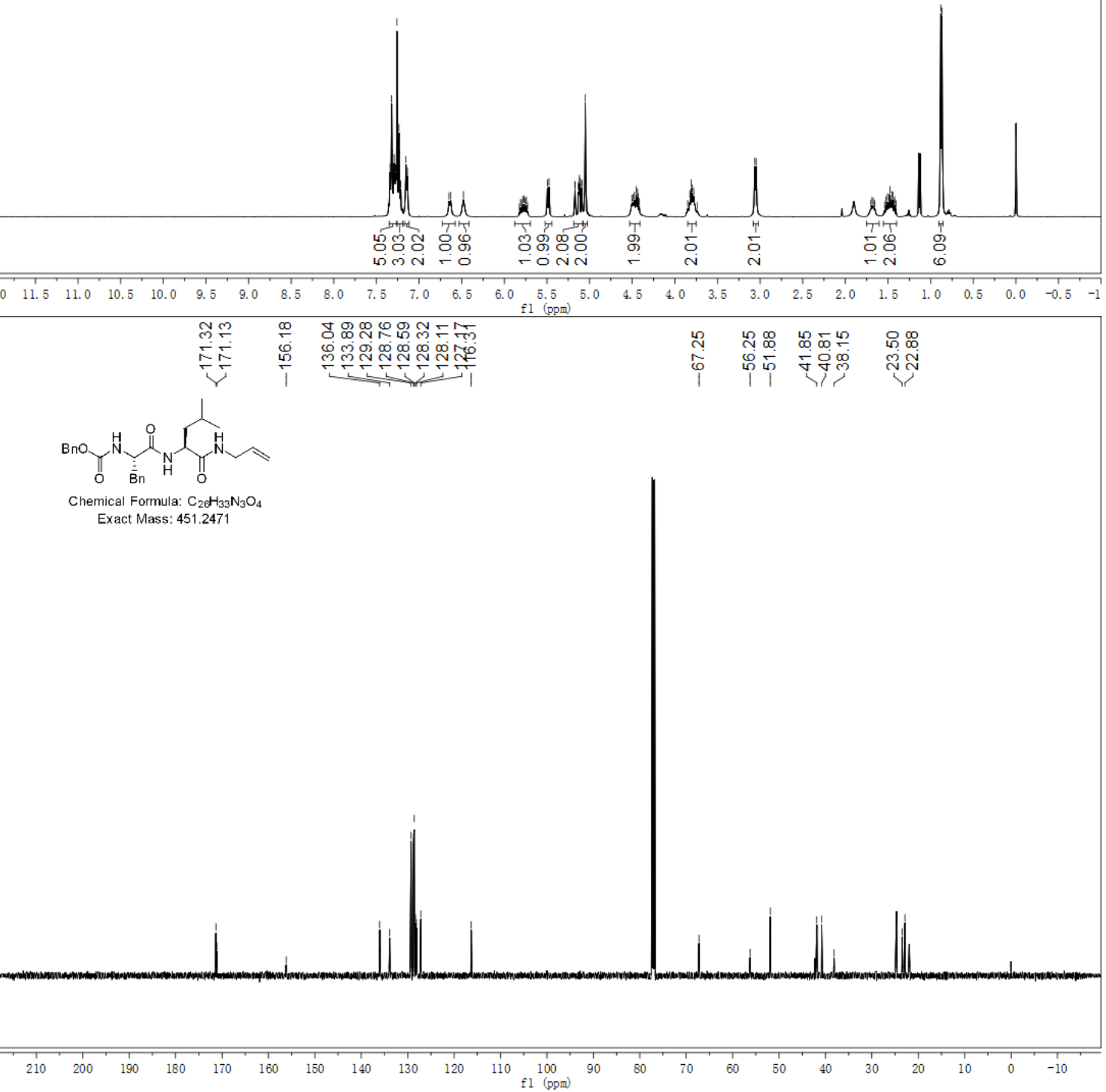


11. Copies of ${ }^{1} \mathrm{H}$ NMR, ${ }^{19}$ F NMR and ${ }^{13} \mathrm{C}$ NMR Spectra of Compounds 2.

Methyl (2-Bromo-2,2-difluoroacetyl)- $L$-alanyl- $L$-phenylalaninate (2e).
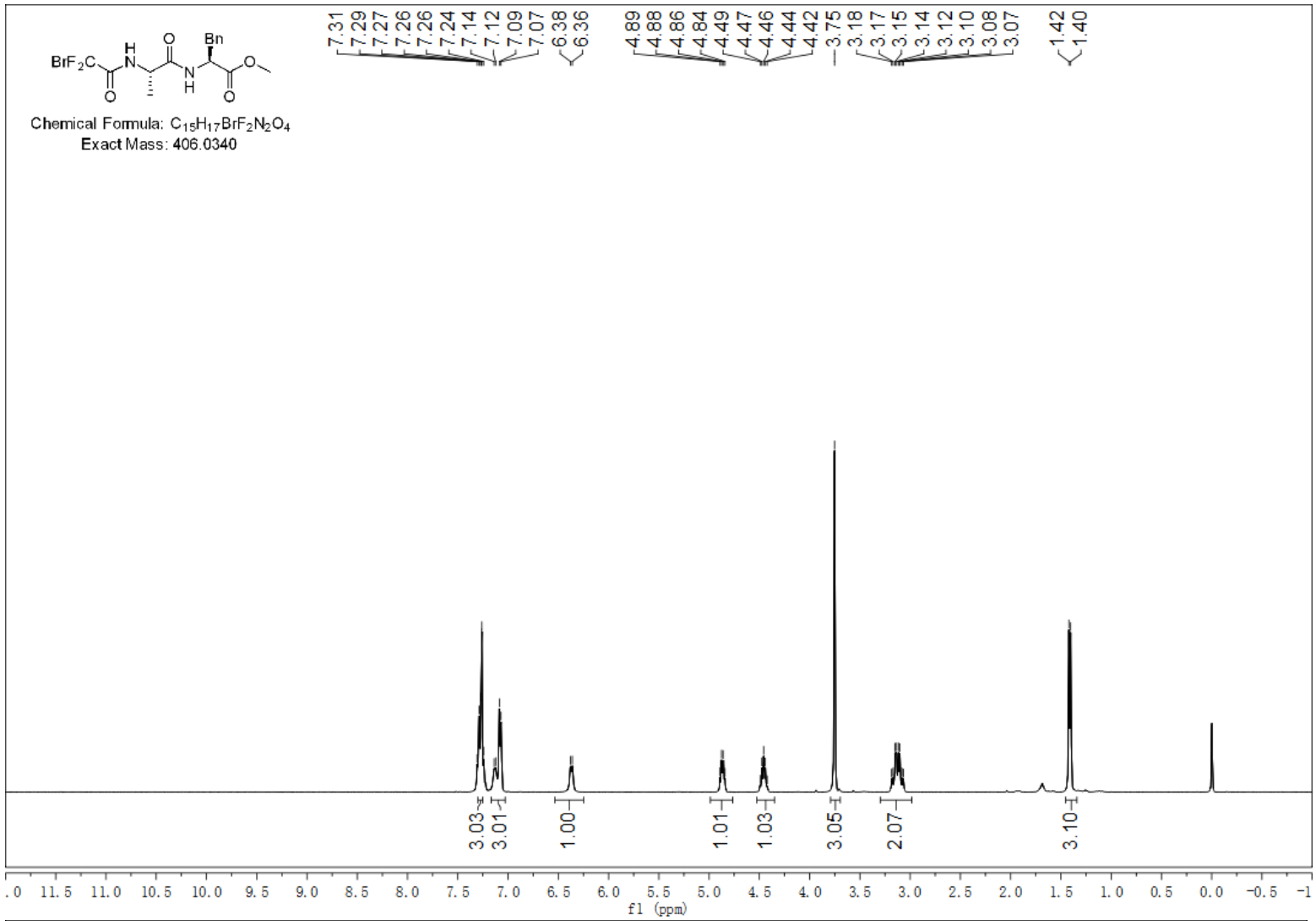

Chemical Formula: $\mathrm{C}_{15} \mathrm{H}_{17} \mathrm{BrF}_{2} \mathrm{~N}_{2} \mathrm{O}_{4}$

o

Exact Mass: 406.0340

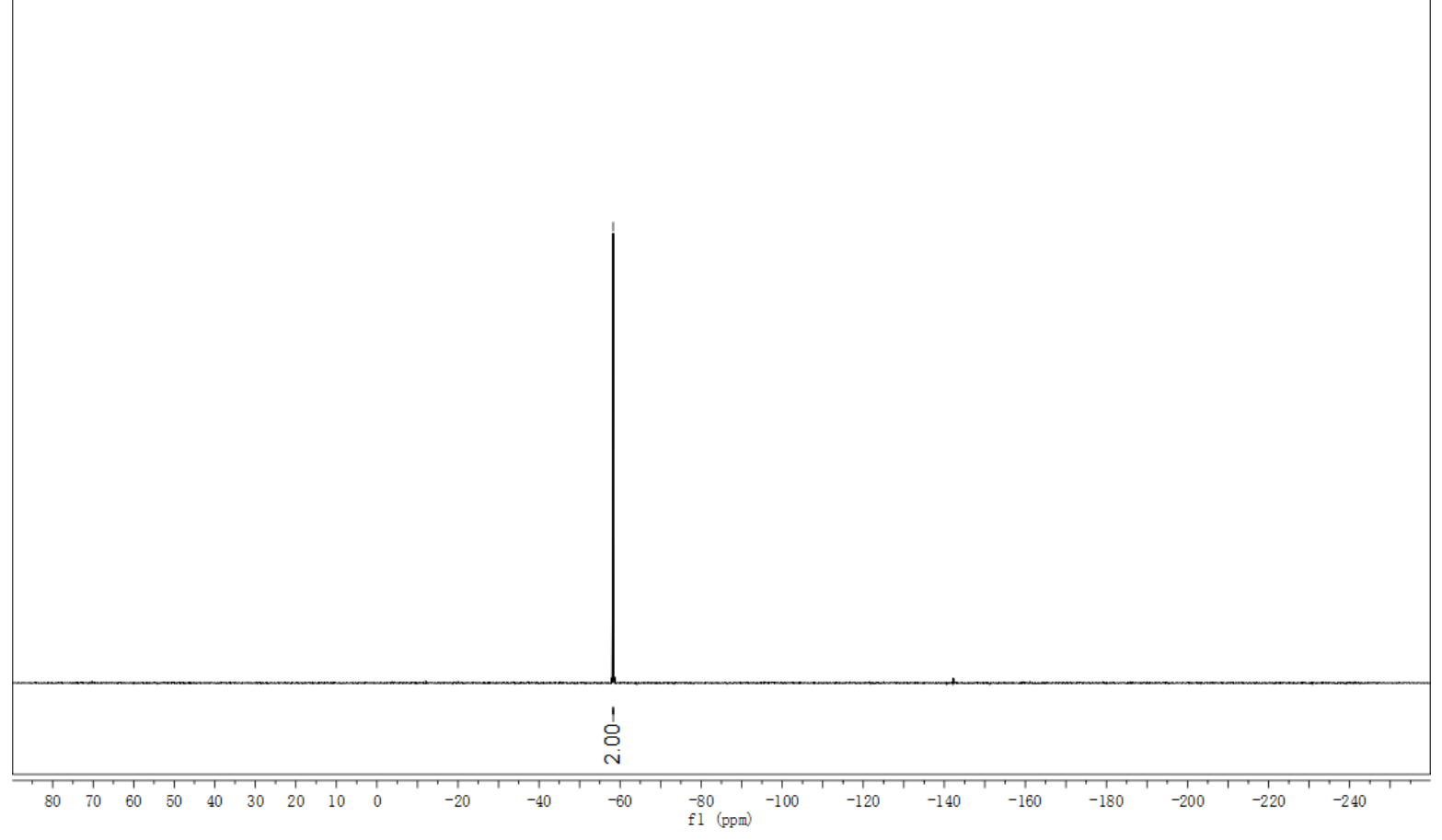




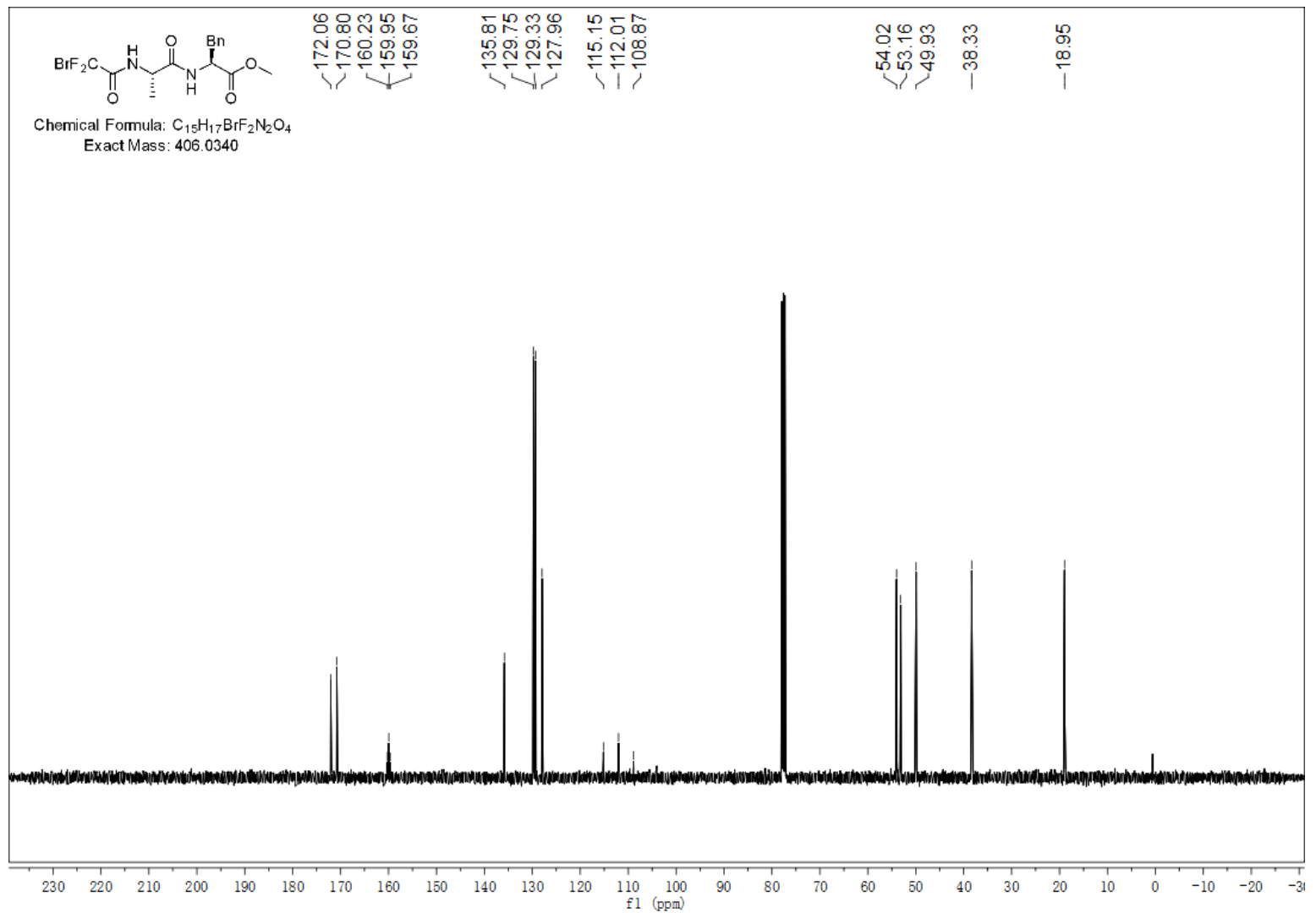

Methyl (S)-3-(2-Bromo-2,2-difluoroacetamido)-4-(((S)-1-methoxy-1-oxo-3-phenylpropan-2yl)amino)-4-oxobutanoate (2f).

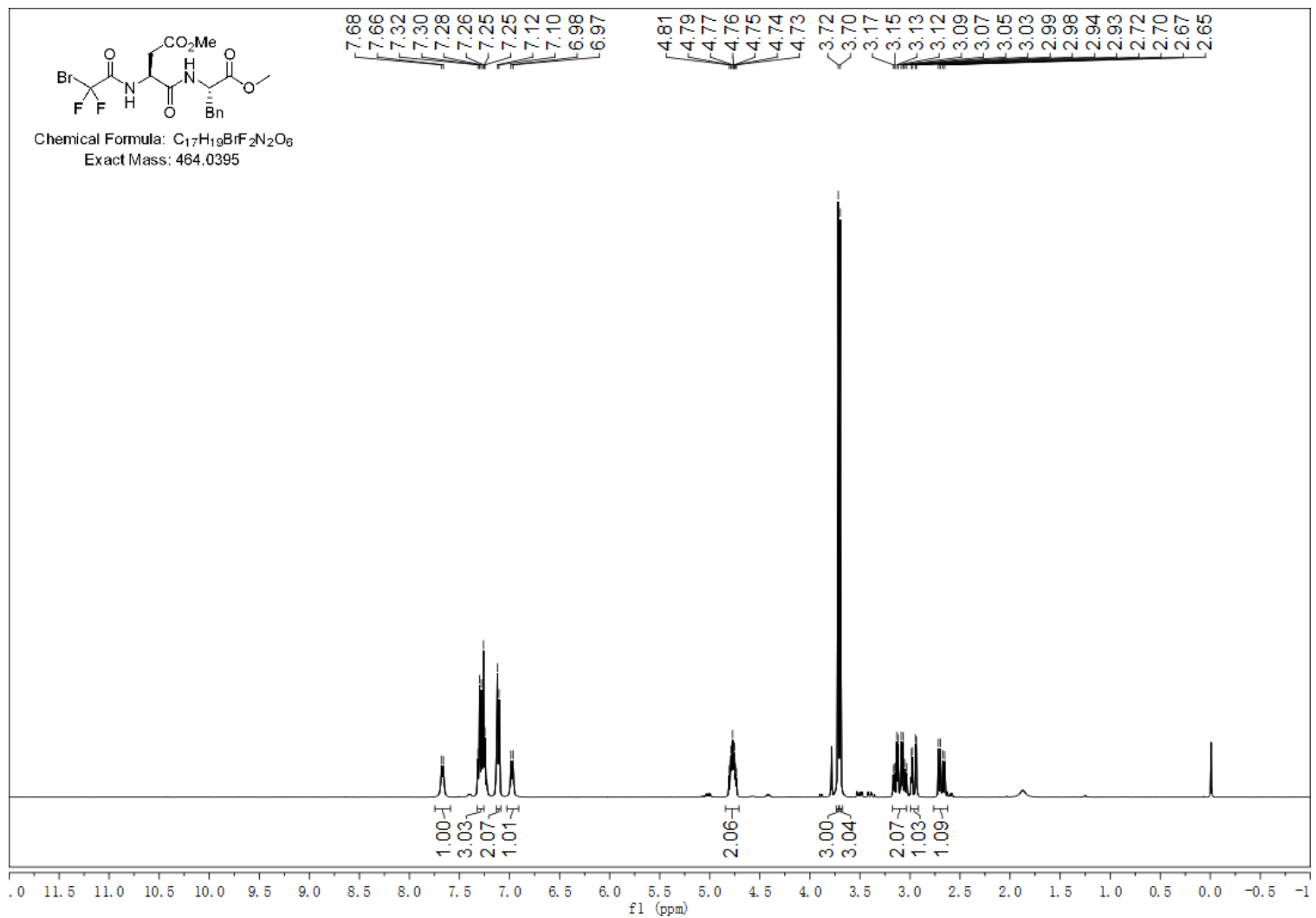




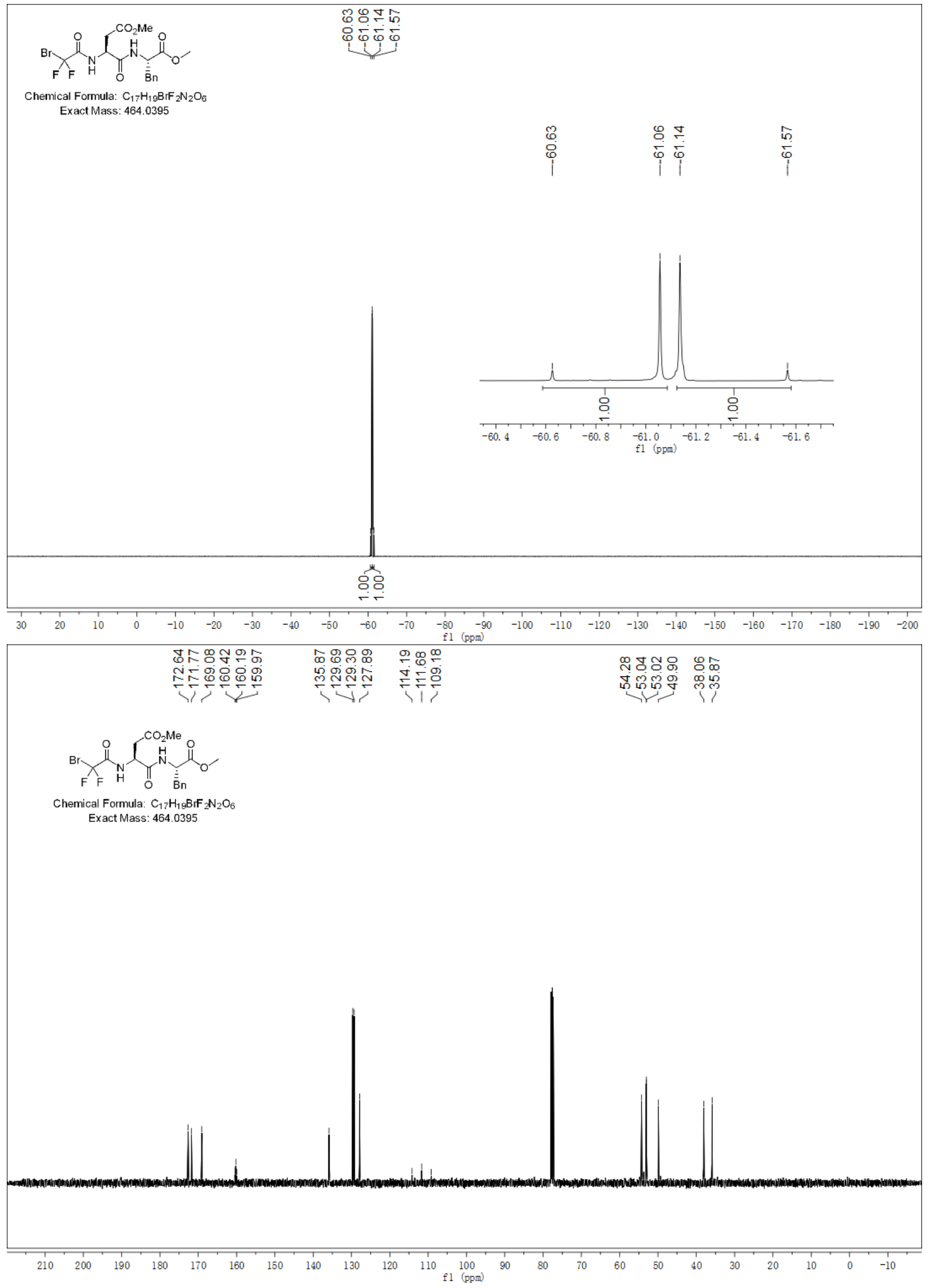


12. Copies of ${ }^{1} \mathrm{H}$ NMR, ${ }^{19}$ F NMR and ${ }^{13}$ C NMR Spectra of Compounds 4-17 and B1.

Ethyl 5-([1,1'-biphenyl]-4-yl)-4-acetamido-2,2-difluoro-5-oxopentanoate (4a).

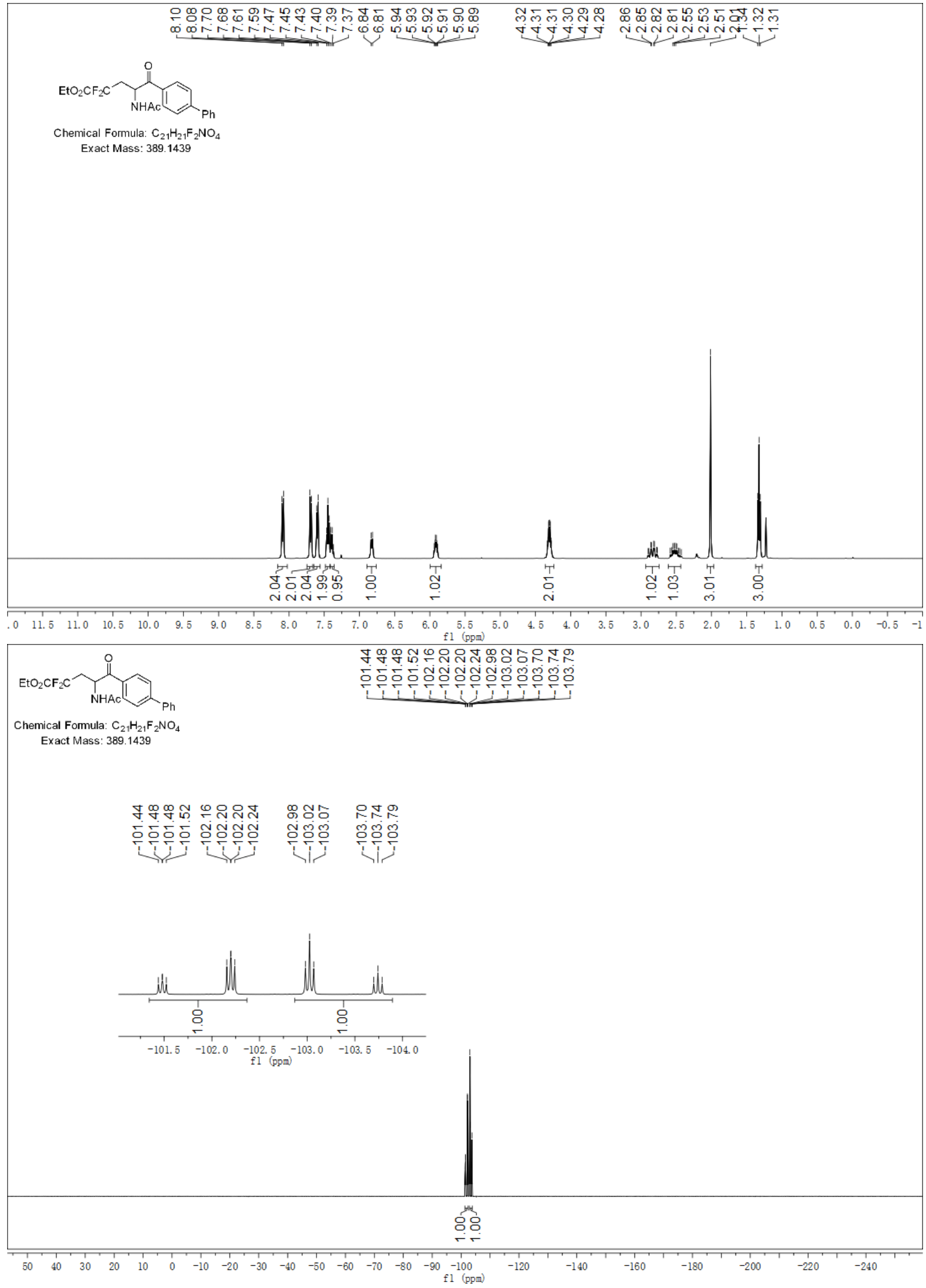




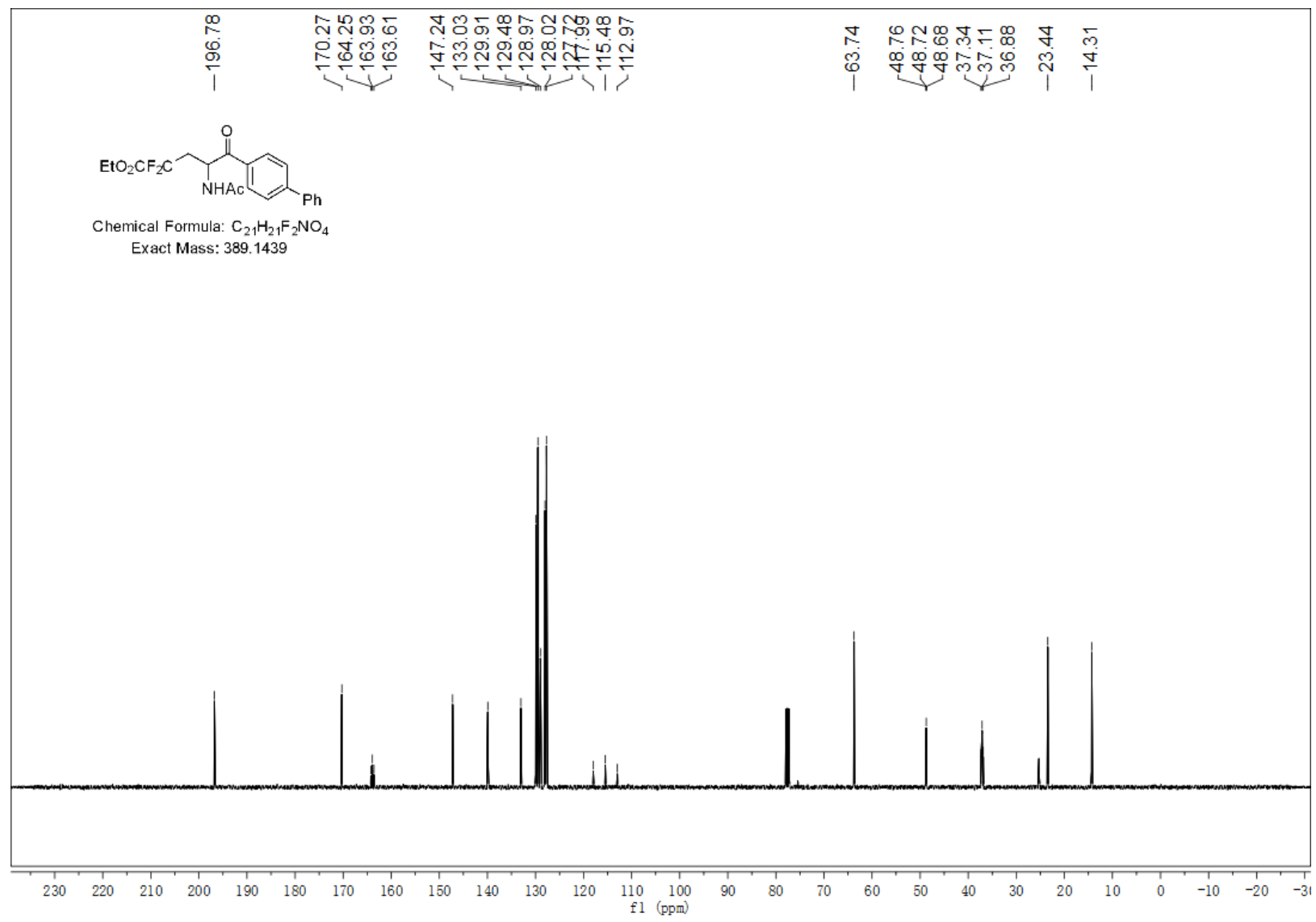

Ethyl 4-acetamido-2,2-difluoro-5-oxo-5-phenylpentanoate (4b).

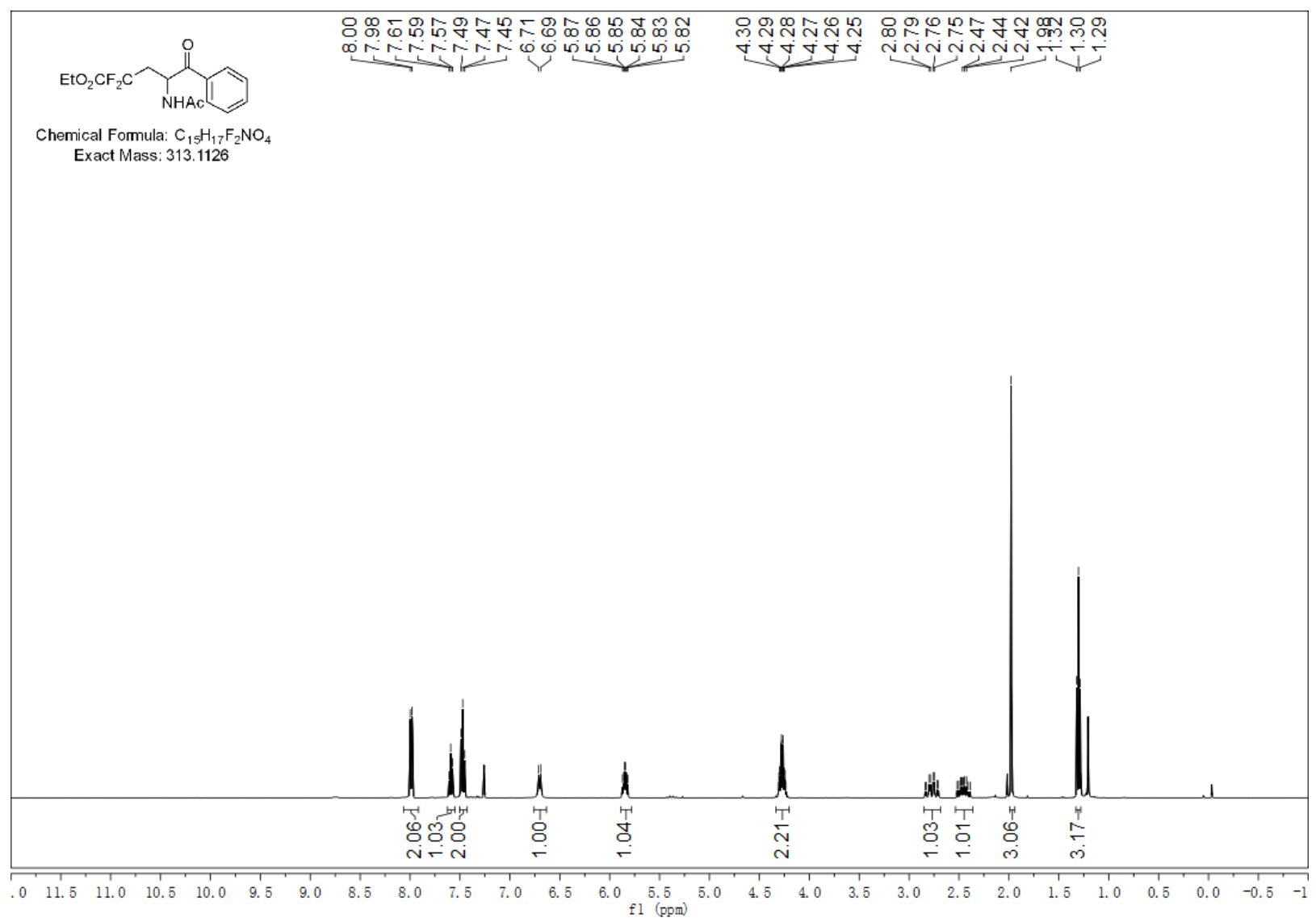




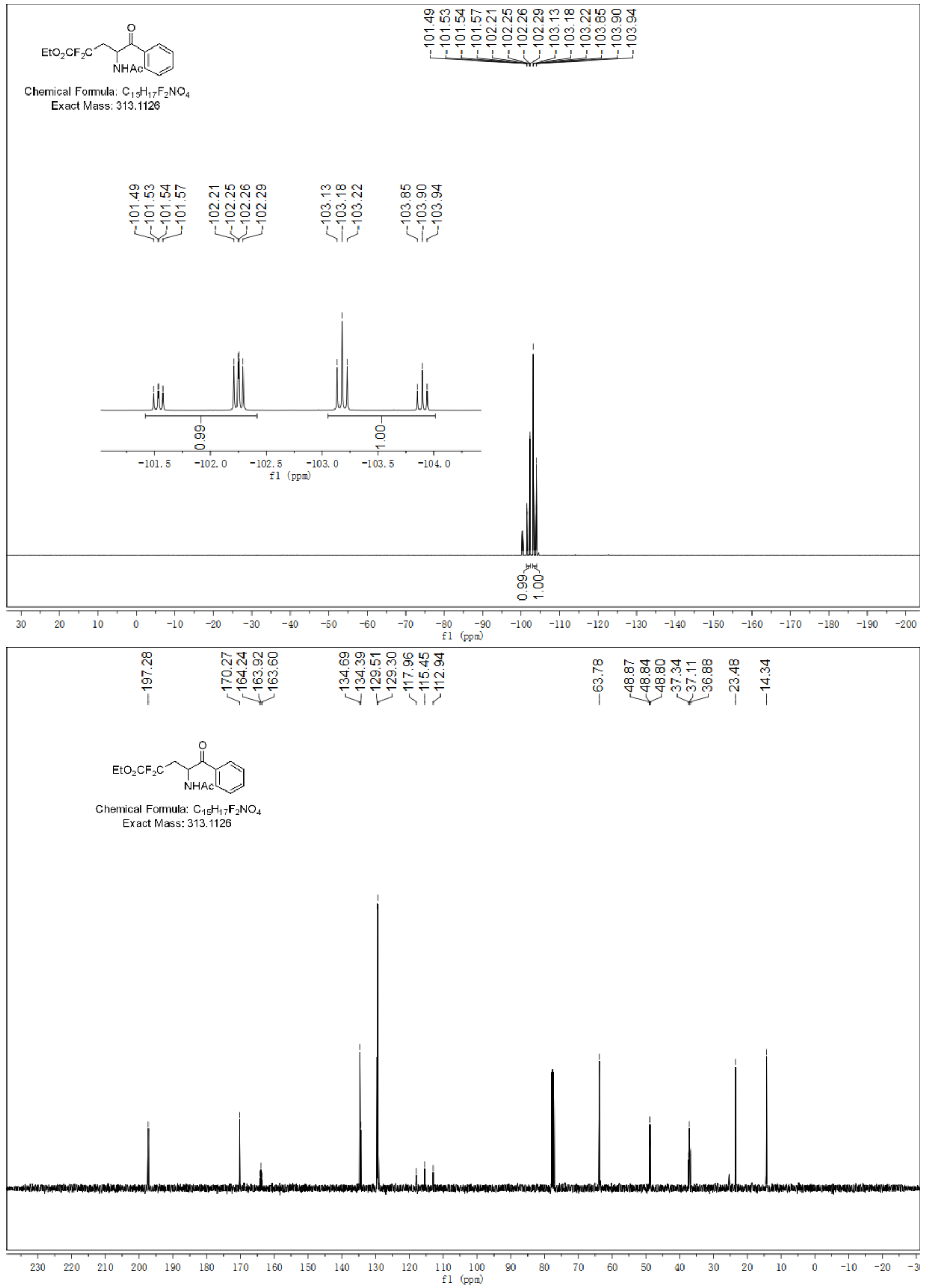


Ethyl 4-acetamido-5-(4-(tert-butyl)phenyl)-2,2-difluoro-5-oxopentanoate (4c).
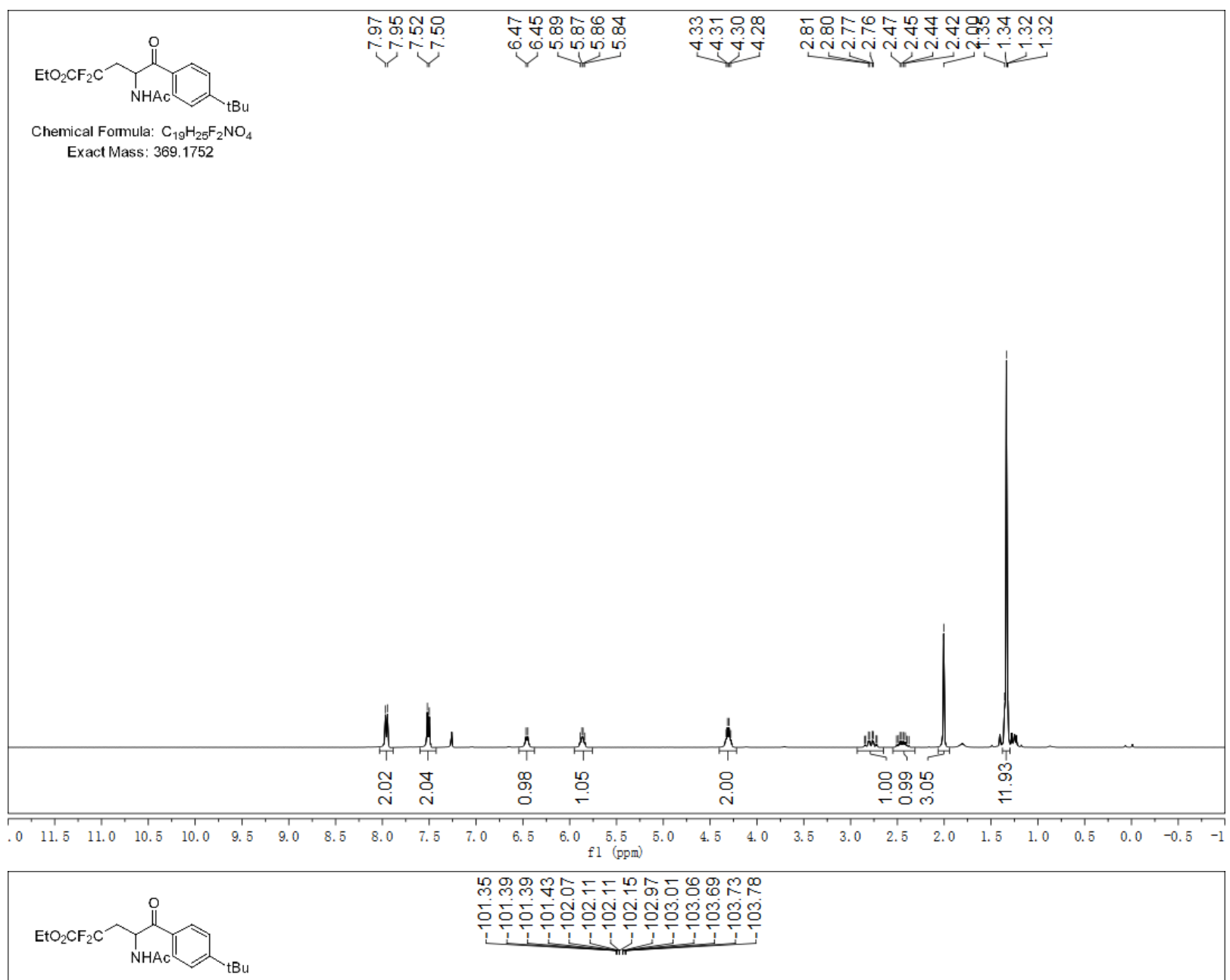

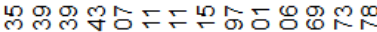

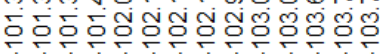

Chemical Formula: $\mathrm{C}_{19} \mathrm{H}_{25} \mathrm{~F}_{2} \mathrm{NO}_{4}$

Exact Mass: 369.1752

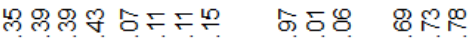

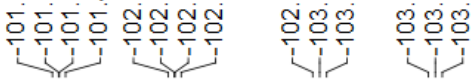

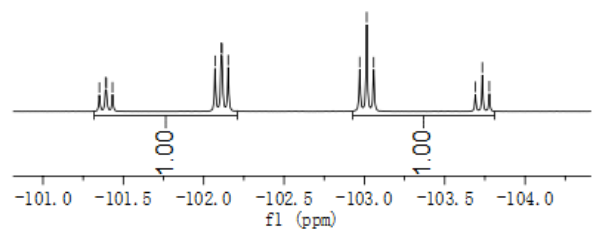

애요
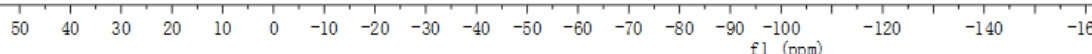


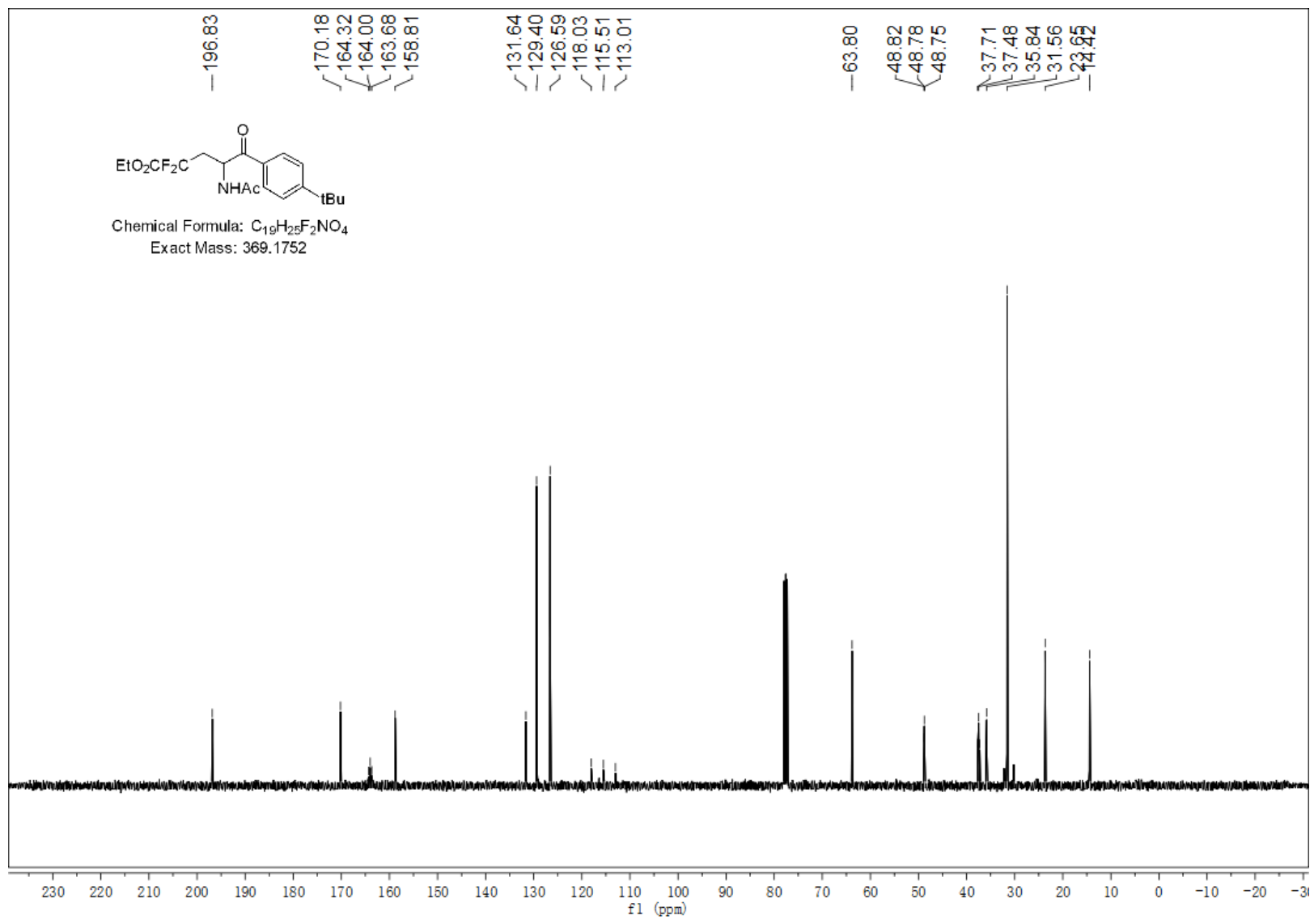

Ethyl 4-acetamido-2,2-difluoro-5-(4-fluorophenyl)-5-oxopentanoate (4d).

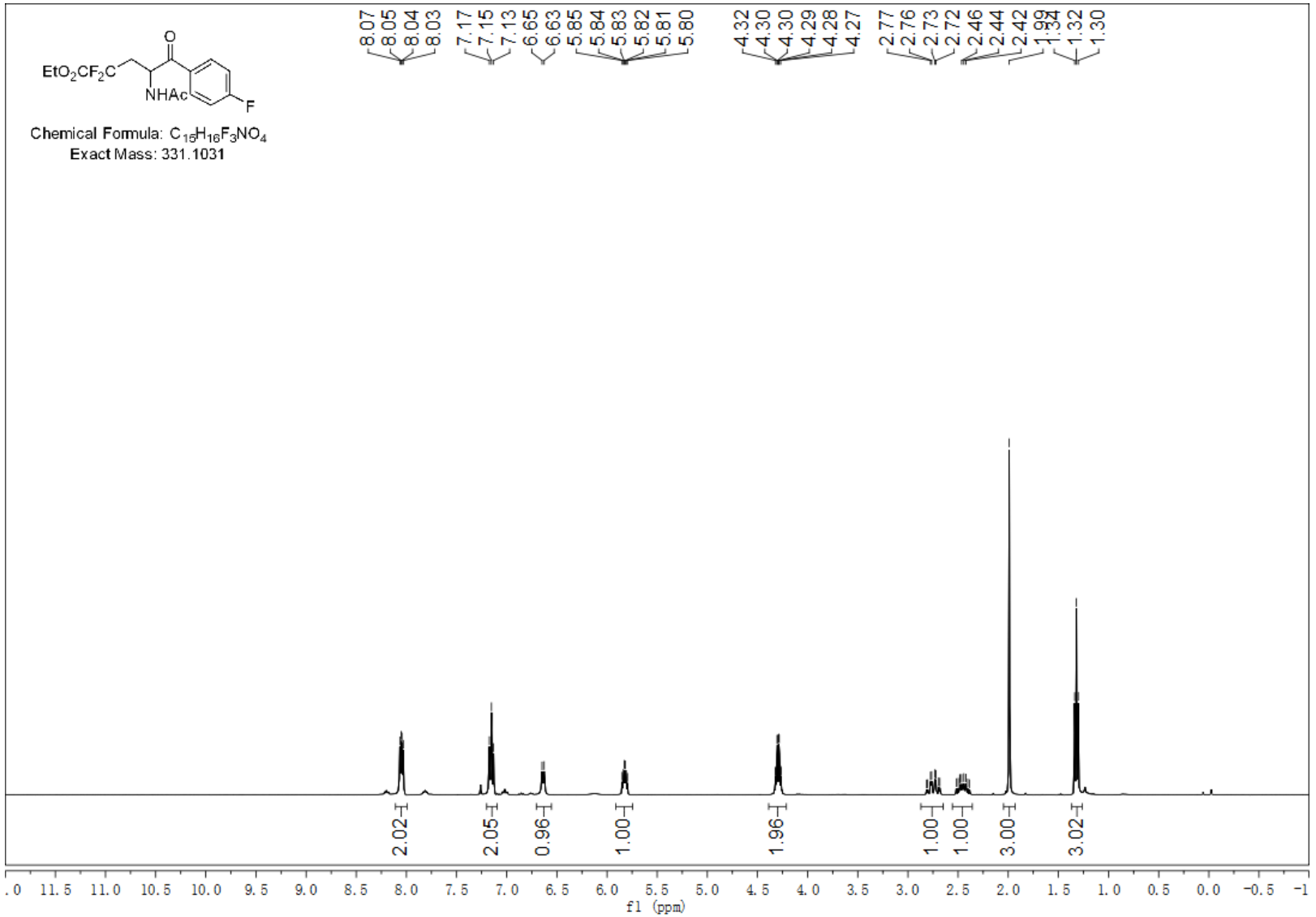




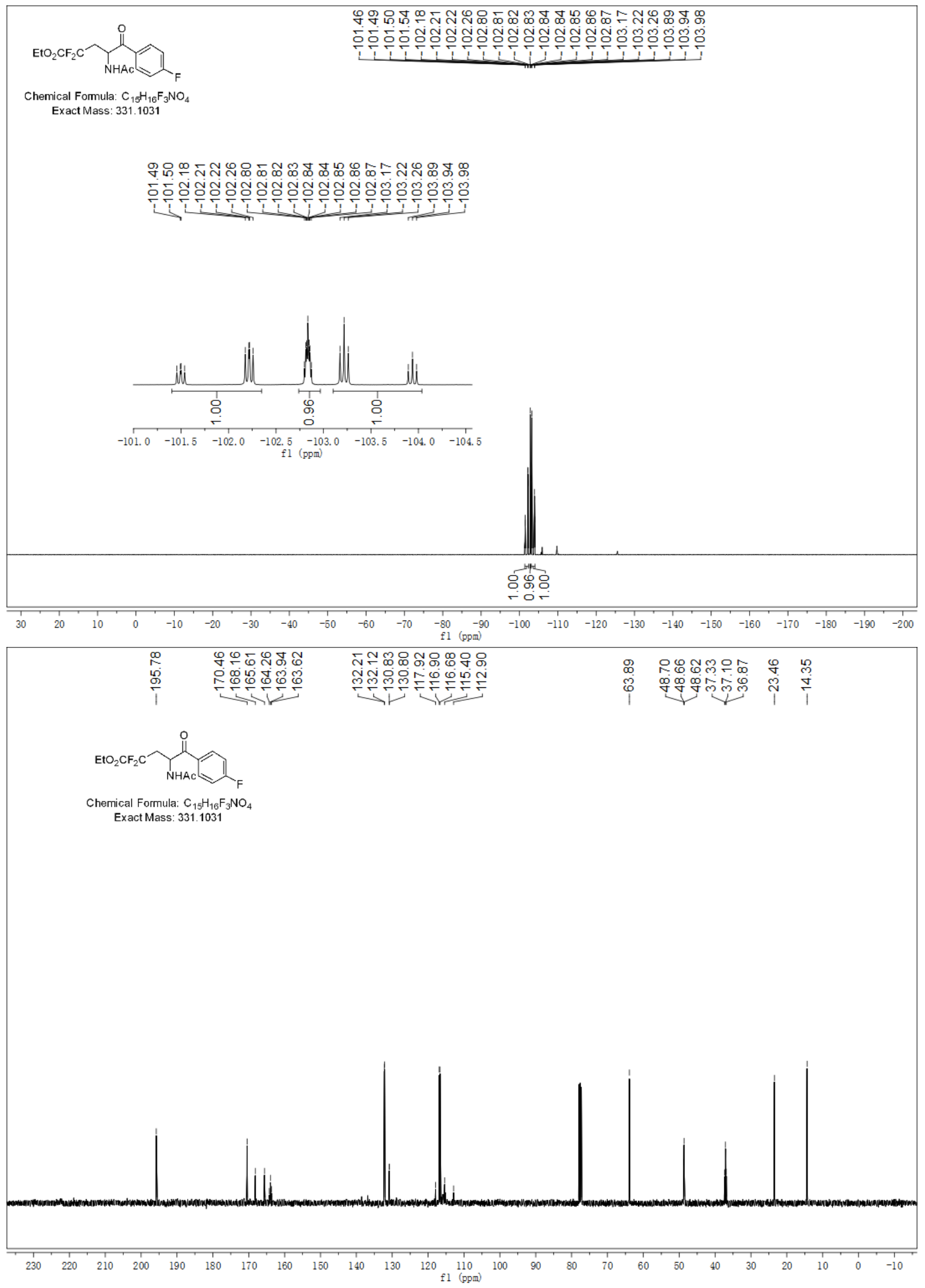


Ethyl 4-acetamido-5-(4-chlorophenyl)-2,2-difluoro-5-oxopentanoate (4e).
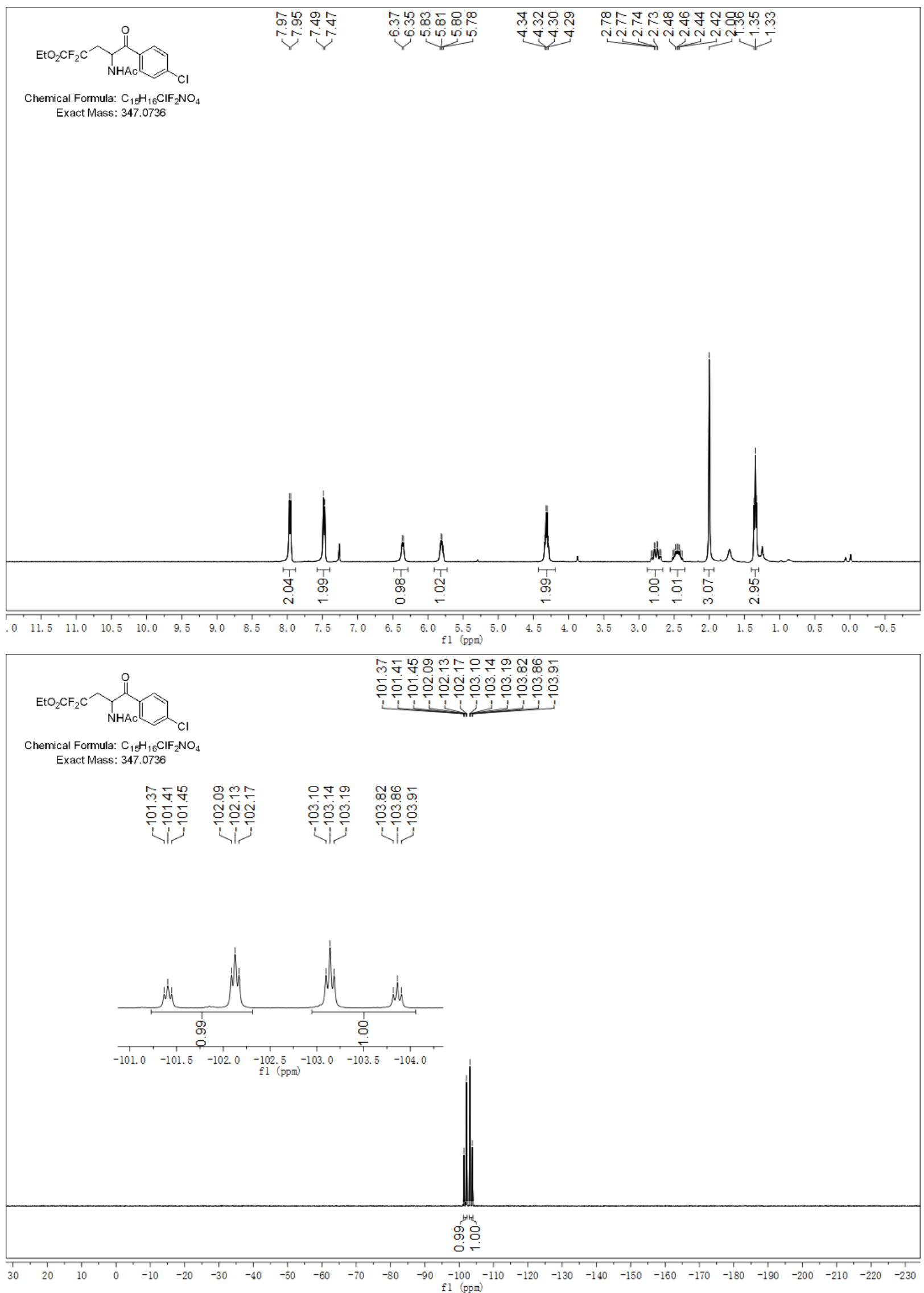


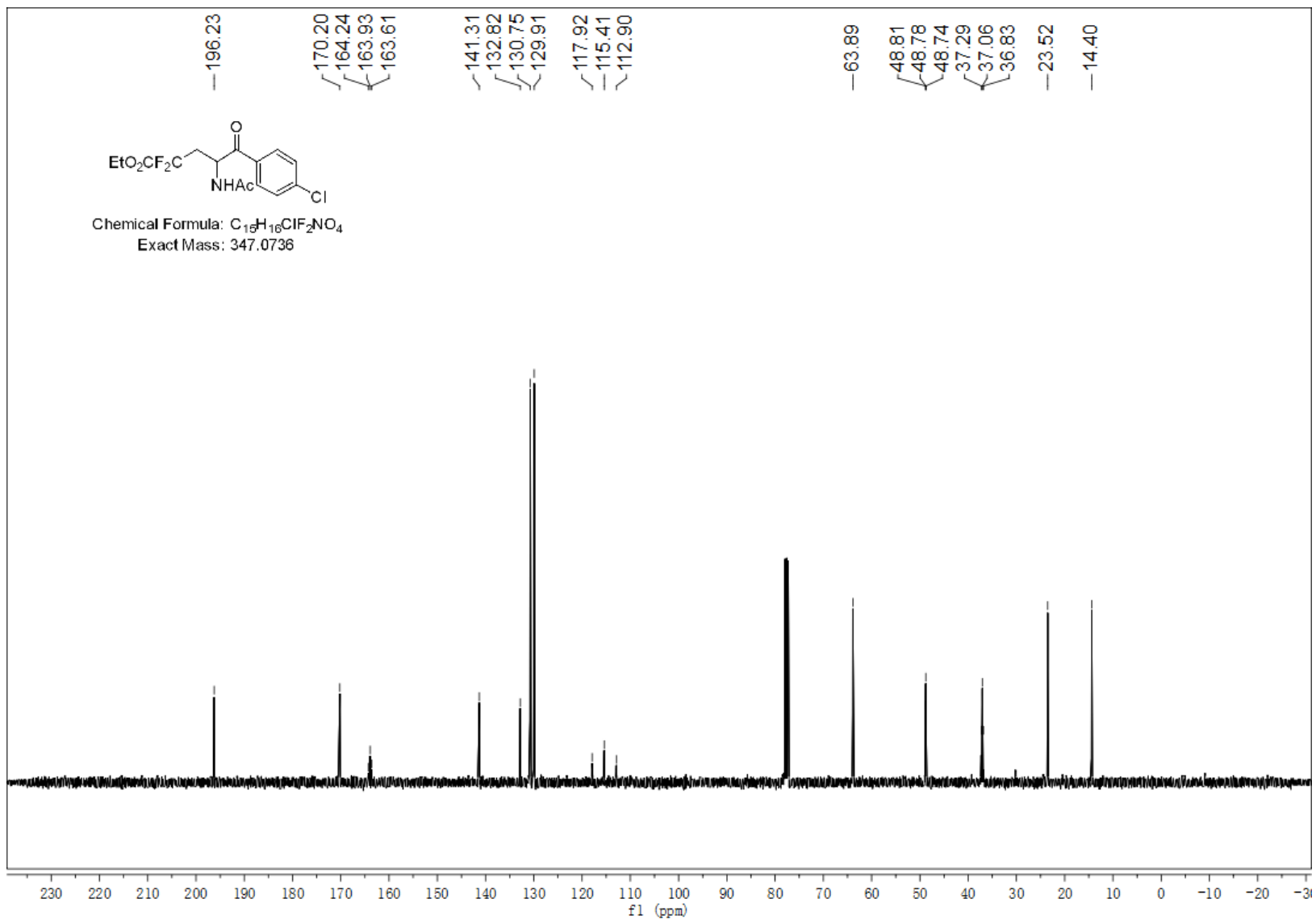

Ethyl 4-acetamido-5-(4-bromophenyl)-2,2-difluoro-5-oxopentanoate (4f).

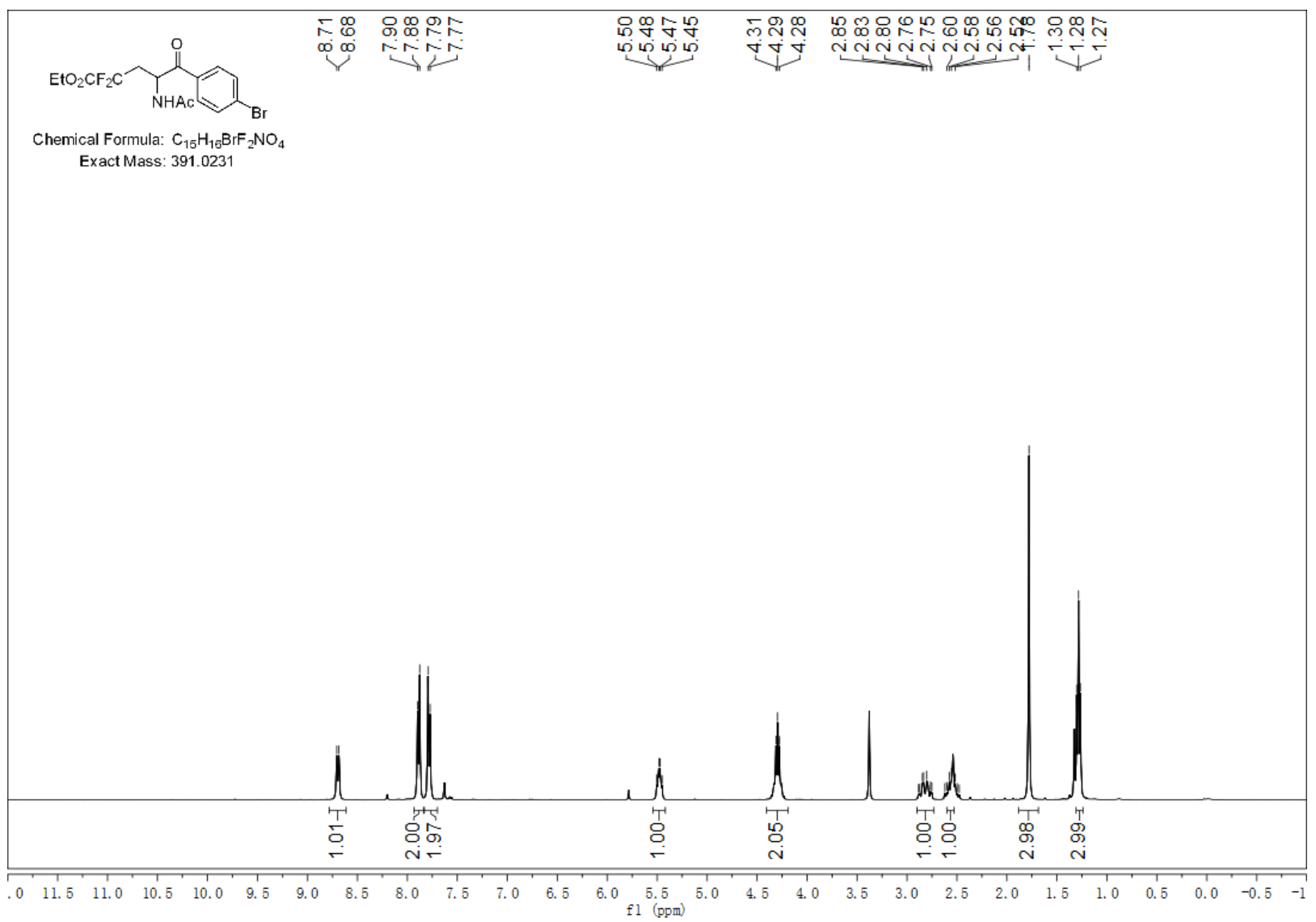




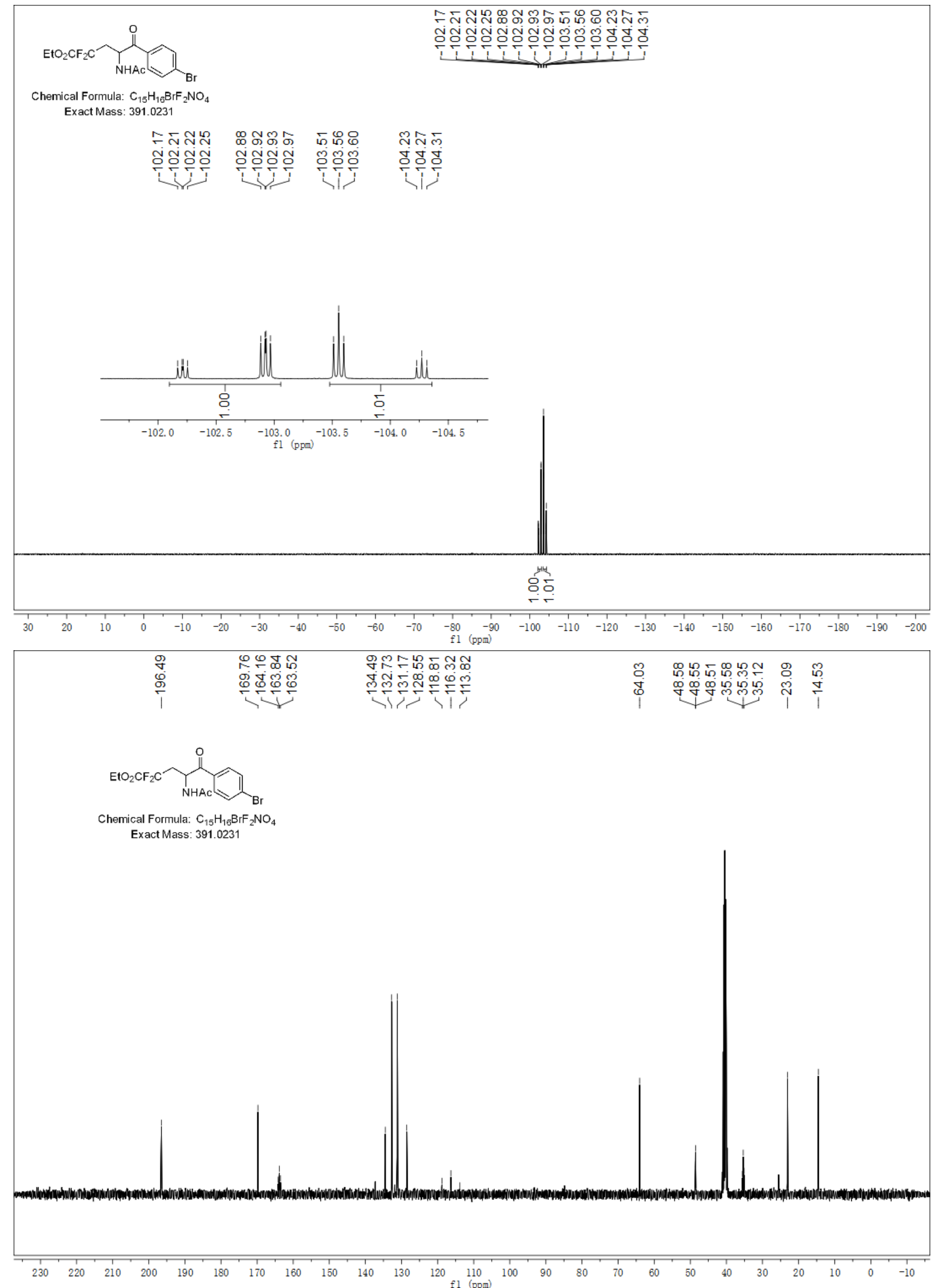


Ethyl 4-acetamido-2,2-difluoro-5-(4-methoxyphenyl)-5-oxopentanoate (4g).
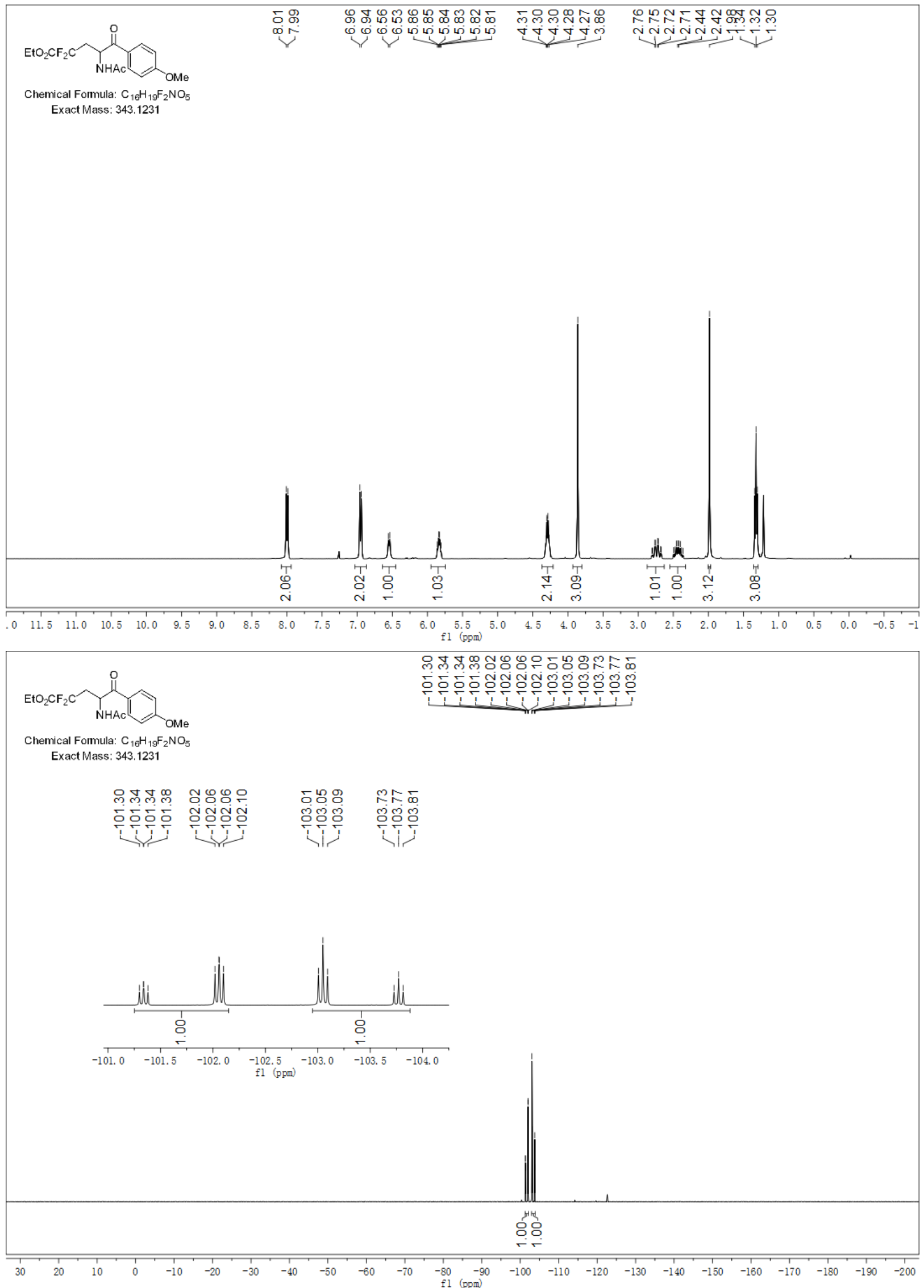


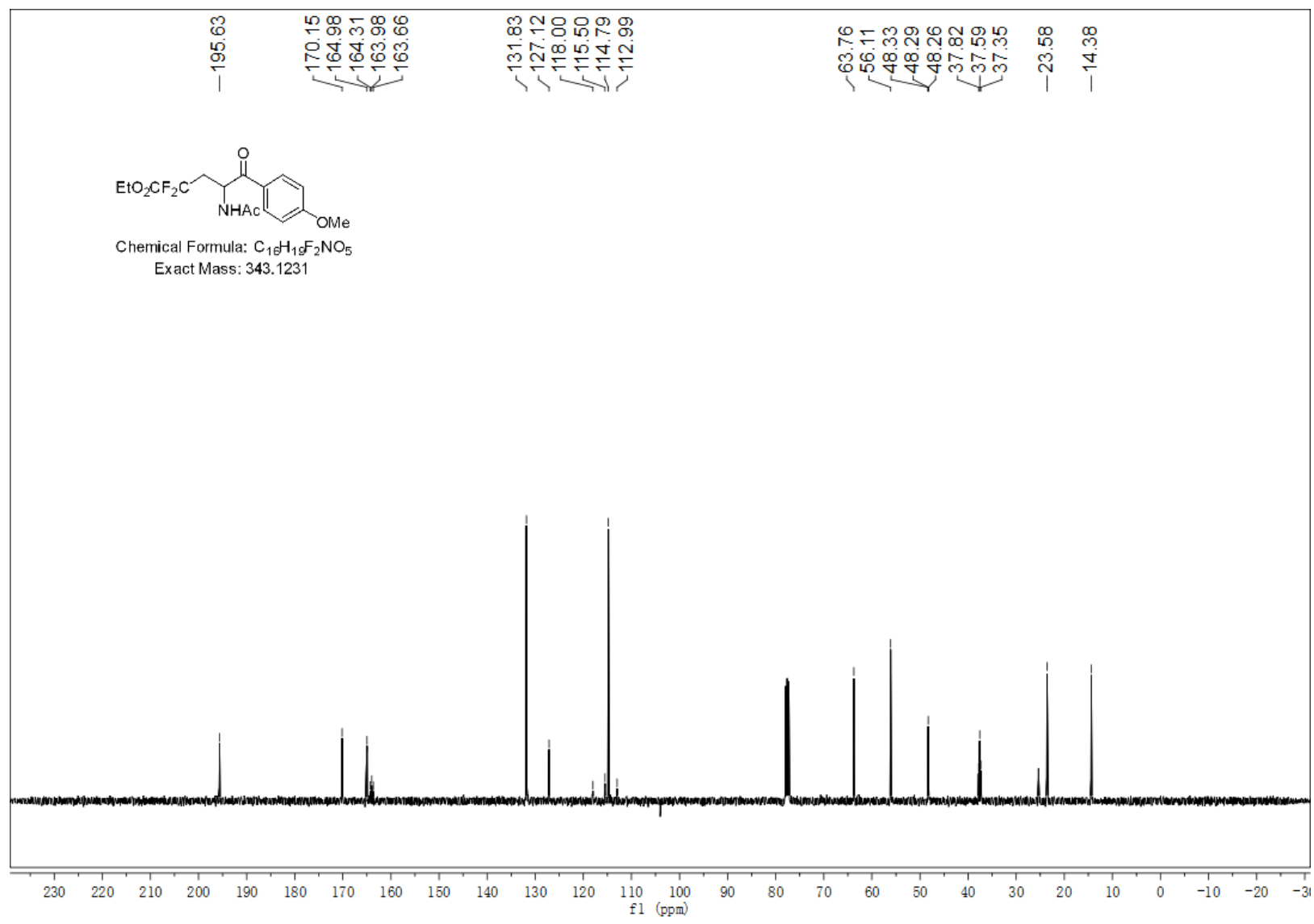

Ethyl 4-acetamido-5-(3,5-dimethoxyphenyl)-2,2-difluoro-5-oxopentanoate (4h).

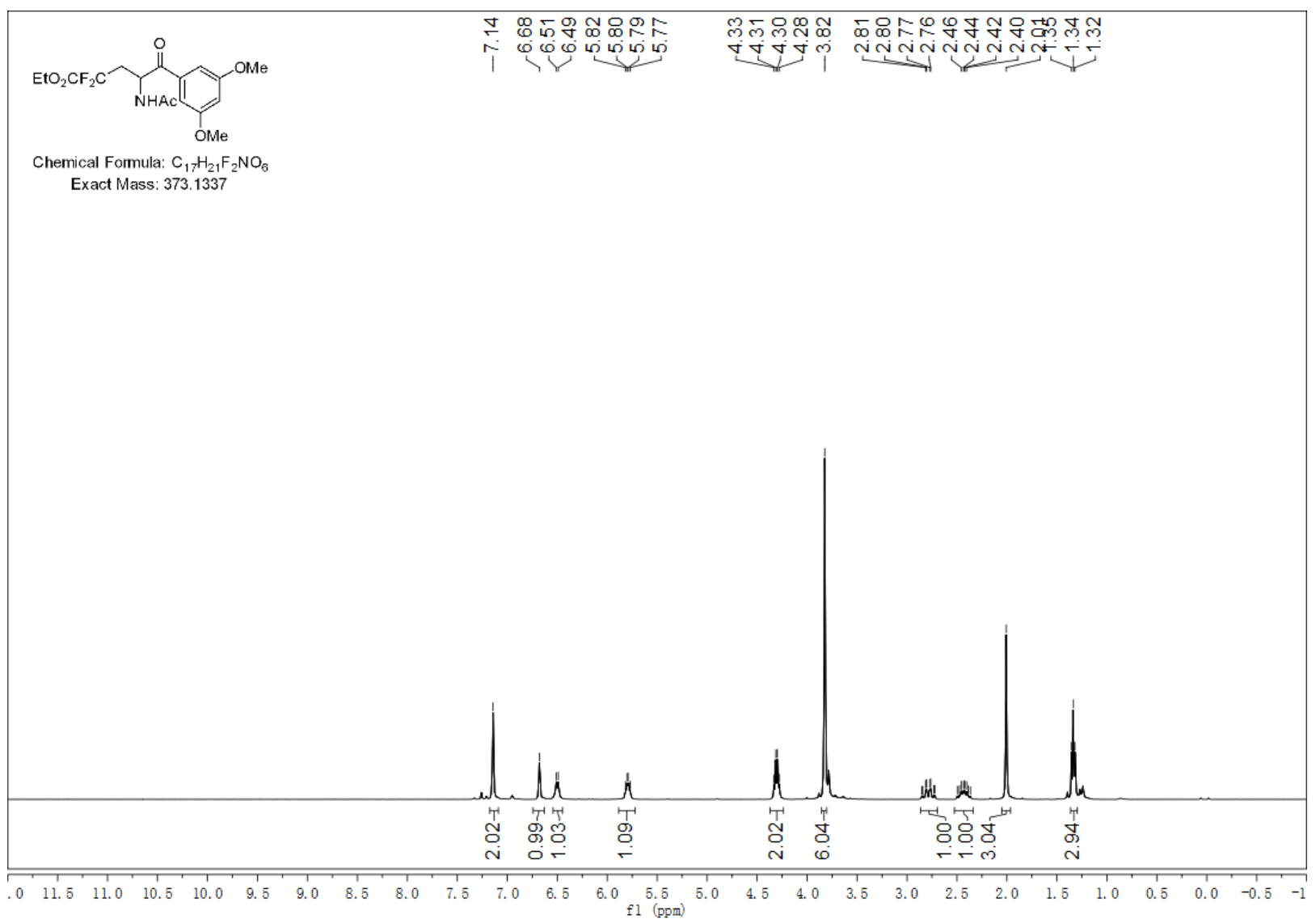




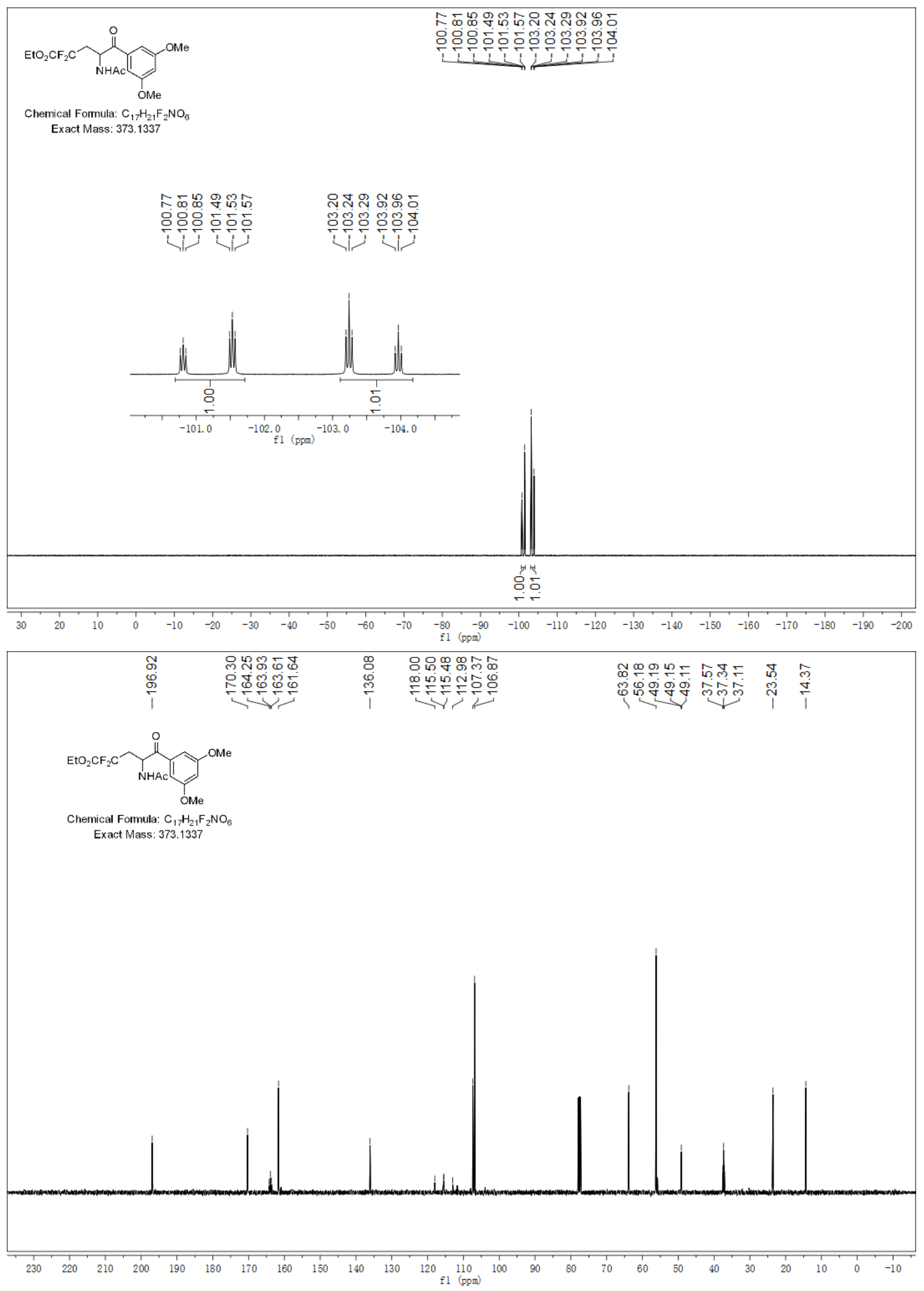


Ethyl 4-acetamido-5-(benzo $[d][1,3]$ dioxol-5-yl)-2,2-difluoro-5-oxopentanoate (4i).
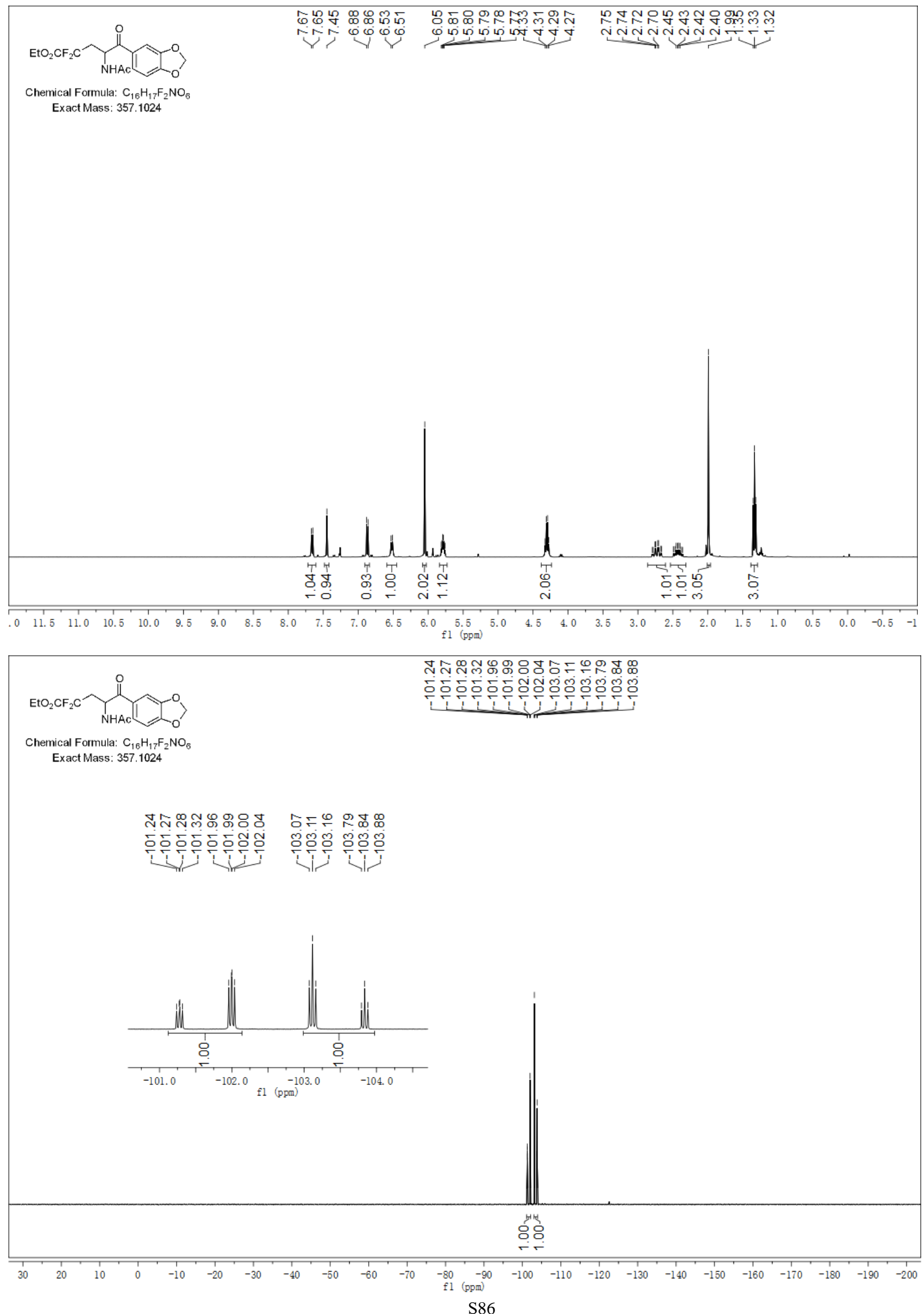


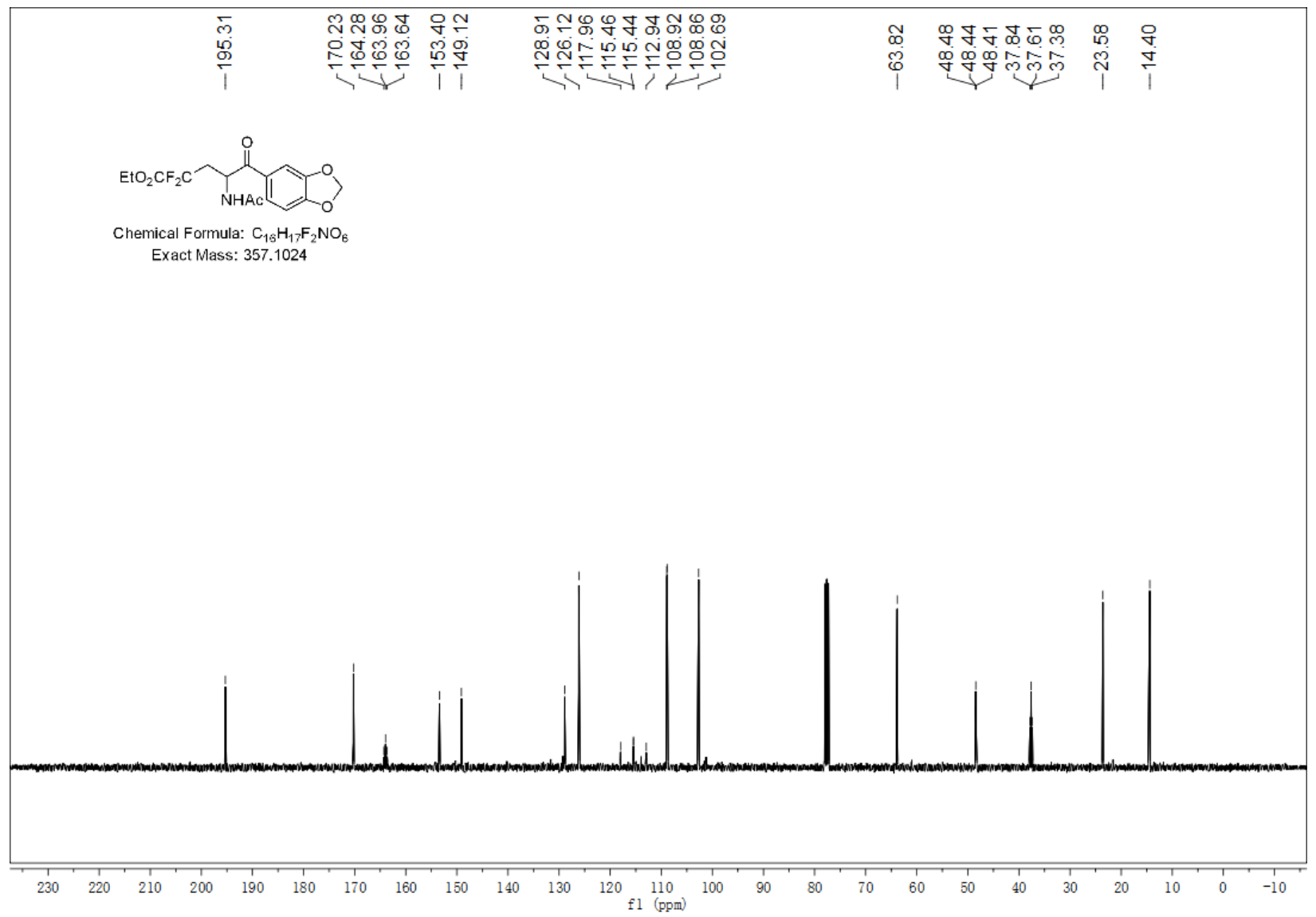

Ethyl 4-acetamido-5-(4-(ethylthio)phenyl)-2,2-difluoro-5-oxopentanoate (4j).

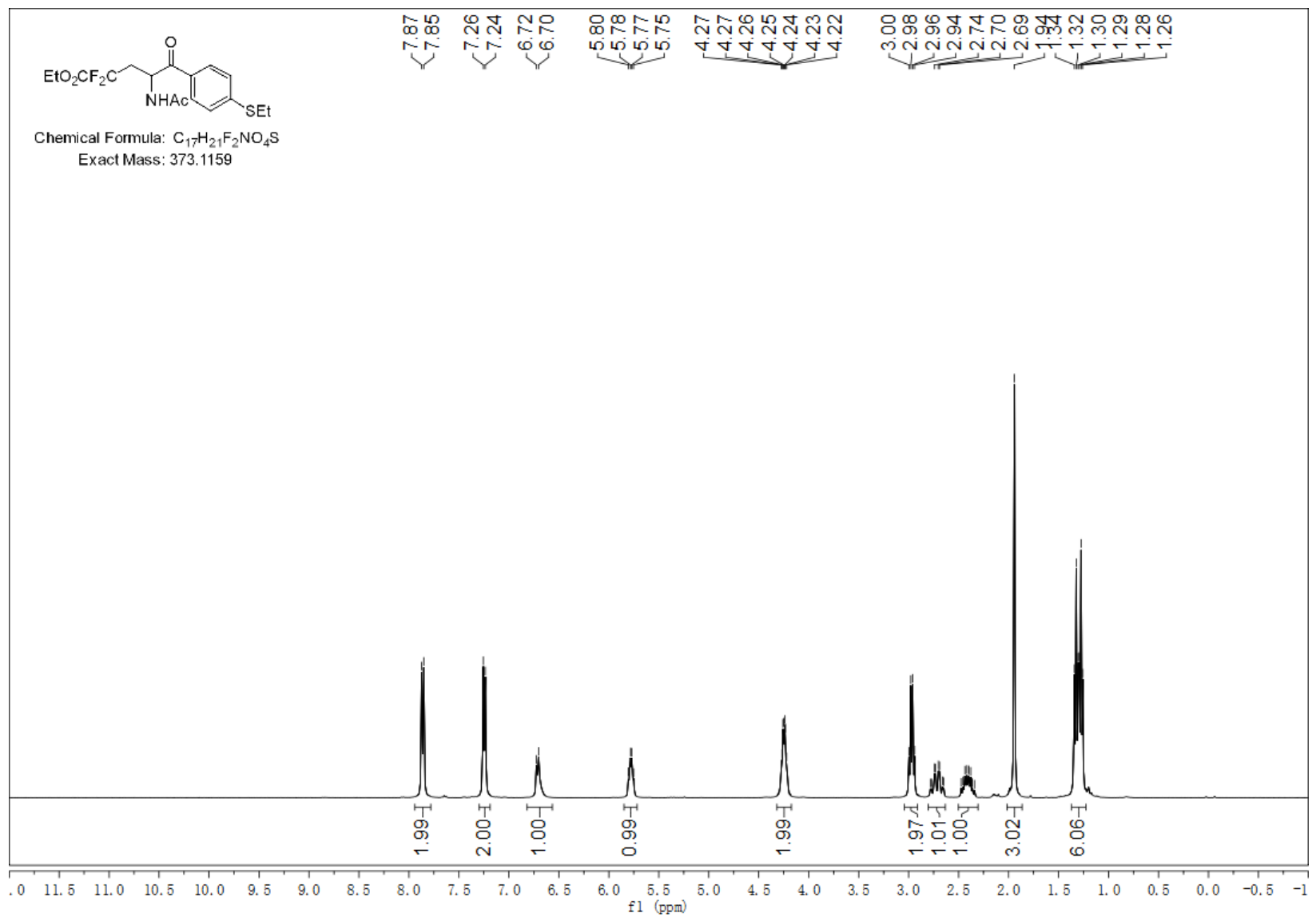



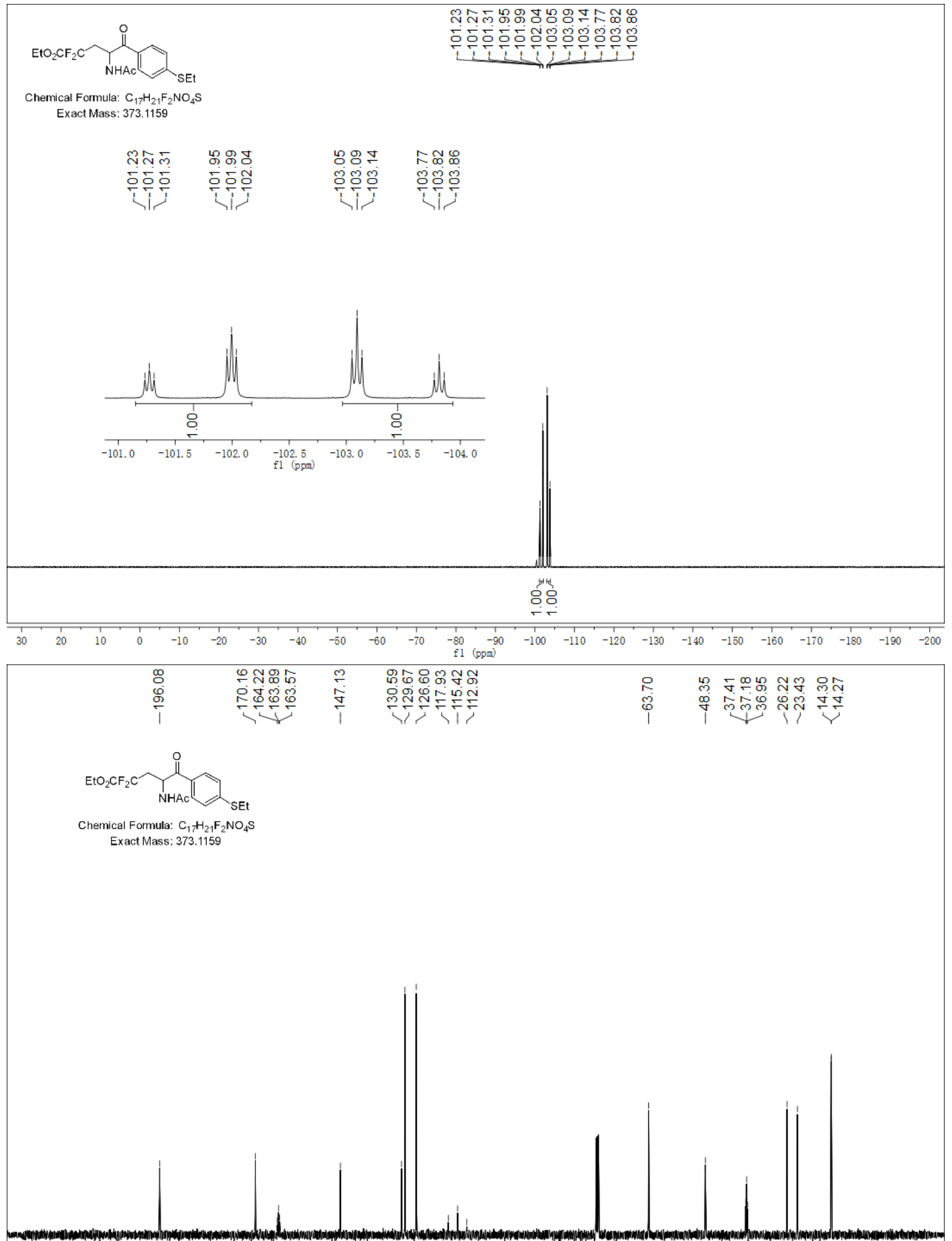

$\begin{array}{llllllllllllllllllllllllll}230 & 220 & 210 & 200 & 190 & 180 & 170 & 160 & 150 & 140 & 130 & 120 & 110 & 100 & 90 & 80 & 70 & 60 & 50 & 40 & 30 & 20 & 10 & 0 & -10\end{array}$ 
Ethyl 4-acetamido-2,2-difluoro-5-(4-morpholinophenyl)-5-oxopentanoate (4k).
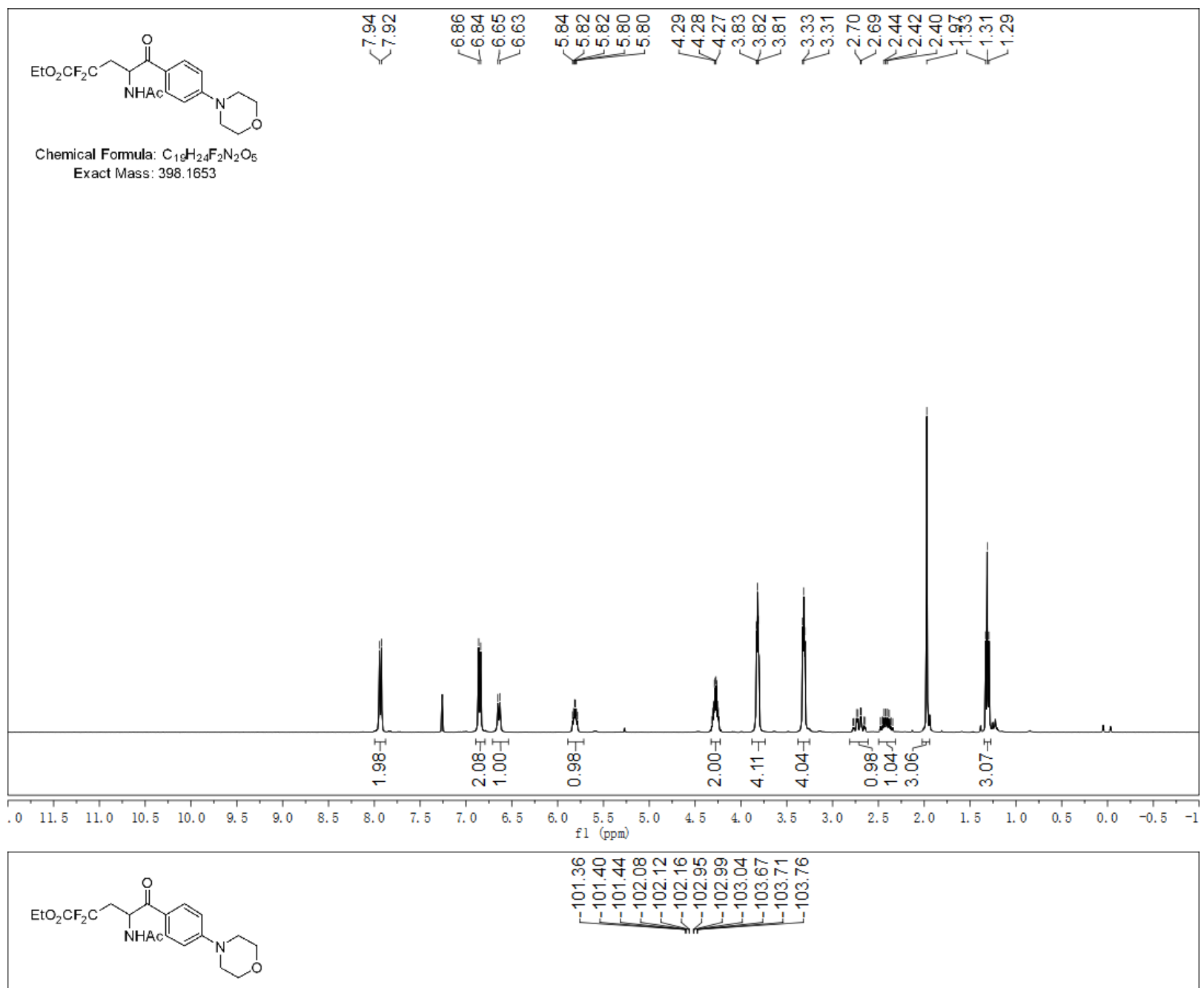

Chemical Formula: $\mathrm{C}_{1} \mathrm{H}_{24} \mathrm{~F}_{2} \mathrm{~N}_{2} \mathrm{O}_{5}$

Exact Mass: 398.1653

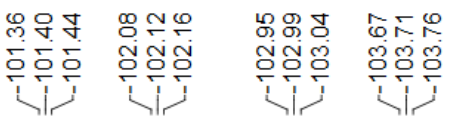

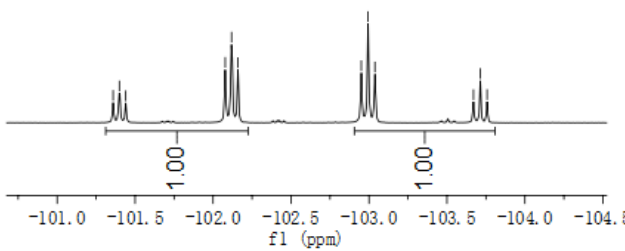

\section{붕응}

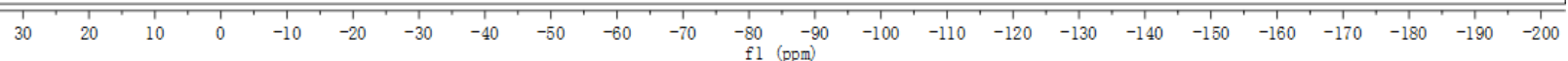




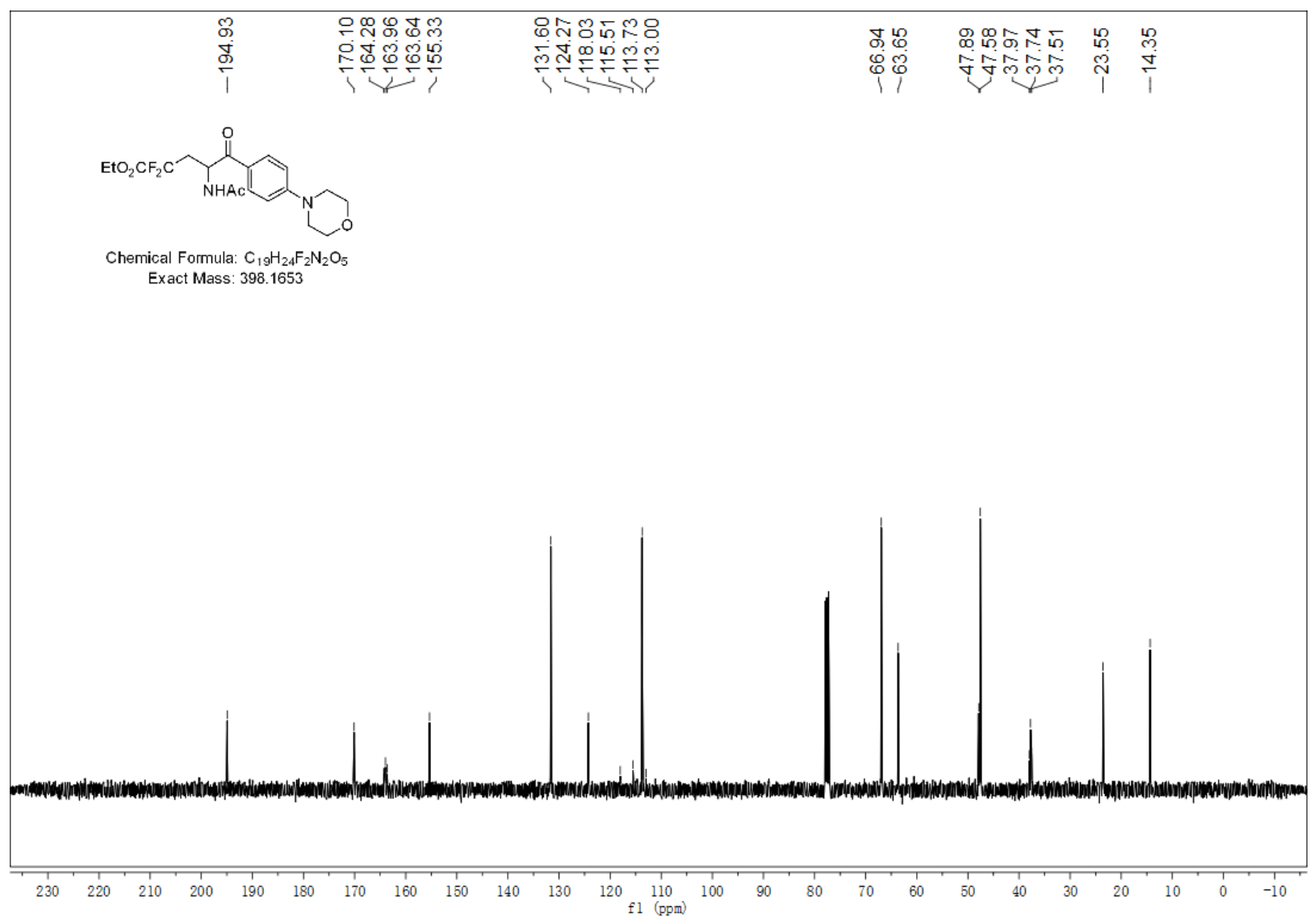

Ethyl 4-acetamido-2,2-difluoro-5-oxo-5-(4-(trimethylsilyl)phenyl)pentanoate (4l).

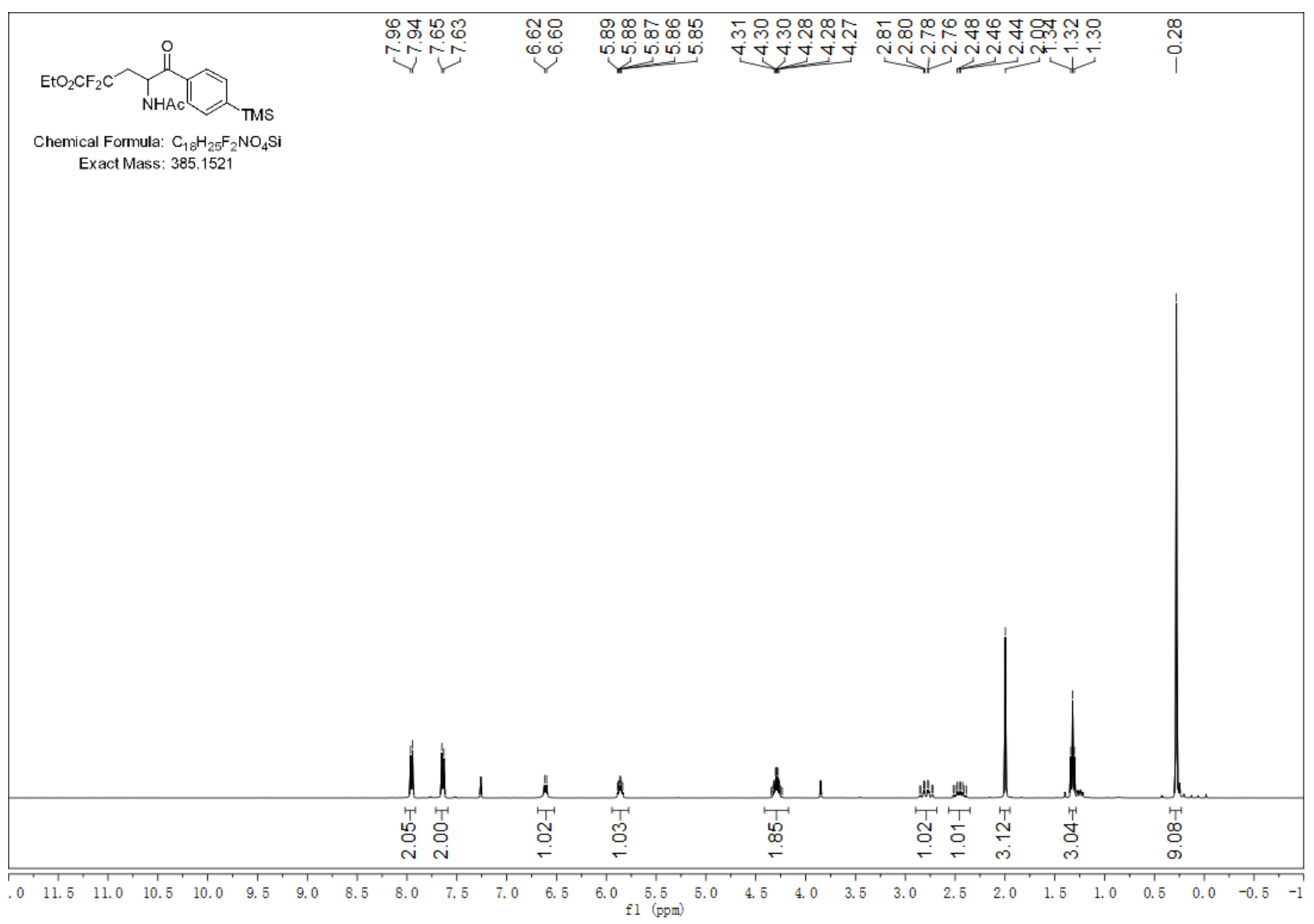



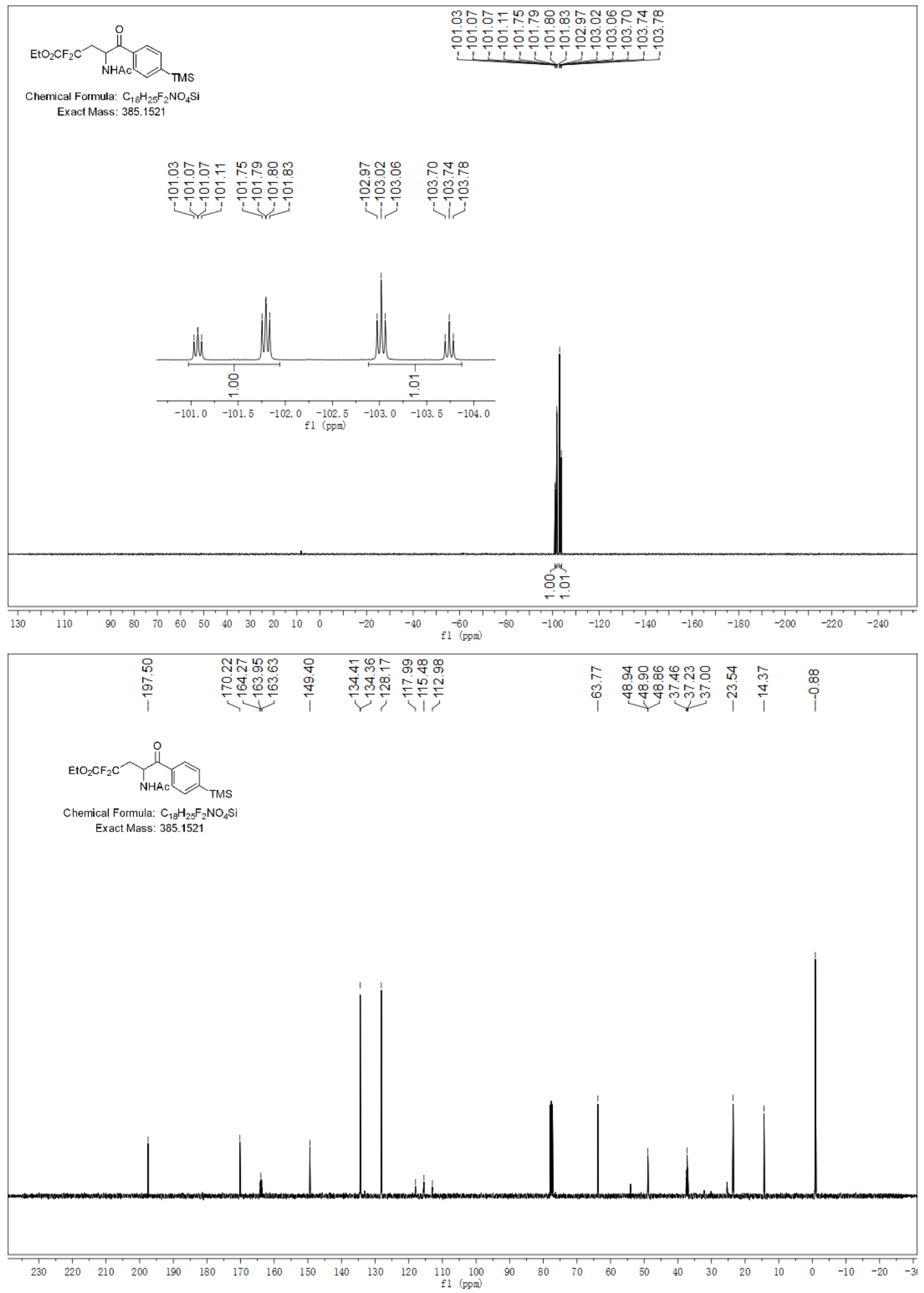
Ethyl 4-acetamido-5-(4-acetylphenyl)-2,2-difluoro-5-oxopentanoate (4m).

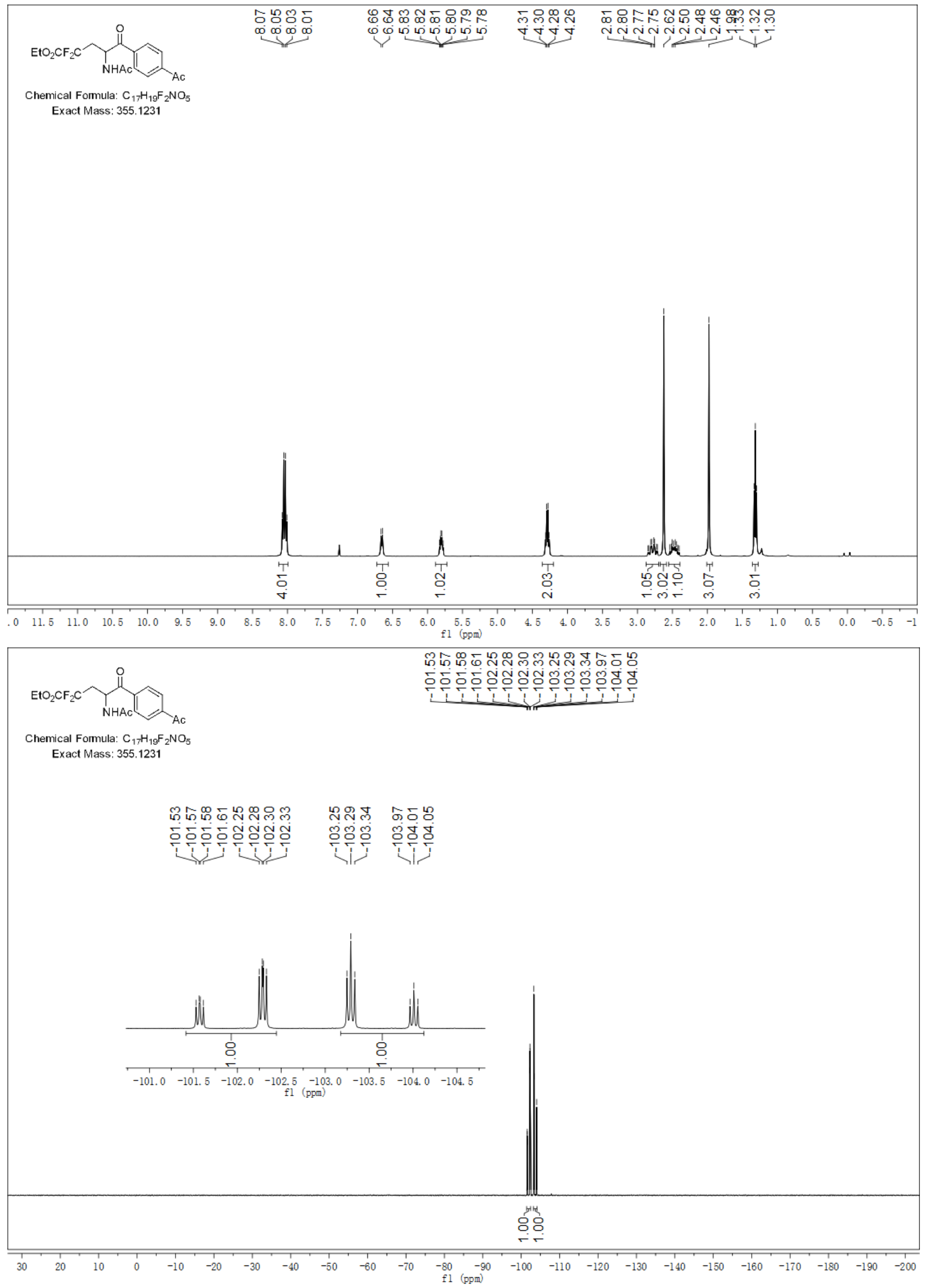




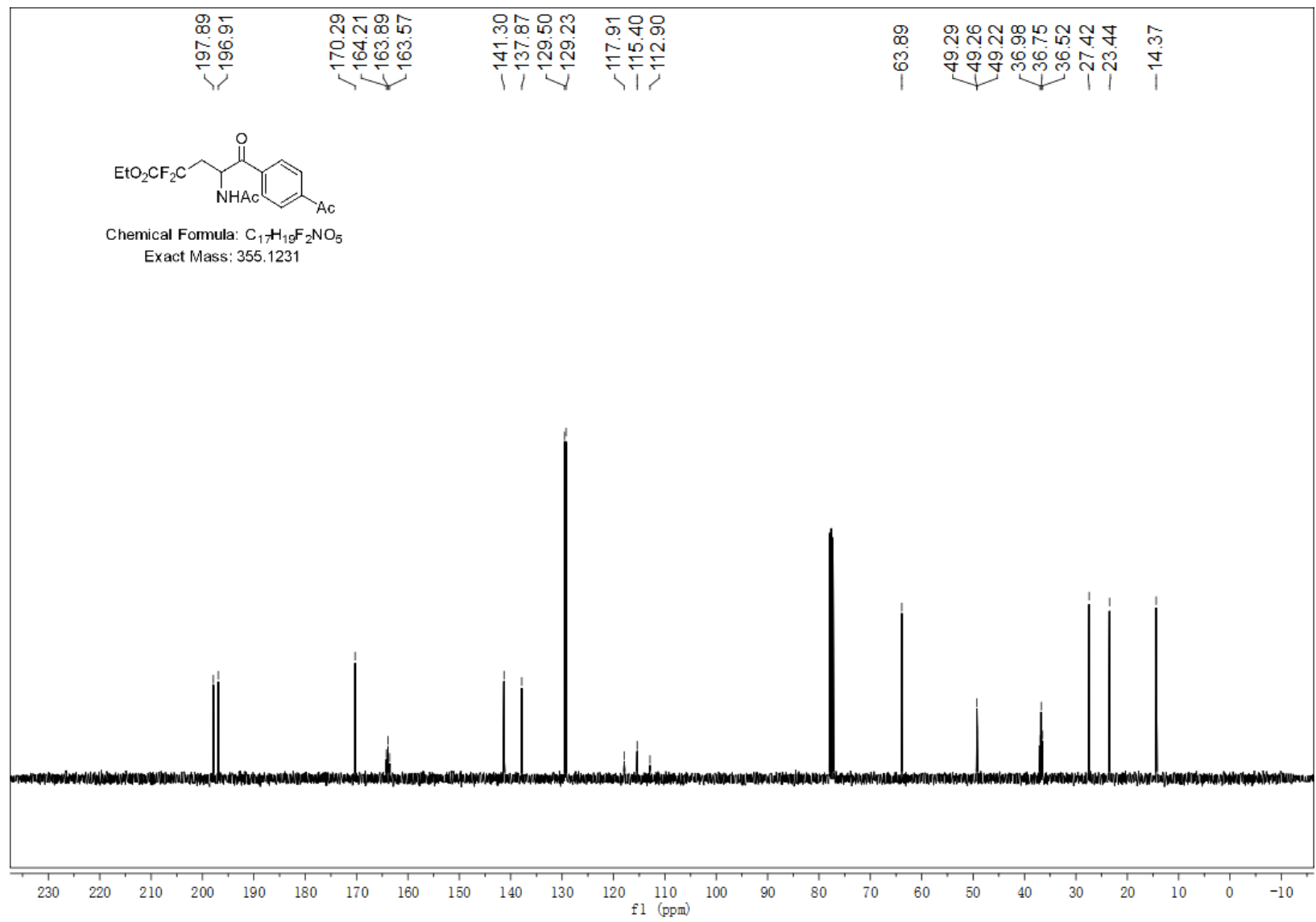

Ethyl 4-acetamido-5-(4-cyanophenyl)-2,2-difluoro-5-oxopentanoate (4n).

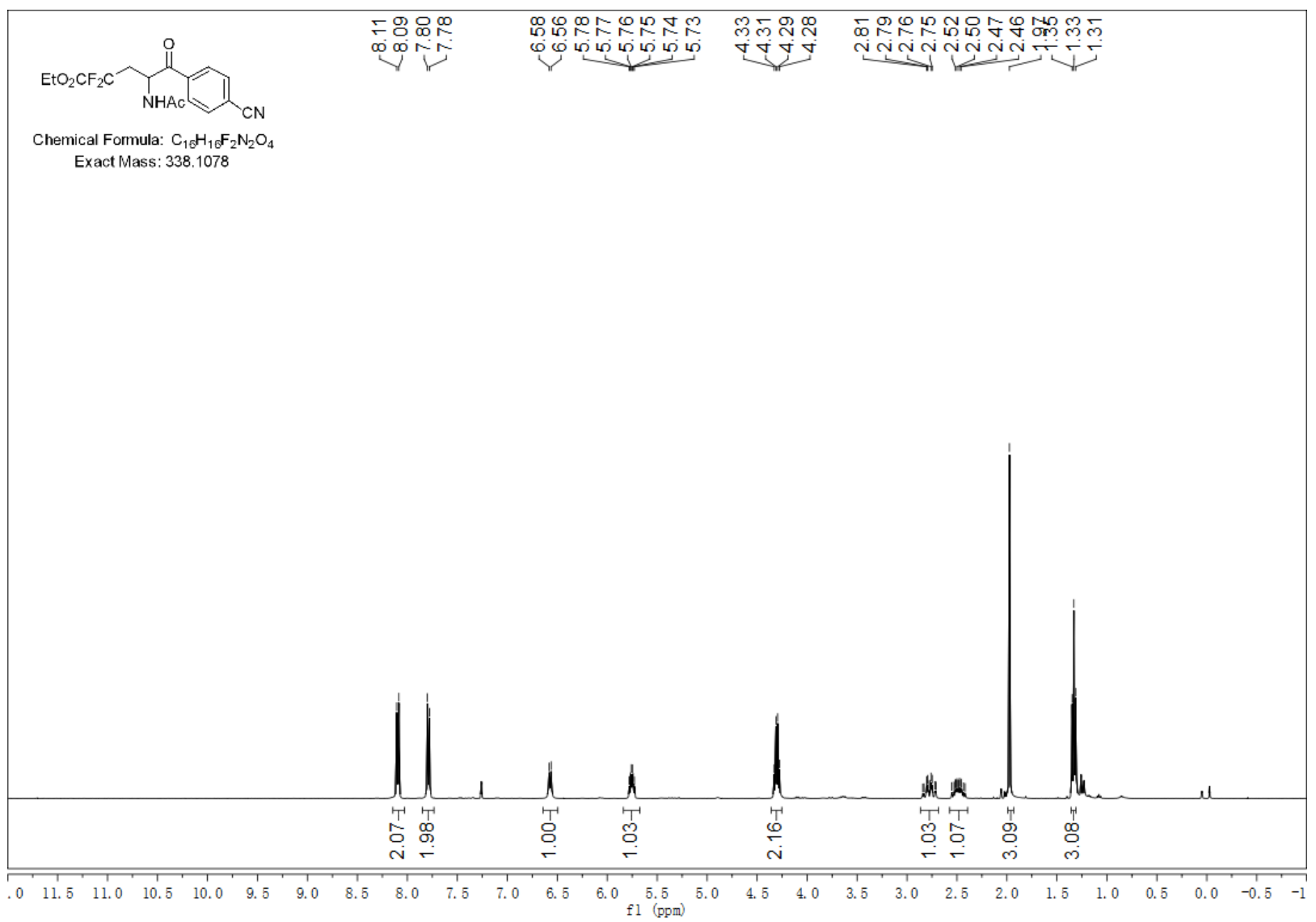




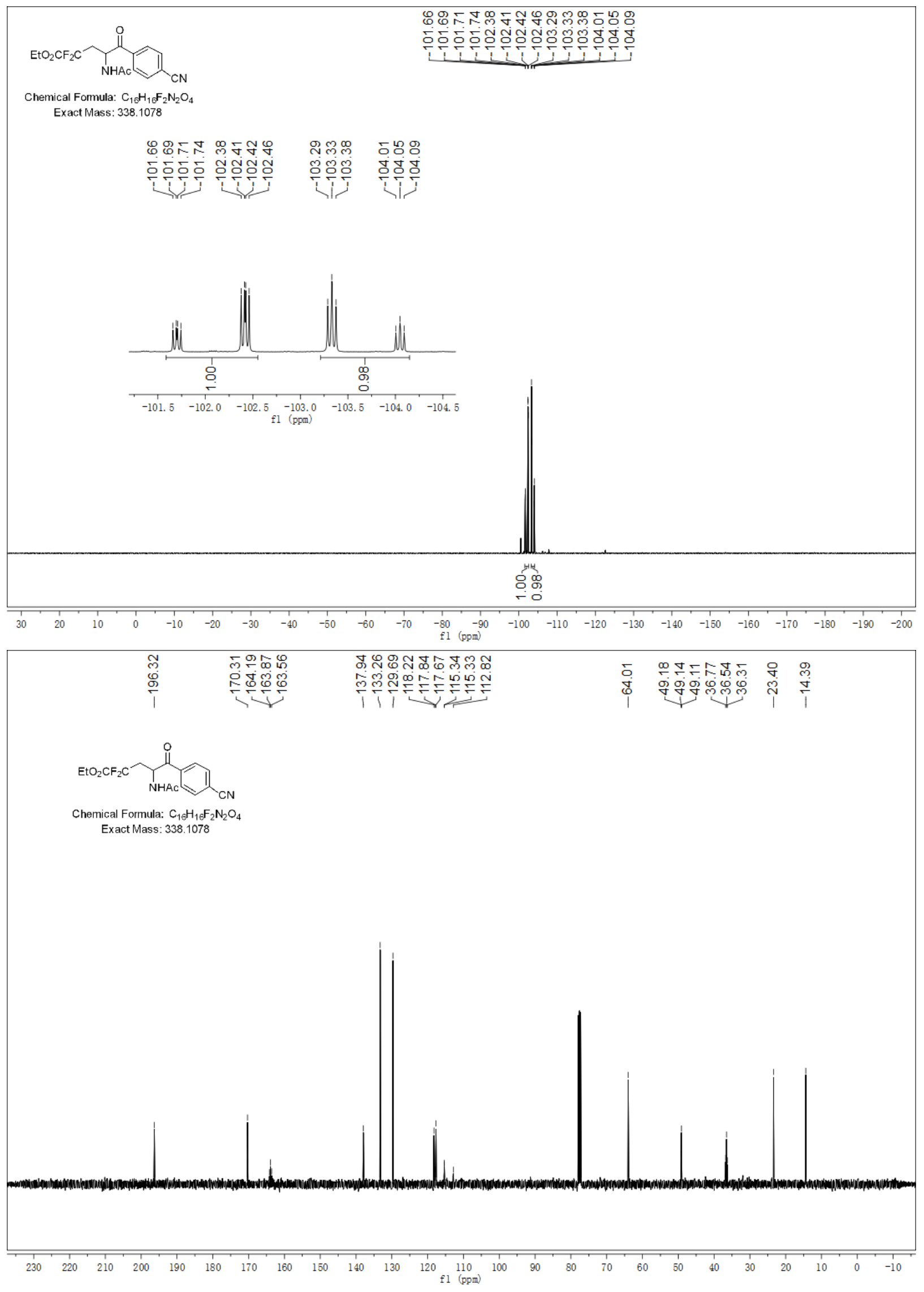


Ethyl 4-(2-acetamido-5-ethoxy-4,4-difluoro-5-oxopentanoyl)benzoate (40).

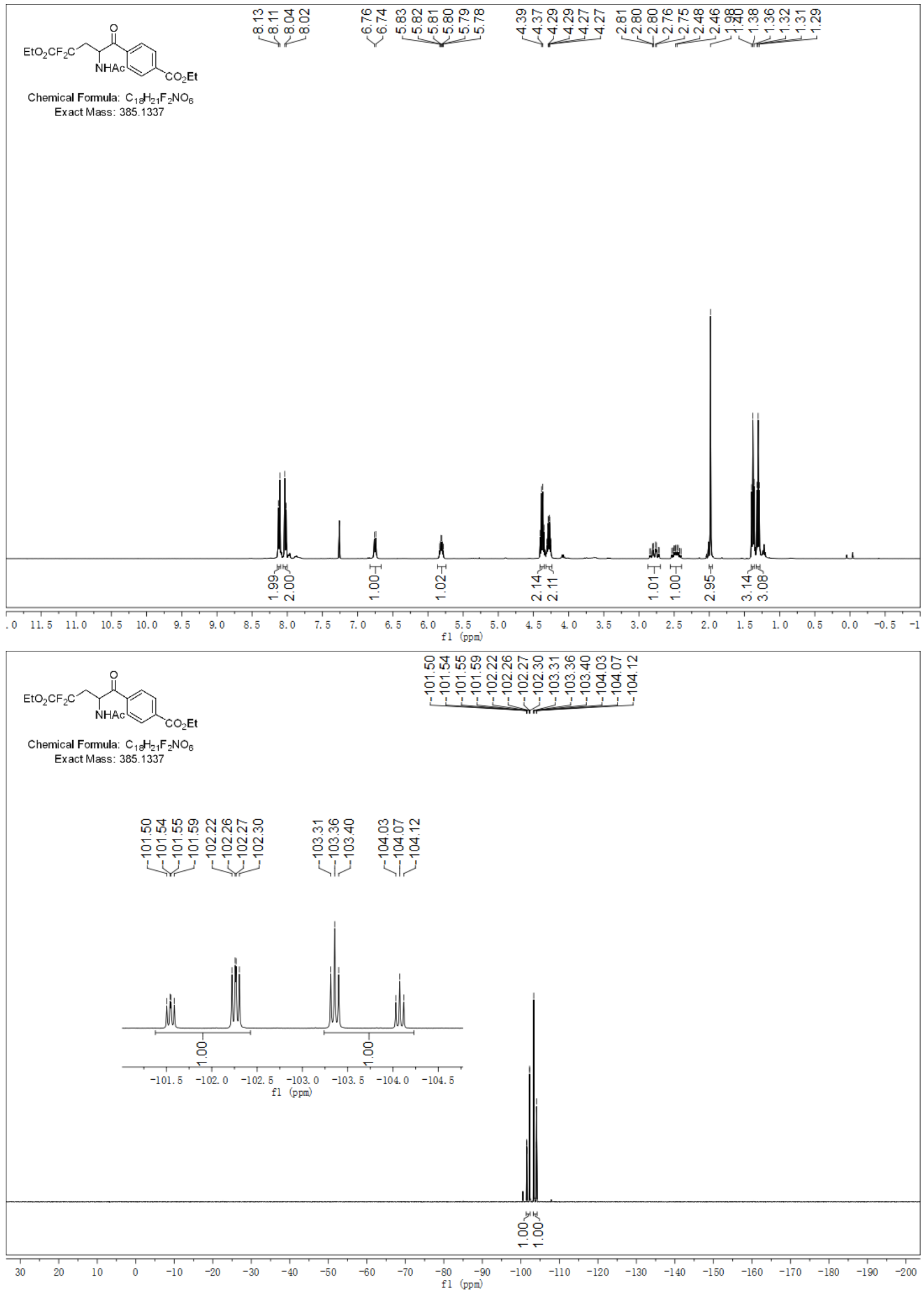




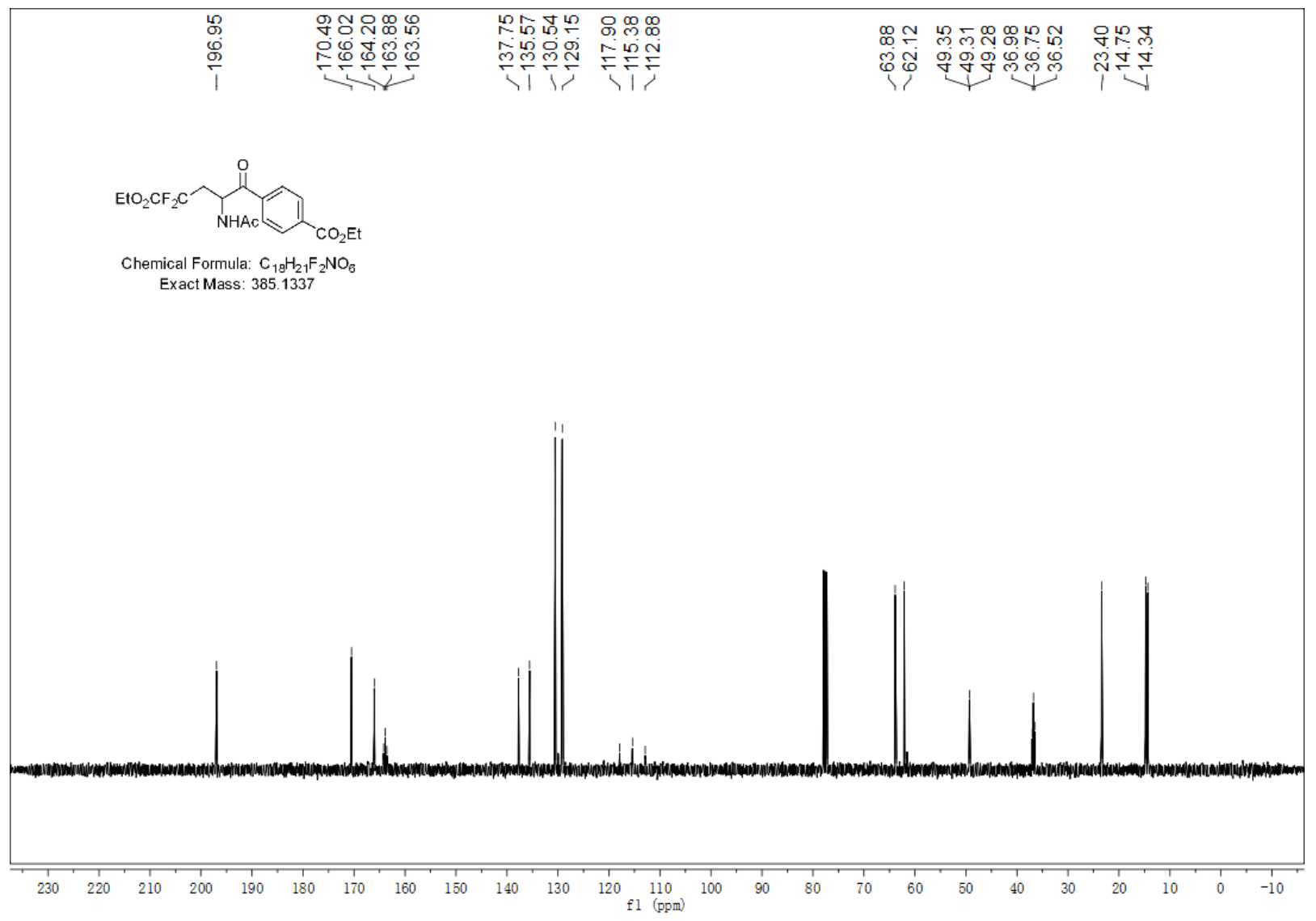

Ethyl 3-(2-acetamido-5-ethoxy-4,4-difluoro-5-oxopentanoyl)benzoate (4p).

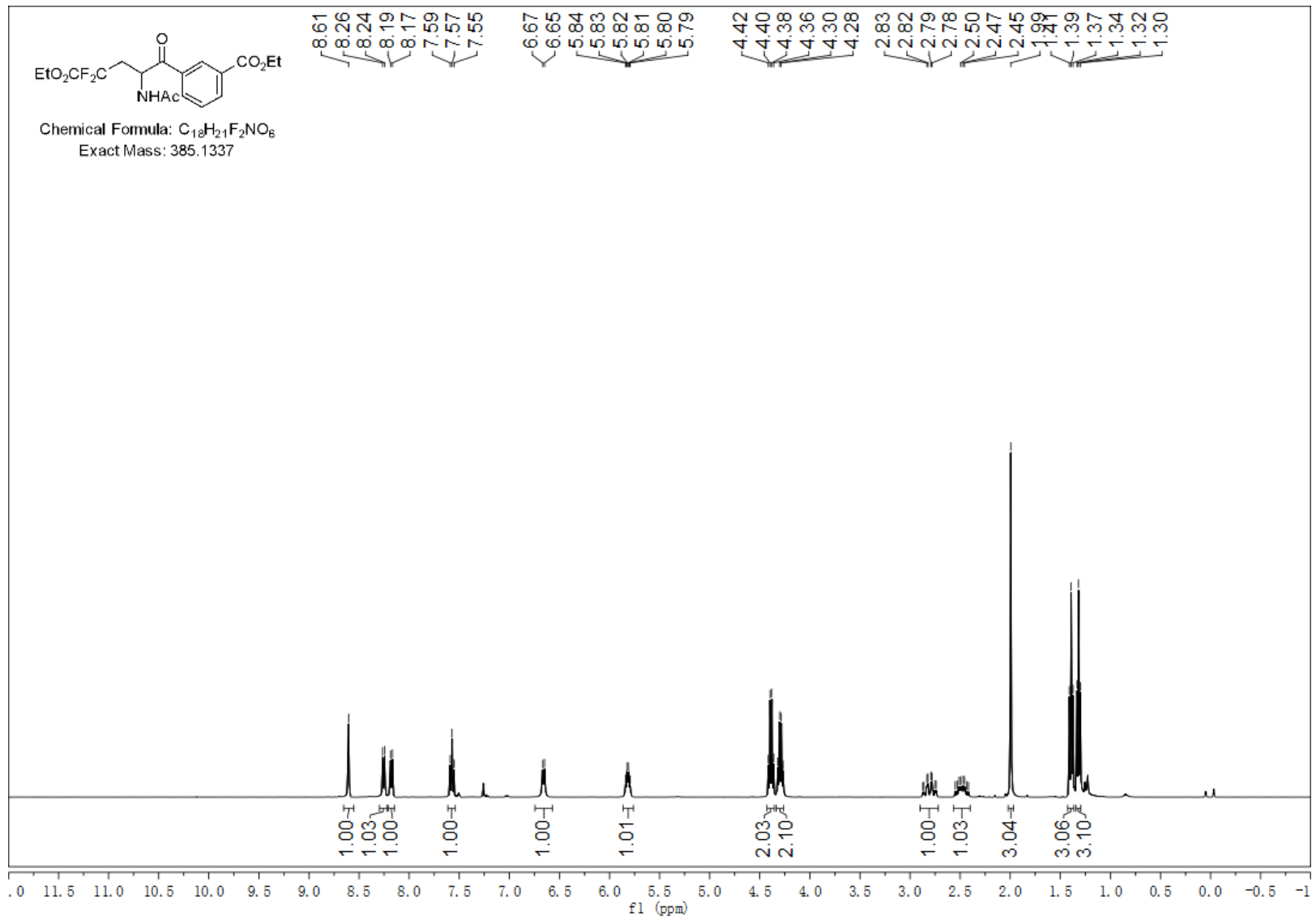



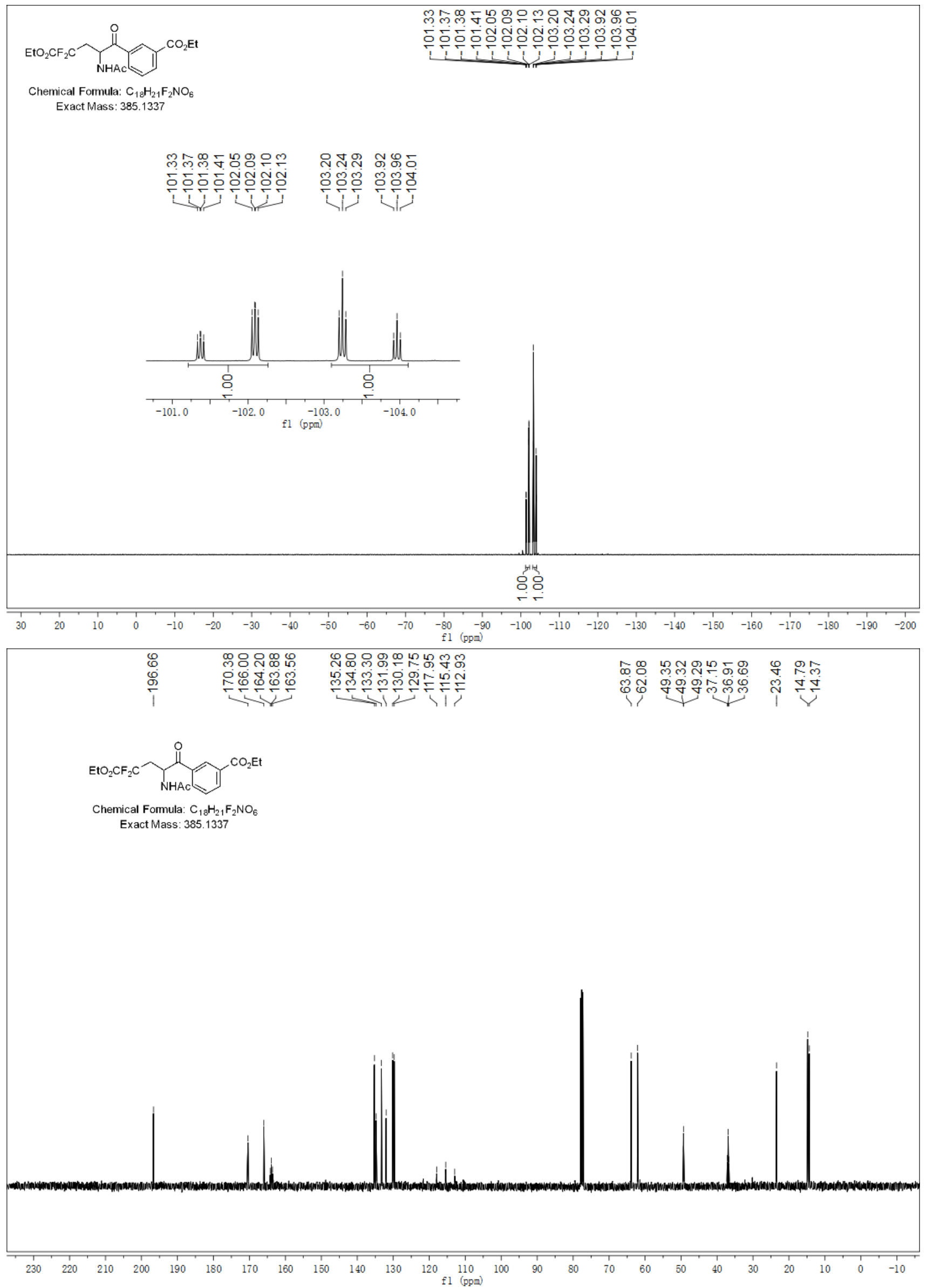
Ethyl 4-acetamido-2,2-difluoro-5-(6-methoxypyridin-3-yl)-5-oxopentanoate (4q).
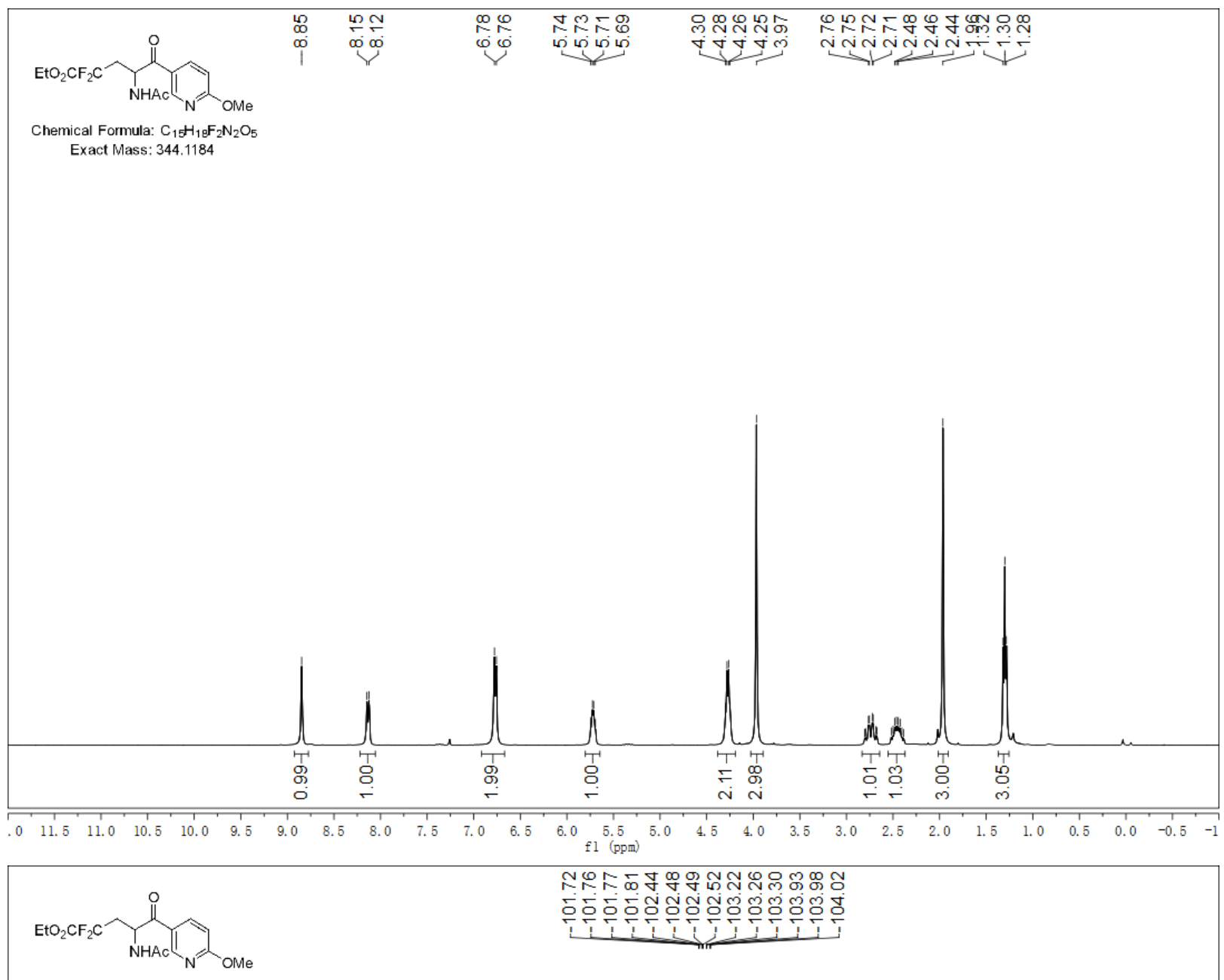

Chemical Formula: $\mathrm{C}_{1} \mathrm{H}_{1} \mathrm{~F}_{2} \mathrm{~N}_{2} \mathrm{O}_{5}$ Exact Mass: 344.1184
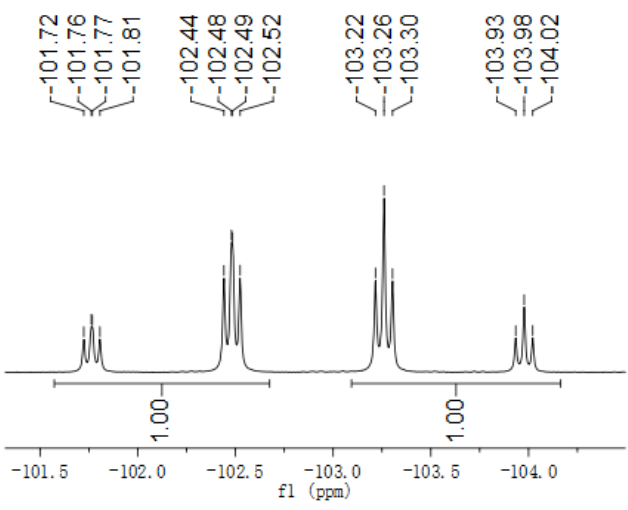

\section{$\stackrel{-10}{0}$}

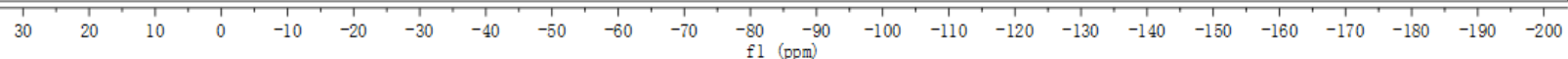




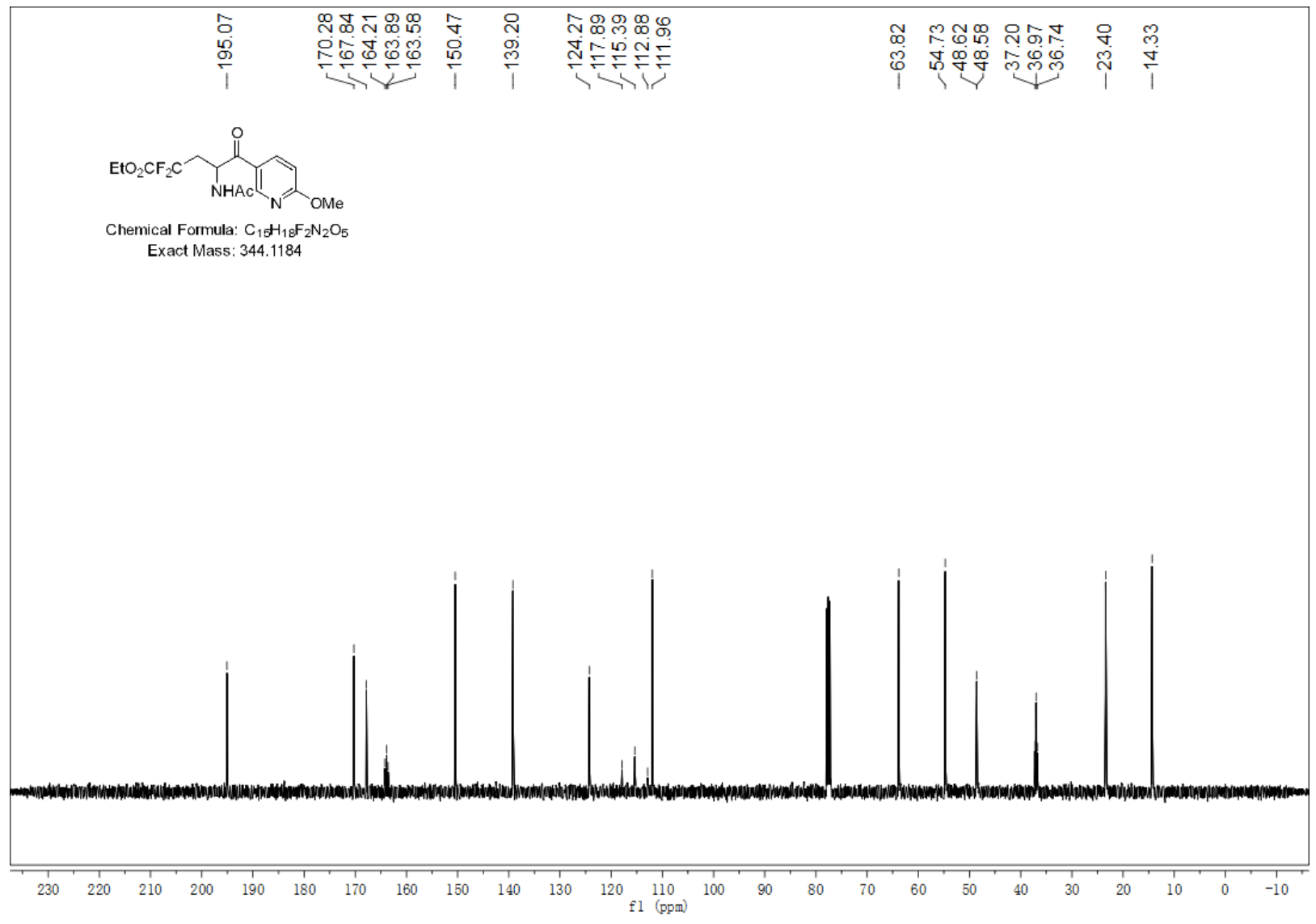

Ethyl 4-acetamido-5-(dibenzo[b,d]furan-2-yl)-2,2-difluoro-5-oxopentanoate (4r).

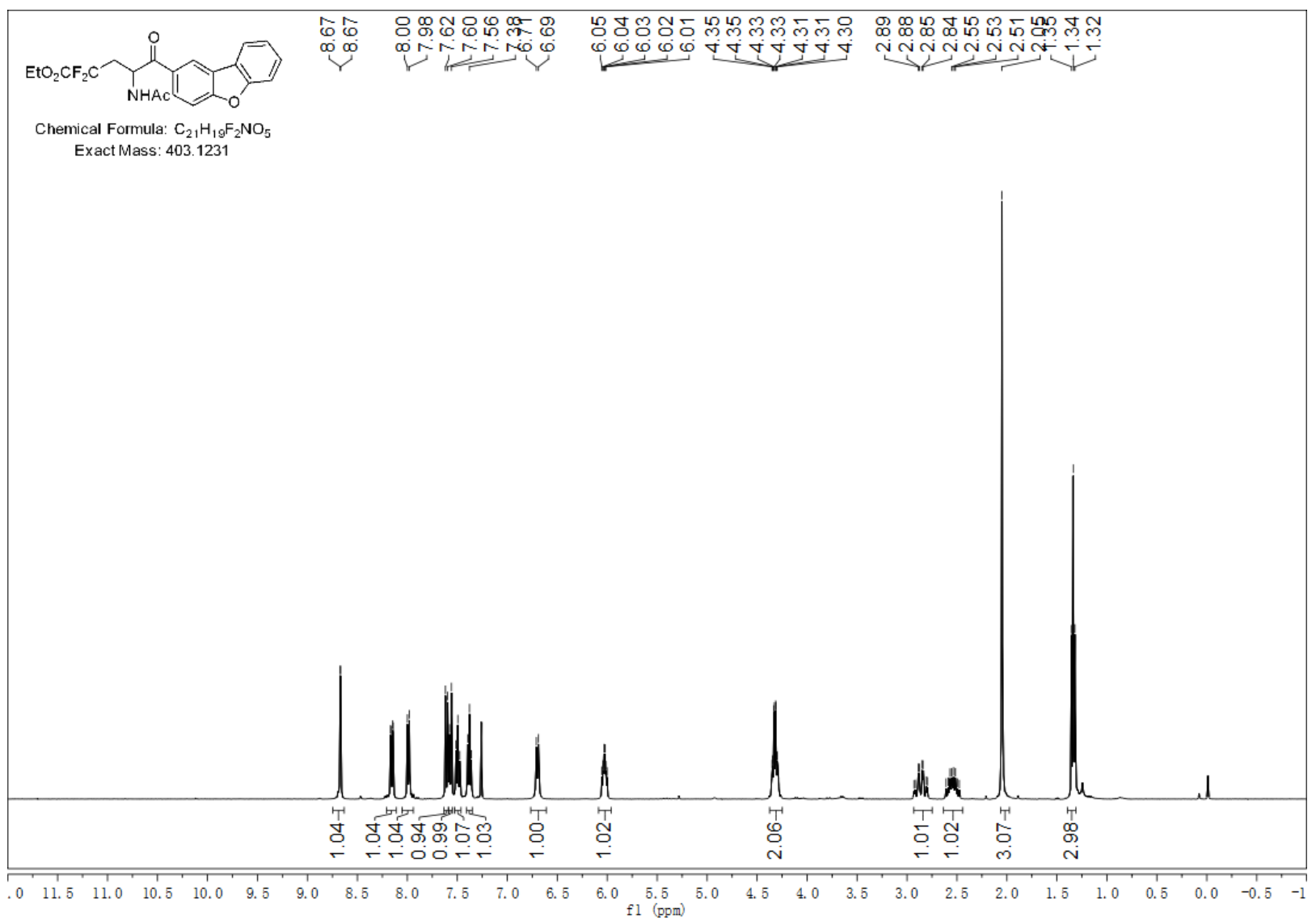



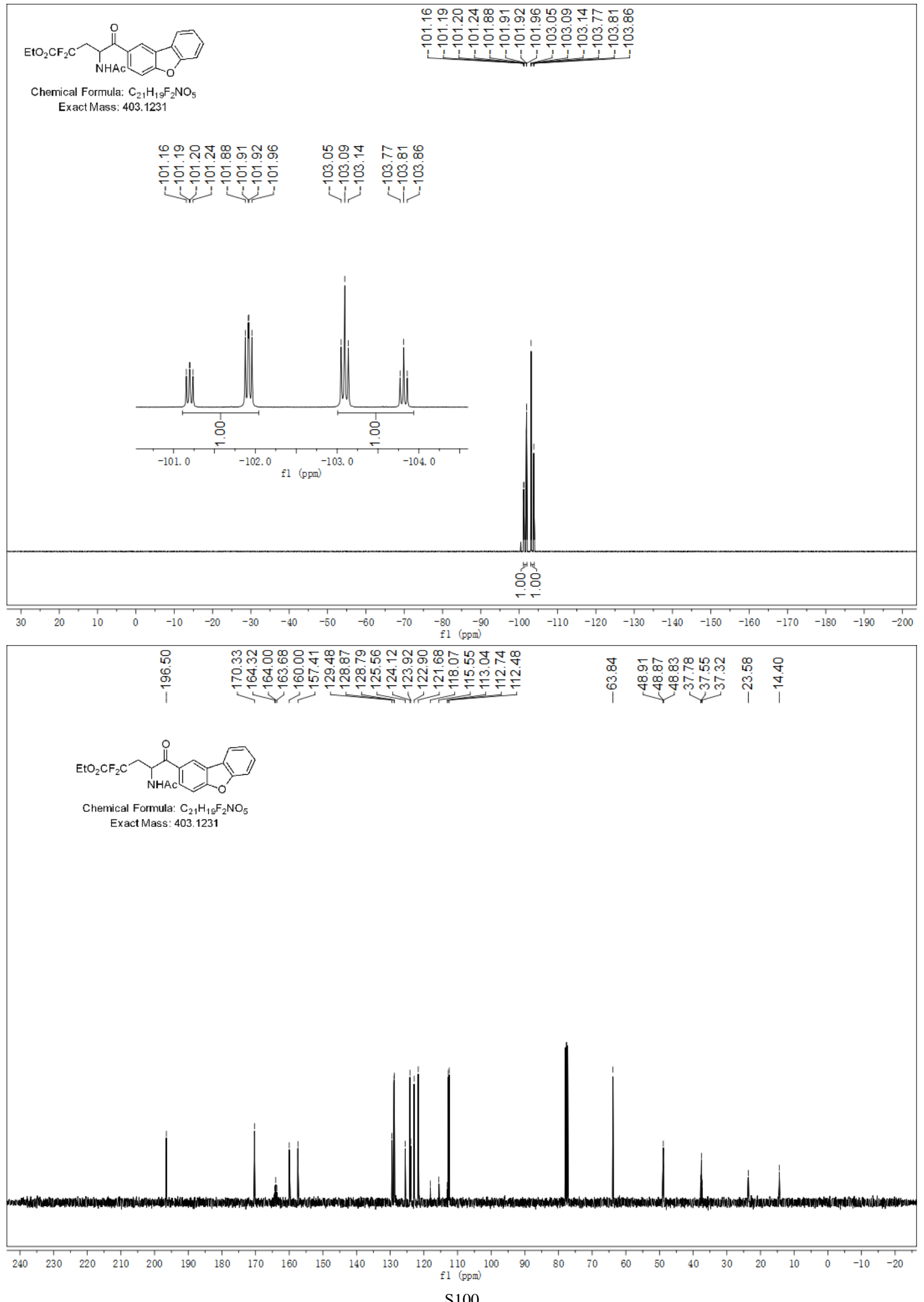
Ethyl 4-acetamido-5-(dibenzo[b,d] thiophen-2-yl)-2,2-difluoro-5-oxopentanoate (4s).
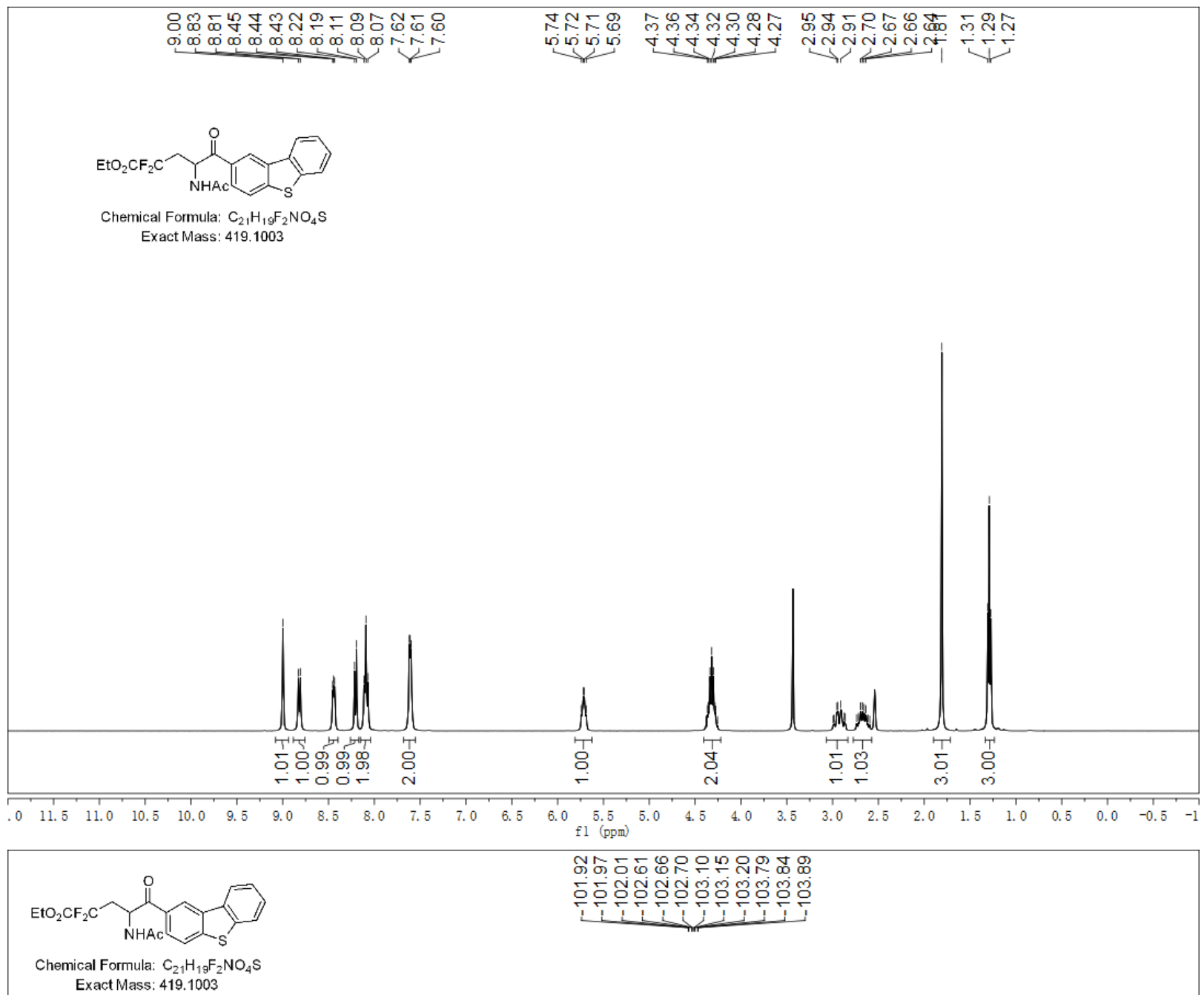

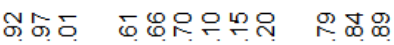

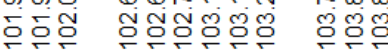

나군

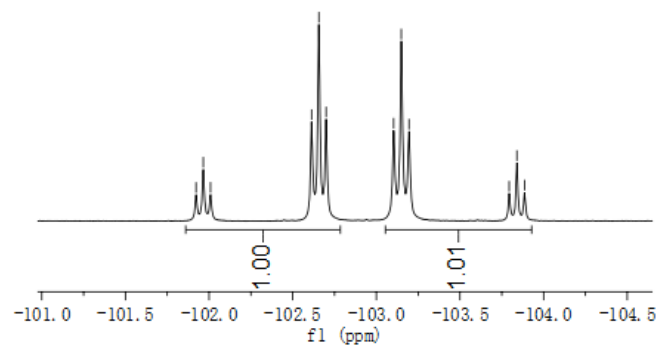

에음

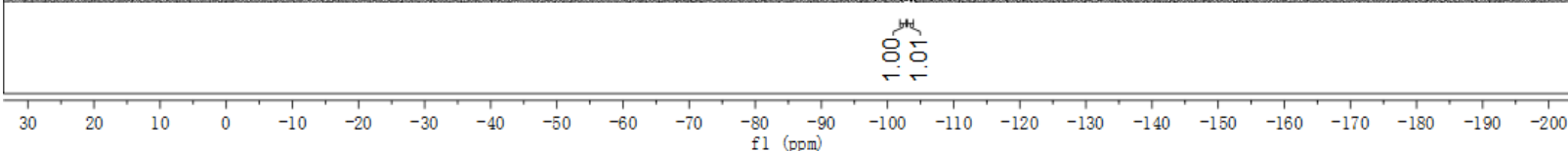




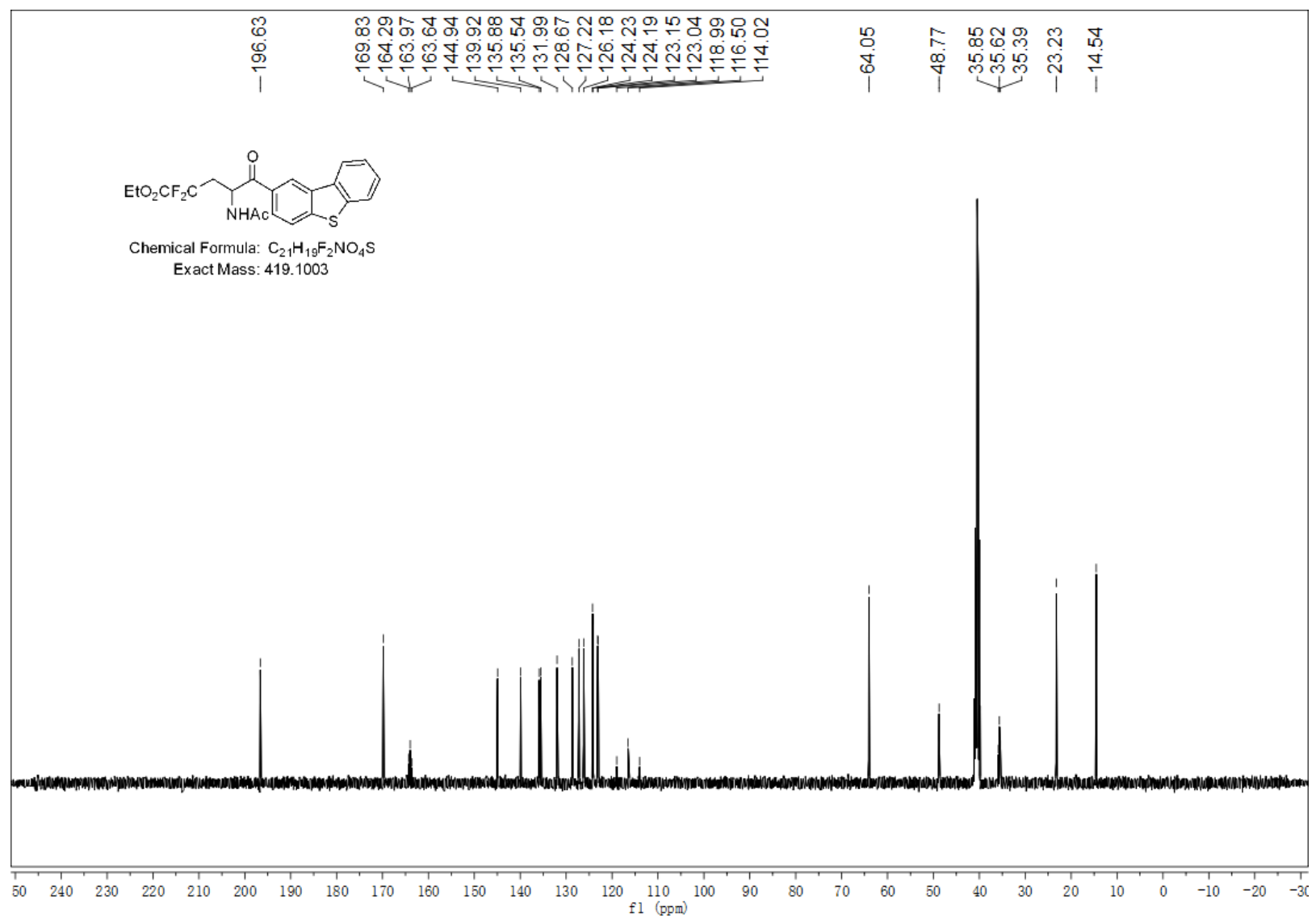

Ethyl 4-acetamido-2,2-difluoro-5-oxo-5-(9-phenyl-9H-carbazol-3-yl)pentanoate (4t).

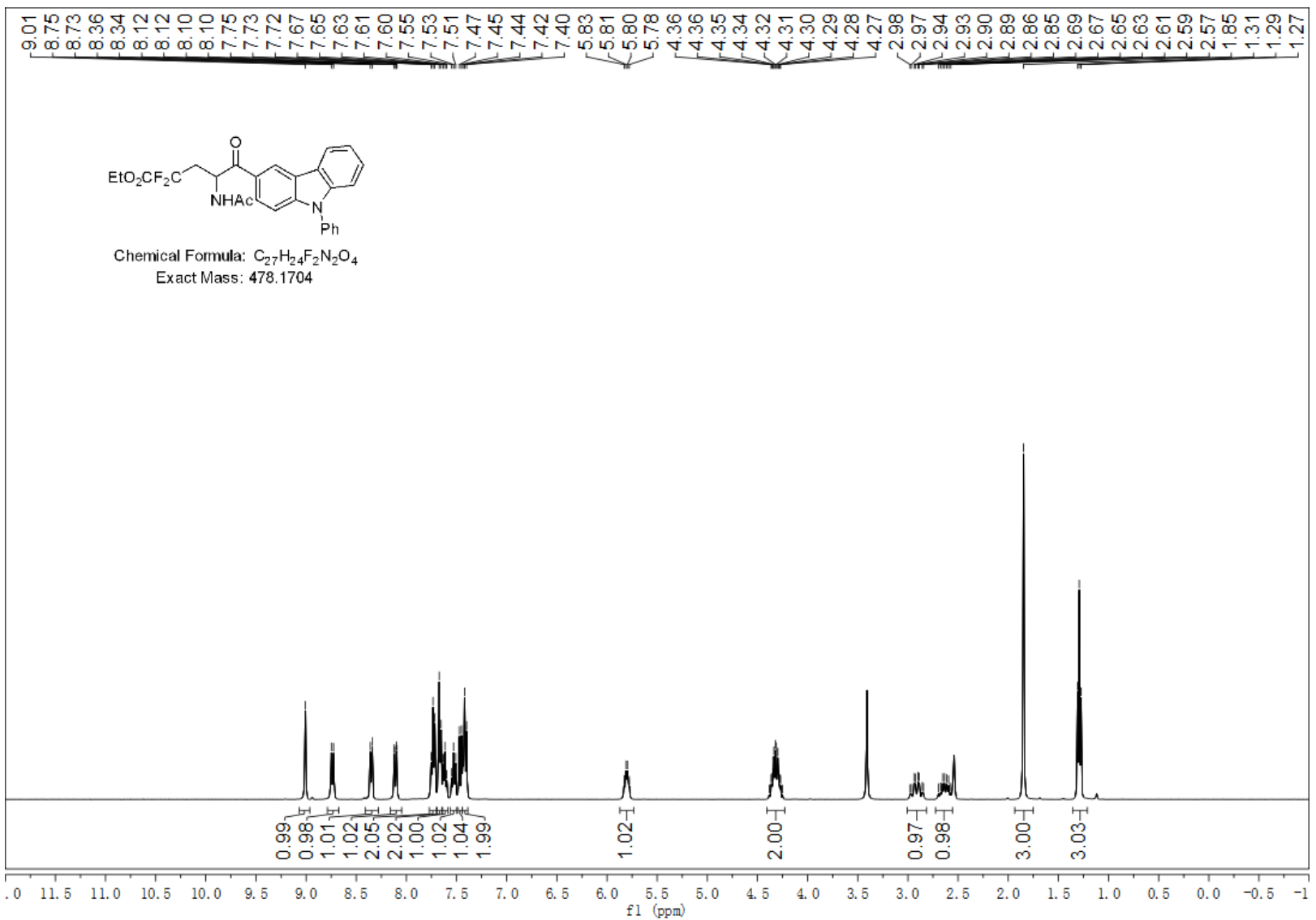



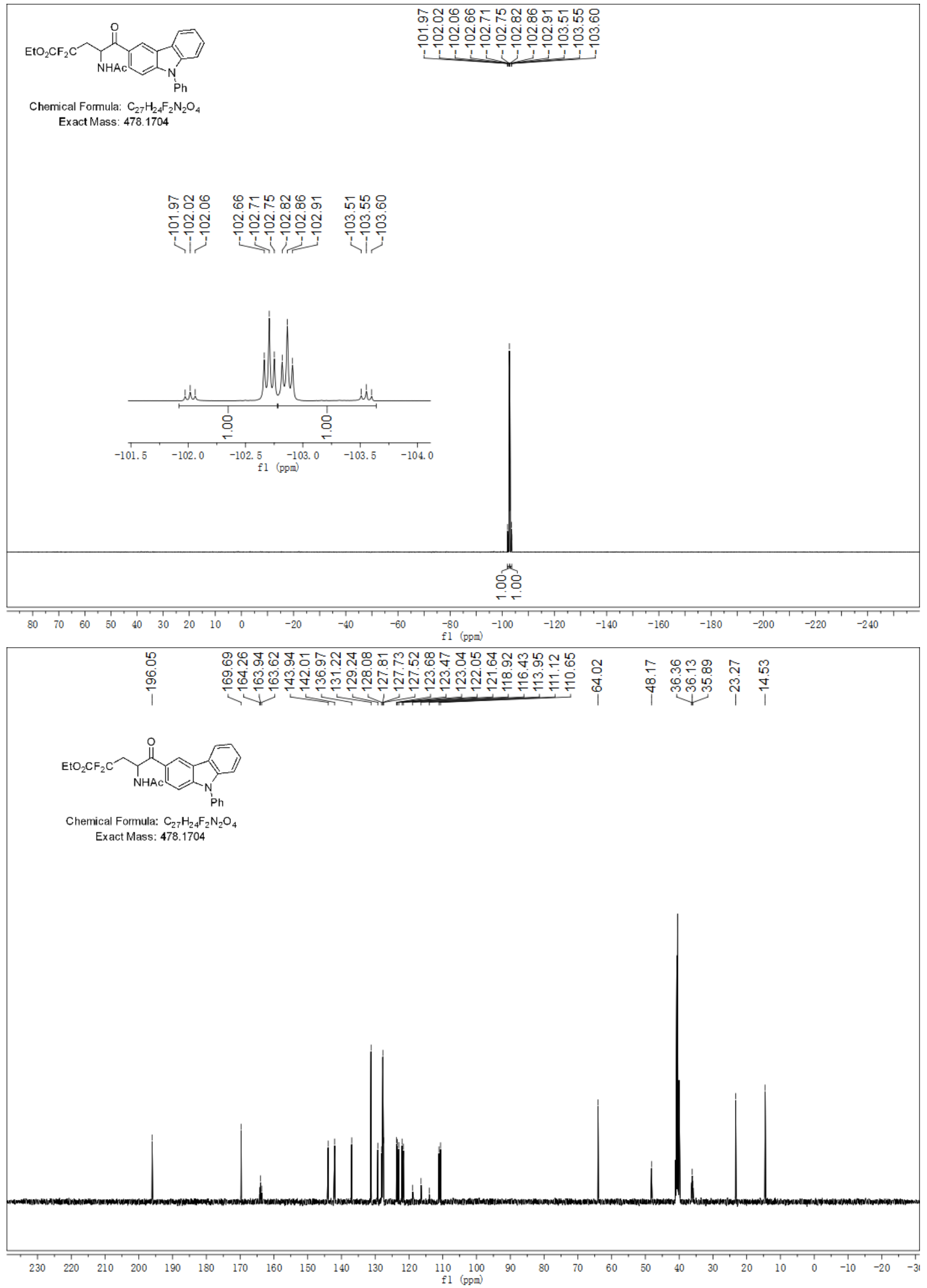
tert-Butyl 2-(2-acetamido-5-ethoxy-4,4-difluoro-5-oxopentanoyl)-10H-phenothiazine-10-

carboxylate (4u).

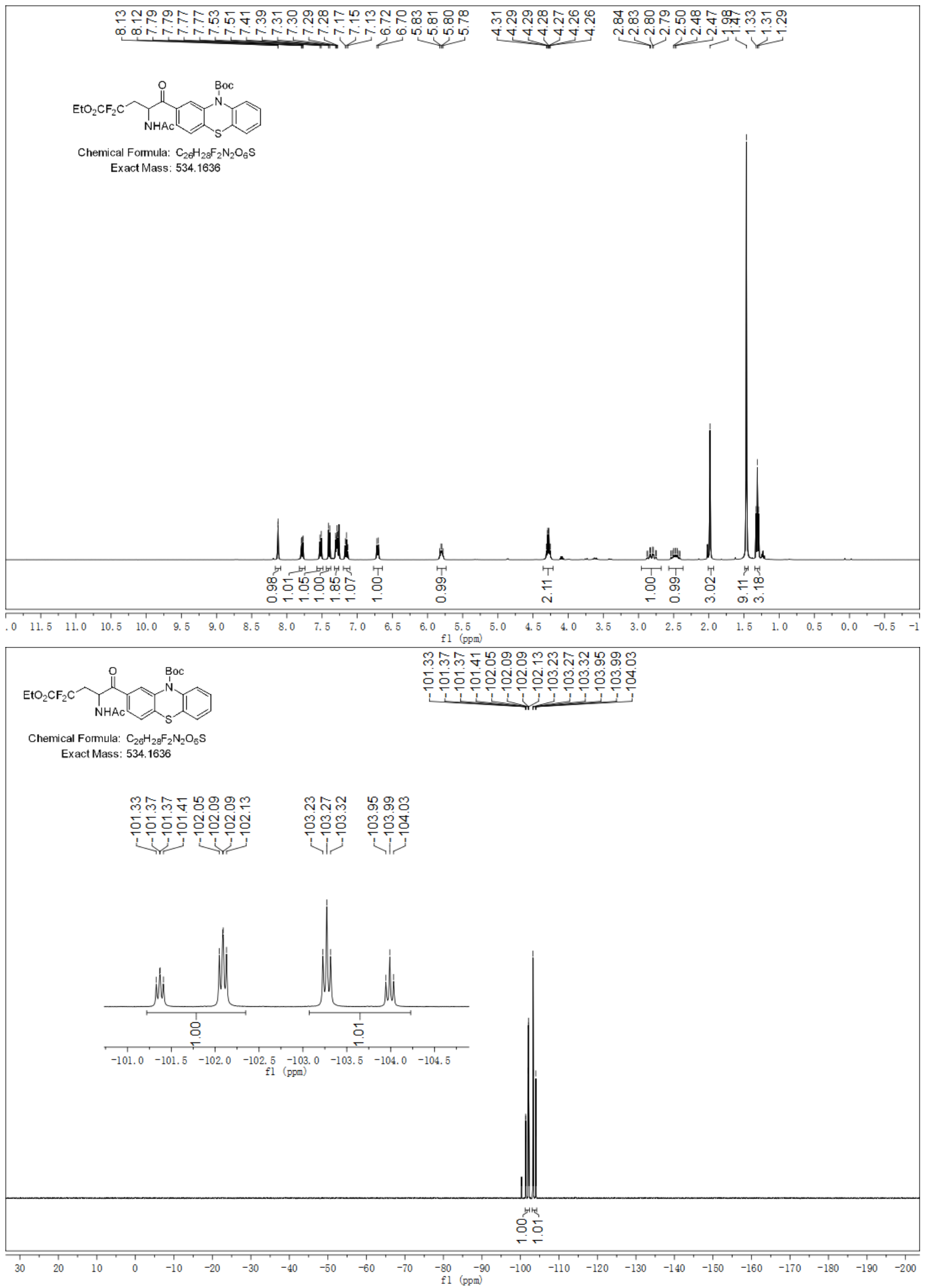




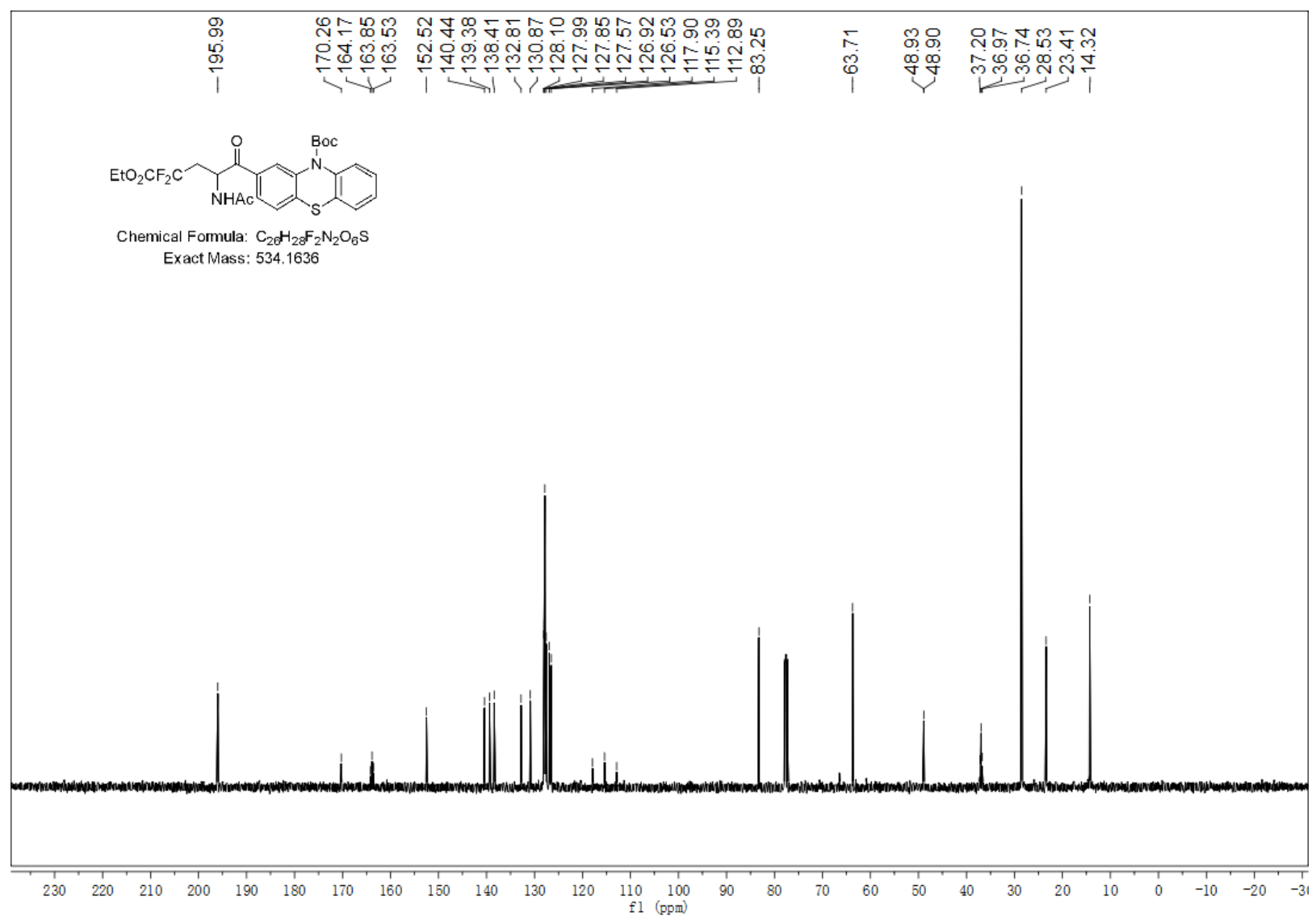

4-Acetamido-5-(benzo[d] [1,3] dioxol-5-yl)- $N, N$-diethyl-2,2-difluoro-5-oxopentanamide (6a).

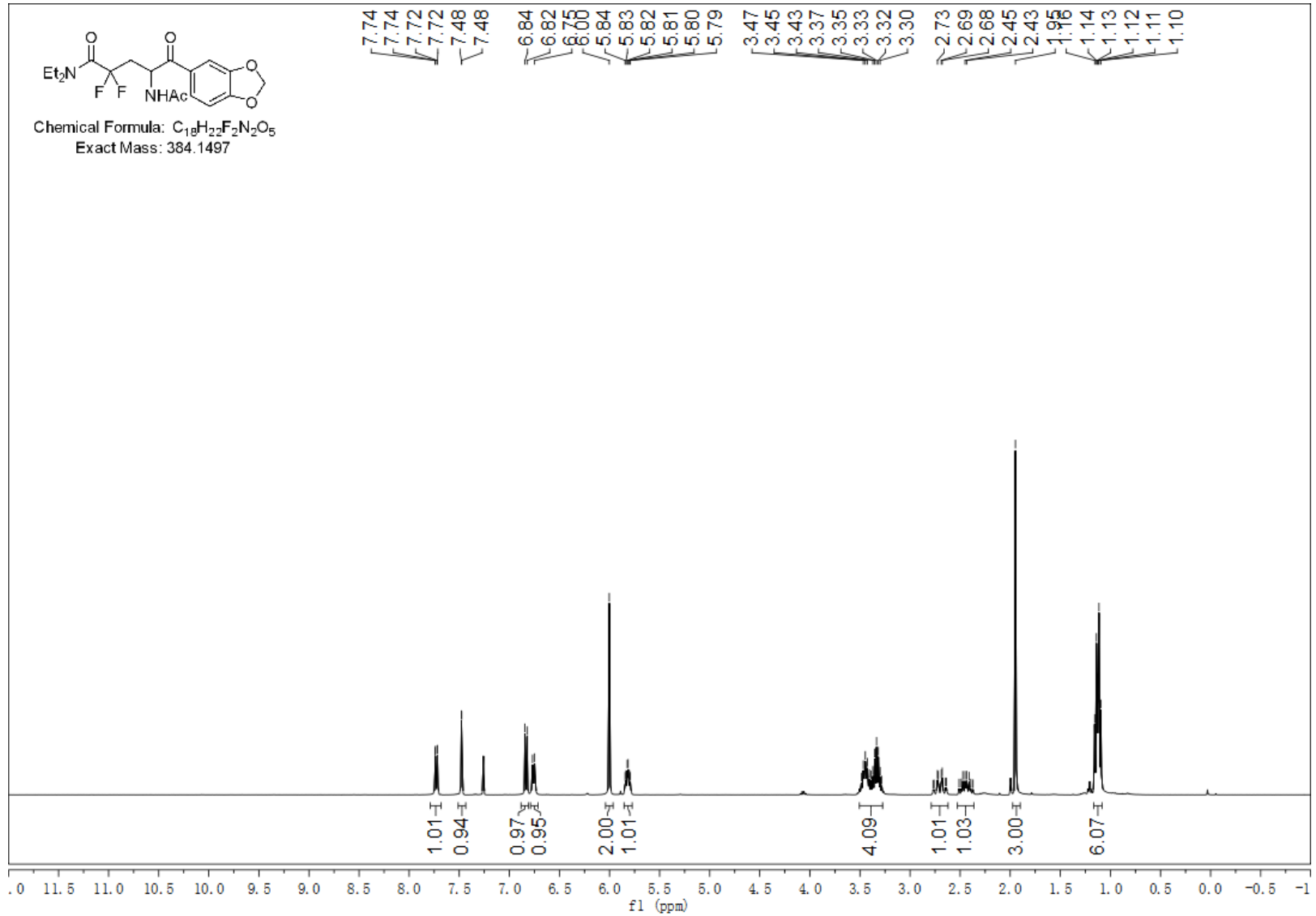




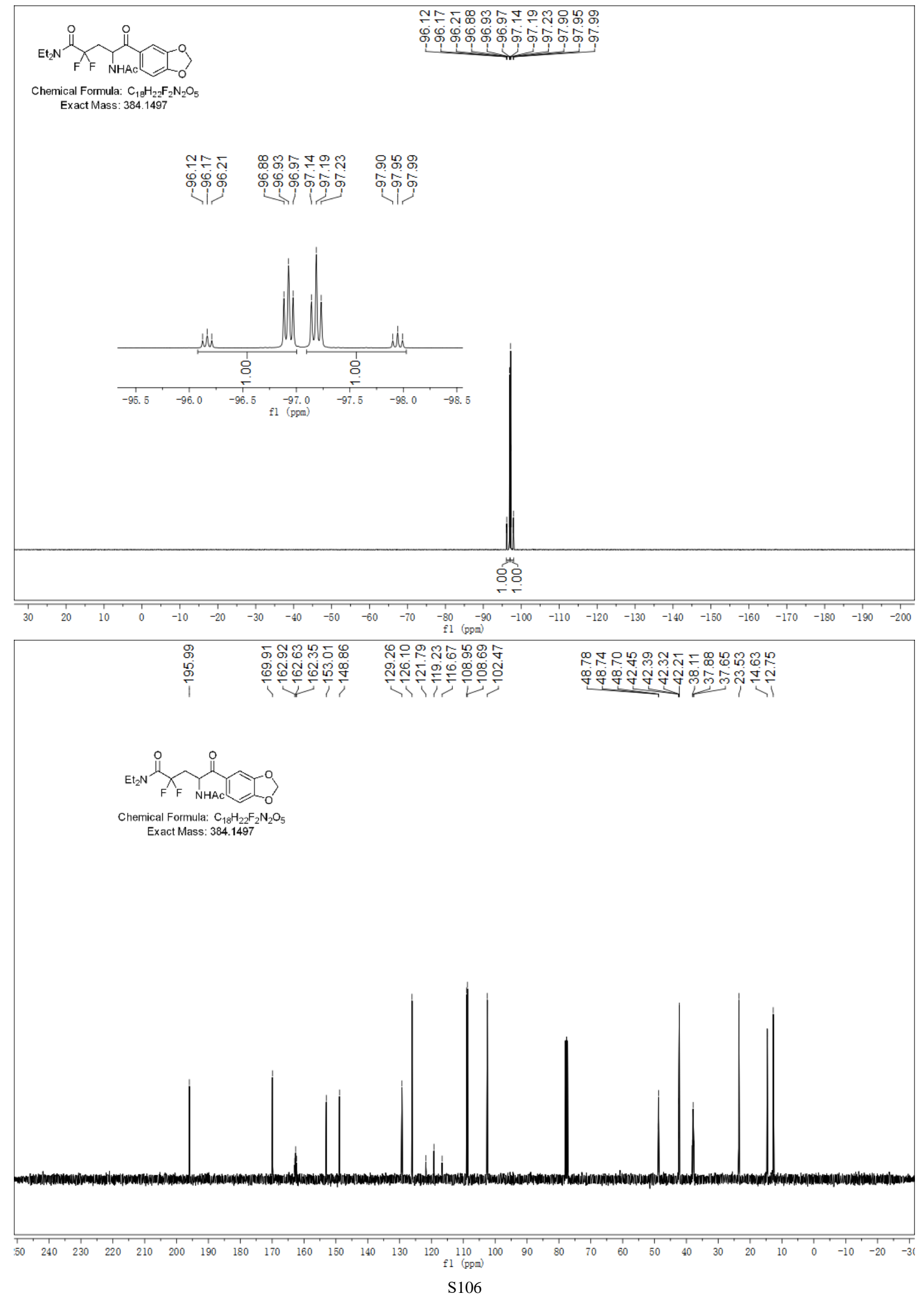


N-(1-([1,1'-Biphenyl]-4-yl)-4,4-difluoro-1-oxo-6-(triisopropylsilyl)hex-5-yn-2-yl)acetamide (6b).
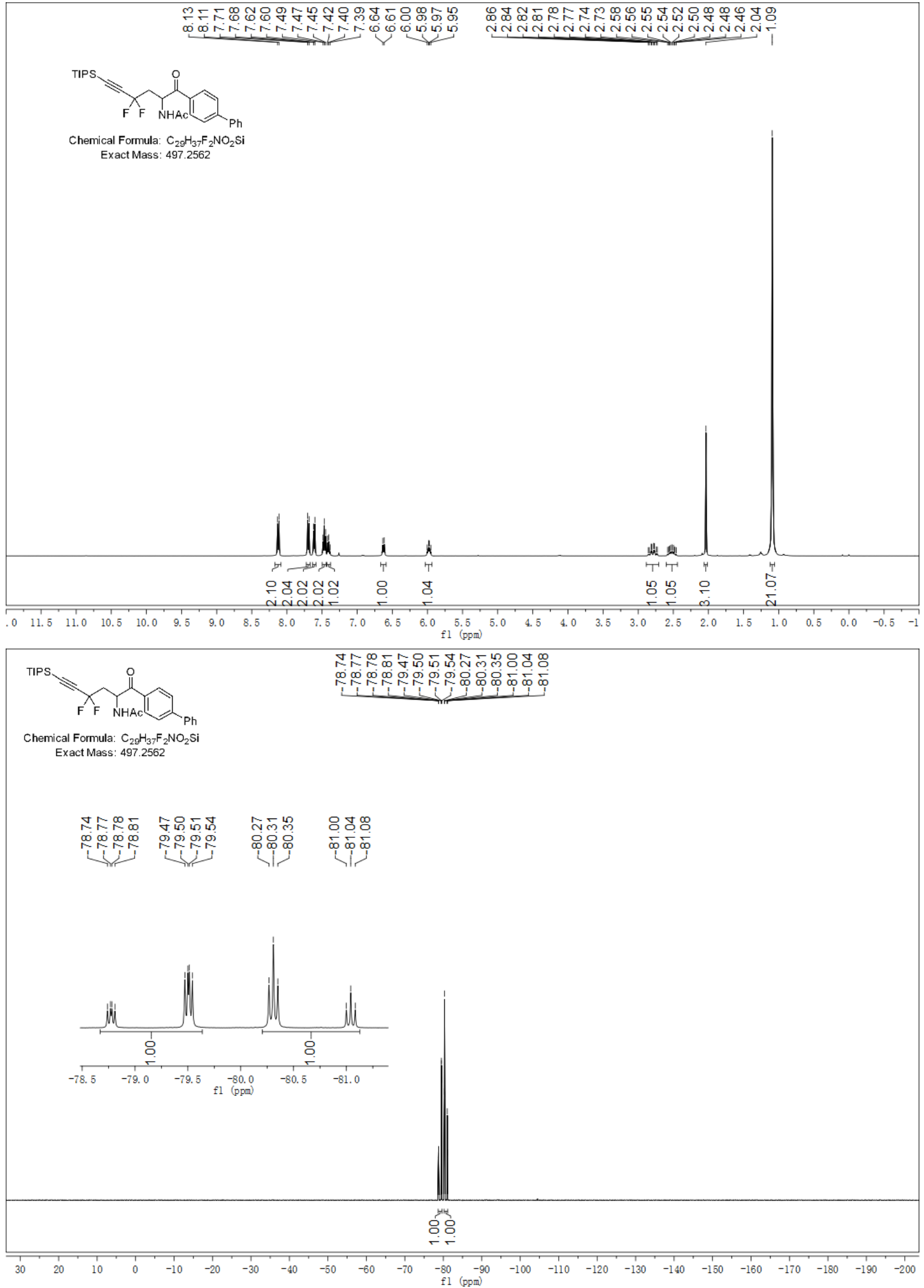


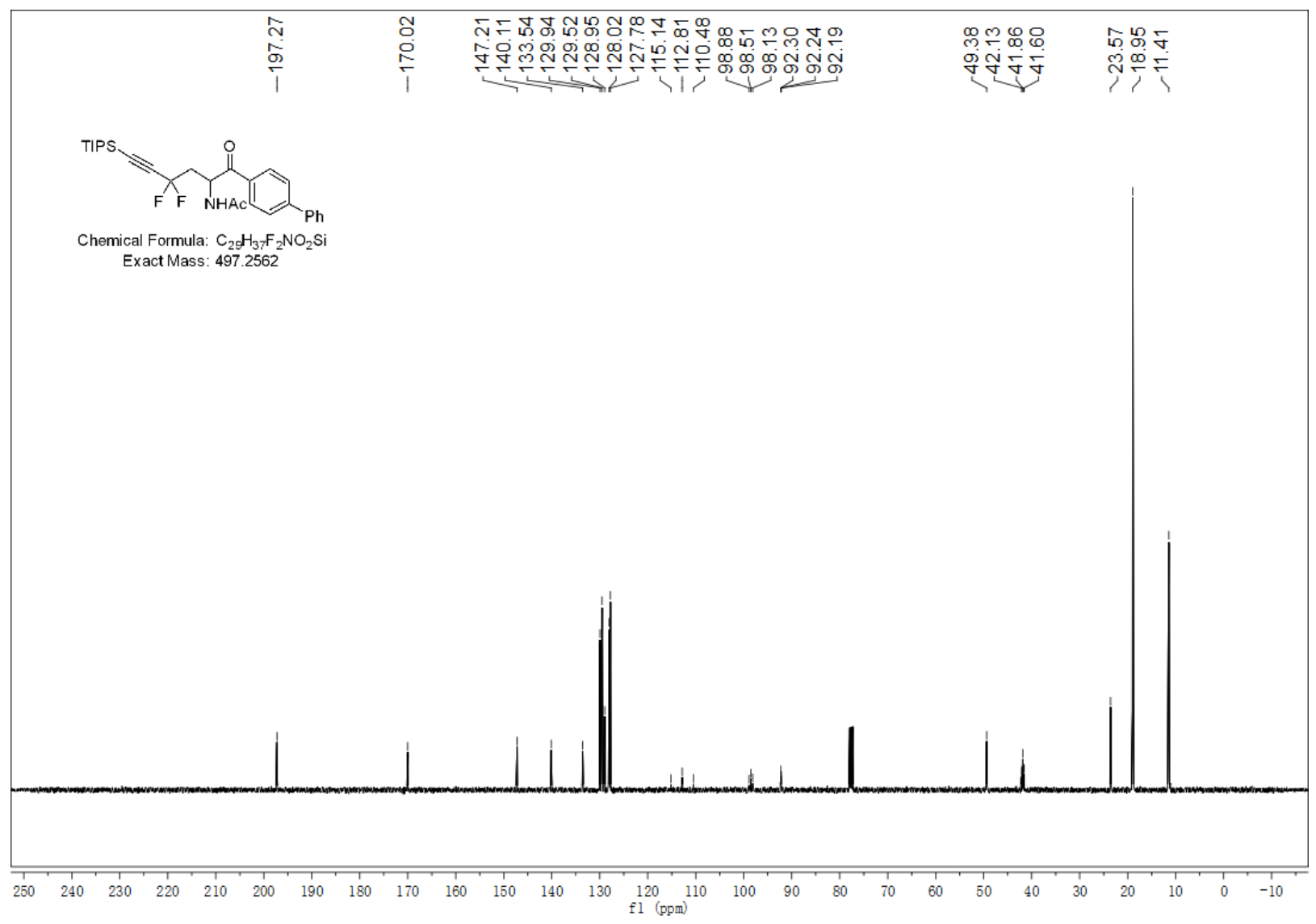

$N$-(1-([1,1'-Biphenyl]-4-yl)-4-(benzo[d] oxazol-2-yl)-4,4-difluoro-1-oxobutan-2-yl)acetamide (6c).

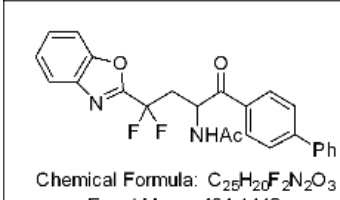

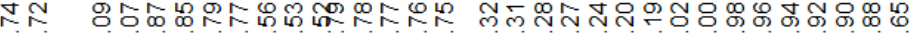

0

Chemical Formula: $\mathrm{C}_{25} \mathrm{H}_{2} \mathrm{~F}_{2} \mathrm{~N}_{2} \mathrm{O}_{3}$

Exact Mass: 434.1442

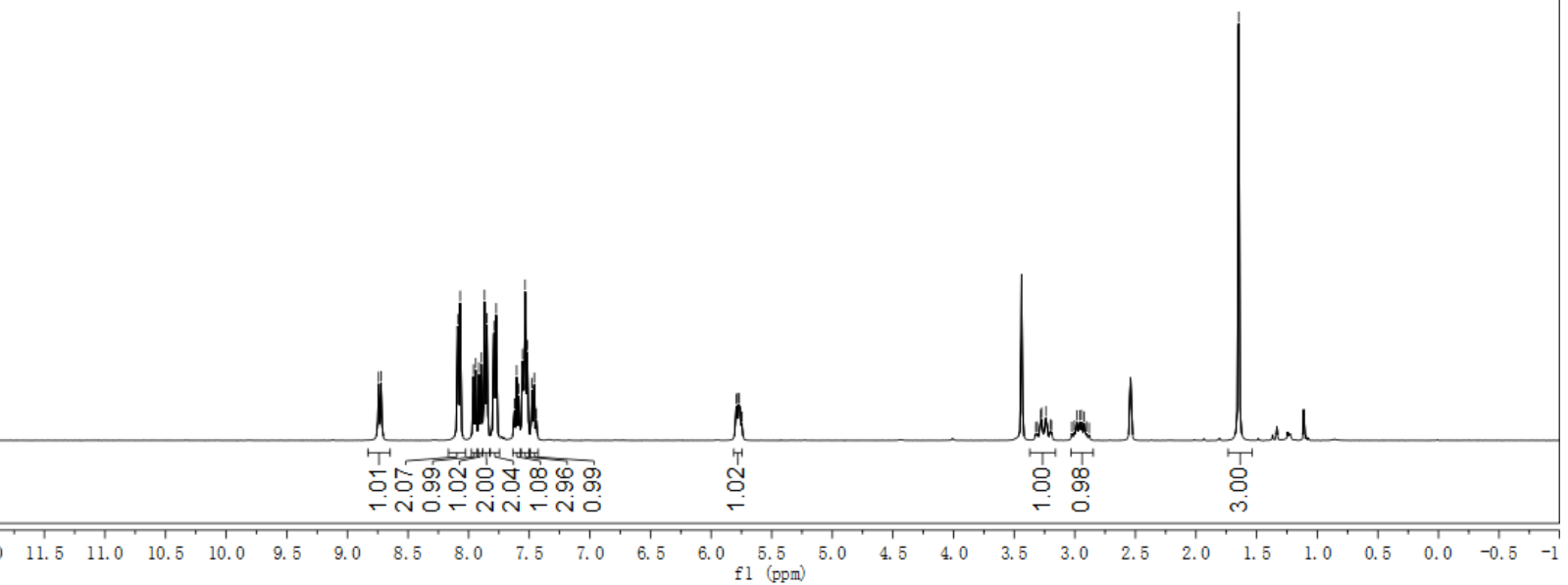




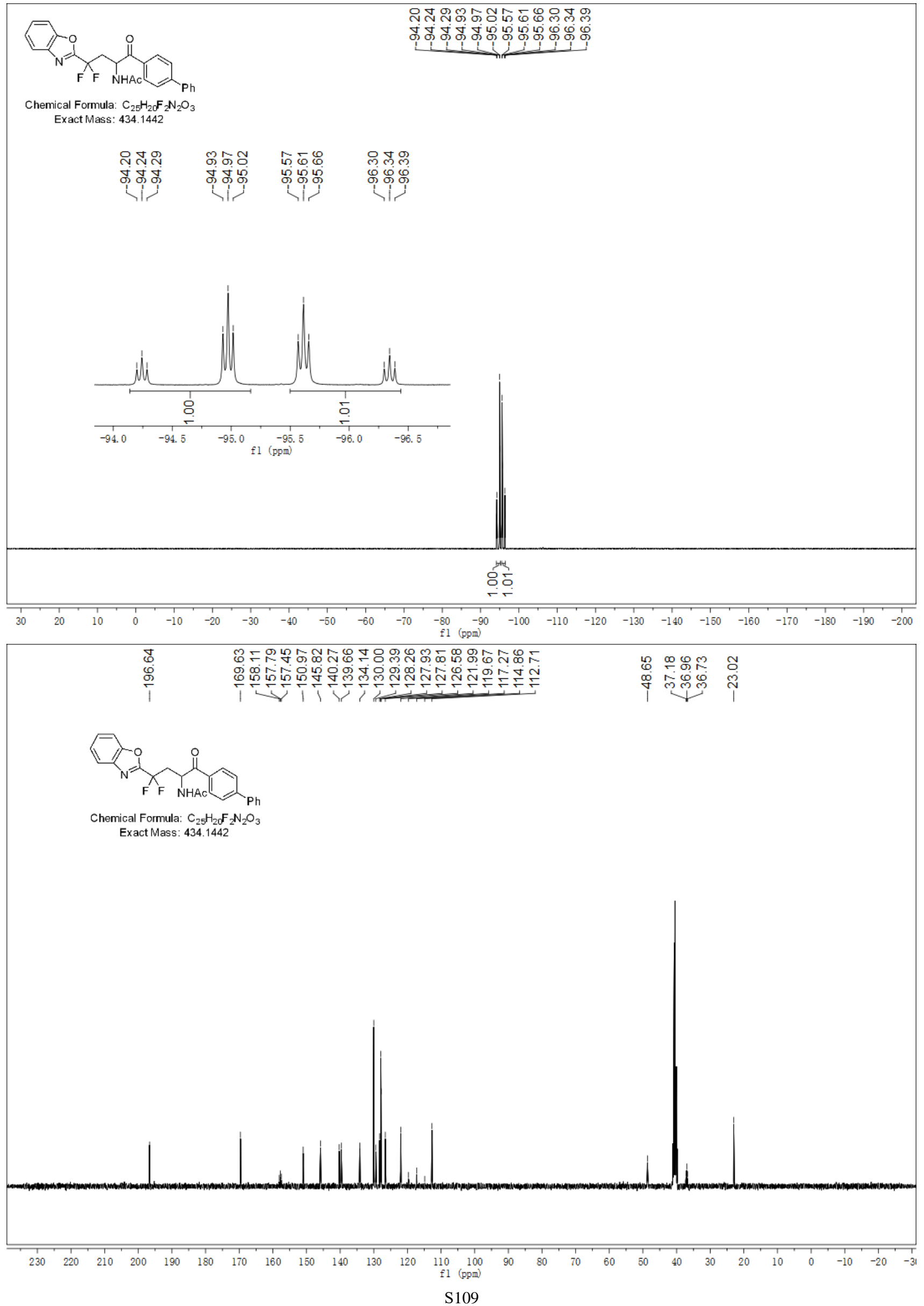


Ethyl 5-([1,1'-biphenyl]-4-yl)-4-((tert-butoxycarbonyl)amino)-2,2-difluoro-5-oxopentanoate (6d).
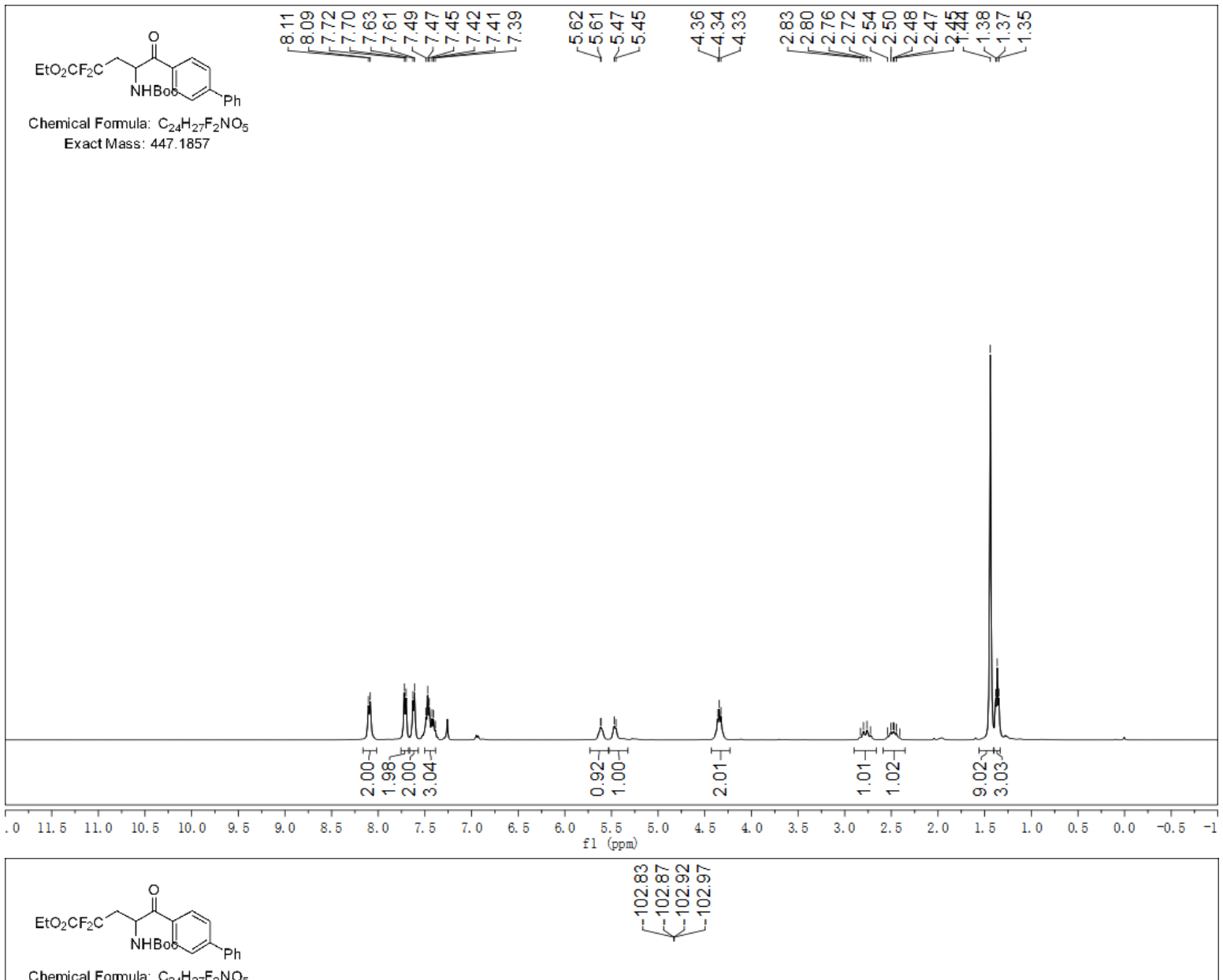

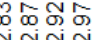

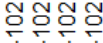

Chemical Formula: $\mathrm{C}_{24} \mathrm{H}_{27} \mathrm{~F}_{2} \mathrm{NO}_{5}$ Exact Mass: 447.1857

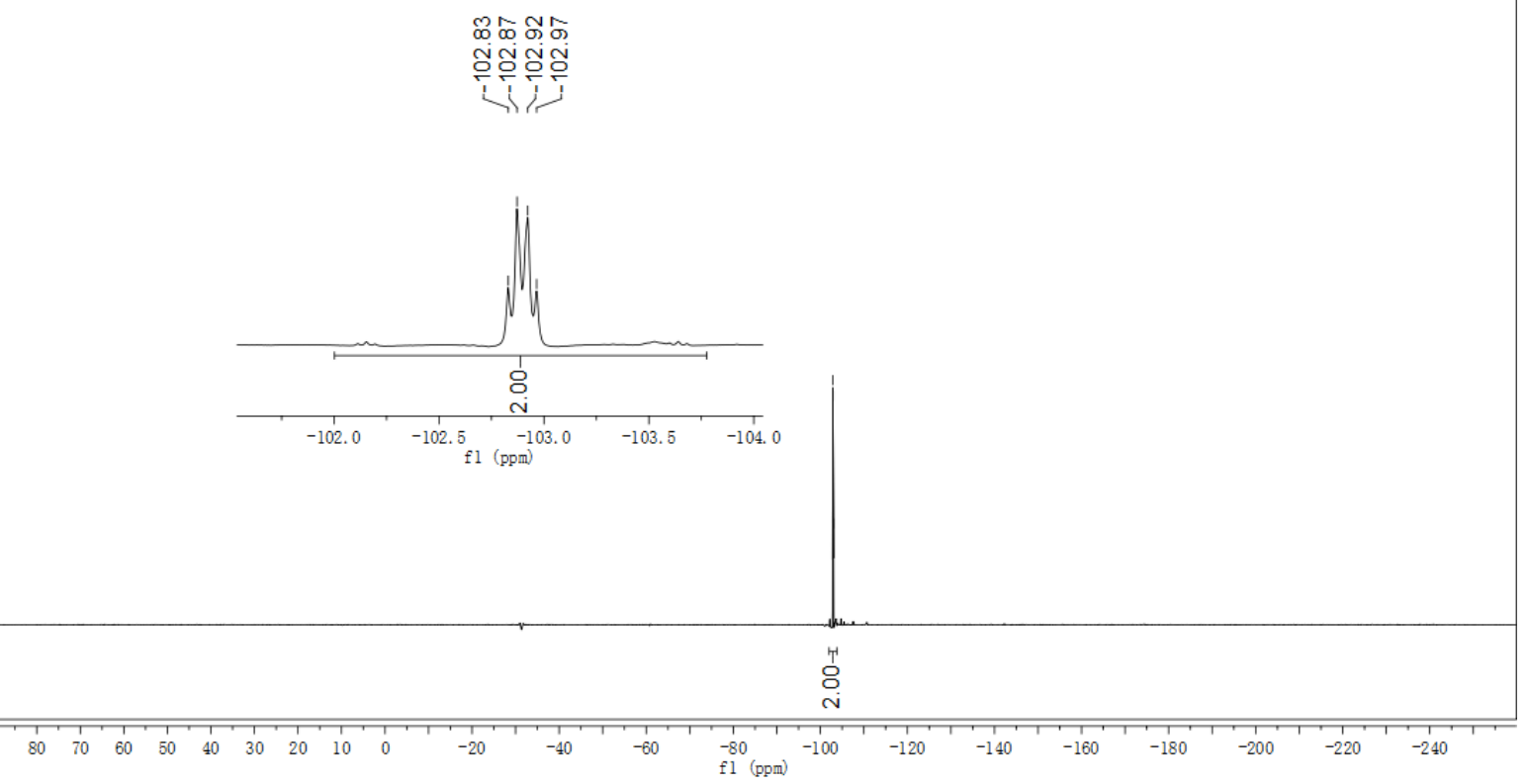




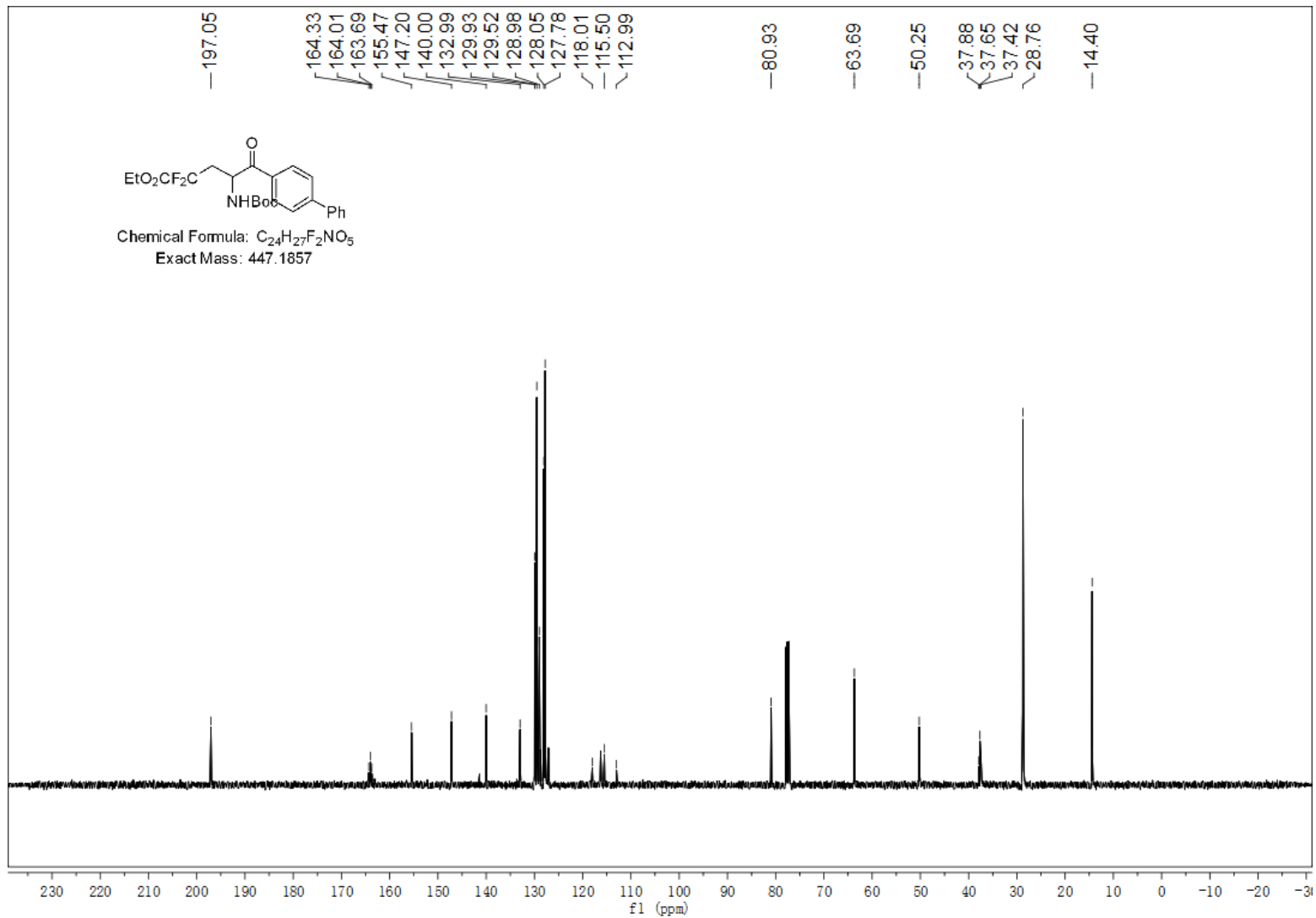

Ethyl 5-(4-(benzyloxy)phenyl)-4-((tert-butoxycarbonyl)amino)-2,2-difluoro-5-oxopentanoate (6e).

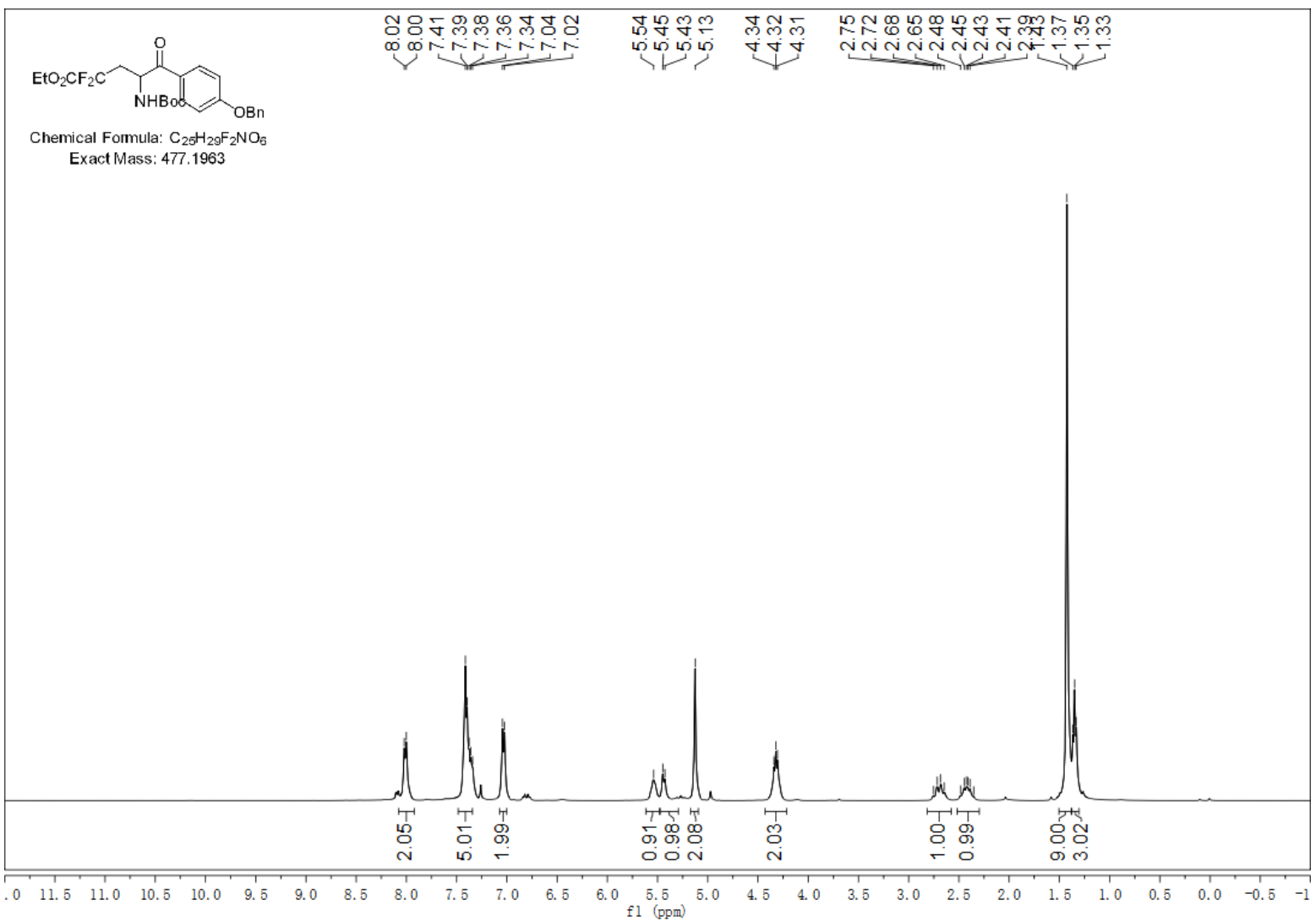



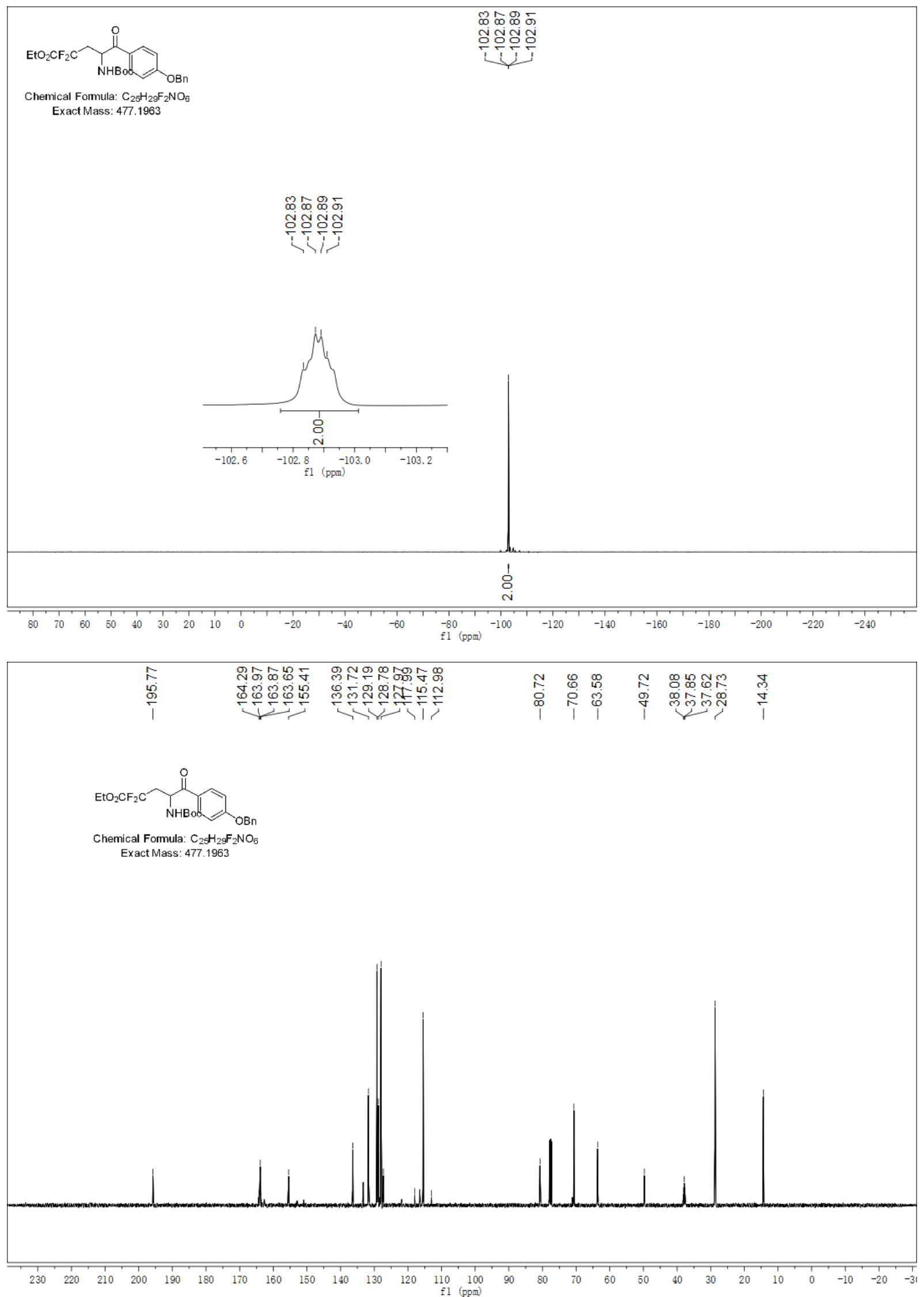
Ethyl 4-benzamido-5-(4-(ethylthio)phenyl)-2,2-difluoro-5-oxopentanoate (6f).

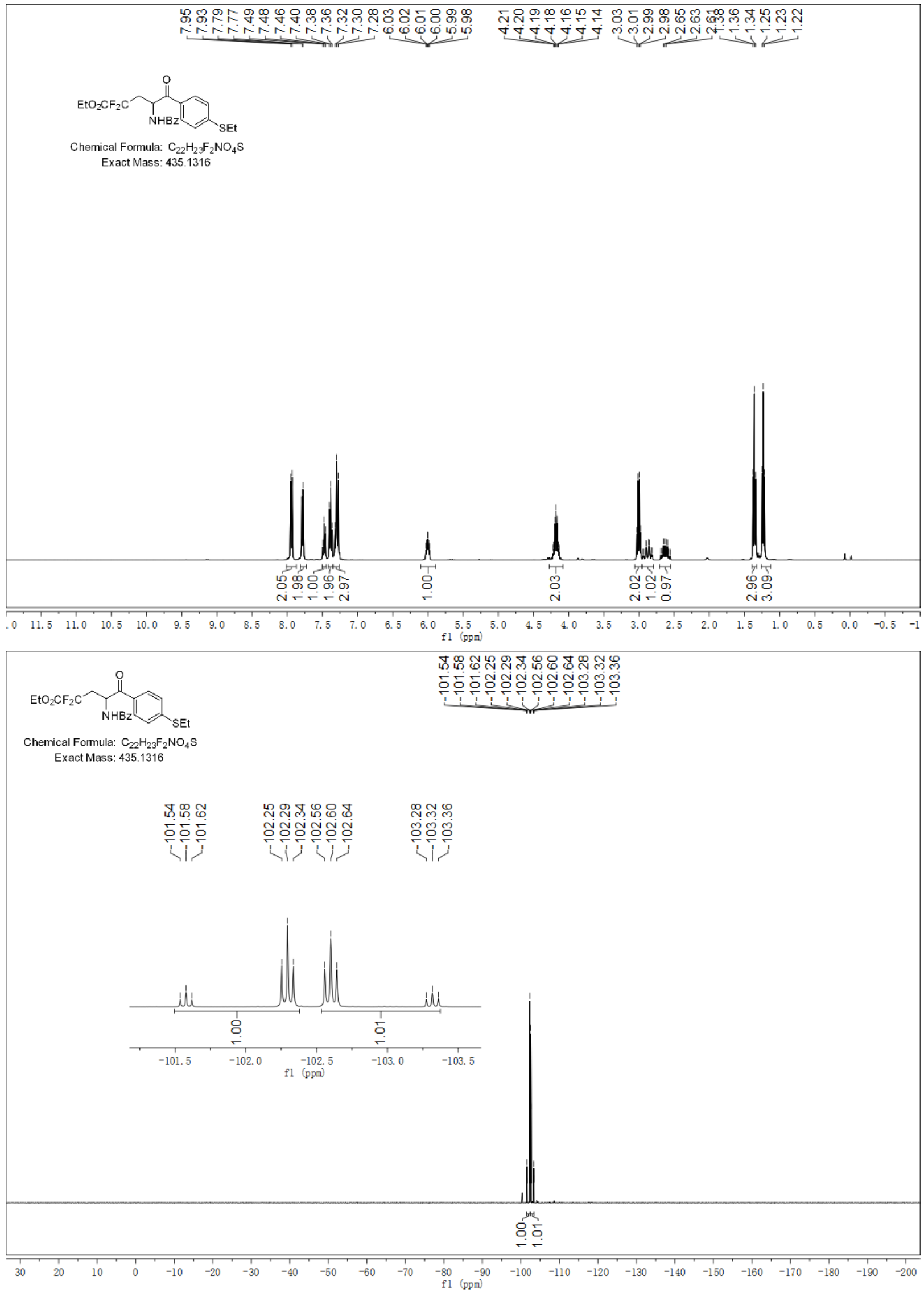




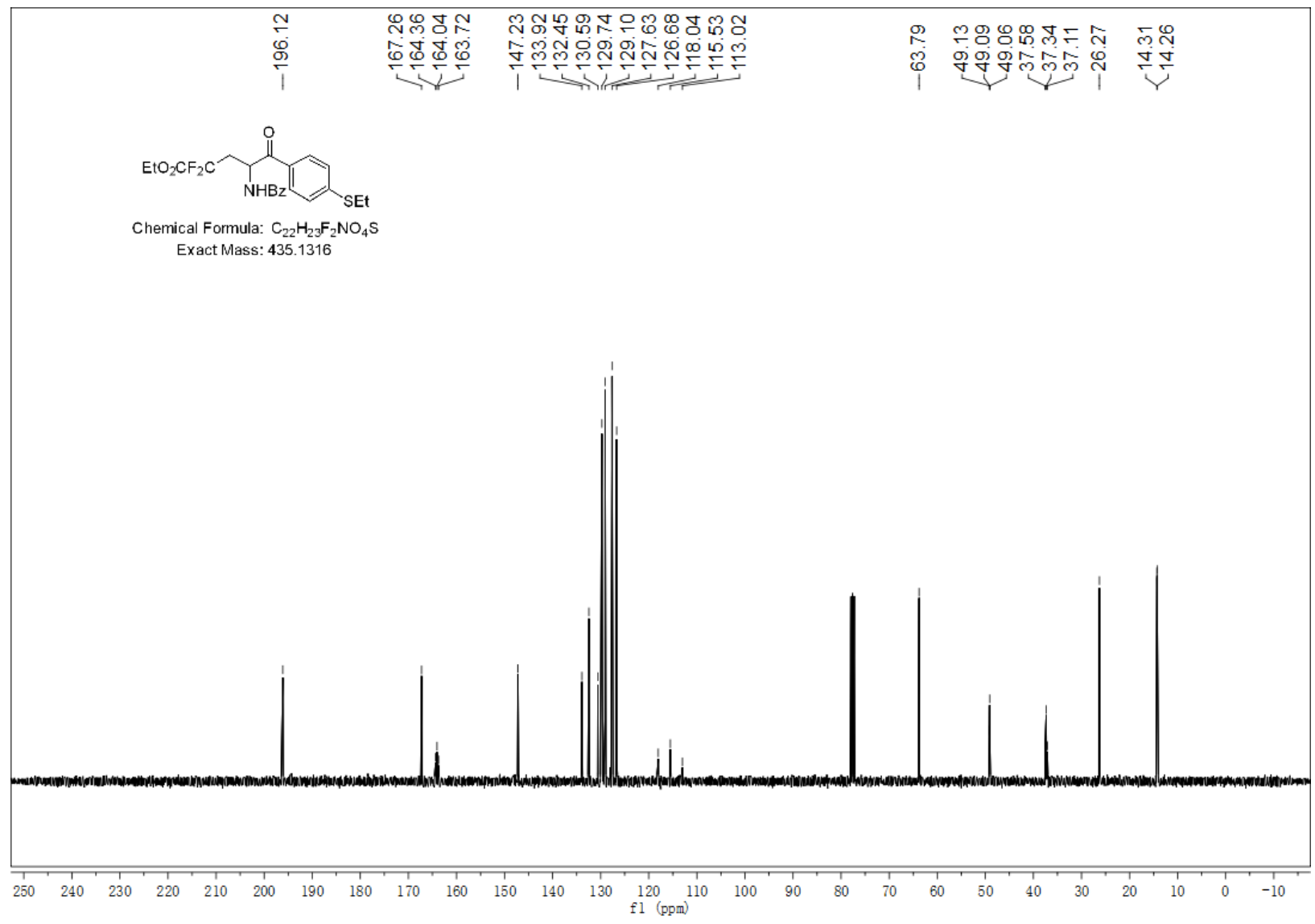

Ethyl 5-([1,1'-biphenyl]-4-yl)-2,2-difluoro-5-oxo-4-(2-oxopyrrolidin-1-yl)pentanoate (6g).

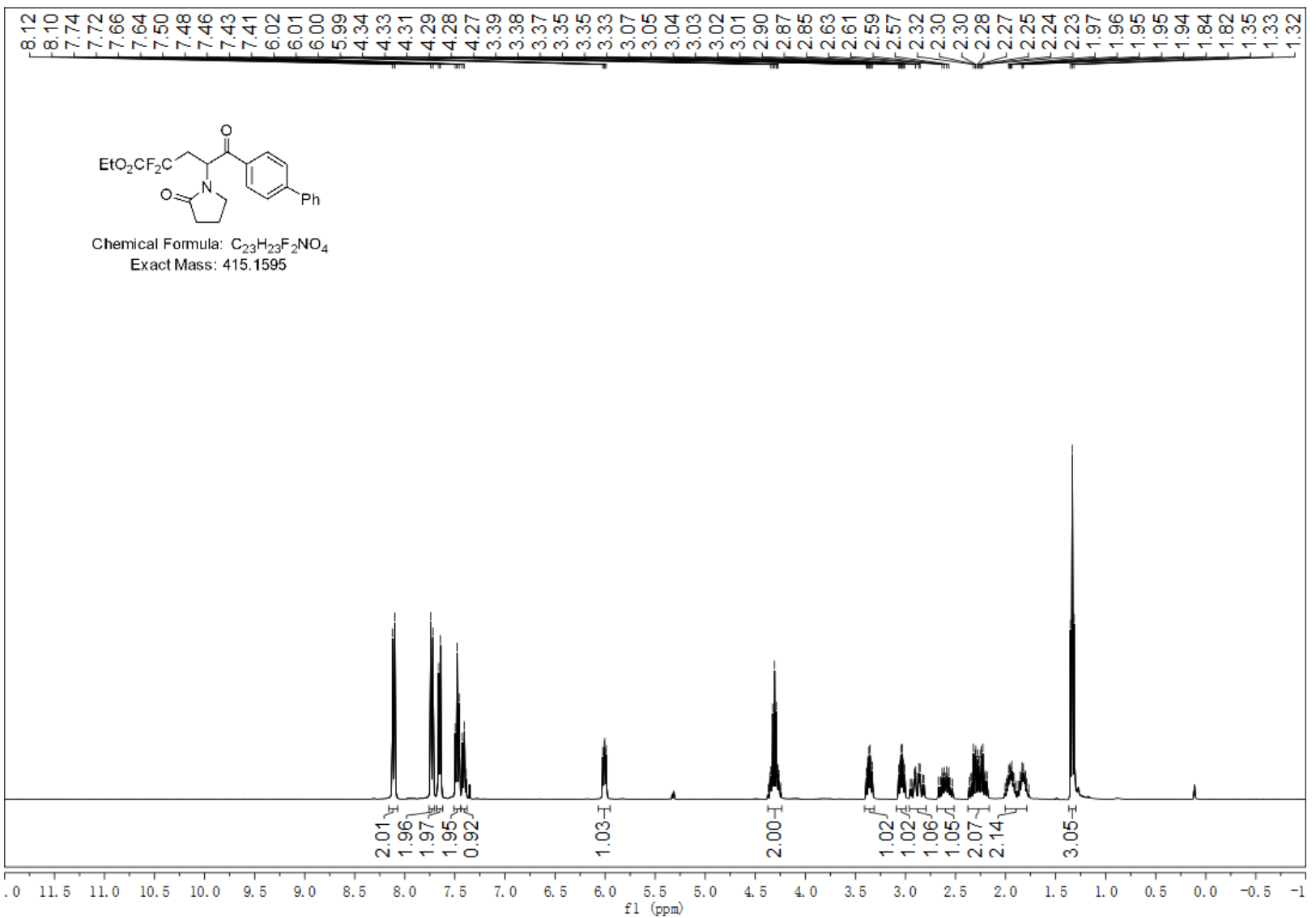



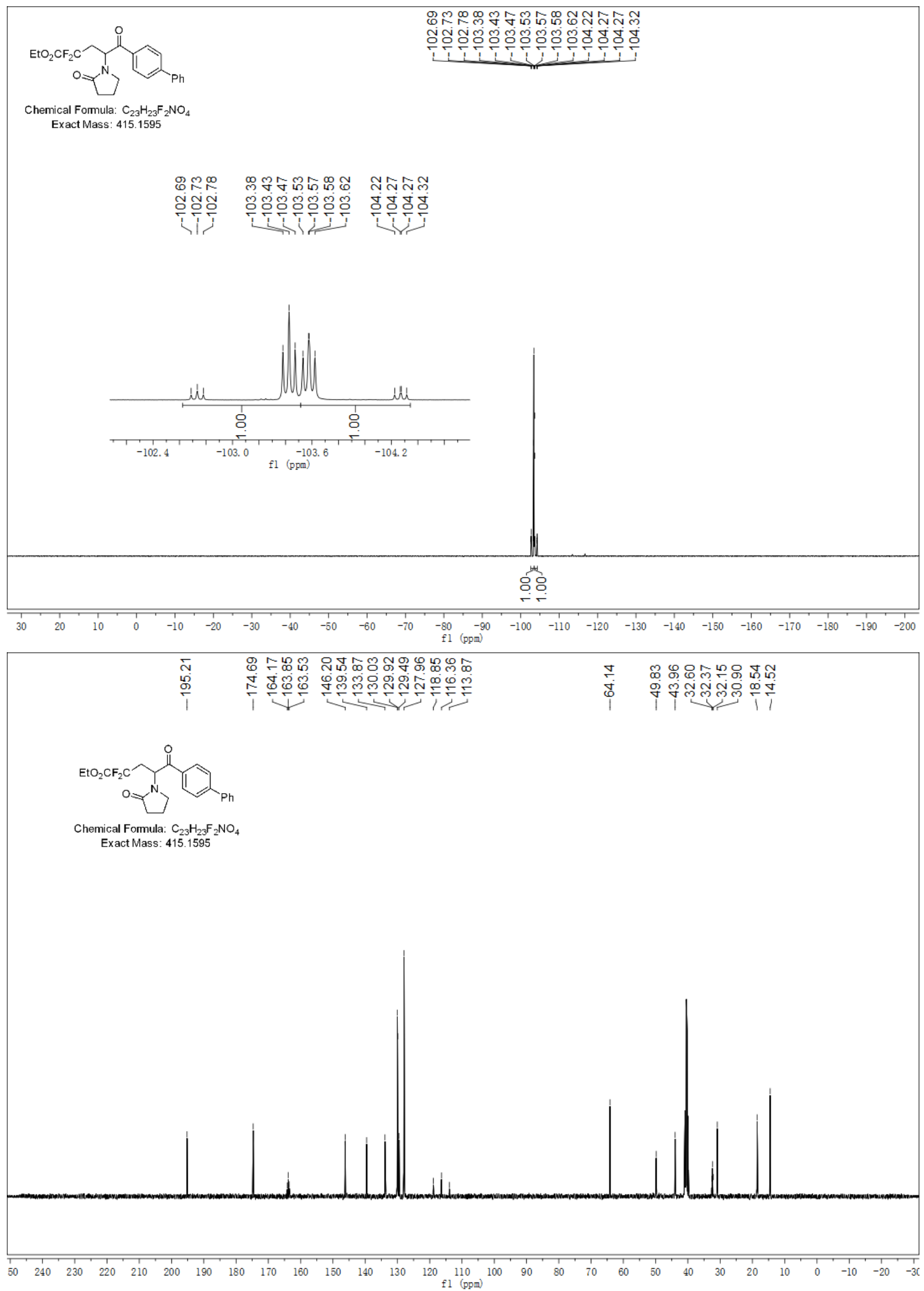
Ethyl 5-(4-chlorophenyl)-2,2-difluoro-5-oxo-4-(2-oxopyrrolidin-1-yl)pentanoate (6h).
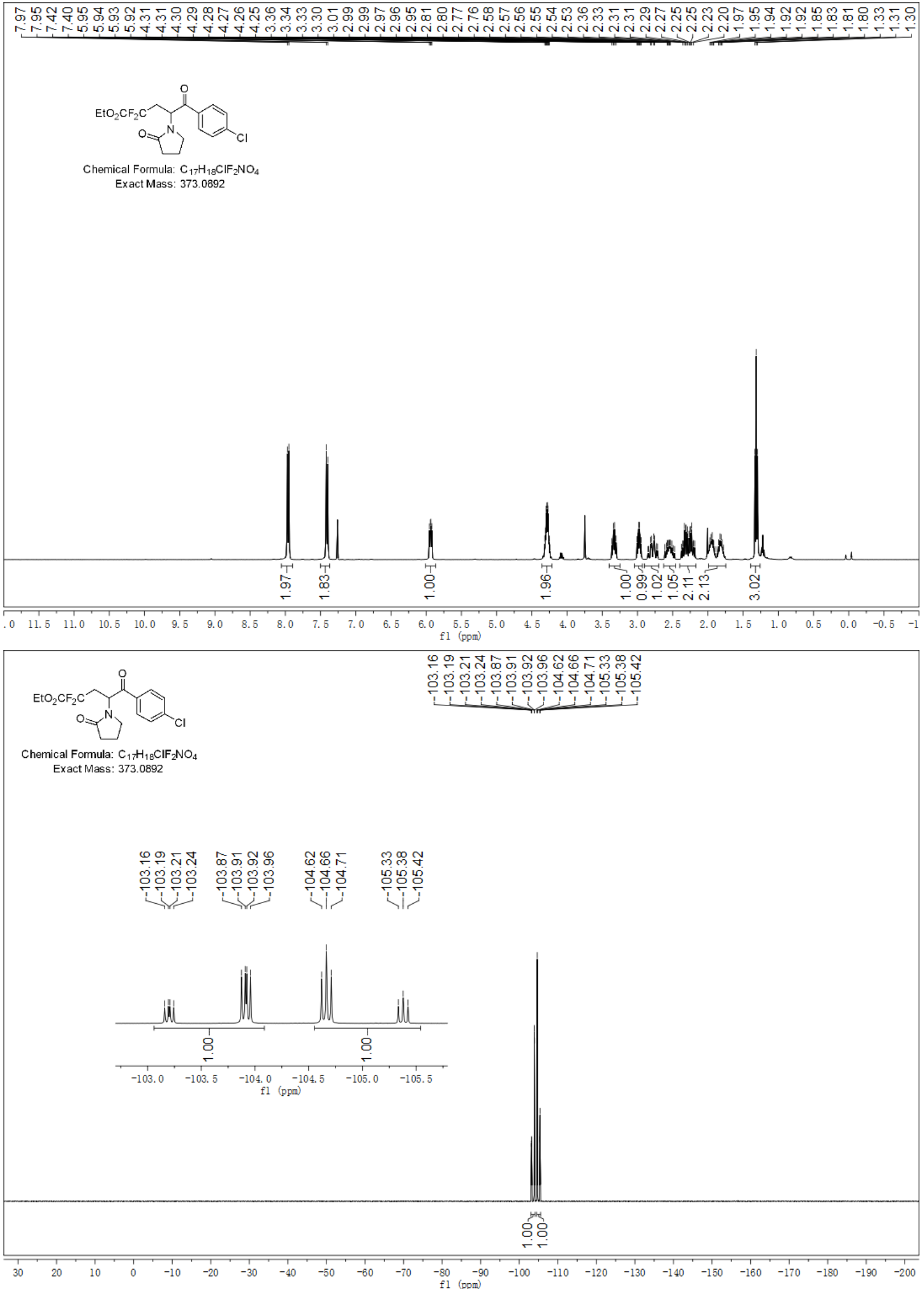


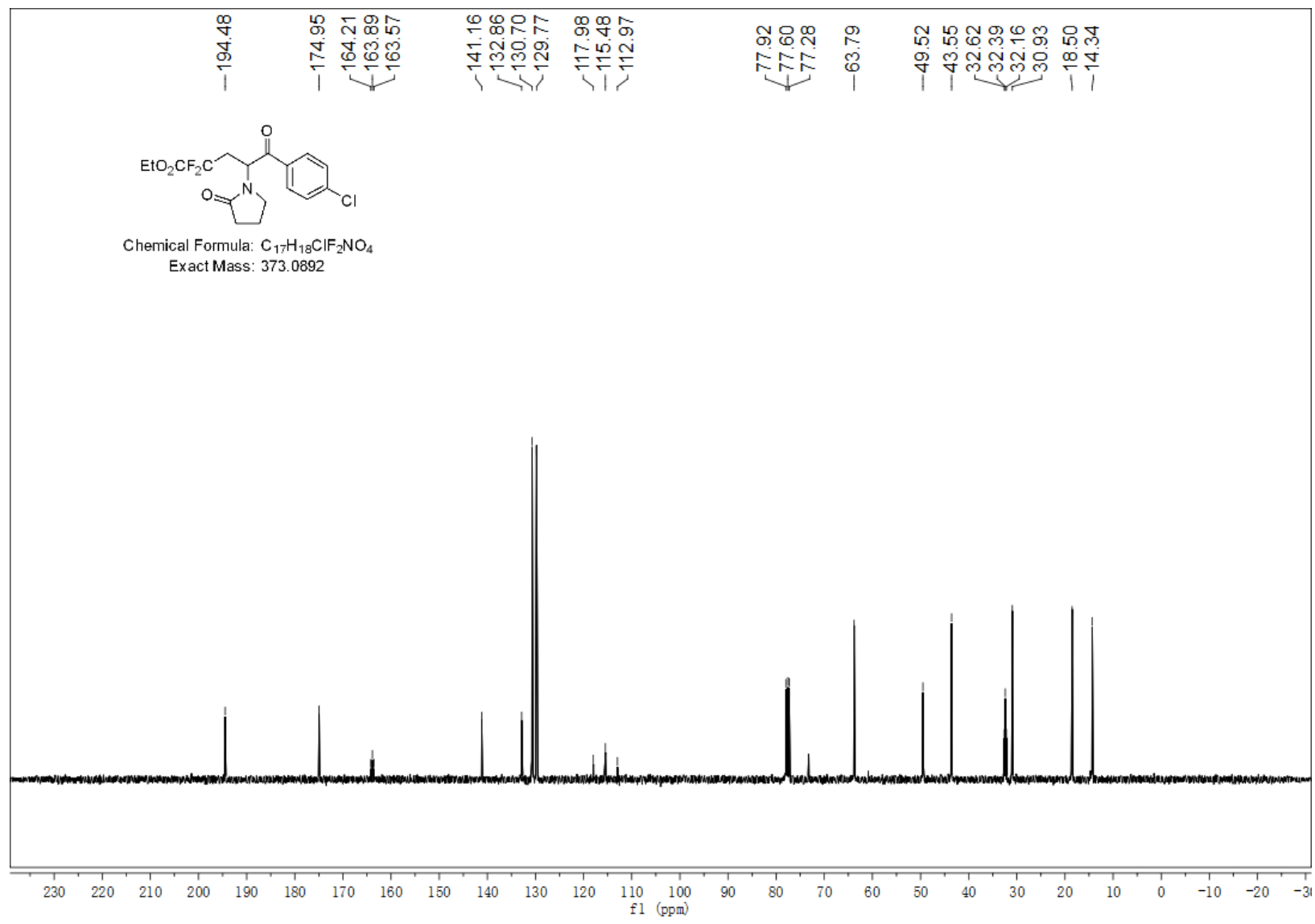

Ethyl 5-([1,1'-biphenyl]-4-yl)-4-acetoxy-2,2-difluoro-5-oxopentanoate (6i).

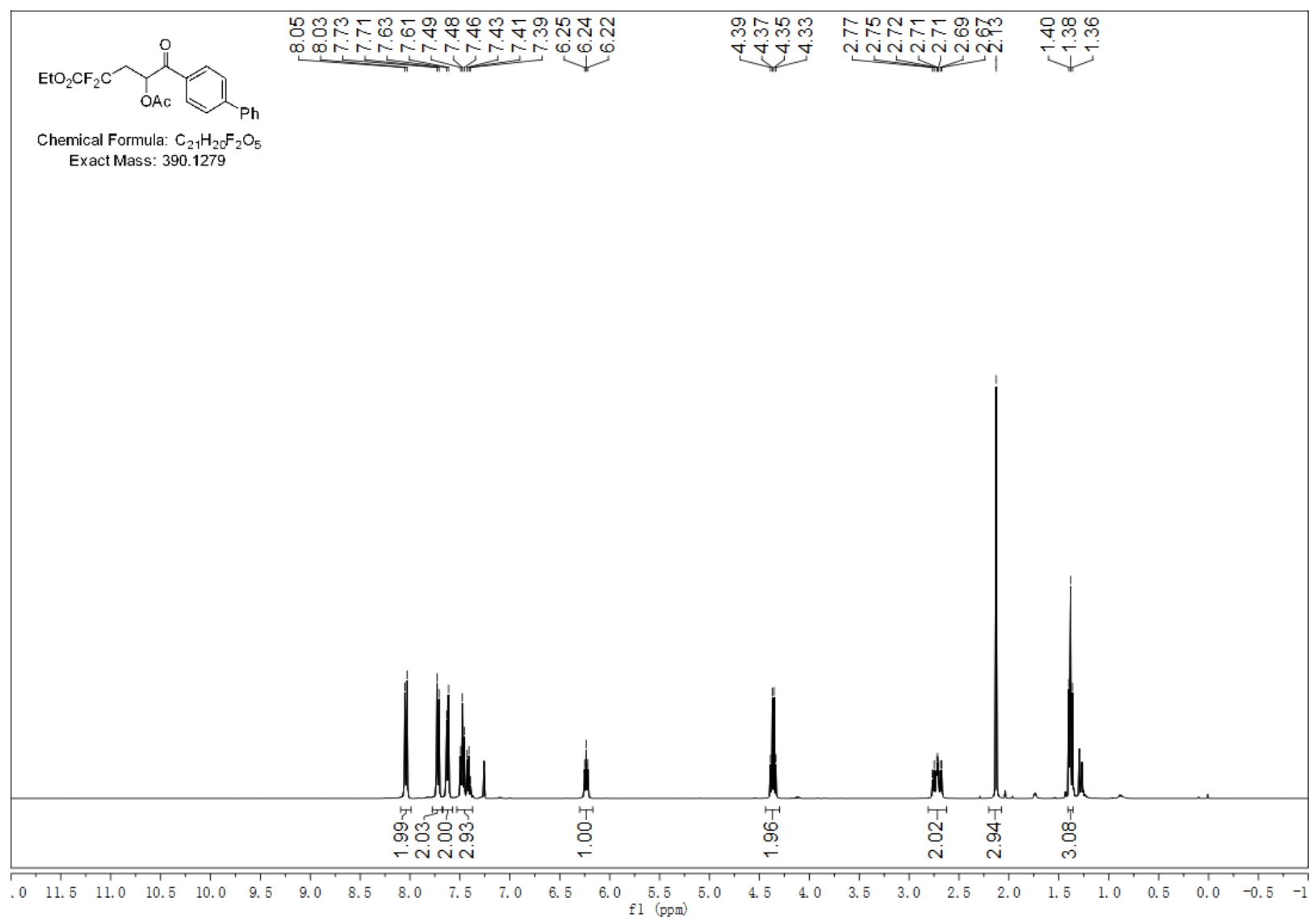




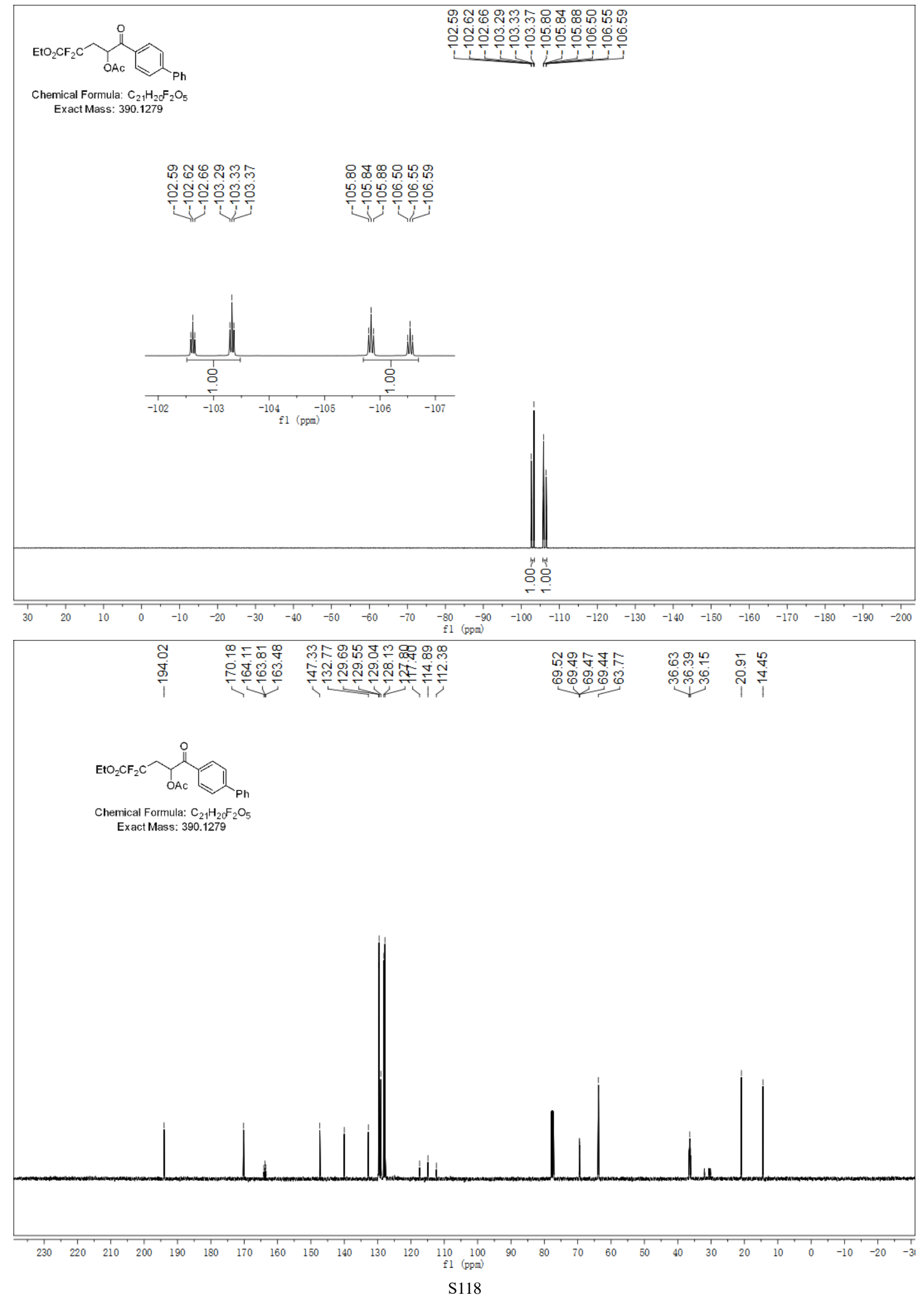


Ethyl 5-([1,1'-biphenyl]-4-yl)-4-acetamido-5-oxopentanoate (6j).
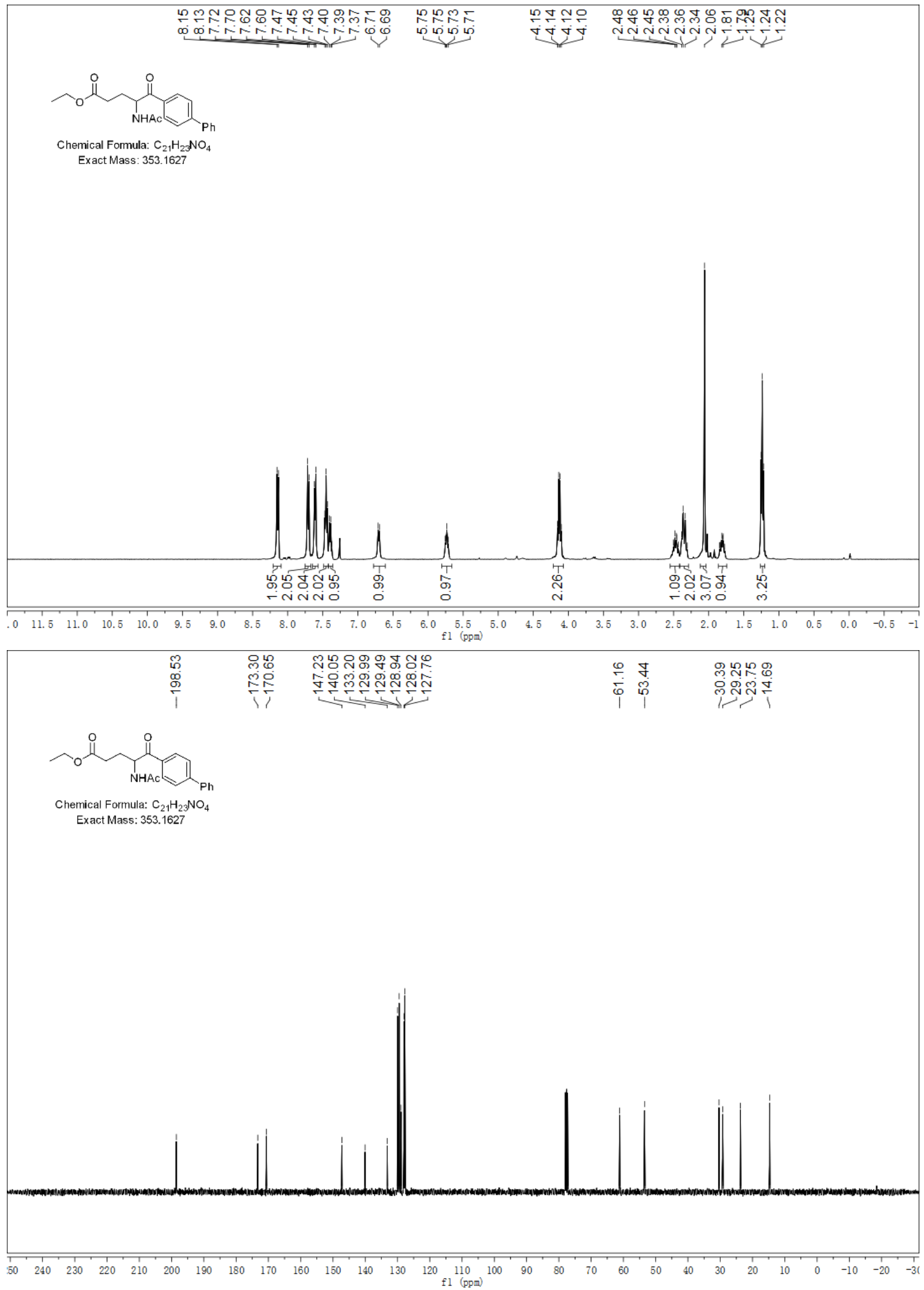
Ethyl 5-([1,1'-biphenyl]-4-yl)-4-acetamido-2,2-dimethyl-5-oxopentanoate (6k).

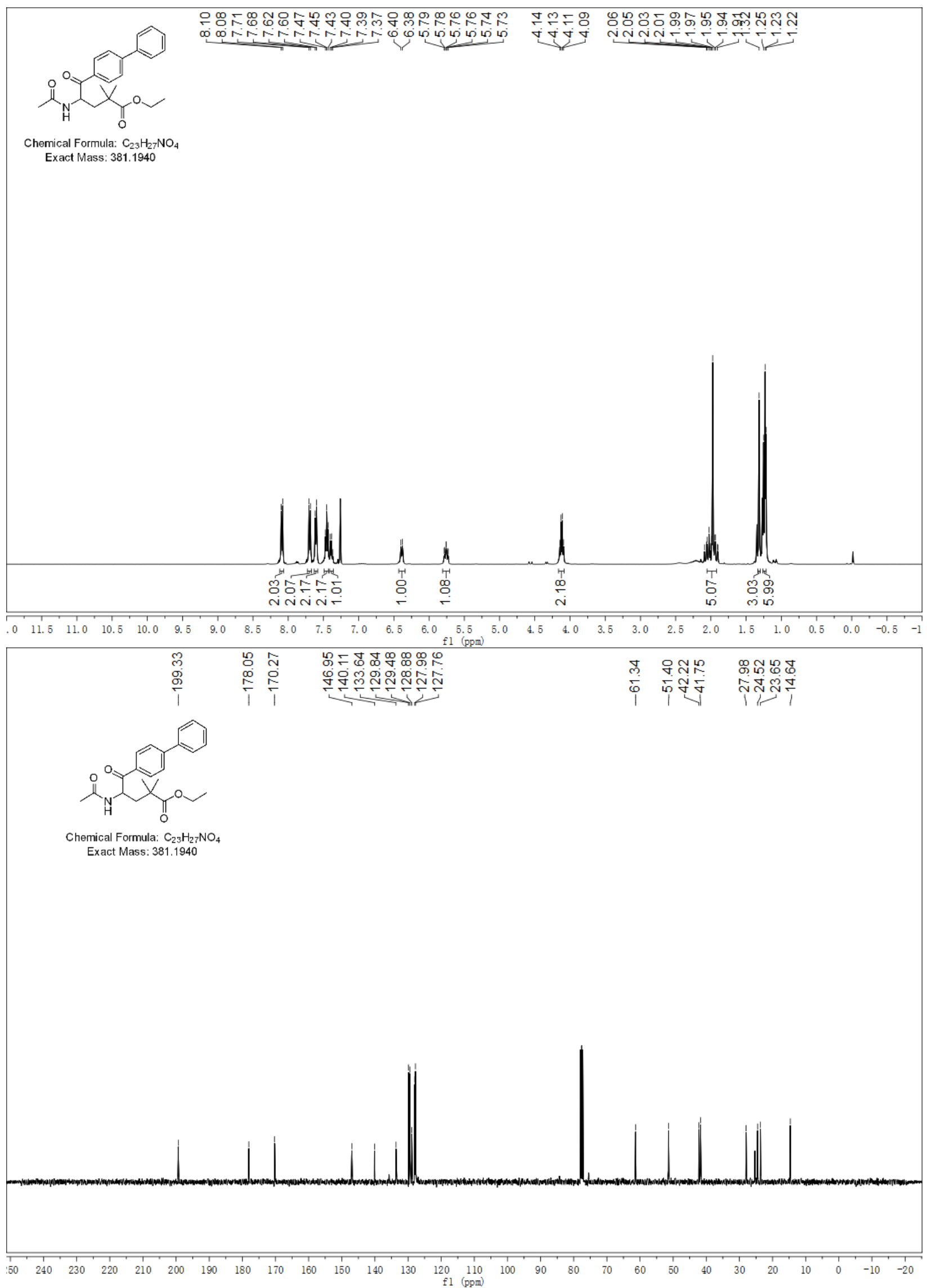


Ethyl 1-(3-([1,1'-biphenyl]-4-yl)-2-acetamido-3-oxopropyl)cyclobutane-1-carboxylate (61).

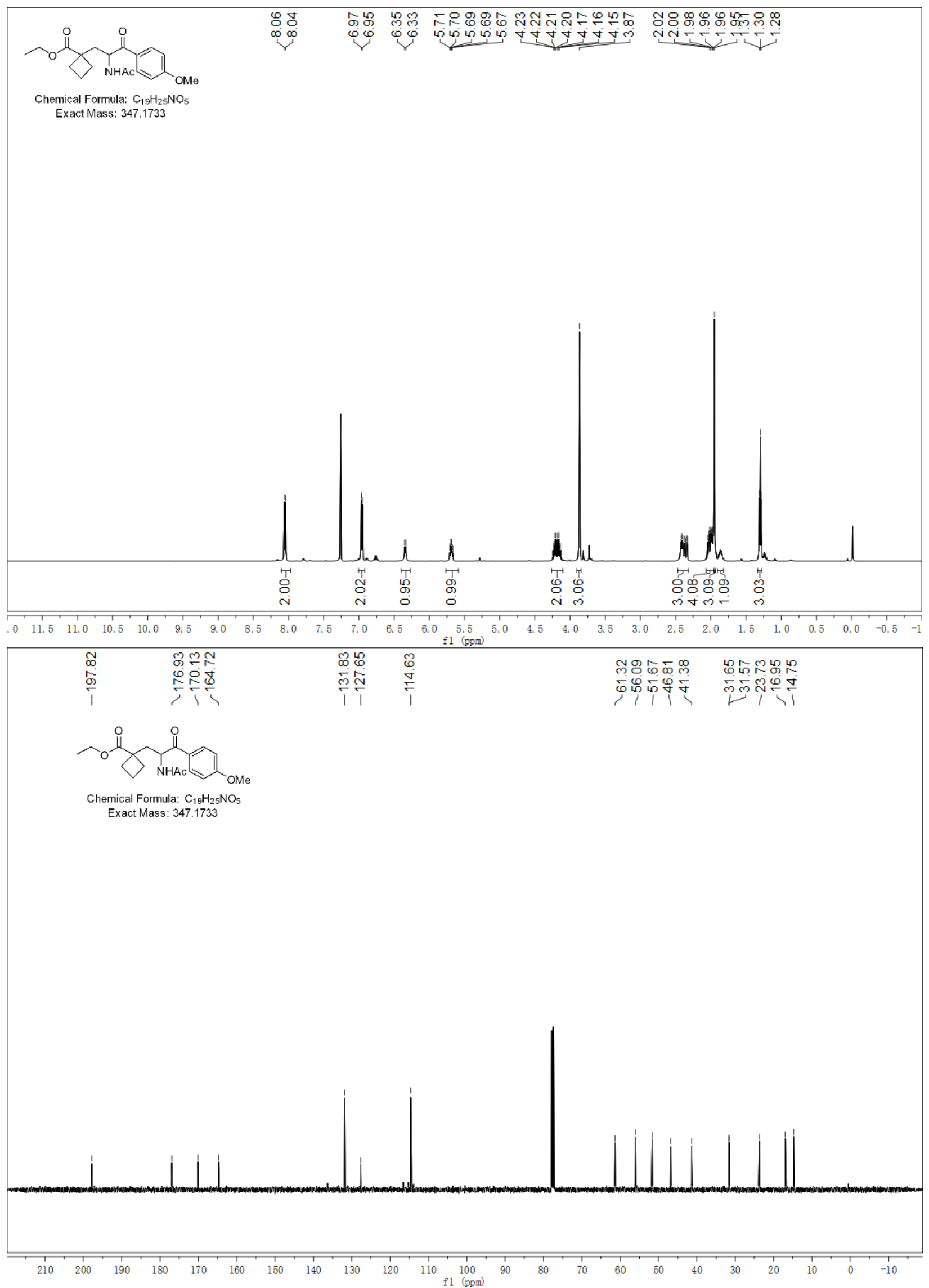


Ethyl 5-([1,1'-biphenyl]-4-yl)-4-(benzamidomethyl)-2,2-difluoro-5-oxopentanoate (7a).

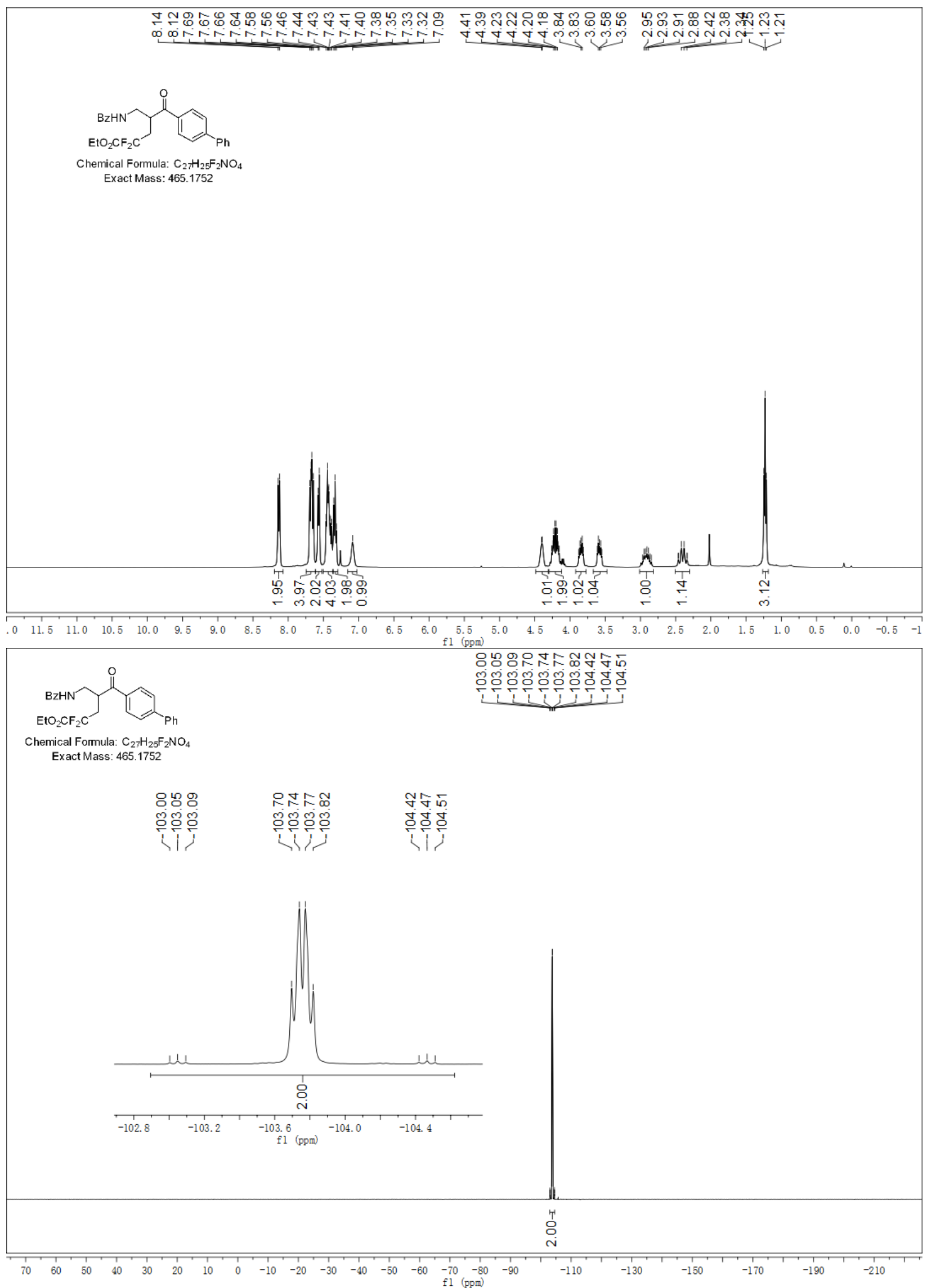




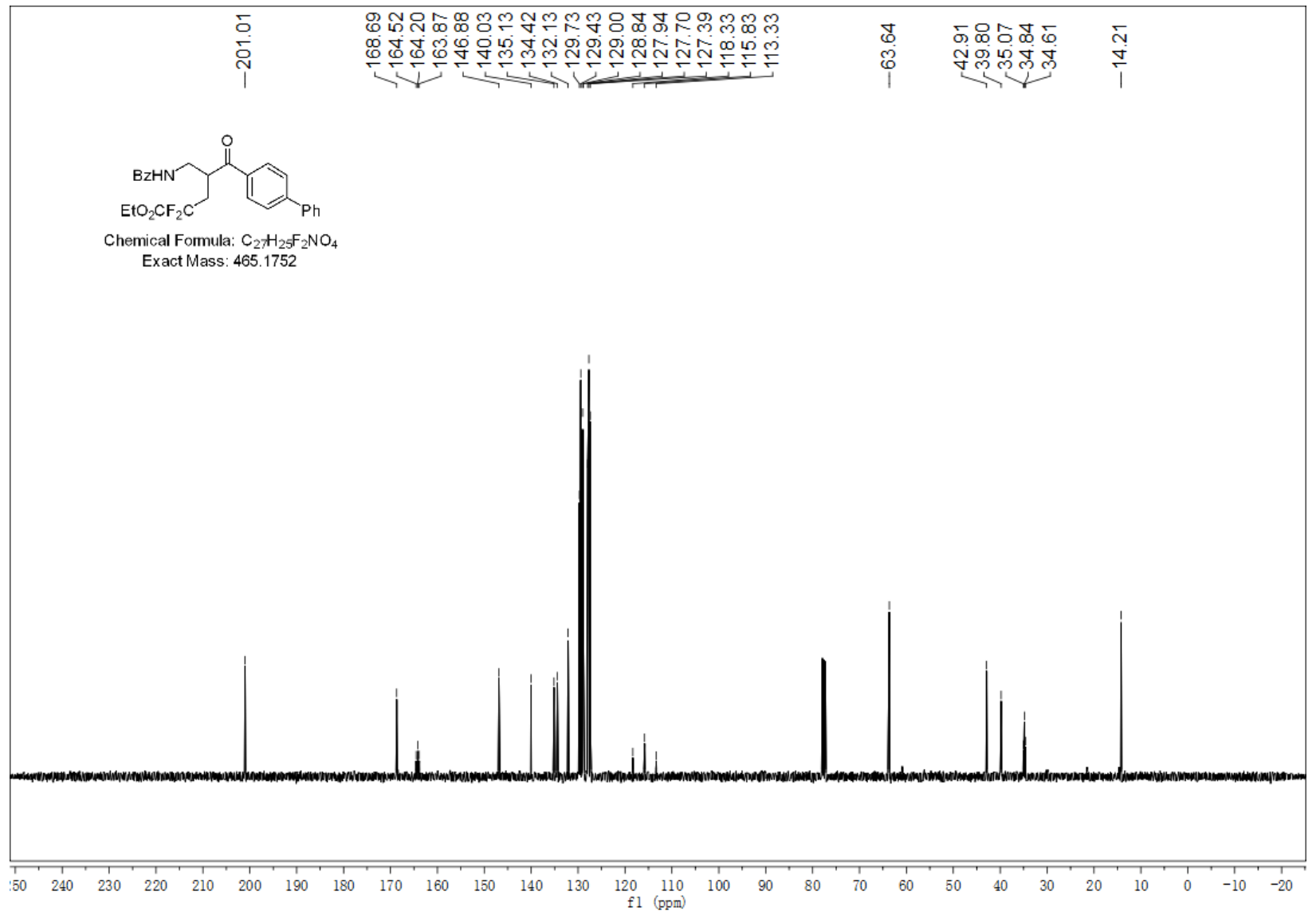

Ethyl 4-(benzamidomethyl)-5-(4-chlorophenyl)-2,2-difluoro-5-oxopentanoate (7b).

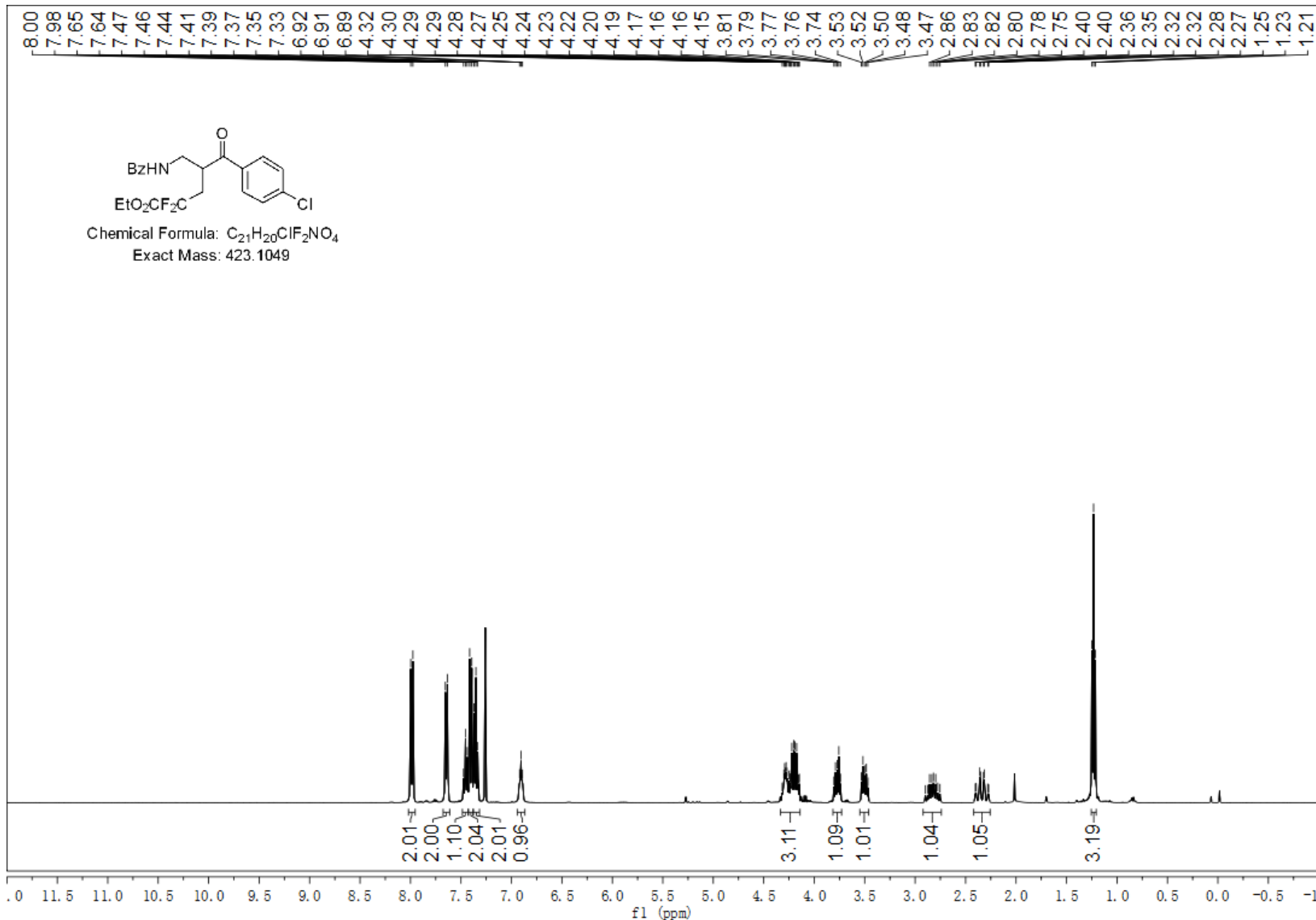




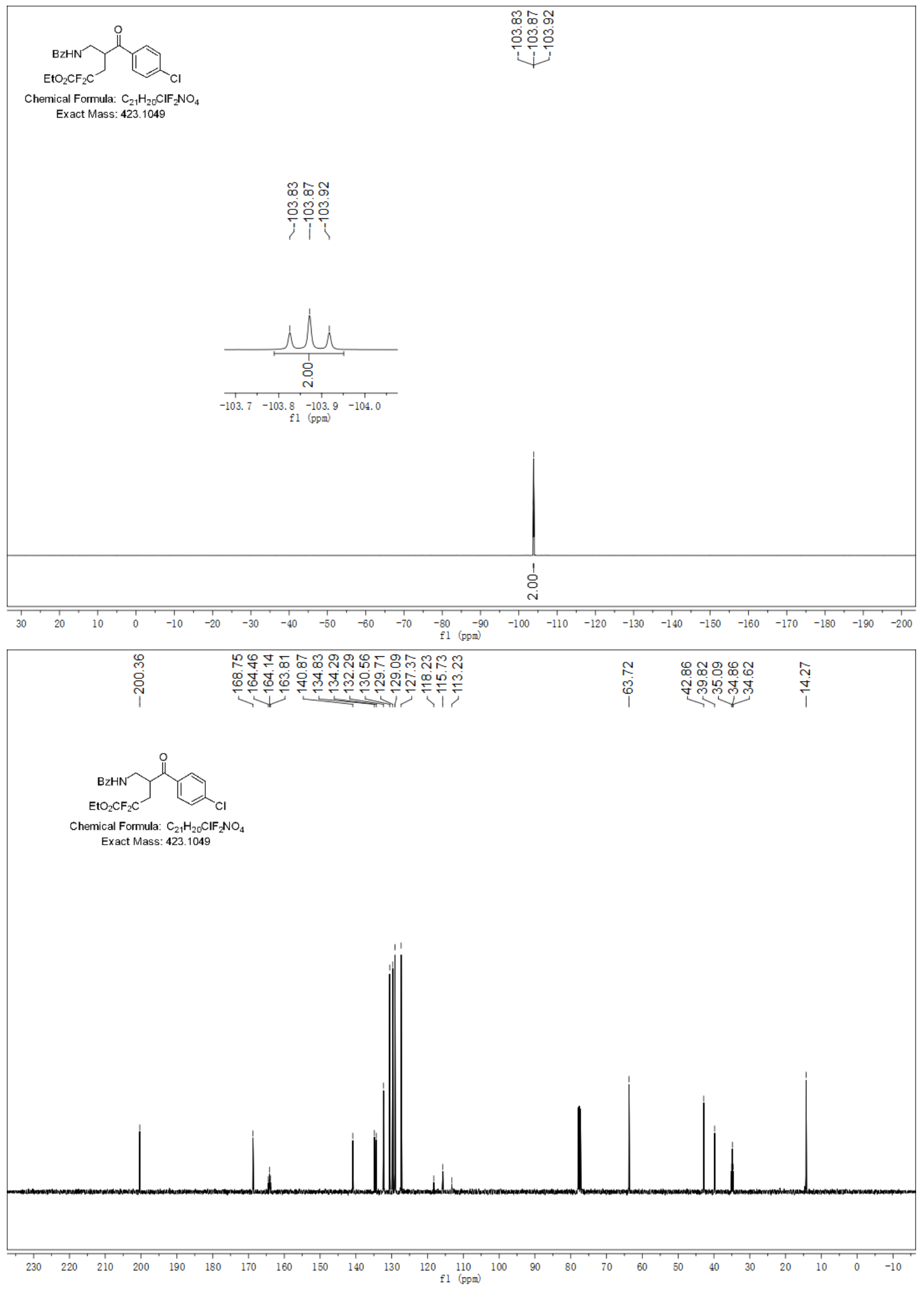


Ethyl 4-(benzamidomethyl)-2,2-difluoro-5-(6-methoxypyridin-3-yl)-5-oxopentanoate (7c).
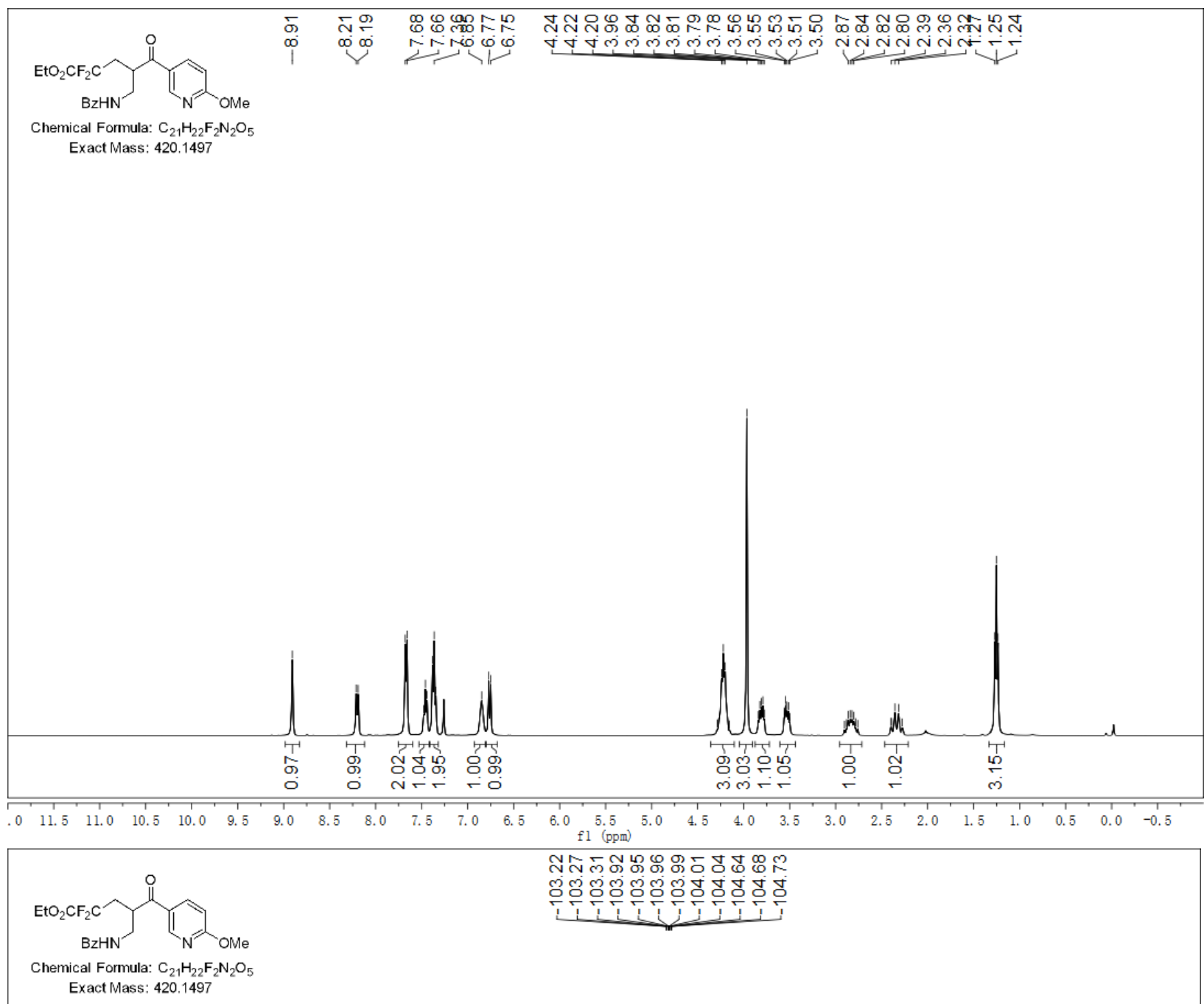

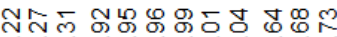

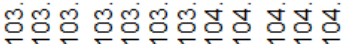

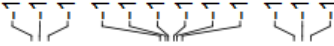

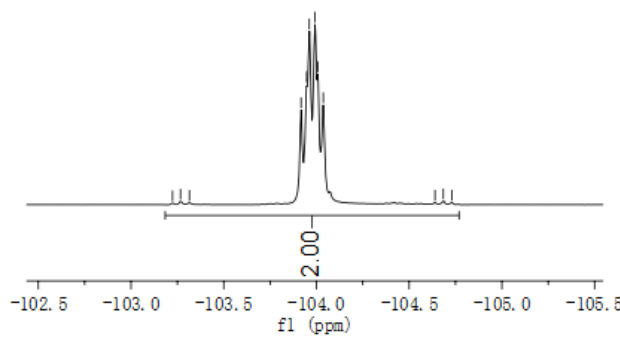

용

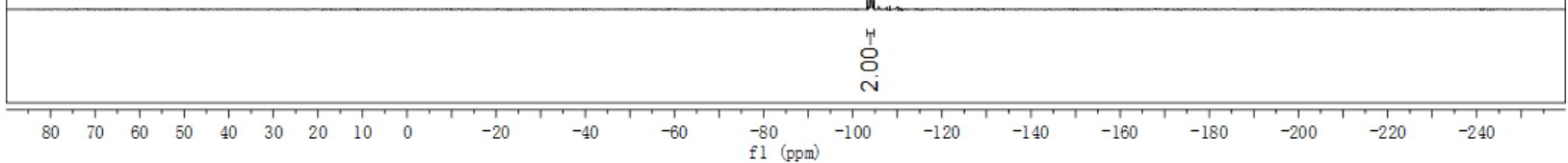




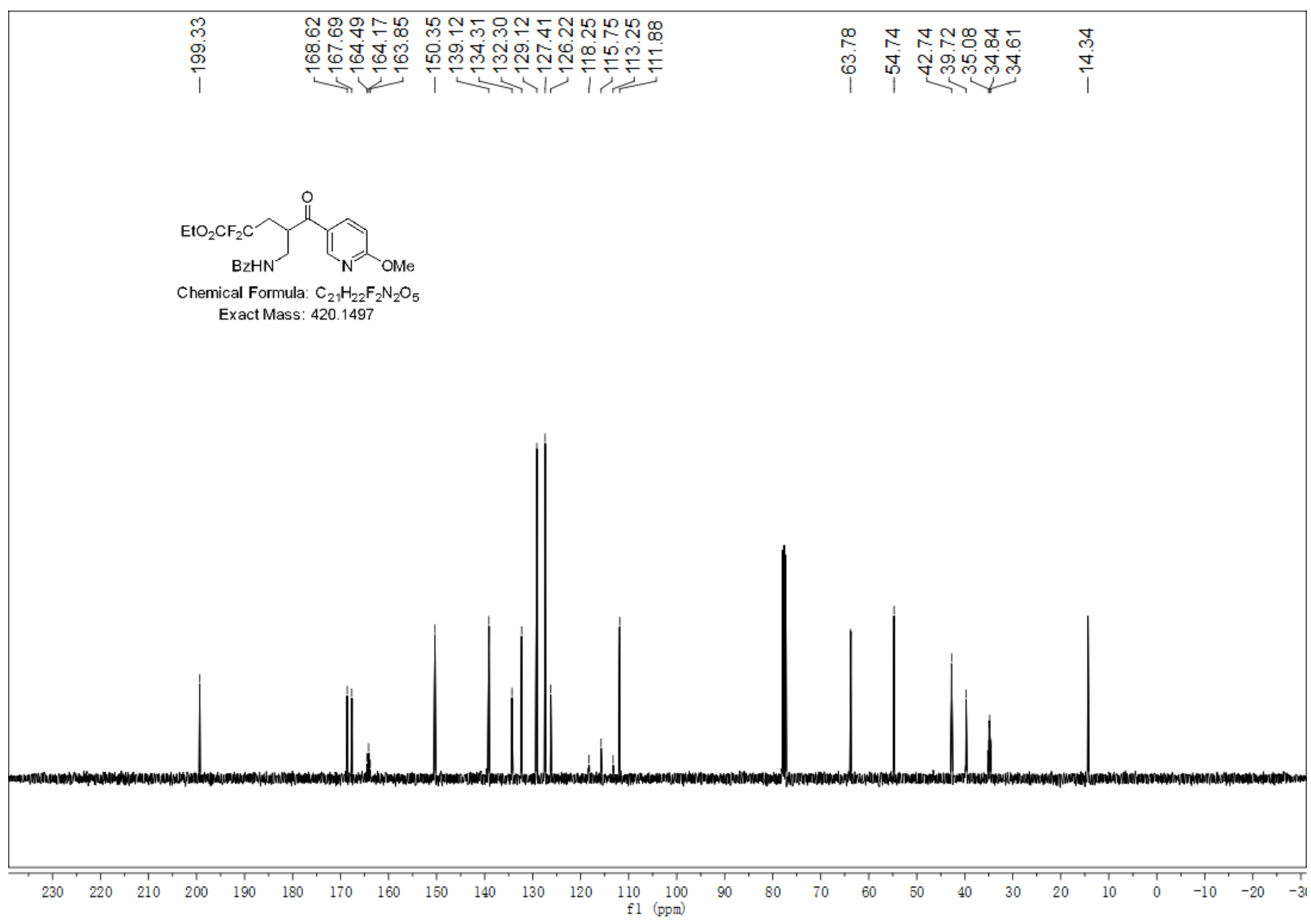

Ethyl 4-((tert-butoxycarbonyl)amino)methyl)-2,2-difluoro-5-oxo-5-phenylpentanoate (7d).

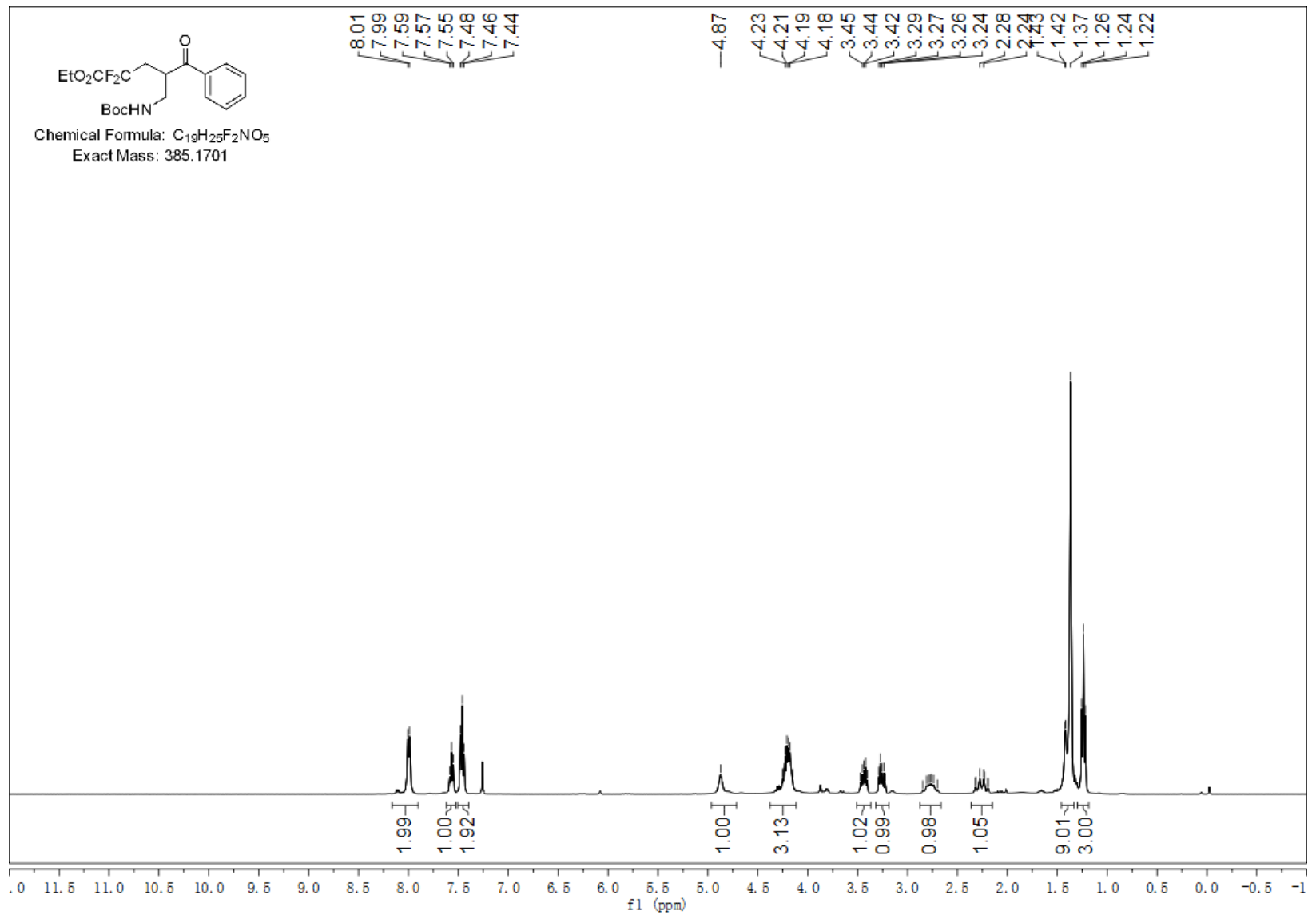




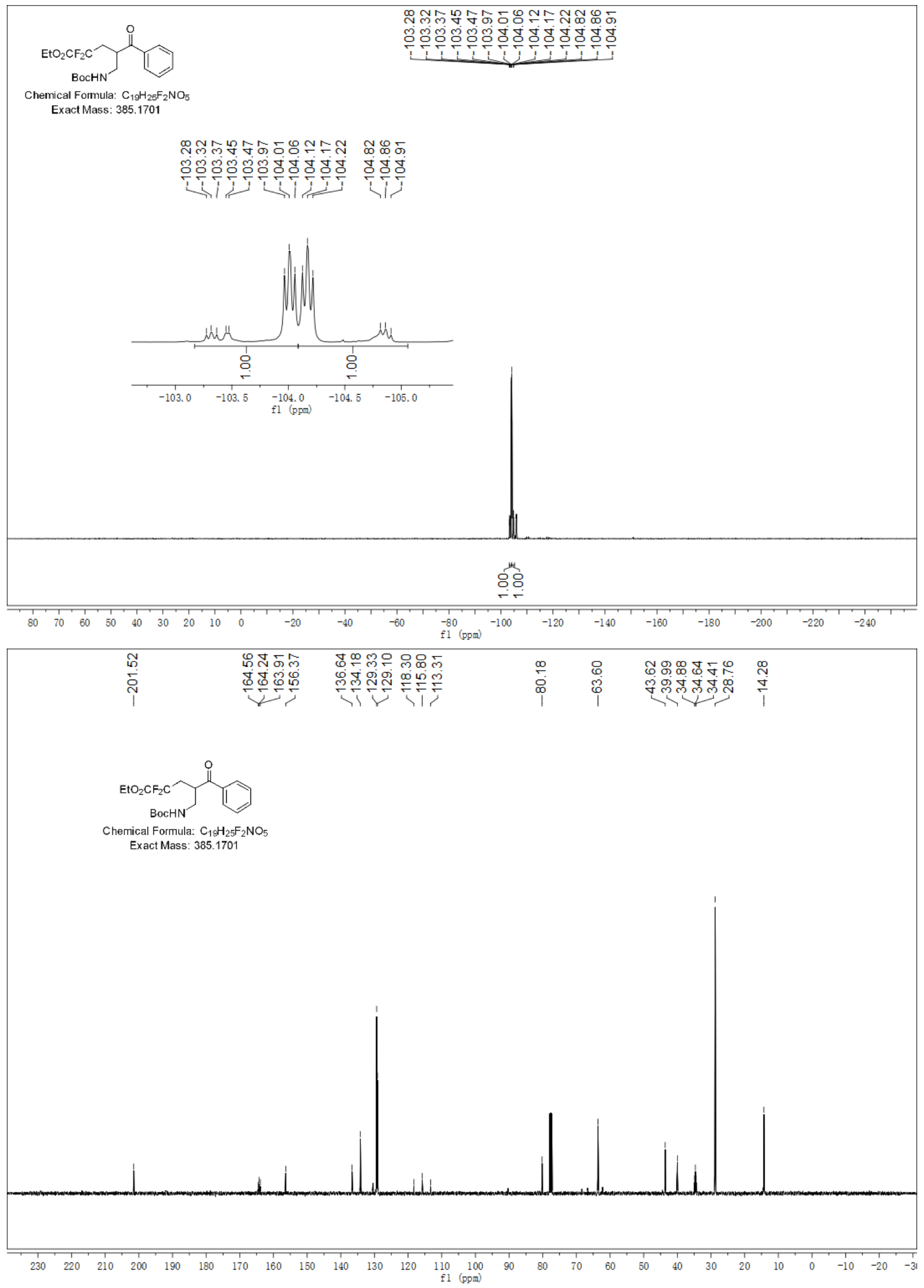


Ethyl 5-([1,1'-biphenyl]-4-yl)-4-(((tert-butoxycarbonyl)amino)methyl)-2,2-difluoro-5-oxopentanoate (7e).
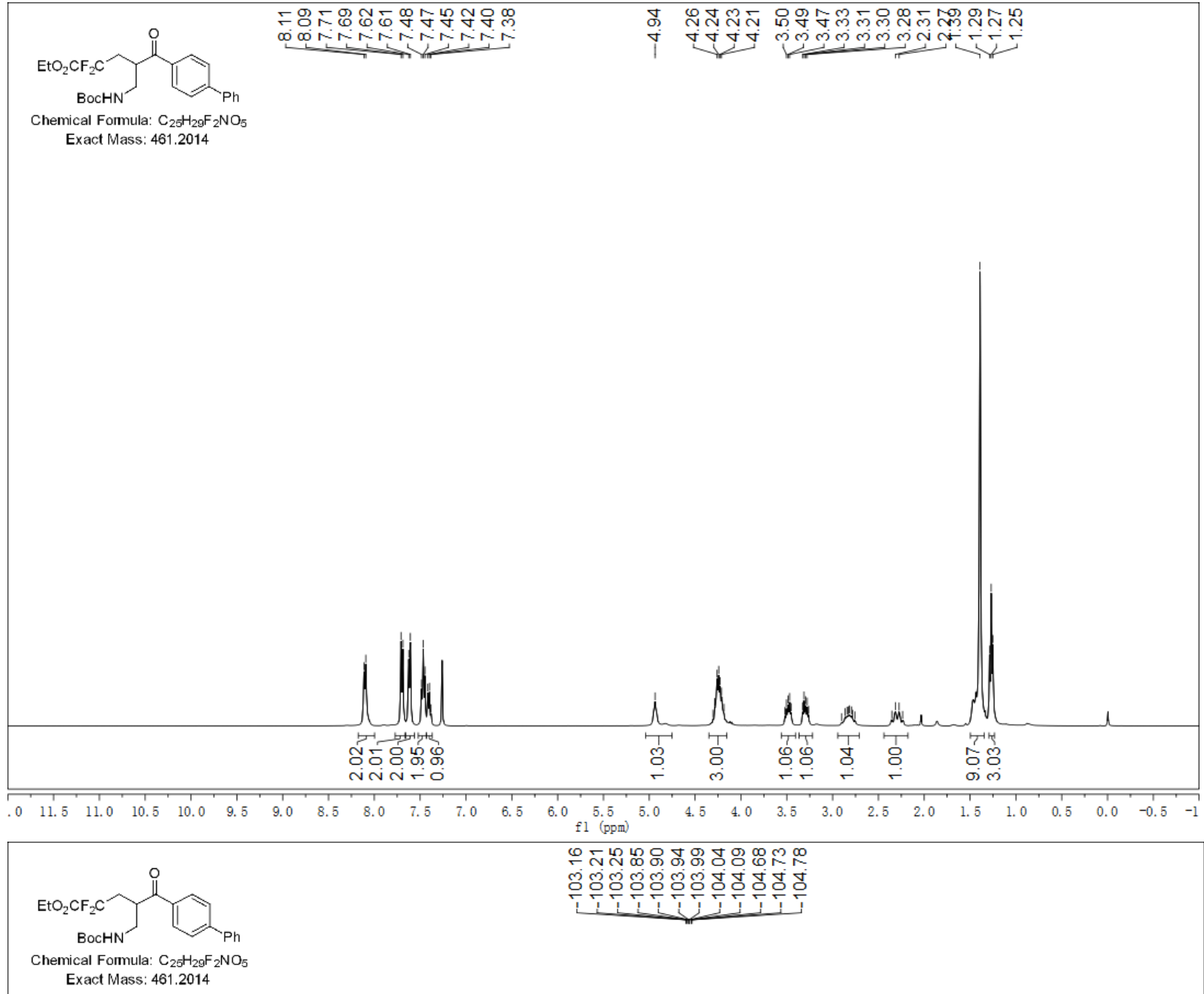

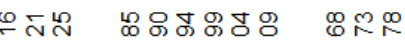

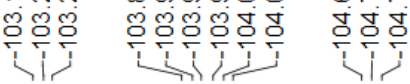

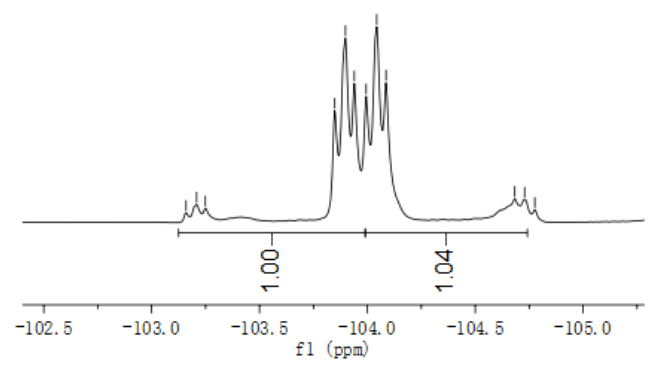

8ृष

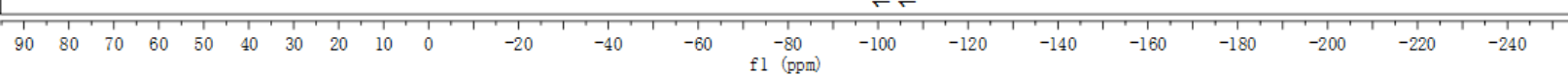




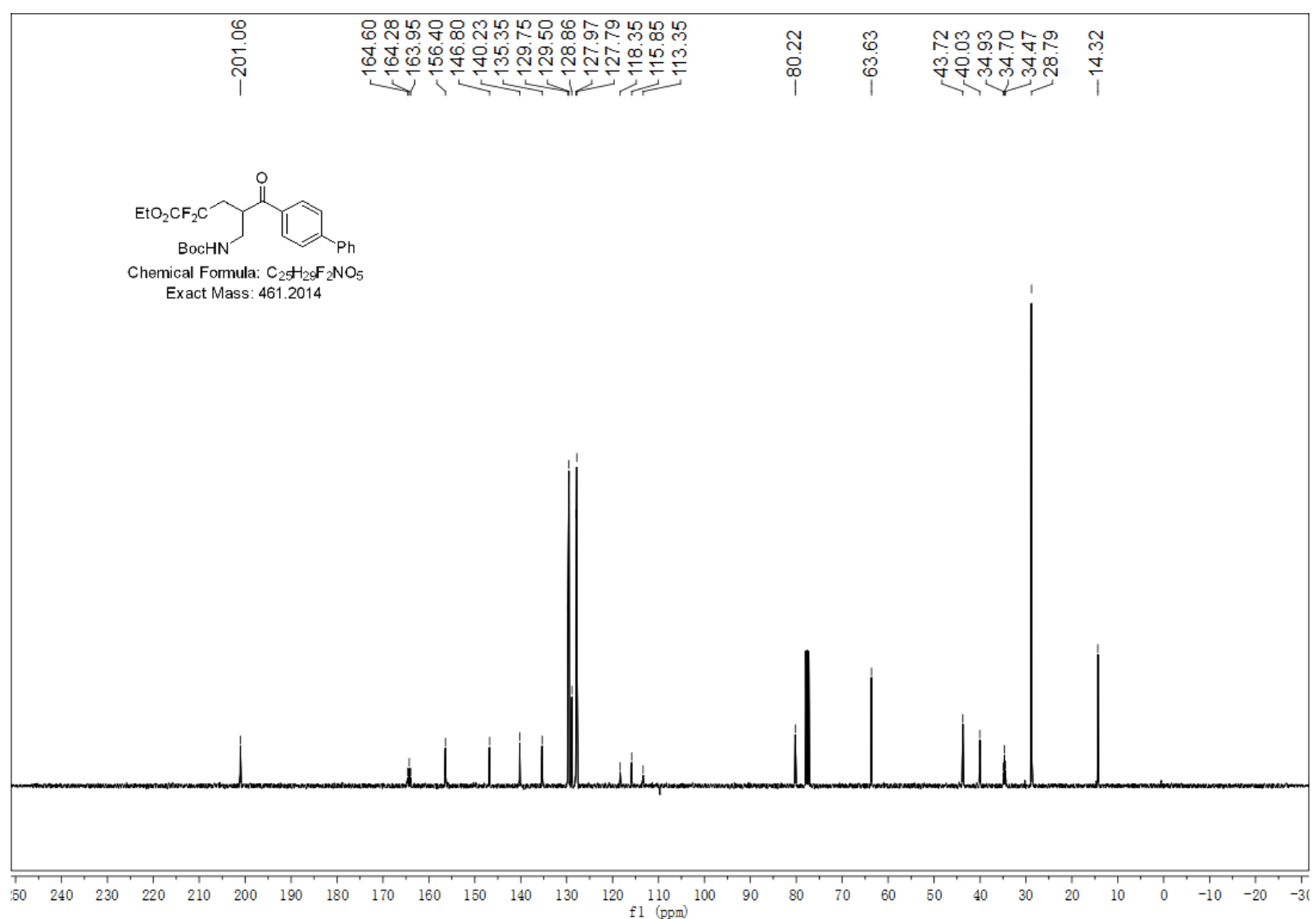

Ethyl 5-(4-(benzyloxy)phenyl)-4-(((tert-butoxycarbonyl)amino)methyl)-2,2-difluoro-5-oxopentanoate (7f).

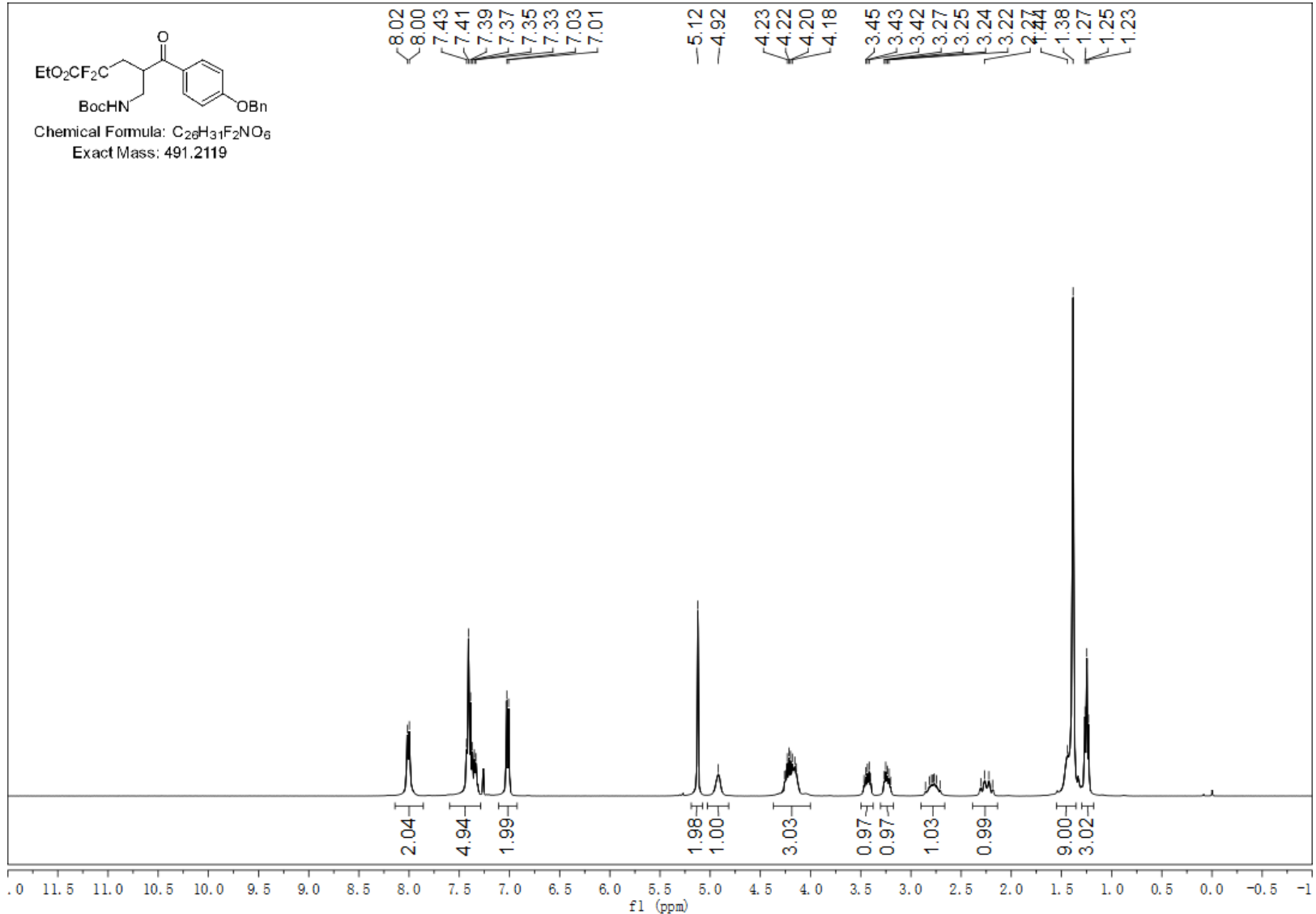



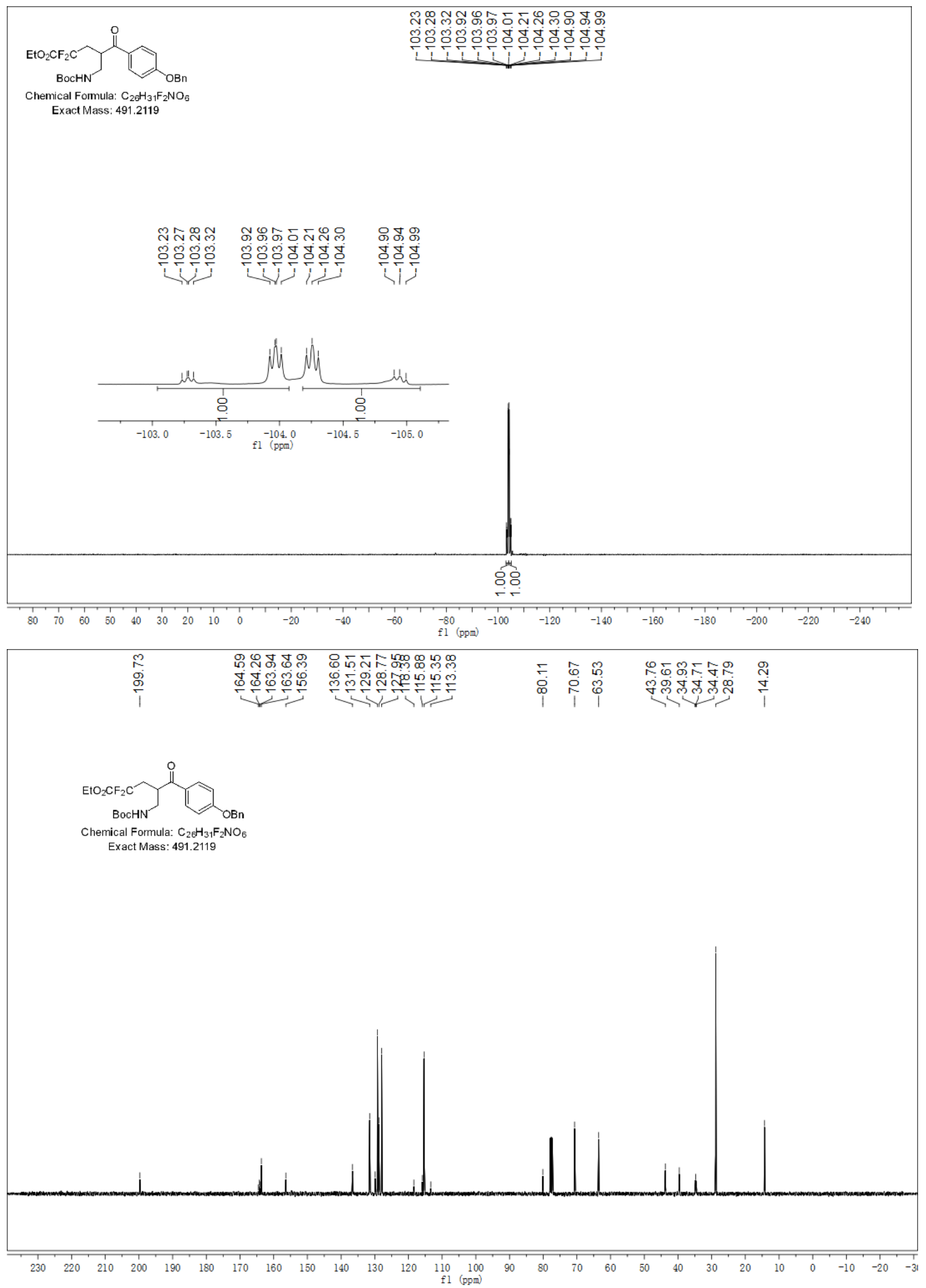
Ethyl 2,2-difluoro-5-(4-methoxyphenyl)-5-oxo-4-((2,2,2-trifluoroacetamido)methyl)pentanoate (7g).
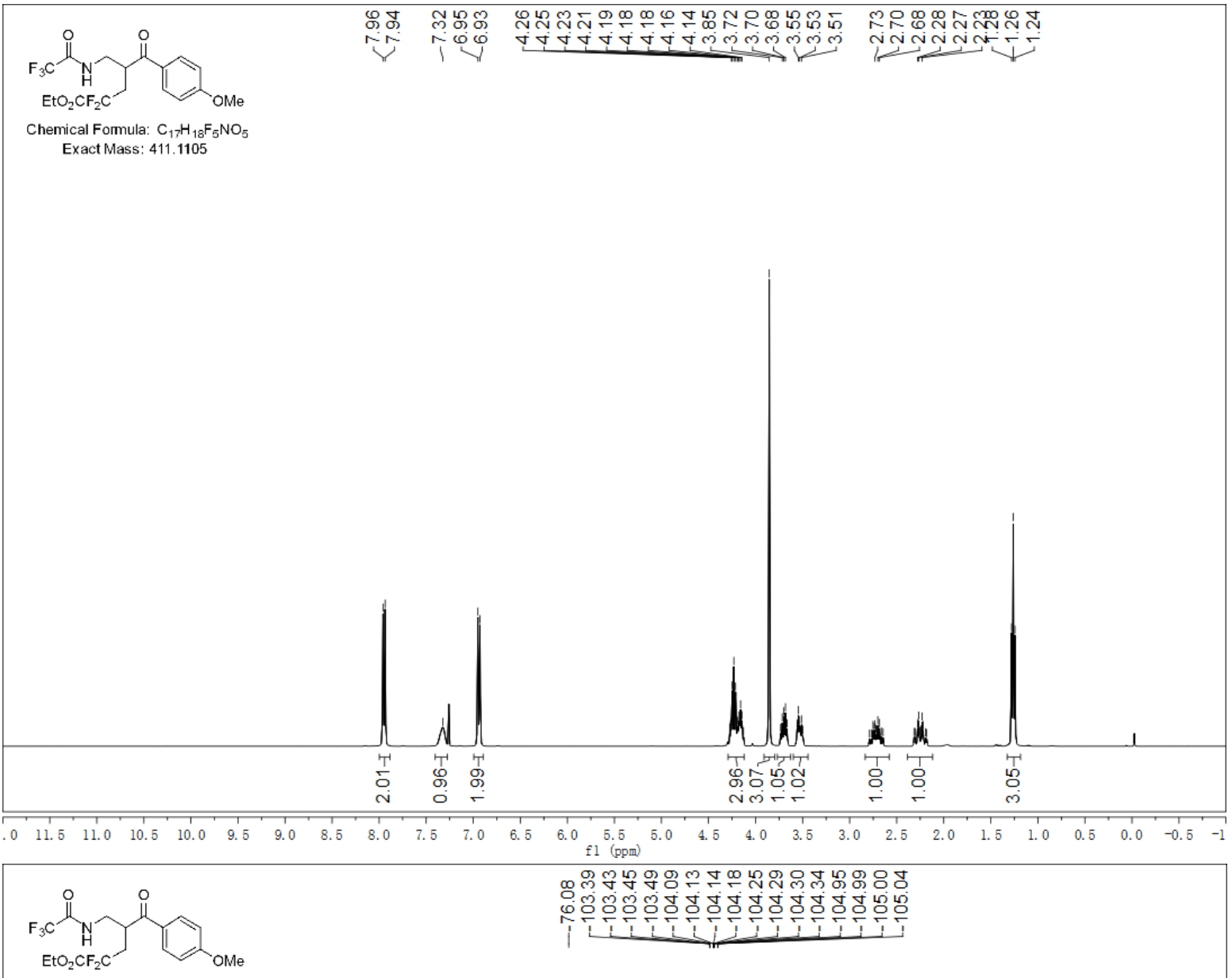

Chemical Formula: $\mathrm{C}_{17} \mathrm{H}_{18} \mathrm{~F}_{5} \mathrm{NO}_{5}$

Exact Mass: 411.1105

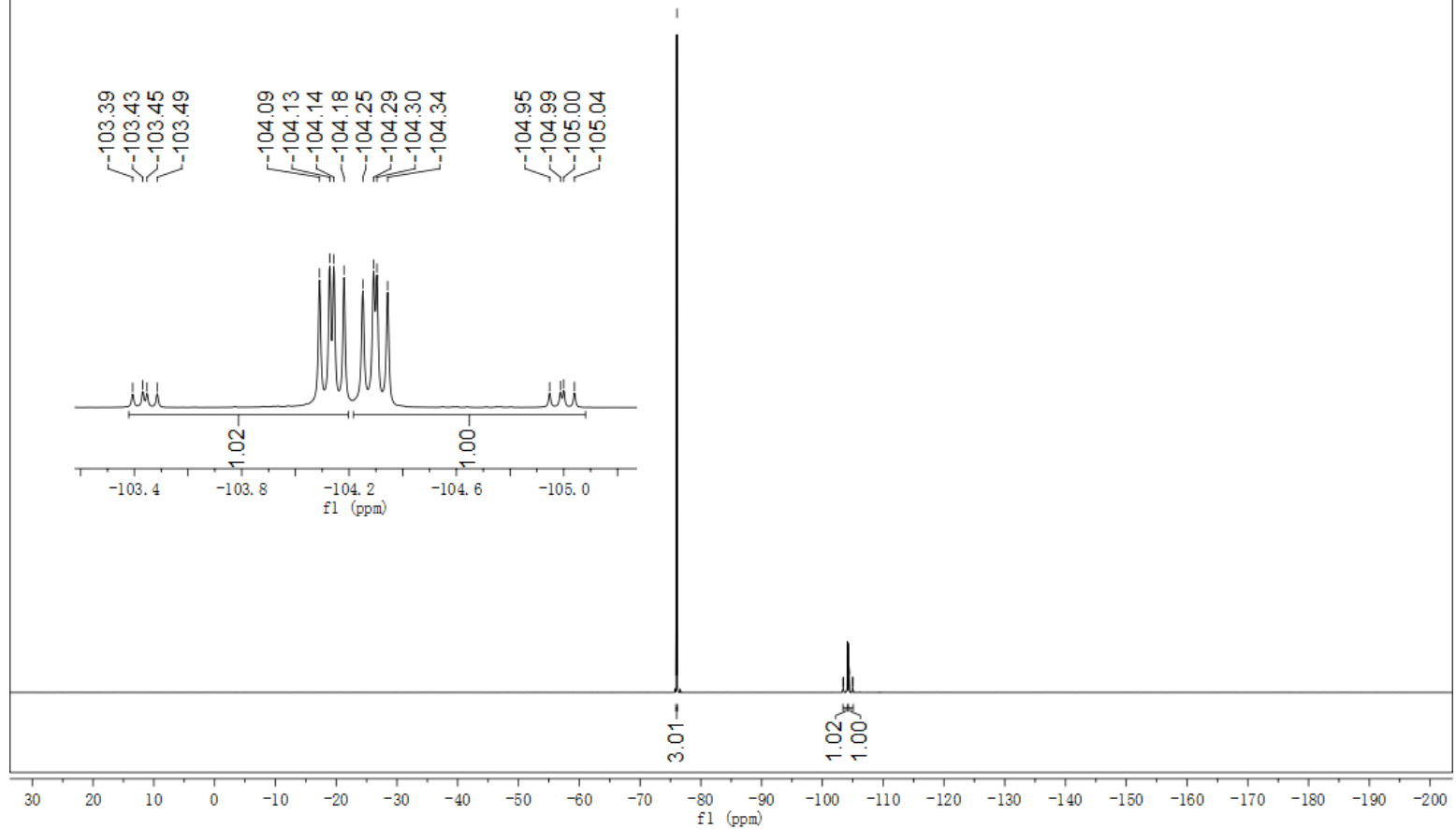




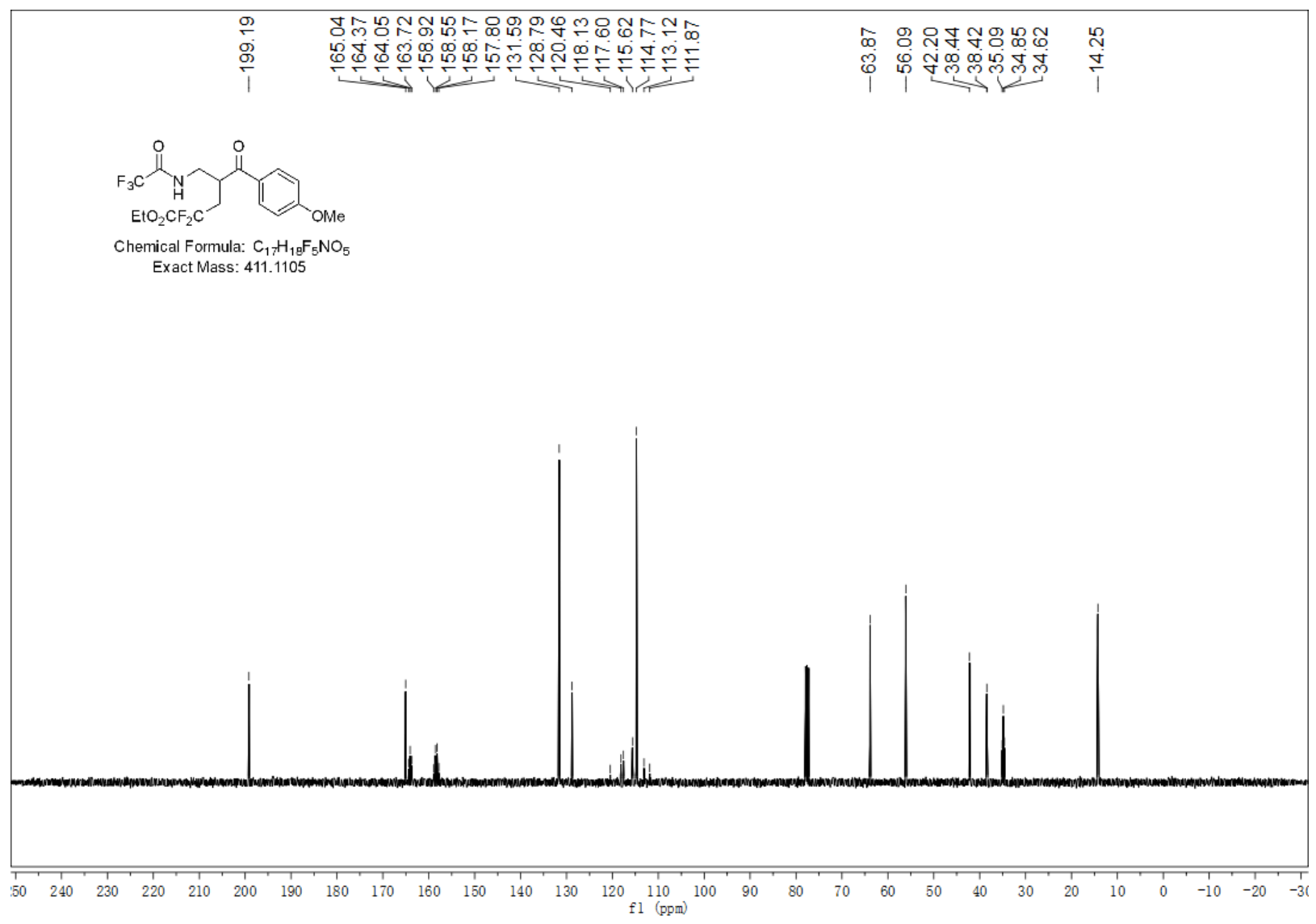

Ethyl 6-benzamido-2,2-difluoro-4-(4-methoxybenzoyl)hexanoate (7h).

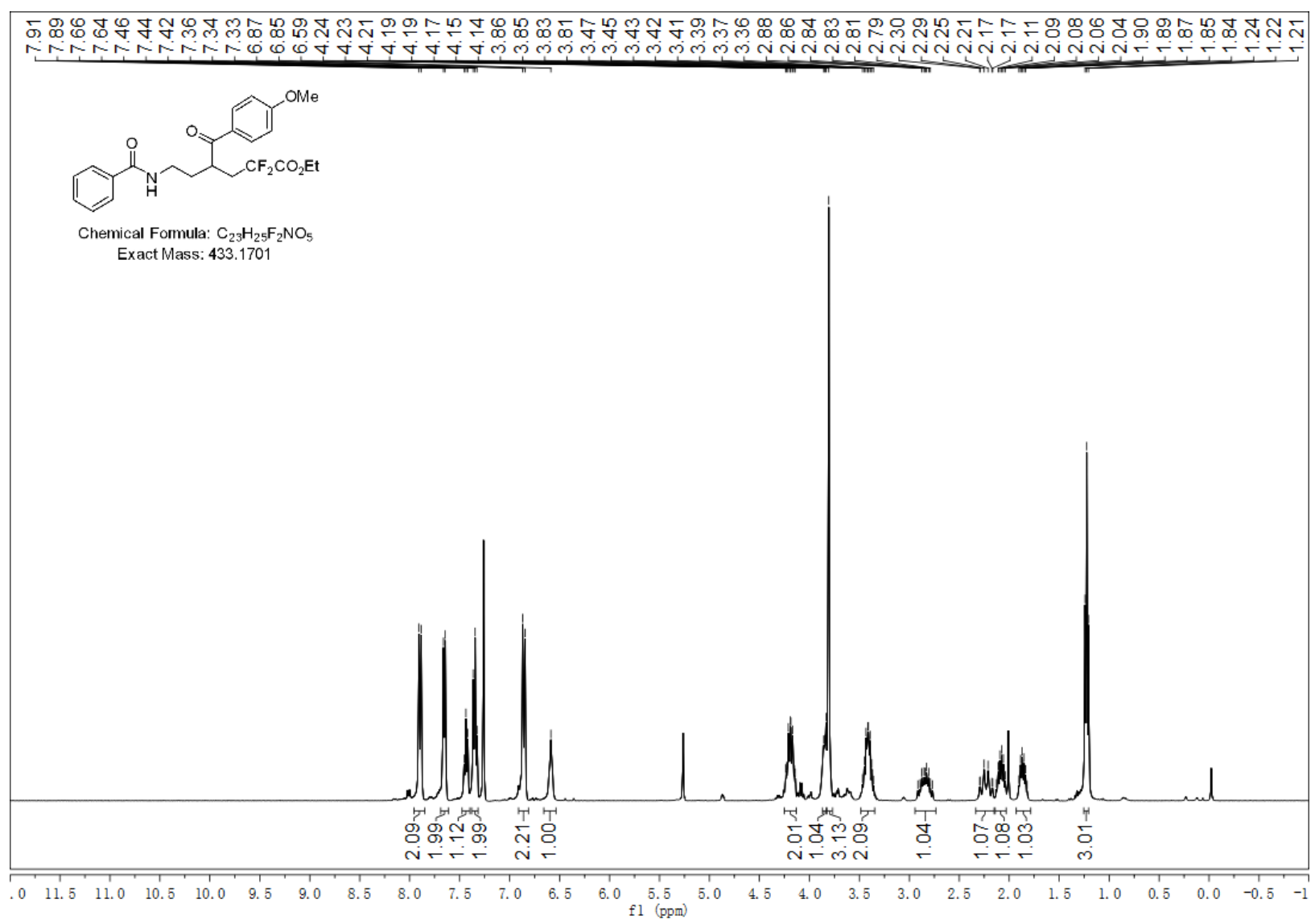



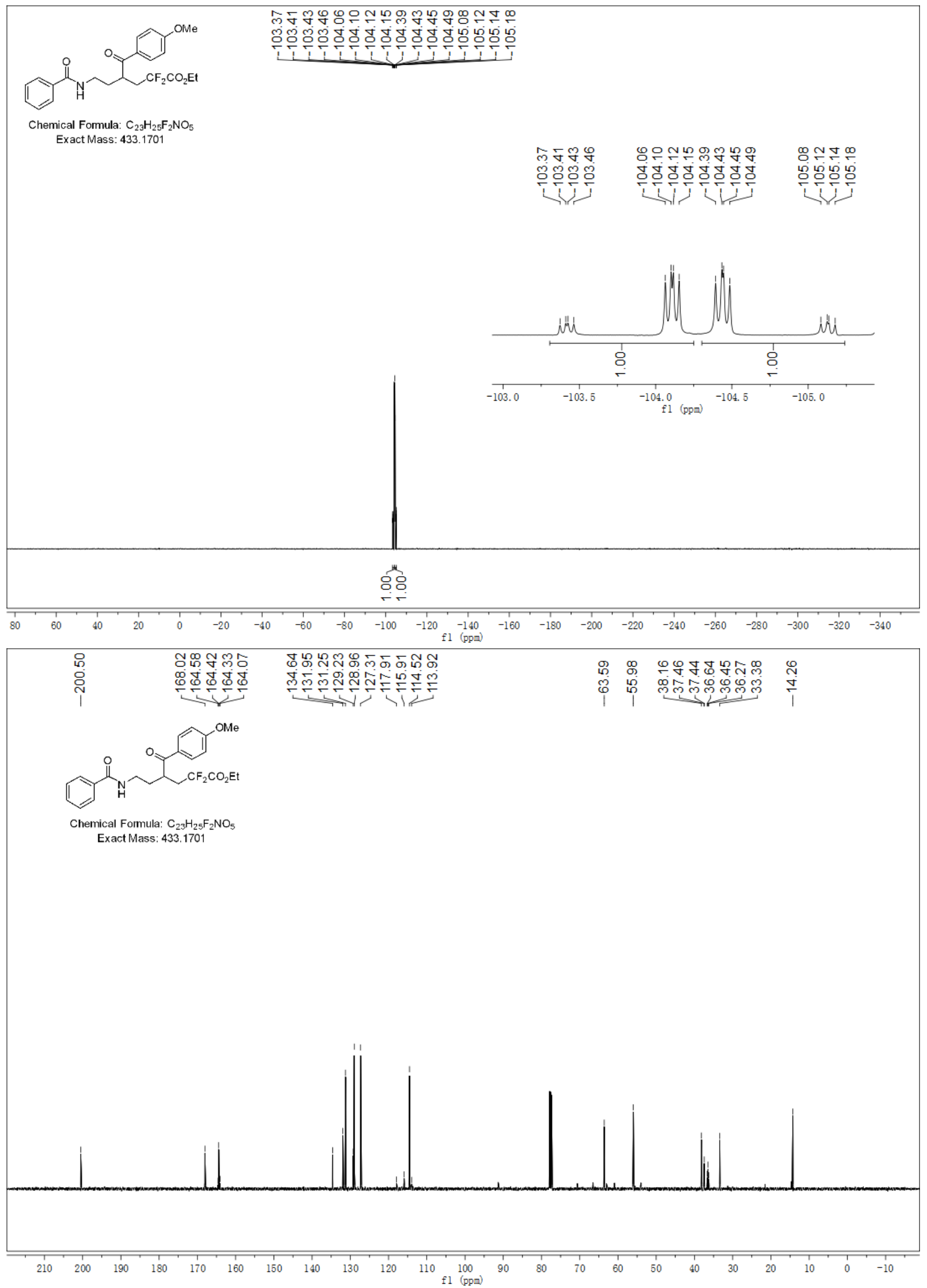


\section{Ethyl 4-(acetoxymethyl)-2,2-difluoro-5-(4-methoxyphenyl)-5-oxopentanoate (7i).}
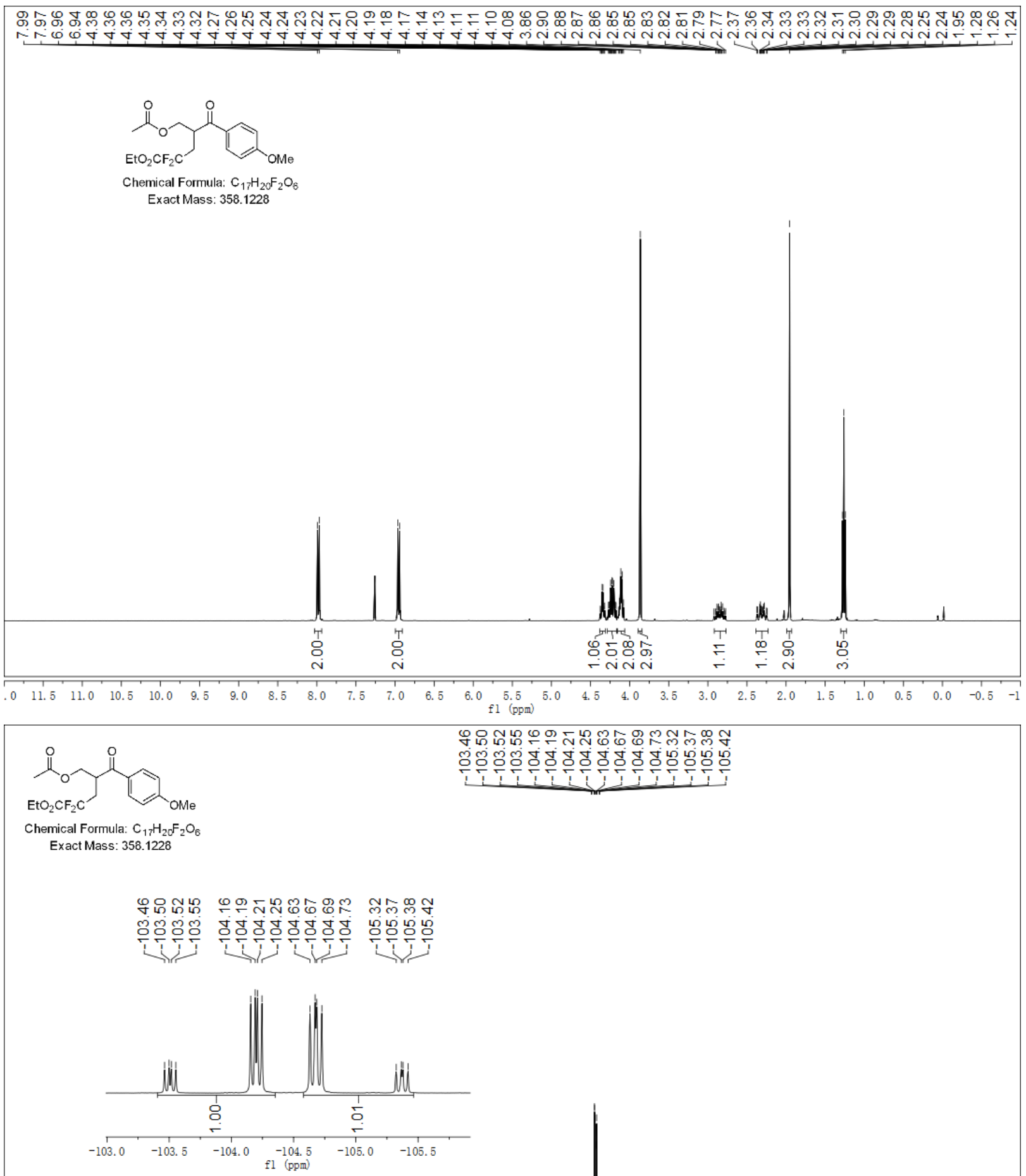

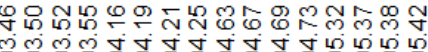

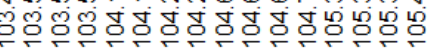

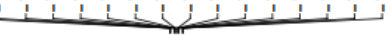

ical Formula: $\mathrm{C}_{17} \mathrm{H}_{20} \mathrm{~F}_{2} \mathrm{O}_{6}$

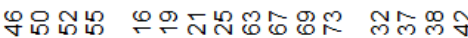

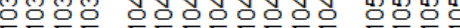

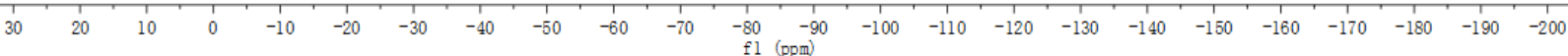




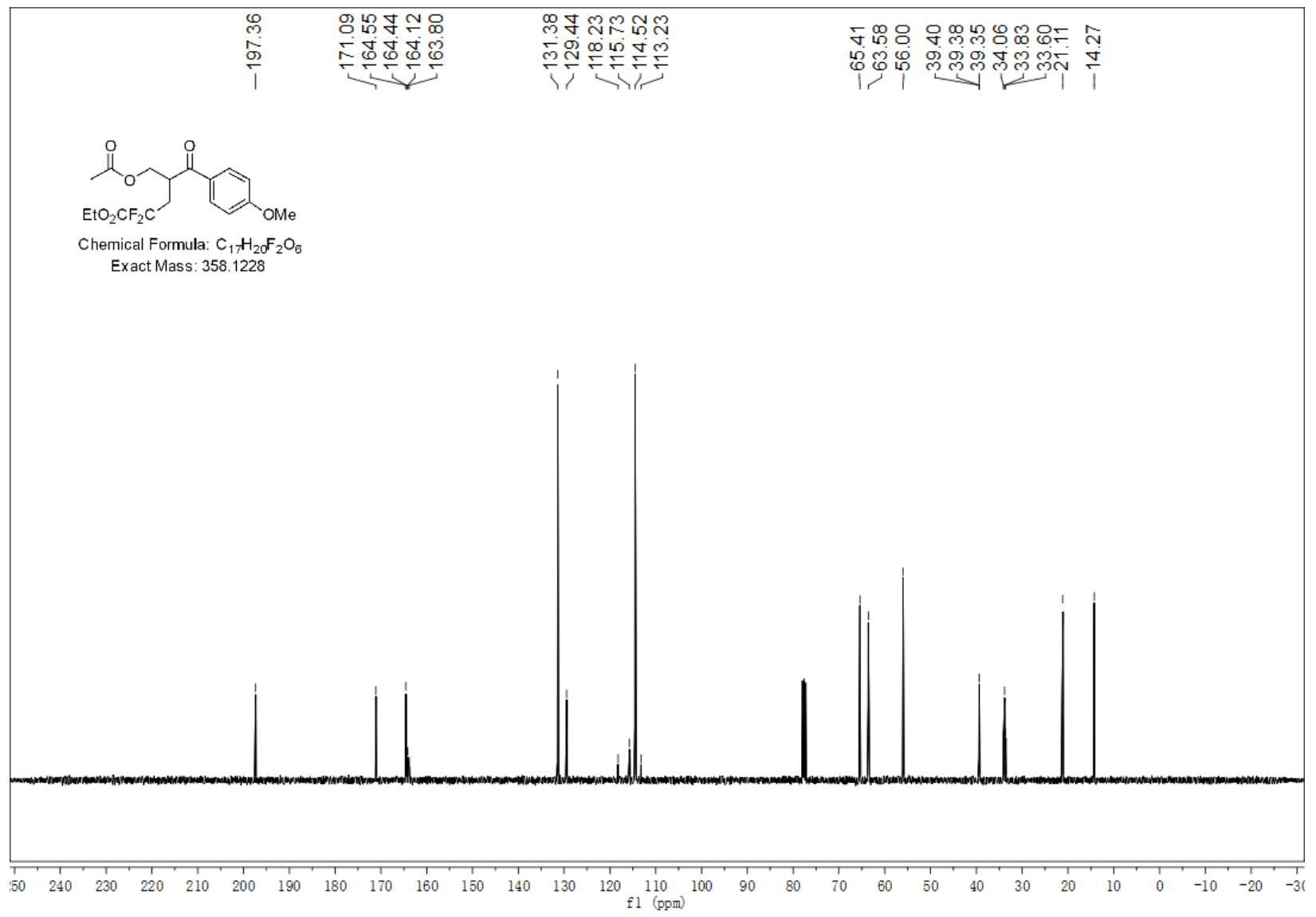

2-([1,1'-Biphenyl]-4-carbonyl)-5-ethoxy-4,4-difluoro-5-oxopentyl benzoate (7j).

뭄

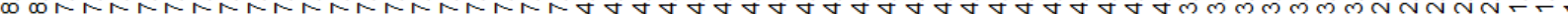

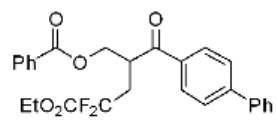

Chemical Formula: $\mathrm{C}_{2} \mathrm{H}_{24} \mathrm{~F}_{2} \mathrm{O}_{5}$ Exact Mass: 466.1592

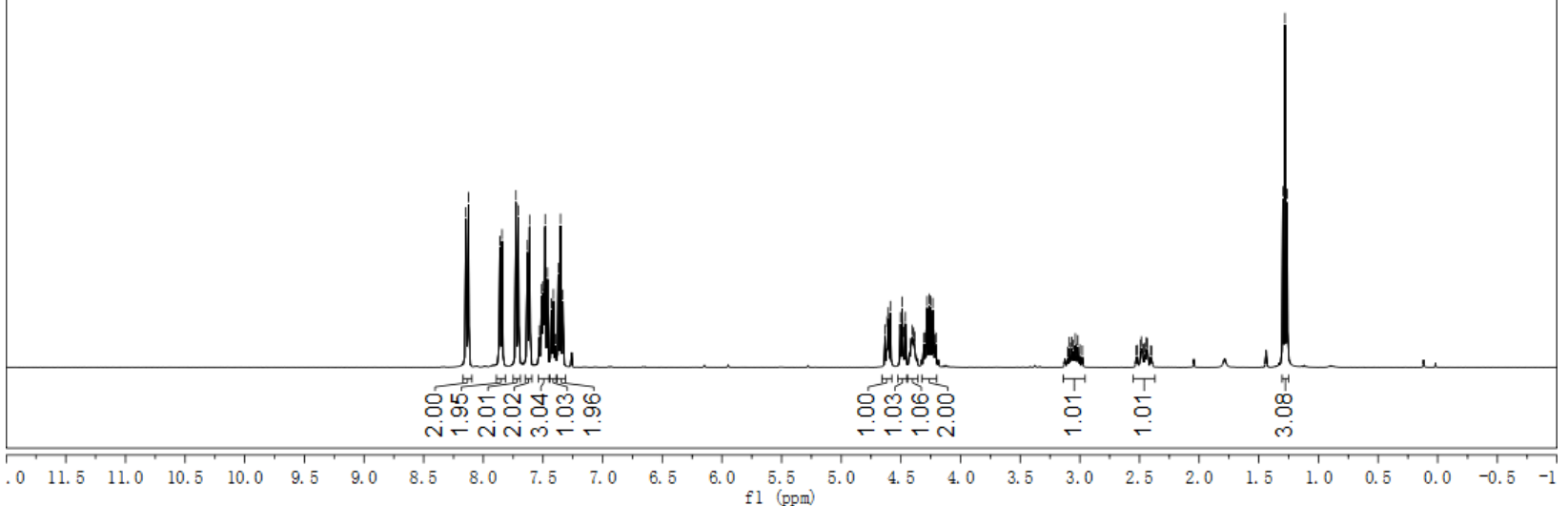




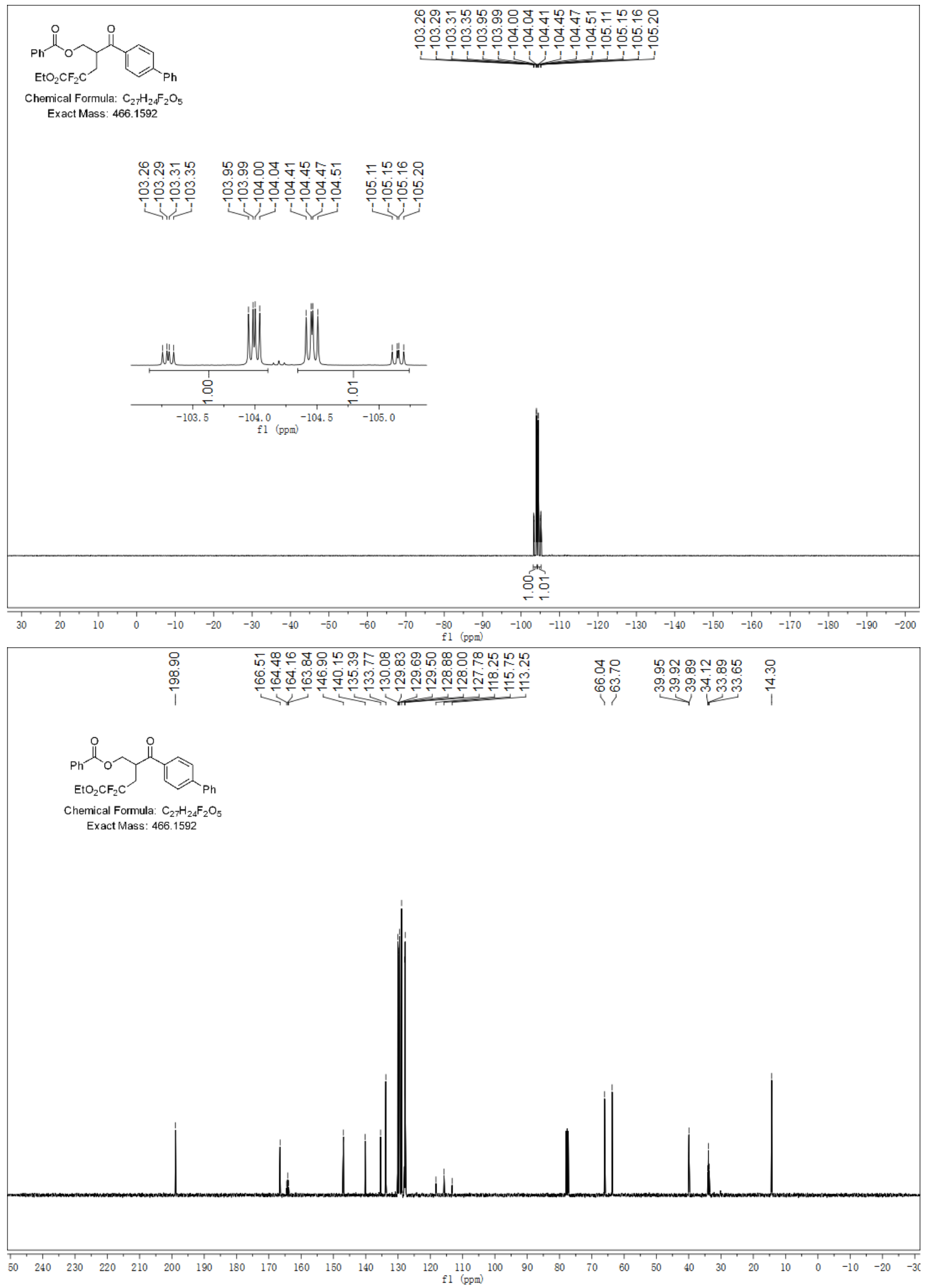


Ethyl 5-([1,1'-biphenyl]-4-yl)-2,2-difluoro-5-oxo-4-((2-phenoxyacetoxy)methyl)pentanoate (7k).

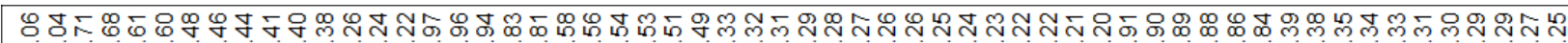

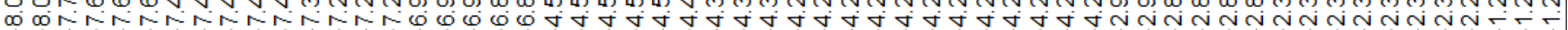

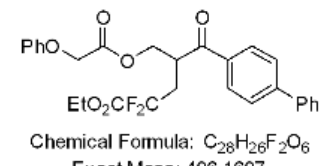

Exact Mass: 496.1697
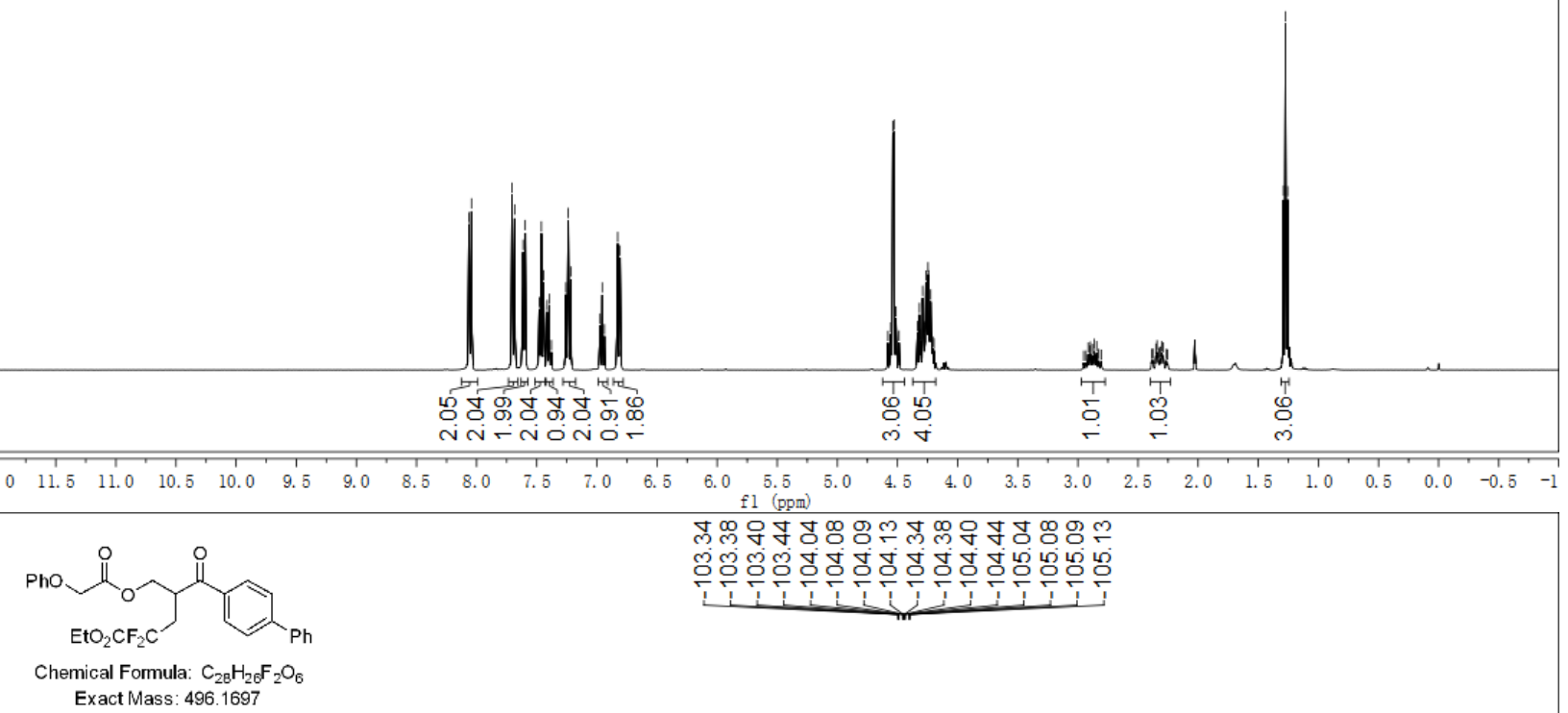

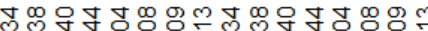

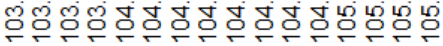
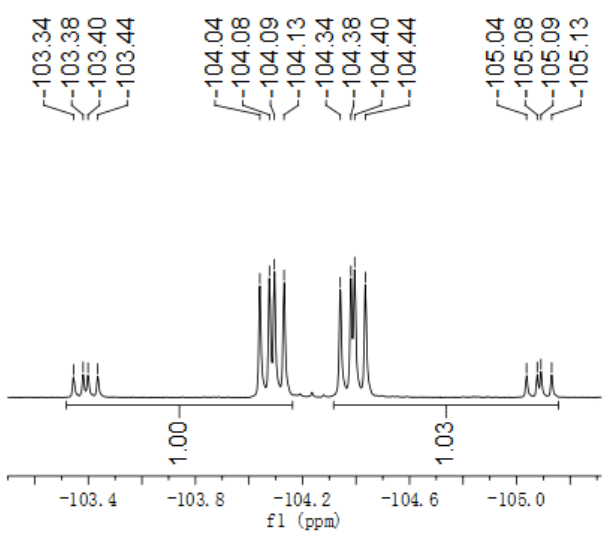

8

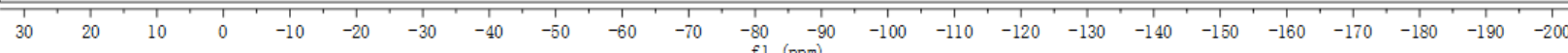




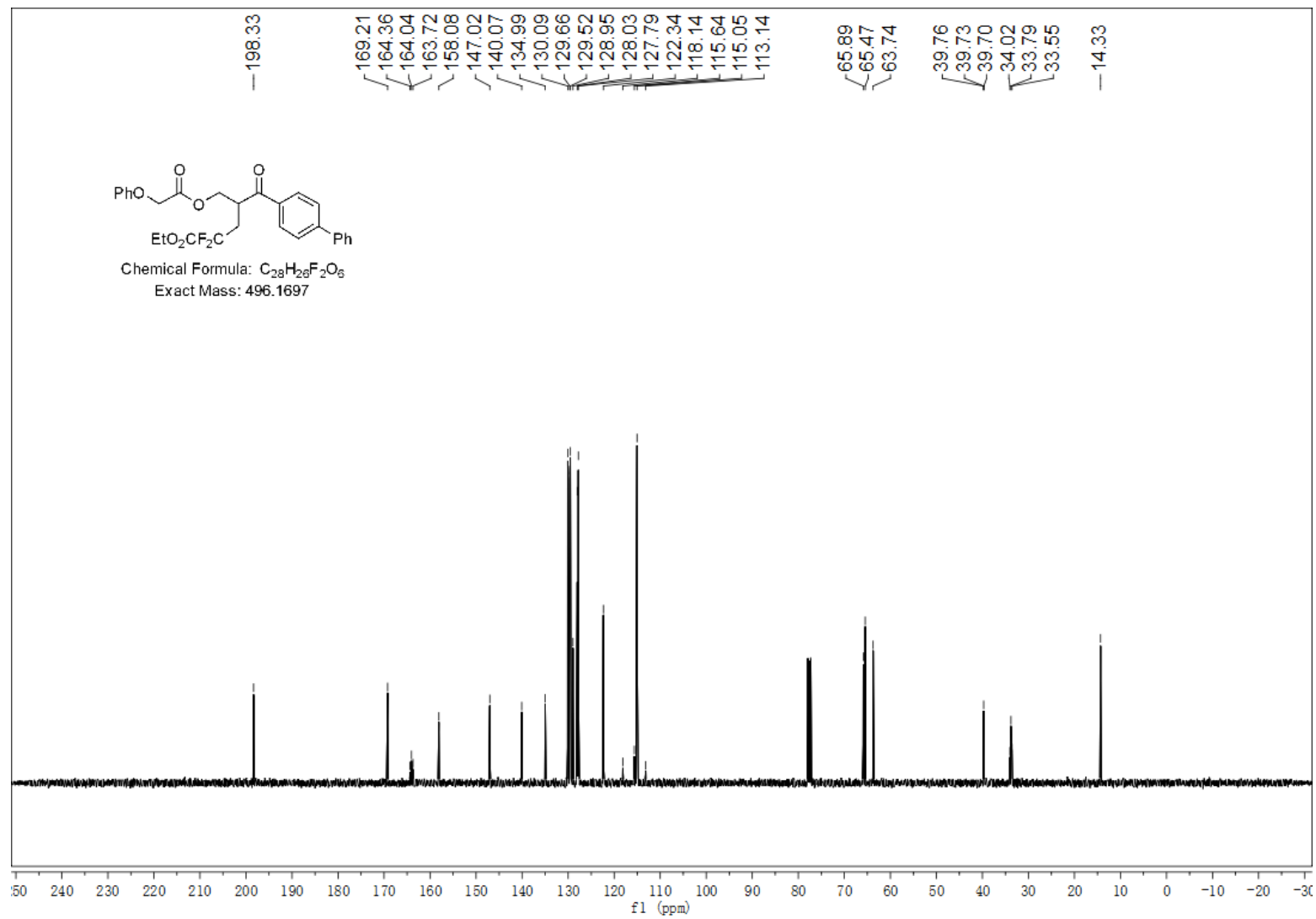

Ethyl 4-acetamido-2,2-difluoro-5-(4-(4-((1-isopropoxy-2-methyl-1-oxopropan-2-yl)oxy)benzoyl) phenyl)-5-oxopentanoate (8a).

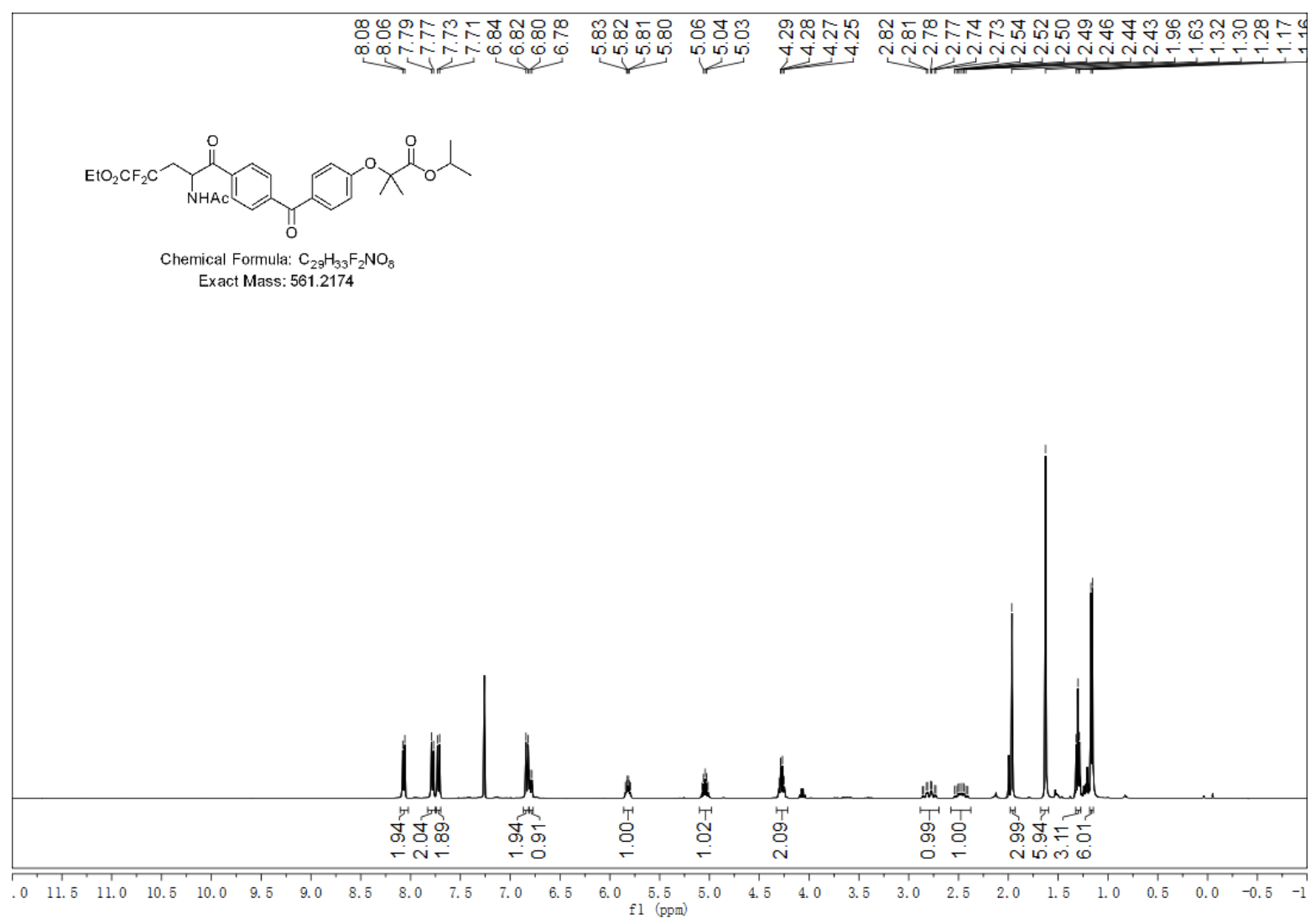




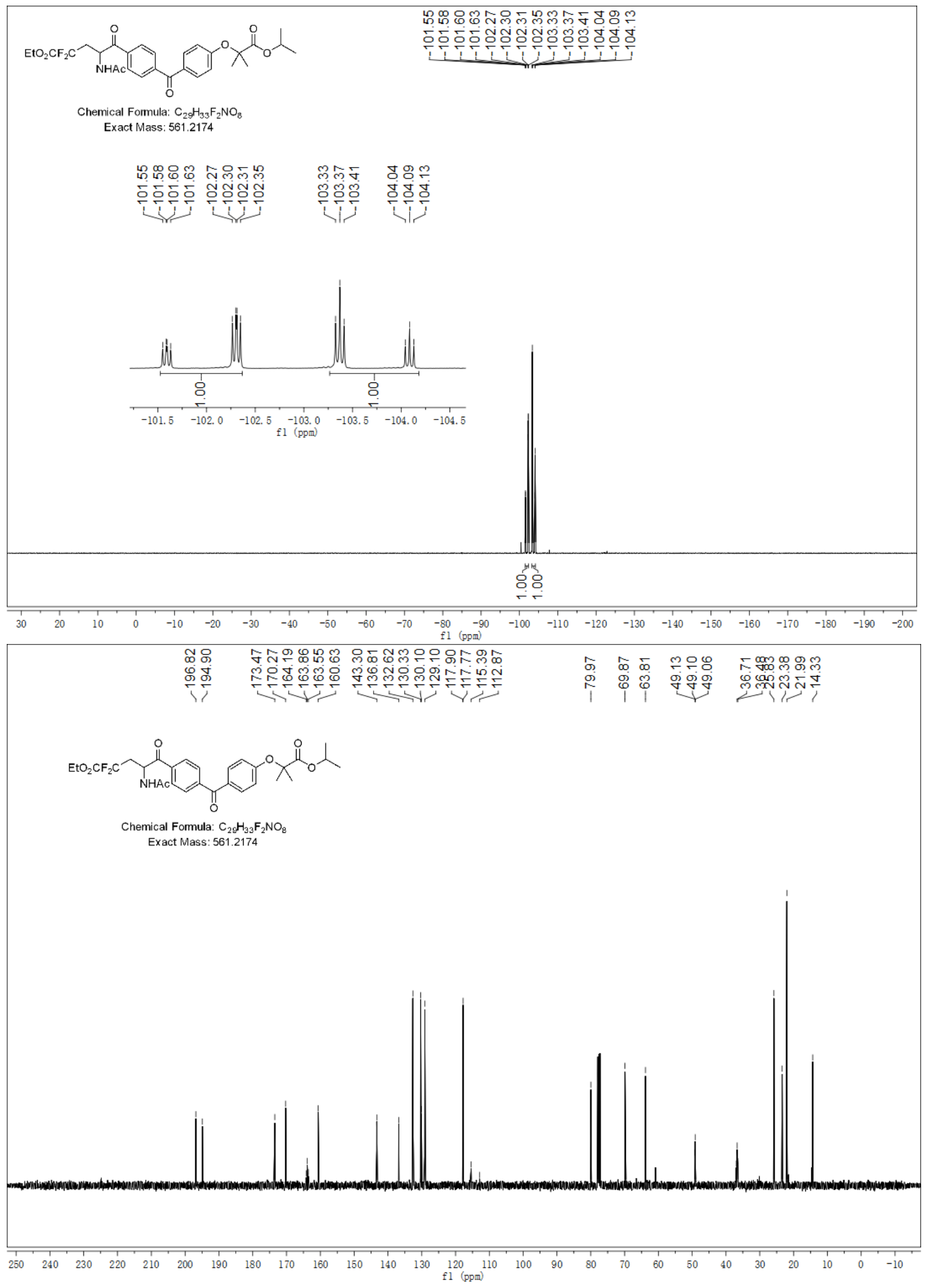


Ethyl 4-acetamido-5-(4-((S)-2-((tert-butoxycarbonyl)amino)-3-methoxy-3-oxopropyl)phenyl)

-2,2-difluoro-5-oxopentanoate (8b).
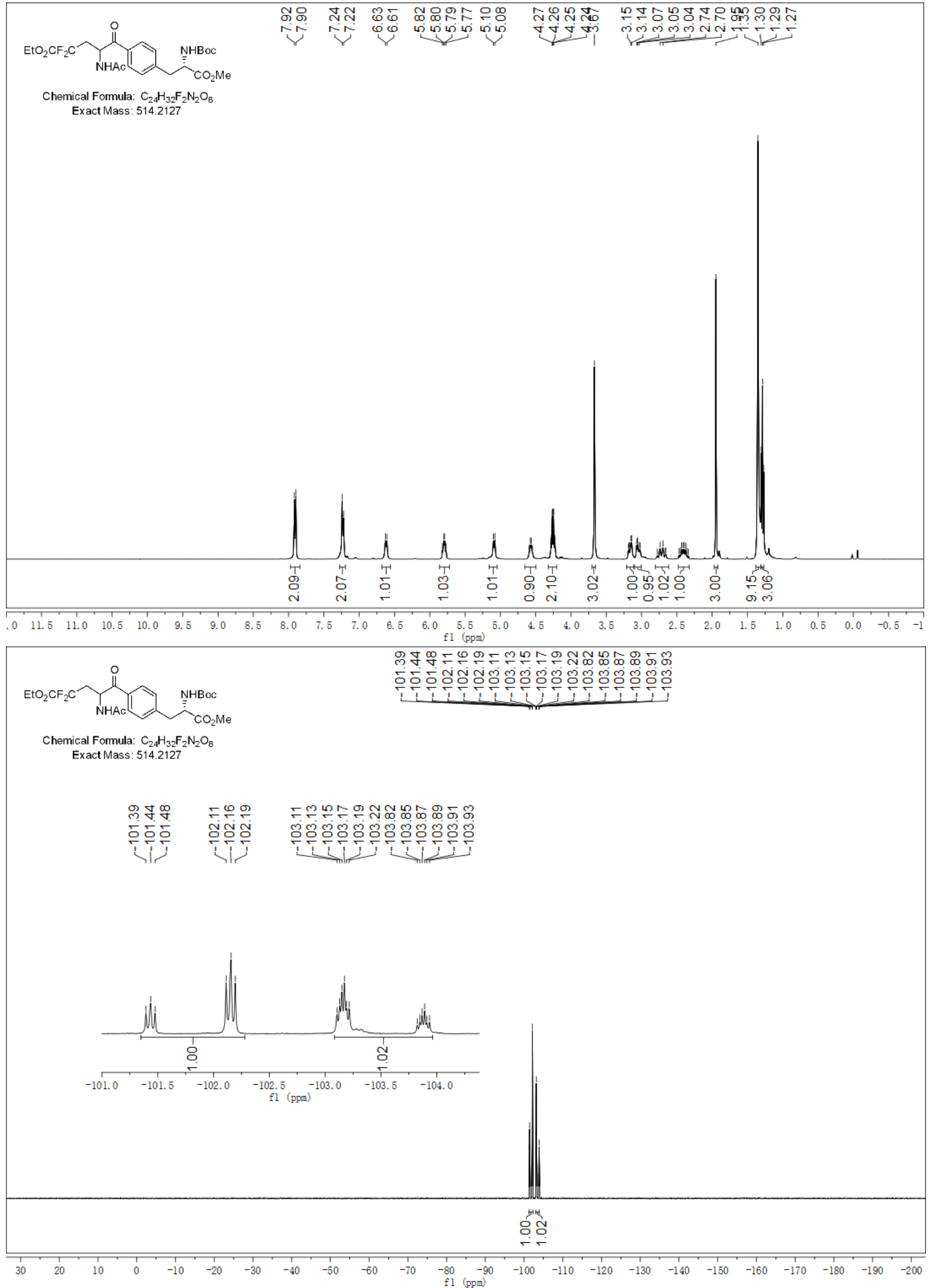


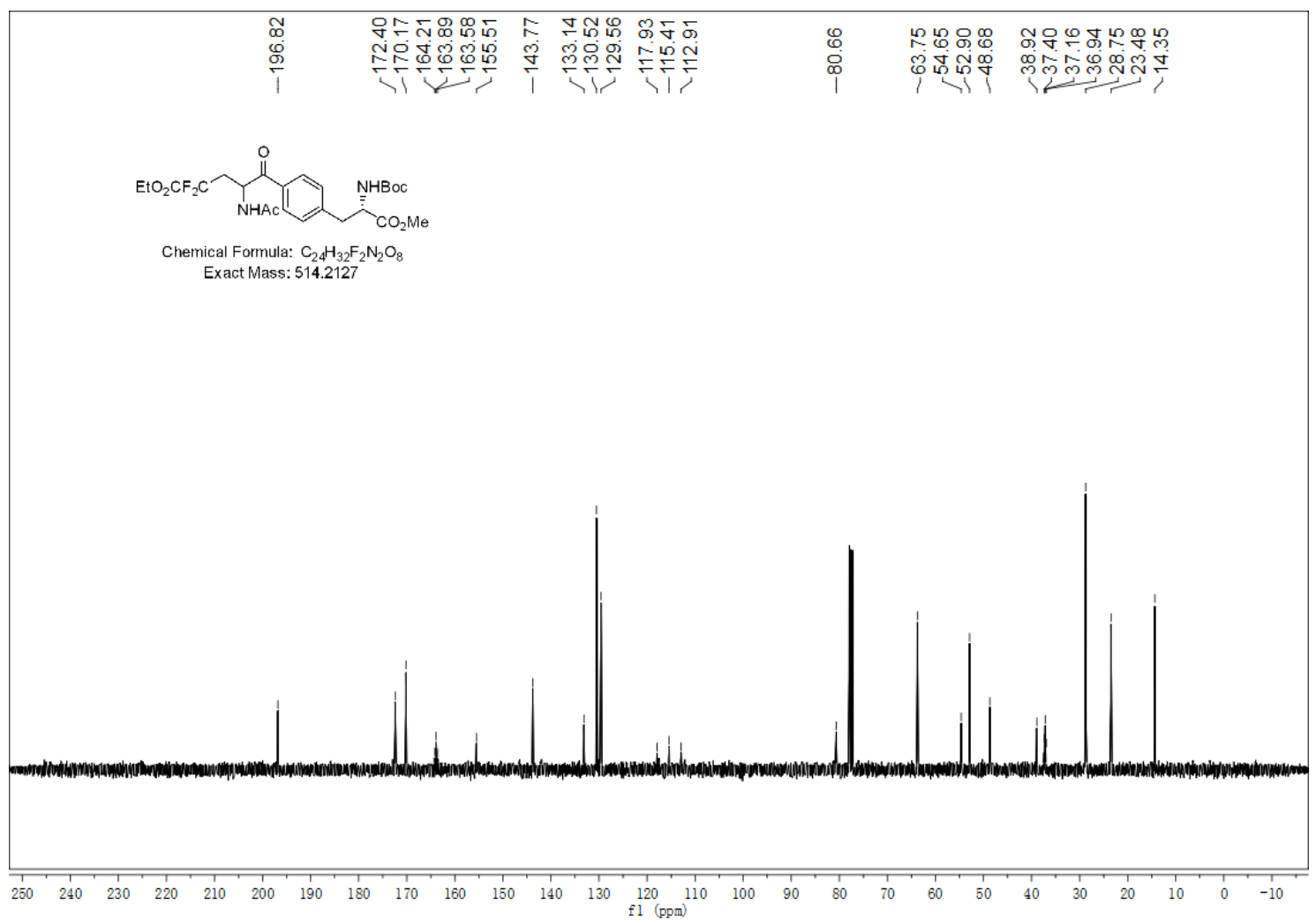

Methyl (4-acetamido-5-(4-chlorophenyl)-2,2-difluoro-5-oxopentanoyl)- $L$-alanyl- $L$ phenylalaninate (8c-1).

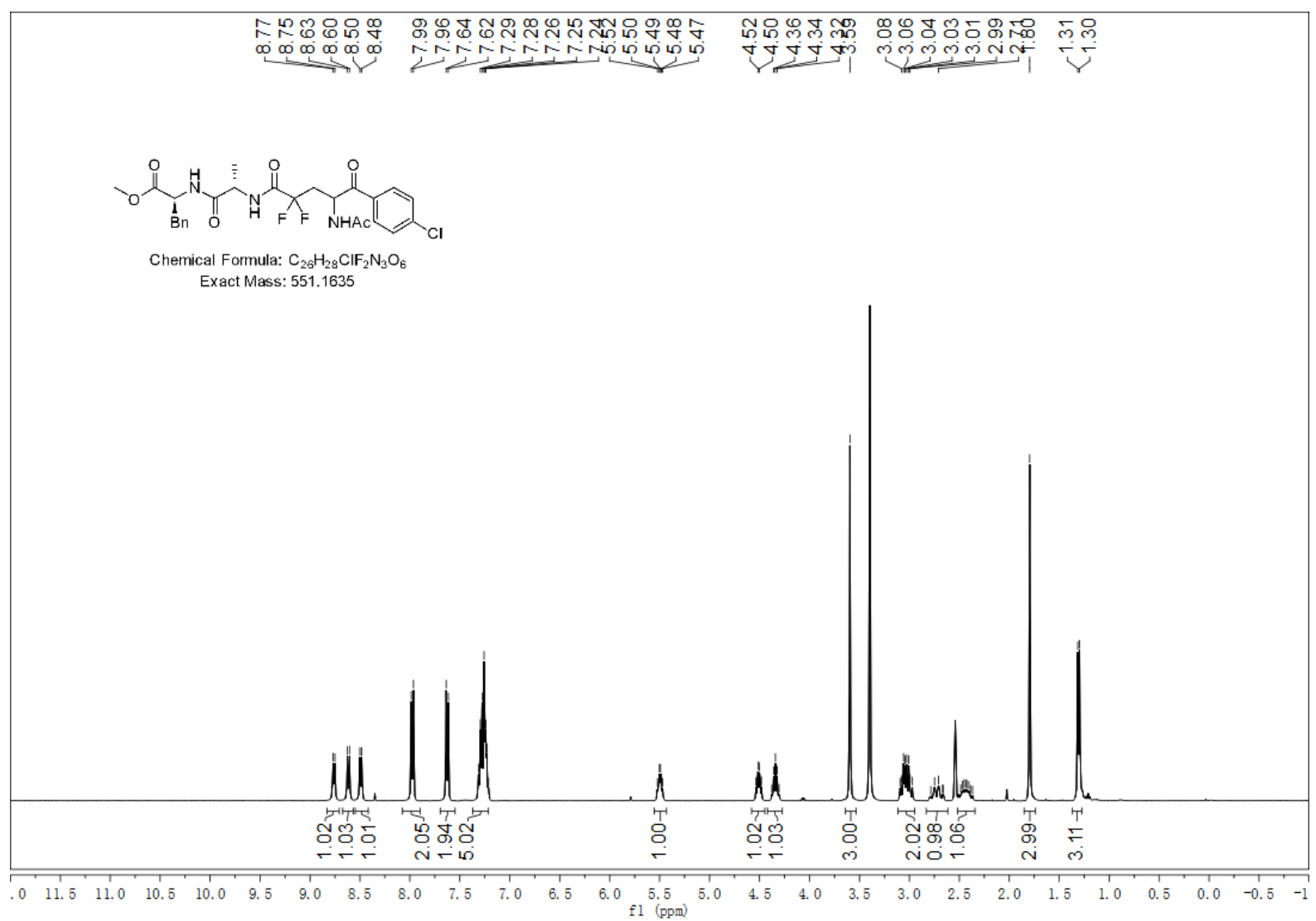




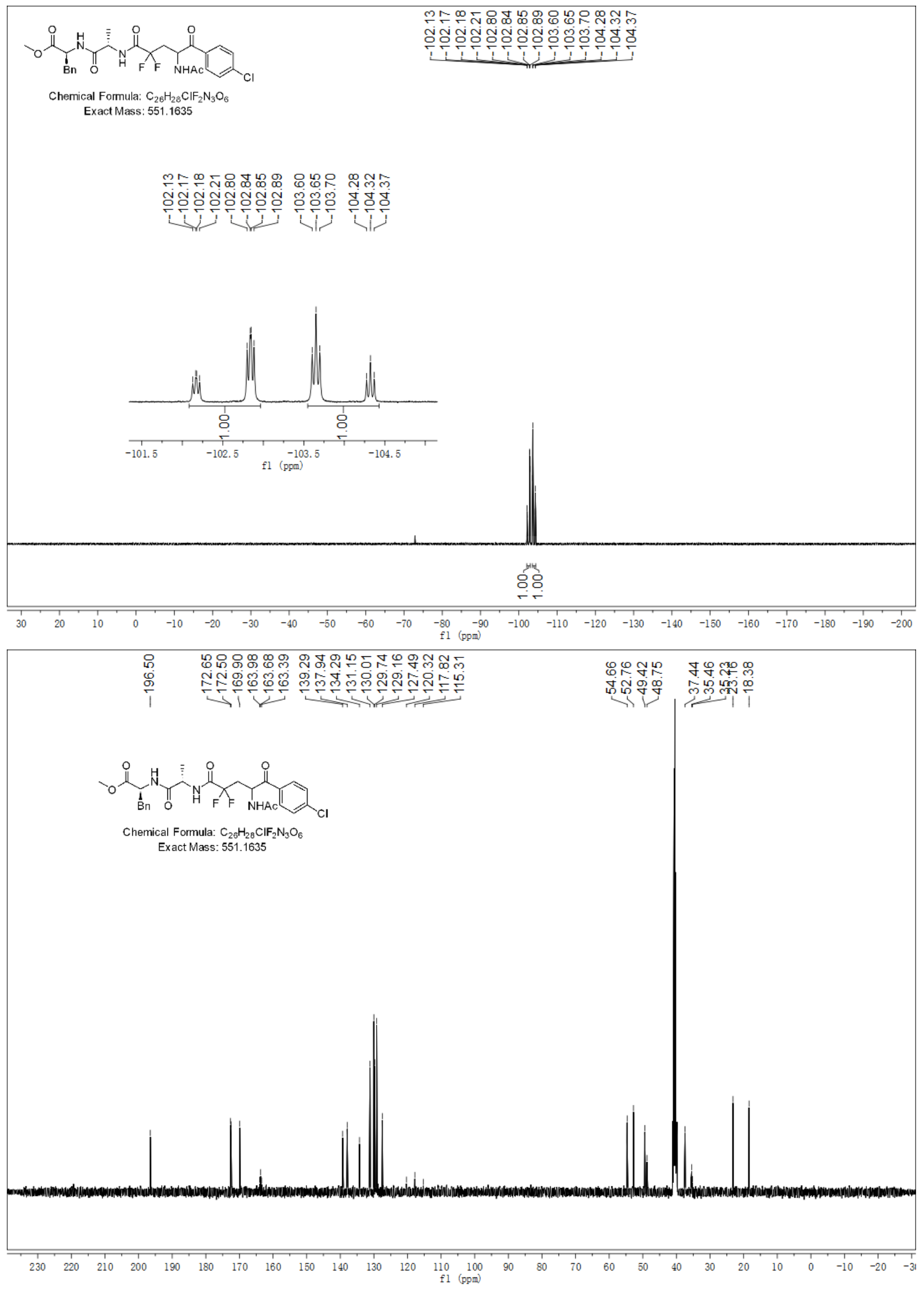


Methyl (4-acetamido-5-(4-chlorophenyl)-2,2-difluoro-5-oxopentanoyl)- $L$-alanyl- $L$ phenylalaninate (8c-2).

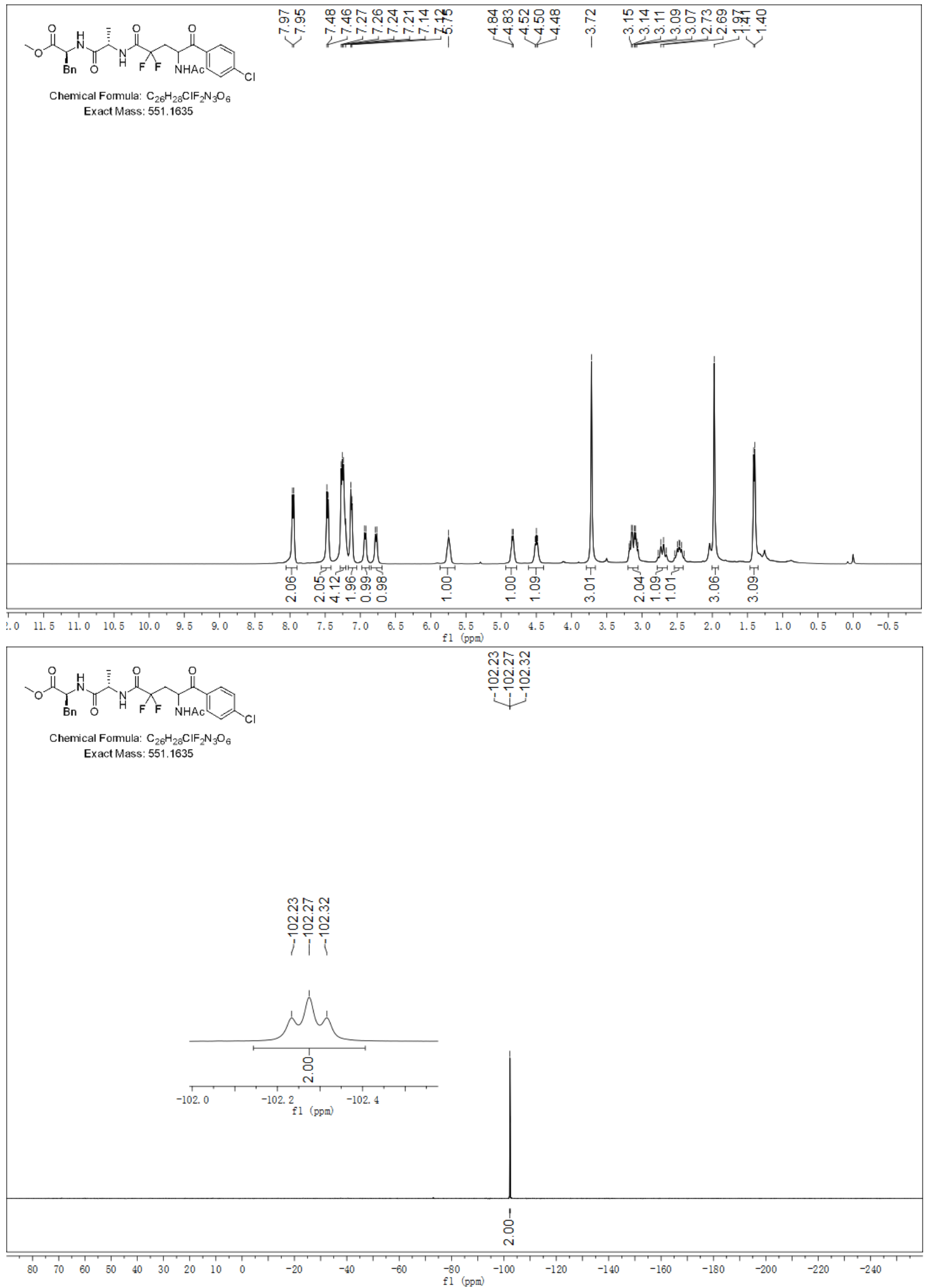




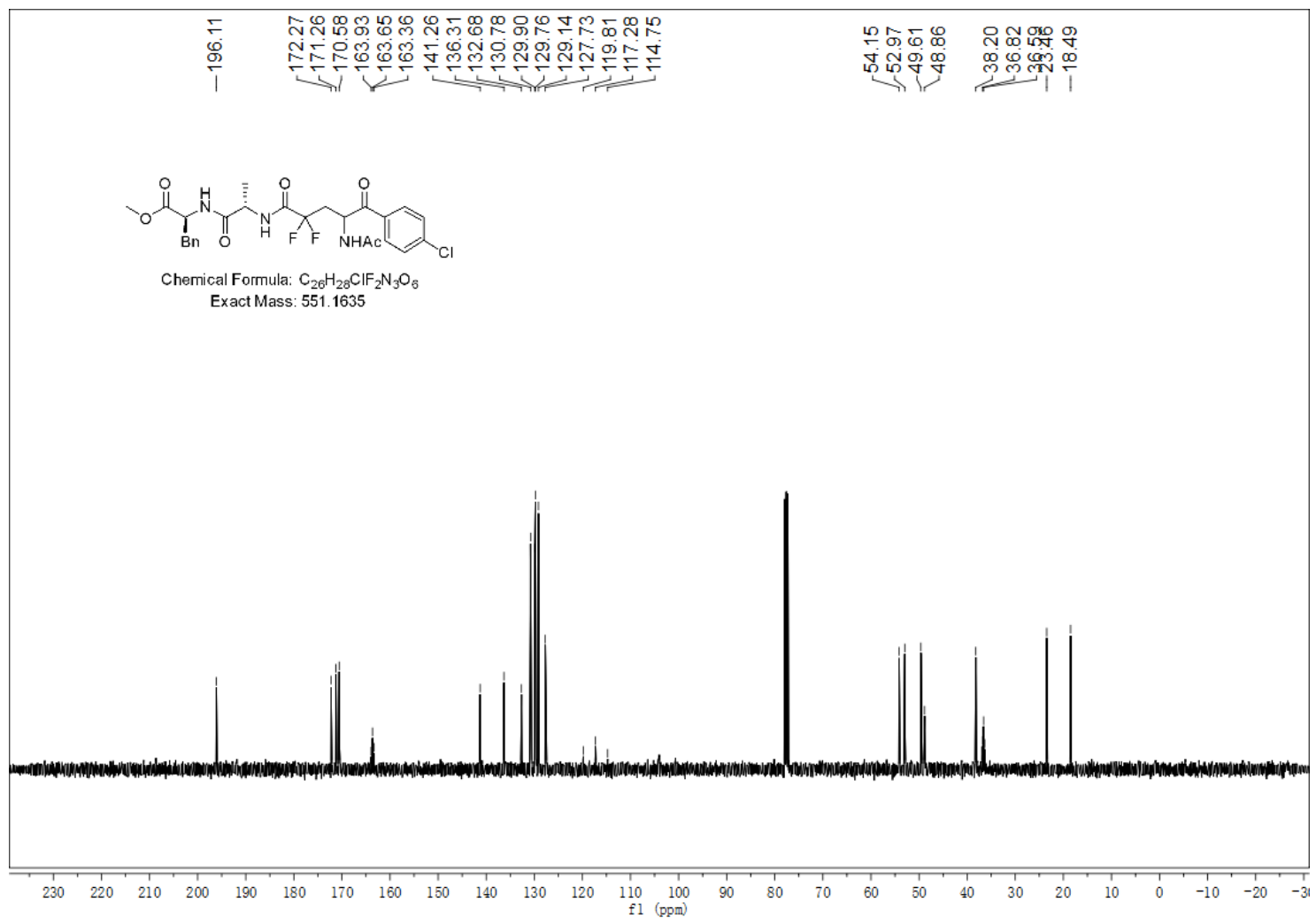

Methyl (4-(((tert-butoxycarbonyl)amino)methyl)-2,2-difluoro-5-(4-methoxyphenyl)-5oxopentanoyl)- $L$-alanyl- $L$-phenylalaninate (8d-1).

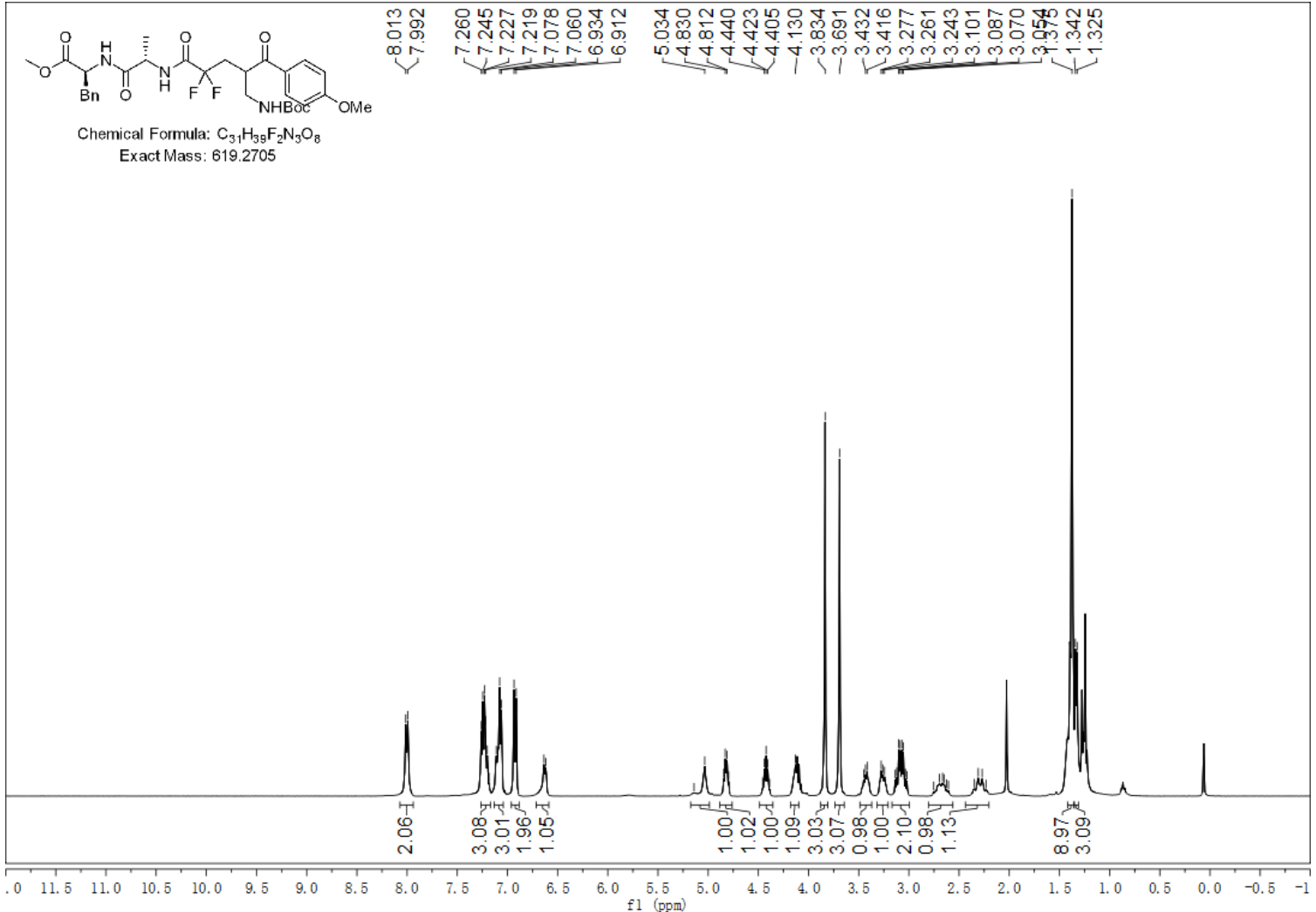



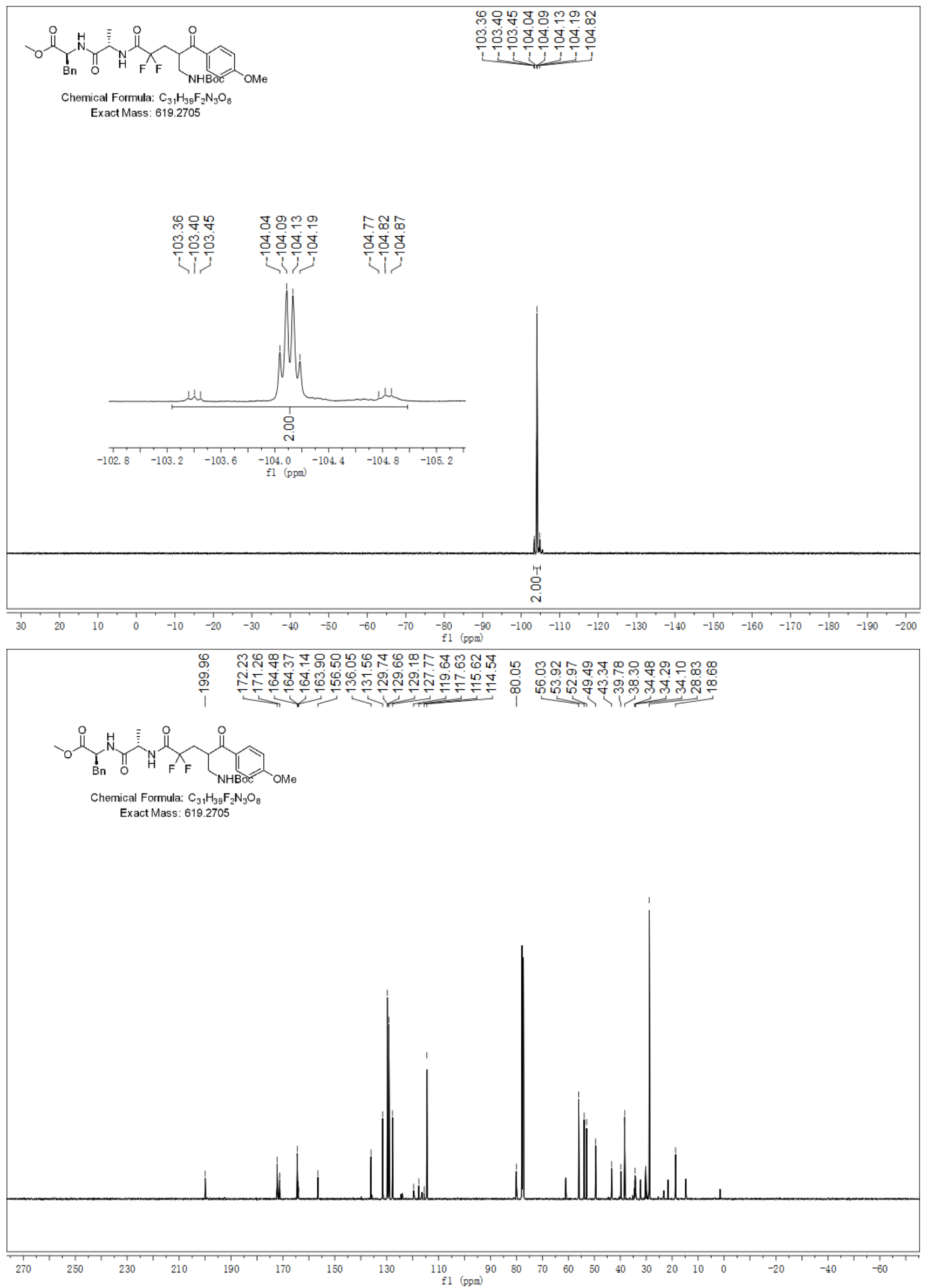
Methyl (4-(((tert-butoxycarbonyl)amino)methyl)-2,2-difluoro-5-(4-methoxyphenyl)-5-

oxopentanoyl)- $L$-alanyl- $L$-phenylalaninate (8d-2).

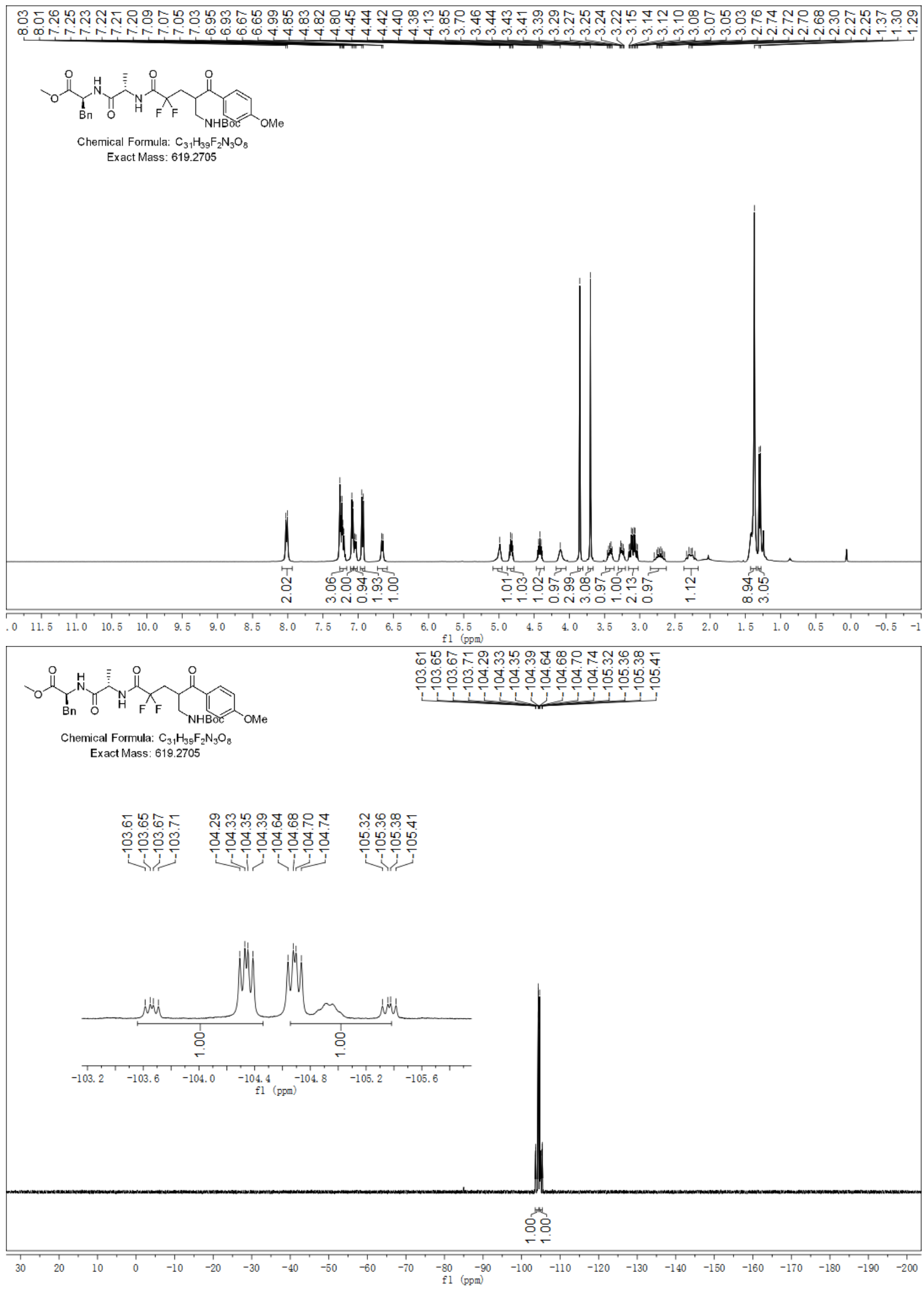




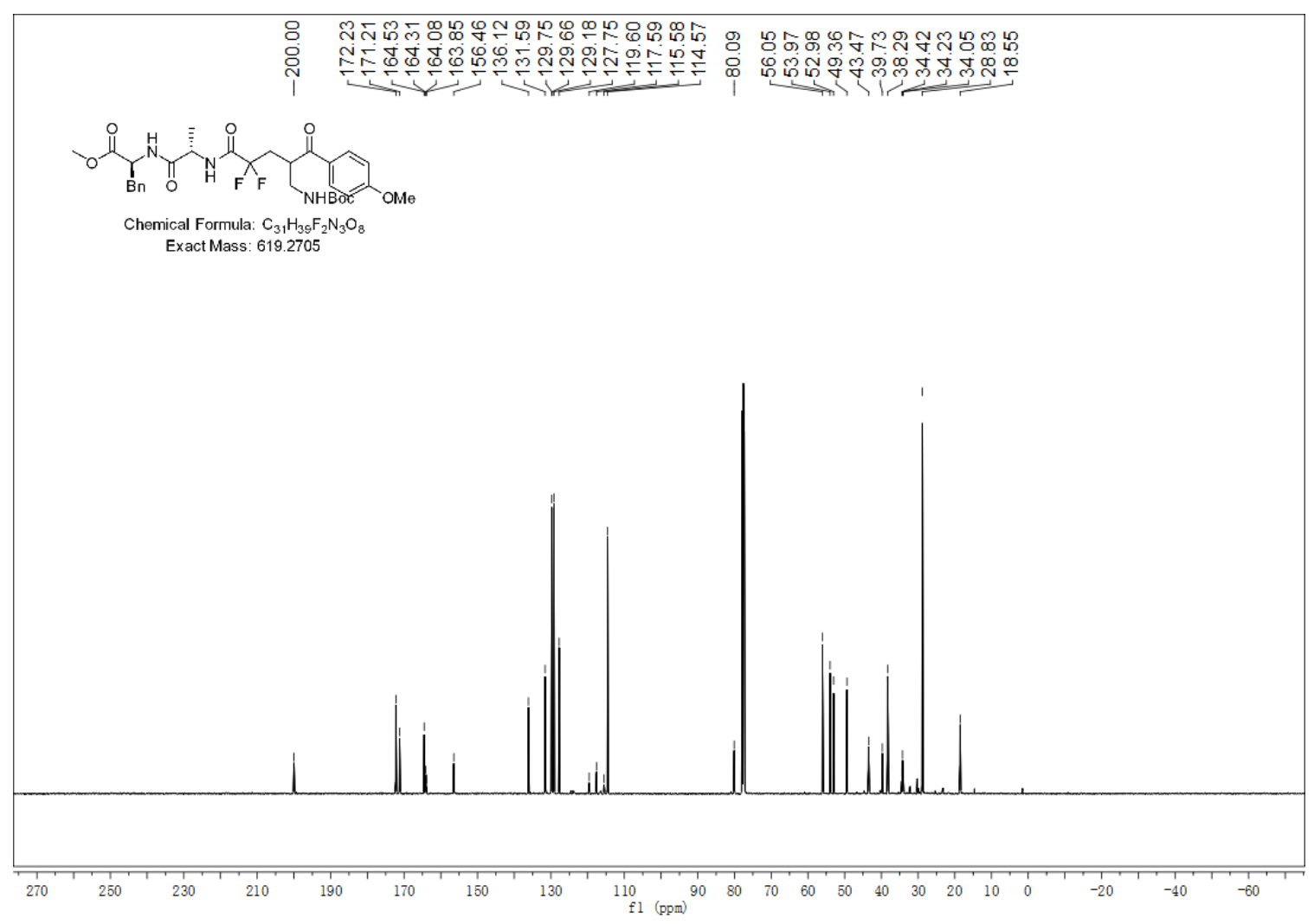

Methyl (5S,14S,17S)-9-([1,1'-biphenyl]-4-carbonyl)-17-benzyl-5-(3-(tert-butoxy)-3-oxopropyl)11,11-difluoro-14-methyl-3,6,12,15-tetraoxo-1-phenyl-2-oxa-4,7,13,16-tetraazaoctadecan-18oate (8e-1).

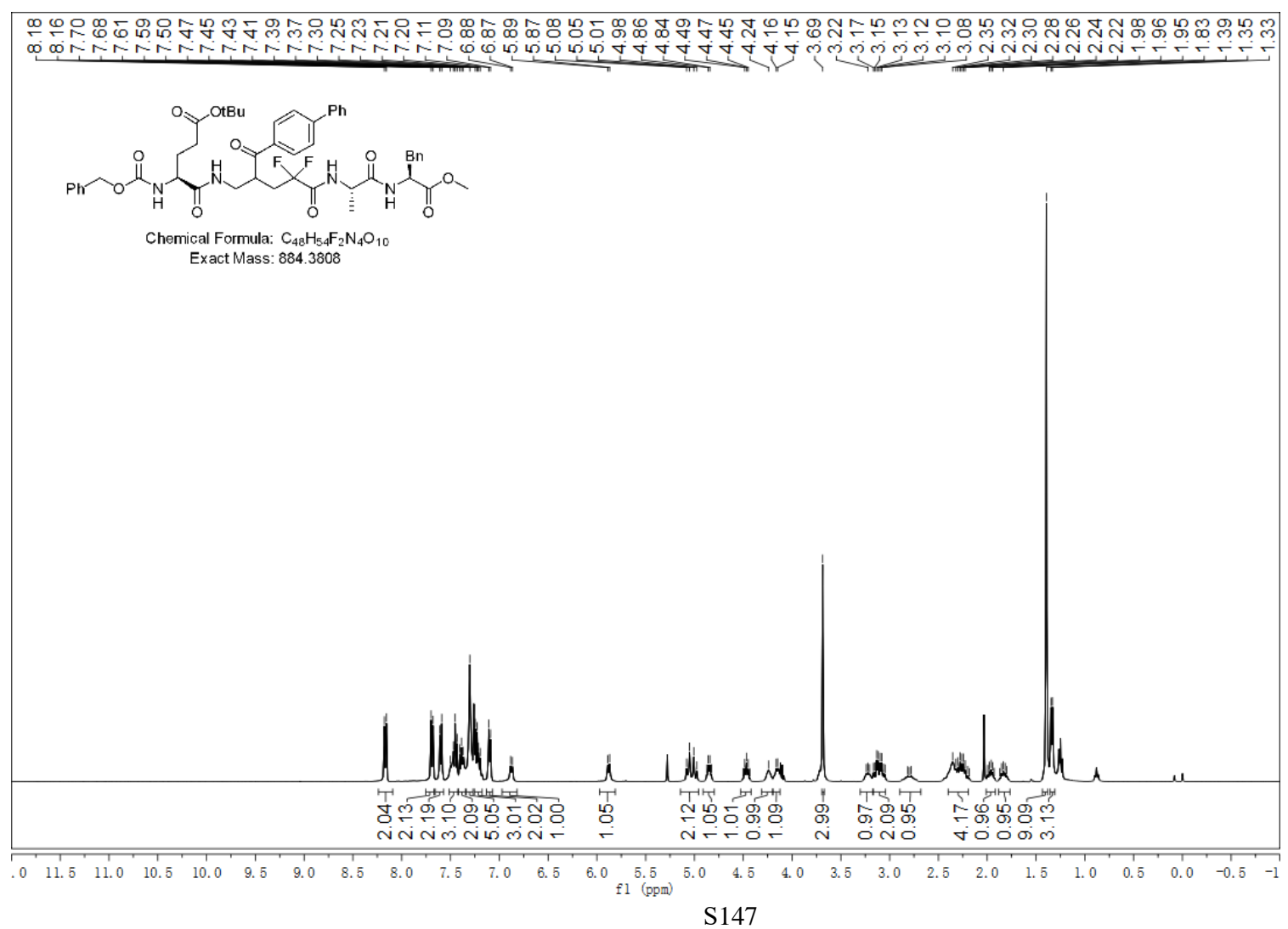



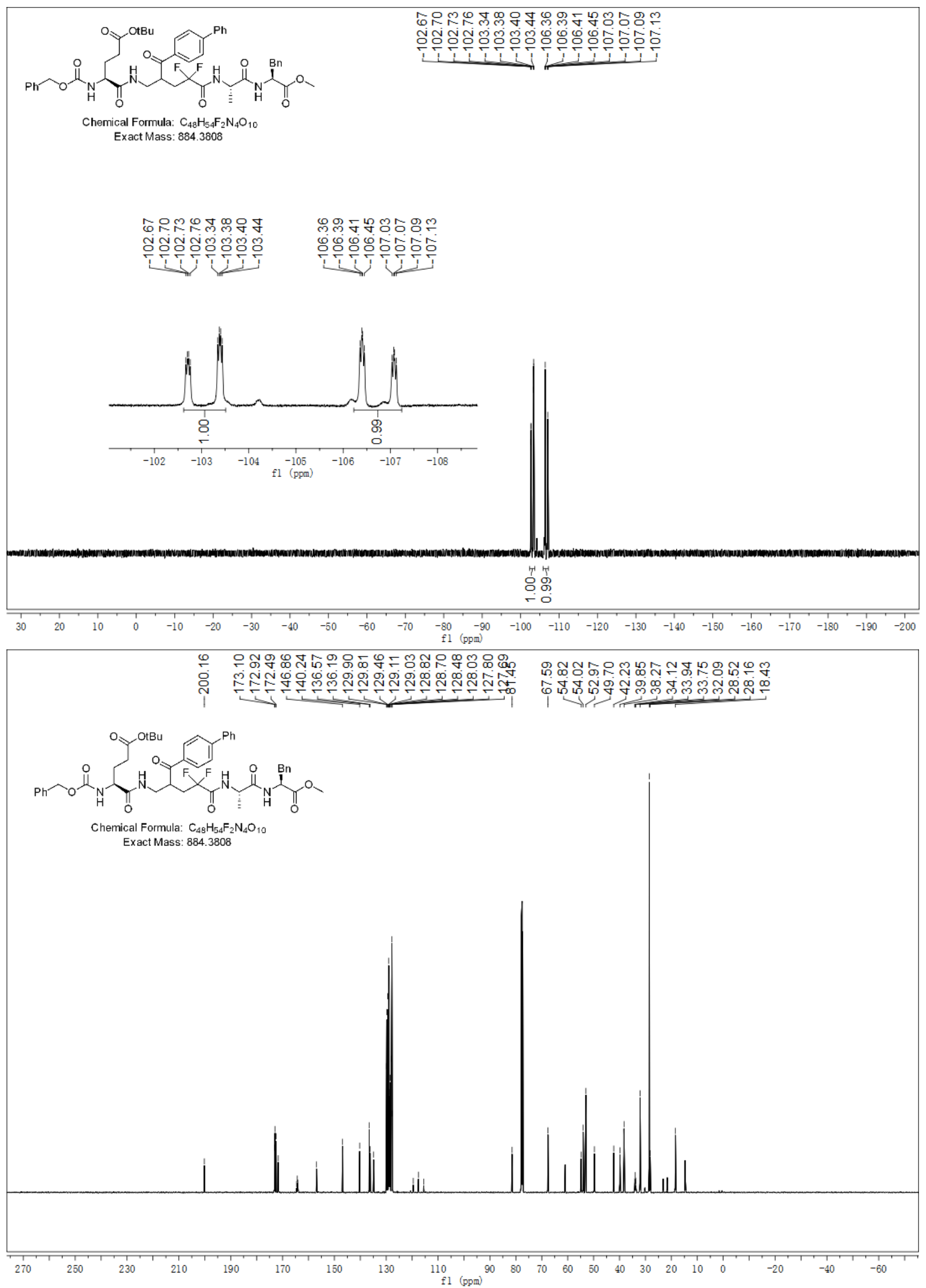
Methyl (5S,14S,17S)-9-([1,1'-biphenyl]-4-carbonyl)-17-benzyl-5-(3-(tert-butoxy)-3-oxopropyl)-

11,11-difluoro-14-methyl-3,6,12,15-tetraoxo-1-phenyl-2-oxa-4,7,13,16-tetraazaoctadecan-18oate (8e-2).
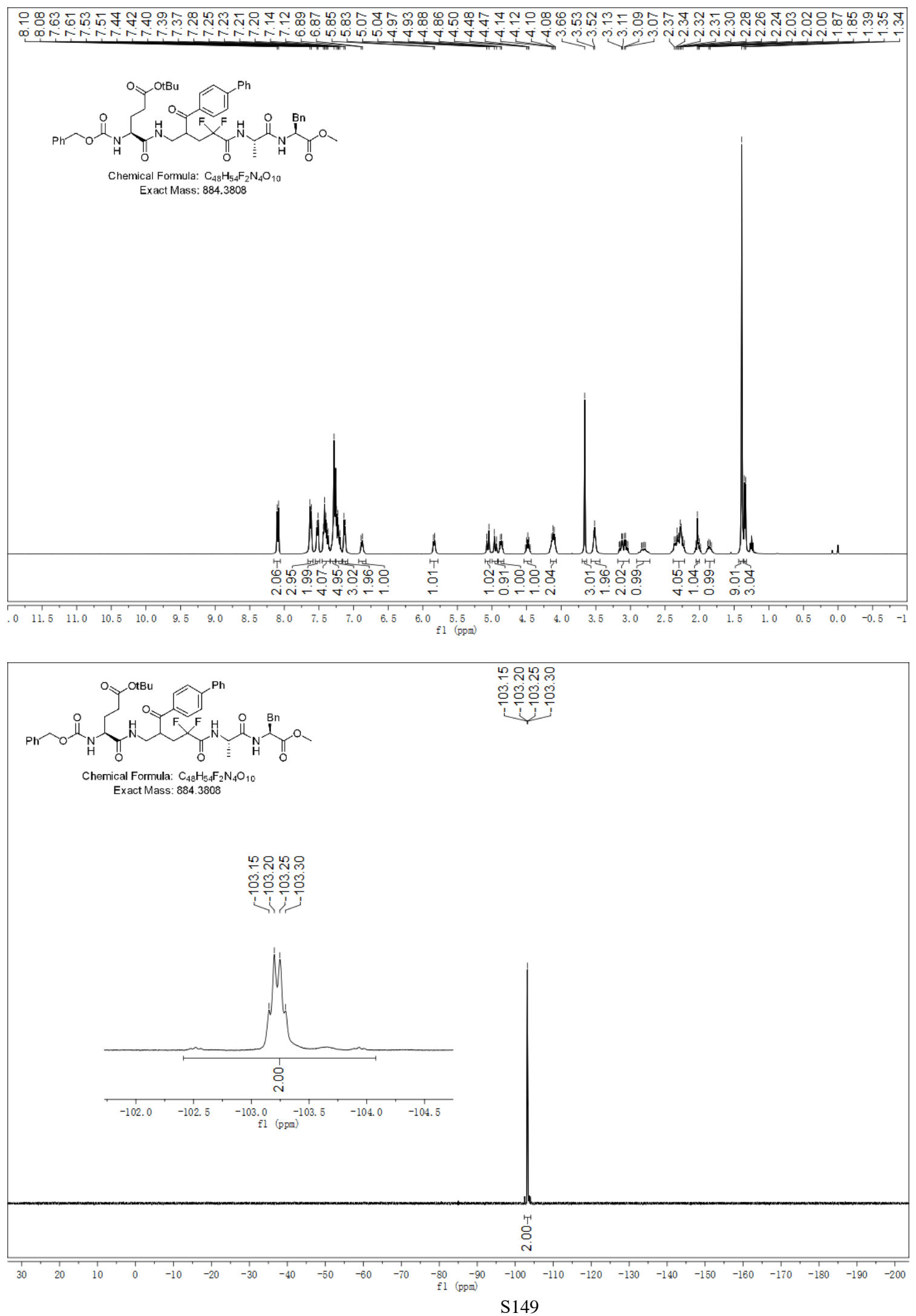


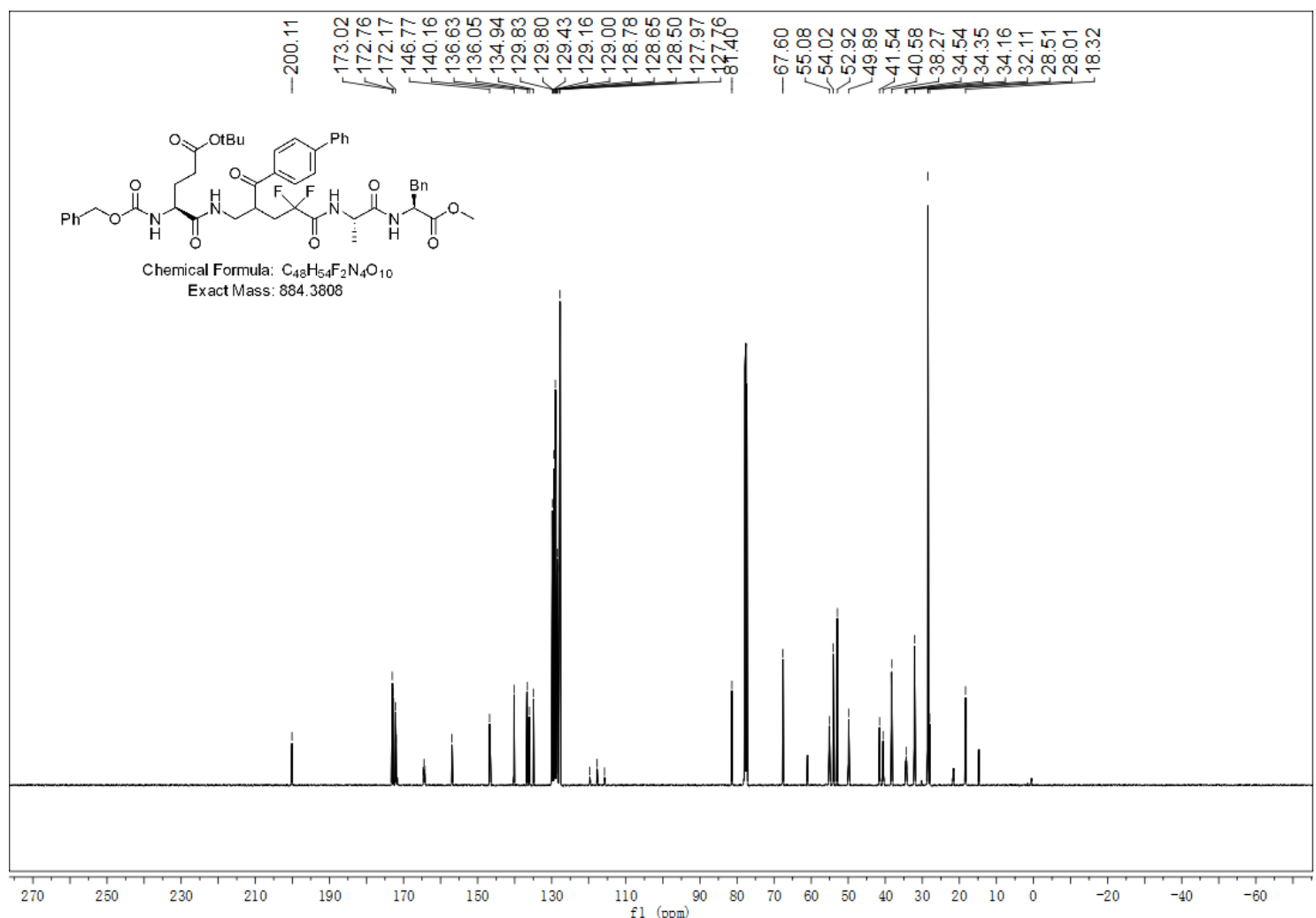

Methyl (6S,15S,18S)-6,18-dibenzyl-10-(4-chlorobenzoyl)-12,12-difluoro-15-(2-methoxy-2-

oxoethyl)-2,2-dimethyl-4,7,13,16-tetraoxo-3-oxa-5,8,14,17-tetraazanonadecan-19-0ate (8f-1).

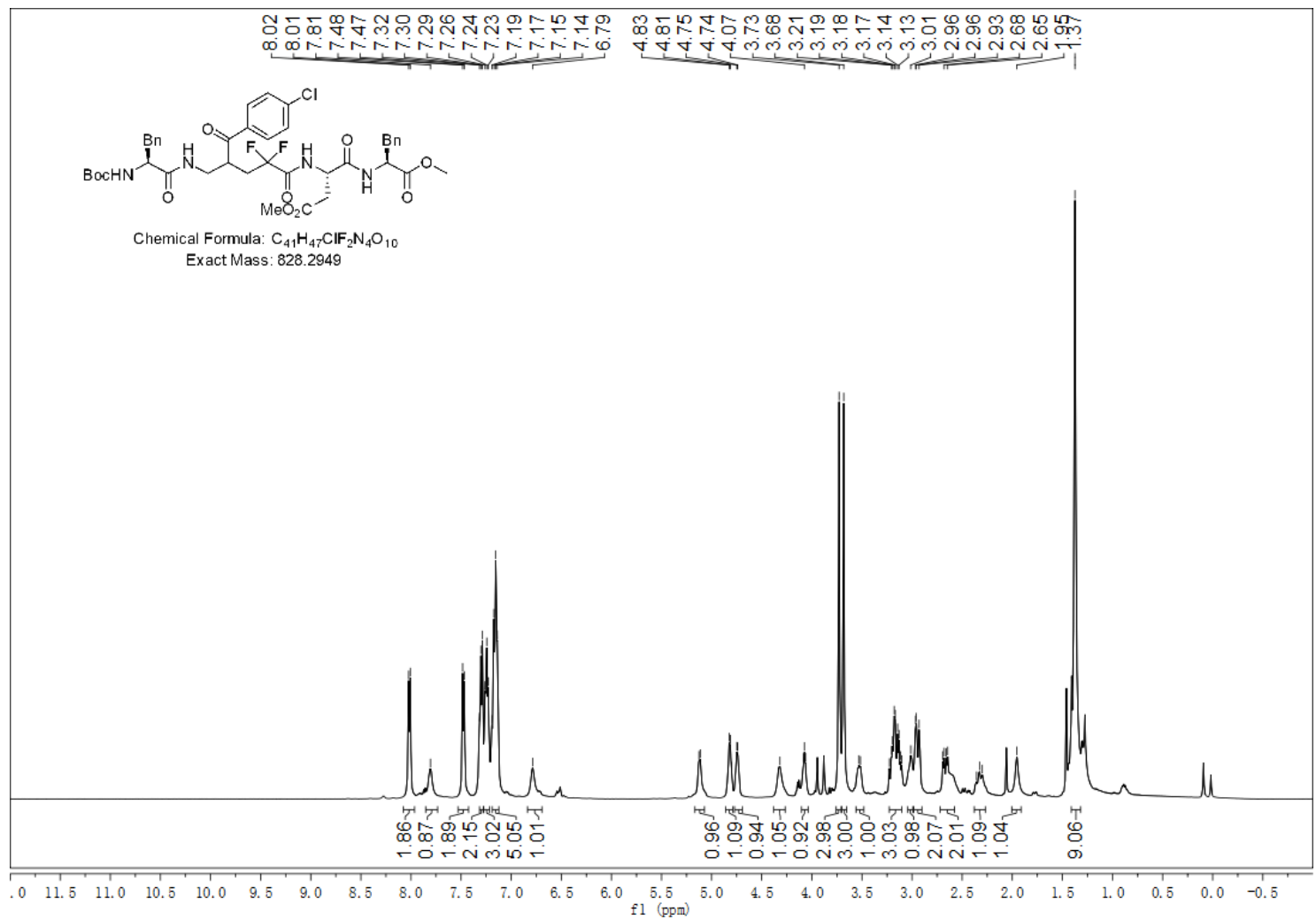



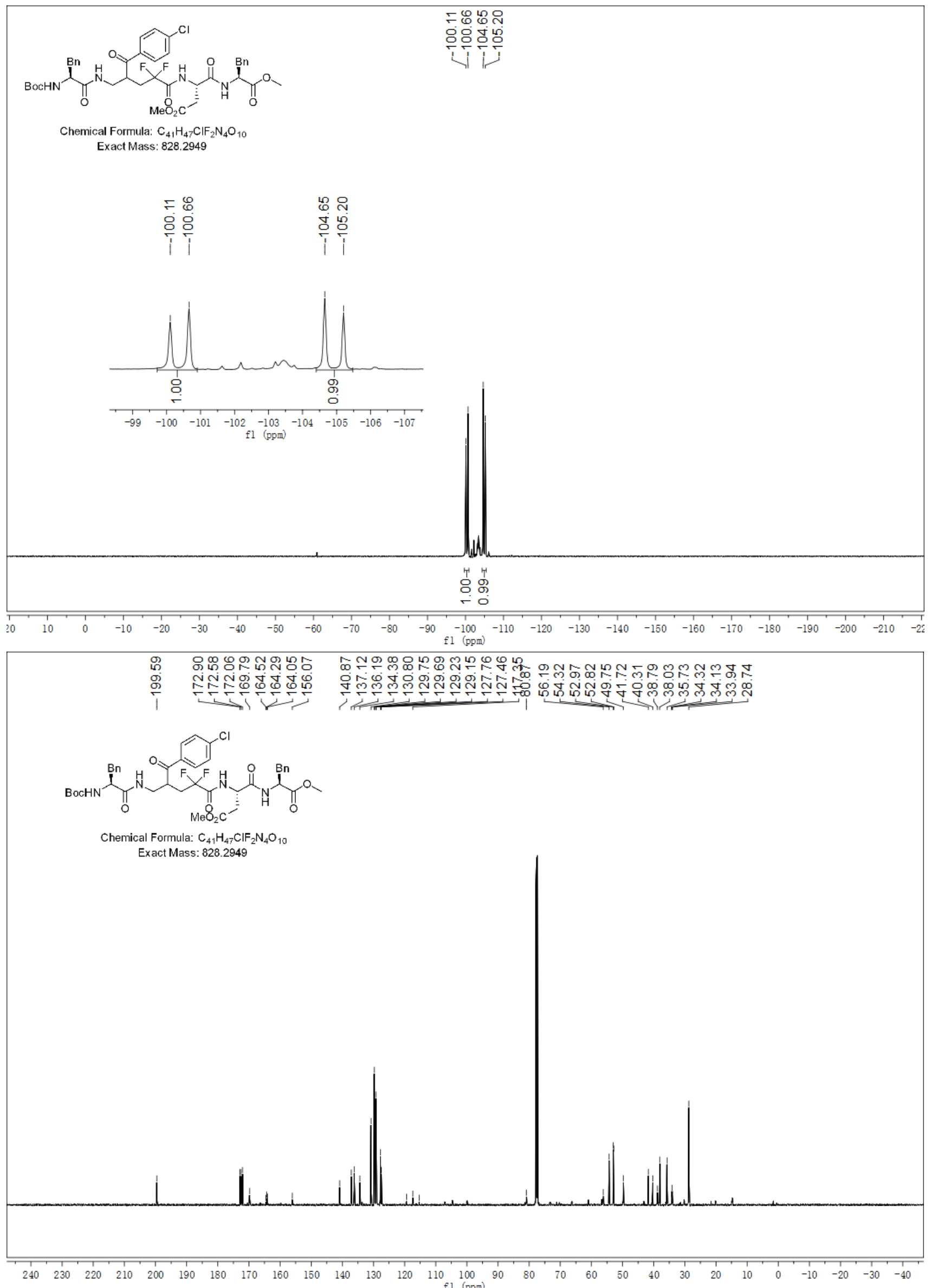


\section{Methyl (6S,15S,18S)-6,18-dibenzyl-10-(4-chlorobenzoyl)-12,12-difluoro-15-(2-methoxy-2-}

oxoethyl)-2,2-dimethyl-4,7,13,16-tetraoxo-3-oxa-5,8,14,17-tetraazanonadecan-19-0ate (8f-2).

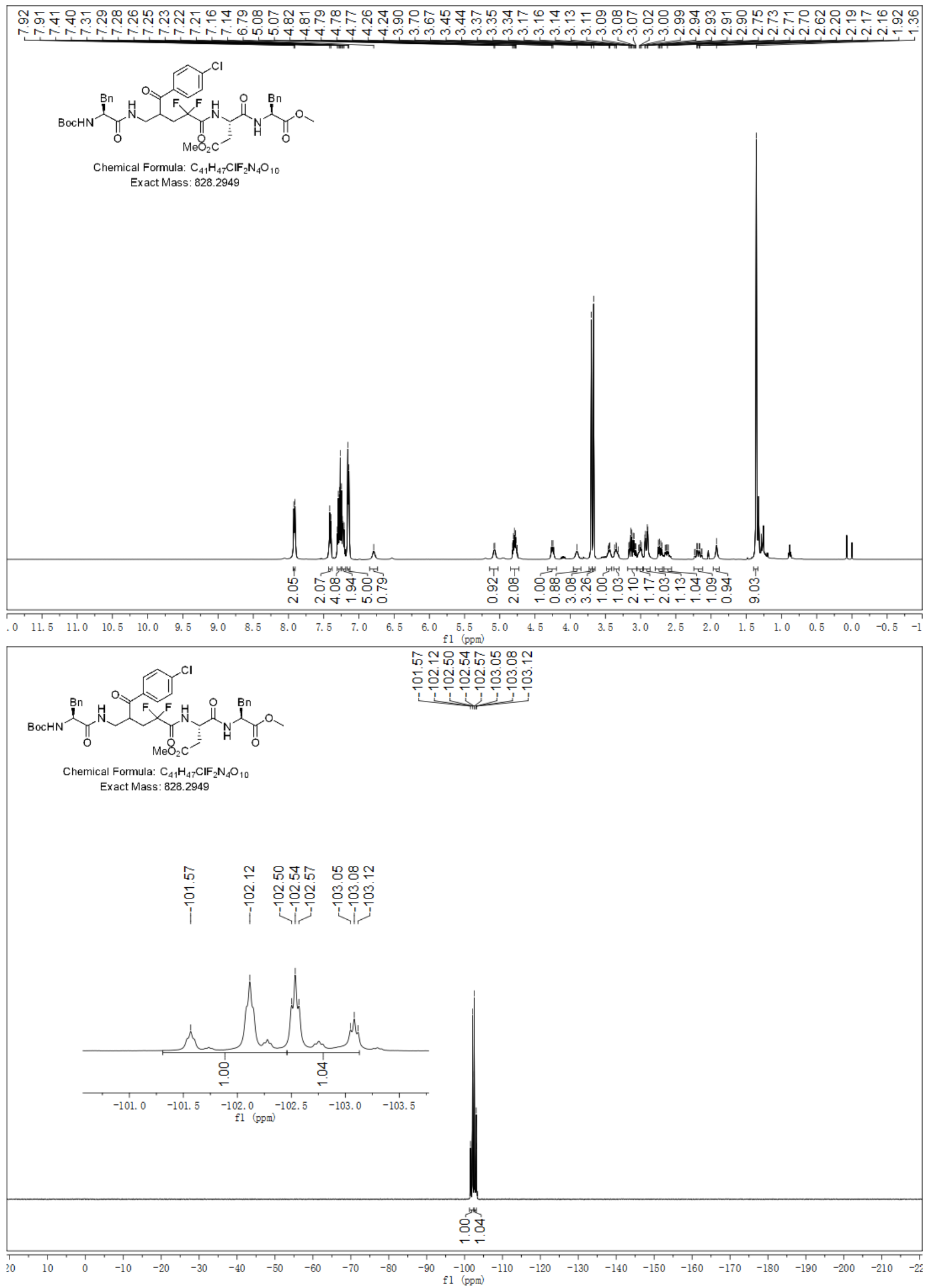




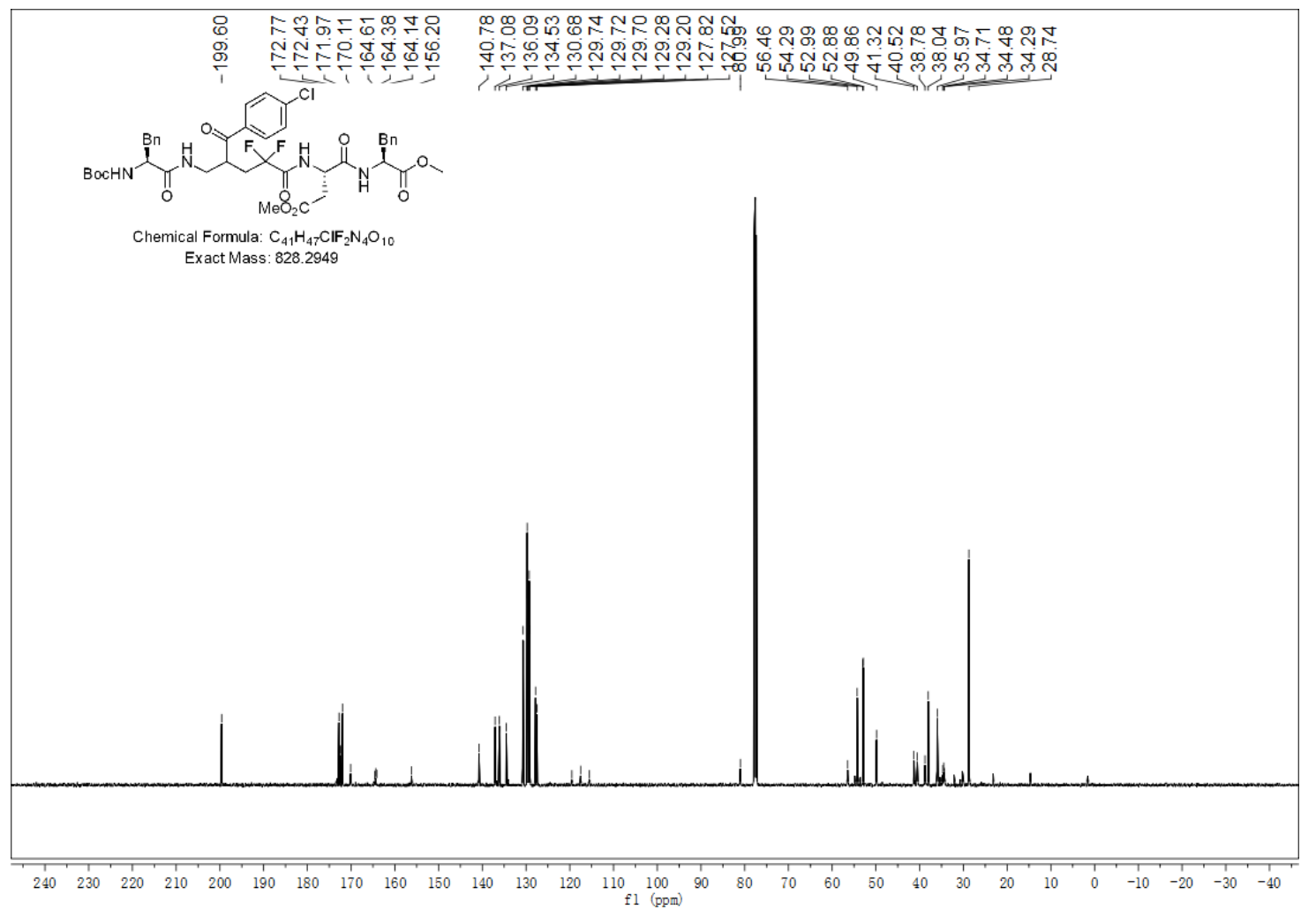

Methyl ((S)-4-(((S)-2-((S)-2-(((benzyloxy)carbonyl)amino)-3-phenylpropanamido)-4methylpentanamido)methyl)-2,2-difluoro-5-(4-methoxyphenyl)-5-oxopentanoyl)- $L$-alanyl- $L$ phenylalaninate (8g-1).

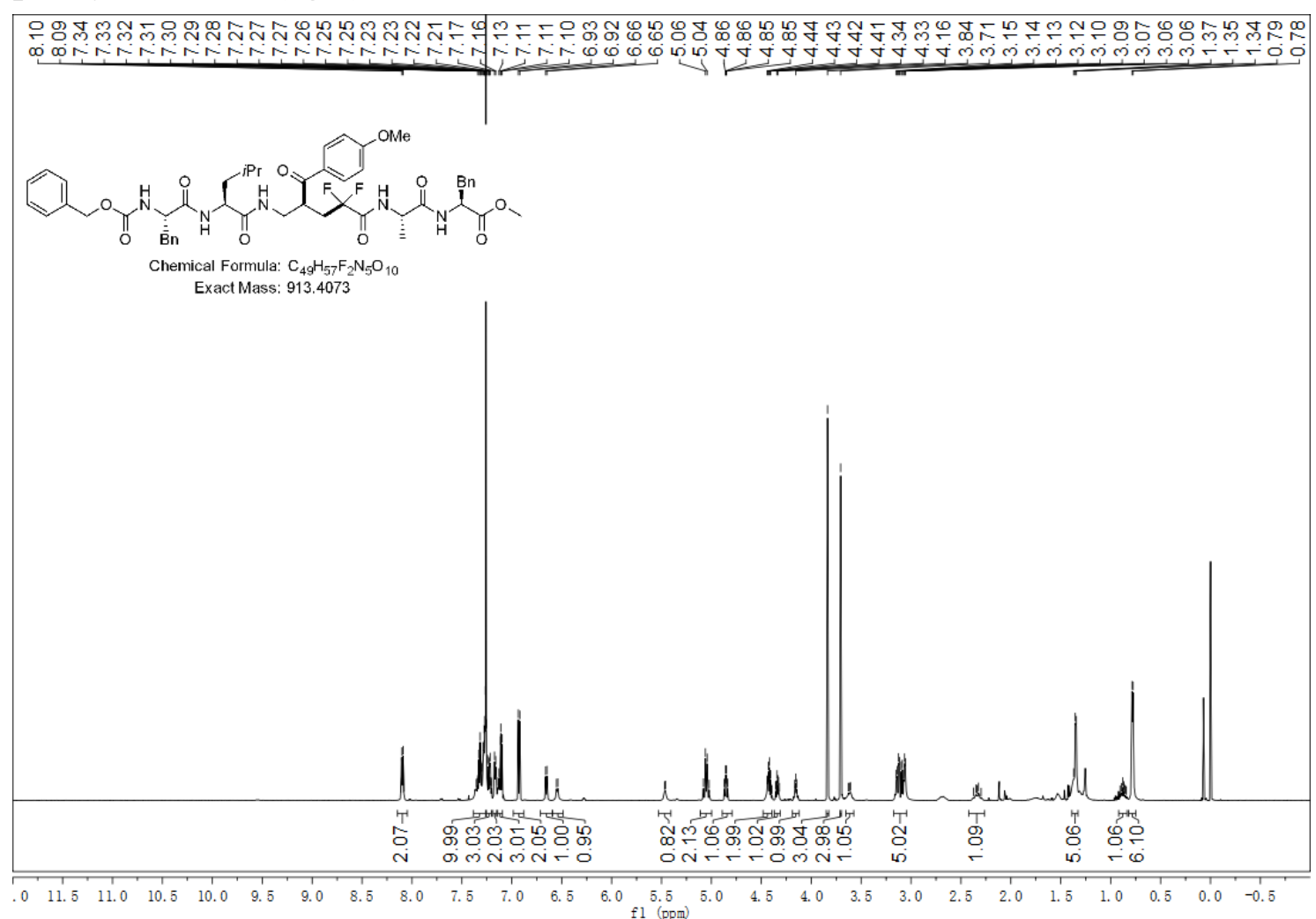




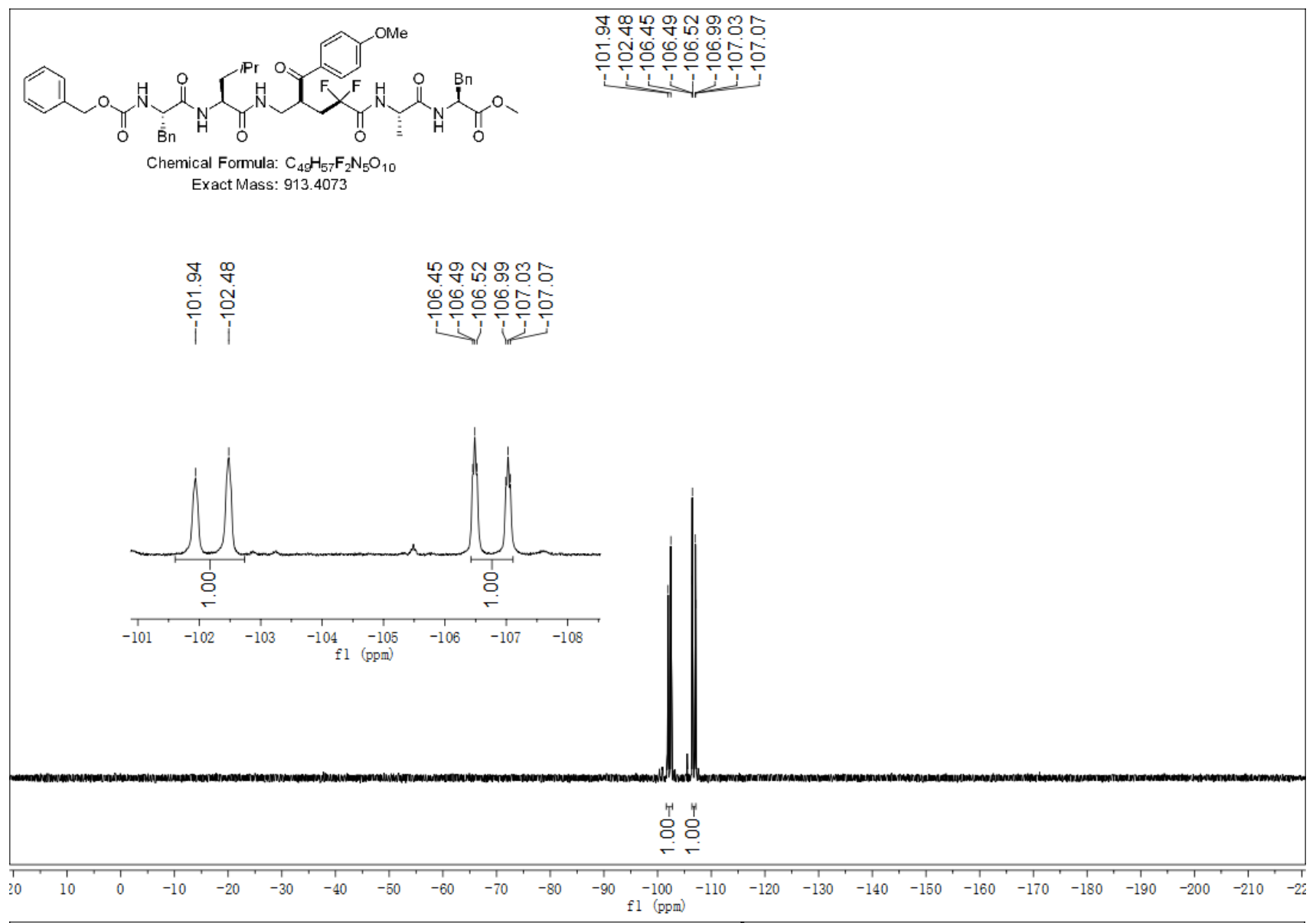

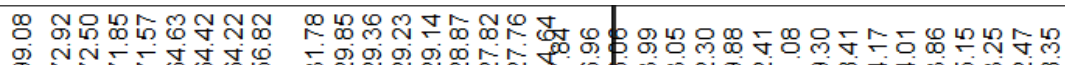

类

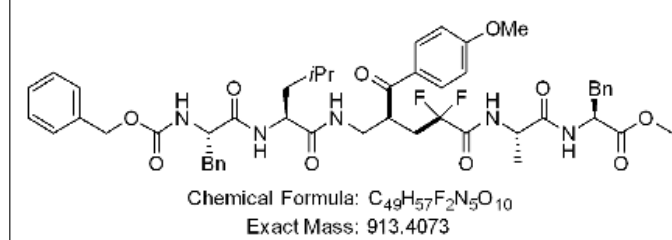

Exact Mass: 913.4073
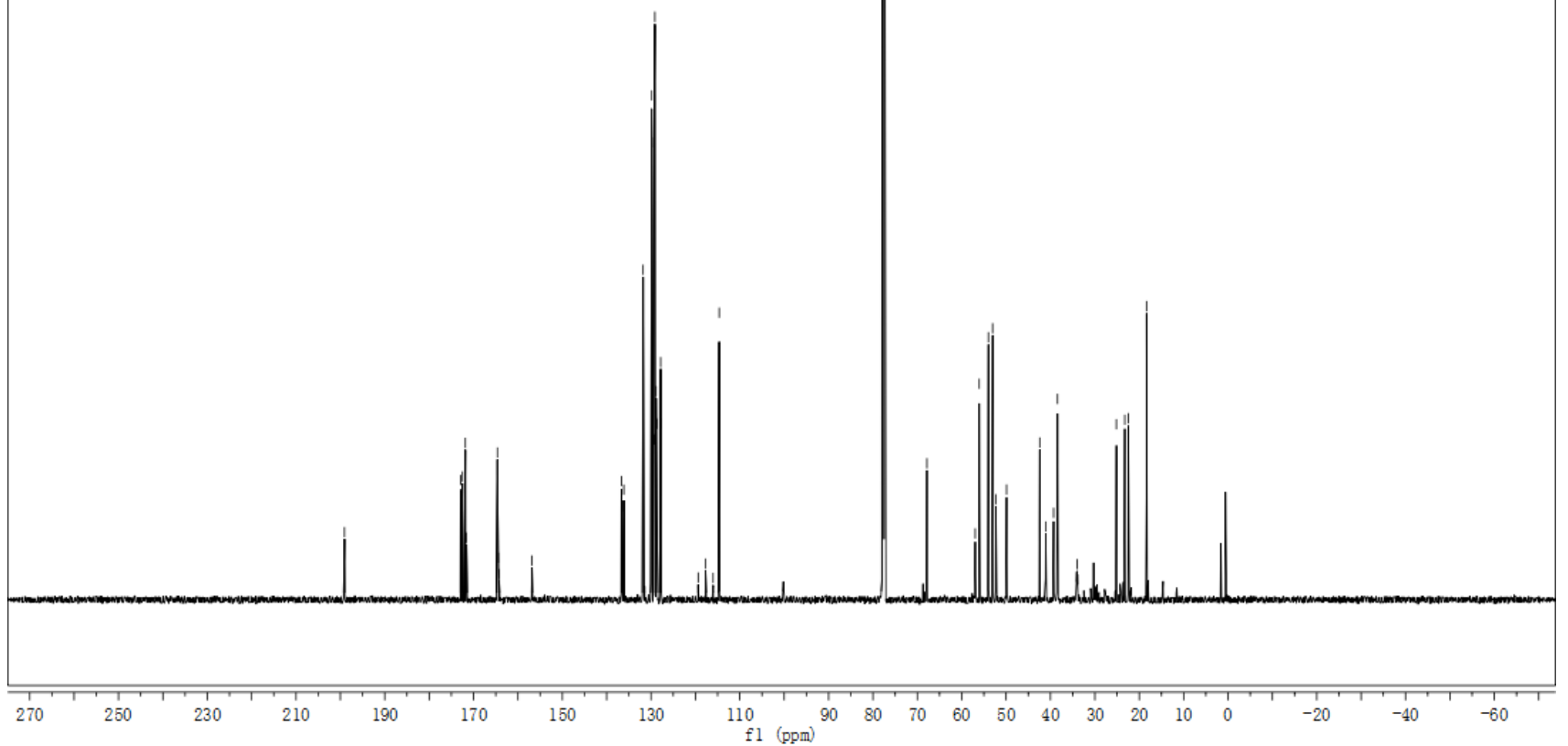
Methyl ((S)-4-(((S)-2-((S)-2-(((benzyloxy)carbonyl)amino)-3-phenylpropanamido)-4methylpentanamido)methyl)-2,2-difluoro-5-(4-methoxyphenyl)-5-oxopentanoyl)- $L$-alanyl- $L$ phenylalaninate (8g-2).

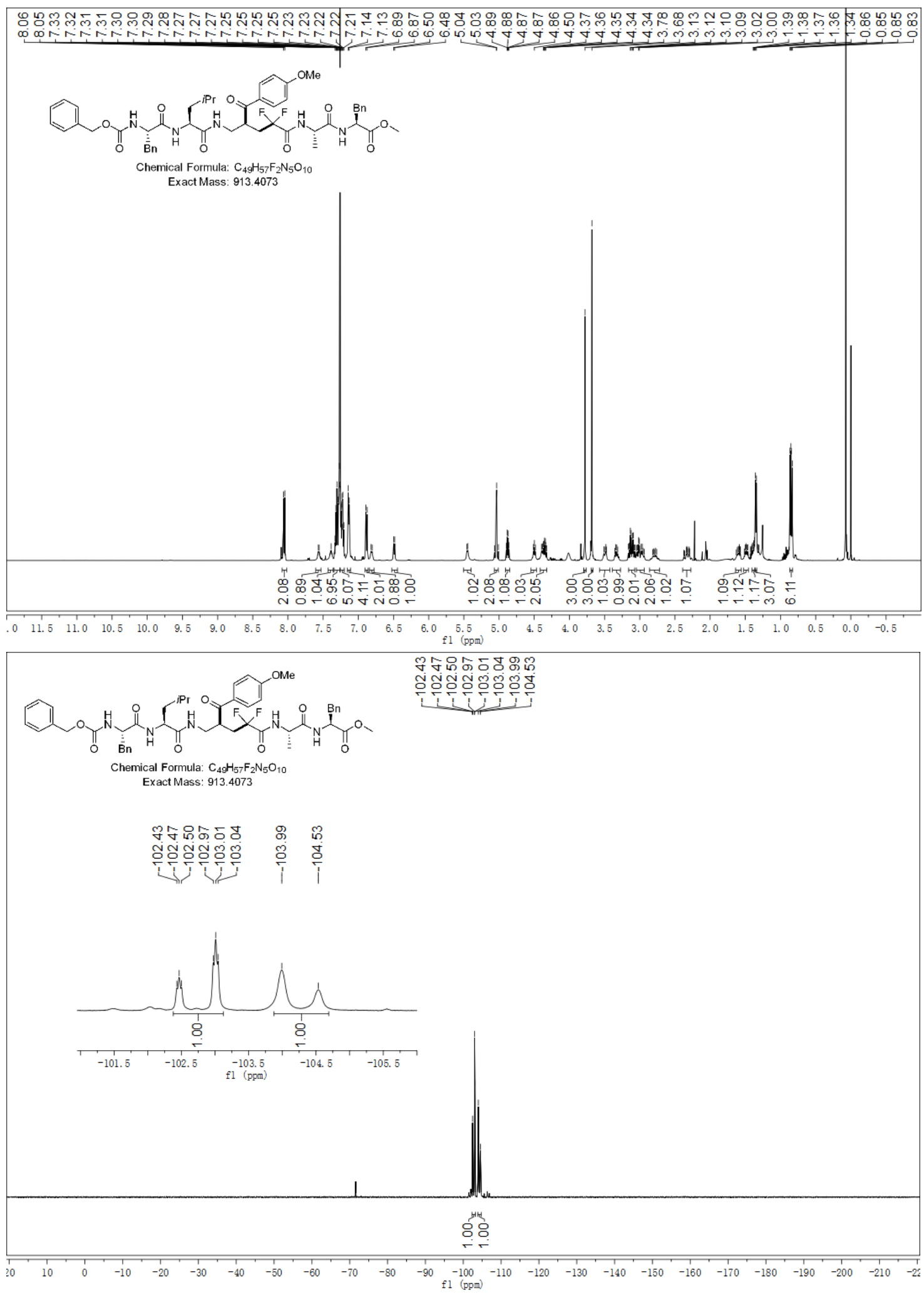




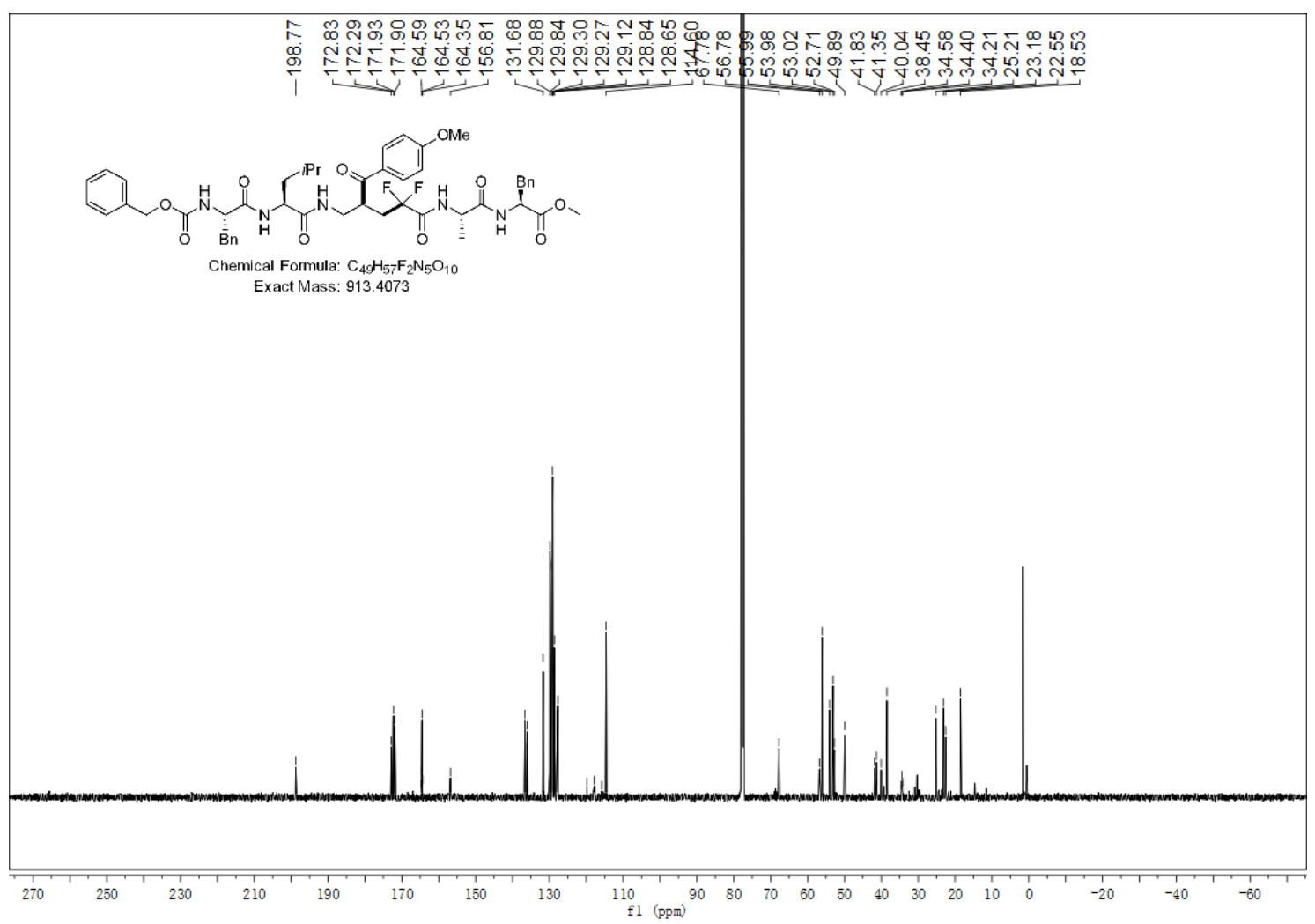

Methyl (5S,14S)-5-(3-(tert-butoxy)-3-oxopropyl)-9-(4-((S)-2-((tert-butoxycarbonyl)amino)-3methoxy-3-oxopropyl)benzoyl)-14-(tert-butyl)-11,11-difluoro-3,6,12-trioxo-1-phenyl-2-oxa4,7,13-triazapentadecan-15-oate (8h-1).

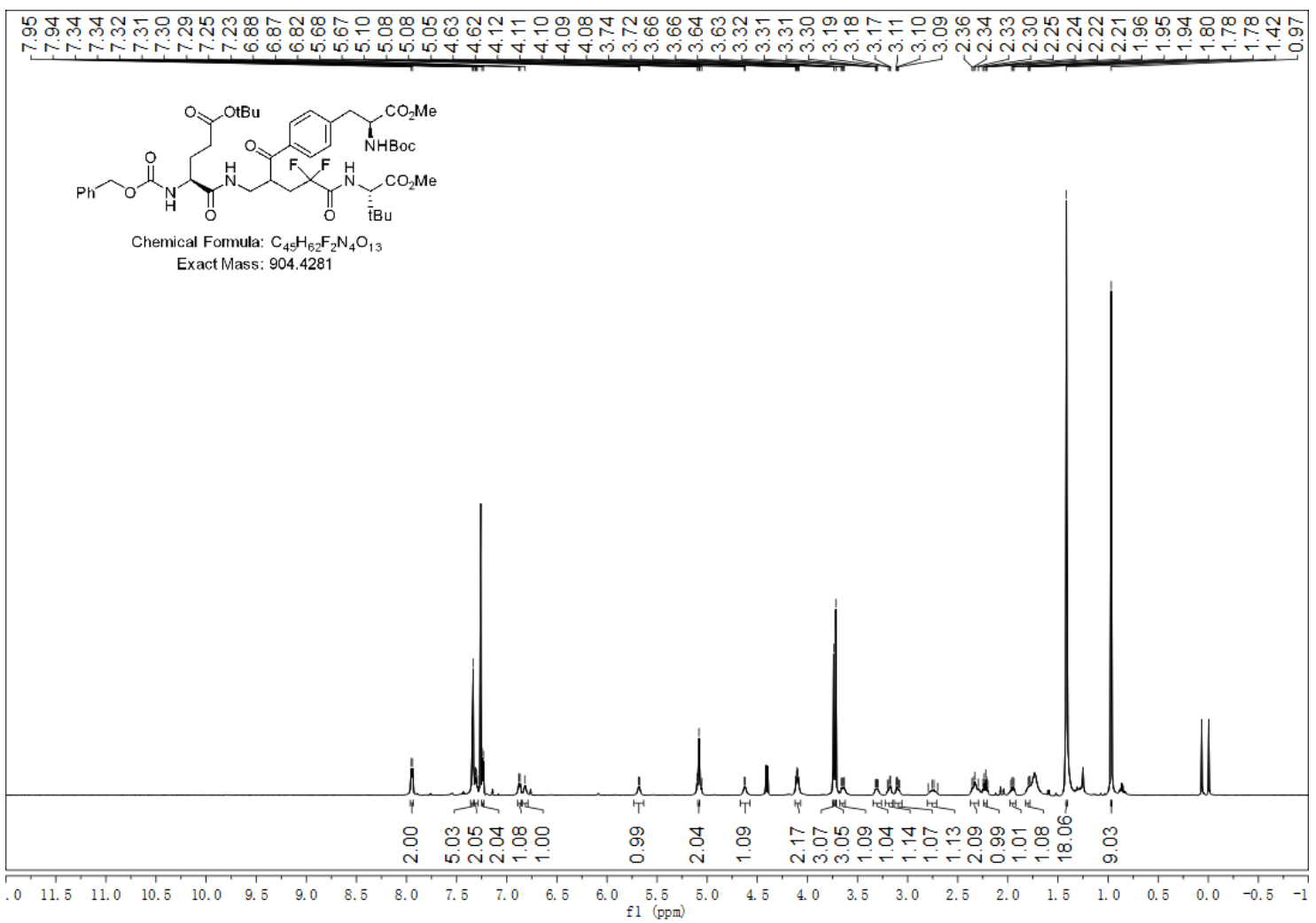




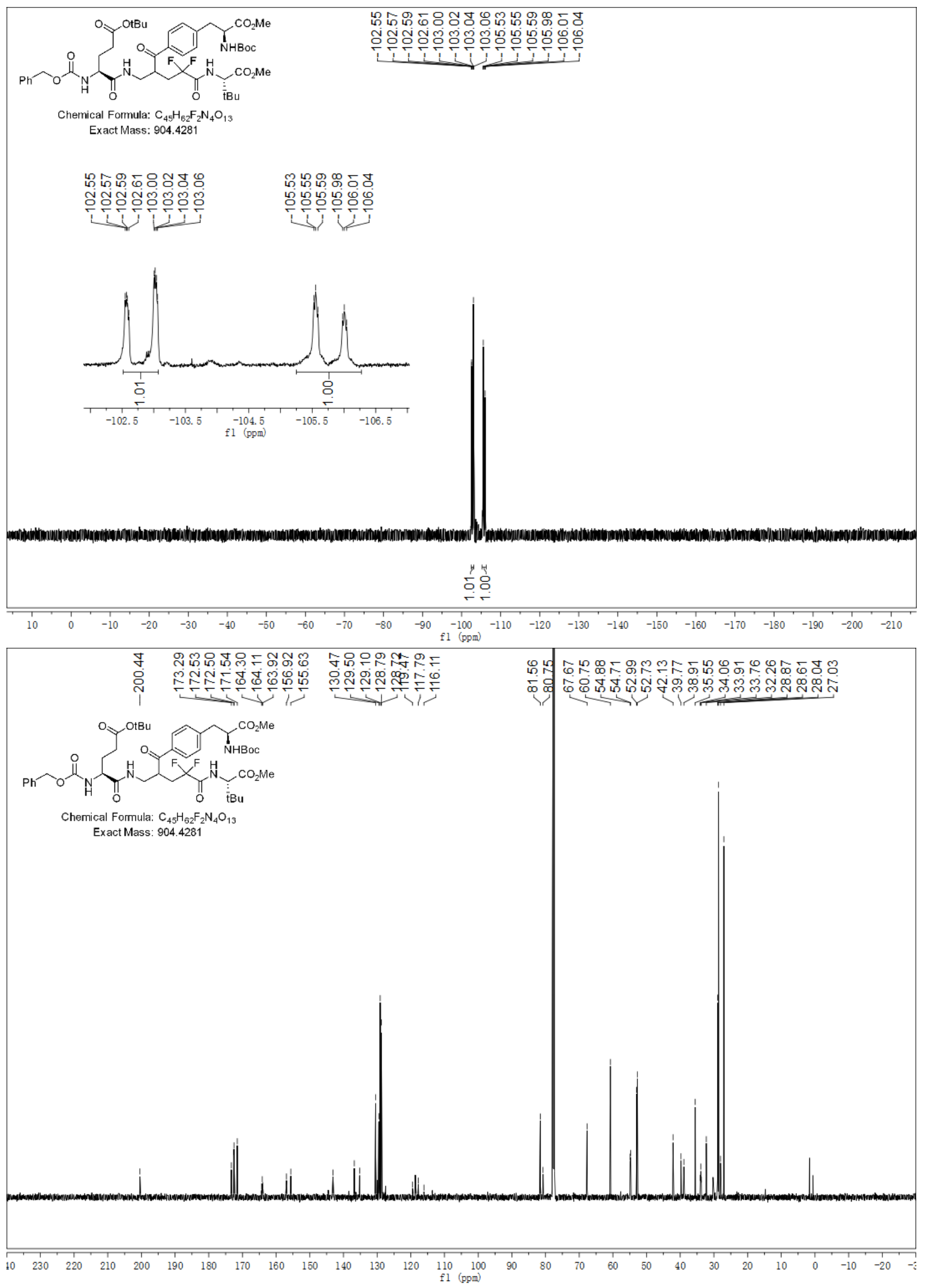


Methyl (5S,14S)-5-(3-(tert-butoxy)-3-oxopropyl)-9-(4-((S)-2-((tert-butoxycarbonyl)amino)-3methoxy-3-oxopropyl)benzoyl)-14-(tert-butyl)-11,11-difluoro-3,6,12-trioxo-1-phenyl-2-oxa4,7,13-triazapentadecan-15-oate $(8 \mathrm{~h}-2)$.

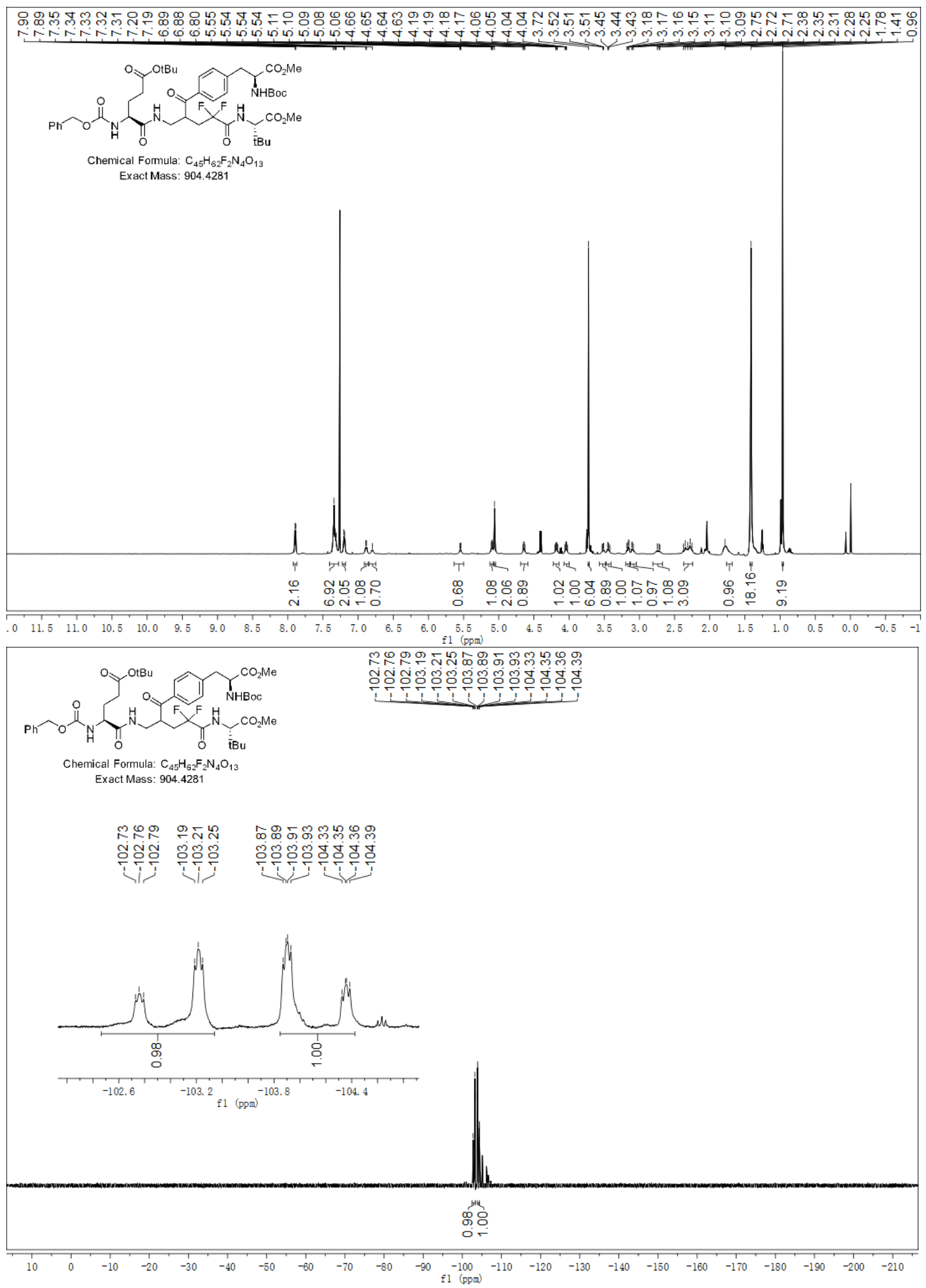




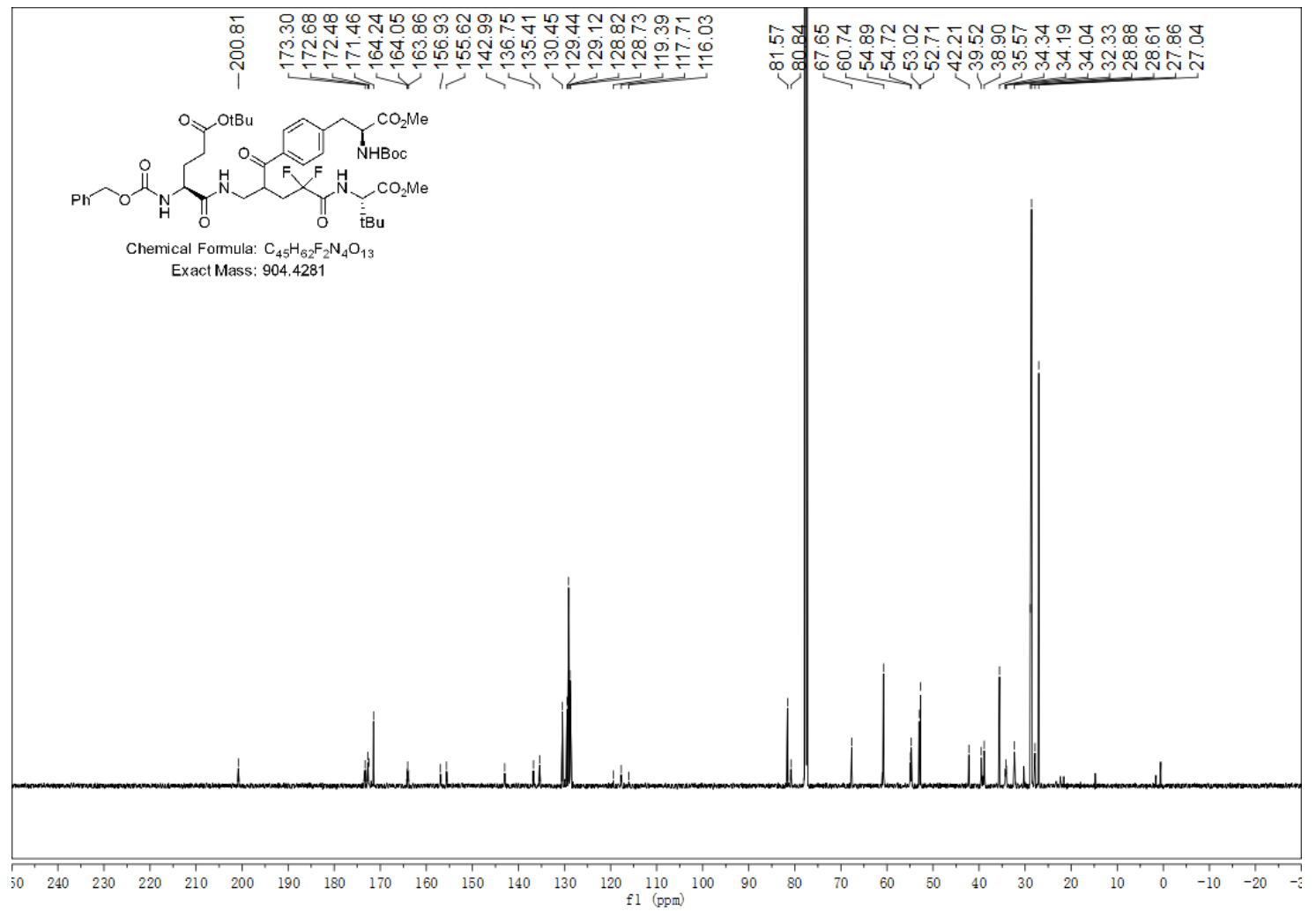

Methyl ((4S)-4-acetamido-5-(4-chlorophenyl)-2,2-difluoro-5-hydroxypentanoyl)- $L$-alanyl- $L$ phenylalaninate (9).

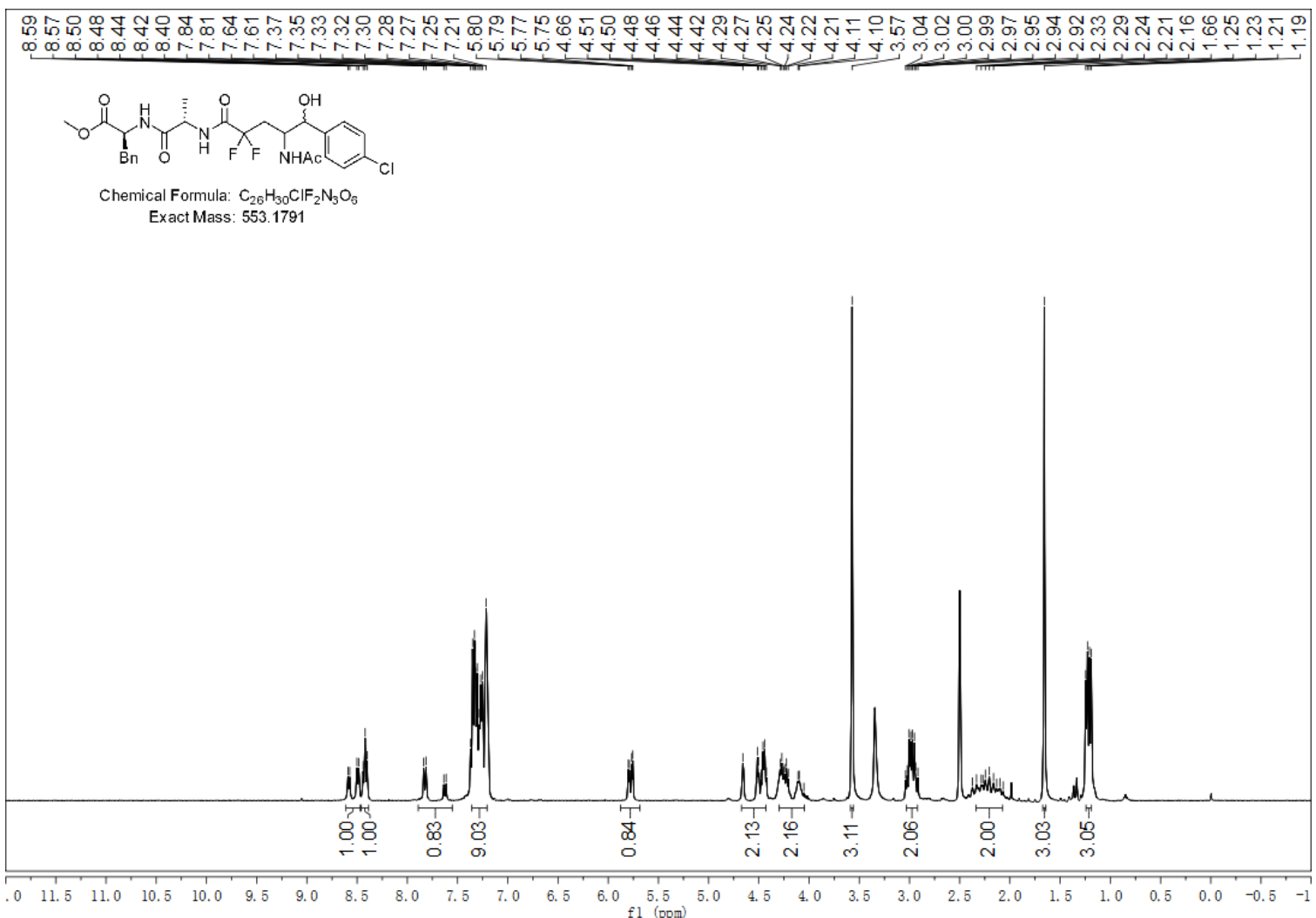




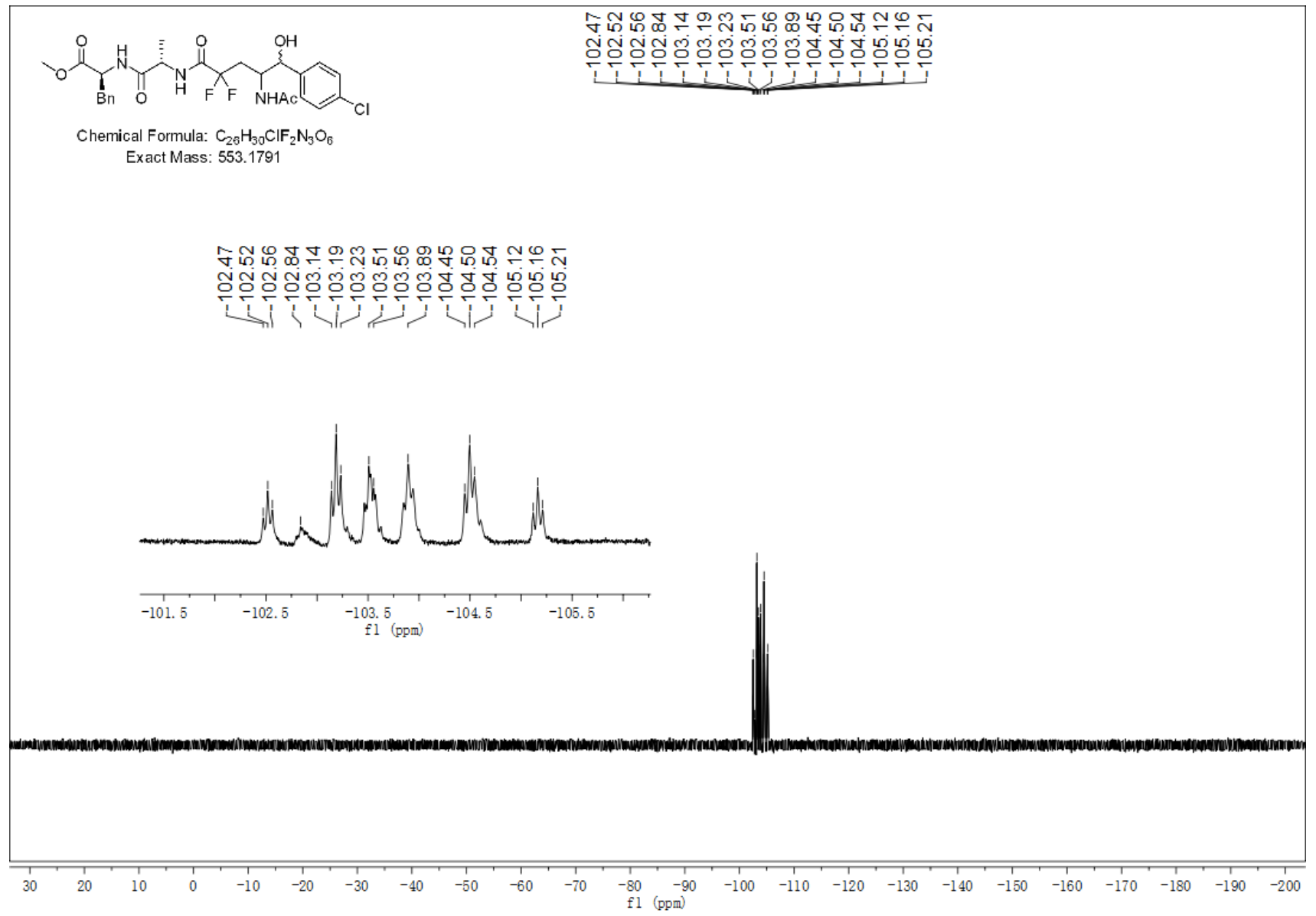

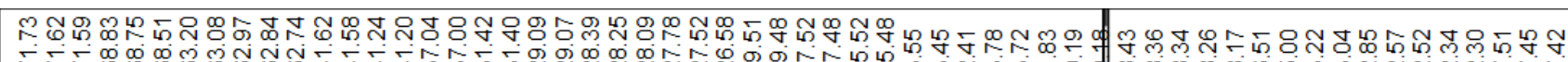

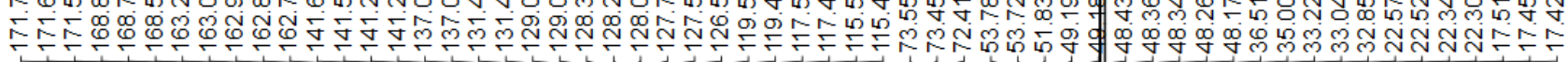

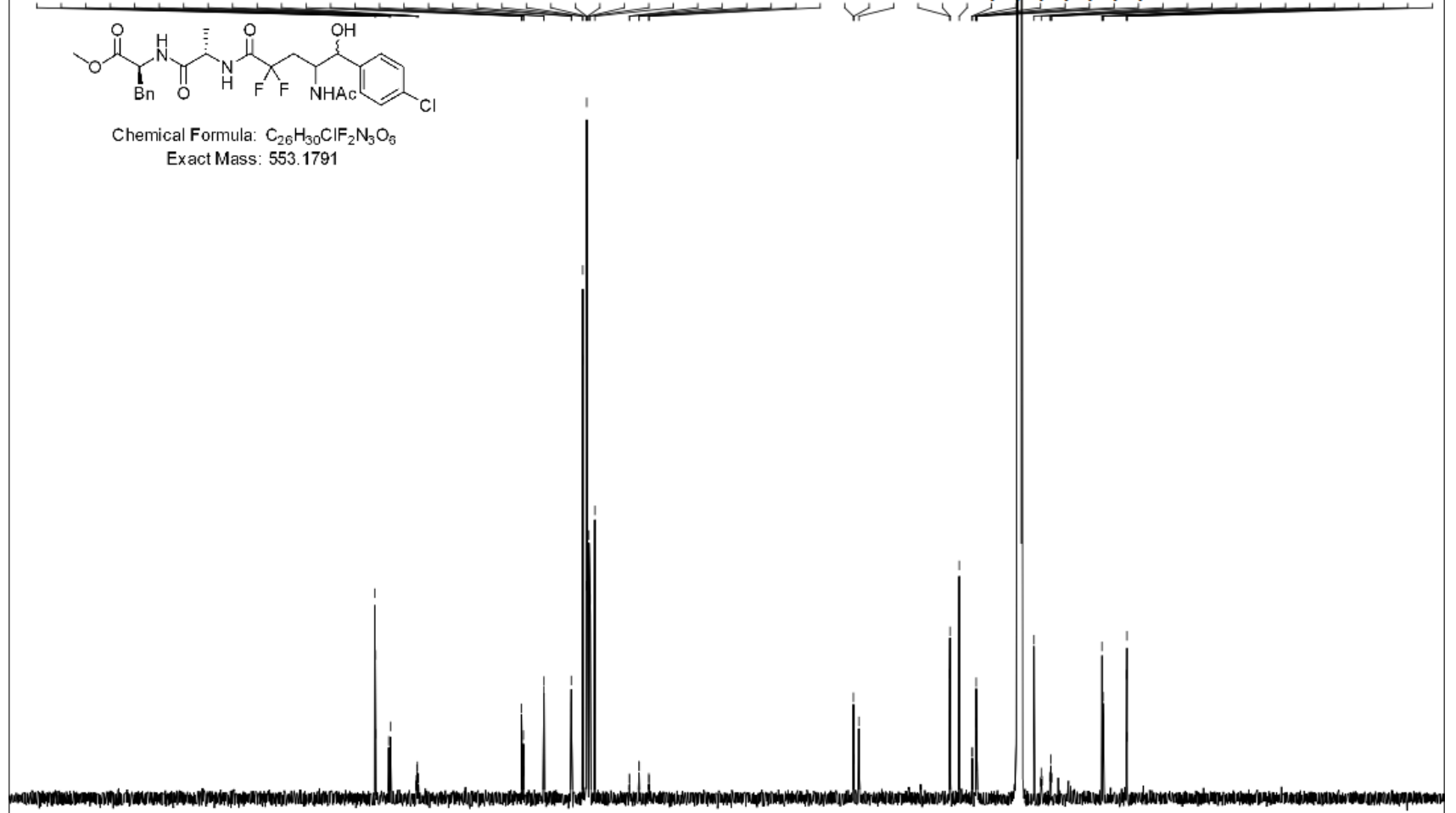

Chemical Formula: $\mathrm{C}_{26} \mathrm{H}_{30} \mathrm{ClF}_{2} \mathrm{~N}_{3} \mathrm{O}_{5}$ Exact Mass: 553.1791

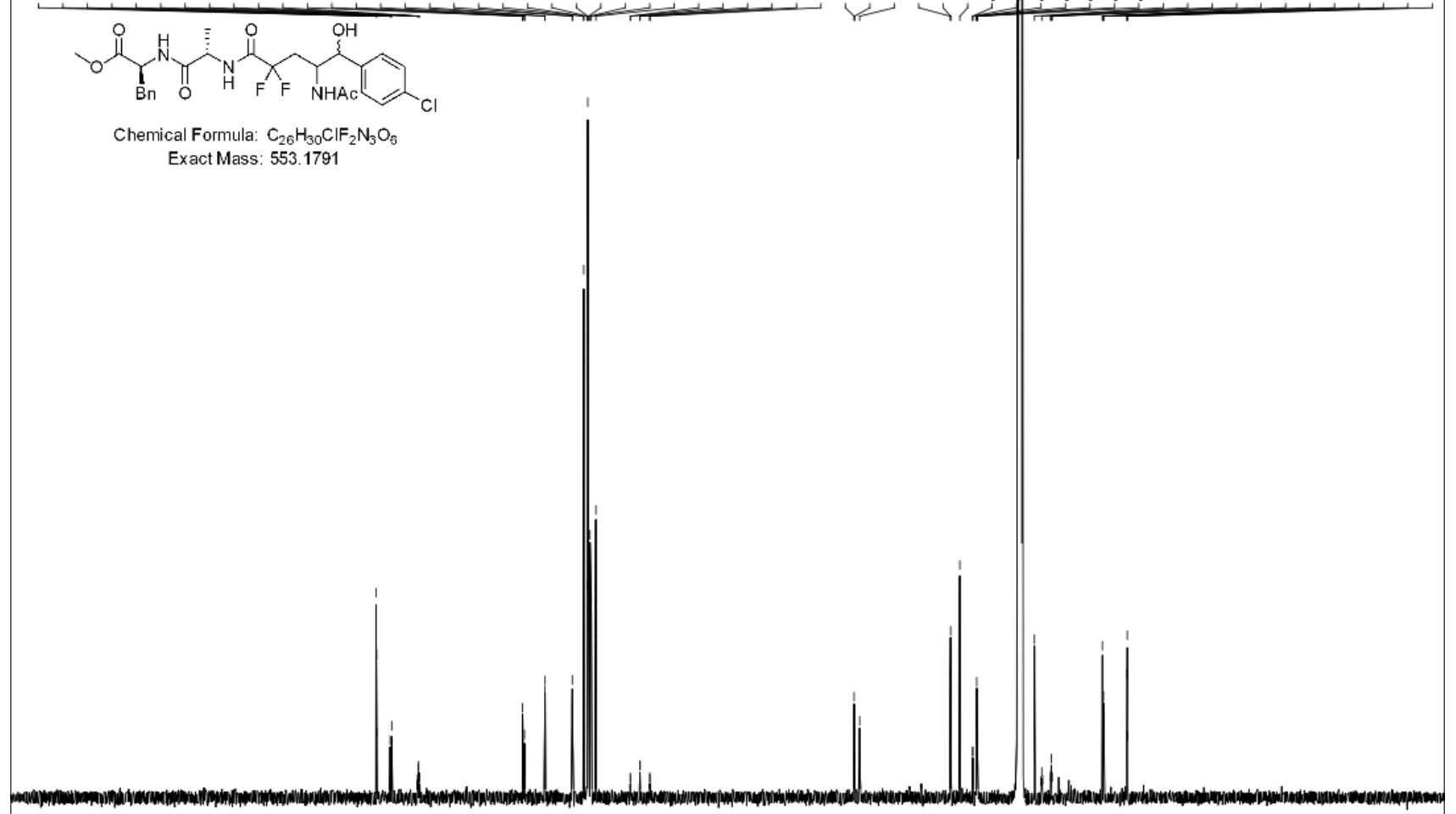

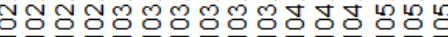

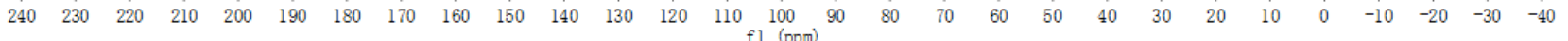




\section{Methyl ((E)-4-acetamido-5-(4-chlorophenyl)-2,2-difluoro-5-(2-(5-((3aS,4S,6aR)-2-}

\section{oxohexahydro-1 $H$-thieno[3,4- $d]$ imidazol-4-yl)pentanoyl)hydrazineylidene)pentanoyl)- $L$-alanyl-}

\section{$L$-phenylalaninate (10).}
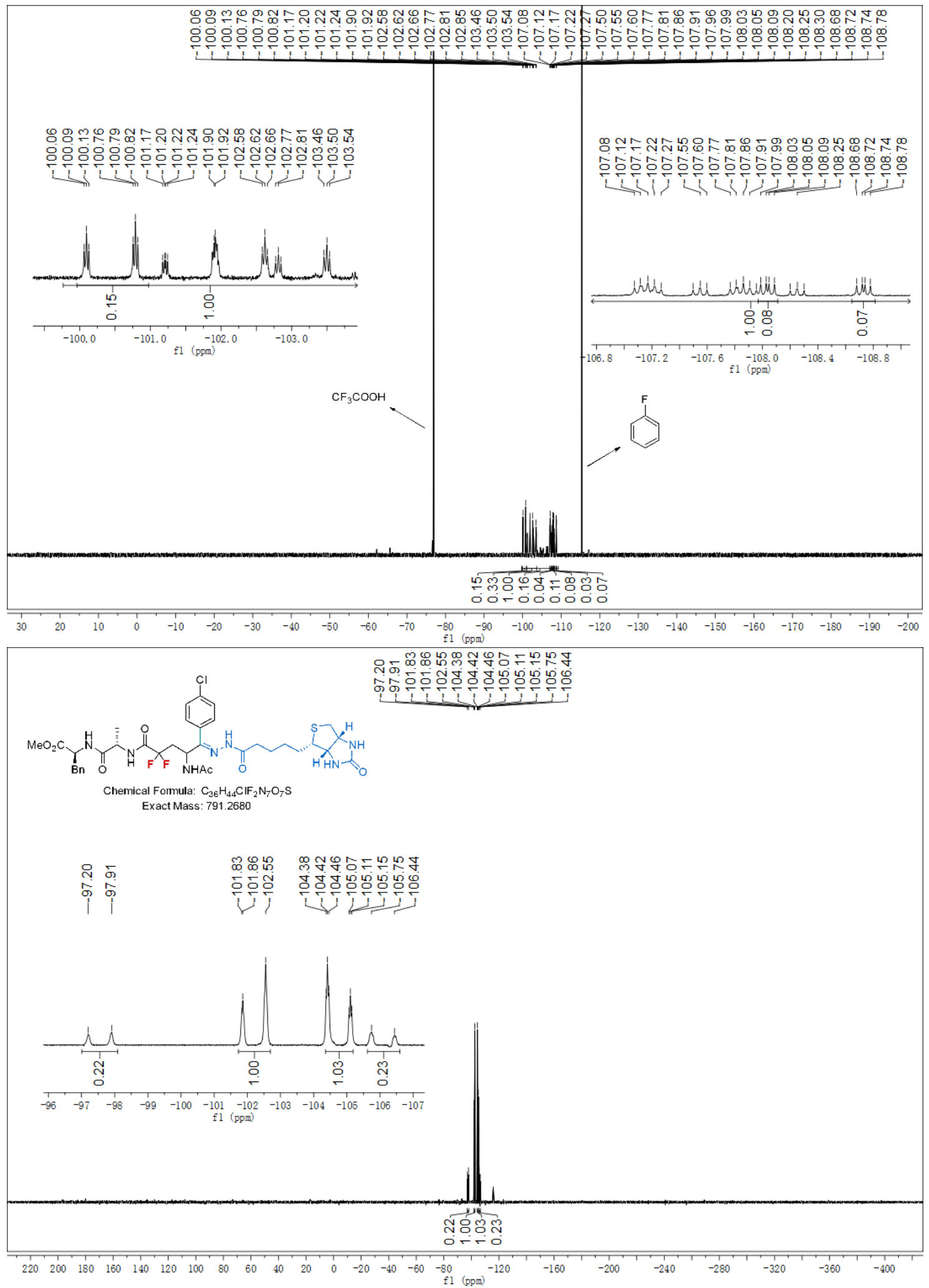
Ethyl 3-(3,4-dihydronaphthalen-1-yl)-2,2-difluoro propanoate (12)
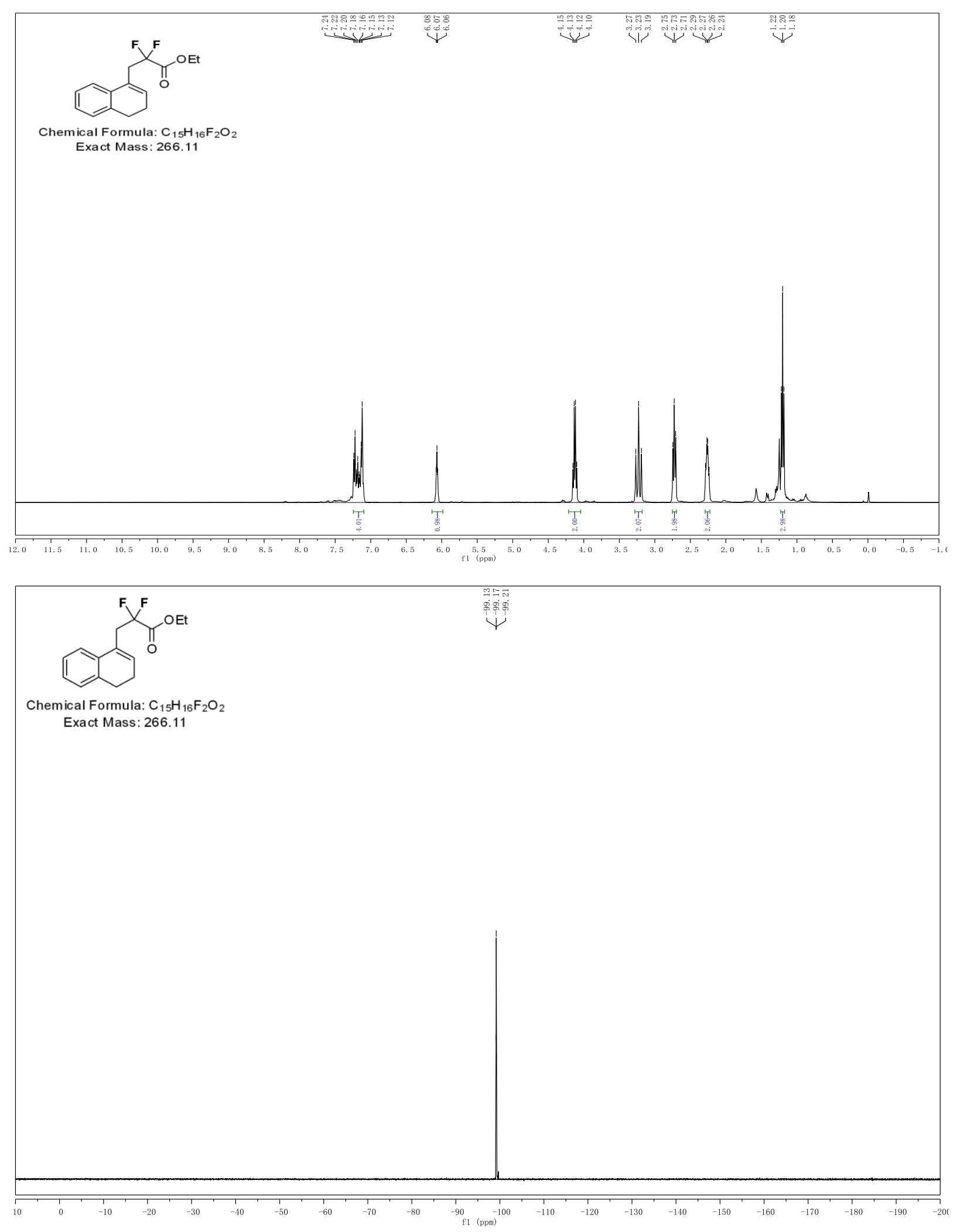

S162 


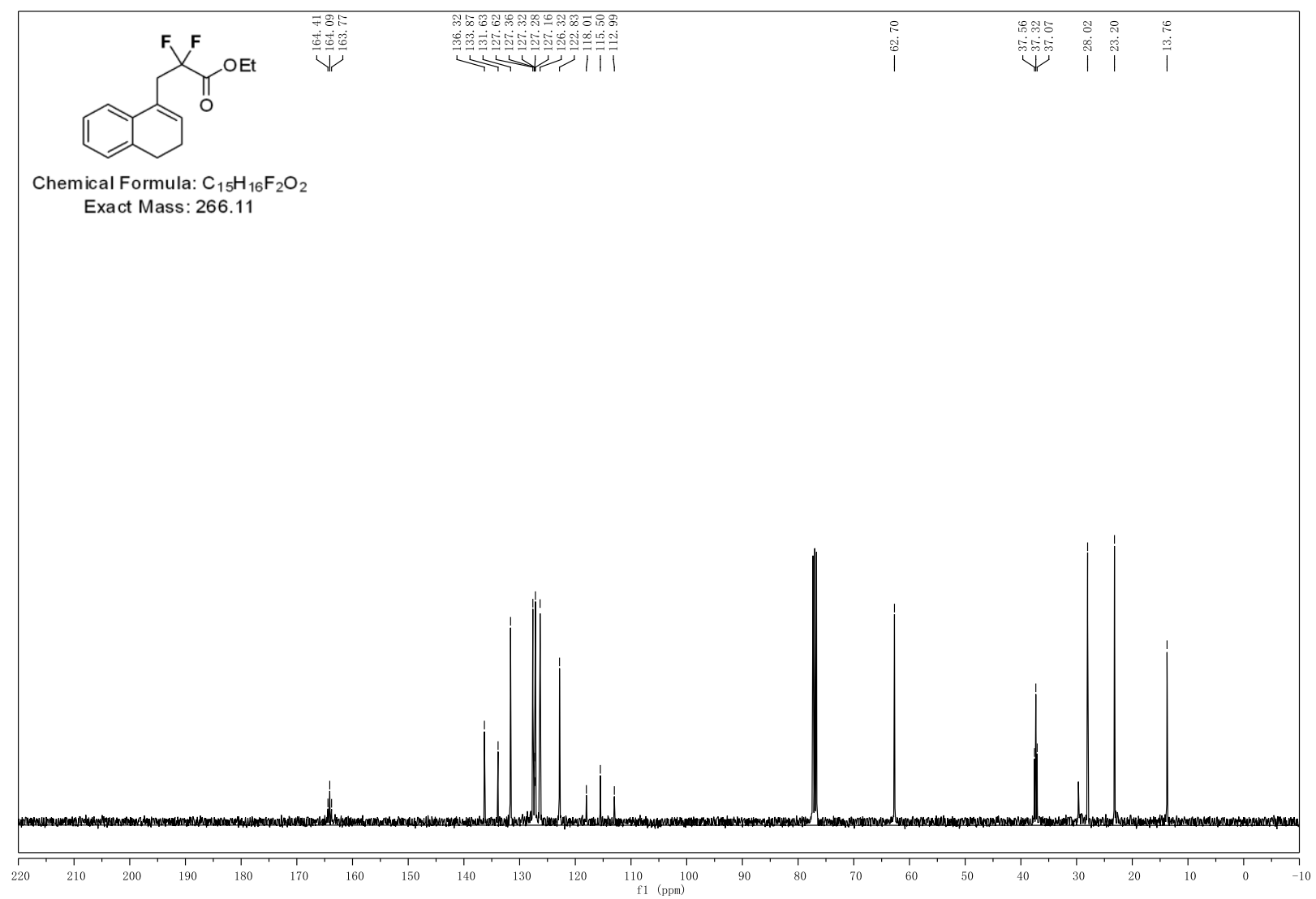

Ethyl 2,2-difluoro-3-(4-fluorophenyl)-3-oxopropanoate (5d)

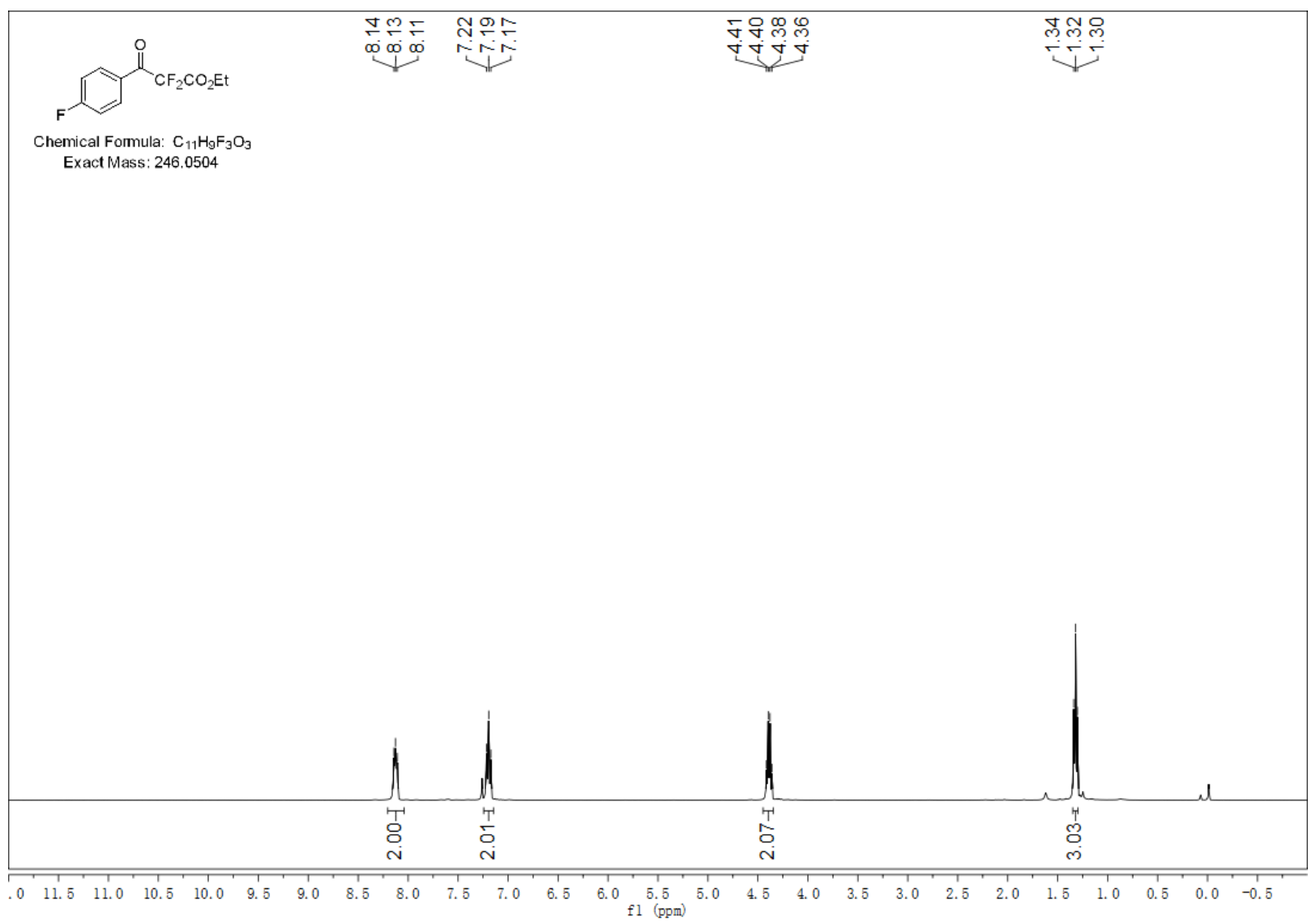



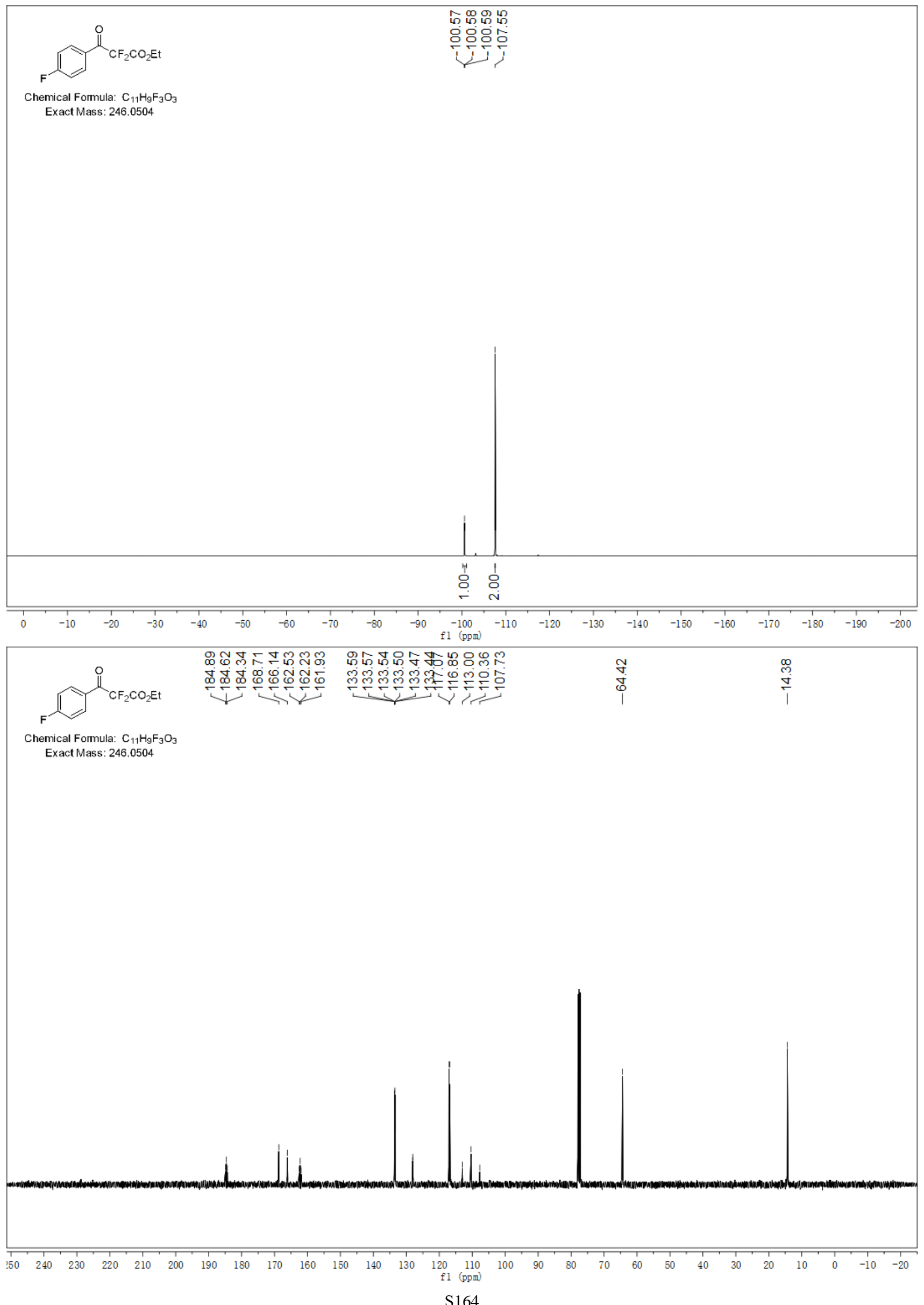
Methyl [1,1'-biphenyl]-4-carboxylate (16)

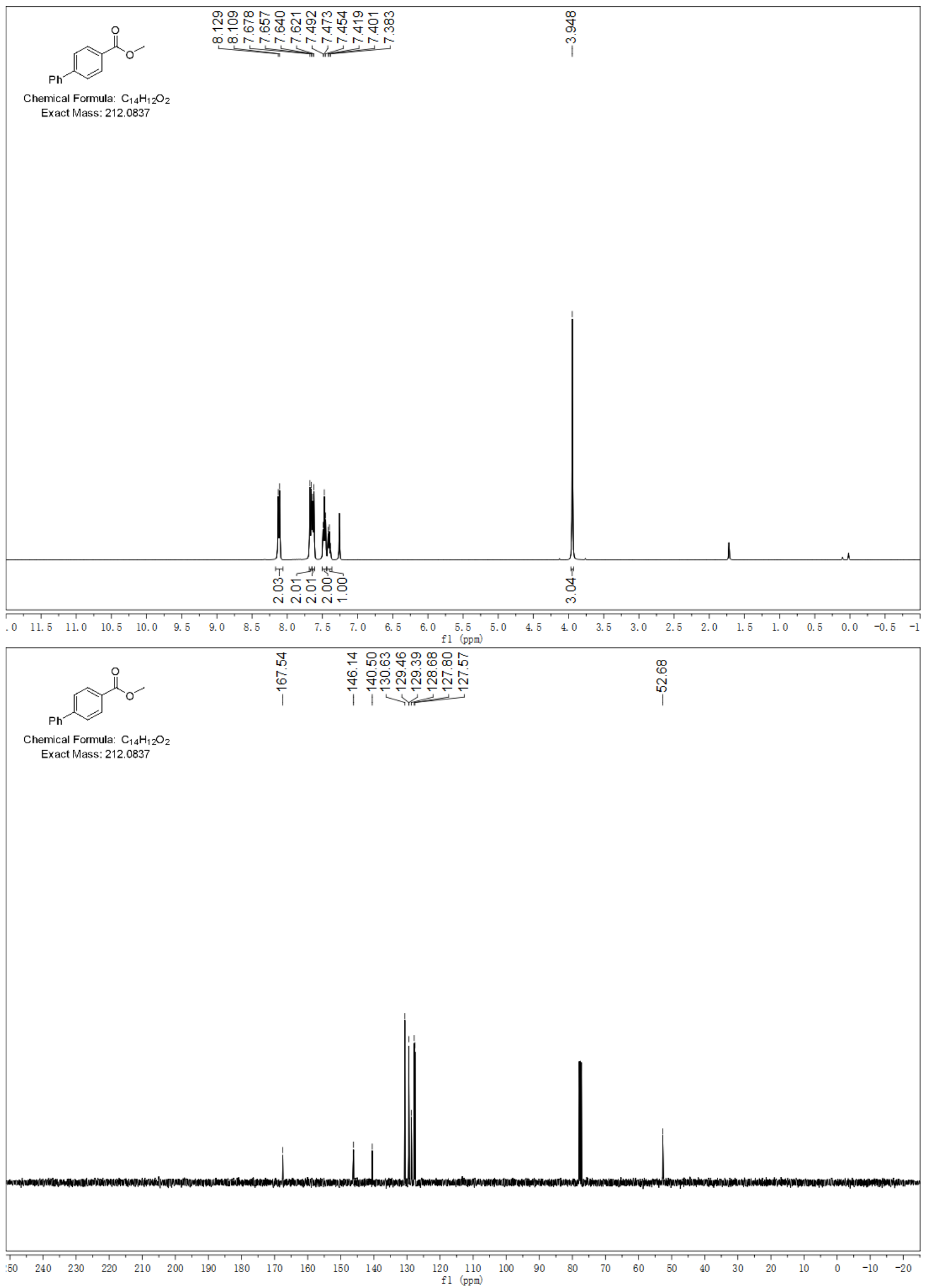


The nickel complex B1
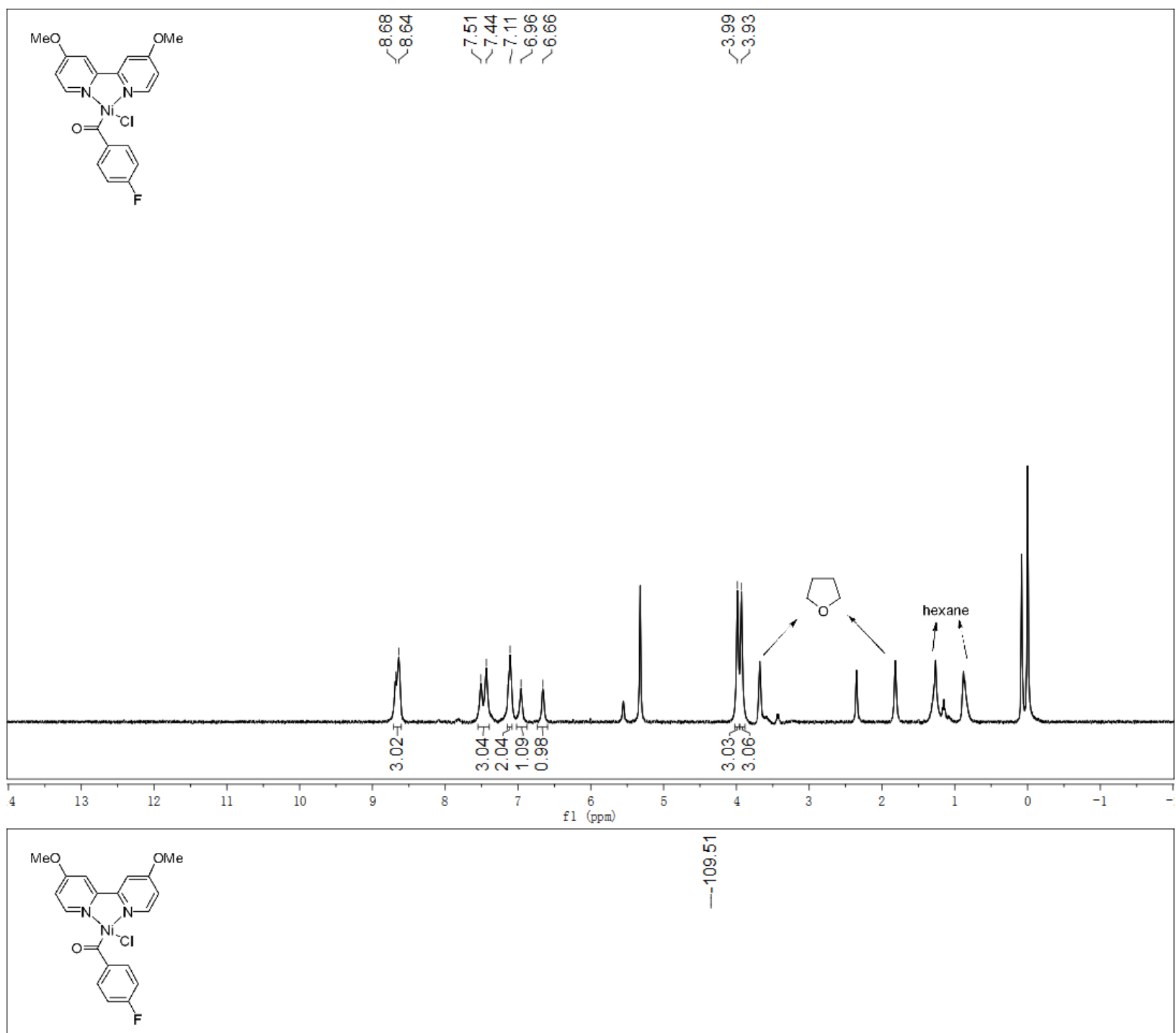

임

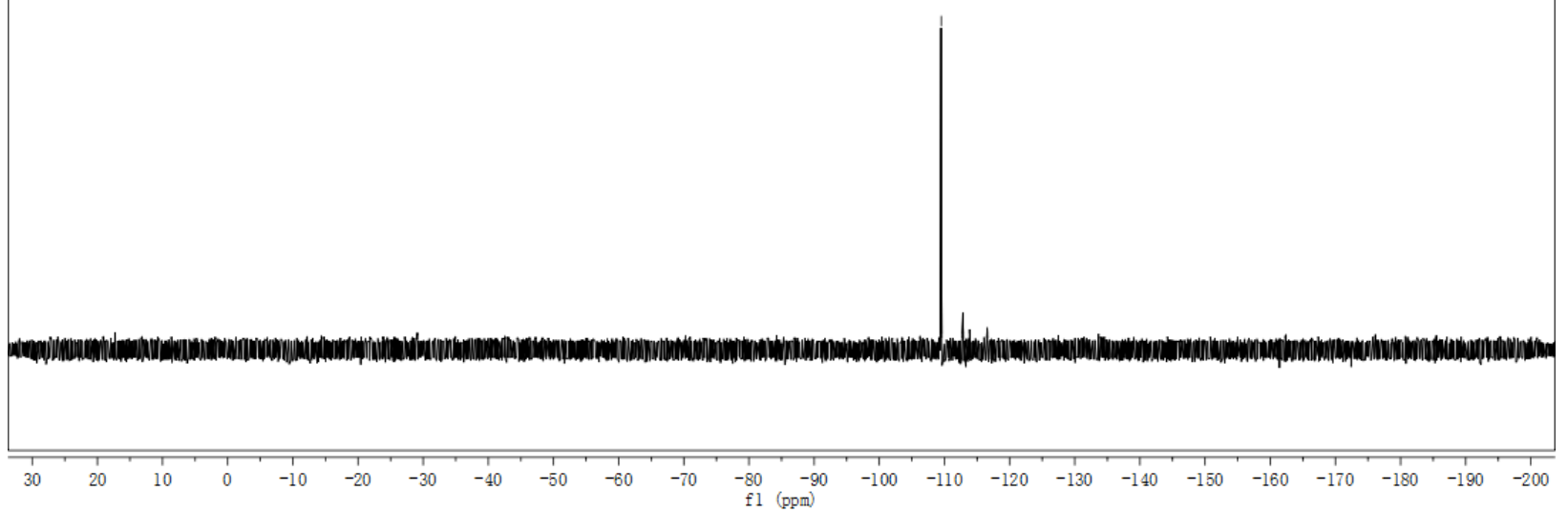

